Francisco Patrick Araujo Almeida

\title{
APLICAÇÃO DO ACOPLAMENTO ENTRE O MEC E O MEF PARA O ESTUDO DA INTERAÇÃO DINÂMICA ELASTOPLÁSTICA ENTRE O SOLO E ESTRUTURAS
}

Tese apresentada à Escola de Engenharia de São Carlos da Universidade de São Paulo, como parte dos requisitos para a obtenção do Título de Doutor em Engenharia de Estruturas.

Orientador: Prof. Dr. Humberto Breves Coda

São Carlos

2003 

A Renata e Rafael, Jesus e Irainê, Patricia e Bruna. 


\section{AGRADECIMENTOS}

Ao meu orientador e amigo, Prof. Humberto Breves Coda.

À Fundação de Amparo à Pesquisa do Estado de São Paulo - FAPESP, pelo financiamento da pesquisa.

À minha esposa Renata.

À minha família e à família da minha esposa.

Aos amigos do Departamento de Engenharia de Estruturas, em especial a Alex, Arthur, Daniel, Leandro, Luciano Barbosa (e Marilene), Marcelo Greco, Rodrigo Paccola, Silvana, Valério Almeida e Valério Souza.

Aos professores e funcionários do Departamento de Engenharia de Estruturas que contribuíram de alguma forma para a realização deste trabalho, em especial aos Professores João Batista de Paiva e Wilson Sérgio Venturini, e aos funcionários Masaki, Nadir e Rosi.

Aos Professores Euclides de Mesquita Neto e Leandro Palermo Júnior da Universidade Estadual de Campinas.

Aos amigos Charles, Célia e Marcinha. 
O que existe de mais importante neste mundo são as pessoas que nele habitam. 


\section{RESUMO}

ALMEIDA, F.P.A. (2003). Aplicação do acoplamento entre o MEC e o MEF para o estudo da interação dinâmica elastoplástica entre o solo e estruturas. 285p. Tese (Doutorado) - Escola de Engenharia de São Carlos, Universidade de São Paulo, São Carlos. 2003.

O objetivo do presente trabalho é o desenvolvimento de um código computacional que possibilite a análise dinâmica de estruturas tridimensionais em regime elástico-linear acopladas ao solo, tratado como meio infinito elastoplástico. As superestruturas são tratadas por elementos finitos simples de casca e de barra geral, as estruturas de fundações são tratadas por elementos de casca que simulam o contato com o solo, modelando radiers, túneis e reservatórios enterrados. Blocos são modelados por elementos de contorno tridimensionais. O solo é modelado de duas maneiras distintas: na região plastificada emprega-se a solução fundamental de Kelvin (estática) e na região não plastificada (elástica) adota-se a solução fundamental do problema de Stokes. O acoplamento entre os meios é feito aplicando-se a técnica de subregiões. Deve ficar claro que todo procedimento estático equivalente foi implementado. Vários exemplos numéricos são apresentados, onde se percebe a eficiência do código computacional desenvolvido.

Palavras-chave: interação solo-estrutura; Método dos Elementos de Contorno; Método dos Elementos Finitos; acoplamento MEC/MEF; dinâmica; nãolinearidade física 


\section{ABSTRACT}

ALMEIDA, F.P.A. (2003). BEM/FEM coupling application to the study of the elastoplastic dynamic interaction between soil and structures. 285p. Ph.D. Thesis - Escola de Engenharia de São Carlos, Universidade de São Paulo, São Carlos. 2003.

The objective of the present work is the development of a computational code that makes possible dynamic analyses of three-dimensional structures in elastic-linear behavior coupled to the soil, modeled as elastoplastic infinite medium. Simple finite elements, shell and general bars, are used to model elastic structures. The structures of foundations are modeled by shell's elements which simulate the contact with the soil, modeling radiers, tunnels and buried reservoirs. Blocks are modeled by three-dimensional boundary elements. The soil is modeled in two different ways: in the plastic region Kelvin's fundamental solution (static) is used and in the elastic region the fundamental solution of the Stoke's problem is adopted. The coupling among the media is done applying the sub-region technique. It is important to note that the equivalent static procedure has been implemented. Several numerical examples are presented, demonstrating the efficiency of the developed computational code.

Keywords: soil-structure interaction; Boundary Element Method; Finite Element Method; BEM/FEM coupling; dynamics; physical non-linearity 


\section{SUMÁRIO}

\section{RESUMO}

ABSTRACT

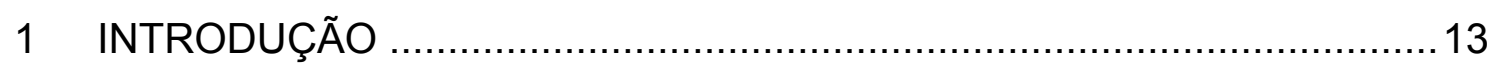

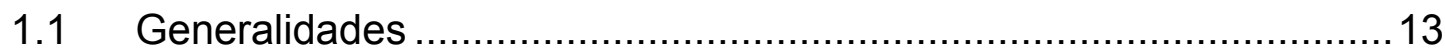

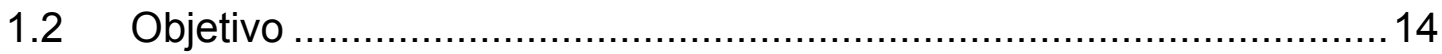

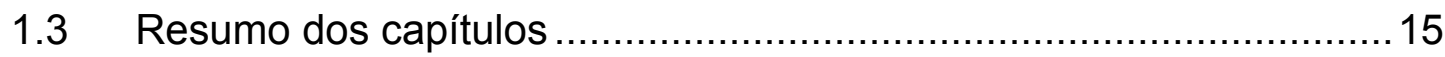

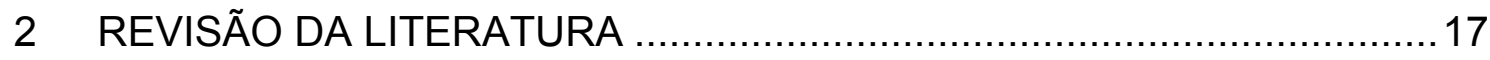

3 EQUAÇÕES INTEGRAIS BÁSICAS DA ELASTOPLASTICIDADE

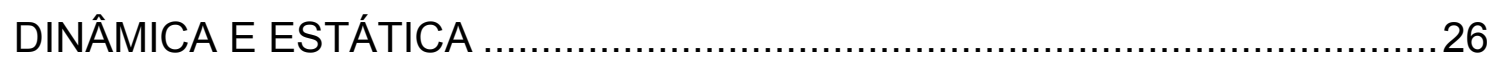

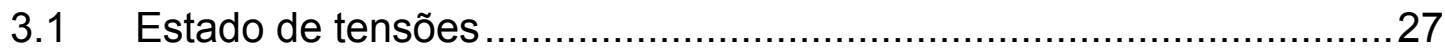

3.2 Estado de deformações lineares …………...................................29

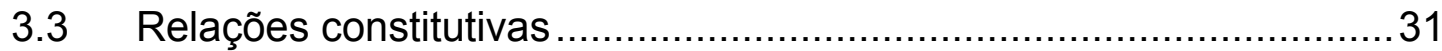

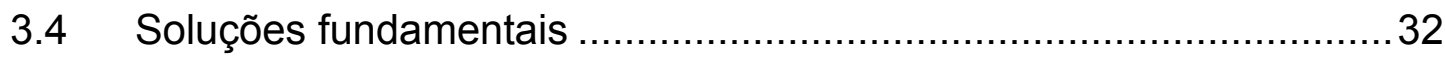

3.5 Equação integral de contorno para deslocamentos..............................34

3.6 Equação integral de contorno para tensões .....................................38

4 ANÁLISE DINÂMICA APLICANDO MATRIZ DE MASSA..........................42

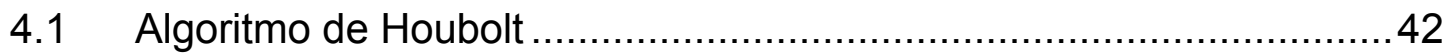

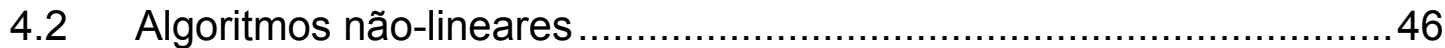

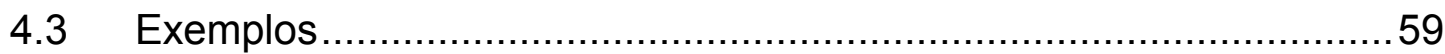

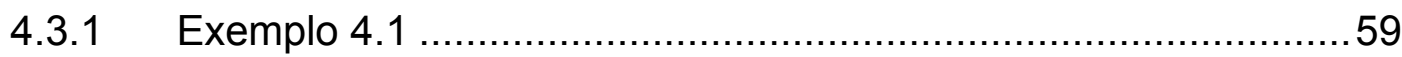

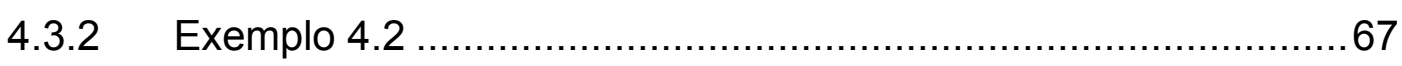

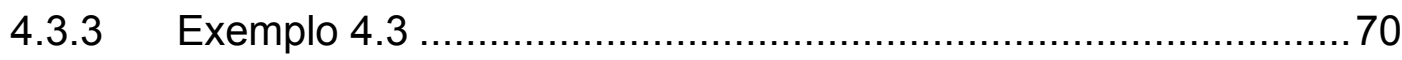

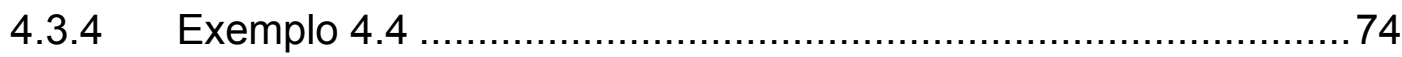

5 INTEGRAÇÕES PARA ELEMENTOS DE CONTORNO E CÉLULAS ......78

5.1 Integração singular para elementos de contorno................................78

5.2 Integração não-singular para elementos de contorno ……….............91

5.3 Integração quase-singular para elementos de contorno.......................93

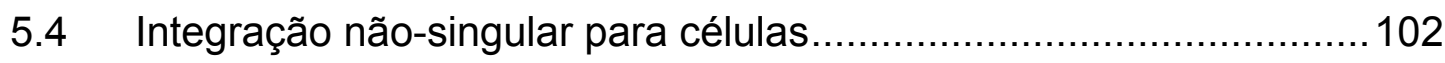

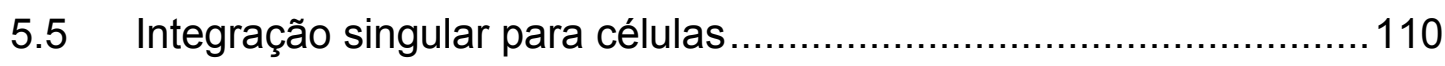

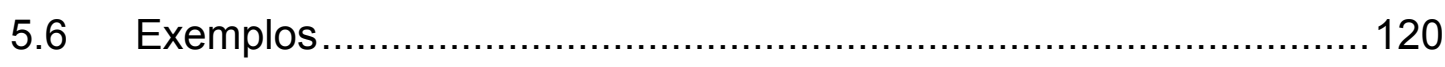




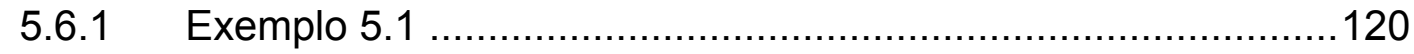

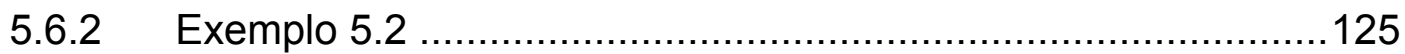

6 O MÉTODO DOS ELEMENTOS DE CONTORNO NO DOMÍNIO DO

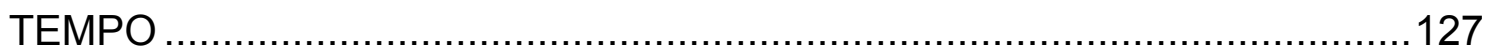

6.1 Equação integral de deslocamentos............................................128

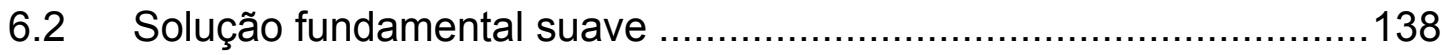

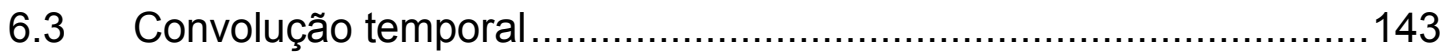

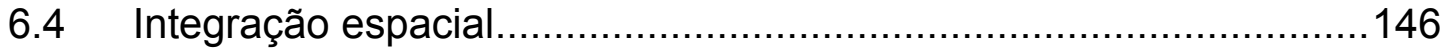

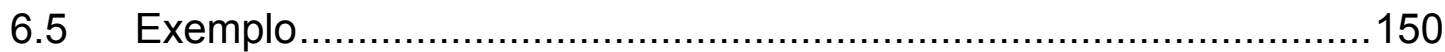

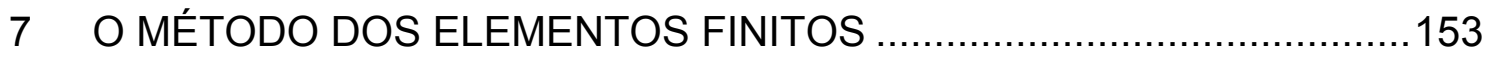

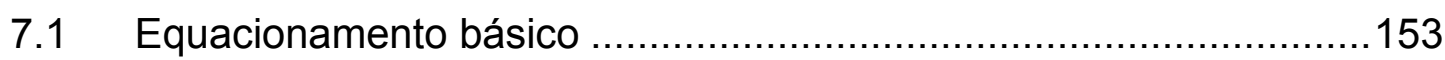

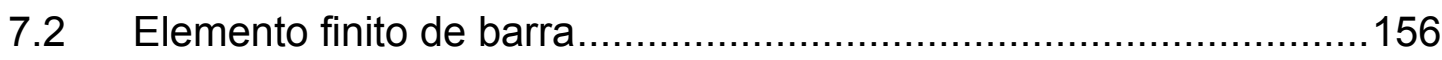

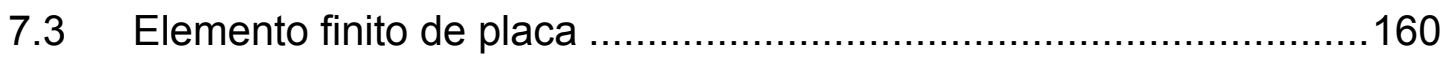

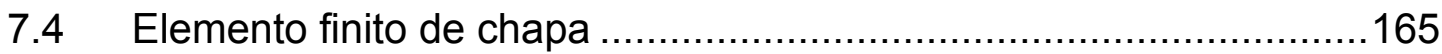

7.5 Algoritmo de Newmark $\beta$ para integração temporal .........................170

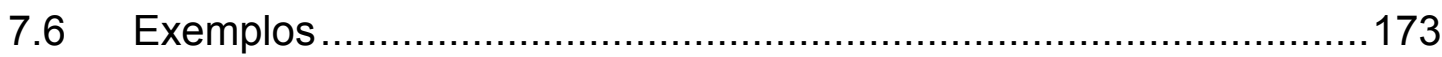

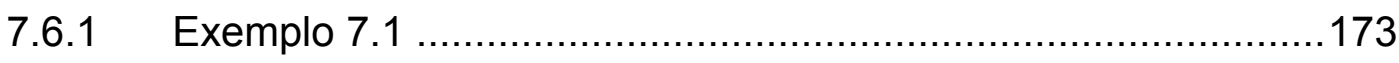

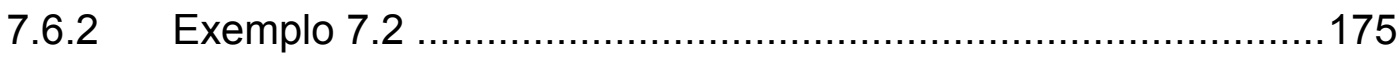

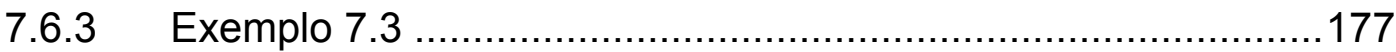

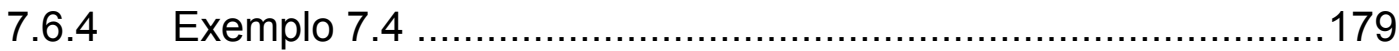

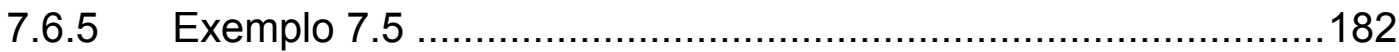

8 O ACOPLAMENTO ENTRE OS MÉTODOS ….......................................187

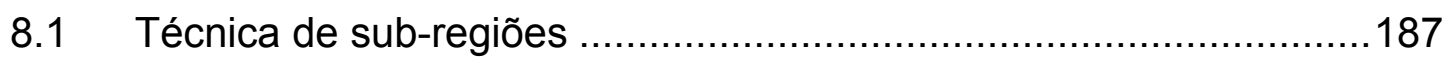

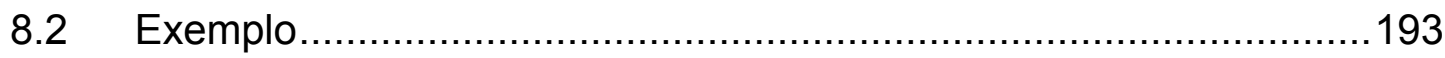

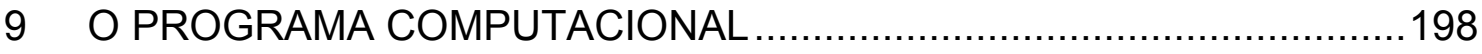

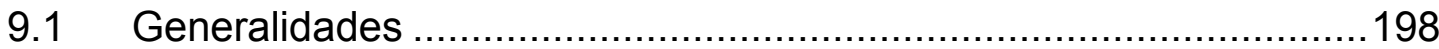

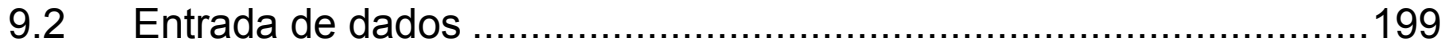

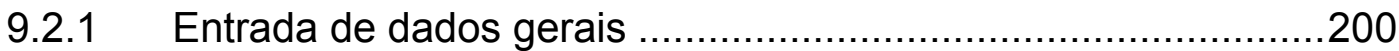

9.2.2 Entrada de dados do MEF.............................................201

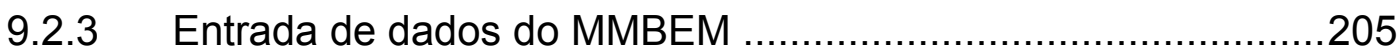

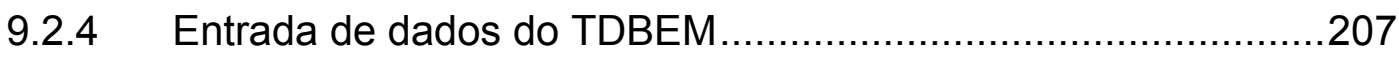

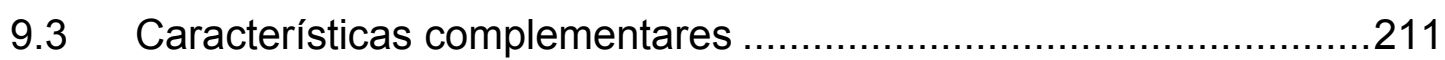

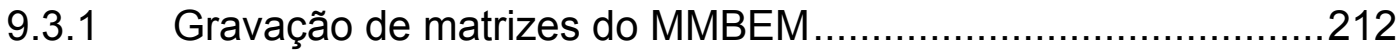

9.3.2 Solução do sistema de equações ...........................................212 
9.3.3 Carregamentos e condições de contorno dinâmicos ..................213

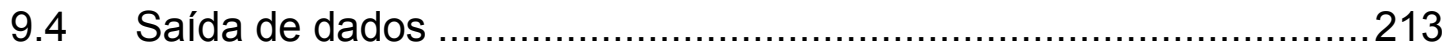

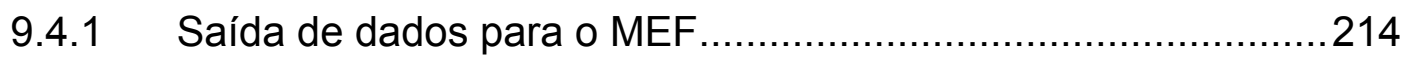

9.4.2 Saída de dados para o MMBEM .................................................. 214

9.4.3 Saída de dados para o TDBEM............................................215

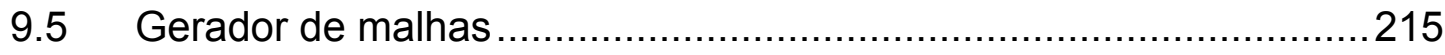

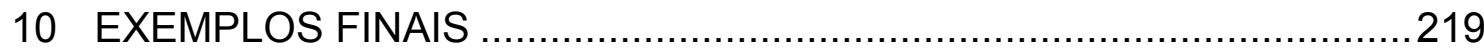

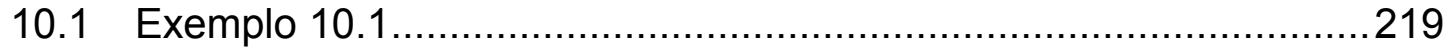

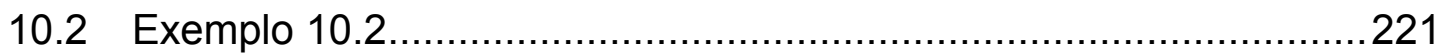

10.2.1 Placa quadrada sobre semiplano infinito.................................222

10.2.2 Placa quadrada com barra sobre semiplano infinito..................234

10.2.3 Reservatório sobre semiplano infinito ...................................240

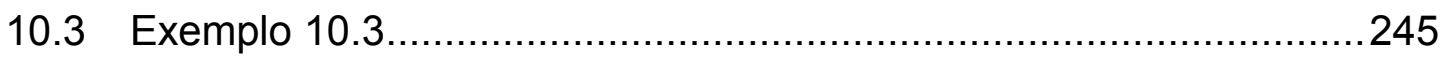

11 CONCLUSÃO

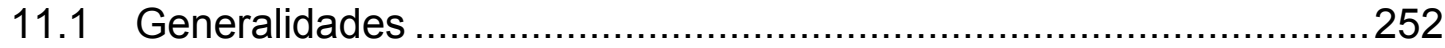

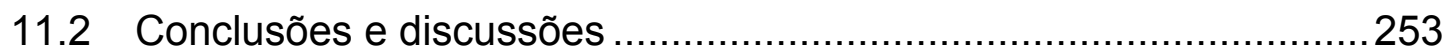

11.3 Sugestões para a continuação da pesquisa ...................................258

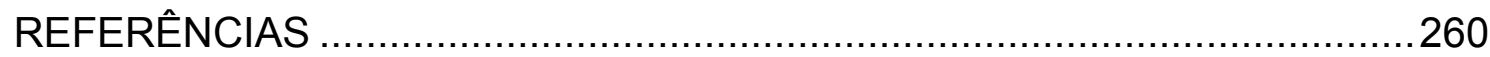

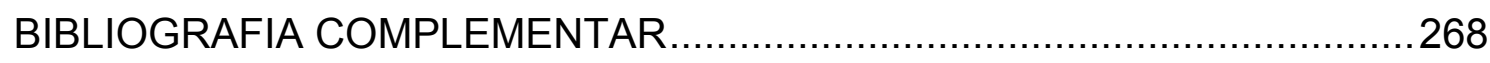

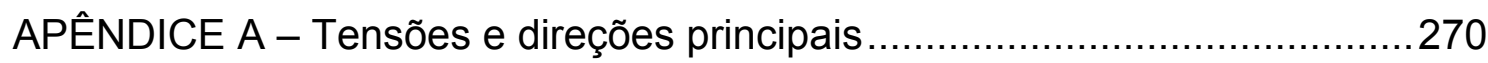

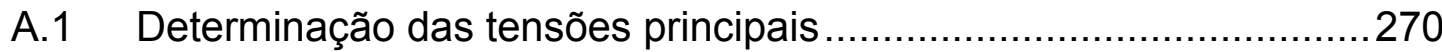

A.2 Determinação das direções principais ............................................272

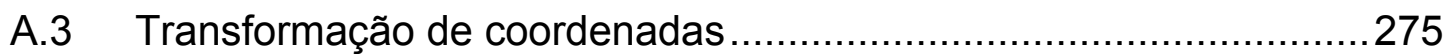

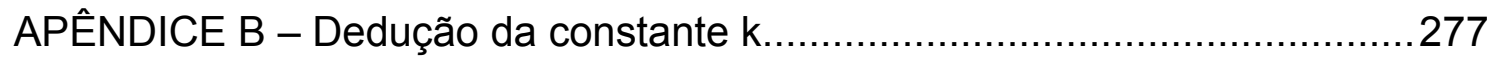

APÊNDICE C - Algoritmo para o cálculo da matriz de rigidez em coordenadas

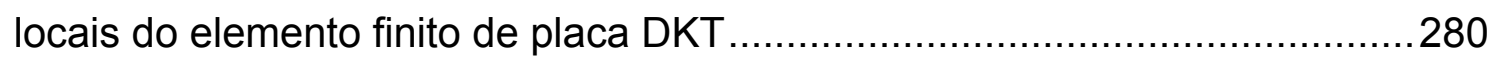
APÊNDICE D - Equacionamento para critério de plastificação de Drucker

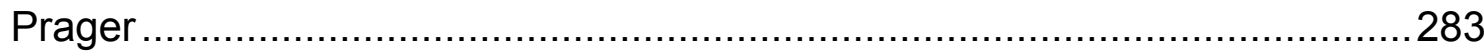




\section{INTRODUÇÃo}

\subsection{GENERALIDADES}

O Método dos Elementos Finitos (MEF) é, atualmente, uma das ferramentas mais difundidas para solucionar diversos problemas da engenharia. Sua aplicação na mecânica estrutural já está consagrada pela prática. (VENTURINI, 1988). É de se observar, contudo, que por ser uma técnica de domínio, traz algumas complicações em análises que envolvem domínios infinitos, pois estes devem ser interrompidos para que se gere uma discretização finita, ocasionando a formação de um contorno fictício que usualmente é eliminado com o uso do Método dos Elementos de Contorno (MEC). Tal problema se agrava ainda mais em análises dinâmicas para domínios infinitos, onde reflexões indesejadas podem ocorrer no final da discretização, ou esta deve ser estendida exaustivamente para comportar o tempo de análise desejado.

Pesquisadores dos mais importantes centros de pesquisa do mundo têm se interessado pelo Método dos Elementos de Contorno, tanto no desenvolvimento de formulações próprias para diversos problemas, como em gerar formulações mistas com o Método dos Elementos Finitos, aproveitando conjuntamente o que cada método tem de melhor. Tal interesse tem resultado em um enorme progresso do método. Praticamente todos os centros importantes de pesquisa dos países mais avançados têm grupos de pesquisadores dedicados ao desenvolvimento desta técnica.

Neste contexto, três vantagens principais são oferecidas pelo Método dos Elementos de Contorno: modelagem própria para domínios infinitos, grande redução do número de equações e volume de dados (principalmente 
quando se trata de meios tridimensionais infinitos como o solo) e a inexistência de erros de interpolação no domínio para problemas lineares.

Em contrapartida, o Método dos Elementos de Contorno não apresenta nenhuma característica vantajosa, no que diz respeito à análise de estruturas reticuladas e cascas gerais, quando comparado ao MEF. Disso se conclui que uma medida natural para a análise da interação solo-estrutura é a composição de uma modelagem onde o MEF é usado para a estrutura (pórticos e cascas) e o MEC para o solo ou semi-espaço infinito.

\subsection{OBJETIVO}

O principal objetivo do trabalho é o desenvolvimento de um código computacional que possibilite a análise dinâmica de estruturas tridimensionais em regime elástico-linear acopladas ao solo, que é tratado como não-linear. Todo o procedimento estático equivalente também foi implementado.

O solo é tratado como meio infinito elastoplástico. As superestruturas são tratadas por elementos finitos simples de casca e de barra geral, as estruturas de fundações são tratadas por elementos de casca que simulam o contato com o solo, modelando radiers e túneis. Blocos são modelados por elementos de contorno tridimensionais.

O solo é modelado de duas maneiras distintas, na região plastificada emprega-se a solução fundamental de Kelvin (estática) acompanhada de integrador temporal apropriado, dando origem ao chamado "non-linear mass matrix BEM' ou MEC não-linear dinâmico via matriz de massa. Na região não plastificada (elástica) adota-se a solução fundamental do problema de Stokes. O acoplamento entre os diferentes meios é feito aplicando-se a técnica de subregiões.

Como justificativas para a escolha do tema da tese, pode-se citar que enquanto os trabalhos voltados para plasticidade bidimensional, estática e dinâmica, através do MEC são abundantes na literatura, não existem muitas publicações disponíveis relativas à plasticidade tridimensional.

Além do mais, acredita-se que o desenvolvimento de código computacional para a simulação numérica tridimensional dinâmica de 
estruturas conectadas ao solo suposto elastoplástico seja de grande importância para a engenharia, pois atualmente não se dispõe de ferramenta tão geral para se fazer tal tipo de análise. Os programas com os quais se poderia tentar tais simulações estão elaborados em elementos finitos, o que limita muito o seu emprego devido ao volume de informações que se precisa gerar e aos problemas relacionados à simulação de meios infinitos ou semiinfinitos quando de análises dinâmicas envolvendo propagação de ondas.

Preocupou-se em desenvolver o código computacional de maneira bem documentada para que seja possível a utilização deste em pesquisas futuras.

O código gerado foi empregado em exemplos simples, para validação científica, e em exemplos mais elaborados, visando uma maior compreensão do comportamento de estruturas gerais (submetidas a ações estáticas e dinâmicas) acopladas ao solo.

Como contribuições do trabalho, pode-se citar: a utilização do algoritmo de Houbolt para a solução de problemas tridimensionais, o emprego do MMBEM para problemas elastoplásticos tridimensionais, os processos de integração para elementos de contorno e células, os estudos sobre a estabilização do acoplamento TDBEM/FEM e a utilização de integrais singulares no TDBEM.

\subsection{RESUMO DOS CAPÍTULOS}

No capítulo 1, apresenta-se o objetivo do trabalho, as justificativas para a escolha do tema da tese, bem como um breve resumo comparativo das técnicas empregadas e justificativas para a utilização das mesmas.

No capítulo 2, descrevem-se resumidamente as principais referências bibliográficas pesquisadas no trabalho, que serviram como base teórica para o mesmo.

No capítulo 3 são apresentadas as equações integrais básicas para a aplicação do Método dos Elementos de Contorno à solução do problema da elastoplasticidade dinâmica e estática para sólidos tridimensionais.

No capítulo 4, descreve-se uma formulação do MEC utilizando-se solução fundamental estática para a análise dinâmica tridimensional de meios 
contínuos, bem como os algoritmos utilizados no trabalho para a solução do problema não-linear. No final do capítulo são apresentados 4 exemplos numéricos com relação à formulação desenvolvida.

No capítulo 5 , os diversos processos de integração para os elementos de contorno, bem como as integrações das células tetraédricas, são apresentados. Dois exemplos numéricos são analisados no final do capítulo.

No capítulo 6, apresenta-se a formulação utilizada para o Método dos Elementos de Contorno no Domínio do Tempo (TDBEM), bem como um exemplo numérico sobre esta técnica.

No sétimo capítulo, apresenta-se toda a formulação utilizada no trabalho para o Método dos Elementos Finitos (MEF), bem como 5 exemplos numéricos, onde foram analisados casos estáticos e dinâmicos.

No capítulo 8, descreve-se a forma de acoplamento entre os diversos meios utilizada no trabalho, e apresenta-se um exemplo numérico da técnica empregada.

O capítulo nono tem como finalidade descrever as principais características do código computacional desenvolvido, em termos gerais, e suas sub-rotinas mais importantes, bem como comentar a respeito da entrada e saída de dados.

No capítulo 10 são apresentados os exemplos finais, que têm como intuito principal mostrar a aplicação dos desenvolvimentos descritos nos capítulos anteriores, bem como a potencialidade do código computacional elaborado na pesquisa.

No último capítulo são apresentadas as conclusões da presente tese de doutoramento e sugestões para a continuação da pesquisa. 


\section{REVISÃO DA LITERATURA}

O Método dos Elementos de Contorno tem sido largamente estudado nas últimas 3 décadas, tornando-se uma ferramenta confiável para a análise de diversos problemas da engenharia. Entre esses problemas, pode-se citar: elasticidade, plasticidade, propagação de ondas, mecânica da fratura, transferência de calor, interação solo-estrutura etc.

O presente trabalho trata da aplicação do acoplamento entre o Método dos Elementos de Contorno (MEC) e o Método dos Elementos Finitos (MEF) para a análise de superestruturas, consideradas em regime elástico-linear, conectadas ao solo, considerando sua não-linearidade física, no caso plasticidade.

Neste sentido, deve-se descrever a evolução do MEC para o tratamento de problemas elastoplásticos estáticos e dinâmicos, bem como o desenvolvimento do acoplamento entre o MEC e o MEF para a análise de estruturas acopladas. No final do capítulo, citam-se - mais resumidamente, uma vez que este não é o foco principal do trabalho - algumas referências a respeito do MEF, seja para a sua aplicação em análises elastostáticas, seja para a solução de problemas elastodinâmicos.

Diversos trabalhos podem ser citados com a utilização do MEC no campo da plasticidade bidimensional em análises estáticas. Banerjee e Cathie (1980) desenvolveram uma formulação direta do MEC com base na introdução de tensões iniciais que era mais geral que outras formulações existentes na época. Telles e Brebbia (1981a) apresentaram uma formulação própria para a plasticidade bidimensional via MEC, utilizando funções interpoladoras lineares para os elementos de contorno e células. Os mesmos autores, ainda em 1981, apresentaram a implementação completa da solução fundamental do 
semiplano (Melan) para a análise de problemas elastoplásticos (TELLES e BREBBIA, 1981b). Martín e Aliabadi (1998) apresentaram uma formulação de elementos de contorno geral para a análise de problemas de contato capaz de tratar efeitos elastoplásticos locais e de fricção. No Departamento de Engenharia de Estruturas da Escola de Engenharia de São Carlos (EESC), USP, os seguintes trabalhos podem ser citados: Venturini (1982), Chueiri (1994) e Fudoli (1999).

Já os trabalhos referentes à não-linearidade tratada pelo MEC no espaço tridimensional, em análises estáticas, não são numerosos. Pode-se citar o trabalho de Tejerina Calderón (1996), cujo objetivo principal foi apresentar fórmulas alternativas do MEC, utilizando-se sua formulação direta, para o estudo da interação de placas com o meio contínuo. A consideração da não-linearidade da reação do solo foi feita adotando-se um critério de plastificação simples e bilinear. No contexto internacional, Cisilino (1997) e Cisilino e Aliabadi (1999) estudaram o cálculo de fatores de intensidade de tensão para meios elastoplásticos tridimensionais utilizando-se células isoparamétricas quadráticas.

Os trabalhos que tratam dos problemas dinâmicos, lineares e nãolineares, pelo MEC, são abundantes na literatura; sendo que a maioria dos problemas não-lineares abordados está definida no espaço bidimensional.

Com relação aos trabalhos sobre análises dinâmicas lineares, Novak e El Hifnawy (1983) compararam dois métodos de avaliação dos efeitos no amortecimento de estruturas sobre fundações elásticas considerando-se a interação solo-estrutura. Os dois métodos citados são: consideração de energia e análise de autovalores complexos. Providakis e Beskos (1986) utilizaram o MEC direto para o estudo de vibrações livres ou forçadas de vigas. Spyrakos e Beskos (1986) apresentaram numericamente a resposta dinâmica de sapatas corridas rígidas sobre (ou parcialmente imersas em) um semi-espaço elástico, linear, isotrópico e homogêneo sob condições de deformação planas sujeitas a forças externas ou ondas sísmicas de incidência oblíqua com variação no tempo arbitrária. Beskos (1987) apresentou uma revisão sobre a utilização do MEC na solução numérica de problemas dinâmicos da elasticidade linear. Considera-se este artigo uma ótima fonte de referência. Em 1997, o mesmo autor (BESKOS, 1997) apresentou uma revisão sobre o MEC para a solução 
numérica de problemas elastodinâmicos referente ao período de 1986 a 1996 . Trata-se da continuação do artigo anterior. Carrer e Mansur (1999) escreveram um artigo referente ao desenvolvimento de equações integrais para a consideração de componentes de tensão e velocidade em análises elastodinâmicas transientes bidimensionais pelo MEC. Posteriormente, Adam, Pflanz e Schmid (2000) compararam duas aproximações para a análise de leito ferroviário (train-track embankment) em um semi-espaço sujeitos a cargas dinâmicas; e Tanrikulu, Yerli e Tanrikulu (2001) apresentaram uma formulação de elementos de contorno, com condições de contorno não-locais, para a análise dinâmica de um corpo com até três regiões, todas lineares.

Considerando o espaço tridimensional em análises dinâmicas lineares, Karabalis e Beskos (1984) apresentaram numericamente a resposta dinâmica de fundações de superfície rígidas tridimensionais de formato qualquer, sobre um semi-espaço homogêneo, isotrópico, elástico e linear representando o solo, sujeitas a forças dinâmicas externas ou ondas sísmicas de vários tipos e direções, com variação no tempo transiente. Os mesmos autores, em 1986, apresentaram os resultados de uma análise semelhante à anterior sendo que desta vez considerando as fundações imersas no semi-espaço, em Karabalis e Beskos (1986). Ahmad e Banerjee (1988b) apresentaram de uma forma mais completa e geral a implementação numérica da formulação direta de elementos de contorno para análises transientes no domínio do tempo de sólidos tridimensionais. Triantafyllidis (1991) apresentou um método direto dos elementos de contorno utilizando as funções de Green em um semi-espaço para a solução de problemas transientes elastodinâmicos tridimensionais no domínio do tempo. Trata-se do primeiro artigo a abordar a análise dinâmica transiente pelo MEC no domínio do tempo utilizando funções de Green em um semi-espaço. Guan e Novak (1994) apresentaram uma solução fechada de resposta transiente para carregamento subitamente aplicado distribuído sobre uma área retangular na superfície de um semi-espaço homogêneo elástico. Tal solução pode ser utilizada para fins especiais como análise dinâmica da interação solo-estrutura ou problemas de contato. Stamos e Beskos (1995) apresentaram resumidamente uma revisão bibliográfica atualizada sobre a dinâmica de estruturas de fundação e, principalmente, estudaram a determinação numérica da resposta dinâmica de estruturas de fundação 
tridimensionais, largas e sujeitas a forças dinâmicas internas ou externas, ou ondas sísmicas, pelo MEC no domínio da freqüência. Tanto a estrutura quanto o solo, considerados em regime elástico-linear ou viscoelástico, foram modelados pelo MEC. Em direção semelhante, podem ser citados os trabalhos de Mesquita Neto e seus orientados: Adolph (2002), Barros e Mesquita (1999), Barros (2001), Mesquita (1989), Mesquita, Adolph e Romanini (2002) e Mesquita e Romanini (1992). Os trabalhos descritos anteriormente, que trataram de problemas transientes lineares, utilizaram soluções fundamentais no domínio do tempo, não sendo necessária a utilização de células para a consideração de forças inerciais. Porém, raros são os trabalhos que utilizando soluções fundamentais transientes considerem não-linearidade. Dentre esses, pode-se citar: Telles, Carrer e Mansur (1999), para problemas bidimensionais, e no espaço tridimensional, Pan, Okada e Atluri (1994). Deve-se mencionar que esse procedimento ainda é muito instável para ser adotado como ferramenta geral de análise não-linear dinâmica.

No Departamento de Engenharia de Estruturas da EESC, no que se refere à utilização do MEC em análises dinâmicas lineares, alguns trabalhos podem ser referenciados. No espaço bidimensional, o trabalho de Coda (1990) teve como objetivo o estudo do problema da vibração livre utilizando-se a formulação direta do MEC, onde a solução fundamental de Kelvin foi empregada. No espaço tridimensional, Coda e Venturini (1995a) desenvolveram uma formulação de elementos de contorno para a análise elastodinâmica transiente empregando-se pontos de colocação externos. A vantagem desse processo é dada pela possibilidade de se evitar a avaliação direta ou indireta de integrais singulares sobre os elementos que contém os pontos fonte. Coda e Venturini (1996a) estudaram a formulação dos elementos de contorno transiente tridimensional juntamente com uma solução fundamental dependente do tempo modificada, obtida com um impulso unitário assumido distribuído sobre um intervalo de tempo. Os mesmos autores, em Coda e Venturini (1996b), compararam essa solução fundamental alternativa com a solução fundamental clássica. Barbirato (1999) desenvolveu uma formulação do MEC para a análise de problemas tridimensionais de fraturamento no regime transiente utilizando solução fundamental estática. 
Os trabalhos sobre análises dinâmicas não-lineares bidimensionais também são abundantes. Porém, utilizam-se de soluções fundamentais estáticas, resultando na integração de células para a consideração das forças inerciais. Kontoni e Beskos (1988) forneceram a formulação completa do MEC direto para a análise dinâmica transiente de sistemas gerais de materiais com comportamento não-linear; porém apenas o equacionamento foi apresentado, sem exemplos numéricos. Carrer e Telles (1992) apresentaram a análise elastoplástica dinâmica transiente bidimensional utilizando-se uma solução fundamental elastostática simples. Utilizou-se o algoritmo de Houbolt para a integração temporal, obtendo-se bons resultados. Israil e Banerjee (1992) apresentaram a formulação e implementação numérica da elastoplasticidade dinâmica transiente bidimensional utilizando-se o MEC. Kontoni e Beskos (1993) apresentaram a técnica da reciprocidade dual para o MEC na análise dinâmica transiente de estruturas em regime elastoplástico. Utilizou-se a solução fundamental elastostática de Kelvin. Os termos inerciais, que são responsáveis pelo aparecimento de integrais de domínio na equação integral, foram transformados para o contorno, resultando em matriz de massa. Alguns algoritmos de integração temporal foram testados, e o algoritmo de Houbolt foi escolhido como o mais adequado. Panzeca, Polizzotto e Zito (1994) empregaram uma formulação do MEC na análise dinâmica de problemas elastoplásticos. Os objetivos do trabalho de Frangi e Maier (1999) são uma primeira investigação do MEC por Galerkin simétrico, com núcleos estáticos, na elastoplasticidade dinâmica, e o desenvolvimento de uma base computacional teórica do método. Telles e Carrer (1994) estudaram a aplicação de técnicas implícitas na solução de problemas elastoplásticos dinâmicos transientes e estáticos. A integral de domínio inercial, que aparece graças à utilização da solução fundamental estática, foi mantida. O algoritmo de Houlbolt foi utilizado. No Departamento de Engenharia de Estruturas da EESC-USP, Coda e Venturini (2000) aplicaram uma formulação dos elementos de contorno elastoplástica na resolução de problemas dinâmicos transientes. Utilizaram-se células para a aproximação de variáveis de domínio. A solução fundamental de Kelvin (estática) foi utilizada na representação integral. Os termos de velocidade e aceleração que aparecem na aproximação do MEC com matriz de massa não-linear foram tratados de maneira mais completa. 
Por fim, o acoplamento entre o MEC e o MEF, que também é utilizado no trabalho, mostra-se como uma técnica já bastante empregada na solução de diversos problemas de engenharia, como plasticidade em análises estáticas, análises dinâmicas lineares e não-lineares e, principalmente, problemas de interação solo-estrutura, ou estrutura e meio contínuo. Deve-se mencionar que em quase todas as referências a plasticidade é considerada na parte modelada pelo MEF. O primeiro trabalho sobre o acoplamento entre o MEC e o MEF foi o de Zienkiewicz, Kelly e Bettess (1977), apud Belytschko, Chang e Lu (1989). Nesse trabalho, os autores apresentam o método das soluções de contorno utilizado no contexto convencional do MEF. Laethem et al. (1984) descreveram uma avaliação estática linear computacional de estruturas de fundações. Von Estorff e Kausel (1989) e Von Estorff (1991) estudaram o comportamento dinâmico linear de blocos envolvidos por um meio sólido infinito e sujeito a carregamentos transientes verticais e horizontais, onde o MEF foi usado para modelar o bloco, enquanto que o MEC no domínio do tempo foi empregado para representar o meio sólido infinito. Pan, Okada e Atluri (1994) analisaram dinamicamente um pavimento em regime elástico-linear sobre um meio sólido elastoplástico, sujeito a uma carga móvel. Utilizou-se o MEF para a análise do pavimento e o MEC não-linear, com solução fundamental no domínio do tempo (Stokes), para a análise do meio sólido. O algoritmo de Newmark $\beta$ foi empregado para a integração temporal para o MEF. Pavlatos e Beskos (1994) desenvolveram um esquema de acoplamento no domínio do tempo para a análise dinâmica de estruturas elastoplásticas sob condições de deformações planas ou tensões planas. O MEF foi utilizado para a discretização da região de plastificação, enquanto que o MEC foi utilizado para a discretização do domínio da região elástica. A integração no tempo foi feita com a utilização do algoritmo de Newmark. Wearing e Burstow (1994) estudaram uma combinação entre o MEC e o MEF para a análise bidimensional de tensões elastoplásticas e de problemas da mecânica da fratura elastoplástica bidimensional. O MEF foi utilizado para a análise da região de plastificação, enquanto que o MEC foi utilizado para a análise da região elástica. Mais recentemente, Yu et al. (2001) utilizaram um método alternativo, chamado linear $\theta$, para melhorar a estabilidade da formulação do acoplamento linear MEC/MEF no domínio do tempo. 
Dentro do Departamento de Engenharia de Estruturas da EESC-USP, Coda (1993) estudou o problema da elastodinâmica transiente tridimensional através da formulação mista do MEF e do MEC, especificamente à ligação entre o solo e estruturas. Também no espaço tridimensional, Coda e Venturini (1995b) exploraram o acoplamento entre o MEC e o MEF no estudo de estruturas de edifícios interagindo com suas fundações. Derivou-se uma solução fundamental modificada no domínio do tempo com o intuito de melhorar a estabilidade do algoritmo numérico desenvolvido. Coda e Venturini (1999a) apresentaram o acoplamento entre estruturas de pórticos, discretizadas por elementos finitos, e corpos tridimensionais tratados pelo MEC. Coda e Venturini (1999b) apresentaram uma formulação do MEC transiente bidimensional com base na aproximação de matriz de massa, onde a formulação implícita do método para a consideração de análise elastoplástica é considerada, bem como os efeitos de amortecimento viscoso. Os processos de integração no tempo foram feitos com base nos algoritmos de Newmark $\beta$ e Houbolt. A integração do domínio para massa, efeitos de amortecimento e elastoplasticidade foi tratada pela técnica de aproximação por células. Coda, Venturini e Aliabadi (1999) apresentaram um procedimento para o acoplamento geral de modelos de elementos finitos (cascas, placas e pórticos) com corpos tridimensionais modelados pelo MEC para a análise de problemas estáticos e dinâmicos. Coda $(2000,2001)$ estudou o MEC, e sua combinação com o MEF, aplicado aos problemas dinâmicos transientes de sólidos contínuos, elásticos ou inelásticos, na análise estrutural.

Além desses trabalhos relacionados com análises dinâmicas, pode-se citar, entre outros, as contribuições de Matos Filho (1999), Mendonça (1997) e Mendonça e Paiva (2000) relacionados com o acoplamento de estacas-solo ou radiers, estaqueados ou não com o solo, também desenvolvidos no Departamento de Engenharia de Estruturas da EESC-USP.

Com relação ao emprego do MEF no estudo de problemas elastostáticos, Batoz, Bathe e Ho (1980) apresentaram os resultados do estudo teórico, numérico e detalhado de elementos de placa com 9 graus de liberdade, sendo 3 por vértice (translação em z $(w)$ e rotações em $x\left(\theta_{x}\right)$ e y $\left(\theta_{y}\right)$ ), onde o objetivo desse estudo foi identificar ou desenvolver um elemento ótimo para a análise linear geral de problemas de placas. Foi dada especial atenção à 
formulação teórica e avaliação numérica de 3 elementos finitos: o DKT (Discrete Kirchhoff Triangle), o HSM (Hybrid Stress Model) e o SRI (Selective Reduced Integration), onde se utilizou o programa de computador ADINA (Automatic Dynamic Incremental Nonlinear Analysis), apresentado por Bathe (1975), no teste desses elementos. Os autores concluíram que os mais eficientes e confiáveis elementos triangulares de placa dentre os estudados eram o DKT e o HSM. Os resultados obtidos com o elemento SRI mostraram que considerando uma placa grossa, o elemento converge para a solução de placas grossas, mas que o mesmo não acontece em se tratando de placas delgadas. Assim, pôde-se dizer que o elemento com integração seletiva reduzida (SRI) não é eficiente quando comparado com os elementos DKT e HSM. No trabalho de Jeyachandrabose, Kirkhope e Ramesh Babu (1985), a matriz de rigidez do elemento finito DKT foi formulada explicitamente em um sistema de coordenadas globais, onde no final do artigo se apresentou uma listagem do respectivo algoritmo. Assan (1999) apresentou com detalhes a formulação clássica para elementos finitos bidimensionais planos, entre eles o CST (Constant Strain Triangle). No livro são apresentados também vários exemplos comparativos e benchmarks. O autor também gostaria de citar nesta revisão suas publicações a respeito do MEF - inclusive sua dissertação de mestrado - (ALMEIDA, 1999; ALMEIDA e ANTUNES, 2000; ANTUNES e ALMEIDA, 1999), onde os elementos finitos de placa DKT e P15N foram empregados na modelagem de lajes que, juntamente com elementos de barras para a discretização de vigas, foram utilizadas para a análise elastostática de pavimentos de edifícios.

O MEF aplicado a análises elastodinâmicas também foi estudado no Departamento de Engenharia de Estruturas da EESC-USP, entre outros, nos trabalhos de Coda (1993) e Coda (2000), já descritos anteriormente.

Uma análise crítica desta revisão bibliográfica revela que a área de estruturas da EESC-USP tem avançado bastante nos modelos dinâmicos nãolineares, via MEC e MEF, e no acoplamento entre o MEC e o MEF para a análise de estruturas interligadas. Deve-se salientar que os trabalhos desenvolvidos no departamento se inserem no contexto internacional de forma atual e inovadora. O trabalho desenvolvido no presente doutoramento é mais um passo na ampliação dos limites do conhecimento na área, garantindo a 
qualidade desta linha de pesquisa na área de estruturas. Além disso, os passos que foram seguidos foram dados com pleno conhecimento dos caminhos mais adequados, tendo em vista os desenvolvimentos internacionais sobre o assunto. 


\section{EQUAÇÕES INTEGRAIS BÁSICAS DA ELASTOPLASTICIDADE DINÂMICA E ESTÁTICA}

O surgimento do Método dos Elementos de Contorno (MEC) como uma alternativa para a resolução de diversos problemas da engenharia é um dos grandes avanços científicos nessa área do conhecimento dos últimos anos. Uma das principais vantagens deste método, para problemas lineares, quando comparado com os métodos de domínio usuais (como por exemplo o Método dos Elementos Finitos (MEF)) é a redução do número de variáveis do problema, pois enquanto nos métodos usuais o domínio a ser tratado precisa ser dividido em vários subdomínios, no Método dos Elementos de Contorno apenas o contorno do mesmo precisa ser discretizado. Além disso, para alguns problemas, já é comprovado que o MEC apresenta respostas mais precisas e confiáveis que os métodos tradicionalmente empregados para análises similares.

Neste capítulo serão apresentadas as equações integrais básicas para a aplicação do Método dos Elementos de Contorno à solução do problema da elastoplasticidade dinâmica para sólidos tridimensionais, sendo o caso estático uma particularização deste. Vale dizer que o desenvolvimento do presente capítulo foi feito principalmente com base em Brebbia e Dominguez (1992) e Coda (2000). 


\subsection{ESTADO DE TENSÕES}

O estado de tensões em um ponto de um corpo pode ser representado, em termos de suas componentes de tensão, pelo tensor de tensões escrito abaixo:

$$
\left[\begin{array}{lll}
\sigma_{11} & \sigma_{12} & \sigma_{13} \\
\sigma_{21} & \sigma_{22} & \sigma_{23} \\
\sigma_{31} & \sigma_{32} & \sigma_{33}
\end{array}\right]=[\sigma]
$$

Ver figura 3.1.

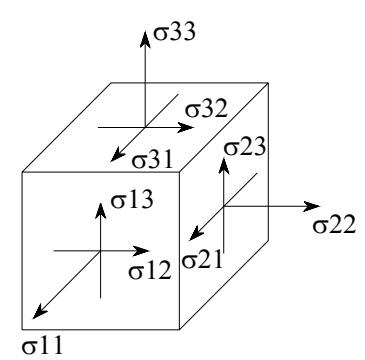

Tensões

$$
\wedge^{\mathrm{x} 3, \mathrm{u} 3}
$$

0

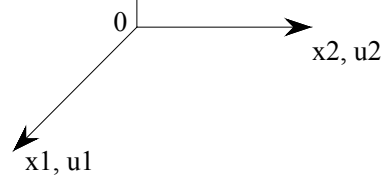

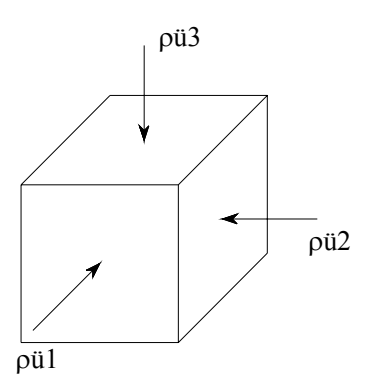

Forças Inerciais

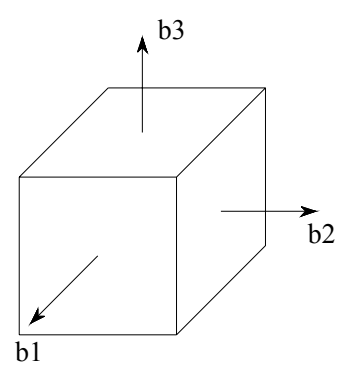

Forças Volumétricas

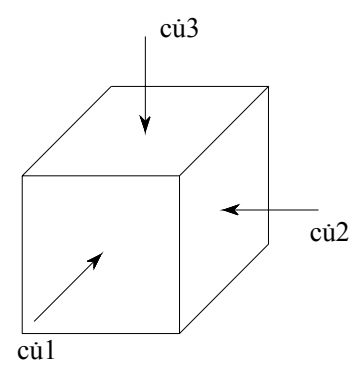

Forças de Amortecimento

Figura 3.1 - Estado de tensões

As componentes de tensão são relacionadas entre si através das equações de equilíbrio de momento, ou teorema de Cauchy, tal como:

$$
\begin{aligned}
& \sigma_{12}=\sigma_{21} \\
& \sigma_{13}=\sigma_{31} \\
& \sigma_{23}=\sigma_{32}
\end{aligned}
$$


Levando-se em consideração a variação das tensões com relação às coordenadas cartesianas, podem ser escritas as seguintes equações diferenciais de equilíbrio:

$$
\begin{aligned}
& \frac{\partial \sigma_{11}}{\partial x_{1}}+\frac{\partial \sigma_{21}}{\partial x_{2}}+\frac{\partial \sigma_{31}}{\partial x_{3}}+b_{1}=\rho \ddot{u}_{1}+c \dot{u}_{1} \\
& \frac{\partial \sigma_{12}}{\partial x_{1}}+\frac{\partial \sigma_{22}}{\partial x_{2}}+\frac{\partial \sigma_{32}}{\partial x_{3}}+b_{2}=\rho \ddot{u}_{2}+c \dot{u}_{2} \\
& \frac{\partial \sigma_{13}}{\partial x_{1}}+\frac{\partial \sigma_{23}}{\partial x_{2}}+\frac{\partial \sigma_{33}}{\partial x_{3}}+b_{3}=\rho \ddot{u}_{3}+c \dot{u}_{3}
\end{aligned}
$$

As eq.(3.5), (3.6) e (3.7) são conhecidas como equações de equilíbrio de forças e são necessariamente satisfeitas no domínio do corpo $(\Omega)$. Utilizandose a notação indicial e as eq.(3.2), (3.3) e (3.4), pode-se escrever:

$$
\frac{\partial \sigma_{i j}}{\partial x_{j}}+b_{i}=\rho \ddot{u}_{i}+c \dot{u}_{i} \quad \text { em } \Omega \quad i, j=1,2,3
$$

ou

$$
\sigma_{i j, j}+b_{i}=\rho \ddot{u}_{i}+c \dot{u}_{i} \quad \text { em } \Omega \quad i, j=1,2,3
$$

Para o caso elastostático, as forças inerciais e de amortecimento não são consideradas, assim a eq.(3.9) pode ser escrita como:

$$
\sigma_{i j, j}+b_{i}=0 \quad \text { em } \Omega \quad i, j=1,2,3
$$

As forças de superfície ' $p_{i}$ ' podem ser escritas da seguinte forma:

$$
\begin{aligned}
& p_{1}=\sigma_{11} n_{1}+\sigma_{12} n_{2}+\sigma_{13} n_{3} \\
& p_{2}=\sigma_{21} n_{1}+\sigma_{22} n_{2}+\sigma_{23} n_{3} \\
& p_{3}=\sigma_{31} n_{1}+\sigma_{32} n_{2}+\sigma_{33} n_{3}
\end{aligned}
$$


onde os ' $\mathrm{n}_{\mathrm{i}}$ ' são os co-senos diretores da direção normal ' $\vec{n}$ ' com relação aos eixos ' $x_{i}$ ', ou seja:

$$
\begin{aligned}
& \mathrm{n}_{1}=\cos \left(\mathrm{n}, \mathrm{x}_{1}\right) \\
& \mathrm{n}_{2}=\cos \left(\mathrm{n}, \mathrm{x}_{2}\right) \\
& \mathrm{n}_{3}=\cos \left(\mathrm{n}, \mathrm{x}_{3}\right)
\end{aligned}
$$

Indicialmente, podem-se escrever as eq.(3.11), (3.12) e (3.13) como:

$\mathrm{p}_{\mathrm{i}}=\sigma_{\mathrm{ij}} \mathrm{n}_{\mathrm{j}} \quad$ em $\Gamma$

onde $\Gamma$ representa o contorno do meio.

As condições de contorno naturais podem ser escritas indicialmente como:

$$
\mathrm{p}_{\mathrm{i}}=\overline{\mathrm{p}}_{\mathrm{i}} \quad \text { em } \Gamma_{2} \quad \mathrm{i}=1,2,3
$$

ou

$$
p_{i}=\sigma_{i j} n_{j}=\bar{p}_{i} \quad \text { em } \Gamma_{2} \quad i, j=1,2,3
$$

onde os $\overline{p_{i}}$ são os valores prescritos das forças de superfície.

\subsection{ESTADO DE DEFORMAÇÕES LINEARES}

As componentes de deformação podem ser divididas em:

Deformações longitudinais: 


$$
\begin{aligned}
& \varepsilon_{11}=\frac{\partial u_{1}}{\partial x_{1}} \\
& \varepsilon_{22}=\frac{\partial u_{2}}{\partial x_{2}} \\
& \varepsilon_{33}=\frac{\partial u_{3}}{\partial x_{3}}
\end{aligned}
$$

Distorções:

$$
\begin{aligned}
& \varepsilon_{12}=\frac{1}{2}\left(\frac{\partial \mathrm{u}_{1}}{\partial \mathrm{x}_{2}}+\frac{\partial \mathrm{u}_{2}}{\partial \mathrm{x}_{1}}\right) \\
& \varepsilon_{13}=\frac{1}{2}\left(\frac{\partial \mathrm{u}_{1}}{\partial \mathrm{x}_{3}}+\frac{\partial \mathrm{u}_{3}}{\partial \mathrm{x}_{1}}\right) \\
& \varepsilon_{23}=\frac{1}{2}\left(\frac{\partial \mathrm{u}_{2}}{\partial \mathrm{x}_{3}}+\frac{\partial \mathrm{u}_{3}}{\partial \mathrm{x}_{2}}\right)
\end{aligned}
$$

Indicialmente, pode-se escrever:

$$
\varepsilon_{\mathrm{ij}}=\frac{1}{2}\left(\mathrm{u}_{\mathrm{i}, \mathrm{j}}+\mathrm{u}_{\mathrm{j}, \mathrm{i}}\right) \quad \mathrm{i}, \mathrm{j}=1,2,3
$$

Utilizando-se as componentes de deformação, pode-se escrever o tensor de deformações, já considerando a simetria deste tensor, da seguinte forma:

$$
\left[\begin{array}{lll}
\varepsilon_{11} & \varepsilon_{12} & \varepsilon_{13} \\
\varepsilon_{12} & \varepsilon_{22} & \varepsilon_{23} \\
\varepsilon_{13} & \varepsilon_{23} & \varepsilon_{33}
\end{array}\right]=[\varepsilon]
$$

As condições de contorno essenciais podem ser escritas da seguinte forma:

$$
\mathrm{u}_{\mathrm{j}}=\overline{\mathrm{u}}_{\mathrm{j}} \quad \text { em } \Gamma_{1} \quad \mathrm{j}=1,2,3
$$


onde os $\overline{\mathrm{u}}_{\mathrm{j}}$ são os valores prescritos dos deslocamentos e $\Gamma_{1}+\Gamma_{2}=\Gamma$.

\subsection{RELAÇÕES CONSTITUTIVAS}

As relações constitutivas são aquelas que relacionam as componentes de tensão com as componentes de deformação.

Utilizando-se as constantes de Lamé ( $\lambda$ e $\mu$ ), essas relações podem ser escritas das seguintes formas:

$$
\begin{aligned}
& \sigma_{\mathrm{ij}}=\lambda \delta_{\mathrm{ij}} \varepsilon_{\mathrm{kk}}+2 \mu \varepsilon_{\mathrm{ij}} \\
& \varepsilon_{\mathrm{ij}}=-\frac{\lambda \delta_{\mathrm{ij}}}{2 \mu(3 \lambda+2 \mu)} \sigma_{\mathrm{kk}}+\frac{1}{2 \mu} \sigma_{\mathrm{ij}}
\end{aligned}
$$

onde:

$$
\begin{aligned}
& \varepsilon_{\mathrm{kk}}=\varepsilon_{11}+\varepsilon_{22}+\varepsilon_{33} \\
& \sigma_{\mathrm{kk}}=\sigma_{11}+\sigma_{22}+\sigma_{33} \\
& \lambda=\frac{v E}{(1+v)(1-2 v)} \\
& \mu=G=\frac{E}{2(1+v)}
\end{aligned}
$$

sendo:

$\delta_{\mathrm{ij}} \quad-\mathrm{o}$ delta de Kroneker $(0$ se $\mathrm{i} \neq \mathrm{j}$ e 1 se $\mathrm{i}=\mathrm{j})$;

$v \quad$ - o coeficiente de Poisson;

E - o módulo de elasticidade longitudinal ou módulo de Young;

G - o módulo de elasticidade transversal ou módulo de elasticidade ao cisalhamento.

Substituindo-se as eq.(3.33) e (3.34) nas eq.(3.29) e (3.30), tem-se: 


$$
\begin{aligned}
\sigma_{i j} & =\frac{E}{(1+v)}\left[\frac{v}{(1-2 v)} \delta_{i j} \varepsilon_{k k}+\varepsilon_{i j}\right] \\
\varepsilon_{i j} & =-\frac{v}{E} \sigma_{k k} \delta_{i j}+\frac{1+v}{E} \sigma_{i j}
\end{aligned}
$$

Substituindo-se as eq.(3.33), (3.34), (3.31) e (3.26) na eq.(3.29), pode-se escrever uma relação para as tensões em função das derivadas dos deslocamentos e das constantes de Lamé. Tal relação será utilizada posteriormente.

$$
\sigma_{i j}=\frac{2 \mu \nu}{1-2 v} \delta_{i j} \frac{\partial u_{k}}{\partial x_{k}}+\mu\left(\frac{\partial u_{i}}{\partial x_{j}}+\frac{\partial u_{j}}{\partial x_{i}}\right)
$$

A partir de todas as equações deduzidas acima, pode-se determinar as variáveis envolvidas no problema elastostático (ou elastodinâmico) linear:

3 equações de equilíbrio

6 relações deslocamento - deformação

6 relações constitutivas
6 componentes de tensão 6 componentes de deformação 3 componentes de deslocamento

\subsection{SOLUÇÕES FUNDAMENTAIS}

Como comentado, neste capítulo, a formulação integral a ser desenvolvida será baseada em Solução Fundamental estática.

Substituindo-se as eq.(3.35) e (3.26) na eq.(3.10), obtém-se a equação de Navier ou equação de equilíbrio em termos de deslocamentos (eq.(3.38)).

$$
\begin{aligned}
& \sigma_{\ell \mathrm{j}}=\frac{\mathrm{E}}{(1+v)}\left[\frac{v}{(1-2 v)} \delta_{\ell j} \varepsilon_{\mathrm{mm}}+\varepsilon_{\ell \mathrm{j}}\right] \\
& \varepsilon_{\ell \mathrm{j}}=\frac{1}{2}\left(\mathrm{u}_{\ell, \mathrm{j}}+\mathrm{u}_{\mathrm{j}, \ell}\right) \\
& \sigma_{\ell \mathrm{j}, \mathrm{j}}+\mathrm{b}_{\ell}=0
\end{aligned}
$$




$$
\left(\frac{1}{1-2 v}\right) u_{\mathrm{j}, \mathrm{j} \ell}+\mathrm{u}_{\ell, \mathrm{j}}+\frac{1}{\mu} \mathrm{b}_{\ell}=0
$$

A solução fundamental de Kelvin, que representa fisicamente o efeito de uma carga unitária estática atuando em um domínio infinito, é obtida a partir da equação de Navier quando uma carga unitária é aplicada em um ponto fonte 's' na direção 'i', ou seja:

$$
\mathrm{b}_{\mathrm{i} \ell}^{*}=\delta_{\mathrm{i} \ell} \delta(\mathrm{s}, \mathrm{q})
$$

onde:

$\mathrm{b}_{\mathrm{i} \ell}^{*} \quad-$ é a carga fundamental aplicada na direção i;

$\delta(s, q) \quad$ - é o delta de Dirac (0 se $s \neq q$ e $+\infty$ se $s=q)$;

q - é um ponto de campo.

Outra propriedade da distribuição delta de Dirac ${ }^{1}$ é que:

$\int_{-\infty}^{+\infty} \delta(\Omega) \mathrm{d} \Omega=1$

Desta forma, o novo conjunto de equações de equilíbrio (eq.(3.10)) e equações de Navier (eq.(3.38)) para os problemas fundamentais 'i' são escritos como:

$$
\begin{aligned}
& \sigma_{i \ell j, j}^{*}+b_{i \ell}^{*}=0 \\
& \left(\frac{1}{1-2 v}\right) u_{i, j, j}^{*}+u_{i \ell, j j}^{*}+\frac{1}{\mu} b_{i \ell}^{*}=0
\end{aligned}
$$

\footnotetext{
1 Maiores detalhes sobre a distribuição delta de Dirac e suas propriedades podem ser encontrados no apêndice 3 de Coda (2000).
} 
Assim, para o espaço tridimensional, chega-se às expressões das soluções fundamentais de deslocamento e força de superfície, respectivamente:

$$
\begin{aligned}
& u_{i j}^{*}=\frac{1}{16 \pi(1-v) G r}\left[(3-4 v) \delta_{i j}+r_{, i} r_{, j}\right] \\
& p_{i j}^{*}=\frac{-1}{8 \pi(1-v) r^{2}}\left\{\left[(1-2 v) \delta_{i j}+3 r_{, i} r_{, j}\right] r_{, n}-(1-2 v)\left(r_{, i} n_{j}-r_{, j} n_{i}\right)\right\}
\end{aligned}
$$

onde:

r $\quad$ - é o raio, ou a distância entre o ponto fonte e o ponto campo;

$r_{i} \quad$ - são as componentes do vetor $r$;

$r, \mathrm{i} \quad$ - são as derivadas parciais do vetor $r$ nas direções $\mathrm{i}$;

$r, n \quad-$ é a derivada parcial do vetor $r$ na direção $\vec{n}$.

\subsection{EQUAÇÃO INTEGRAL DE CONTORNO PARA DESLOCAMENTOS}

Para a dedução da equação integral de deslocamentos foram utilizadas as considerações do método dos resíduos ponderados. Os conceitos apresentados são muito semelhantes àqueles utilizados pelo princípio dos trabalhos virtuais.

Primeiramente, deseja-se minimizar o erro envolvido na aproximação numérica das equações governantes da elastodinâmica, ou seja, eq.(3.9), rescrita abaixo com uma pequena mudança de índice:

$\sigma_{k, j}+b_{k}=\rho \ddot{u}_{k}+\dot{c u}_{k} \quad$ em $\Omega \quad k, j=1,2,3$

satisfazendo as condições de contorno já citadas anteriormente (eq.(3.28) e (3.19)):

$\mathrm{u}_{\mathrm{k}}=\overline{\mathrm{u}}_{\mathrm{k}} \quad$ em $\Gamma_{1} \quad \mathrm{k}=1,2,3$ 
$\mathrm{p}_{\mathrm{k}}=\sigma_{\mathrm{kj}} \mathrm{n}_{\mathrm{j}}=\overline{\mathrm{p}}_{\mathrm{k}} \quad$ em $\Gamma_{2} \quad \mathrm{k}, \mathrm{j}=1,2,3$

Lembrando-se que agora $u, p$, ù e ü são valores que aproximam as variáveis do problema estudado, multiplica-se a eq.(3.9) pela solução fundamental de deslocamento $\mathrm{u}_{\mathrm{ik}}^{*}$, tal como:

$$
\int_{\Omega}\left(\sigma_{\mathrm{kj}, \mathrm{j}}+\mathrm{b}_{\mathrm{k}}-\rho \ddot{u}_{\mathrm{k}}-c \dot{u}_{\mathrm{k}}\right) \cdot \mathrm{u}_{\mathrm{ik}}^{*} \mathrm{~d} \Omega=0
$$

ou

$$
\int_{\Omega} \sigma_{k j, j} u_{i k}^{*} d \Omega+\int_{\Omega} b_{k} u_{i k}^{*} d \Omega-\int_{\Omega} \rho \ddot{u}_{k} u_{i k}^{*} d \Omega-\int_{\Omega} c \dot{u}_{k} u_{i k}^{*} d \Omega=0
$$

onde 'i’ representa a direção da carga fundamental.

Integrando-se por partes a primeira parcela da eq.(3.46), tem-se:

$$
-\int_{\Omega} \sigma_{k j} \varepsilon_{i k j}^{*} d \Omega+\int_{\Omega} b_{k} u_{i k}^{*} d \Omega-\int_{\Omega} \rho \ddot{u}_{k} u_{i k}^{*} d \Omega-\int_{\Omega} c \dot{u}_{k} u_{i k}^{*} d \Omega=-\int_{\Gamma} u_{i k}^{*} p_{k} d \Gamma
$$

Considerando-se que as tensões possam ser decompostas em uma parcela elástica e outra não-elástica, pode-se escrever:

$$
\sigma_{\mathrm{ij}}=\sigma_{\mathrm{ij}}^{\mathrm{e}}-\sigma_{\mathrm{ij}}^{\mathrm{n}}
$$

onde $\sigma_{i j}^{e}$ são as tensões elásticas e $\sigma_{i j}^{n}$ são as tensões não-elásticas.

Substituindo-se a eq.(3.48) na eq.(3.47), tem-se:

$$
\int_{\Omega} \sigma_{k j}^{n} \varepsilon_{i k j}^{*} d \Omega-\int_{\Omega} \sigma_{k j}^{e} \varepsilon_{i k j}^{*} d \Omega+\int_{\Omega} b_{k} u_{i k}^{*} d \Omega-\int_{\Omega} \rho \ddot{u}_{k} u_{i k}^{*} d \Omega-\int_{\Omega} c \dot{u}_{k} u_{i k}^{*} d \Omega+\int_{\Gamma} u_{i k}^{*} p_{k} d \Gamma=0
$$


Integrando-se por partes a integral da tensão elástica da eq.(3.49), obtém-se:

$$
\begin{aligned}
& \int_{\Omega} \sigma_{k j}^{n} \varepsilon_{i k j}^{*} d \Omega-\int_{\Gamma} p_{i k}^{*} u_{k} d \Gamma+\int_{\Omega} u_{k} \sigma_{i k j, j}^{*} d \Omega+\int_{\Omega} b_{k} u_{i k}^{*} d \Omega-\int_{\Omega} \rho \ddot{u}_{k} u_{i k}^{*} d \Omega-\int_{\Omega} c \dot{u}_{k} u_{i k}^{*} d \Omega+ \\
& +\int_{\Gamma} u_{i k}^{*} p_{k} d \Gamma=0
\end{aligned}
$$

A eq.(3.41) pode ser rescrita como:

$$
\sigma_{i \mathrm{kj}, \mathrm{j}}^{*}+b_{\mathrm{ik}}^{*}=0
$$

Substituindo-se a eq.(3.41) na eq.(3.50), tem-se:

$$
\begin{aligned}
& \int_{\Omega} \sigma_{j k}^{n} \varepsilon_{i j k}^{*} d \Omega-\int_{\Gamma} p_{i k}^{*} u_{k} d \Gamma-\int_{\Omega} b_{i k}^{*} u_{k} d \Omega+\int_{\Omega} b_{k} u_{i k}^{*} d \Omega-\int_{\Omega} \rho \ddot{u}_{k} u_{i k}^{*} d \Omega-\int_{\Omega} c \dot{u}_{k} u_{i k}^{*} d \Omega+ \\
& +\int_{\Gamma} u_{i k}^{*} p_{k} d \Gamma=0
\end{aligned}
$$

onde a igualdade $\sigma_{\mathrm{kj}} \varepsilon_{\mathrm{ikj}}^{*}=\sigma_{\mathrm{jk}} \varepsilon_{\mathrm{ijk}}^{*}$ foi utilizada na integral de tensões nãoelásticas.

Aplicando-se as eq.(3.39) e (3.40) na terceira parcela da eq.(3.51), pode-se escrever:

$$
\int_{\Omega} b_{i k}^{*} u_{k} d \Omega=\int_{\Omega} \delta_{i k} \delta(s, q) u_{k} d \Omega=\delta_{i k} u_{k}(s)=u_{i}(s)
$$

onde $u_{i}(s)$ representa a componente 'i' de deslocamento em um ponto fonte $s$.

Substituindo-se a eq.(3.52) na eq.(3.51), pode-se escrever a eq.(3.53) abaixo:

$$
\mathrm{u}_{\mathrm{i}}+\int_{\Gamma} p_{\mathrm{ik}}^{*} \mathrm{u}_{\mathrm{k}} \mathrm{d} \Gamma+\int_{\Omega} \rho \ddot{u}_{\mathrm{k}} \mathrm{u}_{\mathrm{ik}}^{*} \mathrm{~d} \Omega+\int_{\Omega} c \dot{u}_{\mathrm{k}} \mathrm{u}_{\mathrm{ik}}^{*} \mathrm{~d} \Omega=\int_{\Gamma} \mathrm{u}_{\mathrm{ik}}^{*} \mathrm{p}_{\mathrm{k}} \mathrm{d} \Gamma+\int_{\Omega} \mathrm{u}_{\mathrm{ik}}^{*} b_{\mathrm{k}} \mathrm{d} \Omega+\int_{\Omega} \varepsilon_{\mathrm{ijk}}^{*} \sigma_{\mathrm{jk}}^{\mathrm{n}} \mathrm{d} \Omega
$$


válida apenas para pontos internos.

Genericamente, pode-se escrever:

$$
\begin{aligned}
& \mathrm{c}_{\mathrm{ik}} \mathrm{u}_{\mathrm{k}}(\mathrm{s}, \mathrm{t})+\int_{\Gamma} \mathrm{p}_{\mathrm{ik}}^{*}(\mathrm{~s}, \mathrm{q}) \mathrm{u}_{\mathrm{k}}(\mathrm{Q}, \mathrm{t}) \mathrm{d} \Gamma+\int_{\Omega} \rho \ddot{u}_{\mathrm{k}}(\mathrm{q}, \mathrm{t}) \mathrm{u}_{\mathrm{ik}}^{*}(\mathrm{~s}, \mathrm{q}) \mathrm{d} \Omega+\int_{\Omega} \mathrm{cu}_{\mathrm{k}}(\mathrm{q}, \mathrm{t}) \mathrm{u}_{\mathrm{ik}}^{*}(\mathrm{~s}, \mathrm{q}) \mathrm{d} \Omega \\
& =\int_{\Gamma} \mathrm{u}_{\mathrm{ik}}^{*}(\mathrm{~s}, \mathrm{q}) \mathrm{p}_{\mathrm{k}}(\mathrm{Q}, \mathrm{t}) \mathrm{d} \Gamma+\int_{\Omega} \mathrm{u}_{\mathrm{ik}}^{*}(\mathrm{~s}, \mathrm{q}) \mathrm{b}_{\mathrm{k}}(\mathrm{q}, \mathrm{t}) \mathrm{d} \Omega+\int_{\Omega} \varepsilon_{\mathrm{ijk}}^{*}(\mathrm{~s}, \mathrm{q}) \sigma_{\mathrm{jk}}^{\mathrm{n}}(\mathrm{q}, \mathrm{t}) \mathrm{d} \Omega
\end{aligned}
$$

onde:

$\mathrm{c}_{\mathrm{ik}}=\left\{\begin{array}{l}\delta_{\mathrm{ik}}, \text { para pontos fonte internos ao problema estudado } \\ 0, \text { para pontos fonte externos ao problema estudado } \\ \mathrm{c}_{\mathrm{ik}}, \text { para pontos fonte no contorno do problema estudado }\end{array}\right.$

e $\varepsilon_{\mathrm{ijk}}^{*}$ é dado pela seguinte expressão:

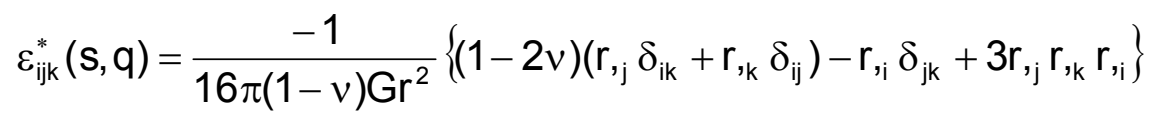

A eq.(3.54) pode ser chamada de equação integral de contorno para deslocamentos dinâmicos. Para o caso estático, pode-se escrever:

$$
\mathrm{c}_{i k} u_{k}(s)+\int_{\Gamma} p_{i k}^{*}(s, q) u_{k}(Q) d \Gamma=\int_{\Gamma} u_{i k}^{*}(s, q) p_{k}(Q) d \Gamma+\int_{\Omega} u_{i k}^{*}(s, q) b_{k}(q) d \Omega+\int_{\Omega} \varepsilon_{i j k}^{*}(s, q) \sigma_{j k}^{n}(q) d \Omega
$$

Considerando que só existam tensões elásticas, tem-se:

$$
c_{i k} u_{k}(s)+\int_{\Gamma} p_{i k}^{*}(s, q) u_{k}(Q) d \Gamma=\int_{\Gamma} u_{i k}^{*}(s, q) p_{k}(Q) d \Gamma+\int_{\Omega} u_{i k}^{*}(s, q) b_{k}(q) d \Omega
$$

Para o cálculo de deslocamentos em pontos internos $\left(\mathrm{c}_{\mathrm{ik}}=\delta_{\mathrm{ik}}\right)$, a eq.(3.57) é conhecida como Identidade de Somigliana (eq.(3.58)) e fornece os 
valores dos deslocamentos internos em função dos valores de $u_{k}$ e $p_{k}$ no contorno, $b_{k}$ e soluções fundamentais.

$u_{i}+\int_{\Gamma} p_{i k}^{*} u_{k} d \Gamma=\int_{\Gamma} u_{i k}^{*} p_{k} d \Gamma+\int_{\Omega} u_{i k}^{*} b_{k} d \Omega$

Matricialmente, a eq.(3.54) poderia ser escrita como:

$H U(t)+C \dot{U}(t)+M U ̈(t)=G P(t)+B b(t)+Q \sigma^{n}(t)$

onde para densidade ' $\rho$ ' e parâmetro de amortecimento ' $c$ ' constantes sobre o domínio, escreve-se:

$\mathrm{C}=\mathrm{C} \cdot \mathrm{B}$

$M=\rho \cdot B$

A matriz $C$ é conhecida como matriz de amortecimento e a matriz $M$, como matriz de massa.

A montagem das matrizes envolvidas na equação matricial eq.(3.59) serão descritas no capítulo 5 .

\subsection{EQUAÇÃO INTEGRAL DE CONTORNO PARA TENSÕES}

Neste item, será apresentado um processo que permite o cálculo das tensões, internas ou no contorno, dinamicamente, utilizando-se uma única equação matricial (eq.(3.62)). Tal processo foi feito para o caso bidimensional por Coda (2000). Parte da formulação apresentada a seguir foi feita com base em Brebbia e Dominguez (1992).

$$
\sigma(t)=G^{\prime} P(t)-H^{\prime} U(t)+B^{\prime} b(t)-C^{\prime} \dot{U}(t)-M^{\prime} U ̈(t)+Q^{\prime} \sigma^{n}(t)
$$

Utilizando-se a eq.(3.53), pode-se determinar os deslocamentos nos pontos internos do sólido: 


$$
u_{i}=\int_{\Gamma} u_{i k}^{*} p_{k} d \Gamma-\int_{\Gamma} p_{i k}^{*} u_{k} d \Gamma+\int_{\Omega} u_{i k}^{*} b_{k} d \Omega-\int_{\Omega} c \dot{u}_{k} u_{i k}^{*} d \Omega-\int_{\Omega} \rho \ddot{u}_{k} u_{i k}^{*} d \Omega+\int_{\Omega} \varepsilon_{i j k}^{*} \sigma_{j k}^{n} d \Omega
$$

Por sua vez, as tensões no sólido podem ser calculadas utilizando-se a eq.(3.37), rescrita abaixo:

$$
\sigma_{m n}(s)=\frac{2 G v}{1-2 v} \delta_{m n} \frac{\partial u_{\ell}(s)}{\partial x_{\ell}}+G\left(\frac{\partial u_{m}(s)}{\partial x_{n}}+\frac{\partial u_{n}(s)}{\partial x_{m}}\right)
$$

onde a relação $\mu=G$ foi utilizada, e a derivada deve ser entendida segundo variação da posição do ponto fonte.

Observa-se que:

$$
\frac{\partial \mathrm{u}_{\ell}(\mathrm{s})}{\partial \mathrm{x}_{\ell}}=\frac{\partial \mathrm{u}_{1}(\mathrm{~s})}{\partial \mathrm{x}_{1}}+\frac{\partial \mathrm{u}_{2}(\mathrm{~s})}{\partial \mathrm{x}_{2}}+\frac{\partial \mathrm{u}_{3}(\mathrm{~s})}{\partial \mathrm{x}_{3}}
$$

Porém, considerando-se a presença de tensões não-elásticas (eq.(3.48)), a eq.(3.37) pode ser escrita como:

$$
\sigma_{\mathrm{mn}}(\mathrm{s})=\frac{2 \mathrm{G} v}{1-2 v} \delta_{\mathrm{mn}} \frac{\partial \mathrm{u}_{\ell}(\mathrm{s})}{\partial \mathrm{x}_{\ell}}+\mathrm{G}\left(\frac{\partial \mathrm{u}_{\mathrm{m}}(\mathrm{s})}{\partial \mathrm{x}_{\mathrm{n}}}+\frac{\partial \mathrm{u}_{\mathrm{n}}(\mathrm{s})}{\partial \mathrm{x}_{\mathrm{m}}}\right)-\sigma_{\mathrm{mn}}^{\mathrm{n}}(\mathrm{s})
$$

Substituindo-se a eq.(3.53) na eq.(3.64), tem-se:

$$
\begin{aligned}
& \sigma_{\mathrm{mn}}=\int_{\Gamma}\left\{\frac{2 \mathrm{G} v}{1-2 v} \delta_{\mathrm{mn}} \frac{\partial \mathrm{u}_{\ell \mathrm{k}}^{*}}{\partial \mathrm{x}_{\ell}}+\mathrm{G}\left(\frac{\partial \mathrm{u}_{\mathrm{mk}}^{*}}{\partial \mathrm{x}_{\mathrm{n}}}+\frac{\partial \mathrm{u}_{\mathrm{nk}}^{*}}{\partial \mathrm{x}_{\mathrm{m}}}\right)\right\} \mathrm{p}_{\mathrm{k}} \mathrm{d} \Gamma-\int_{\Gamma}\left\{\frac{2 \mathrm{Gv}}{1-2 v} \delta_{\mathrm{mn}} \frac{\partial \mathrm{p}_{\ell \mathrm{k}}^{*}}{\partial \mathrm{x}_{\ell}}+\mathrm{G}\left(\frac{\partial \mathrm{p}_{\mathrm{mk}}^{*}}{\partial \mathrm{x}_{\mathrm{n}}}+\frac{\partial \mathrm{p}_{\mathrm{nk}}^{*}}{\partial \mathrm{x}_{\mathrm{m}}}\right)\right\} \mathrm{u}_{\mathrm{k}} \mathrm{d} \Gamma+ \\
& +\int_{\Omega}\left\{\frac{2 \mathrm{G} v}{1-2 v} \delta_{\mathrm{mn}} \frac{\partial \mathrm{u}_{\ell \mathrm{k}}^{*}}{\partial \mathrm{x}_{\ell}}+\mathrm{G}\left(\frac{\partial \mathrm{u}_{\mathrm{mk}}^{*}}{\partial \mathrm{x}_{\mathrm{n}}}+\frac{\partial \mathrm{u}_{\mathrm{nk}}^{*}}{\partial \mathrm{x}_{\mathrm{m}}}\right)\right\} \mathrm{b}_{\mathrm{k}} \mathrm{d} \Omega-\mathrm{c} \cdot \int_{\Omega}\left\{\frac{2 \mathrm{G} v}{1-2 v} \delta_{\mathrm{mn}} \frac{\partial \mathrm{u}_{\ell \mathrm{k}}^{*}}{\partial \mathrm{x}_{\ell}}+\mathrm{G}\left(\frac{\partial \mathrm{u}_{\mathrm{mk}}^{*}}{\partial \mathrm{x}_{\mathrm{n}}}+\frac{\partial \mathrm{u}_{\mathrm{nk}}^{*}}{\partial \mathrm{x}_{\mathrm{m}}}\right)\right\} \dot{\mathrm{u}}_{\mathrm{k}} \mathrm{d} \Omega+ \\
& -\rho \cdot \int_{\Omega}\left\{\frac{2 \mathrm{G} v}{1-2 v} \delta_{\mathrm{mn}} \frac{\partial \mathrm{u}_{\ell \mathrm{k}}^{*}}{\partial \mathrm{x}_{\ell}}+\mathrm{G}\left(\frac{\partial \mathrm{u}_{\mathrm{mk}}^{*}}{\partial \mathrm{x}_{\mathrm{n}}}+\frac{\partial \mathrm{u}_{\mathrm{nk}}^{*}}{\partial \mathrm{x}_{\mathrm{m}}}\right)\right\} \ddot{\mathrm{u}}_{\mathrm{k}} \mathrm{d} \Omega+\int_{\Omega}\left\{\frac{2 \mathrm{Gv}}{1-2 v} \delta_{\mathrm{mn}} \frac{\partial \varepsilon_{\ell \mathrm{k} \mathrm{k}}^{*}}{\partial \mathrm{x}_{\ell}}+\mathrm{G}\left(\frac{\partial \varepsilon_{\mathrm{mjk}}^{*}}{\partial \mathrm{x}_{\mathrm{n}}}+\frac{\partial \varepsilon_{\mathrm{njk}}^{*}}{\partial \mathrm{x}_{\mathrm{m}}}\right)\right\} \sigma_{\mathrm{jk}}^{\tilde{n}} \mathrm{~d} \Omega+ \\
& +\overline{\mathrm{g}}_{\mathrm{mnjk}} \sigma_{\mathrm{jk}}^{\tilde{n}}(\mathrm{~s})-\sigma_{\mathrm{mn}}^{\tilde{n}}(\mathrm{~s})
\end{aligned}
$$


onde o símbolo $\sigma_{\mathrm{jk}}^{\tilde{n}}$ foi utilizado no lugar de $\sigma_{\mathrm{jk}}^{\mathrm{n}}$ para que o índice ' $\tilde{n}$ ' (referente a não-elástico) não fosse confundido com o índice ' $n$ ' das tensões $\sigma_{m n}$, e o termo livre $\overline{\mathrm{g}}_{\mathrm{mnjk}}$ será descrito no capítulo 5 .

Aplicando-se as expressões das soluções fundamentais (eq.(3.43), (3.44) e (3.55)) na eq.(3.65), escreve-se:

$$
\begin{aligned}
& \sigma_{i j}=\int_{\Gamma} D_{k j j} p_{k} d \Gamma-\int_{\Gamma} S_{k j i} u_{k} d \Gamma+\int_{\Omega} D_{k j i j} b_{k} d \Omega-c \cdot \int_{\Omega} D_{k i j} \dot{u}_{k} d \Omega-\rho \cdot \int_{\Omega} D_{k j i j} \ddot{u}_{k} d \Omega+ \\
& +\int_{\Omega} \bar{E}_{i j k k} \sigma_{\ell k}^{\tilde{n}}(q) d \Omega+\overline{\bar{g}}_{i j k k} \sigma_{\ell k}^{\tilde{n}}(s)
\end{aligned}
$$

onde:

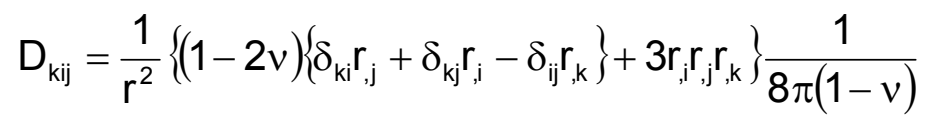

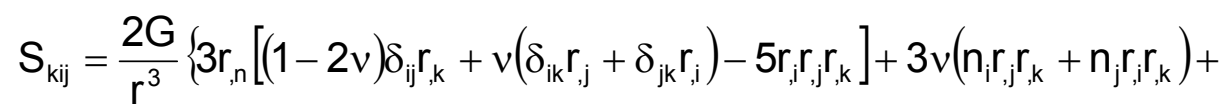

$$
\begin{aligned}
& \left.+(1-2 v)\left(3 n_{k} r_{,} r_{j}+n_{j} \delta_{i k}+n_{i} \delta_{j k}\right)-(1-4 v) n_{k} \delta_{i j}\right\} \frac{1}{8 \pi(1-v)} \\
& \overline{\mathrm{E}}_{\mathrm{ij} / \mathrm{k}}=\frac{1}{8 \pi(1-v) \mathrm{r}^{3}}\left\{(1-2 v)\left(\delta_{\mathrm{i} \ell} \delta_{\mathrm{kj}}+\delta_{\mathrm{j} \ell} \delta_{\mathrm{ki}}-\delta_{\mathrm{ij}} \delta_{\ell \mathrm{k}}+3 \mathrm{r}, \ell \mathrm{r}_{, \mathrm{k}} \delta_{\mathrm{ij}}\right)+3 v\left(\mathrm{r}_{, \mathrm{i}} \mathrm{r}_{\ell} \delta_{\mathrm{kj}}+\mathrm{r}_{, j} \mathrm{r}_{\ell} \delta_{\mathrm{ki}}+\right.\right. \\
& \left.\left.+r_{, j} r_{{ }_{k}} \delta_{j \ell}+r_{, j} r_{, k} \delta_{i \ell}\right)+3 r,{ }_{, i} r_{, j} \delta_{\ell k}-15 r,{ }_{, i} r_{, j} r_{, \ell} r_{, k}\right\} \\
& \overline{\bar{g}}_{\mathrm{ij} \ell \mathrm{k}}=\frac{1}{30(1-v)}\left[(-7+5 v)\left(\delta_{\mathrm{i} \ell} \delta_{\mathrm{jk}}+\delta_{\mathrm{ik}} \delta_{\mathrm{j} \ell}\right)-(2-10 v) \delta_{\mathrm{ij}} \delta_{\ell \mathrm{k}}\right]
\end{aligned}
$$

A notação indicial da eq.(3.65) foi modificada com o intuito de se continuar seguindo a notação apresentada nas equações anteriores. Observase que $\overline{\bar{g}}_{\mathrm{ij} / \mathrm{k}}$ é conhecido como termo livre da eq.(3.66) e sua dedução completa pode ser encontrada na seção 5.5 .

Matricialmente, a eq.(3.66) pode ser escrita como a eq.(3.62), onde se observa que:

$$
C^{\prime}=\mathrm{C} \cdot \mathrm{B}^{\prime}
$$


$M^{\prime}=\rho \cdot B^{\prime}$

e os termos livres já estão implícitos na matriz Q'.

Para o cálculo de tensões em pontos do contorno, deve-se adaptar o procedimento apresentado por Brebbia e Dominguez (1992) para a eq.(3.62).

Assim, pode-se escrever o vetor de tensões em um ponto no contorno como:

$$
\left\{\begin{array}{c}
\sigma_{11} \\
\sigma_{12} \\
\sigma_{13} \\
\sigma_{22} \\
\sigma_{23} \\
\sigma_{33}
\end{array}\right\}=\left[G L^{\prime}\right]_{6 \times 9}\{P\}_{9}-\left[H L^{\prime}\right]_{6 \times 9}\{U\}_{9}-\left\{\begin{array}{c}
\bar{\sigma}_{11} \\
\bar{\sigma}_{12} \\
0 \\
\bar{\sigma}_{22} \\
0 \\
0
\end{array}\right\}
$$

Os elementos da matriz GL' são determinados em função dos co-senos diretores de cada elemento e do coeficiente de Poisson ' $v$ '; enquanto que os elementos da matriz $\mathrm{HL}^{\prime}$ podem ser determinados em função das coordenadas dos nós de vértice do elemento e do módulo de elasticidade transversal ' $G$ ', além dos co-senos diretores do elemento e do coeficiente de Poisson.

Os elementos das matrizes $\mathrm{GL}^{\prime}, \mathrm{HL}^{\prime}$ e a incidência dos elementos do vetor de tensões não-elásticas $\left(\bar{\sigma}_{i j}\right)$ são alocados nas matrizes $G^{\prime}, H^{\prime}$ e $Q^{\prime}$, respectivamente. Mais detalhes podem ser encontrados no capítulo 5 . 


\section{ANÁLISE DINÂMICA APLICANDO MATRIZ DE MASSA}

Neste capítulo, descreve-se uma formulação do MEC para a análise dinâmica transiente tridimensional de meios contínuos - baseada em solução fundamental estática - bem como os algoritmos desenvolvidos no trabalho para a solução do problema não-linear. A integração temporal é feita empregandose o algoritmo de Houbolt, já utilizado com bastante sucesso em análises bidimensionais por outros pesquisadores, como por exemplo: Carrer e Telles (1992), Coda e Venturini (1999b), Kontoni e Beskos (1993) e Telles e Carrer (1994). Os procedimentos relativos à integração temporal foram feitos com base principalmente em Coda (2000). No final do capítulo são apresentados 4 exemplos numéricos com relação à formulação desenvolvida.

\subsection{ALGORITMO DE HOUBOLT}

Segundo Argyris \& Mlejnek (1991), o vetor de acelerações e o vetor de velocidades em um instante atual $(s+1)$ podem ser, respectivamente, aproximados pelas seguintes expressões:

$$
\begin{aligned}
& \ddot{U}_{s+1}=\frac{2 U_{s+1}}{\Delta t^{2}}+a_{s} \\
& \dot{U}_{s+1}=\frac{11}{6} \cdot \frac{U_{s+1}}{\Delta t}+v_{s}
\end{aligned}
$$

onde: 


$$
\begin{aligned}
& a_{s}=\frac{1}{\Delta t^{2}}\left[-5 U_{s}+4 U_{s-1}-U_{s-2}\right] \\
& v_{s}=\frac{1}{6 \Delta t}\left[-18 U_{s}+9 U_{s-1}-2 U_{s-2}\right]
\end{aligned}
$$

Deve-se observar que $\Delta$ t é o intervalo de tempo e que é constante durante toda a análise.

Substituindo-se as eq.(4.3) e (4.4) nas eq.(4.1) e (4.2), tem-se:

$$
\begin{aligned}
& \ddot{U}_{s+1}=\frac{1}{\Delta t^{2}}\left[2 U_{s+1}-5 U_{s}+4 U_{s-1}-U_{s-2}\right] \\
& \dot{U}_{s+1}=\frac{1}{6 \Delta t}\left[11 U_{s+1}-18 U_{s}+9 U_{s-1}-2 U_{s-2}\right]
\end{aligned}
$$

Substituindo-se as eq.(4.5) e (4.6) na eq.(3.59), pode-se chegar a:

$$
\overline{\mathrm{H}} \mathrm{U}_{\mathrm{s}+1}=\Delta \mathrm{t}^{2} \mathrm{GP} \mathrm{P}_{\mathrm{s}+1}+\Delta \mathrm{t}^{2} \mathrm{Q} \sigma_{\mathrm{s}+1}^{\mathrm{n}}+\mathrm{F}_{\mathrm{s}}
$$

onde:

$$
\begin{aligned}
& \bar{H}=2 \mathrm{M}+\frac{11}{6} \Delta \mathrm{tC}+\Delta \mathrm{t}^{2} \mathrm{H} \\
& \mathrm{F}_{\mathrm{s}}=[5 \mathrm{M}+3 \Delta \mathrm{tC}] \mathrm{U}_{\mathrm{s}}-\left[4 \mathrm{M}+\frac{3}{2} \Delta \mathrm{tC}\right] \mathrm{U}_{\mathrm{s}-1}+\left[\mathrm{M}+\frac{1}{3} \Delta \mathrm{tC}\right] \mathrm{U}_{\mathrm{s}-2}+\Delta \mathrm{t}^{2} \mathrm{Bb} \mathrm{b}_{\mathrm{s}+1}
\end{aligned}
$$

Substituindo-se as eq.(3.60) e (3.61) nas eq.(4.8) e (4.9), tem-se:

$$
\begin{aligned}
& \bar{H}=\left(2 \rho+\frac{11}{6} \Delta t c\right) B+\Delta t^{2} H \\
& F_{s}=[5 \rho+3 \Delta t c] B U_{s}-[8 \rho+3 \Delta t c] \frac{1}{2} B U_{s-1}+[3 \rho+\Delta t c] \frac{1}{3} B U_{s-2}+\Delta t^{2} B b_{s+1}
\end{aligned}
$$

As prescrições do sólido do problema analisado são aplicadas à eq.(4.7) de maneira usual, onde as colunas das matrizes $\bar{H}$ e $\Delta t^{2} G$ são trocadas com 
sinal contrário, para cada grau de liberdade de deslocamento prescrito naquele passo de tempo.

$$
A X_{s+1}=F_{s+1}+\Delta t^{2} Q \sigma_{s+1}^{n}
$$

onde:

A - é a matriz referente às variáveis desconhecidas (matriz $\overline{\mathrm{H}}$ modificada);

$\mathrm{X}_{\mathrm{s}+1}$ - é o vetor de incógnitas.

e

$F_{s+1}=F_{s}+G_{h} y_{s+1}$

sendo:

$\mathrm{G}_{\mathrm{h}} \quad$ - a matriz referente às variáveis conhecidas (matriz $\Delta \mathrm{t}^{2} \mathrm{G}$ modificada);

$\mathrm{y}_{\mathrm{s}+1} \quad-\mathrm{o}$ vetor dos valores conhecidos.

Resolvendo-se o sistema de equações representado na eq.(4.12), determina-se o vetor $X_{s+1}$, ou seja, são determinados os deslocamentos $e$ forças de superfície incógnitos, no instante atual.

Deve-se acrescentar que na implementação do desenvolvimento acima, foram consideradas condições iniciais nulas para deslocamentos e velocidades.

$\mathrm{u}\left(\mathrm{q}, \mathrm{t}_{0}\right)=\mathrm{U}_{0}=0$

$\dot{\mathrm{u}}\left(\mathrm{q}, \mathrm{t}_{0}\right)=\dot{\mathrm{U}}_{0}=0$

Apenas para se ilustrar o processo desenvolvido e apresentado acima, observa-se o seguinte esquema: 


\section{Para $s=0$}

$$
\left\{\begin{array} { l } 
{ F _ { 0 } = \Delta t ^ { 2 } B b _ { 1 } } \\
{ F _ { 1 } = F _ { 0 } + G _ { h } y _ { 1 } } \\
{ A X _ { 1 } = F _ { 1 } + \Delta t ^ { 2 } Q \sigma _ { 1 } ^ { n } }
\end{array} \Rightarrow \text { Determina - se } X _ { 1 } \left\{\begin{array}{l}
U_{1} \\
P_{1}
\end{array}\right.\right.
$$

\section{Para $s=1$}

$$
\left\{\begin{array} { l } 
{ F _ { 1 } = [ 5 \rho + 3 \Delta t c ] B U _ { 1 } + \Delta t ^ { 2 } B b _ { 2 } } \\
{ F _ { 2 } = F _ { 1 } + G _ { h } y _ { 2 } } \\
{ A X _ { 2 } = F _ { 2 } + \Delta t ^ { 2 } Q \sigma _ { 2 } ^ { n } }
\end{array} \Rightarrow \text { Determina - se } X _ { 2 } \left\{\begin{array}{l}
U_{2} \\
P_{2}
\end{array}\right.\right.
$$

\section{Para $\mathrm{s}=2$}

$$
\left\{\begin{array} { l } 
{ F _ { 2 } = [ 5 \rho + 3 \Delta t c ] B U _ { 2 } - [ 8 \rho + 3 \Delta t c ] \frac { 1 } { 2 } B U _ { 1 } + \Delta t ^ { 2 } B _ { 3 } } \\
{ F _ { 3 } = F _ { 2 } + G _ { h } y _ { 3 } } \\
{ A X _ { 3 } = F _ { 3 } + \Delta t ^ { 2 } Q \sigma _ { 3 } ^ { n } }
\end{array} \Rightarrow \text { Determina - se } X _ { 3 } \left\{\begin{array}{l}
U_{3} \\
P_{3}
\end{array}\right.\right.
$$

Para $s=3$

$$
\left\{\begin{array} { l } 
{ F _ { 3 } = [ 5 \rho + 3 \Delta t c ] B U _ { 3 } - [ 8 \rho + 3 \Delta t c ] \frac { 1 } { 2 } B U _ { 2 } + [ 3 \rho + \Delta t c ] \frac { 1 } { 3 } B U _ { 1 } + \Delta t ^ { 2 } B ^ { 2 } b _ { 4 } } \\
{ F _ { 4 } = F _ { 3 } + G _ { h } y _ { 4 } } \\
{ A X _ { 4 } = F _ { 4 } + \Delta t ^ { 2 } Q \sigma _ { 4 } ^ { n } }
\end{array} \Rightarrow \text { Determina - se } X _ { 4 } \left\{\begin{array}{l}
U_{4} \\
P_{4}
\end{array}\right.\right.
$$

Para $s=n-1$

$$
\left\{\begin{array} { l } 
{ F _ { n - 1 } = [ 5 \rho + 3 \Delta t c ] B U _ { n - 1 } - [ 8 \rho + 3 \Delta t c ] \frac { 1 } { 2 } B U _ { n - 2 } + [ 3 \rho + \Delta t c ] \frac { 1 } { 3 } B U _ { n - 3 } + \Delta t ^ { 2 } B b _ { n } } \\
{ F _ { n } = F _ { n - 1 } + G _ { n } y _ { n } } \\
{ A X _ { n } = F _ { n } + \Delta t ^ { 2 } Q \sigma _ { n } ^ { n } }
\end{array} \Rightarrow \text { Determina - se } X _ { n } \left\{\begin{array}{l}
U_{n} \\
P_{n}
\end{array}\right.\right.
$$

onde 'n' é o número de passos de tempo, dado por:

$$
\mathrm{n}=\frac{\mathrm{t}}{\Delta \mathrm{t}}
$$


sendo ' $\mathrm{t}$ ' o tempo total da análise.

Por fim, para o cálculo das tensões, a eq.(3.62) é resolvida de maneira direta, para cada passo de tempo, levando-se em consideração os resultados temporais de força de superfície e deslocamento e as eq.(4.5) e (4.6).

\subsection{ALGORITMOS NÃO-LINEARES}

Nesta seção será apresentada a formulação para análise elastoplástica estática e dinâmica.

O autor procurou ser o mais direto possível na apresentação dos conceitos inseridos nesta seção, dando mais ênfase a aspectos de implementação e a uma ou outra novidade proposta nesse tipo de análise. Detalhes a respeito da formulação utilizada podem ser encontrados nos trabalhos de Mesquita (2002) e Simo e Hughes (1986). Deve-se salientar que a aplicação e adaptação dos modelos desenvolvidos em Mesquita (2002) para aplicações dinâmicas são feitas originalmente no presente trabalho.

Inicialmente, considera-se o caso elastoplástico estático para depois se apresentar o caso dinâmico.

Em análises não-lineares físicas, sabe-se que, para espaços multiaxiais, a região elástica é limitada no espaço das tensões por uma superfície conhecida por superfície de plastificação. Essa superfície pode ser descrita pela seguinte equação:

$f(\sigma, \kappa)=\bar{f}(\sigma)-\bar{\sigma}(\kappa)=0$

onde:

$\sigma \quad$ - representa o estado de tensão;

$\kappa \quad$ - o parâmetro de encruamento;

$\bar{f} \quad-$ uma função do estado de tensão $\sigma_{i j}$;

$\bar{\sigma} \quad$ - uma tensão equivalente. 
Considerando-se, por exemplo, a superfície de plastificação como sendo a de von Mises, a eq.(4.17) pode ser rescrita como:

$f=\bar{f}(\sigma)-\frac{1}{\sqrt{3}}\left(\sigma_{y}+H \cdot \kappa\right)$

onde:

$\sigma_{y} \quad$ - é o limite de plastificação inicial;

H $\quad$ - é o módulo plástico.

Observa-se que a tensão equivalente $\bar{\sigma}$ foi definida segundo uma lei de encruamento isotrópico linear, figura 4.1.

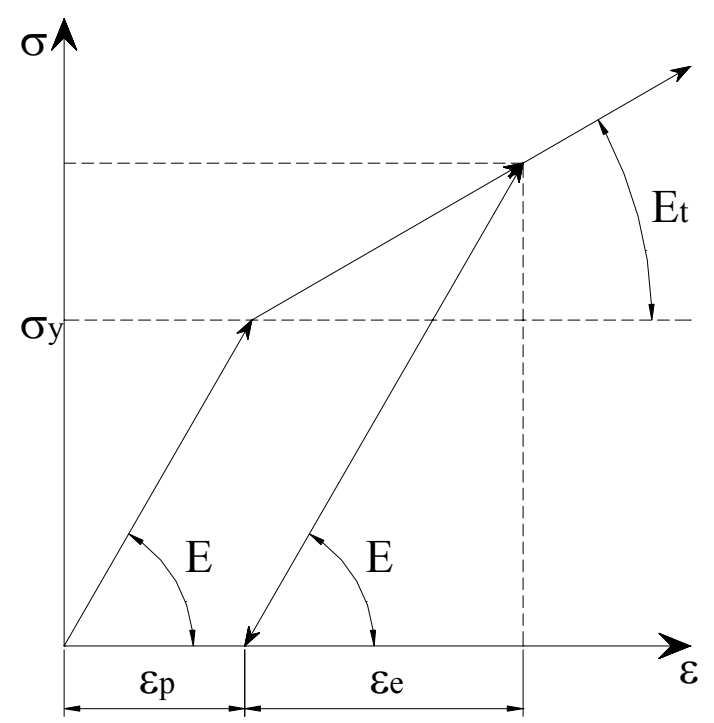

Figura 4.1 - Lei de encruamento isotrópico linear

onde:

$\begin{array}{ll}\varepsilon_{\mathrm{e}} & - \text { é a deformação elástica; } \\ \varepsilon_{\mathrm{p}} & - \text { a deformação plástica; } \\ \mathrm{E} & - \text { o módulo de elasticidade; } \\ \mathrm{E}_{\mathrm{t}} & - \text { o módulo de elasticidade tangente. }\end{array}$ 
Observa-se que:

$\varepsilon=\varepsilon_{\mathrm{e}}+\varepsilon_{\mathrm{p}}$

e

$E_{t}=\frac{E \cdot H}{E+H}$

A função $\bar{f}(\sigma)$ foi definida como:

$\bar{f}(\sigma)=\|\sigma\|$

onde a norma $\|\sigma\|$ é calculada pela seguinte equação:

$$
\|\sigma\|=\frac{1}{\sqrt{3}} \sqrt{\sigma_{1}^{2}+\sigma_{2}^{2}+\sigma_{3}^{2}-\sigma_{1} \sigma_{2}-\sigma_{1} \sigma_{3}-\sigma_{2} \sigma_{3}}
$$

Como se pode observar pela eq.(4.22), o estado de tensão $\sigma$ foi considerado no espaço das tensões principais. Para a implementação da transformação das tensões do espaço cartesiano para o espaço principal, e sua transformação inversa, ver o apêndice $A$.

Substituindo a eq.(4.21) na eq.(4.18), pode-se escrever:

$f=\|\sigma\|-\frac{1}{\sqrt{3}}\left(\sigma_{y}+H \cdot \kappa\right)$

Utilizou-se um procedimento incremental-iterativo na análise não-linear, onde o carregamento da estrutura foi aplicado em incrementos de carga e o equilíbrio foi obtido iterativamente dentro de limites de precisão préestabelecidos. 
De uma maneira simplificada, o procedimento não-linear é iniciado com um incremento de carga, onde são calculados os deslocamentos e forças de superfície, utilizando-se a eq.(4.24), e tensões, eq.(4.25).

$$
\begin{aligned}
& H U=G P+B b+Q \sigma^{p} \\
& \sigma=G^{\prime} P-H^{\prime} U+B^{\prime} b+Q^{\prime} \sigma^{p}
\end{aligned}
$$

Observa-se que o vetor $\sigma^{p}$ é o vetor de tensões não-lineares, acumulado durante toda a análise, e o vetor $\sigma$ é o vetor de tensões, tanto no contorno quanto no domínio. $O$ vetor $\sigma^{p}$ deve ser atualizado nas eq.(4.24) e (4.25) até que a convergência seja obtida, como descrito mais adiante.

De posse das tensões no sólido, verifica-se se esse estado de tensões é elástico ou não, nos pontos da região de plastificação (nós das células de discretização da região definida como de plastificação), utilizando-se a eq.(4.23); onde:

Se $\left\{\begin{array}{lll}\mathrm{f}_{\mathrm{i}+1}^{\text {ten }}=\left\|\sigma_{\mathrm{i}+1}^{\text {ten }}\right\|-\frac{1}{\sqrt{3}}\left(\sigma_{\mathrm{y}}+\mathrm{H} \cdot \kappa_{\mathrm{i}}\right)<0 & \Rightarrow & \text { Incremento elástico } \\ \mathrm{f}_{\mathrm{i}+1}^{\text {ten }}=\left\|\sigma_{\mathrm{i}+1}^{\text {ten }}\right\|-\frac{1}{\sqrt{3}}\left(\sigma_{\mathrm{y}}+\mathrm{H} \cdot \kappa_{\mathrm{i}}\right) \geq 0 & \Rightarrow & \text { Incremento plástico }\end{array}\right.$

Observa-se que o índice 'ten' é referente a 'tentativa', uma vez que não se sabe se esse estado de tensão é verdadeiro ou não; $\sigma_{i+1}^{\text {ten }}$ é o vetor de tensões calculado na eq.(4.25); $\kappa_{i}$ é o valor de $\kappa$ no incremento de carga anterior, para cada nó analisado (também acumulativo em toda a análise). Vale dizer que o índice ' $i+1$ ' será utilizado para referenciar a iteração atual, enquanto que o símbolo ' $\Delta$ ' será utilizado quando se quiser referir a incremento.

$\mathrm{O}$ caso em que $\mathrm{f}_{\mathrm{i}+1}^{\text {ten }}>0$ representa um estado inadmissível de tensão para $\sigma^{\text {ten }}$. É necessário que se retorne desse estado de tensão para aquele da superfície de plastificação. Existem diversos processos que tratam desse retorno. Optou-se por um tipo de algoritmo de retorno não-associativo, ou seja, 
em que a direção do retorno é não-perpendicular à superfície de plastificação, com base no trabalho de Mesquita (2002). Sendo assim:

$\sigma_{i+1}=\sigma_{i+1}^{\text {ten }}-\Delta \sigma_{i+1}^{p}$

A parcela de deformação plástica em uma iteração atual pode ser escrita como:

$\varepsilon_{\mathrm{i}+1}^{\mathrm{p}}=\varepsilon_{\mathrm{i}}^{\mathrm{p}}+\Delta \varepsilon_{\mathrm{i}+1}^{\mathrm{p}}$

Em termos de tensões, pode-se escrever:

$\sigma_{i+1}^{p}=\sigma_{i}^{p}+\Delta \sigma_{i+1}^{p}$

O fluxo de deformação plástica pode ser escrito como:

$\Delta \varepsilon_{\mathrm{i}+1}^{\mathrm{p}}=\Delta \lambda_{\mathrm{i}+1} \cdot \mathrm{n}$

onde $\Delta \lambda_{i+1}$ é um escalar e 'n' representa um tensor unitário qualquer.

É interessante que o tensor ' $n$ ' esteja relacionado com a direção da tensão tentativa; assim, pode-se escrever 'n' como:

$\mathrm{n}=\frac{\mathrm{C}^{*} \cdot \sigma^{\text {ten }}}{\left|\mathrm{C}^{*} \cdot \sigma^{\text {ten }}\right|}$

onde a matriz $\mathrm{C}^{*}$, semelhantemente à matriz constitutiva elástica, pode representar qualquer grau de compressibilidade do material plastificado. No trabalho, adotou-se $C^{*}=\left(C^{e}\right)^{-1}$. Levando-se em consideração a igualdade anterior na eq.(4.30), tem-se:

$n=\frac{\left(C^{e}\right)^{-1} \cdot \sigma^{\text {ten }}}{\left|\left(C^{e}\right)^{-1} \cdot \sigma^{\text {ten }}\right|}$ 
Vale observar que a norma da eq.(4.31) é a Euclidiana, ou seja:

$$
|x|=\sqrt{x_{1}^{2}+x_{2}^{2}+x_{3}^{2}}
$$

Substituindo-se a eq.(4.31) na eq.(4.29), tem-se:

$$
\Delta \varepsilon_{i+1}^{p}=\Delta \lambda_{i+1} \cdot \frac{\left(C^{e}\right)^{-1} \cdot \sigma_{i+1}^{\text {ten }}}{\left|\left(C^{e}\right)^{-1} \cdot \sigma_{i+1}^{\text {ten }}\right|}
$$

Sabe-se que:

$$
\Delta \sigma_{\mathrm{i}+1}^{\mathrm{p}}=\mathrm{C}^{\mathrm{e}} \cdot \Delta \varepsilon_{\mathrm{i}+1}^{\mathrm{p}}
$$

Substituindo-se a eq.(4.33) na eq.(4.34), pode-se escrever que:

$$
\Delta \sigma_{i+1}^{p}=C^{e} \cdot \Delta \lambda_{i+1} \cdot \frac{\left(C^{e}\right)^{-1} \cdot \sigma_{i+1}^{\text {ten }}}{\left|\left(C^{e}\right)^{-1} \cdot \sigma_{i+1}^{\text {ten }}\right|}
$$

ou

$$
\Delta \sigma_{i+1}^{p}=\Delta \lambda_{i+1} \cdot \frac{\sigma_{i+1}^{\text {ten }}}{\left|\left(C^{e}\right)^{-1} \cdot \sigma_{i+1}^{\text {ten }}\right|}
$$

Substituindo a eq.(4.36) na eq.(4.26), tem-se:

$$
\sigma_{i+1}=\left(1-\frac{\Delta \lambda_{i+1}}{\left|\left(C^{e}\right)^{-1} \cdot \sigma_{i+1}^{\text {ten }}\right|}\right) \cdot \sigma_{i+1}^{\text {ten }}
$$

O cálculo de $\Delta \lambda_{i+1}$ pode ser feito utilizando-se a equação da superfície de plastificação escrita abaixo: 
$\mathrm{f}_{\mathrm{i}+1}=\left\|\sigma_{\mathrm{i}+1}\right\|-\frac{1}{\sqrt{3}}\left(\sigma_{\mathrm{y}}+\mathrm{H} \cdot \kappa_{\mathrm{i}+1}\right)=0$

O parâmetro de encruamento na iteração ' $i+1$ ' pode ser escrito com:

$\kappa_{\mathrm{i}+1}=\kappa_{\mathrm{i}}+\Delta \kappa_{\mathrm{i}+1}$

Substituindo a eq.(4.39) na eq.(4.38), tem-se:

$\left\|\sigma_{i+1}\right\|-\frac{\sigma_{y}}{\sqrt{3}}-\frac{H}{\sqrt{3}}\left(\kappa_{i}+\Delta \kappa_{i+1}\right)=0$

O escalar $\Delta \kappa$ é definido como sendo igual a um fluxo de deformação plástica equivalente escalar, conforme eq.(4.41) abaixo.

$\Delta \kappa_{\mathrm{i}+1}=\Delta \varepsilon_{\mathrm{i}+1}^{\mathrm{peq}}=\mathrm{k} \cdot\left|\Delta \varepsilon_{\mathrm{i}+1}^{\mathrm{p}}\right|$

onde k é um termo cujo valor deve ser escolhido de tal forma que a partir do modelo generalizado se possa recuperar o caso unidimensional. Com base em Mesquita (2002), o valor de k pode ser obtido a partir do coeficiente de Poisson (v) segundo a eq.(4.42) abaixo. Ver apêndice B.

$k=\frac{1}{\sqrt{1+2 v^{2}}}$

Substituindo-se as eq.(4.33) e (4.42) na eq.(4.41), tem-se:

$$
\Delta \kappa_{\mathrm{i}+1}=\mathrm{k} \cdot\left|\Delta \lambda_{\mathrm{i}+1}\right|=\mathrm{k} \cdot \Delta \lambda_{\mathrm{i}+1}=\frac{\Delta \lambda_{\mathrm{i}+1}}{\sqrt{1+2 v^{2}}}
$$

Substituindo-se a eq.(4.43) na eq.(4.40), tem-se: 
$\left\|\sigma_{i+1}\right\|-\frac{\sigma_{y}}{\sqrt{3}}-\frac{H}{\sqrt{3}}\left(\kappa_{i}+\frac{\Delta \lambda_{i+1}}{\sqrt{1+2 v^{2}}}\right)=0$

Substituindo a eq.(4.37) na eq.(4.44), tem-se:

$\left(1-\frac{\Delta \lambda_{i+1}}{\left|\left(\mathrm{C}^{\mathrm{e}}\right)^{-1} \cdot \sigma_{\mathrm{i}+1}^{\mathrm{ten}}\right|}\right) \cdot\left\|\sigma_{\mathrm{i}+1}^{\mathrm{ten}}\right\|-\frac{\sigma_{\mathrm{y}}}{\sqrt{3}}-\frac{\mathrm{H}}{\sqrt{3}} \cdot \kappa_{\mathrm{i}}-\frac{\mathrm{H}}{\sqrt{3}} \cdot \frac{\Delta \lambda_{\mathrm{i}+1}}{\sqrt{1+2 v^{2}}}=0$

ou

$\left\|\sigma_{i+1}^{\text {ten }}\right\|-\frac{\sigma_{y}}{\sqrt{3}}-\frac{H}{\sqrt{3}} \cdot \kappa_{i}-\frac{\Delta \lambda_{i+1}}{\left|\left(C^{e}\right)^{-1} \cdot \sigma_{i+1}^{\text {ten }}\right|} \cdot\left\|\sigma_{i+1}^{\text {ten }}\right\|-\frac{H \cdot \Delta \lambda_{i+1}}{\sqrt{3+6 v^{2}}}=0$

Observa-se que as 3 primeiras parcelas da eq.(4.46) formam a expressão de $\mathrm{f}^{\text {ten: }}$

$f^{\text {ten }}-\frac{\Delta \lambda_{i+1}}{\left|\left(C^{e}\right)^{-1} \cdot \sigma_{i+1}^{\text {ten }}\right|} \cdot\left\|\sigma_{i+1}^{\text {ten }}\right\|-\frac{H \cdot \Delta \lambda_{i+1}}{\sqrt{3+6 v^{2}}}=0$
$f^{\text {ten }}=\left(\frac{\left\|\sigma_{i+1}^{\text {ten }}\right\|}{\left|\left(C^{e}\right)^{-1} \cdot \sigma_{i+1}^{\text {ten }}\right|}+\frac{H}{\sqrt{3+6 v^{2}}}\right) \cdot \Delta \lambda_{i+1}$

Por fim, obtém-se a expressão para $\Delta \lambda_{i+1}$ :

$$
\Delta \lambda_{i+1}=\frac{f^{\text {ten }}}{\left(\frac{\| \sigma_{i+1}^{\text {ten }} \mid}{\left|\left(C^{e}\right)^{-1} \cdot \sigma_{i+1}^{\text {ten }}\right|}+\frac{H}{\sqrt{3+6 v^{2}}}\right)}
$$

Assim, o multiplicador plástico é obtido de forma fechada; retornando-se à eq.(4.28) para se atualizar as tensões residuais (plásticas), aplicando-as nas eq.(4.24) e (4.25). 
O processo iterativo é repetido até que determinadas condições de convergência sejam satisfeitas. No trabalho, 2 condições de convergência foram adotadas: convergência por deslocamento e convergência por tensão, ambas apresentadas a seguir.

i) Convergência por Tensão

Este critério de convergência é testado ao final de cada iteração:

$\frac{\left|\Delta \sigma_{i}^{p}\right|}{\left|\sigma_{1}^{p}\right|} \leq e_{t}$

onde $\sigma_{1}^{p}$ é o vetor de tensões plásticas da primeira iteração de cada incremento de carga.

Vale ressaltar que a norma presente na eq.(4.50) é a Euclidiana, ou seja, para o cálculo dessa norma deve-se somar os quadrados de todos os elementos do vetor e depois calcular a raiz quadrada desse número.

ii) Convergência por Deslocamento

No critério de convergência por deslocamento, a seguinte equação deve ser verificada, também ao final de cada iteração:

$$
\frac{\left|\Delta U_{i}\right|}{\left|U_{1}\right|} \leq e_{d}
$$

ou melhor:

$$
\frac{\left|U_{i}-U_{i-1}\right|}{\left|U_{1}\right|} \leq e_{d}
$$


Com o objetivo de ilustrar o processo de análise não-linear utilizado no trabalho, apresenta-se o algoritmo abaixo:

FAÇA IC = 1 ATÉ NIC

APLICA INCREMENTO DE CARGA

ENQUANTO NÃO HOUVER CONVERGÊNCIA FAÇA

CALCULA U $U_{i+1} \mathrm{EP}_{\mathrm{i}+1} \mathrm{EM} \mathrm{HU} \mathrm{U}_{\mathrm{i}+1}=\mathrm{GP}_{\mathrm{i}+1}+\mathrm{Bb}_{\mathrm{i}+1}+\mathrm{Q \sigma}_{\mathrm{i}}^{\mathrm{p}}$

$\sigma_{i+1}^{\text {ten }}=G^{\prime} P_{i+1}-H^{\prime} U_{i+1}+B^{\prime} b_{i+1}+Q^{\prime} \sigma_{i}^{p}$

FAÇA J = 1 ATÉ NNNL

CALCULA $\sigma_{i+1}^{\text {ten }}$ EM TENSÕES PRINCIPAIS

CALCULA f $f^{\text {ten }}$

SE $\mathrm{f}^{\text {ten }}>0$ ENTÃO

CALCULA $\Delta \lambda_{\mathrm{i}+1}\{$ eq.(4.49)\}

CALCULA $\Delta \sigma_{i+1}^{\mathrm{p}}\{$ eq.(4.36)\}

DETERMINA DIREÇÕES PRINCIPAIS

TRANSFORMA $\Delta \sigma_{i+1}^{p}$ PARA COORD. CARTESIANAS

CALCULA $\Delta \kappa_{i+1}\{$ eq. $(4.43)\}$

CALCULA $\kappa_{i+1}\{$ eq. $(4.39)\}$

CALCULA $\sigma_{i+1}\{$ eq. $(4.37)\}$

FIM SE

FIM FAÇA

CALCULA $\sigma_{\mathrm{i}+1}^{\mathrm{p}}$ \{eq.(4.28)\}

VERIFICA CONVERGÊNCIA

FIM FAÇA

FIM FAÇA

onde:

IC - é o número do incremento de carga;

NIC - é o número de incrementos de carga fornecido na análise;

NNNL - é o número de nós da região de plastificação. 
Deve-se observar que o acúmulo de tensão plástica é feito em coordenadas cartesianas (não principais) respeitando-se a propriedade de soma tensorial.

Para a análise elastoplástica dinâmica, considerou-se o carregamento sendo aplicado totalmente no passo de tempo, e dentro deste, uma análise iterativa considerando-se a não-linearidade física do material. Da maneira como a análise foi implementada no código computacional desenvolvido, a análise estática é realizada como um caso particular da análise dinâmica (da mesma forma como se fez para a análise elástica).

A seguir, serão apresentadas as particularidades do processo utilizado no trabalho, dando atenção também às analogias com o processo estático.

Inicialmente, o tempo total da análise é dividido em passos de tempo e o carregamento nesse passo de tempo é aplicado totalmente.

Em seguida, os carregamentos e deslocamentos dinâmicos prescritos são calculados para o tempo atual. Ver seção 9.3.3.

$\mathrm{O}$ vetor $\mathrm{F}_{\mathrm{s}+1}$ é calculado e somado com a parcela referente às tensões plásticas, segundo eq.(4.53) abaixo:

$$
F_{s+1}+\Delta t^{2} Q \sigma_{s+1}^{p}=F_{s}+G_{h} y_{s+1}+\Delta t^{2} Q \sigma_{s+1}^{p}
$$

Em seguida, inicia-se o processo iterativo até que as condições de convergência, que são as mesmas da análise estática, sejam satisfeitas.

No processo iterativo, inicialmente são determinados os deslocamentos e forças de superfície com a resolução do sistema de equações representado na eq.(4.12) rescrita abaixo:

$$
A X_{s+1}=F_{s+1}+\Delta t^{2} Q \sigma_{s+1}^{p}
$$

Com os deslocamentos calculados na iteração, obtém-se os vetores de aceleração e velocidade no passo de tempo atual, utilizando respectivamente as eq.(4.5) e (4.6) também rescritas: 


$$
\begin{aligned}
& \ddot{U}_{s+1}=\frac{1}{\Delta t^{2}}\left[2 U_{s+1}-5 U_{s}+4 U_{s-1}-U_{s-2}\right] \\
& \dot{U}_{s+1}=\frac{1}{6 \Delta t}\left[11 U_{s+1}-18 U_{s}+9 U_{s-1}-2 U_{s-2}\right]
\end{aligned}
$$

O vetor de tensões é calculado a partir dos vetores determinados anteriormente utilizando-se a eq.(3.62):

$$
\sigma(t)=G^{\prime} P(t)-H^{\prime} U(t)+B^{\prime} b(t)-C^{\prime} \dot{U}(t)-M^{\prime} U^{\prime}(t)+Q^{\prime} \sigma^{p}(t)
$$

O algoritmo de análise não-linear é o mesmo utilizado para o caso estático, descrito anteriormente, ou seja, para cada nó de célula da discretização da região definida como de plastificação, transformam-se as tensões de tentativa (obtidas na eq.(3.62)) para tensões principais; calcula-se a função de tentativa $f^{\text {ten; }}$ se houver plastificação ( $f^{\text {ten }}>0$ ), calcula-se $\Delta \lambda_{i+1}$ e $\Delta \sigma_{i+1}^{p}$; determinam-se as direções principais; transforma-se $\Delta \sigma_{i+1}^{p}$ para coordenadas cartesianas e calcula-se $\Delta \kappa_{i+1}, \kappa_{i+1}$ e $\sigma_{i+1}$. Por fim, acumula-se $\Delta \sigma_{i+1}^{p}$ em $\sigma_{i+1}^{p}$ e verifica-se a convergência.

Da mesma forma como se fez para o caso estático, apresenta-se o algoritmo para a análise não-linear dinâmica desenvolvido. 


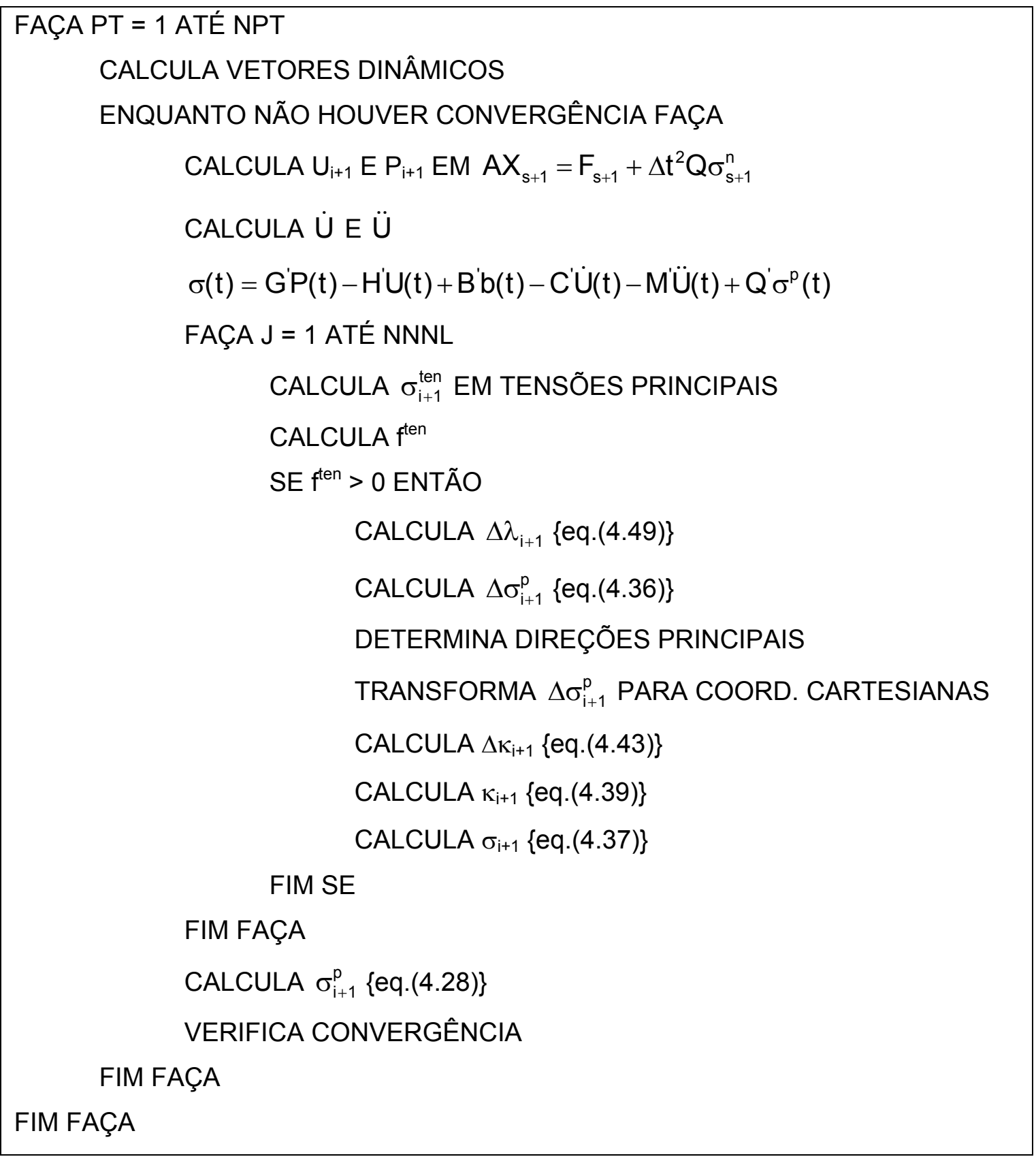

Para se realizar uma análise estática utilizando o código computacional desenvolvido, basta que se forneça valores nulos para a densidade ' $\rho$ ' e coeficiente de amortecimento 'c'; o número de passos de tempo será entendido como número de incrementos de carga, e pode-se fornecer um valor igual ao tempo total da análise, assim, o valor de $\Delta$ t será 1 (ver eq.(4.54)); o carregamento pode ser aplicado linearmente no tempo para simular sua aplicação incremental. Ver figura 4.2. O programa desenvolvido também dispõe de um recurso que economiza tempo de processamento evitando o "looping" 
dos nós de plastificação: basta que simplesmente se forneça valor nulo para a tensão de plastificação $\sigma_{y}$.

$$
\Delta \mathrm{t}=\frac{\mathrm{t}}{\mathrm{n}}
$$

sendo 't' o tempo total e 'n' o número de passos de tempo.
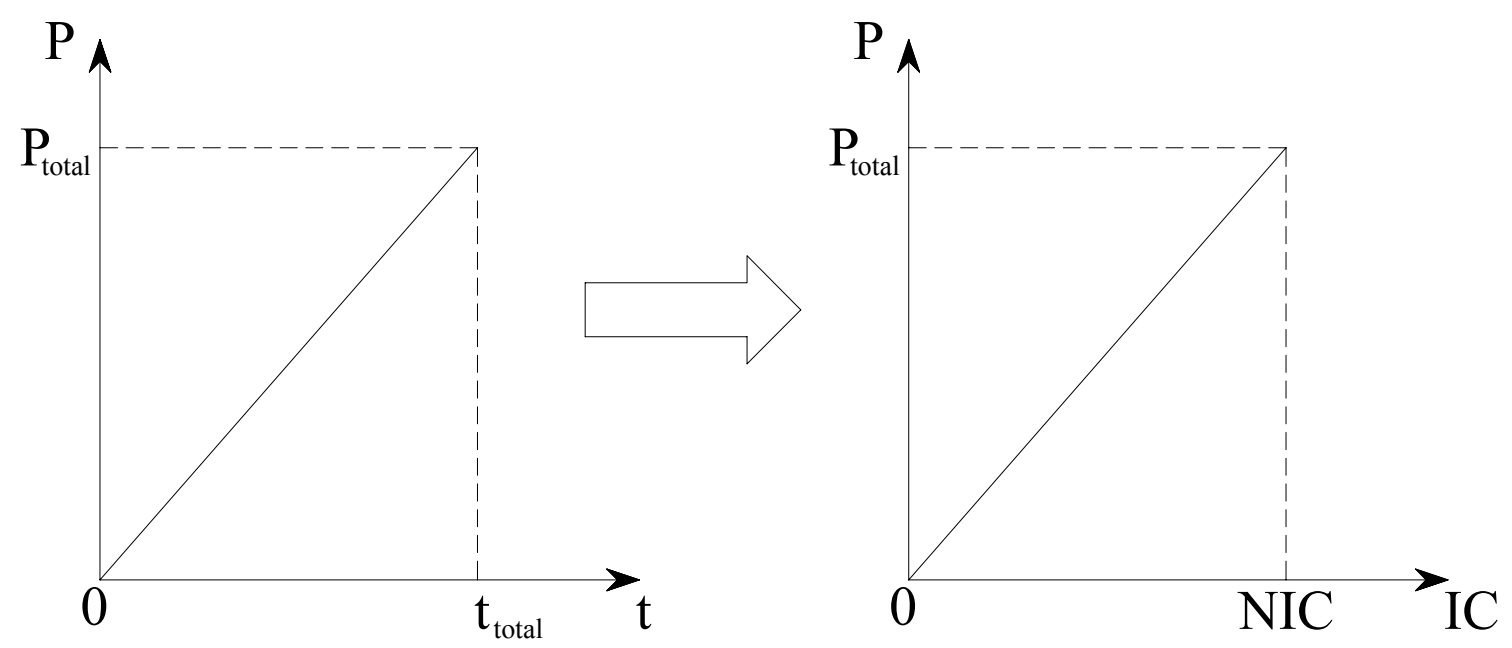

Figura 4.2 - Simulação de carregamento incremental

\subsection{EXEMPLOS}

Nesta seção serão apresentados 4 exemplos com o intuito de validar as técnicas desenvolvidas e o programa implementado.

\subsubsection{Exemplo 4.1}

No exemplo 4.1, analisa-se uma viga submetida à ação de uma carga súbita de tração, de valor constante com o tempo. Figura 4.3. Os resultados obtidos são comparados com os valores analíticos apresentados por Mansur e Brebbia (1984). 


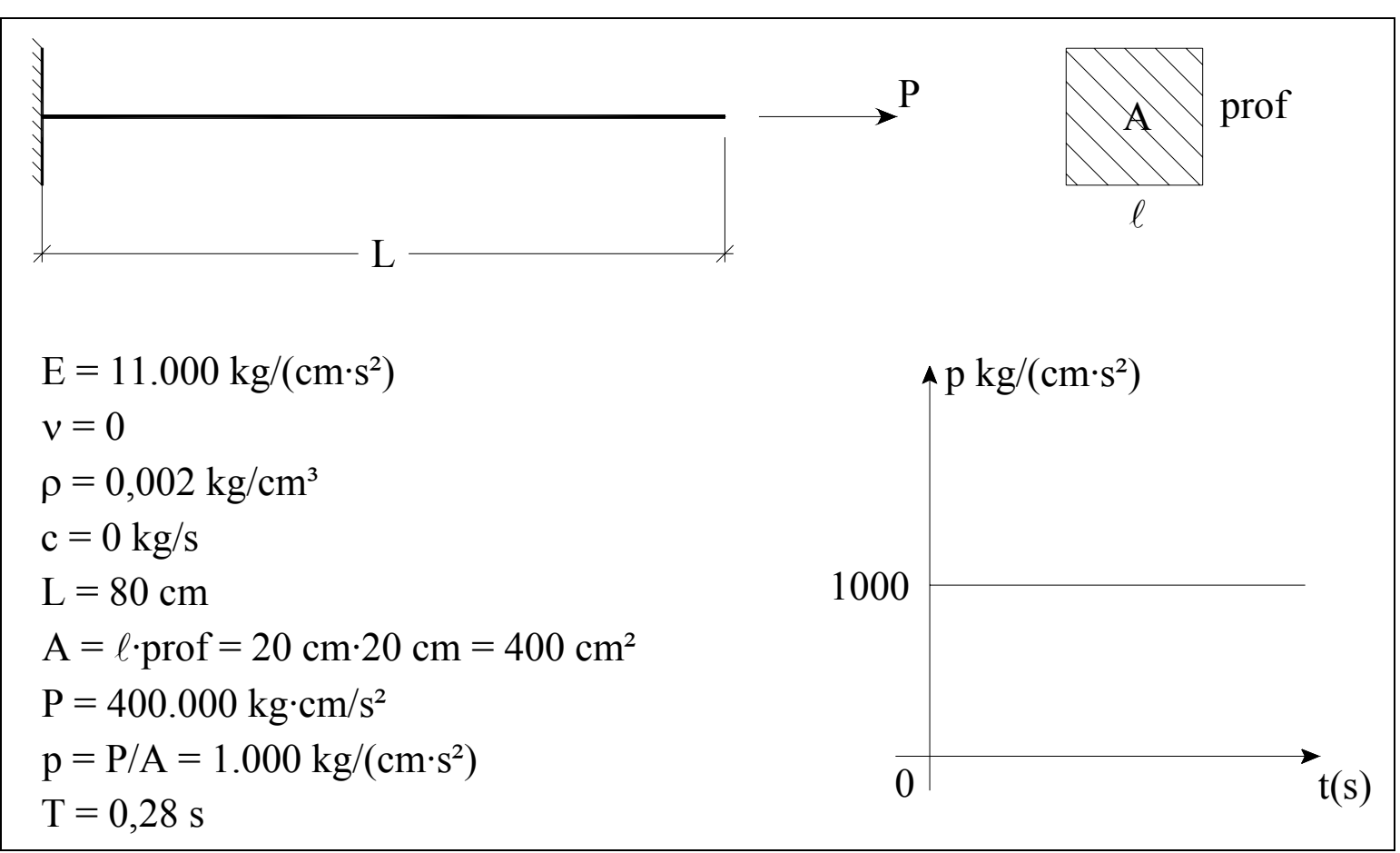

Figura 4.3 - Exemplo 4.1

São apresentados resultados de deslocamentos e reações de apoio, onde foram utilizadas várias possibilidades de discretização e intervalos de tempo $\Delta$ t. Criou-se um código para as discretizações envolvidas: os 3 primeiros números são relativos à discretização do contorno (nd $\ell$, ndp e ndc) e os 3 números seguintes são relativos à discretização do domínio (nd $\ell$ c, ndpc e ndcc). Caso o número de divisões seja o mesmo para contorno e domínio, só são apresentados os 3 números de divisões. Ver capítulo 9.

Para a geração das discretizações, utilizou-se um gerador de malhas desenvolvido no trabalho, onde maiores detalhes podem ser vistos no capítulo 9.

As figuras 4.4 a 4.8 apresentam o deslocamento em pontos do contorno do sólido, para discretizações 224 (figura 4.4) e 2210 (demais) para o contorno e $\Delta \mathrm{t}=0,001 \mathrm{~s}$, onde se variou a discretização para o domínio do sólido. 


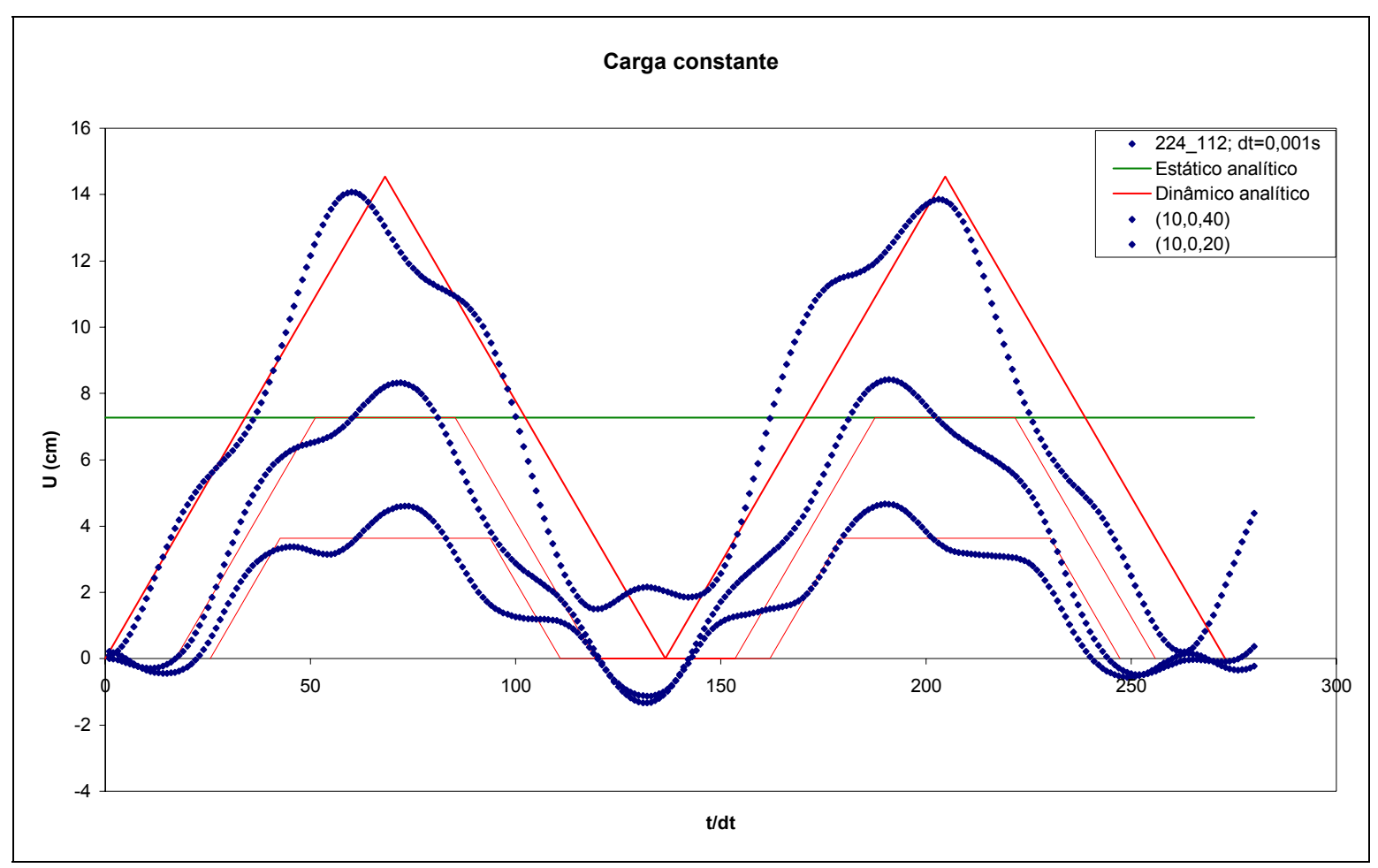

Figura 4.4 - Deslocamentos no contorno - 224_112; $\mathrm{dt}=0,001 \mathrm{~s}$

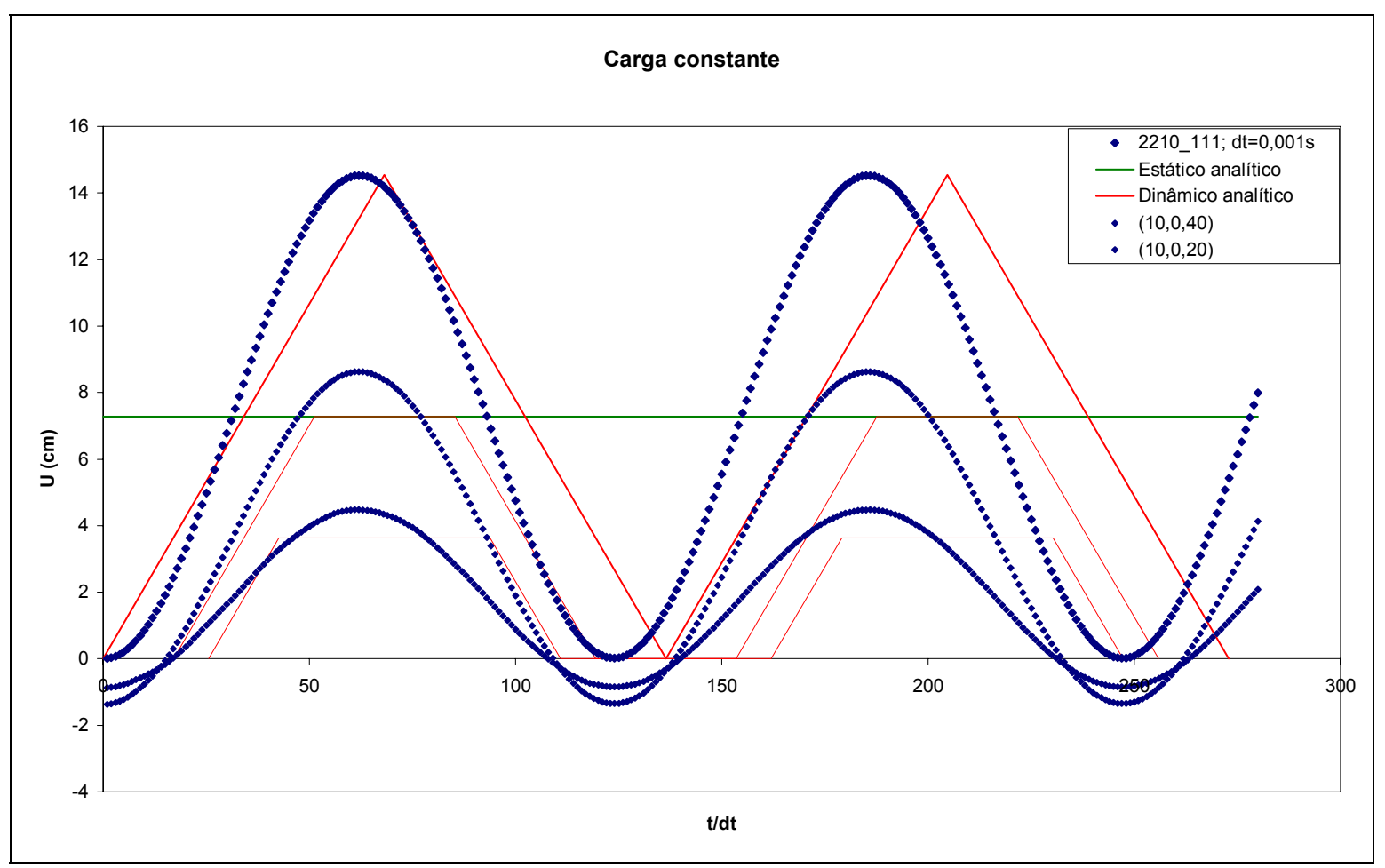

Figura 4.5 - Deslocamentos no contorno $-2210 \_111 ; \mathrm{dt}=0,001 \mathrm{~s}$ 


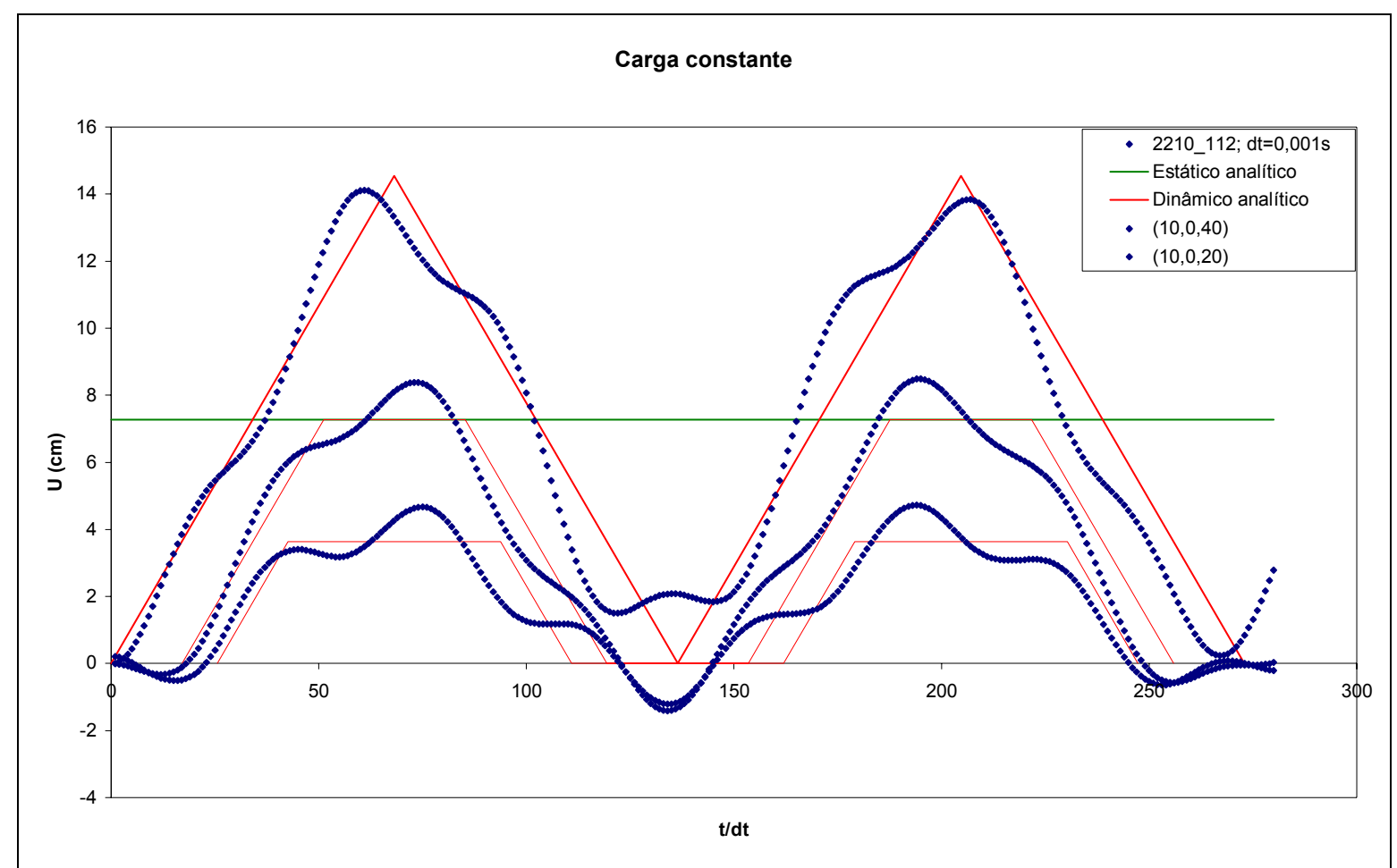

Figura 4.6 - Deslocamentos no contorno - 2210_112; dt =0,001 s

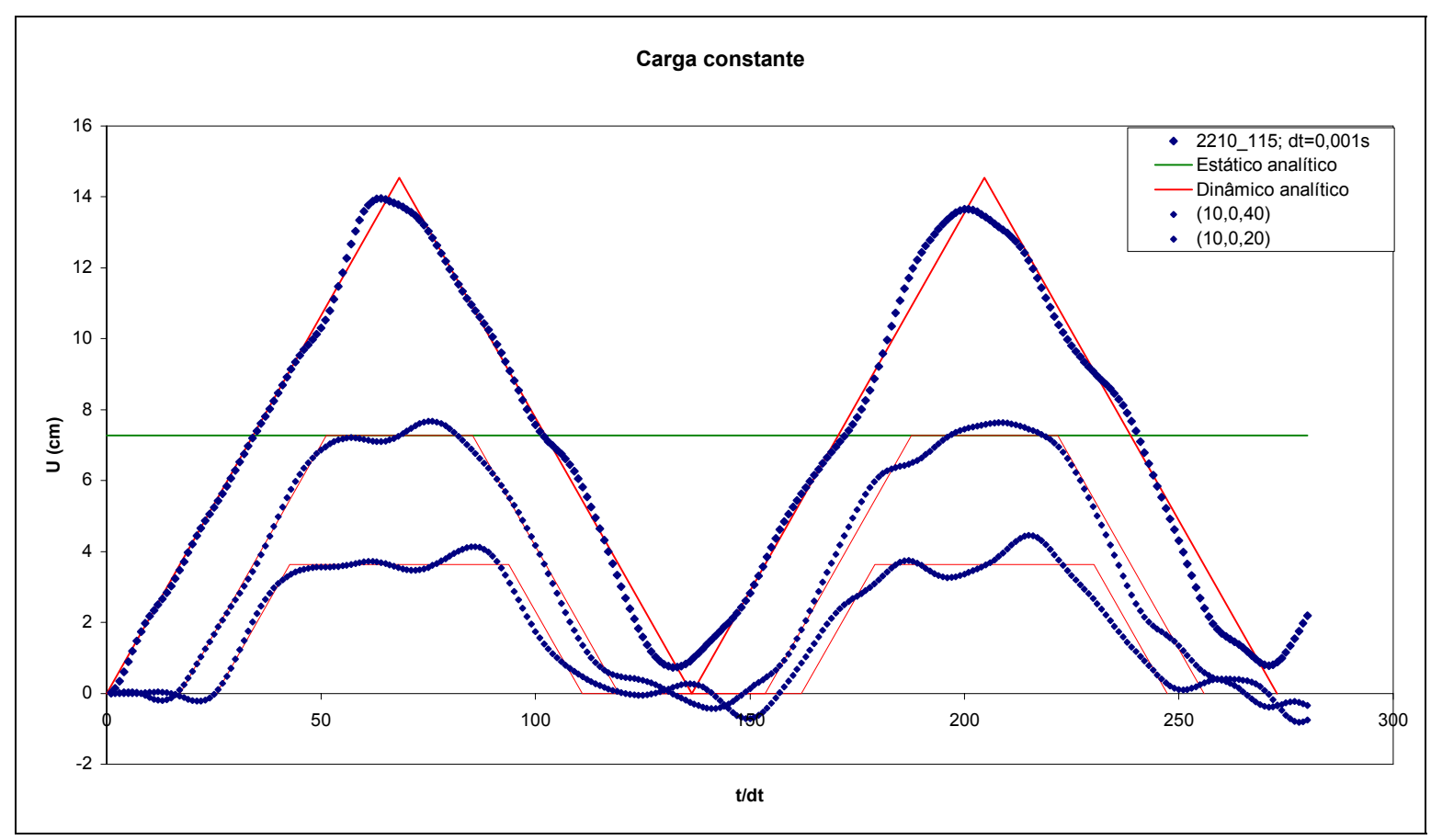

Figura 4.7 - Deslocamentos no contorno - 2210_115; $\mathrm{dt}=0,001 \mathrm{~s}$ 


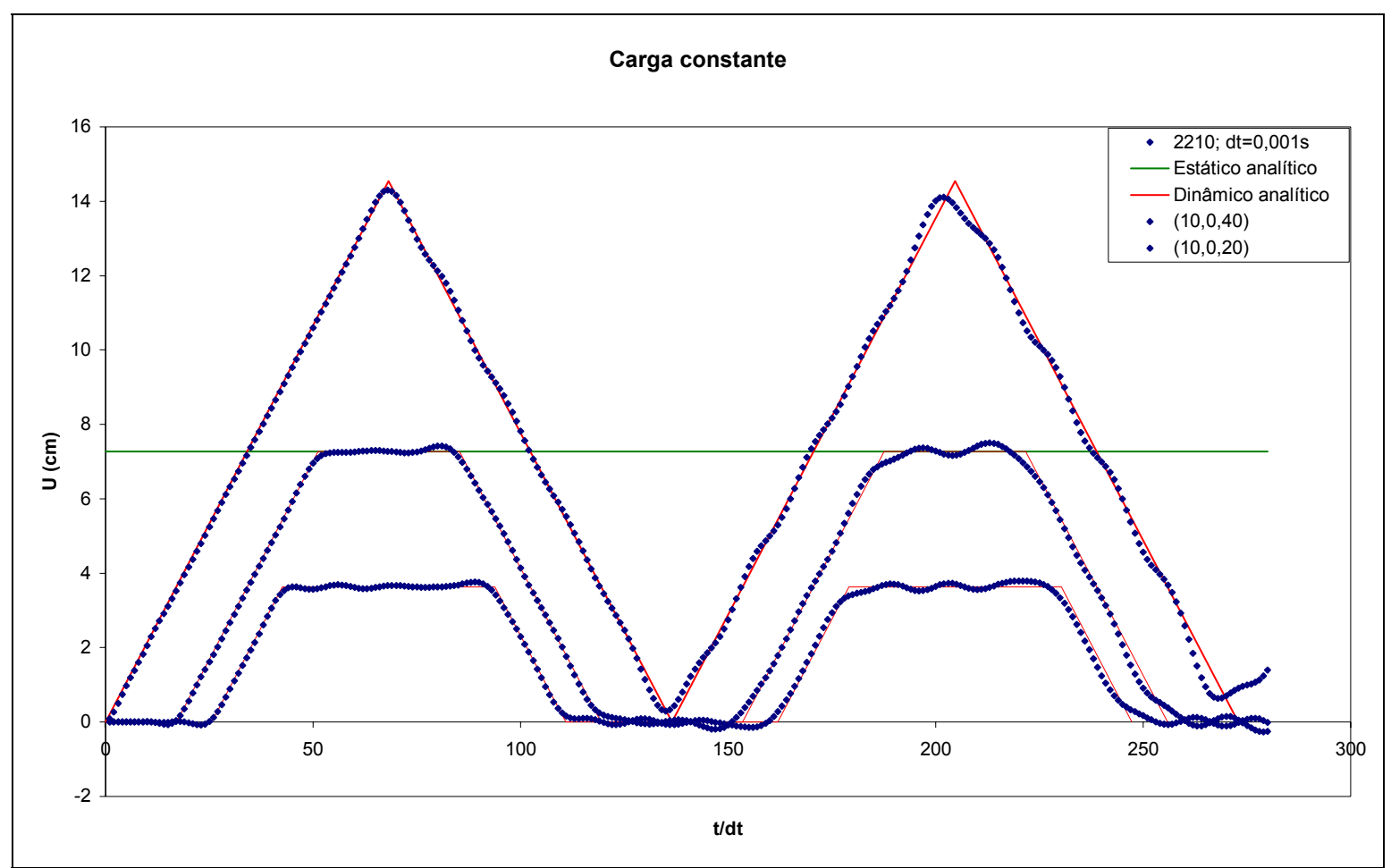

Figura 4.8 - Deslocamentos no contorno $-2210 ; \mathrm{dt}=0,001 \mathrm{~s}$

Observando-se as 5 figuras acima, nota-se uma perfeita convergência para os valores analíticos, com o aumento do refinamento da discretização do domínio para uma discretização adequada no contorno do sólido.

A figura 4.9 apresenta o deslocamento em pontos internos do sólido, ainda para a discretização 2210 para o contorno, e 2210 para o domínio; e $\Delta t=$ $0,001 \mathrm{~s}$. Também se observa uma excelente qualidade dos resultados obtidos. Comparando-se esta figura com a 4.8 , observa-se que os resultados para os dois pontos que não estão na extremidade livre $(X 3=20$ e X3 $=40)$, são semelhantes, estando no contorno ou no domínio do mesmo.

A figura 4.10 apresenta os resultados para os deslocamentos no contorno para uma discretização do tipo 2210_1110. Observa-se que para esse tipo de discretização, houve perda de estabilidade na análise; por esse motivo, foram apresentados apenas os resultados até o passo de tempo de número 143. Isto ocorre pois o quadrado do intervalo de tempo adotado é um número muito pequeno e, quando multiplicado pela matriz $G$ também muito pequena, leva à perda de precisão no processo numérico que relaciona esta matriz com a matriz $\overline{\mathrm{H}}$. 


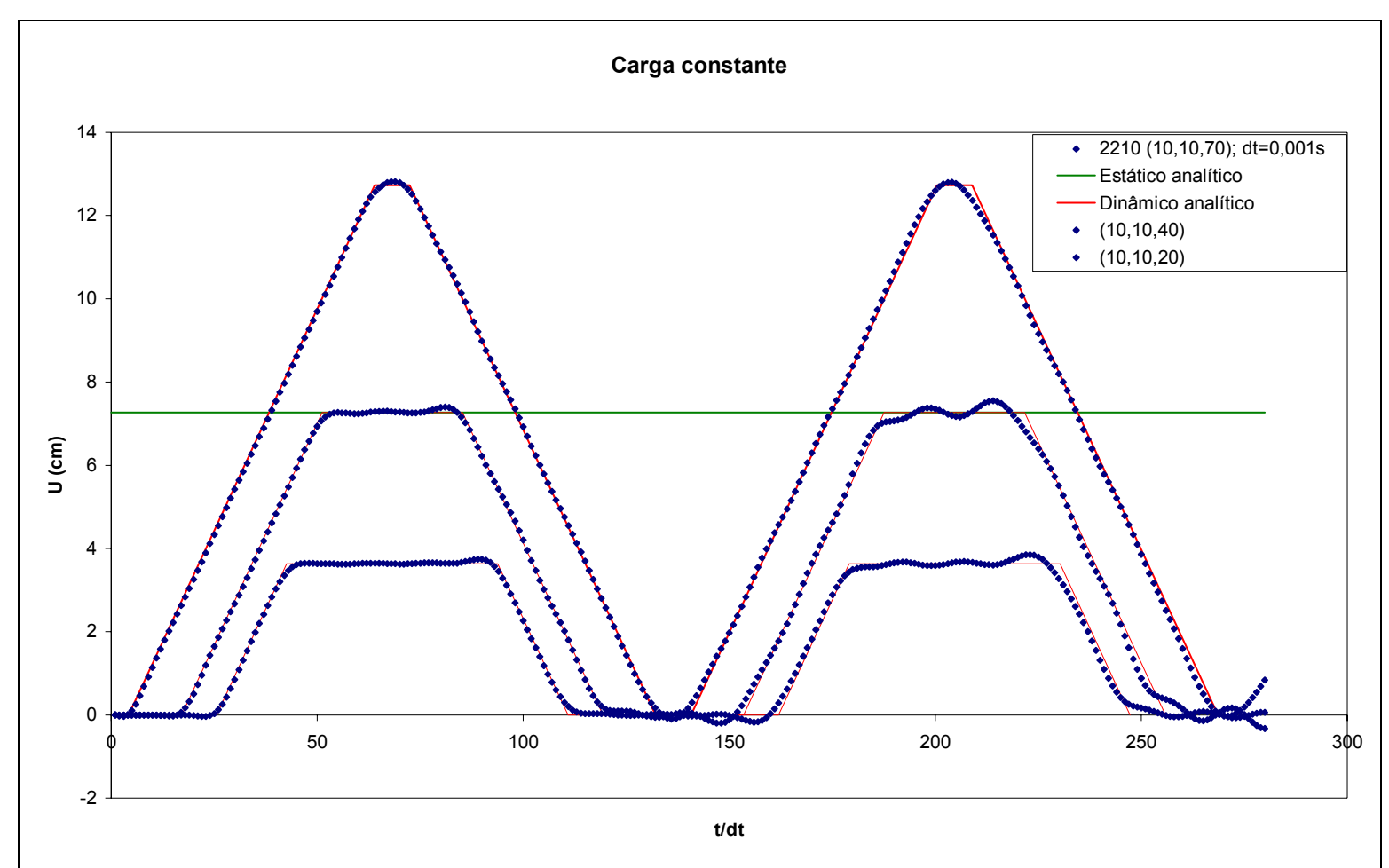

Figura 4.9 - Deslocamentos internos $-2210 ; \mathrm{dt}=0,001 \mathrm{~s}$

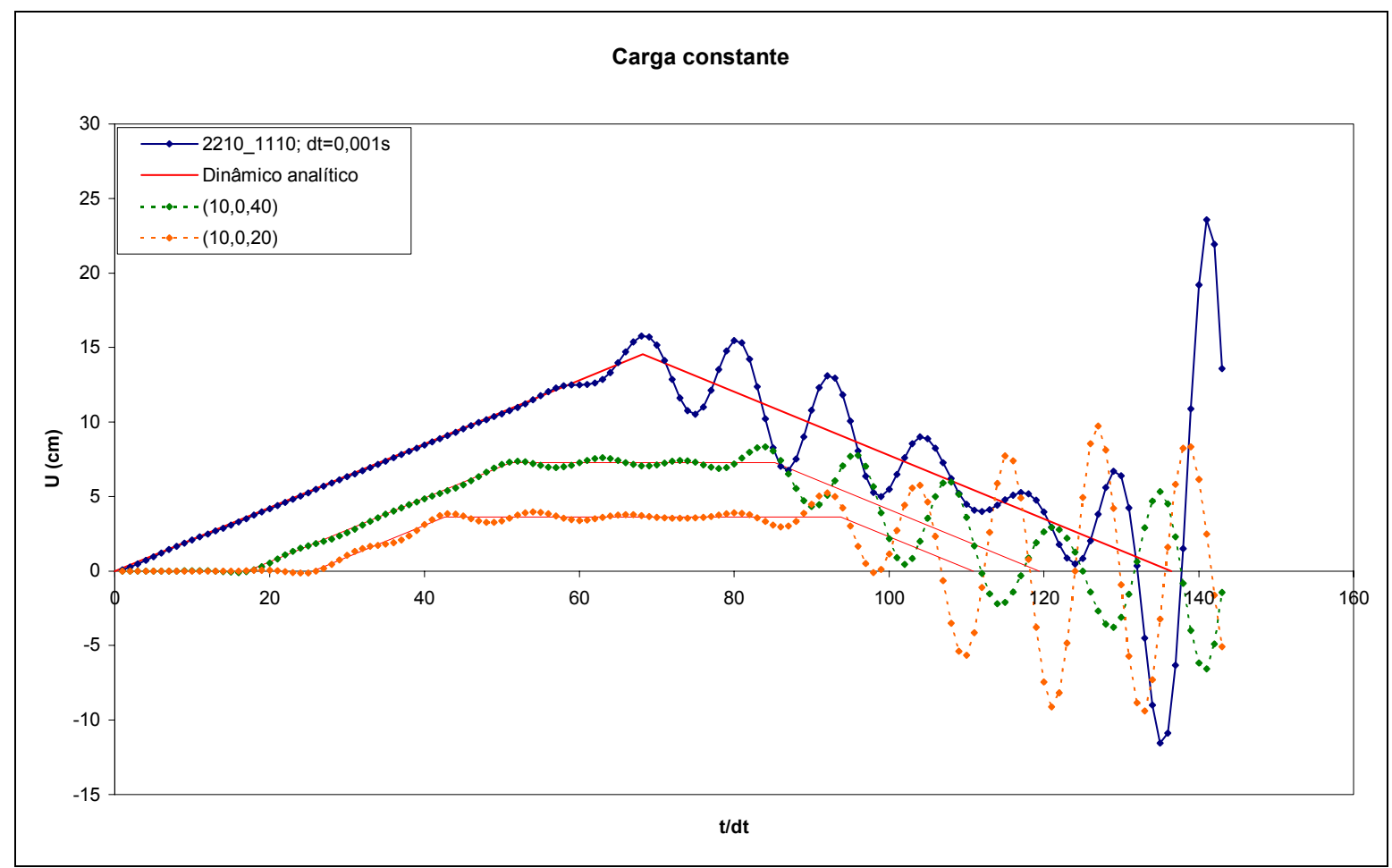

Figura 4.10 - Deslocamentos no contorno - 2210_1110; $\mathrm{dt}=0,001 \mathrm{~s}$ 
As figuras 4.11 e 4.12 apresentam os resultados para as reações de apoio para as discretizações do tipo 2210_115 e 2210, onde também se observa a boa precisão dos resultados obtidos.

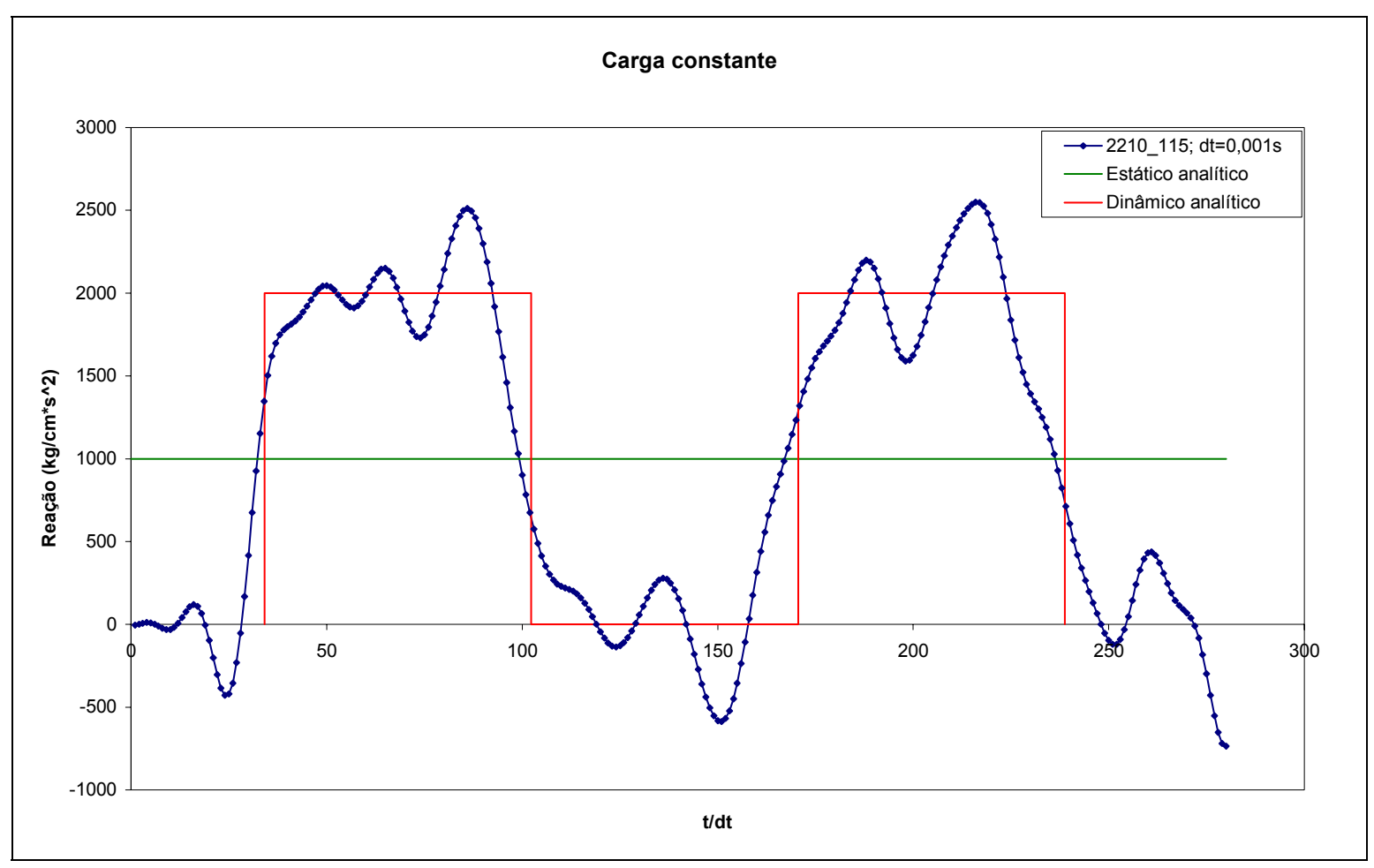

Figura 4.11 - Reações de apoio - 2210_115; dt =0,001 s

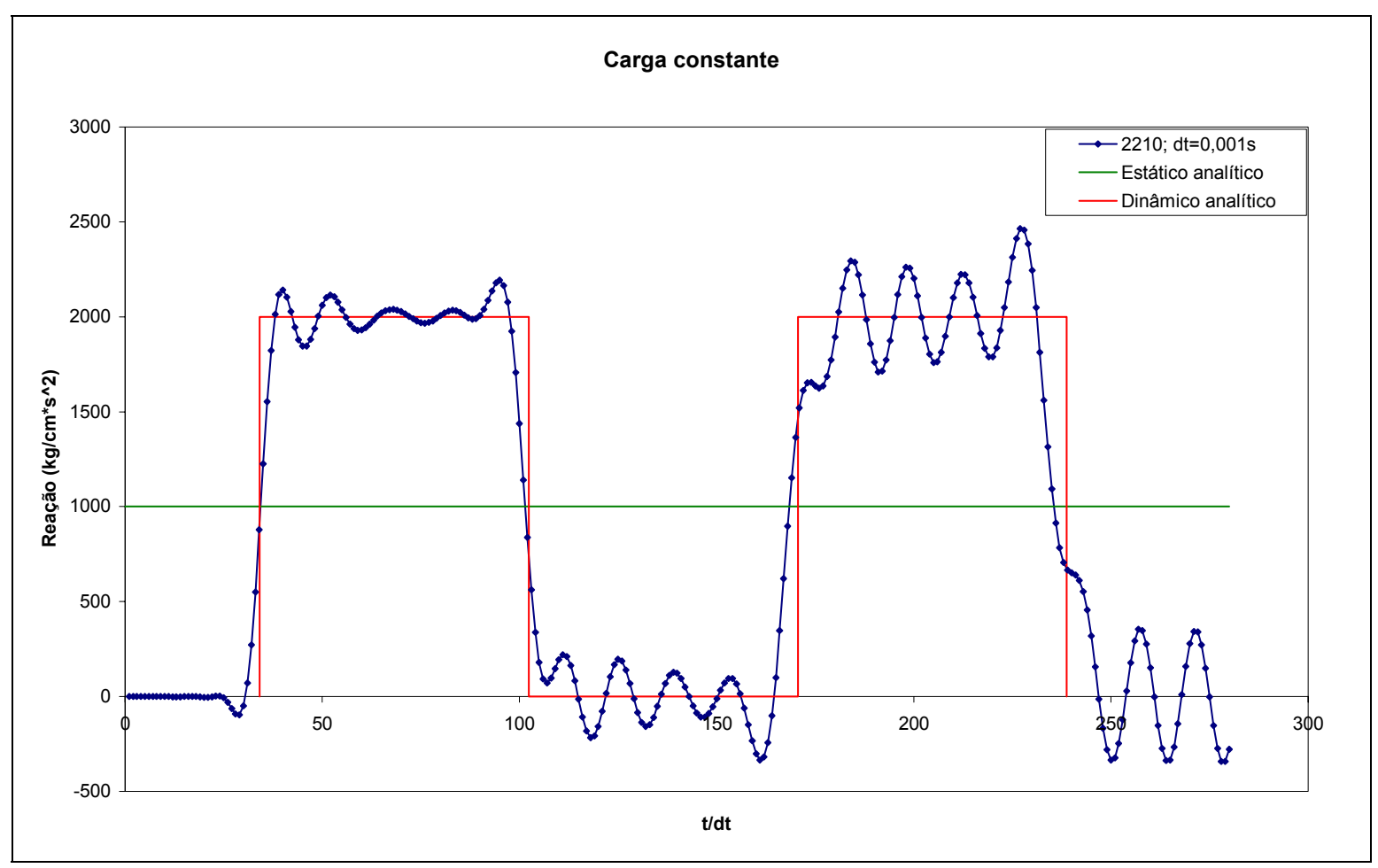

Figura 4.12 - Reações de apoio $-2210 ; \mathrm{dt}=0,001 \mathrm{~s}$ 
As figuras 4.13 e 4.14 representam os deslocamentos no contorno para as discretizações 2210_1110 e 2210; para $\Delta t=0,002 \mathrm{~s}$, onde para este valor de $\Delta t$, não houve perda de estabilidade na análise, como aconteceu com $\Delta t=$ 0,001 s, para a discretização 2210_1110.

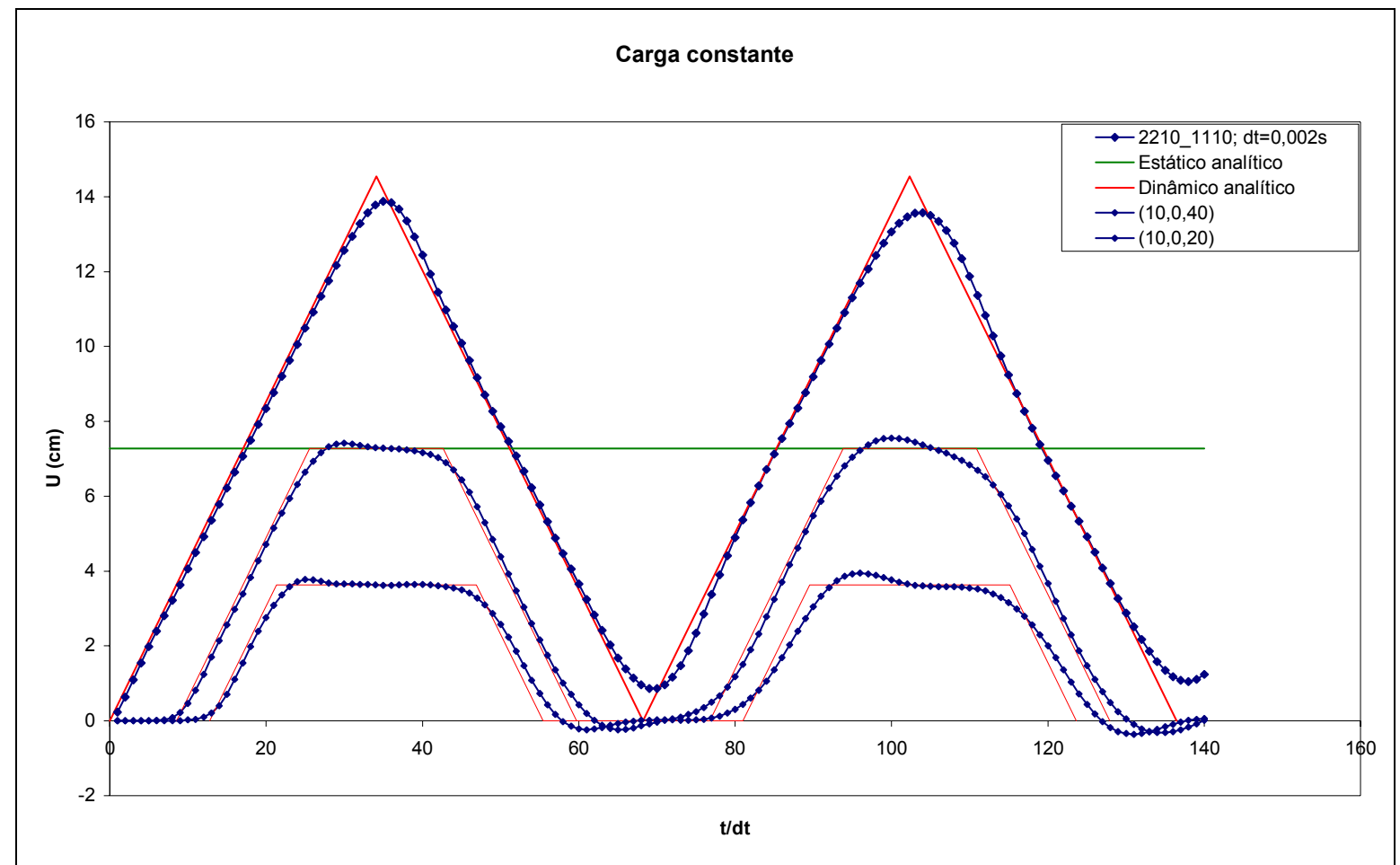

Figura 4.13 - Deslocamentos no contorno $-2210 \_1110 ; \mathrm{dt}=0,002 \mathrm{~s}$

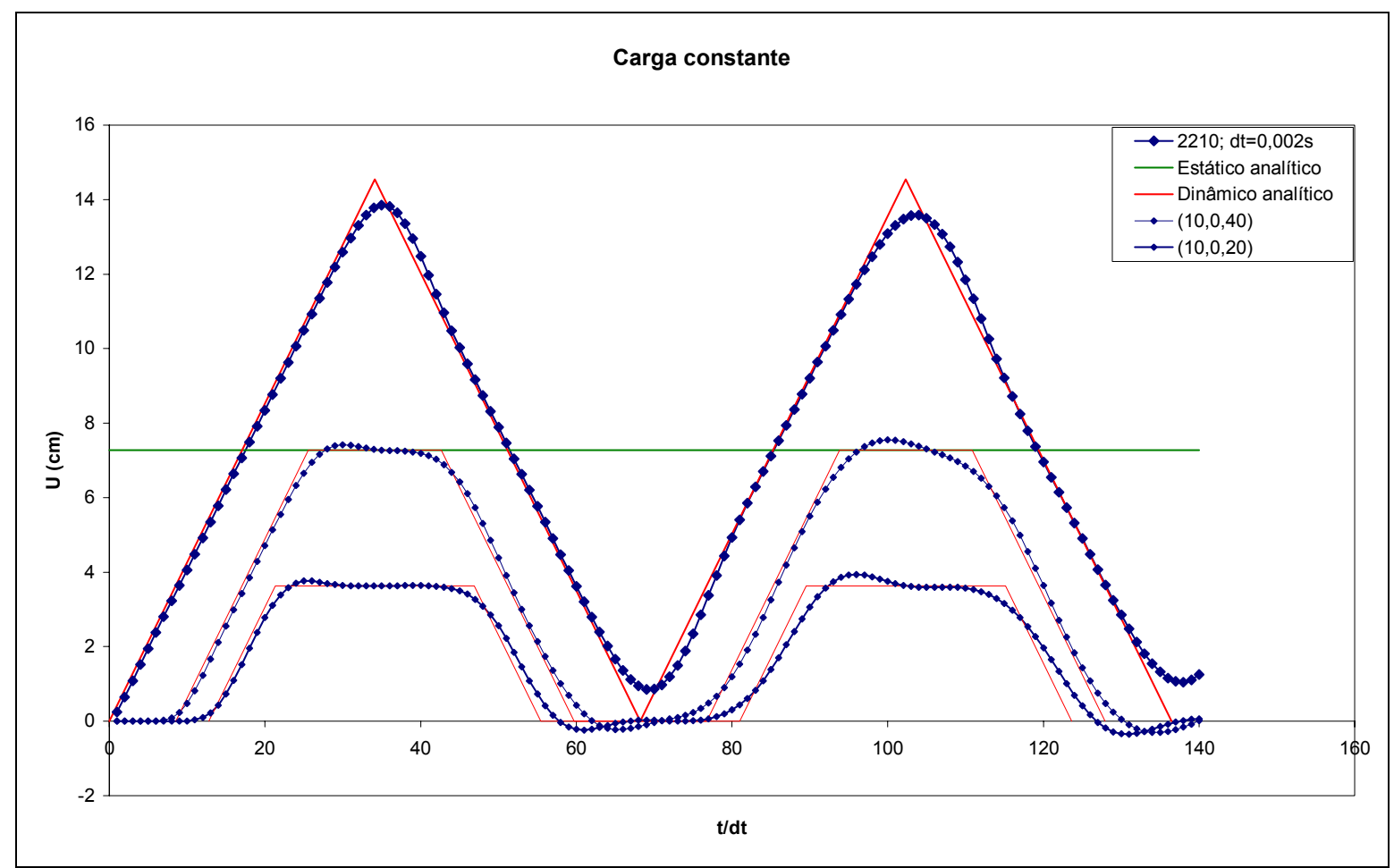

Figura 4.14 - Deslocamentos no contorno $-2210 ; \mathrm{dt}=0,002 \mathrm{~s}$ 
De maneira geral, os resultados para este problema são fortemente influenciados pela densidade de células na direção predominante do movimento (longitudinal). Pode-se afirmar também que a discretização do contorno também influencia significativamente na análise. No próximo exemplo será mostrado que, para problemas de baixa freqüência (no caso, flexão), a dependência em relação à densidade de células diminui significativamente.

\subsubsection{Exemplo 4.2}

O segundo exemplo analisa uma viga engastada sujeita a ação de uma carga transversal, subitamente aplicada, na extremidade livre. Os dados envolvidos no problema estão apresentados na figura 4.15. Este exemplo foi apresentado na sua versão bidimensional em Coda e Venturini (1999b).

Utilizou-se uma malha do tipo 2410 para a discretização do contorno da viga, onde a largura ( $\ell$ ), profundidade (prof) e comprimento (L) estão representados na figura 4.15 .

A figura 4.16 apresenta os valores dos deslocamentos transversais (direção do carregamento) no nó central da extremidade livre da viga. Foram admitidos valores de amortecimento nulo (c $=0 \mathrm{~kg} / \mathrm{s}$ ) e $\mathrm{c}=0,25 \mathrm{~kg} / \mathrm{s}$. $\mathrm{O}$ domínio da viga foi discretizado com as seguintes densidades de células: 2410 , 1110, 115 e 111. Por uma questão de comparação, são apresentados ainda resultados de análises utilizando-se o Método dos Elementos Finitos para a análise dinâmica de barras, com o integrador temporal de Newmark $\beta$, que é bastante adequado para este método ${ }^{1}$.

\footnotetext{
${ }^{1}$ O Método dos Elementos Finitos é apresentado no capítulo 7.
} 


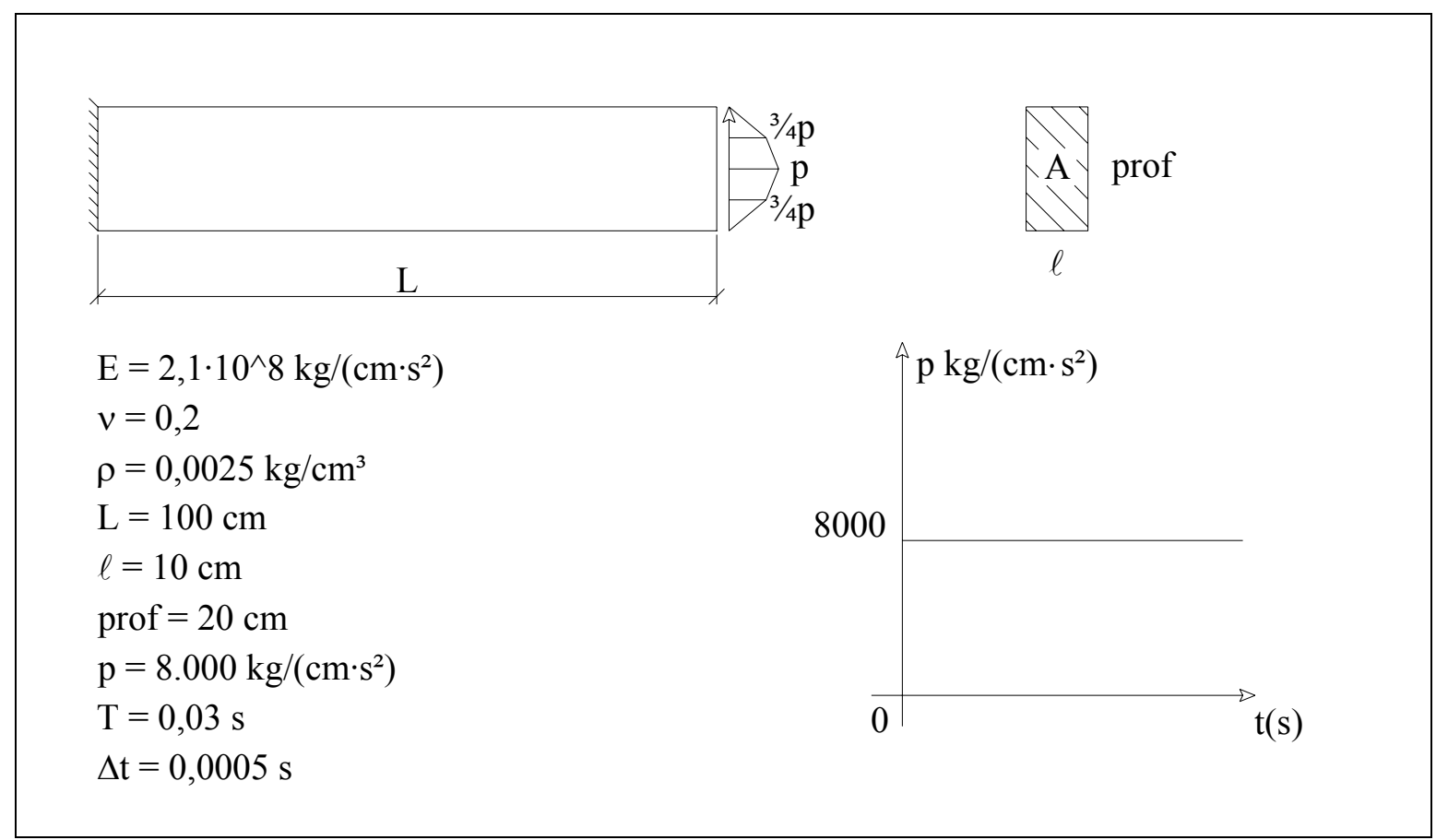

Figura 4.15 - Exemplo 4.2

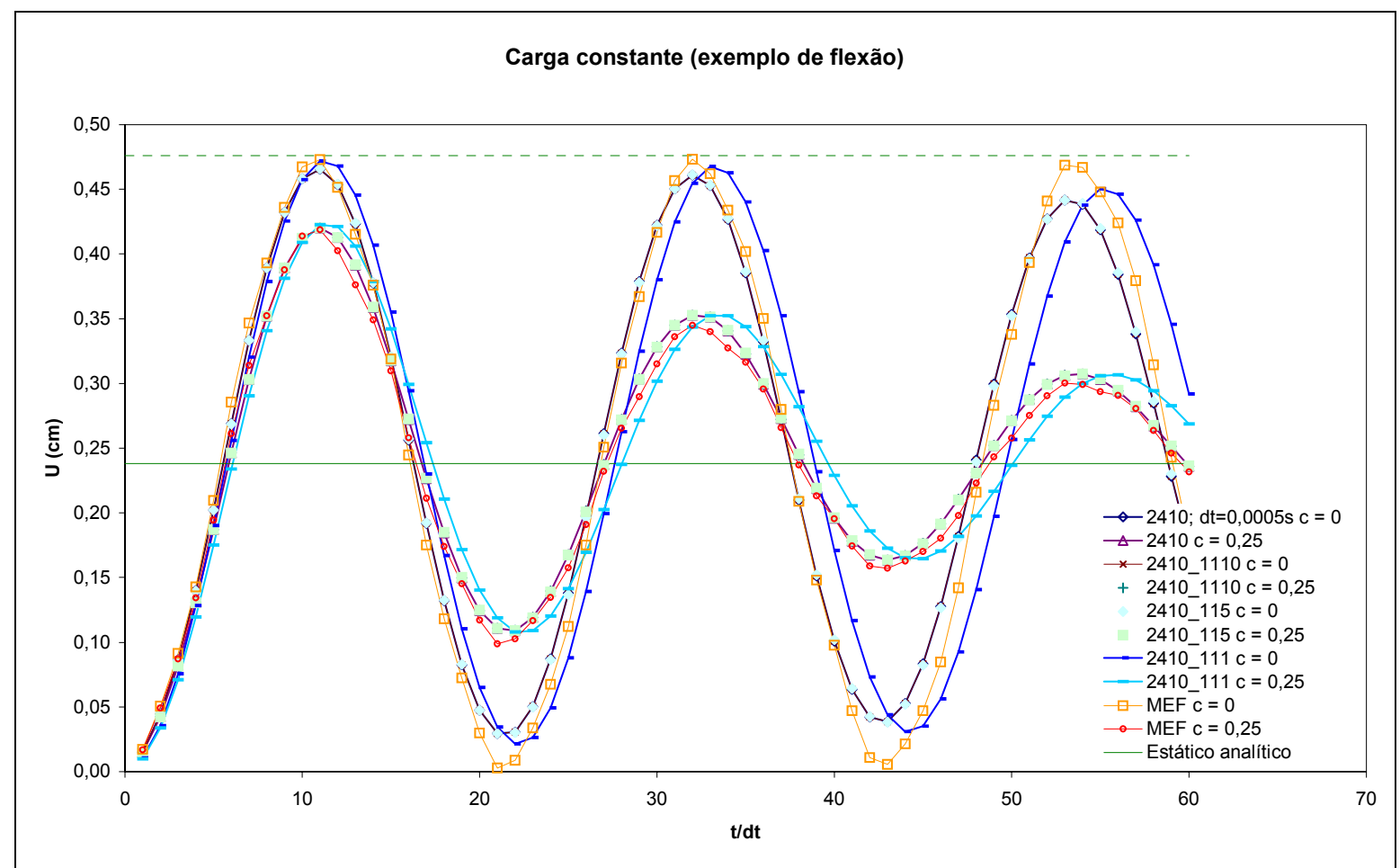

Figura 4.16 - Deslocamentos na extremidade livre da viga

Analisando-se os resultados da figura 4.16, pode-se notar que estes foram bastante próximos, o que mostra que a influência da discretização do domínio da viga, para este caso de sólido sob flexão, é menor que a influência da discretização do contorno. 
As figuras 4.17 e 4.18 apresentam, separadamente, os resultados da figura 4.16, para $\mathrm{c}=0 \mathrm{~kg} / \mathrm{s}$ e $\mathrm{c}=0,25 \mathrm{~kg} / \mathrm{s}$, respectivamente.

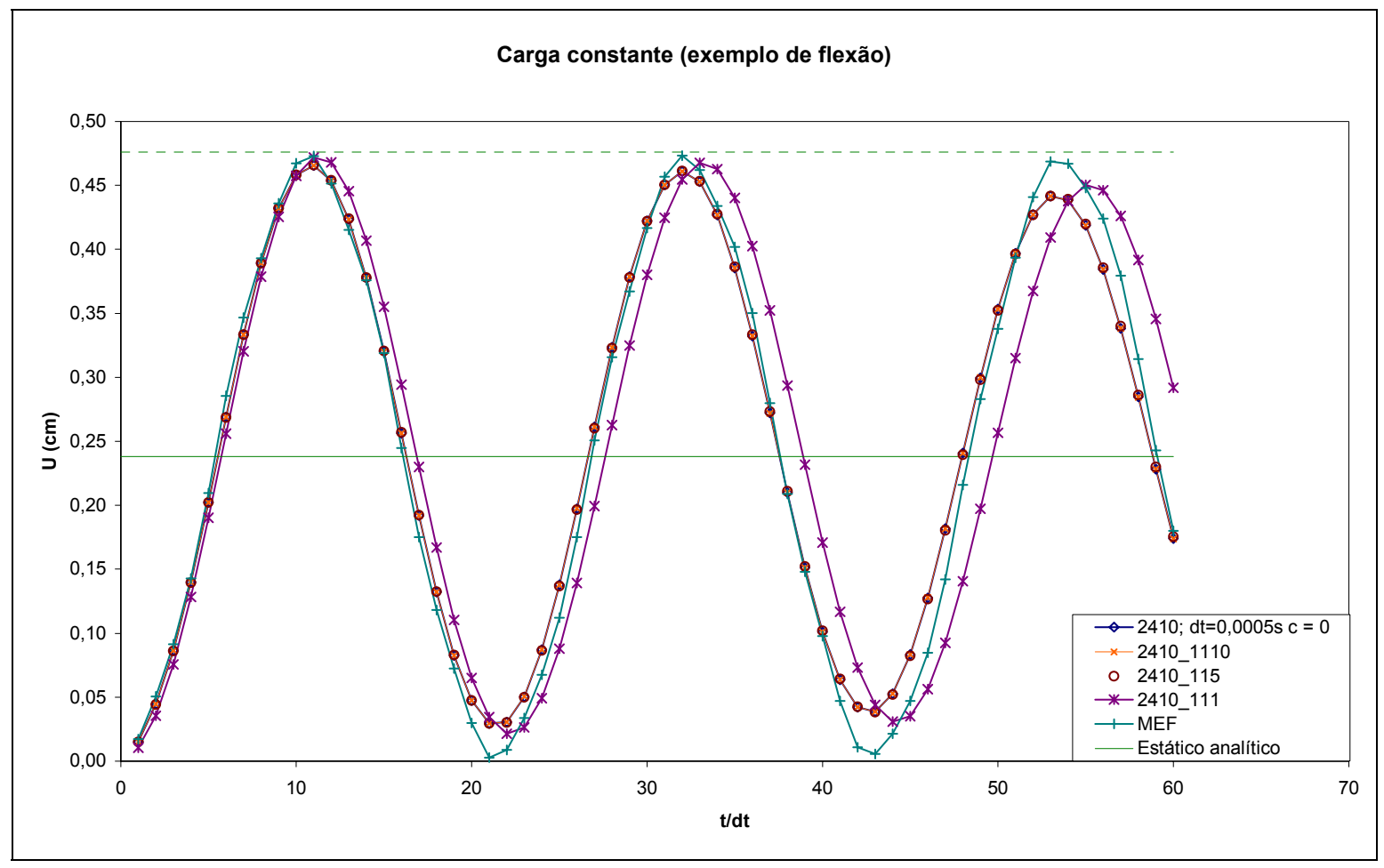

Figura 4.17 - Deslocamentos na extremidade livre da viga $-\mathrm{c}=0$

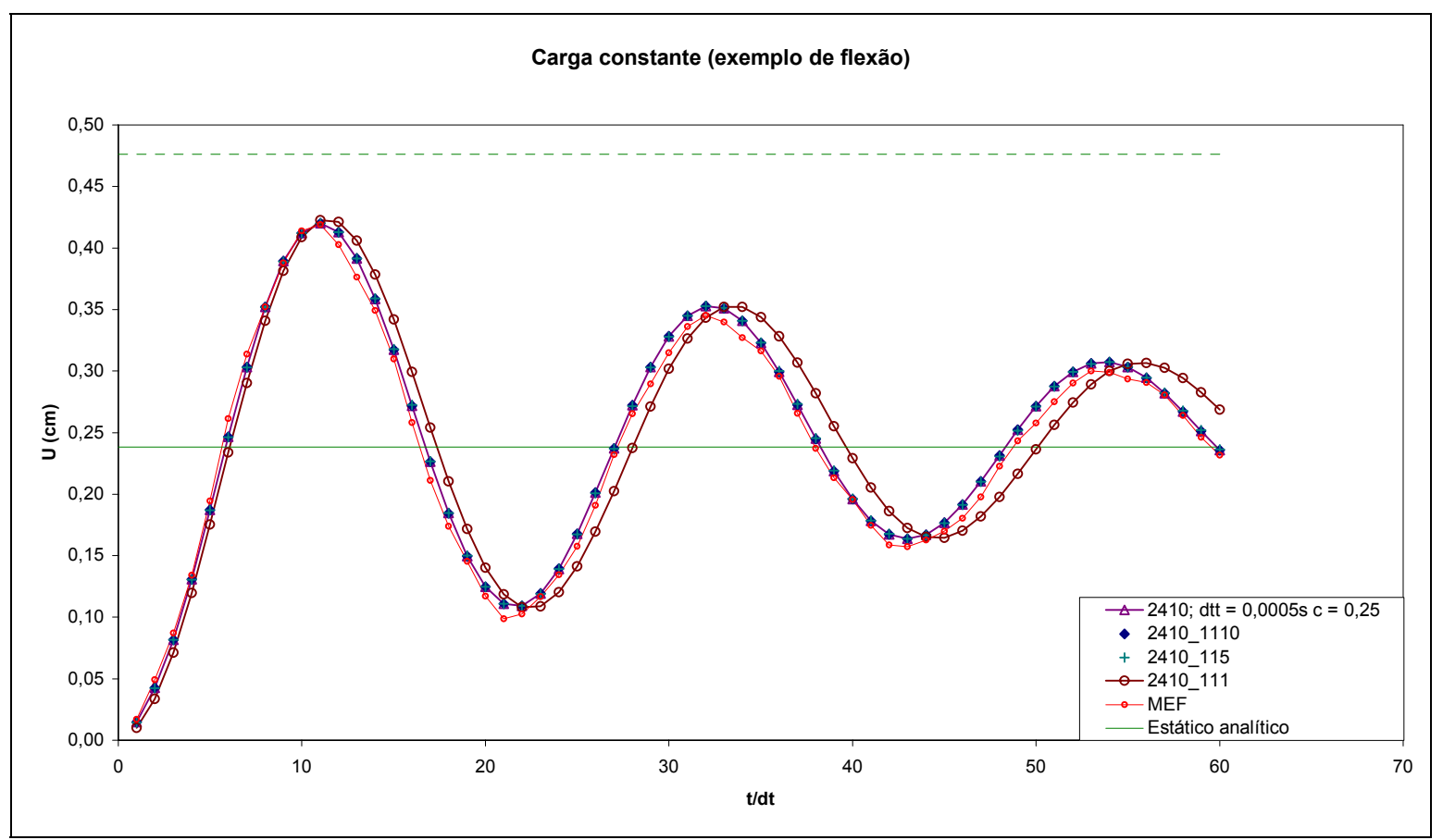

Figura 4.18 - Deslocamentos na extremidade livre da viga $-\mathrm{c}=0,25 \mathrm{~kg} / \mathrm{s}$ 
Observando-se a figura 4.17, onde o amortecimento físico é nulo (c = 0), nota-se que o amortecimento numérico do Método dos Elementos de Contorno (Houbolt) é considerável, em comparação com os resultados obtidos com o Método dos Elementos Finitos. Porém, é de notório saber, apesar de não citado explicitamente na literatura, que o algoritmo de Newmark $\beta$ é instável para aplicações com o MEC. Além disso, também aparece movimento longitudinal e transversal devido à análise ser tridimensional. Estes movimentos causam uma redistribuição da energia envolvida no movimento transversal da viga, ajudando na redução do pico inicial da formulação.

\subsubsection{Exemplo 4.3}

O terceiro exemplo trata da análise estática elastoplástica de um sólido paralelepipédico engastado em uma face (livre para se deformar transversalmente) e sujeito a uma carga de tração uniformemente distribuída na face oposta, conforme figura 4.19, onde são apresentados dados complementares a respeito do problema analisado.

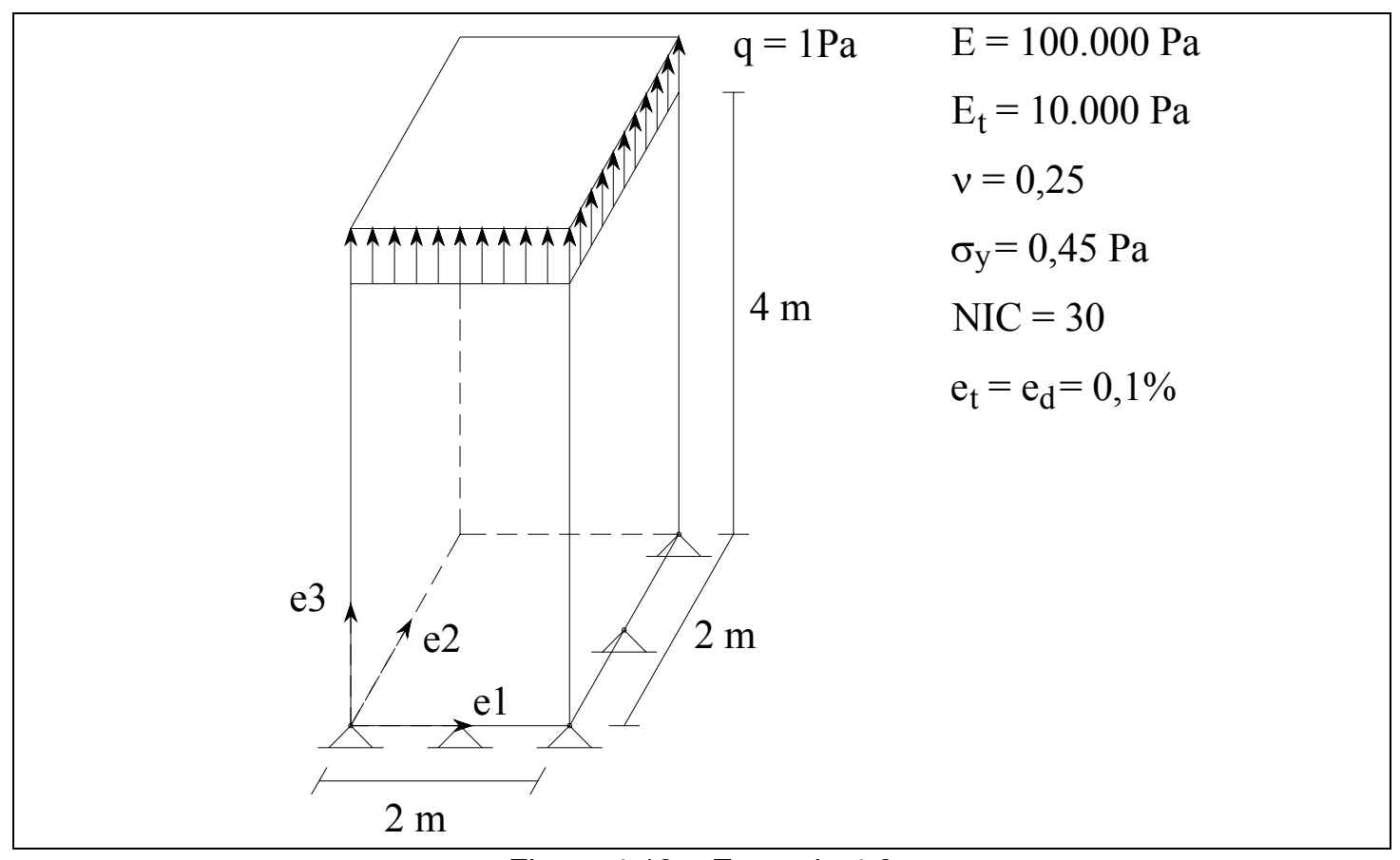

Figura 4.19 - Exemplo 4.3 
A respeito da figura 4.19, observa-se que $\sigma_{y}$ é a tensão de plastificação, NIC é o número de incrementos de carga, $e_{t}$ e $e_{d}$ são os parâmetros dos critérios de convergência e $E_{t}$ é o módulo de elasticidade tangente (ver seção 4.2).

Utilizou-se uma discretização de 24 elementos de contorno e 29 células tetraédricas, onde todo o domínio do sólido foi considerado como passível de plastificação. A figura 4.20 apresenta a discretização do contorno do sólido. As discretizações utilizadas no exemplo foram geradas pelo pré-processador de um outro programa de análise estrutural (ANSYS).

Figura 4.20 - Discretização do contorno do sólido do exemplo 4.3

A figura 4.21 apresenta os deslocamentos longitudinais (direção e3 na figura 4.19) em um ponto localizado no centro da face carregada do sólido, com a aplicação do carregamento. A resposta analítica foi determinada considerando-se o caso de uma viga sujeita a carga concentrada equivalente. Observa-se que os resultados obtidos foram bastante satisfatórios.

A figura 4.22 apresenta as deformações longitudinais (no mesmo ponto da figura 4.21) pelas tensões. Também se observa uma perfeita concordância entre os resultados obtidos e os valores analíticos.

Nas figuras 4.23 e 4.24 , procedeu-se a um controle de deslocamentos nos nós do topo do sólido, com um valor total de $3,6 \cdot 10^{-5} \mathrm{~m}$, e calculou-se as reações na base do mesmo. Para o caso da figura 4.23 , considerou-se um comportamento elastoplástico perfeito para o material, ou seja, o módulo de 
elasticidade tangente $\left(\mathrm{E}_{\mathrm{t}}\right)$ foi considerado nulo na análise; enquanto que na figura 4.24, considerou-se encruamento negativo (softening) definido por $E_{t}=$ $5,0 \cdot 10^{4} \mathrm{~Pa}$. Mais uma vez, os resultados obtidos foram bastante satisfatórios.

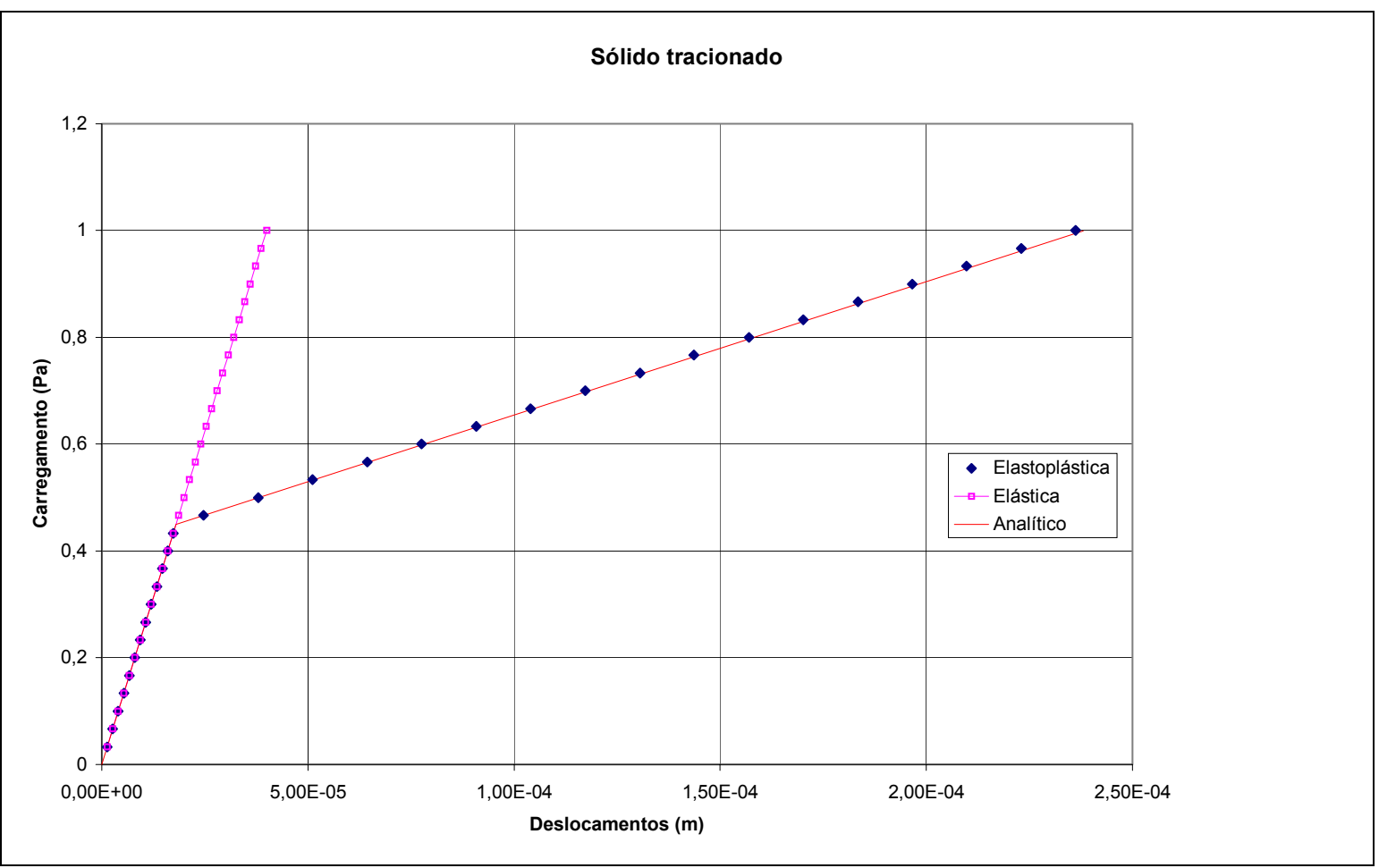

Figura 4.21 - Deslocamento x carregamento

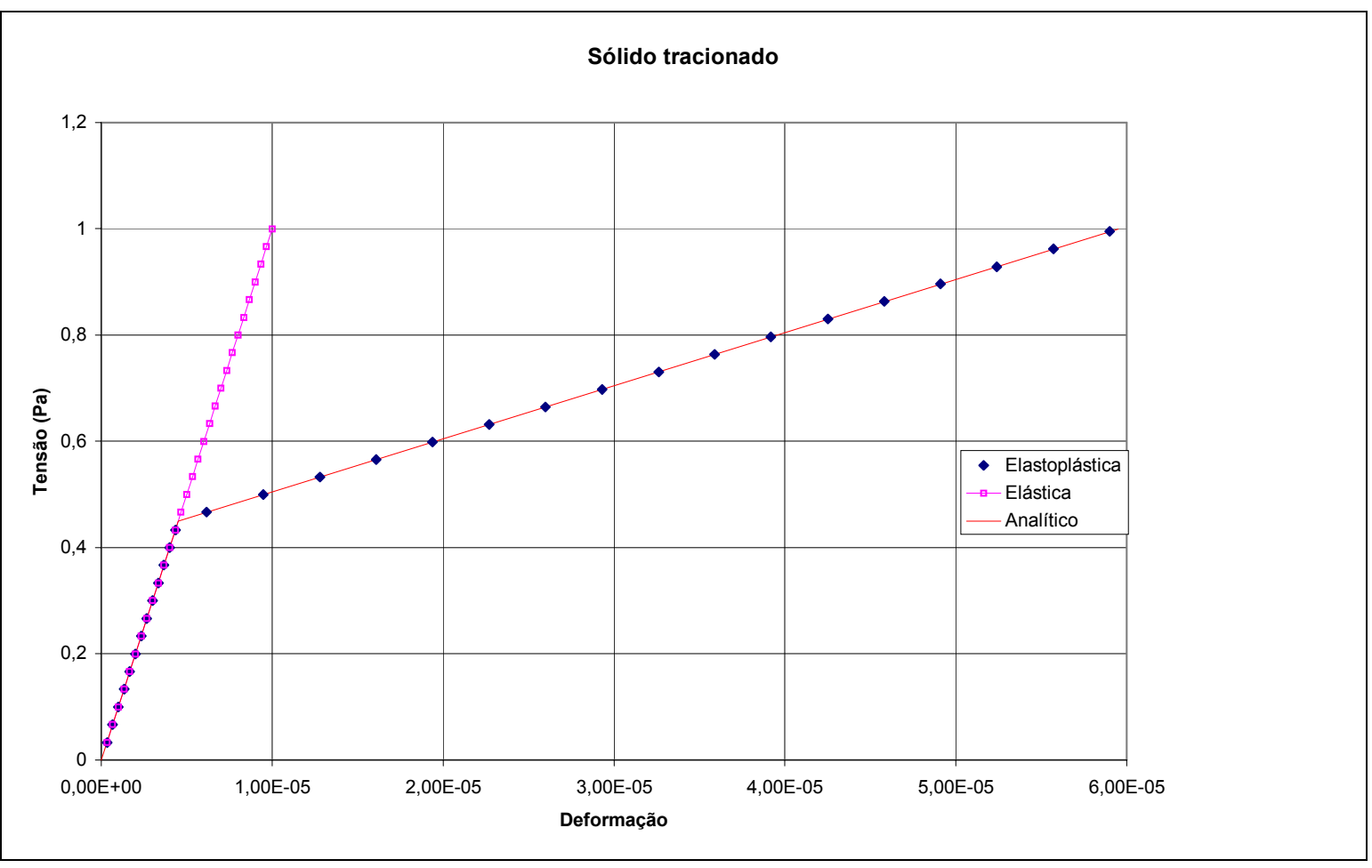

Figura 4.22 - Deformação $x$ tensão 


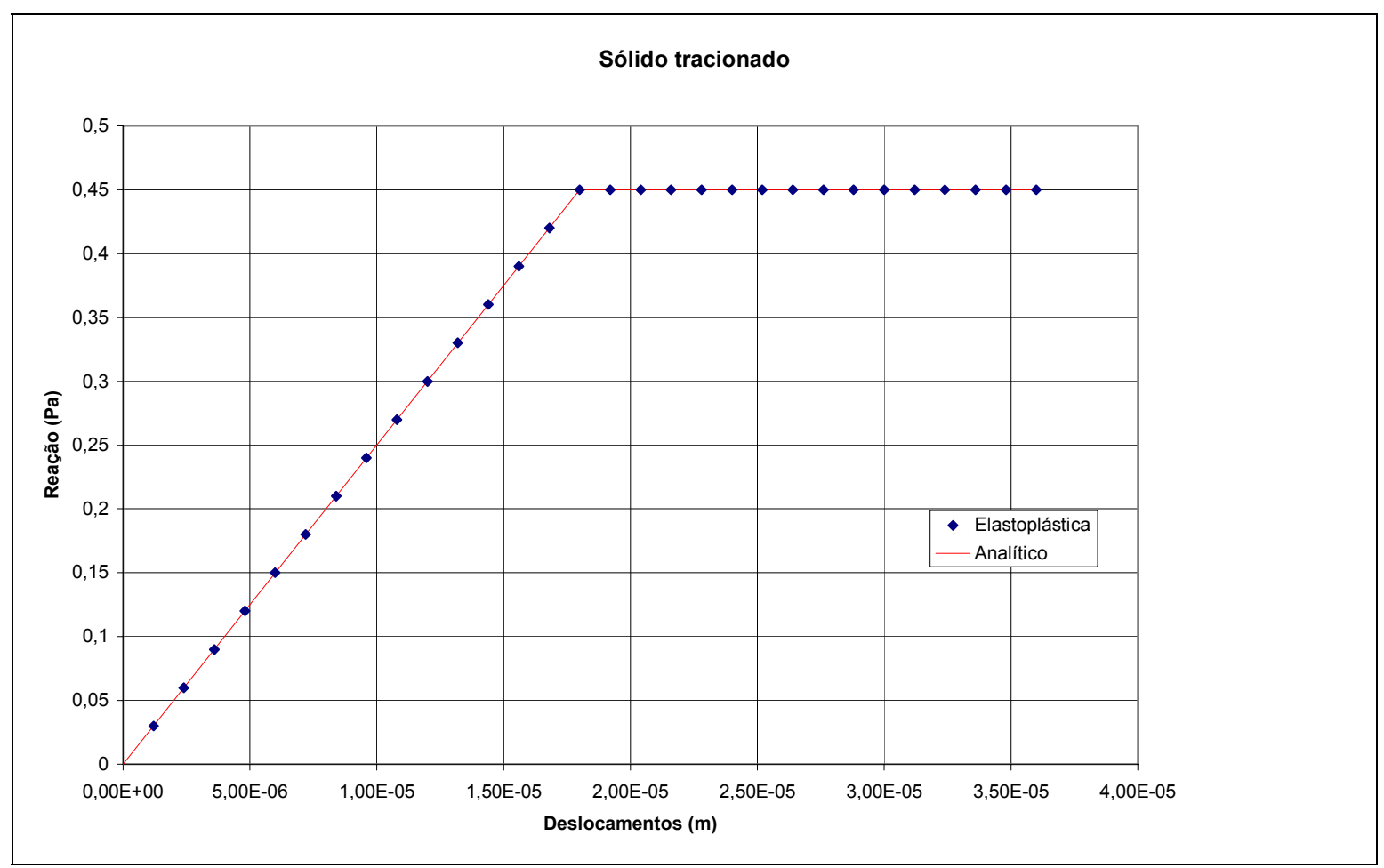

Figura 4.23 - Deslocamento x reação - encruamento nulo

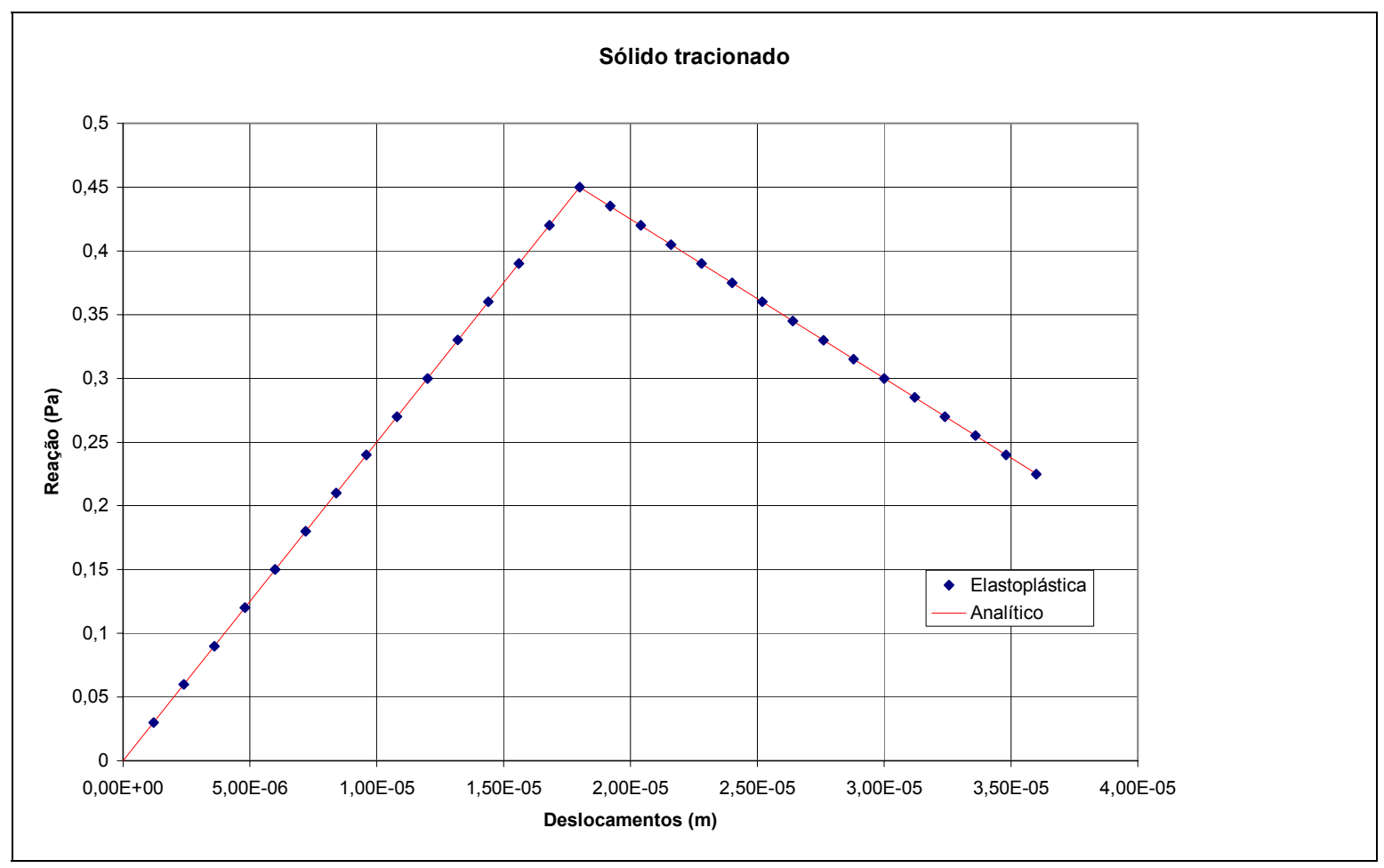

Figura 4.24 - Deslocamento $\mathrm{x}$ reação - encruamento negativo 
Através dos resultados apresentados neste exemplo simples, pode-se concluir que o código desenvolvido está funcionando corretamente para análises elastoplásticas estáticas, com diferentes tipos de encruamento. Este exemplo mostra a capacidade do MEC em tratar problemas não-lineares com bastante precisão. Observa-se que as células tetraédricas possibilitam variação linear de tensões (4 nós), o mesmo só seria possível no MEF para 8 nós. Além disso, para problemas onde uma pequena porção do corpo plastifica, tal como para o solo que circunda a estrutura de fundação, apenas naquela região é necessária a utilização de células.

\subsubsection{Exemplo 4.4}

O quarto exemplo trata da análise elastoplástica dinâmica do sólido apresentado no exemplo 4.3, onde os parâmetros dinâmicos foram calibrados de tal forma a convergir monotonicamente ao resultado de deslocamento estático no ponto localizado no centro do topo do sólido. Foi utilizado um valor de densidade $\rho=0,002 \mathrm{~kg} / \mathrm{m}^{3}$ para o material, tempo total de análise de $\mathrm{t}=$ 0,036s e número de passos de tempo NPT $=240$. Utilizou-se 3 tipos de carregamento: de impacto (ou carga constante), crescente e 'platô'. Os 3 tipos de carregamento utilizados podem ser vistos na figura 4.25.

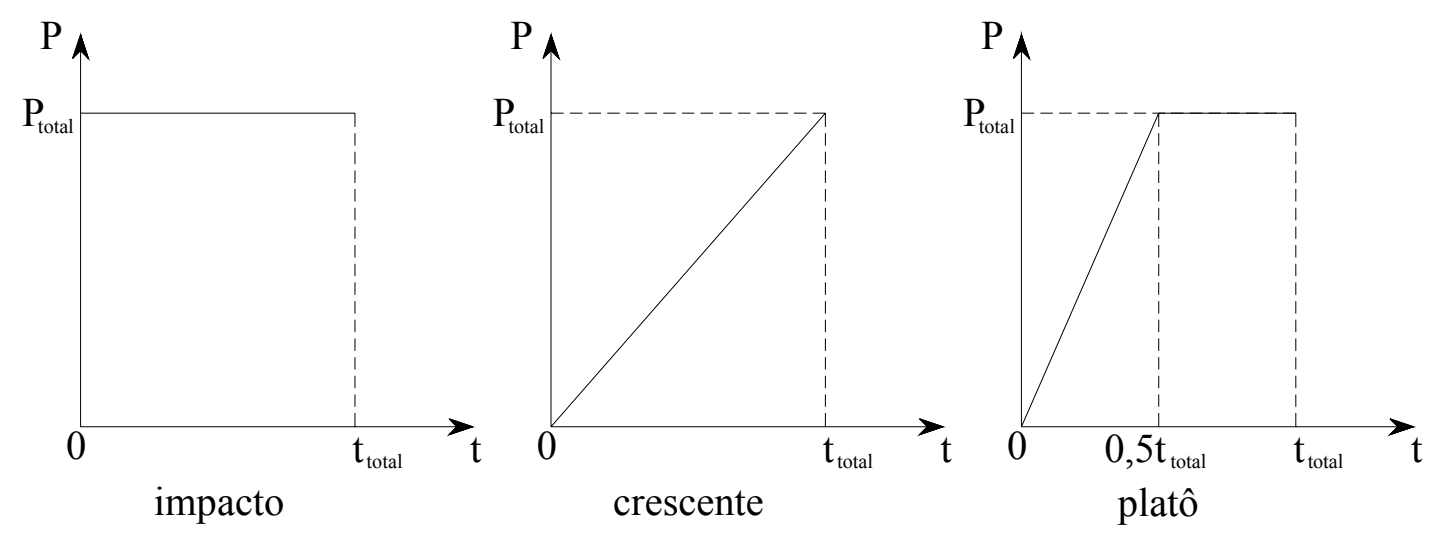

Figura 4.25 - Tipos de carregamento

Na figura 4.26 são apresentados os deslocamentos por passo de tempo para os 3 tipos de carregamento citados anteriormente. Vale salientar que o valor adotado para o coeficiente de amortecimento dinâmico 'c' não foi o mesmo para todos os tipos de carregamento, procurou-se apresentar aquele 
que forneceu o comportamento desejado para a análise em questão. Observase que os deslocamentos obtidos realmente convergem para a resposta analítica não-linear para todos os tipos de carregamento, sendo aqueles obtidos com o carregamento de impacto os que apresentaram melhor comportamento.

Na figura 4.27 são apresentadas as curvas de deslocamento por passo de tempo para valores do coeficiente de amortecimento dinâmico variando de 0,25 a $25 \mathrm{~kg} / \mathrm{s}$. Observa-se uma considerável variação dos resultados obtidos com o valor do coeficiente de amortecimento adotado, em que, para $\mathrm{c}=$ $2,5 \mathrm{~kg} / \mathrm{s}$, foi obtido um comportamento elastoplástico típico.

$\mathrm{Na}$ figura 4.28, o carregamento utilizado foi o crescente e variou-se o valor do amortecimento de 0,2 a 20kg/s. Para esse tipo de carregamento, também houve uma grande variação dos resultados obtidos com o valor do amortecimento. Considerou-se o melhor comportamento para $\mathrm{c}=2 \mathrm{~kg} / \mathrm{s}$.

Por fim, a figura 4.29 apresenta os resultados de deslocamentos para a carga denominada de 'platô', onde se variou o valor de 'c' de 0,2 a 20kg/s e se chegou às mesmas conclusões do caso da figura anterior.

Com este quarto exemplo, pode-se perceber que o código elastoplástico dinâmico desenvolvido está fornecendo resultados consistentes com o esperado. Deve-se notar também a existência de um amortecimento numérico na solução dinâmica do MEC, tal como observado no exemplo 4.2. Tal amortecimento pode ser responsável pela pequena diferença entre os valores estático e dinâmico não-lineares finais.

Para a aferição do procedimento empregado no trabalho, utilizou-se este exemplo simples, porém, no capítulo 10 são apresentados exemplos mais rebuscados e com um maior grau de complexidade com o objetivo de mostrar um pouco mais das potencialidades do código desenvolvido. 


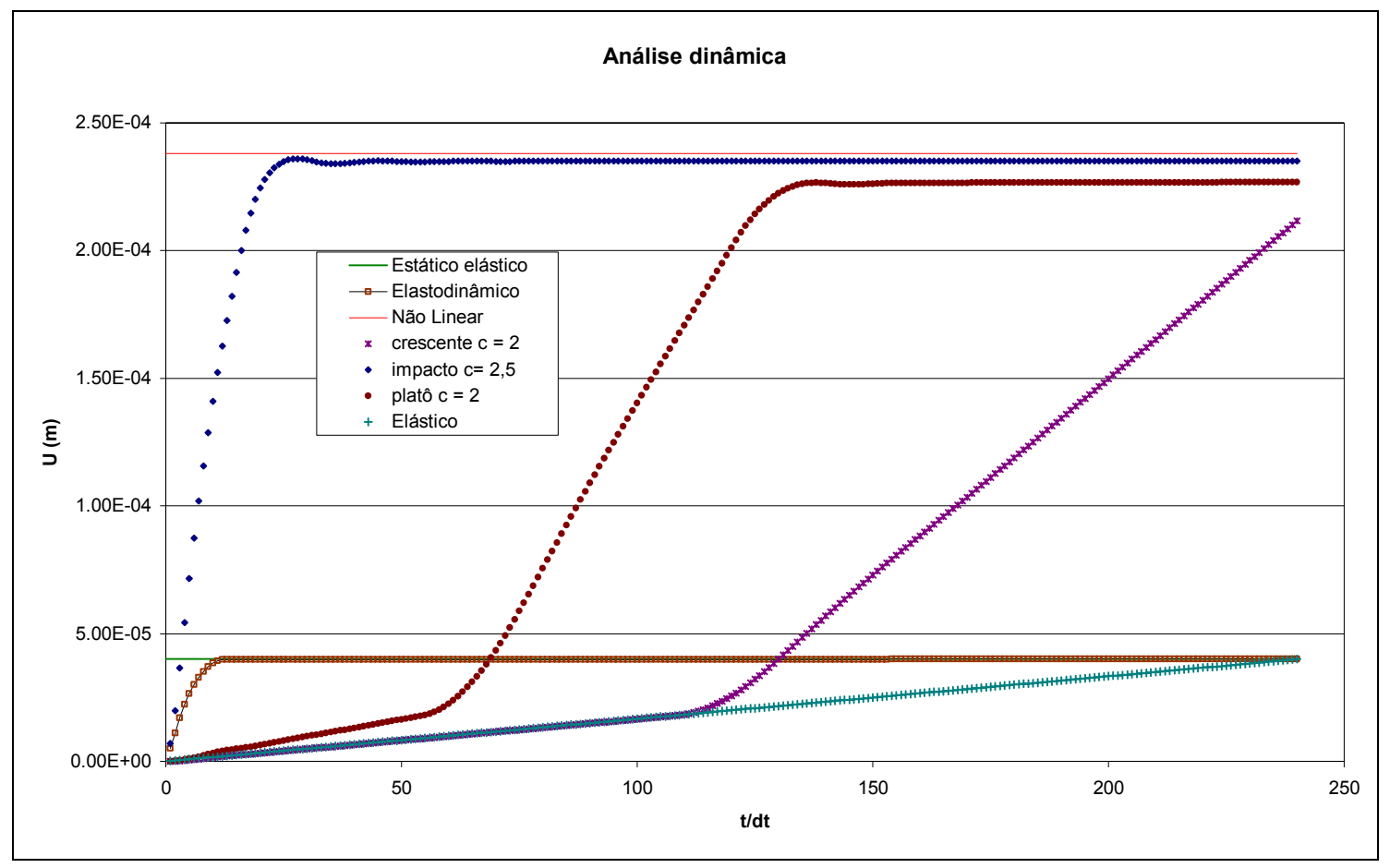

Figura 4.26 - Análise dinâmica

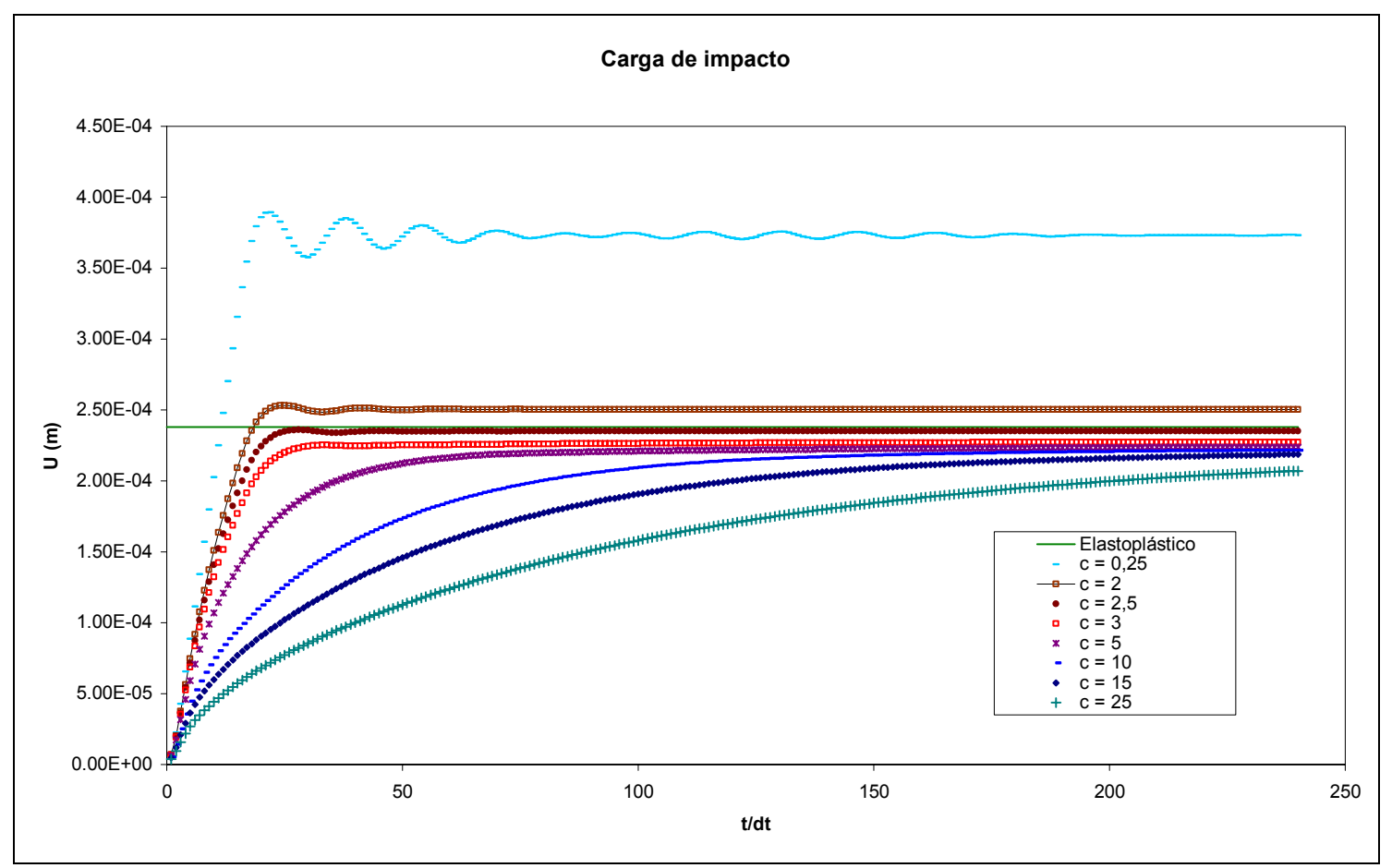

Figura 4.27 - Carga de impacto 


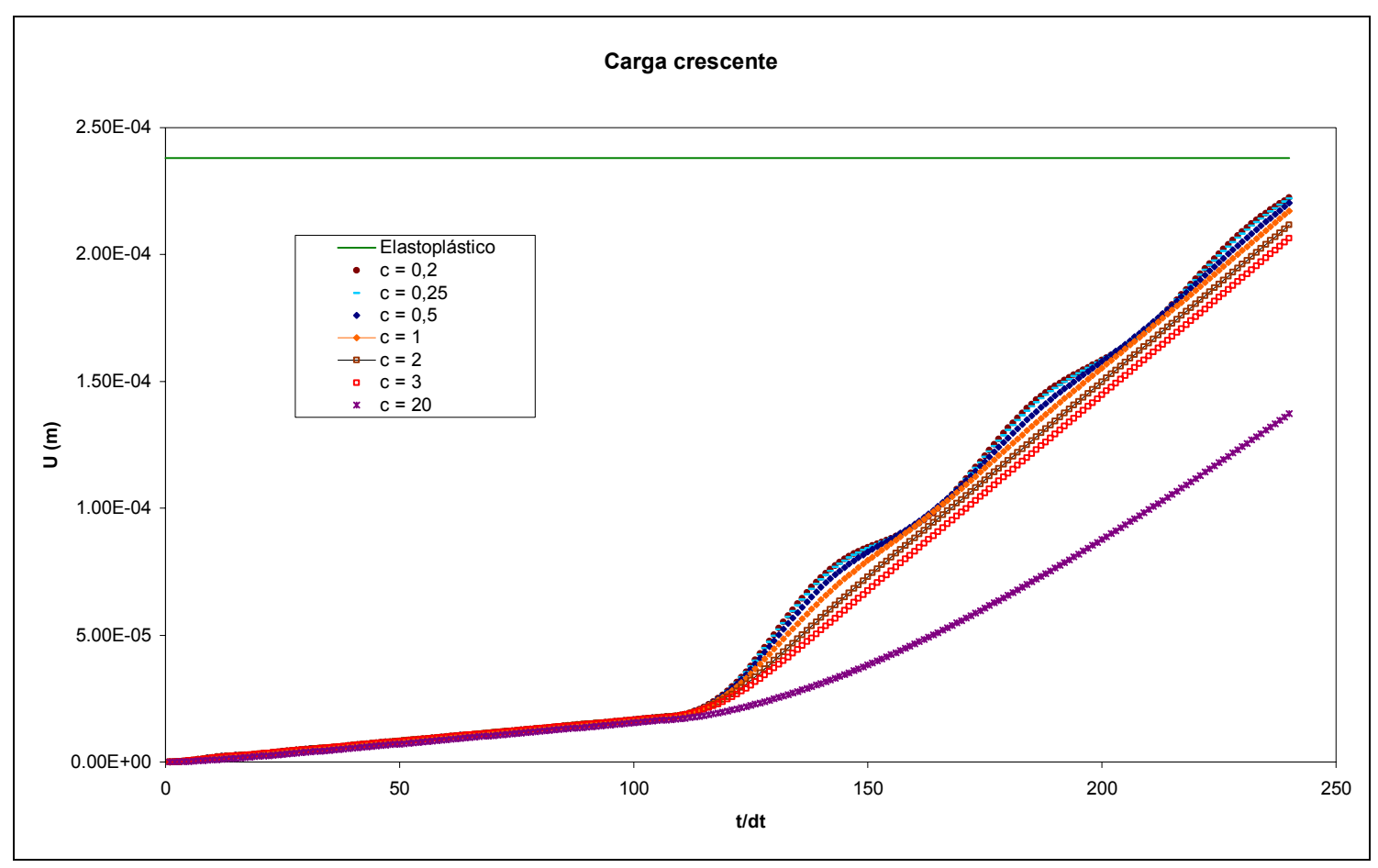

Figura 4.28 - Carga crescente

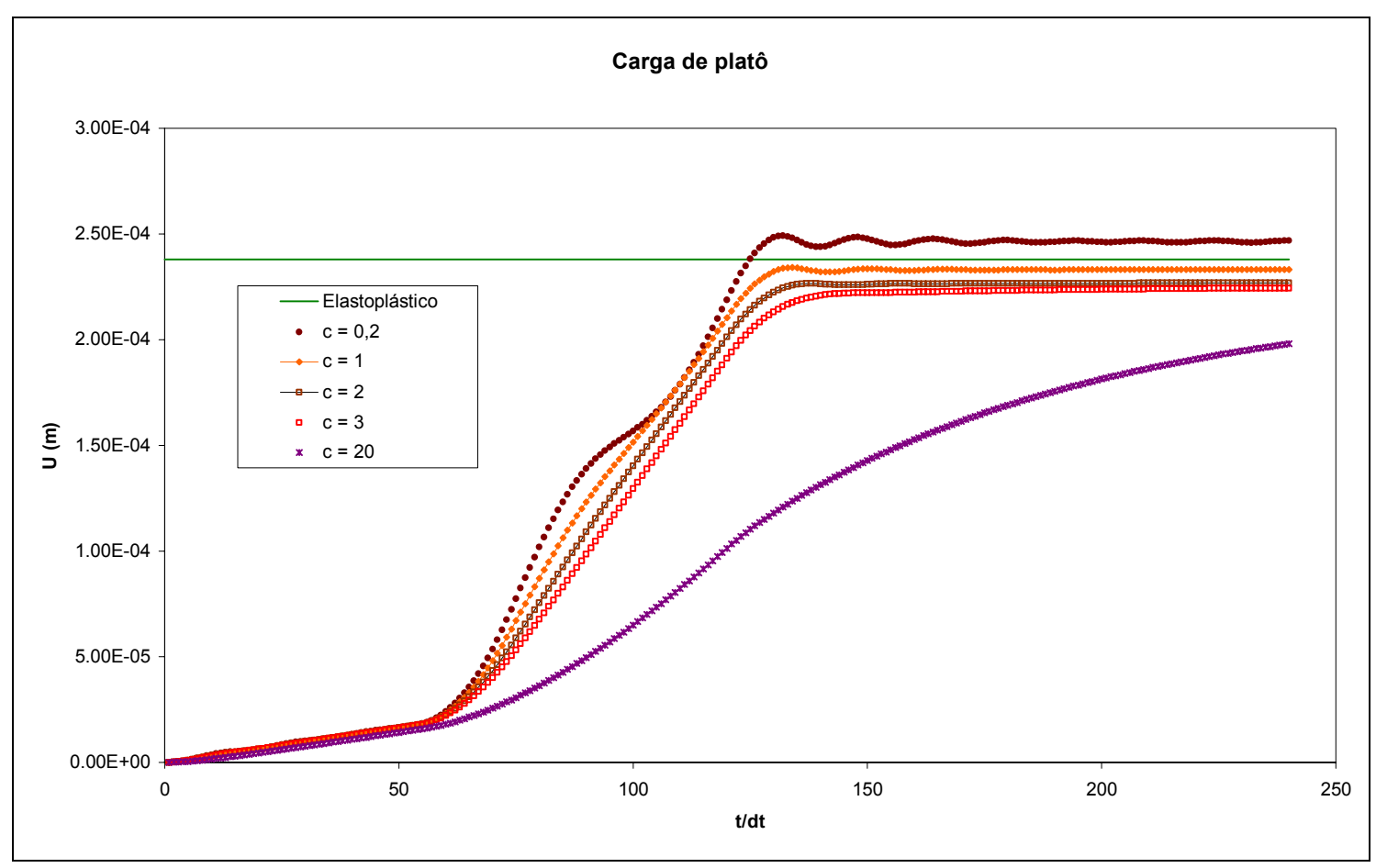

Figura 4.29 - Carga de platô 


\section{INTEGRAÇÕES PARA ELEMENTOS DE CONTORNO E CÉLULAS}

Neste capítulo, apresentam-se os diversos processos de integração (singular, não-singular e quase-singular) para os elementos de contorno triangulares planos utilizados na discretização do contorno dos sólidos, bem como as integrações das células tetraédricas. Apresenta-se também o tratamento específico da integral singular das tensões residuais para células. Acredita-se que tais técnicas representam grande importância no desenvolvimento do presente trabalho, bem como de trabalhos futuros. No final do capítulo, são apresentados dois exemplos numéricos.

\subsection{INTEGRAÇÃO SINGULAR PARA ELEMENTOS DE CONTORNO}

Na presente seção será apresentado o processo de integração singular para elementos de contorno, onde o ponto fonte pode estar localizado em qualquer lugar do domínio do elemento.

Esse procedimento permite a colocação de pontos no contorno do sólido analisado, sem que esses pontos sejam necessariamente nós de elementos de contorno, determinando-se seus deslocamentos, forças de superfície e tensões como variáveis independentes. A colocação de tais pontos possibilita uma independência entre os nós das células e os nós dos elementos de contorno, ou seja, pode acontecer de haver um nó de célula no contorno do sólido e esse nó de célula não ser nó de elemento de contorno, sendo, assim, um "ponto 
especial" (PE). Observa-se que esses pontos especiais podem pertencer a 1 ou a 2 elementos de contorno. Figura 5.1.

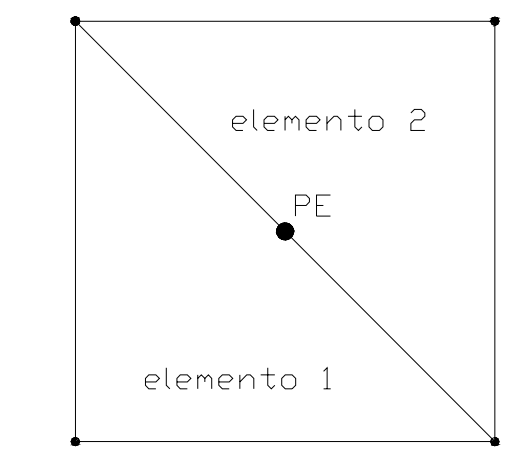

Ponto especial pertencente a 2 elementos

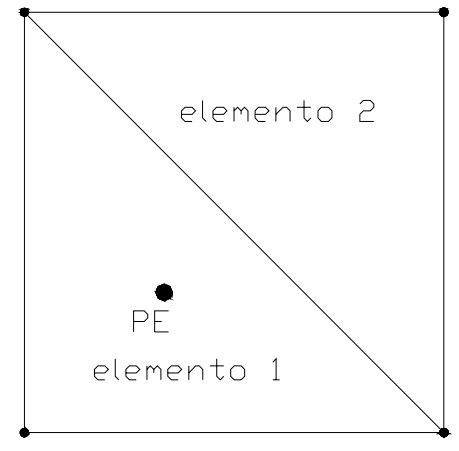

Ponto especial pertencente a 1 elemento

Figura 5.1 - Pontos especiais

O processo de integração é iniciado com a divisão do domínio do elemento de contorno em 3 subdomínios. Figura 5.2.

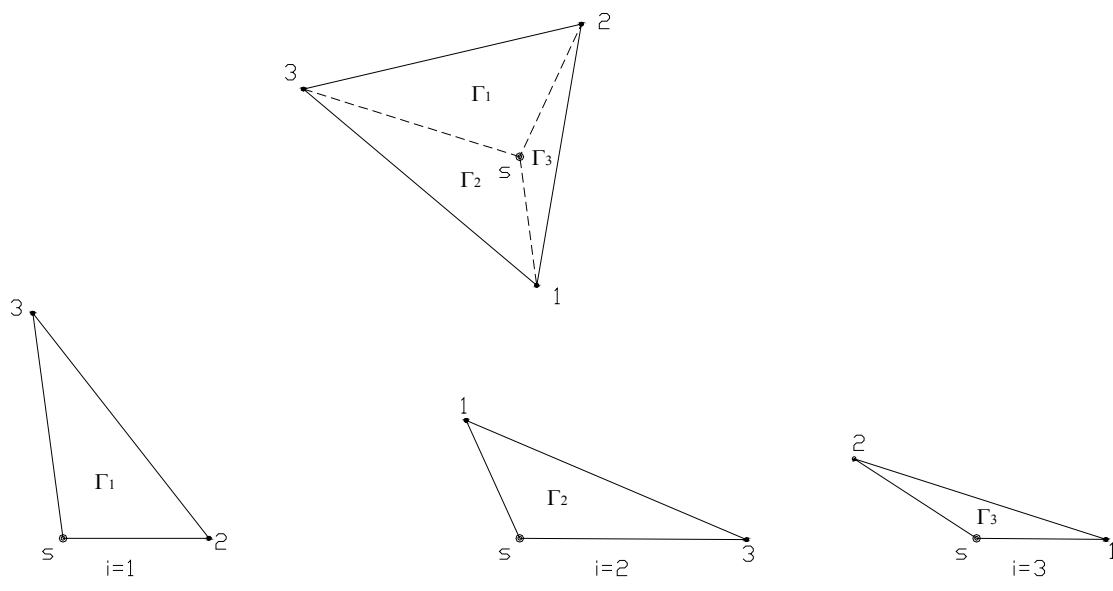

Figura 5.2 - Subdivisão de elemento de contorno (integração singular)

Assim, pode-se escrever:

$I=\int_{\Gamma} f \cdot d \Gamma=\sum_{i=1}^{3} \int_{\Gamma_{i}} f \cdot d \Gamma_{i}$

onde a função $f$ tanto pode ser $\mathrm{u}_{\mathrm{kj}}^{*}(\mathrm{~s}, \mathrm{Q}) \phi_{\ell_{\mathrm{i}}}$ para a determinação da matriz $\mathrm{g}$, como $p_{\mathrm{kj}}^{*}(\mathrm{~s}, \mathrm{Q}) \phi_{\ell_{\mathrm{i}}}$, para a determinação da matriz $\mathrm{h}$. 
A eq.(5.1) pode ser escrita também como:

$I=\sum_{i=1}^{3} I_{i} \quad i=1,2,3$

onde:

$\mathrm{I}_{\mathrm{i}}=\int_{\Gamma_{\mathrm{i}}} \mathrm{f} \cdot \mathrm{d} \Gamma_{\mathrm{i}} \quad \mathrm{i}=1,2,3$

Cada integral $\mathrm{l}_{\mathrm{i}}$ é resolvida, tanto para a montagem da matriz $\mathrm{g}$ como para a da matriz $h$, segundo um procedimento em coordenadas polares no plano do elemento, considerando-se cada subdivisão de elemento de contorno como se fosse um elemento separadamente.

Sendo assim, inicialmente considera-se a dedução da expressão para a matriz g:

$\mathrm{g}_{\mathrm{ij} \ell}^{\mathrm{e}}=\int_{\Gamma_{\mathrm{e}}} \mathrm{u}_{\mathrm{ij}}^{*}(\mathrm{~s}, \mathrm{Q}) \phi_{\ell} \mathrm{d} \Gamma$

onde $\Gamma_{e}$ é o domínio do elemento 'e' e $u_{i j}^{*}(s, Q)$ é a solução fundamental de deslocamento, segundo Kelvin:

$u_{i j}^{*}(s, Q)=\frac{1}{16 \pi(1-v) G r}\left[(3-4 v) \delta_{i j}+r_{, i} r_{, j}\right]$

Substituindo-se a eq.(5.5) na eq.(5.4):

$g_{i j \ell}^{e}=\int_{\Gamma_{e}} \frac{1}{16 \pi(1-v) G r}\left[(3-4 v) \delta_{i j}+r_{, i}, r_{j}\right] \cdot \phi_{\ell} d \Gamma$

A eq.(5.6) acima deve ser escrita em um sistema de coordenadas polares no plano do elemento como segue: 


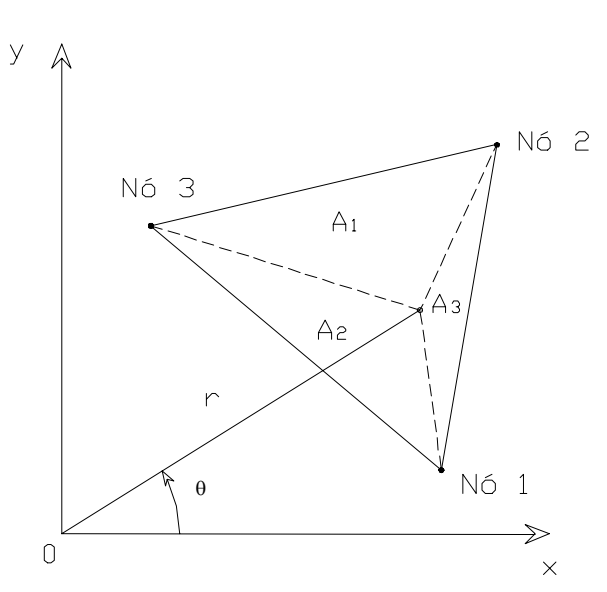

$$
\begin{aligned}
& \xi_{\ell}=\frac{1}{2 \mathrm{~A}}\left[\alpha_{\ell}+\beta_{\ell} \mathrm{x}+\gamma_{\ell} \mathrm{y}\right] \\
& \left\{\begin{array}{l}
\alpha_{\ell}=\mathrm{x}_{\mathrm{j}} \mathrm{y}_{\mathrm{k}}-\mathrm{x}_{\mathrm{k}} \mathrm{y}_{\mathrm{j}} \\
\beta_{\ell}=\mathrm{y}_{\mathrm{j}}-\mathrm{y}_{\mathrm{k}} \\
\gamma_{\ell}=\mathrm{x}_{\mathrm{k}}-\mathrm{x}_{\mathrm{j}}
\end{array}\right. \\
& \begin{array}{l|l|l}
\ell & \mathrm{j} & \mathrm{k} \\
\hline 1 & 2 & 3
\end{array} \\
& 2301 \\
& 3|1| 2
\end{aligned}
$$

Figura 5.3 - Sistema de coordenadas polares

onde:

A - é a área total do elemento;

$\xi_{\ell} \quad$ - é a coordenada homogênea $\ell$ do ponto $(\mathrm{x}, \mathrm{y})$, que também pode ser calculada como sendo a razão entre a área $\ell$ e a área total $\left(\xi_{\ell}=\mathrm{A}_{\ell} / \mathrm{A}\right)$.

Observa-se que:

$x=r \cos \theta$

$y=r \operatorname{sen} \theta$

E ainda, para o caso em questão, que:

Para $\mathrm{x}=\mathrm{y}=0 \Rightarrow \xi_{\ell}=\xi_{\ell}^{0}=\frac{\alpha_{\ell}}{2 \mathrm{~A}}$

onde $\xi_{\ell}^{0}$ é a coordenada homogênea $\ell$ do ponto de origem $(0,0)$.

Substituindo-se as eq.(5.8) e (5.9) na eq.(5.7), tem-se:

$\xi_{\ell}=\frac{1}{2 A}\left[\alpha_{\ell}+\beta_{\ell} r \cos \theta+\gamma_{\ell} r \operatorname{sen} \theta\right]$ 
e, substituindo-se a eq.(5.10) na eq.(5.11) acima, pode-se escrever:

$\xi_{\ell}=\xi_{\ell}^{0}+\frac{r}{2 A}\left[\beta_{\ell} \cos \theta+\gamma_{\ell} \operatorname{sen} \theta\right]$

Vale a pena observar ainda, que se o ponto de origem coincidir com o ponto fonte (figura 5.4), tem-se:

$\xi_{\ell}=\delta_{\ell s}+\frac{r}{2 A}\left[\beta_{\ell} \cos \theta+\gamma_{\ell} \operatorname{sen} \theta\right]$

onde:

$\delta_{\ell \mathrm{s}} \quad-$ é o delta de Kroneker ( 0 se $\ell \neq s$ e 1 se $\ell=s$ ), já citado anteriormente.

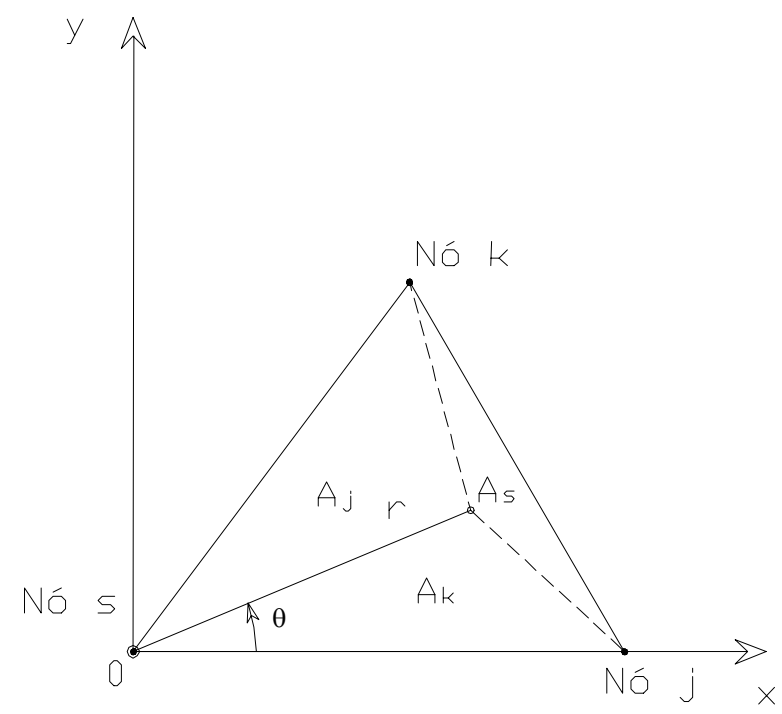

Figura 5.4 - Ponto origem coincidente com ponto fonte

Também se pode escrever que:

$\mathrm{d} \Gamma=\operatorname{rdrd} \theta$

Substituindo-se as eq.(5.11) e (5.14) na eq.(5.6), tem-se: 


$$
\begin{aligned}
& g_{\mathrm{ij} \ell}^{\mathrm{e}}=\int_{\theta} \int_{\mathrm{r}} \frac{1}{16 \pi(1-v) \mathrm{Gr}}\left[(3-4 v) \delta_{\mathrm{ij}}+\mathrm{r}_{\mathrm{i}}, r_{\mathrm{j}}\right] \frac{1}{2 \mathrm{~A}}\left[\alpha_{\ell}+\beta_{\ell} r \cos \theta+\gamma_{\ell} r \operatorname{sen} \theta\right] \mathrm{rdrd} \theta \\
& \mathrm{g}_{\mathrm{ij} \ell}^{\mathrm{e}}=\frac{1}{32 \pi(1-v) \mathrm{GA}} \int_{\theta} \int_{\mathrm{r}}\left[(3-4 v) \delta_{\mathrm{ij}}+\mathrm{r}_{, \mathrm{i}} \mathrm{r}_{\mathrm{j}}\right] \cdot\left[\alpha_{\ell}+\beta_{\ell} r \cos \theta+\gamma_{\ell} r \operatorname{sen} \theta\right] \mathrm{drd} \theta
\end{aligned}
$$

Deve-se observar que $\phi_{\ell}=\xi_{\ell}$ para o elemento com aproximação linear. Como a expressão resultante não é singular, optou-se por uma integração numérica, tanto no ângulo $\theta$, como no raio.

Assim, a eq.(5.16) será escrita como:

$$
\begin{aligned}
& g_{\mathrm{ij} \ell}^{\mathrm{e}}=\frac{1}{32 \pi(1-v) G A} \int_{\theta} \frac{R}{2} \sum_{\mathrm{igr}=1}^{\mathrm{ngr}}\left[(3-4 v) \delta_{\mathrm{ij}}+\mathrm{r}_{, \mathrm{i}} \mathrm{r}_{\mathrm{j}}\right] \cdot\left[\alpha_{\ell}+\beta_{\ell} r \cos \theta+\gamma_{\ell} r \operatorname{sen} \theta\right] \omega_{\mathrm{igr}} \mathrm{d} \theta \\
& \mathrm{g}_{\mathrm{ij} \ell}^{\mathrm{e}}=\frac{\theta_{\mathrm{f}}}{128 \pi(1-v) \mathrm{GA}} \sum_{\mathrm{ig} \theta=1 \mathrm{igr}=1}^{\mathrm{ng} \theta} \sum^{\mathrm{ngr}} \mathrm{R}\left[(3-4 v) \delta_{\mathrm{ij}}+\mathrm{r}_{\mathrm{i}} \mathrm{r}_{\mathrm{j}}\right] \cdot\left[\alpha_{\ell}+\beta_{\ell} r \cos \theta+\gamma_{\ell} r \operatorname{sen} \theta\right] \omega_{\mathrm{igr}} \omega_{\mathrm{ig} \theta}
\end{aligned}
$$

onde:

R - função de $\theta$, é o limite superior do raio $r$;

igr - são os pontos de Gauss em r e variam de 1 até ngr;

ngr - é o número de pontos de Gauss em r;

$\omega_{\text {igr }} \quad$ - é o peso do ponto de Gauss igr;

$\theta_{\mathrm{f}} \quad$ - é o ângulo que o lado 'ks' faz com o eixo x (local ao elemento). Ver figura 5.4;

ig $\theta$ - são os pontos de Gauss em $\theta$ e variam de 1 até ng $\theta$;

ng $\theta$ - é o número de pontos de Gauss em $\theta$;

$\omega_{\text {ige }} \quad$ - é o peso do ponto de Gauss ig $\theta$.

$\mathrm{O}$ valor de $\mathrm{R}$ pode ser facilmente calculado utilizando-se a eq.(5.13) e considerando-se que, para $\ell=\mathrm{s}, \xi_{\ell}=0$ no lado 'jk'. Assim, a eq.(5.13) é escrita como: 


$$
\begin{aligned}
& 0=1+\frac{R}{2 A}\left[\beta_{s} \cos \theta+\gamma_{s} \operatorname{sen} \theta\right] \\
& R=\frac{-2 A}{\beta_{s} \cos \theta+\gamma_{s} \operatorname{sen} \theta}
\end{aligned}
$$

Deve-se notar que a integração numérica utilizada para a expressão da matriz g, como foi apresentado acima, só foi possível por ser essa expressão não-singular (singularidade fraca); para a determinação da matriz h é que se faz necessário o uso de um processo de integração singular, uma vez que no núcleo desta integral se encontra singularidade de ordem $1 / r^{2}$.

Assim, pode-se escrever que:

$\mathrm{h}_{\mathrm{ij}}=\int_{\Gamma} \mathrm{p}_{\mathrm{ij}}^{*}(\mathrm{~s}, \mathrm{Q}) \mathrm{u}_{\mathrm{j}}(\mathrm{Q}) \mathrm{d} \Gamma$

onde $\mathrm{p}_{\mathrm{ij}}^{*}(\mathrm{~s}, \mathrm{Q})$ é a solução fundamental de força de superfície, segundo Kelvin:

$\left.p_{i j}^{*}(s, Q)=\frac{-1}{8 \pi(1-v) r^{2}}\left\{(1-2 v) \delta_{i j}+3 r,{ }_{, i} r_{j}\right] r_{n}-(1-2 v)\left(r_{, j} n_{j}-r, n_{j} n_{i}\right)\right\}$

e a integral da eq.(5.21) é feita em todo o contorno do sólido $(\Gamma)$.

No elemento, o vetor de deslocamentos no ponto campo $Q$, pode ser escrito como:

$\mathrm{u}_{\mathrm{j}}(\mathrm{Q})=\phi_{\ell} \mathrm{U}_{\mathrm{j}}^{\ell}$

onde $\bigcup_{j}^{\ell}$ é o vetor dos deslocamentos na direção 'j' dos vértices do elemento a ser integrado.

Substituindo-se as eq.(5.22), (5.23) e (5.13) na eq.(5.21) e levando-se em consideração que $r_{n}=0$, tem-se: 


$$
h_{\mathrm{ij}}=\frac{(1-2 v)}{8 \pi(1-v)} \int_{\Gamma} \frac{1}{\mathrm{r}^{2}}\left(\mathrm{r}_{\mathrm{i}} \mathrm{n}_{\mathrm{j}}-\mathrm{r}_{\mathrm{j}, \mathrm{j}} \mathrm{n}_{\mathrm{i}}\right)\left\{\delta_{\ell \mathrm{s}}+\frac{\mathrm{r}}{2 \mathrm{~A}}\left[\beta_{\ell} \cos \theta+\gamma_{\ell} \operatorname{sen} \theta\right]\right\} \mathrm{U}_{\mathrm{j}}^{\ell} \mathrm{d} \Gamma
$$

Escrevendo-se em coordenadas polares, tem-se:

$$
\begin{aligned}
& h_{\mathrm{ij}}=\frac{(1-2 v)}{8 \pi(1-v)} \int_{\theta} \int_{\mathrm{r}} \frac{1}{\mathrm{r}}\left(\mathrm{r}_{\mathrm{i}} \mathrm{n}_{\mathrm{j}}-\mathrm{r}_{, \mathrm{j}} \mathrm{n}_{\mathrm{i}}\right)\left\{\delta_{\ell \mathrm{s}}+\frac{\mathrm{r}}{2 \mathrm{~A}}\left[\beta_{\ell} \cos \theta+\gamma_{\ell} \operatorname{sen} \theta\right]\right\} \mathrm{U}_{\mathrm{j}}^{\ell} \mathrm{drd} \theta \\
& \mathrm{h}_{\mathrm{ij}}=\frac{(1-2 v)}{8 \pi(1-v)}\left\{\int_{\theta} \int_{\mathrm{r}} \frac{1}{r}\left(\mathrm{r}_{{ }_{\mathrm{i}}} \mathrm{n}_{\mathrm{j}}-\mathrm{r}_{{ }_{\mathrm{j}}} \mathrm{n}_{\mathrm{i}}\right) \delta_{\ell \mathrm{s}} \mathrm{U}_{\mathrm{j}}^{\ell} \mathrm{drd} \theta+\int_{\theta} \int_{\mathrm{r}} \frac{1}{2 \mathrm{~A}}\left[\beta_{\ell} \cos \theta+\gamma_{\ell} \operatorname{sen} \theta\right] \mathrm{U}_{\mathrm{j}}^{\ell} \mathrm{drd} \theta\right\}
\end{aligned}
$$

Nota-se que a eq.(5.26) pode ser dividida em duas integrais: uma singular, com ordem de singularidade de $r^{-1}$, e outra regular.

$$
h=h^{s}+h^{r}
$$

A integral regular pode ser resolvida numericamente em $r$ e $\theta$ como segue:

$$
\begin{aligned}
& h_{\mathrm{ij}}^{r}=\frac{(1-2 v)}{16 \pi(1-v) A} \int_{\theta} \int_{\mathrm{r}} r\left[\beta_{\ell} \cos \theta+\gamma_{\ell} \operatorname{sen} \theta\right] \mathrm{drd} \theta \mathrm{U}_{\mathrm{j}}^{\ell} \\
& \mathrm{h}_{\mathrm{ij}}^{\mathrm{r}}=\frac{\theta_{\mathrm{f}}(1-2 v)}{64 \pi(1-v) \mathrm{A}} \sum_{\mathrm{ig} \theta=1 \mathrm{igr}=1}^{\mathrm{ng} \theta} \sum^{\mathrm{ngr}} \operatorname{Rr}\left[\beta_{\ell} \cos \theta+\gamma_{\ell} \operatorname{sen} \theta\right] \omega_{\mathrm{igr}} \omega_{\mathrm{ig} \theta} \mathrm{U}_{\mathrm{j}}^{\ell}
\end{aligned}
$$

A integral singular propriamente dita, será resolvida analiticamente.

$$
\mathrm{h}_{\mathrm{ij}}^{\mathrm{s}}=\frac{(1-2 v)}{8 \pi(1-v)} \int_{\theta} \int_{\mathrm{r}} \frac{1}{r}\left(\mathrm{r}_{{ }_{\mathrm{i}}} \mathrm{n}_{\mathrm{j}}-\mathrm{r}_{\mathrm{j}} \mathrm{n}_{\mathrm{i}}\right) \delta_{\ell \mathrm{s}} \mathrm{U}_{\mathrm{j}}^{\ell} \mathrm{drd} \theta
$$

Sendo que:

$$
\delta_{\ell \mathrm{s}} \mathrm{U}_{\mathrm{j}}^{\ell}=\mathrm{U}_{\mathrm{j}}^{\mathrm{s}}
$$

onde $U_{j}^{s}$ é o vetor dos deslocamentos na direção 'j' no ponto fonte ' $s$ '. 
Substituindo-se a eq.(5.31) na eq.(5.30), tem-se:

$h_{i j}^{s}=\frac{(1-2 v)}{8 \pi(1-v)} \int_{\theta} \int_{r} \frac{1}{r}\left(r_{, j} n_{j}-r, n_{j} n_{i}\right) d r d \theta U_{j}^{s}$

Resolvendo-se analiticamente em r, tem-se:

$$
\begin{aligned}
& h_{i j}^{s}=\frac{(1-2 v)}{8 \pi(1-v)} \int_{\theta}\left(r_{, i} n_{j}-r,{ }_{j} n_{i}\right) \ln R(\theta) d \theta U_{j}^{s} \\
& h_{i j}^{s}=\frac{(1-2 v)}{8 \pi(1-v)}\left\{\int_{\theta} r_{, j} n_{j} \ln R d \theta-\int_{\theta} r_{j} n_{i} \ln R(\theta) d \theta\right\}_{j}^{s} \\
& h_{i j}^{s}=\frac{(1-2 v)}{8 \pi(1-v)}\left\{n_{j} \int_{\theta} r_{, i} \ln R d \theta-n_{i} \int_{\theta} r_{, j} \ln R(\theta) d \theta\right\}_{j}^{s}
\end{aligned}
$$

onde o limite inferior, para $r \rightarrow 0$, desaparece, pois a integral em $\theta$ se anula para $\theta$ variando de 0 a $2 \pi$.

Sendo que $r_{, i}$ pode ser escrito da seguinte forma:

$$
r_{, i}=\cos \theta \frac{\partial x}{\partial X_{i}}+\operatorname{sen} \theta \frac{\partial y}{\partial X_{i}}
$$

onde $X_{i}$ é a coordenada global 'i'.

Maiores detalhes a respeito da eq.(5.36), apresentada acima, podem ser vistos no capítulo 6 de Souza (2001).

Substituindo-se a eq.(5.36) na eq.(5.35), tem-se:

$$
\mathrm{h}_{\mathrm{ij}}^{\mathrm{s}}=\frac{(1-2 v)}{8 \pi(1-v)}\left\{\mathrm{n}_{\mathrm{j}} \int_{\theta}\left(\cos \theta \frac{\partial \mathrm{x}}{\partial \mathrm{X}_{\mathrm{i}}}+\operatorname{sen} \theta \frac{\partial \mathrm{y}}{\partial \mathrm{X}_{\mathrm{i}}}\right) \ln \operatorname{Rd} \theta-\mathrm{n}_{\mathrm{i}} \int_{\theta}\left(\cos \theta \frac{\partial \mathrm{x}}{\partial \mathrm{X}_{\mathrm{j}}}+\operatorname{sen} \theta \frac{\partial \mathrm{y}}{\partial \mathrm{X}_{\mathrm{j}}}\right) \ln \mathrm{Rd} \theta\right\} \mathrm{U}_{\mathrm{j}}^{\mathrm{s}}
$$

$$
\mathrm{h}_{\mathrm{ij}}^{\mathrm{s}}=\frac{(1-2 v)}{8 \pi(1-v)}\left\{\mathrm{n}_{\mathrm{j}} \frac{\partial \mathrm{x}}{\partial \mathrm{X}_{\mathrm{i}}} \int_{\theta} \cos \theta \ln R \mathrm{~d} \theta+\mathrm{n}_{\mathrm{j}} \frac{\partial \mathrm{y}}{\partial \mathrm{X}_{\mathrm{i}}} \int_{\theta} \operatorname{sen} \theta \ln R \mathrm{~d} \theta-\mathrm{n}_{\mathrm{i}} \frac{\partial \mathrm{x}}{\partial \mathrm{X}_{\mathrm{j}}} \int_{\theta} \cos \theta \ln R \mathrm{~d} \theta+\right.
$$




$$
\begin{aligned}
& \left.-\mathrm{n}_{\mathrm{i}} \frac{\partial \mathrm{y}}{\partial \mathrm{X}_{\mathrm{j}}} \int_{\theta} \operatorname{sen} \theta \ln R \mathrm{~d} \theta\right\} \mathrm{U}_{\mathrm{j}}^{\mathrm{s}} \\
& \mathrm{h}_{\mathrm{ij}}^{\mathrm{s}}=\frac{(1-2 v)}{8 \pi(1-v)}\left\{\left(\mathrm{n}_{\mathrm{j}} \frac{\partial \mathrm{x}}{\partial \mathrm{X}_{\mathrm{i}}}-\mathrm{n}_{\mathrm{i}} \frac{\partial \mathrm{x}}{\partial \mathrm{X}_{\mathrm{j}}}\right) \int_{\theta} \cos \theta \ln R \mathrm{~d} \theta+\left(\mathrm{n}_{\mathrm{j}} \frac{\partial \mathrm{y}}{\partial \mathrm{X}_{\mathrm{i}}}-\mathrm{n}_{\mathrm{i}} \frac{\partial \mathrm{y}}{\partial \mathrm{X}_{\mathrm{j}}}\right) \int \operatorname{sen} \theta \ln R \mathrm{~d} \theta\right\} \mathrm{U}_{\mathrm{j}}^{\mathrm{s}}
\end{aligned}
$$

Substituindo-se a eq.(5.20) na eq.(5.39), tem-se:

$$
\begin{aligned}
& \mathrm{h}_{\mathrm{ij}}^{\mathrm{s}}=\frac{(1-2 v)}{8 \pi(1-v)}\left\{\left(\mathrm{n}_{\mathrm{j}} \frac{\partial \mathrm{x}}{\partial \mathrm{X}_{\mathrm{i}}}-\mathrm{n}_{\mathrm{i}} \frac{\partial \mathrm{x}}{\partial \mathrm{X}_{\mathrm{j}}}\right) \int_{\theta} \cos \theta \ln \left(\frac{-2 \mathrm{~A}}{\beta_{\mathrm{s}} \cos \theta+\gamma_{\mathrm{s}} \operatorname{sen} \theta}\right) \mathrm{d} \theta+\right. \\
& \left.+\left(\mathrm{n}_{\mathrm{j}} \frac{\partial \mathrm{y}}{\partial \mathrm{X}_{\mathrm{i}}}-\mathrm{n}_{\mathrm{i}} \frac{\partial \mathrm{y}}{\partial \mathrm{X}_{\mathrm{j}}}\right) \iint_{\theta} \operatorname{sen} \theta \ln \left(\frac{-2 \mathrm{~A}}{\beta_{\mathrm{s}} \cos \theta+\gamma_{\mathrm{s}} \operatorname{sen} \theta}\right) \mathrm{d} \theta\right\} \mathrm{U}_{\mathrm{j}}^{\mathrm{s}}
\end{aligned}
$$

Apenas por uma questão de simplificação, pode-se fazer:

$$
\begin{aligned}
& \bar{A}=-2 A \\
& B=n_{j} \frac{\partial x}{\partial X_{i}}-n_{i} \frac{\partial x}{\partial X_{j}} \\
& C=n_{j} \frac{\partial y}{\partial X_{i}}-n_{i} \frac{\partial y}{\partial X_{j}}
\end{aligned}
$$

Integrando-se por partes as duas integrais da eq.(5.40), tem-se respectivamente:

$$
\begin{aligned}
& \int_{\theta} \cos \theta \ln R d \theta=\left.\operatorname{sen} \theta \ln \left(\frac{\bar{A}}{\beta_{s} \cos \theta+\gamma_{s} \operatorname{sen} \theta}\right)\right|_{0} ^{\theta_{f}}+\int_{0}^{\theta_{f}} \frac{\operatorname{sen} \theta\left(\gamma_{s} \cos \theta-\beta_{s} \operatorname{sen} \theta\right)}{\beta_{s} \cos \theta+\gamma_{s} \operatorname{sen} \theta} d \theta \\
& \int_{\theta} \operatorname{sen} \theta \ln \operatorname{Rd} \theta=-\left.\cos \theta \ln \left(\frac{\bar{A}}{\beta_{s} \cos \theta+\gamma_{s} \operatorname{sen} \theta}\right)\right|_{0} ^{\theta_{f}}-\int_{0}^{\theta_{f}} \frac{\cos \theta\left(\gamma_{s} \cos \theta-\beta_{s} \operatorname{sen} \theta\right)}{\beta_{s} \cos \theta+\gamma_{s} \operatorname{sen} \theta} d \theta
\end{aligned}
$$

Substituindo-se as eq.(5.44) e (5.45) e considerando-se as simplificações feitas, pode-se escrever: 


$$
\begin{aligned}
& h_{i j}^{s}=\frac{(1-2 v)}{8 \pi(1-v)}\left\{\left.B \operatorname{sen} \theta \ln \left(\frac{\bar{A}}{\beta_{s} \cos \theta+\gamma_{s} \operatorname{sen} \theta}\right)\right|_{0} ^{\theta_{f}}+B \int_{0}^{\theta_{f}} \frac{\operatorname{sen} \theta\left(\gamma_{s} \cos \theta-\beta_{s} \operatorname{sen} \theta\right)}{\beta_{s} \cos \theta+\gamma_{s} \operatorname{sen} \theta} d \theta+\right. \\
& \left.-\left.C \cos \theta \ln \left(\frac{\bar{A}}{\beta_{s} \cos \theta+\gamma_{s} \operatorname{sen} \theta}\right)\right|_{0} ^{\theta_{f}}-C \int_{0}^{\theta_{f}} \frac{\cos \theta\left(\gamma_{s} \cos \theta-\beta_{s} \operatorname{sen} \theta\right)}{\beta_{s} \cos \theta+\gamma_{s} \operatorname{sen} \theta} d \theta\right\} U_{j}^{s} \\
& h_{i j}^{s}=\frac{(1-2 v)}{8 \pi(1-v)}\left\{\left.(B \operatorname{sen} \theta-C \cos \theta) \ln \left(\frac{\bar{A}}{\beta_{s} \cos \theta+\gamma_{s} \operatorname{sen} \theta}\right)\right|_{0} ^{\theta_{f}}+B \int_{0}^{\theta_{f}} \frac{\operatorname{sen} \theta\left(\gamma_{s} \cos \theta-\beta_{s} \operatorname{sen} \theta\right)}{\beta_{s} \cos \theta+\gamma_{s} \operatorname{sen} \theta} d \theta+\right. \\
& \left.-C \int_{0}^{\theta_{f}} \frac{\cos \theta\left(\gamma_{s} \cos \theta-\beta_{s} \operatorname{sen} \theta\right)}{\beta_{s} \cos \theta+\gamma_{s} \operatorname{sen} \theta} d \theta\right\} U_{j}^{s}
\end{aligned}
$$

As equações integrais presentes na eq.(5.47) foram resolvidas com o auxílio do software Mathcad7 Professional:

$\int_{0}^{\theta_{f}} \frac{\operatorname{sen} \theta\left(\gamma_{s} \cos \theta-\beta_{s} \operatorname{sen} \theta\right)}{\beta_{s} \cos \theta+\gamma_{s} \operatorname{sen} \theta} d \theta=\frac{1}{D_{s}}\left[D_{s} \operatorname{sen} \theta+2 \beta_{s} a \tanh \left(\frac{-\beta_{s}+\beta_{s} \cos \theta+\gamma_{s} \operatorname{sen} \theta}{D_{s} \operatorname{sen} \theta}\right)\right]_{0}^{\theta_{f}}$

$\int_{0}^{\theta_{f}} \frac{\cos \theta\left(\gamma_{s} \cos \theta-\beta_{s} \operatorname{sen} \theta\right)}{\beta_{s} \cos \theta+\gamma_{s} \operatorname{sen} \theta} d \theta=\frac{1}{D_{s}}\left[D_{s} \cos \theta+D_{s}-2 \gamma_{s} \operatorname{atanh}\left(\frac{-\beta_{s}+\beta_{s} \cos \theta+\gamma_{s} \operatorname{sen} \theta}{D_{s} \operatorname{sen} \theta}\right)\right]_{0}^{\theta_{f}}$

onde $D_{s}=\sqrt{\beta_{s}^{2}+\gamma_{s}^{2}}$.

Substituindo-se as eq.(5.48) e (5.49) na eq.(5.47), pode-se chegar a:

$$
\begin{aligned}
& h_{i j}^{s}=\frac{(1-2 v)}{8 \pi(1-v)}\left\{(B \operatorname{sen} \theta-C \cos \theta)\left[\ln \left(\frac{\bar{A}}{\beta_{s} \cos \theta+\gamma_{s} \operatorname{sen} \theta}\right)+1\right]-E_{s}+\right. \\
& \left.+2\left(E_{s} \beta_{s}+F_{s} \gamma_{s}\right) \operatorname{atanh}\left(\frac{-\beta_{s}+\beta_{s} \cos \theta+\gamma_{s} \operatorname{sen} \theta}{D_{s} \operatorname{sen} \theta}\right)\right\}\left.\right|_{0} ^{\theta_{f}} U_{j}^{s}
\end{aligned}
$$

onde: 


$$
\begin{aligned}
& E_{s}=\frac{B}{D_{s}} \\
& F_{s}=\frac{C}{D_{s}}
\end{aligned}
$$

Por fim, a eq.(5.50) foi calculada como:

$h_{i j}^{s}=\left(h_{i j}^{s \theta_{f}}-h_{i j}^{s 0}\right) U_{j}^{s}$

onde:

$$
\begin{aligned}
& h_{i j}^{s \theta_{f}}=\frac{(1-2 v)}{8 \pi(1-v)}\left\{\left(B \operatorname{sen} \theta_{f}-C \cos \theta_{f}\right)\left[\ln \left(\frac{\bar{A}}{\beta_{s} \cos \theta_{f}+\gamma_{s} \operatorname{sen} \theta_{f}}\right)+1\right]-E_{s}+\right. \\
& \left.+2\left(E_{s} \beta_{s}+F_{s} \gamma_{s}\right) \operatorname{atanh}\left(\frac{-\beta_{s}+\beta_{s} \cos \theta_{f}+\gamma_{s} \operatorname{sen} \theta_{f}}{D_{s} \operatorname{sen} \theta_{f}}\right)\right\} \\
& h_{i j}^{s 0}=\frac{(1-2 v)}{8 \pi(1-v)}\left\{-E_{s}\left[\ln \left(\frac{\bar{A}}{\beta_{s}}\right)+2\right]+2\left(E_{s} \beta_{s}+F_{s} \gamma_{s}\right) \operatorname{atanh}\left[\frac{\gamma_{s}}{D_{s}}\right]\right\}
\end{aligned}
$$

Vale uma pequena observação a respeito do arco tangente hiperbólica presente nas eq.(5.52) e (5.53); para que esse fosse calculado, dentro do código computacional desenvolvido, criou-se uma função dada como segue:

$$
\operatorname{atanh}(x)=\frac{1}{2} \ln \left(\frac{1+x}{1-x}\right)
$$

Utilizando-se o procedimento desenvolvido e apresentado nesta seção, não se pode calcular as forças de superfície incógnitas nos pontos especiais, ou seja, não se pode aplicar a condição de deslocamento conhecido em tais pontos, pois isso levaria a um problema de singularidade na resolução do sistema de equações. Os valores de força de superfície devem ser calculados utilizando-se as funções de forma do elemento, para os pontos especiais. Figura 5.5 . 


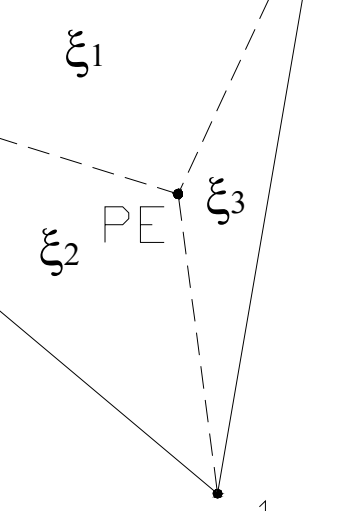

Figura 5.5 - Coordenadas adimensionais de ponto especial

$\mathrm{P}_{1}=\xi_{1} \cdot \mathrm{P}_{1}^{1}+\xi_{2} \cdot \mathrm{P}_{1}^{2}+\xi_{3} \cdot \mathrm{P}_{1}^{3}$

$P_{2}=\xi_{1} \cdot P_{2}^{1}+\xi_{2} \cdot P_{2}^{2}+\xi_{3} \cdot P_{2}^{3}$

$P_{3}=\xi_{1} \cdot P_{3}^{1}+\xi_{2} \cdot P_{3}^{2}+\xi_{3} \cdot P_{3}^{3}$

ou simplesmente:

$P_{i}=\xi_{j} \cdot P_{i}^{j} \quad$ i e j $=1,2,3$

onde $P_{i}^{j}$ é a força de superfície na direção 'i', do nó 'j', referente ao elemento a que pertence o ponto especial.

Se o ponto especial pertencer a 2 elementos (figura 5.1), calculam-se os valores das forças de superfície deste ponto para cada um dos elementos e depois se pode fazer a média entre os dois valores.

Com relação ao cálculo das tensões no contorno para os pontos especiais, segue-se o procedimento apresentado na seção 3.6 sem maiores problemas, uma vez que as forças de superfície já foram devidamente calculadas utilizando-se as eq.(5.55), (5.56) e (5.57). Mais uma vez, se o ponto especial pertencer a 2 elementos (figura 5.1), calculam-se os valores das tensões deste ponto para cada um dos elementos e depois se pode fazer a média entre os dois valores. 


\subsection{INTEGRAÇÃO NÃO-SINGULAR PARA ELEMENTOS DE CONTORNO}

Para as integrações não-singulares, utilizou-se o esquema de integração de Hammer com subelementação, feito com base em Tejerina Calderón (1996).

O número de subelementos (NS) em que será dividido o elemento é função de uma relação entre a distância do ponto fonte ao centróide do elemento $(\bar{R})$ e o comprimento médio dos lados do mesmo $(\bar{L})$.

$0 \leq \overline{\mathrm{R}} \leq \overline{\mathrm{L}} \quad-25$ subelementos

$\overline{\mathrm{L}}<\overline{\mathrm{R}} \leq 2 \overline{\mathrm{L}} \quad-16$ subelementos

$2 \overline{\mathrm{L}}<\overline{\mathrm{R}} \leq 4 \overline{\mathrm{L}}-9$ subelementos

$\overline{\mathrm{R}}>4 \overline{\mathrm{L}} \quad-4$ subelementos

No código computacional, após a determinação do número de subelementos, procede-se a um looping de 1 até NS e, mais interno a este, outro looping de 1 até o número de pontos de integração de Hammer, que no trabalho foi escolhido 7. Para cada ponto de integração, serão determinadas as coordenadas homogêneas $\xi_{1}, \xi_{2}$ e $\xi_{3}$ globais em função das mesmas coordenadas homogêneas locais (valores tabelados). Para maiores detalhes, ver Tejerina Calderón (1996).

De posse das coordenadas homogêneas globais do ponto de integração, pode-se determinar as coordenadas globais $X_{1}, X_{2}$ e $X_{3}$ desse ponto utilizando-se as equações abaixo:

$$
\begin{aligned}
& X_{1}=\xi_{1} \cdot X_{1}^{1}+\xi_{2} \cdot X_{1}^{2}+\xi_{3} \cdot X_{1}^{3} \\
& X_{2}=\xi_{1} \cdot X_{2}^{1}+\xi_{2} \cdot X_{2}^{2}+\xi_{3} \cdot X_{2}^{3} \\
& X_{3}=\xi_{1} \cdot X_{3}^{1}+\xi_{2} \cdot X_{3}^{2}+\xi_{3} \cdot X_{3}^{3}
\end{aligned}
$$

onde $X_{i}^{j}$ são as coordenadas globais na direção 'i' do nó 'j' do elemento a ser integrado. 
A partir das coordenadas do ponto de integração, determinam-se outros parâmetros necessários ao cálculo dos elementos das matrizes g e h:
$r_{1}=X_{1}-X F_{1}^{\prime}$$$
r_{, 1}=\frac{r_{1}}{r}
$$
$r_{2}=X_{2}-X F_{2}^{\prime}$$$
r_{2}=\frac{r_{2}}{r}
$$
$r_{3}=X_{3}-X F_{3}^{1}$$$
r_{3}=\frac{r_{3}}{r}
$$
$r=\sqrt{r_{1}^{2}+r_{2}^{2}+r_{3}^{2}}$

$$
r, n=r, 1 \cdot n_{1}+r, 2 \cdot n_{2}+r_{, 3} \cdot n_{3}
$$

onde:

r - é o raio, ou a distância entre o ponto fonte e o ponto de integração;

$r_{i} \quad$ - são as componentes do vetor $r$;

$r_{, i} \quad$ - são as derivadas parciais do vetor $r$ nas direções $i$;

$r, n \quad$ é a derivada parcial do vetor $r$ na direção normal ao elemento.

As expressões das soluções fundamentais deduzidas na seção 3.4 (eq.(3.43) e (3.44)) geram duas matrizes de dimensão 3x3 para cada ponto de integração. Essas matrizes devem ser então armazenadas em outras duas matrizes de dimensões 3x9, denominadas no trabalho por LG e LH:

$L G=\frac{A \cdot w}{N S}\left[\begin{array}{lll:lll:lll}u_{11}^{*} \xi_{1} & u_{12}^{*} \xi_{1} & u_{13}^{*} \xi_{1} & u_{11}^{*} \xi_{2} & u_{12}^{*} \xi_{2} & u_{13}^{*} \xi_{2} & u_{11}^{*} \xi_{3} & u_{12}^{*} \xi_{3} & u_{13}^{*} \xi_{3} \\ u_{21}^{*} \xi_{1} & u_{22}^{*} \xi_{1} & u_{23}^{*} \xi_{1} & u_{21}^{*} \xi_{2} & u_{22}^{*} \xi_{2} & u_{23}^{*} \xi_{2} & u_{21}^{*} \xi_{3} & u_{22}^{*} \xi_{3} & u_{23}^{*} \xi_{3} \\ u_{31}^{*} \xi_{1} & u_{32}^{*} \xi_{1} & u_{33}^{*} \xi_{1} & u_{31}^{*} \xi_{2} & u_{32}^{*} \xi_{2} & u_{33}^{*} \xi_{2} & u_{31}^{*} \xi_{3} & u_{32}^{*} \xi_{3} & u_{33}^{*} \xi_{3}\end{array}\right]$

$\mathrm{LH}=\frac{\mathrm{A} \cdot \mathrm{w}}{\mathrm{NS}}\left[\begin{array}{llllllllll}\mathrm{p}_{11}^{*} \xi_{1} & \mathrm{p}_{12}^{*} \xi_{1} & \mathrm{p}_{13}^{*} \xi_{1} & \mathrm{p}_{11}^{*} \xi_{2} & \mathrm{p}_{12}^{*} \xi_{2} & \mathrm{p}_{13}^{*} \xi_{2} & \mathrm{p}_{11}^{*} \xi_{3} & \mathrm{p}_{12}^{*} \xi_{3} & \mathrm{p}_{13}^{*} \xi_{3} \\ \mathrm{p}_{21}^{*} \xi_{1} & \mathrm{p}_{22}^{*} \xi_{1} & \mathrm{p}_{23}^{*} \xi_{1} & \mathrm{p}_{21}^{*} \xi_{2} & \mathrm{p}_{22}^{*} \xi_{2} & \mathrm{p}_{23}^{*} \xi_{2} & \mathrm{p}_{21}^{*} \xi_{3} & \mathrm{p}_{22}^{*} \xi_{3} & \mathrm{p}_{23}^{*} \xi_{3} \\ \mathrm{p}_{31}^{*} \mathrm{p}_{32}^{*} \xi_{1} & \mathrm{p}_{33}^{*} \xi_{1} & \mathrm{p}_{31}^{*} \xi_{2} & \mathrm{p}_{32}^{*} \xi_{2} & \mathrm{p}_{33}^{*} \xi_{2} & \mathrm{p}_{31}^{*} \xi_{3} & \mathrm{p}_{32}^{*} \xi_{3} & \mathrm{p}_{33}^{*} \xi_{3}\end{array}\right]$

onde w é o "peso" atribuído ao ponto de integração (local ao subelemento), tabelado. 
Após a contribuição de todos os subelementos (integração do elemento). Procede-se à alocação das matrizes LG e LH nas matrizes $g$ e $h$, respectivamente, globais. Esse processo é feito de acordo com o esquema apresentado a seguir:

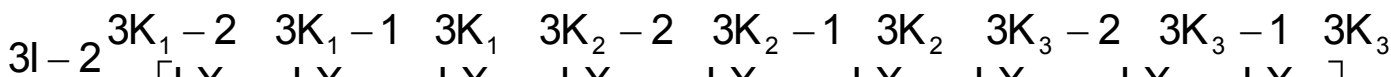

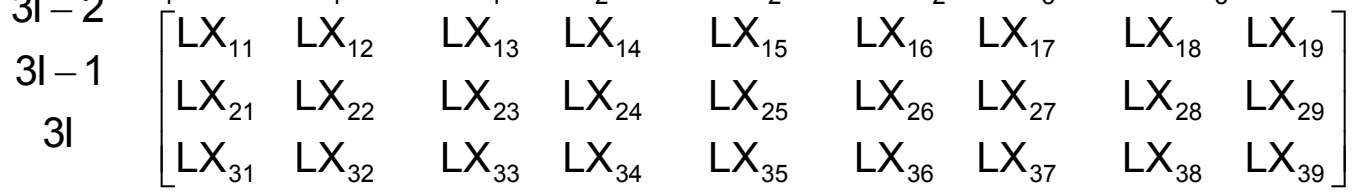

onde:

I - é o número do ponto fonte;

$\mathrm{K}_{\mathrm{A}} \quad-$ é o nó $\mathrm{A}$ do elemento que está sendo integrado;

LX $X_{B C}$ - são os elementos das matrizes LG ou LH.

Observa-se que as matrizes $g$ e $\mathrm{h}$ terão dimensão de $3 \mathrm{NNT} \times 3 \mathrm{NNT}$, onde NNT é o número total de nós.

\subsection{INTEGRAÇÃO QUASE-SINGULAR PARA ELEMENTOS DE CONTORNO}

A presente seção apresenta uma nova forma de integração quasesingular, ou seja, quando o ponto fonte estiver muito próximo ao elemento a ser integrado. Sabe-se que a precisão obtida pela integração de Hammer é inversamente proporcional a uma potência da distância entre o ponto fonte e o ponto campo $(r)$, devido ao problema de singularidade e o número de pontos de integração. Assim, para valores pequenos de ' $r$ ', faz-se necessário um tratamento especial de tais integrais.

No código computacional desenvolvido, considerou-se a utilização da integração quase-singular quando a inequação abaixo for satisfeita.

$\overline{\mathrm{R}}<$ fator $\cdot \mathrm{L}_{\max }$ 
onde:

$\bar{R} \quad$ - é a distância do ponto fonte ao centróide do elemento;

fator - é um fator de multiplicação (adotado 0,4 no programa);

$\mathrm{L}_{\max } \quad$ - é o comprimento do maior lado do elemento a ser integrado.

O primeiro passo deste processo é a determinação das coordenadas do ponto base do integrador. Para tal, deve-se projetar o ponto fonte no plano do elemento a ser integrado.

$\mathrm{Na}$ figura 5.6, nota-se que as coordenadas $\mathrm{X} 1, \mathrm{X} 2$ e $\mathrm{X} 3$ são globais à análise do problema; o ponto s' é a projeção do ponto fonte $s$ no plano do elemento, e o vetor $\vec{n}$ é normal ao elemento. O ponto b é o ponto base do integrador. A determinação de sua localização será apresentada mais à frente.

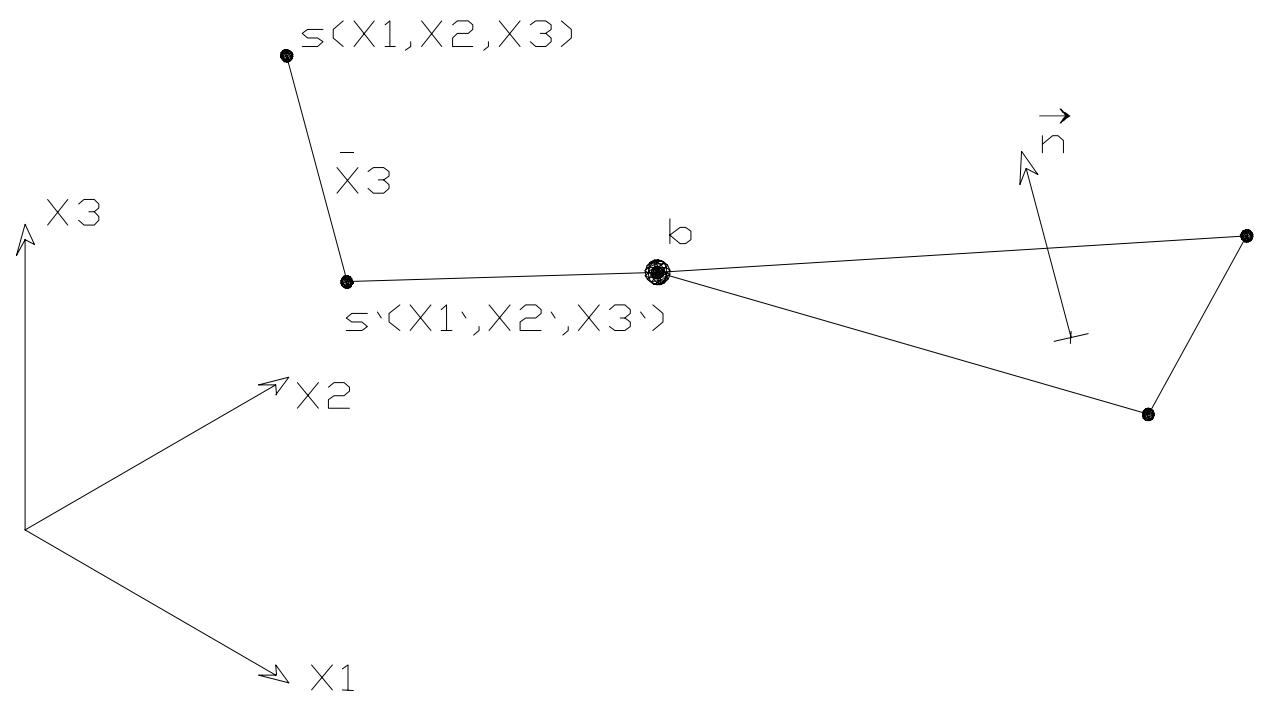

Figura 5.6 - Coordenadas do ponto s'

As coordenadas do ponto s' são calculadas utilizando-se a seguinte equação:

$\left\{\begin{array}{l}X_{1}^{\prime} \\ X_{2}^{\prime} \\ X_{3}^{\prime}\end{array}\right\}=\left\{\begin{array}{l}X_{1} \\ X_{2} \\ X_{3}\end{array}\right\}-\bar{X}_{3}\left\{\begin{array}{l}n_{1} \\ n_{2} \\ n_{3}\end{array}\right\}$ 
onde:

$$
\bar{X}_{3}=n_{1}\left(X_{1}-X_{1}^{0}\right)+n_{2}\left(X_{2}-X_{2}^{0}\right)+n_{3}\left(X_{3}-X_{3}^{0}\right)
$$

sendo $n_{1}, n_{2}$ e $n_{3}$ as componentes do vetor $\vec{n}$ nas direções 1,2 e 3 globais, respectivamente, $\mathrm{e}\left(\mathrm{X}_{1}^{0}, \mathrm{X}_{2}^{0}, \mathrm{X}_{3}^{0}\right)$ as coordenadas globais do ponto origem do sistema de coordenadas locais.

De posse das coordenadas do ponto s', pode-se determinar as coordenadas homogêneas deste ponto; porém, antes, é necessário que se faça uma transformação de coordenadas: do sistema global para o local.

$$
\xi_{\ell}^{\prime}=\frac{1}{2 A}\left[\alpha_{\ell}+\beta_{\ell} x+\gamma_{\ell} y\right]
$$

As coordenadas homogêneas do ponto base podem ser determinadas de acordo com o esquema abaixo:

$$
\text { Se } \xi_{i}^{\prime} \text { e } \xi_{j}^{\prime} \leq 0 \Rightarrow \xi_{k}^{b}=1 ; \xi_{i}^{b}=0 ; \xi_{j}^{b}=0
$$

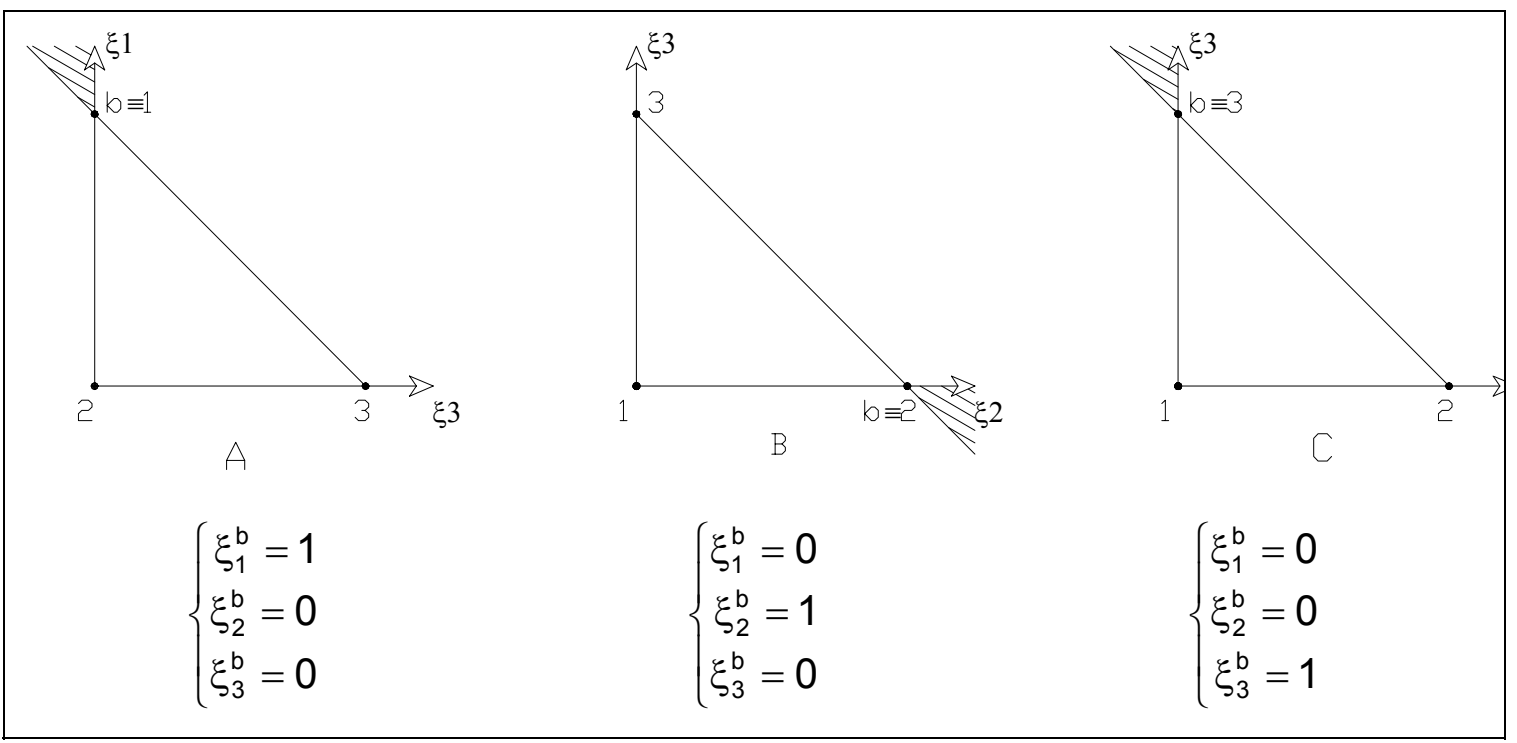

Figura 5.7 - Ponto base no vértice do elemento

Se apenas $\xi_{i}^{\prime} \leq 0 \Rightarrow a=\xi_{j}^{\prime} / \xi_{k}^{\prime} ; \xi_{k}^{b}=1 /(a+1) ; \xi_{j}^{b}=a \cdot \xi_{k}^{b} ; \xi_{i}^{b}=0$ 
onde j e k são determinados por permutação cíclica dos índices do elemento de contorno:
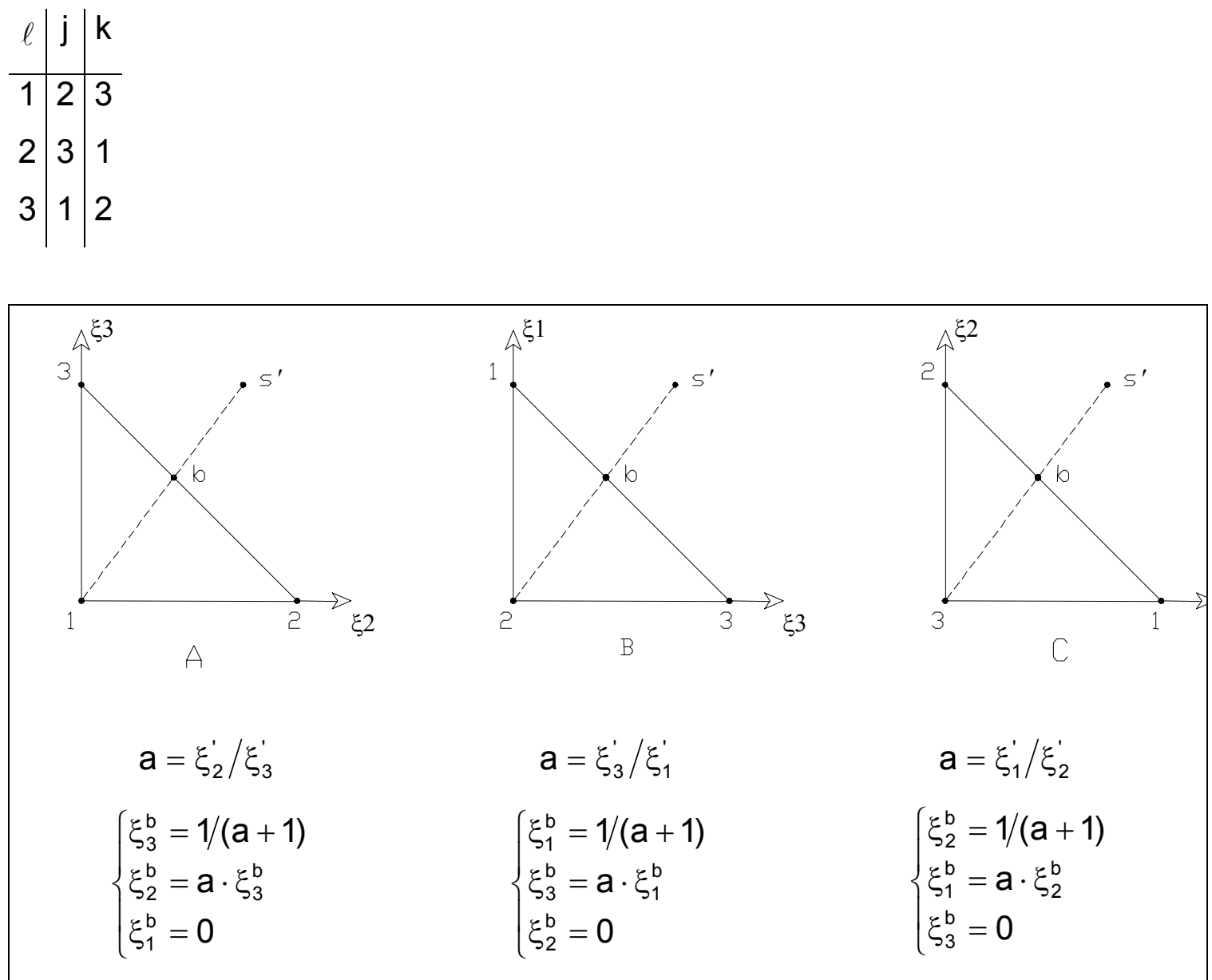

Figura 5.8 - Ponto base no lado do elemento

Se $\xi_{i}^{\prime}, \xi_{j}^{\prime}$ e $\xi_{k}^{\prime}>0 \Rightarrow \xi_{i}^{b}=\xi_{i}^{\prime} ; \xi_{j}^{b}=\xi_{j}^{\prime} ; \xi_{k}^{b}=\xi_{k}^{\prime}$

Este último é o caso em que o ponto s' está localizado no domínio do elemento de contorno, assim, o ponto base coincide com o ponto s'.

De posse das coordenadas homogêneas do ponto base, pode-se finalmente se determinar as coordenadas globais desse ponto utilizando-se as equações abaixo:

$$
\begin{aligned}
& X_{1}^{\mathrm{b}}=\xi_{1}^{\mathrm{b}} \cdot \mathrm{X}_{1}^{1}+\xi_{2}^{\mathrm{b}} \cdot \mathrm{X}_{1}^{2}+\xi_{3}^{\mathrm{b}} \cdot \mathrm{X}_{1}^{3} \\
& \mathrm{X}_{2}^{\mathrm{b}}=\xi_{1}^{\mathrm{b}} \cdot \mathrm{X}_{2}^{1}+\xi_{2}^{\mathrm{b}} \cdot \mathrm{X}_{2}^{2}+\xi_{3}^{\mathrm{b}} \cdot \mathrm{X}_{2}^{3} \\
& \mathrm{X}_{3}^{\mathrm{b}}=\xi_{1}^{\mathrm{b}} \cdot \mathrm{X}_{3}^{1}+\xi_{2}^{\mathrm{b}} \cdot \mathrm{X}_{3}^{2}+\xi_{3}^{\mathrm{b}} \cdot \mathrm{X}_{3}^{3}
\end{aligned}
$$


ou simplesmente:

$$
X_{i}^{b}=\xi_{j}^{b} \cdot X_{i}^{j} \quad i, j=1,2,3
$$

onde as coordenadas globais $X_{i}^{j}$ são referentes ao elemento a ser integrado.

Após a determinação das coordenadas globais do ponto base, procedese a uma transformação de coordenadas: do sistema cartesiano global para o sistema adimensional homogêneo. Figura 5.9.

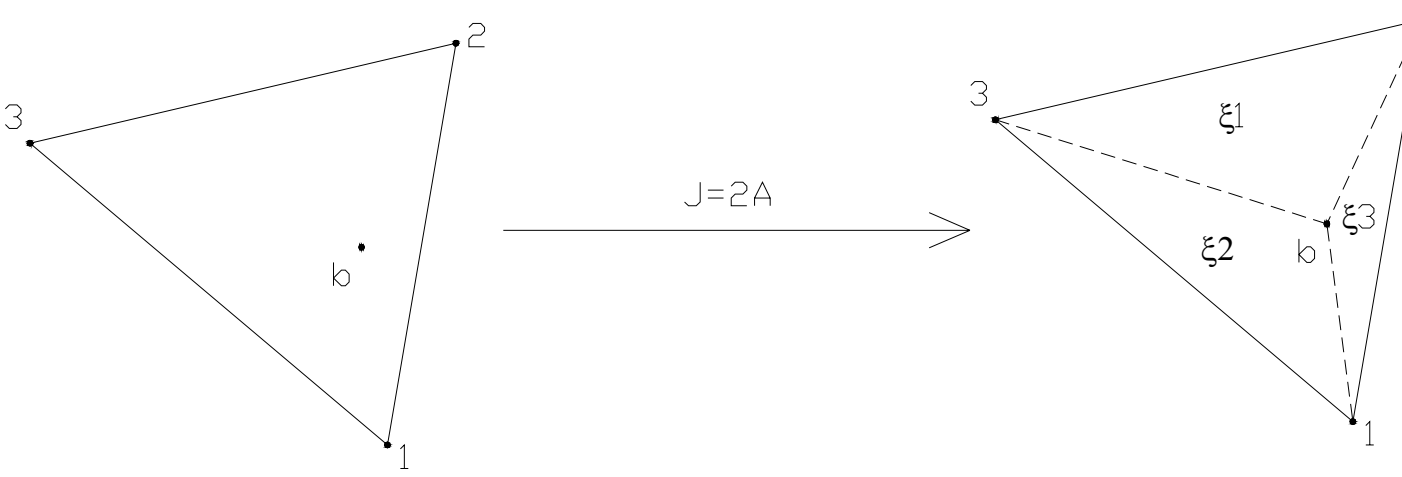

Figura 5.9 - Transformação de coordenadas

onde J é o Jacobiano da transformação. Assim:

$I=\int_{A} f d A=2 A \int_{\xi_{2}} \int_{\xi_{1}} f d \xi_{1} d \xi_{2}$

onde a função f pode ser $u_{i j}^{*}(s, Q) \phi_{\ell}$ para a determinação da matriz $g, p_{i j}^{*}(s, Q) \phi_{\ell}$ para a determinação da matriz $h, D_{\mathrm{ijk}} \phi_{\ell}$ para a matriz g' e $S_{\mathrm{ijk}} \phi_{\ell}$ para a matriz h'.

O domínio da segunda integral da eq.(5.79) é dividido genericamente em 3 subdomínios como segue: 


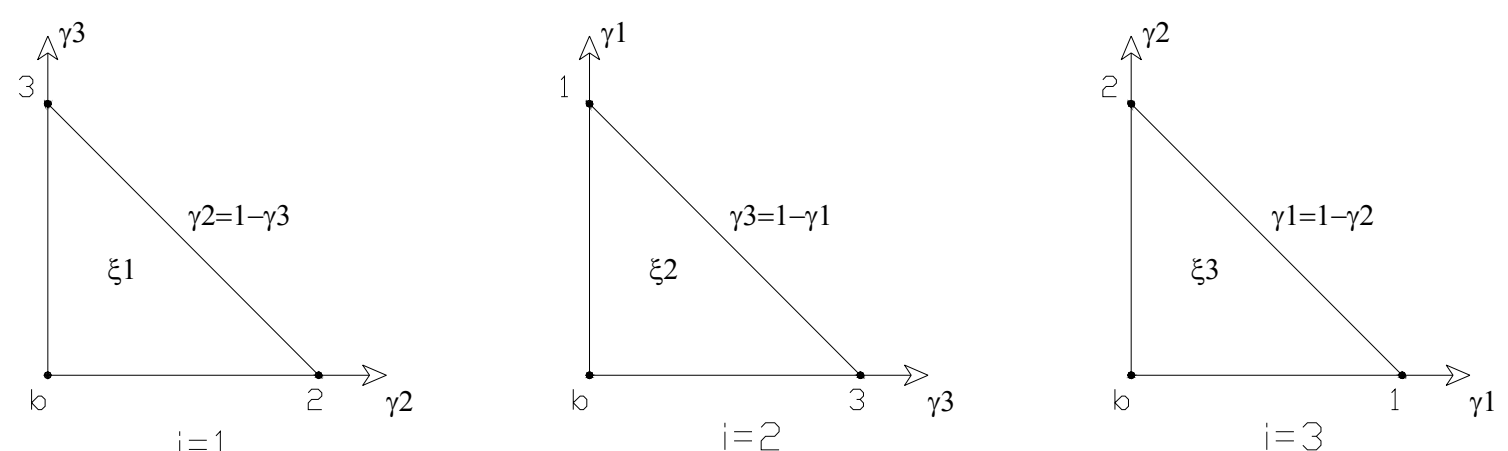

Figura 5.10 - Subdivisão de elemento de contorno

onde:

$I=2 A \sum_{i=1}^{3} \xi_{i}^{b} \int_{0}^{1} \int_{0}^{1-\gamma_{j}} f d \gamma_{j} d \gamma_{k}$

sendo:

i - o subelemento;

$\xi_{\mathrm{i}}^{\mathrm{b}} \quad$ - o Jacobiano da transformação;

j e k - a numeração local dos nós do lado oposto ao ponto base (b);

$\gamma \quad$ - a coordenada homogênea no sistema de coordenadas do subelemento.

As coordenadas homogêneas $\xi_{\ell}$ de um ponto qualquer q pertencente ao subelemento i, podem ser calculadas utilizando-se as equações abaixo:

Para $\mathrm{i}=1 \Rightarrow \xi_{\ell}^{\mathrm{a}}=\xi_{\ell}^{\mathrm{b}} \gamma_{1}+\xi_{\ell}^{2} \gamma_{2}+\xi_{\ell}^{3} \gamma_{3}$

Para $\mathrm{i}=2 \Rightarrow \xi_{\ell}^{\mathrm{a}}=\xi_{\ell}^{1} \gamma_{1}+\xi_{\ell}^{\mathrm{b}} \gamma_{2}+\xi_{\ell}^{3} \gamma_{3}$

Para $\mathrm{i}=3 \Rightarrow \xi_{\ell}^{\mathrm{q}}=\xi_{\ell}^{1} \gamma_{1}+\xi_{\ell}^{2} \gamma_{2}+\xi_{\ell}^{\mathrm{b}} \gamma_{3}$

onde $\ell$ varia de 1 a 3.

A eq.(5.80) será escrita segundo um sistema de coordenadas polares adimensional $(\rho, \theta)$ de acordo com a figura 5.11 abaixo. 


$$
\begin{aligned}
\gamma_{j} & =\rho \cdot \cos \theta \\
\gamma_{k} & =\rho \cdot \operatorname{sen} \theta \\
\gamma=1-\gamma \quad & \bar{\rho}=\frac{1}{\operatorname{sen} \theta+\cos \theta}
\end{aligned}
$$

Figura 5.11 - Coordenadas polares no espaço 3D adimensional (subelemento i)

Assim, a eq.(5.80) pode ser escrita como:

$I=2 A \sum_{i=1}^{3} \xi_{i}^{b} \int_{0}^{\frac{\pi}{2}} \int_{0}^{\bar{\rho}} f \cdot \rho \cdot d \rho d \theta$

A equação integral em $\theta$ será escrita em outro sistema adimensional mais adequado à quadratura de Gauss:

$I=\frac{\pi A}{2} \sum_{i=1}^{3} \xi_{i}^{b} \int_{-1}^{1} \int_{0}^{\bar{\rho}} f \cdot \rho \cdot d \rho d \zeta_{\theta}$

onde:

$\theta\left(\zeta_{\theta}\right)=\frac{\pi}{4}\left(1+\zeta_{\theta}\right)$

Com o objetivo de melhorar a qualidade da integração da eq.(5.88), divide-se o raio adimensional $\rho$ progressivamente. Figura 5.12. Esta técnica foi apresentada para elementos unidimensionais por Mon-Ma, Venturini e Coda (1996). 

$\rho 1 \quad \rho 2$
ค3
$\rho$
$\bar{\rho}$
$\rho$

Figura 5.12 - Subdivisão de raio

O valor de $\rho_{1}$,é calculado como sendo:

$\rho_{1}=\left(\frac{d}{L_{\max }}\right)^{(g s+1)}$

onde:

d - é a distância entre o ponto fonte (s) e o ponto base (b);

$L_{\max } \quad$ - é o comprimento do maior lado do elemento a ser integrado;

gs - é o grau de singularidade da função $f^{1}$.

Os próximos valores de $\rho$ são calculados pela eq.(5.91) abaixo:

$\rho_{j}=\rho_{(j-1)}+\left(\rho_{(j-1)}-\rho_{(j-2)}\right) R P$

onde RP é o valor da razão de progressão (adotado 1,5 no programa).

$$
\begin{aligned}
& I=\frac{\pi A}{2} \sum_{i=1}^{3} \xi_{i}^{b} \int_{-1}^{1}\left\{\int_{0}^{\rho_{1}} f \cdot \rho \cdot d \rho+\int_{\rho_{1}}^{\rho_{2}} f \cdot \rho \cdot d \rho+\ldots+\int_{\rho_{n-1}}^{\rho_{n}} f \cdot \rho \cdot d \rho\right\} d \zeta_{\theta} \\
& I=\frac{\pi A}{2} \sum_{i=1}^{3} \xi_{i}^{b} \int_{-1}^{1} \sum_{z=1}^{n} \int_{\rho_{z-1}}^{\rho_{z}} f \cdot \rho \cdot d \rho d \zeta_{\theta}
\end{aligned}
$$

\footnotetext{
${ }^{1}$ No código desenvolvido, foi adotado gs=2 para deslocamento e força de superfície, e gs=3 para tensões internas.
} 
onde:

z - é o número da subdivisão do raio $\rho$;

$\mathrm{n} \quad-$ é o número de subdivisões do raio $\rho$.

e:

$\left\{\begin{array}{l}\rho_{z_{0}}=0 \\ \rho_{z_{n}}=\bar{\rho}\left(\theta\left(\zeta_{\theta}\right)\right)\end{array}\right.$

Transformando-se $\rho$ em uma outra coordenada adimensional mais adequada, tem-se:

$I=\frac{\pi A}{4} \sum_{i=1}^{3} \xi_{i}^{b} \int_{-1}^{1} \sum_{z=1}^{n}\left(\rho_{z}-\rho_{z-1}\right) \int_{-1}^{1} f \cdot \rho\left(\zeta_{\rho}\right) \cdot d \zeta_{\rho} d \zeta_{\theta}$

onde $\rho\left(\zeta_{\rho}\right)$ pode ser calculado utilizando-se a eq.(5.96) abaixo:

$\rho\left(\zeta_{\rho}\right)=\left(\frac{\rho_{\mathrm{f}}-\rho_{\mathrm{i}}}{2}\right) \zeta_{\rho}+\frac{\rho_{\mathrm{f}}+\rho_{\mathrm{i}}}{2}$

onde $\rho_{\mathrm{f}}$ e $\rho_{\mathrm{i}}$ são referentes ao sub-raio.

Resolvendo-se numericamente a eq.(5.95), pode-se escrever:

$I=\frac{\pi A}{4} \sum_{i=1}^{3} \xi_{i}^{b} \sum_{i g \rho=1 z=1}^{n g \rho} \sum_{z}^{n}\left(\rho_{z}-\rho_{z-1}\right) \sum_{i g \theta=1}^{n g \theta} f \cdot \rho\left(\zeta_{\rho}\right) \omega_{\text {ig } \rho} \omega_{\text {ig } \theta}$

Foram adotados valores de $\mathrm{ng} \rho=3$ e $\mathrm{ng} \theta=6$ no programa desenvolvido. 


\subsection{INTEGRAÇÃO NÃO-SINGULAR PARA CÉLULAS}

Nesta seção será apresentada uma integração geral para células. Entenda-se por geral, uma integração regular em que o ponto fonte pode estar em qualquer lugar em relação ao elemento (inclusive no seu interior). Será utilizado um procedimento bastante semelhante ao que foi apresentado na seção anterior. Assim, o primeiro passo seria a determinação do ponto base de integração. Observa-se a figura 5.13.

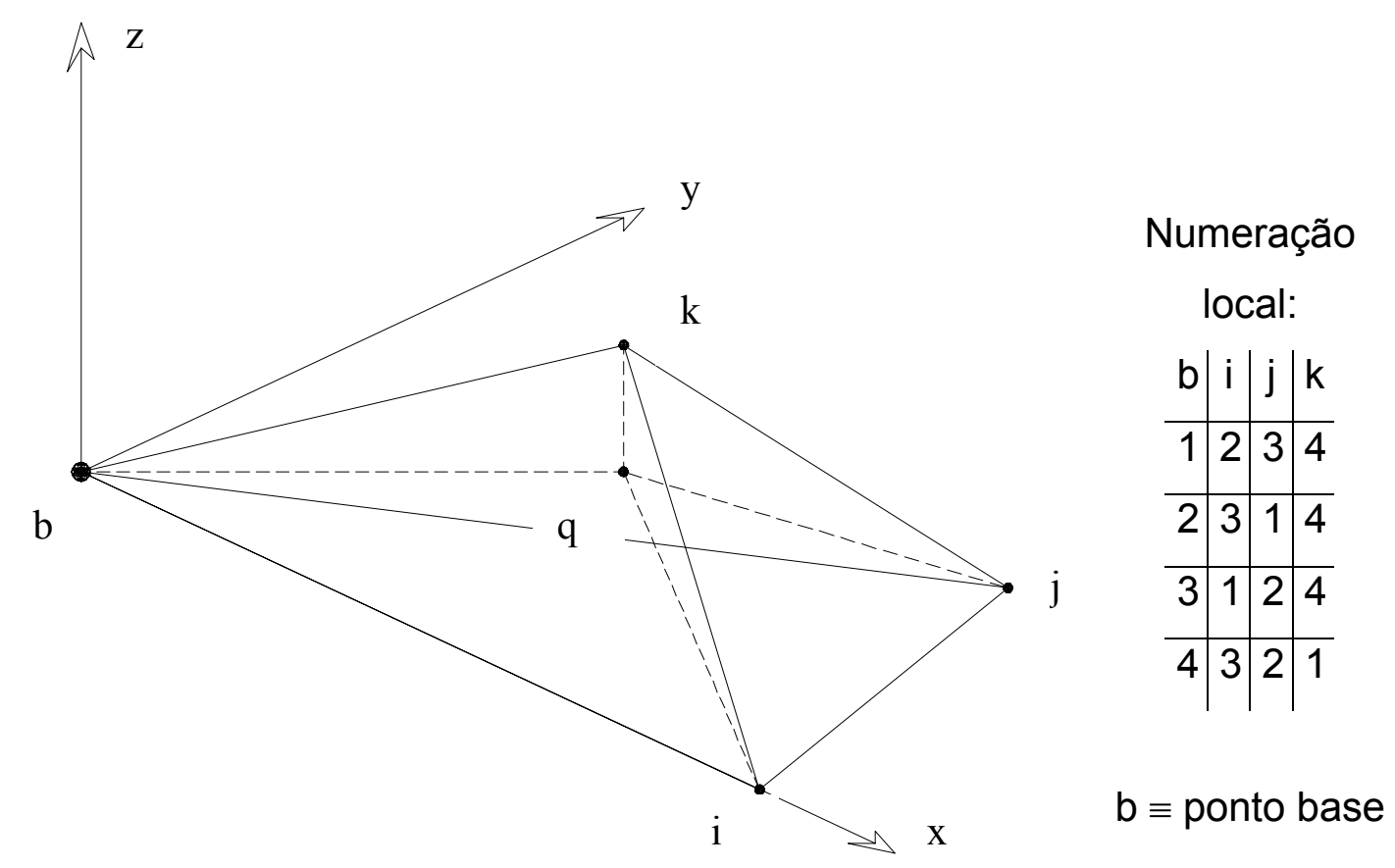

Figura 5.13 - Célula tetraédrica

Para uma aproximação linear, pode-se escrever que:

$\phi_{\ell}=\xi_{\ell}=\frac{1}{6 \mathrm{~V}}\left[\alpha_{\ell}+\beta_{\ell} \mathrm{x}+\gamma_{\ell} \mathrm{y}+\delta_{\ell} \mathrm{z}\right]$

onde $\vee$ é o volume da célula, e: 


$$
\begin{aligned}
& \alpha_{\ell}=(-1)^{\ell+1}\left[\mathrm{x}_{\mathrm{i}} \mathrm{y}_{\mathrm{j}} \mathrm{z}_{\mathrm{k}}+\mathrm{x}_{\mathrm{k}} \mathrm{y}_{\mathrm{i}} \mathrm{z}_{\mathrm{j}}+\mathrm{x}_{\mathrm{j}} \mathrm{y}_{\mathrm{k}} \mathrm{z}_{\mathrm{i}}-\mathrm{x}_{\mathrm{k}} \mathrm{y}_{\mathrm{j}} \mathrm{z}_{\mathrm{i}}-\mathrm{x}_{\mathrm{j}} \mathrm{y}_{\mathrm{i}} \mathrm{z}_{\mathrm{k}}-\mathrm{x}_{\mathrm{i}} \mathrm{y}_{\mathrm{k}} \mathrm{z}_{\mathrm{j}}\right] \\
& \beta_{\ell}=(-1)^{\ell}\left[\mathrm{y}_{\mathrm{j}} \mathrm{z}_{\mathrm{k}}+\mathrm{y}_{\mathrm{i}} \mathrm{z}_{\mathrm{j}}+\mathrm{y}_{\mathrm{k}} \mathrm{z}_{\mathrm{i}}-\mathrm{y}_{\mathrm{j}} \mathrm{z}_{\mathrm{i}}-\mathrm{y}_{\mathrm{i}} \mathrm{z}_{\mathrm{k}}-\mathrm{y}_{\mathrm{k}} \mathrm{z}_{\mathrm{j}}\right] \\
& \gamma_{\ell}=(-1)^{\ell}\left[\mathrm{x}_{\mathrm{i}} \mathrm{z}_{\mathrm{k}}+\mathrm{x}_{\mathrm{k}} \mathrm{z}_{\mathrm{j}}+\mathrm{x}_{\mathrm{j}} \mathrm{z}_{\mathrm{i}}-\mathrm{x}_{\mathrm{k}} \mathrm{z}_{\mathrm{i}}-\mathrm{x}_{\mathrm{j}} \mathrm{z}_{\mathrm{k}}-\mathrm{x}_{\mathrm{i}} \mathrm{z}_{\mathrm{j}}\right] \\
& \delta_{\ell}=(-1)^{\ell}\left[\mathrm{x}_{\mathrm{i}} \mathrm{y}_{\mathrm{j}}+\mathrm{x}_{\mathrm{k}} \mathrm{y}_{\mathrm{i}}+\mathrm{x}_{\mathrm{j}} \mathrm{y}_{\mathrm{k}}-\mathrm{x}_{\mathrm{k}} \mathrm{y}_{\mathrm{j}}-\mathrm{x}_{\mathrm{j}} \mathrm{y}_{\mathrm{i}}-\mathrm{x}_{\mathrm{i}} \mathrm{y}_{\mathrm{k}}\right]
\end{aligned}
$$

sendo i, j e k determinados por permutação cíclica de índices para células:

$$
\left\{\begin{array}{l}
\ell=1,2,3,4 \\
i=2,3,4,1 \\
j=3,4,1,2 \\
k=4,1,2,3
\end{array}\right.
$$

Substituindo-se as coordenadas locais do ponto fonte na eq.(5.98), calculam-se as coordenadas homogêneas desse ponto.

As coordenadas homogêneas do ponto base são determinadas a partir das coordenadas homogêneas do ponto fonte, seguindo o esquema abaixo:

$$
\text { Se } \xi_{i}^{s}, \xi_{j}^{s} \text { e } \xi_{k}^{s} \leq 0 \Rightarrow \xi_{\ell}^{b}=1 ; \xi_{i}^{b}=0 ; \xi_{j}^{b}=0 ; \xi_{k}^{b}=0
$$

ou seja:

$$
\begin{aligned}
& \text { Para } \xi_{2}^{\mathrm{s}}, \xi_{3}^{\mathrm{s}} \mathrm{e} \xi_{4}^{\mathrm{s}} \leq 0 \Rightarrow \xi_{1}^{\mathrm{b}}=1 \mathrm{e} \xi_{2}^{\mathrm{b}}=\xi_{3}^{\mathrm{b}}=\xi_{4}^{\mathrm{b}}=0 \\
& \text { Para } \xi_{1}^{\mathrm{s}}, \xi_{3}^{\mathrm{s}} \mathrm{e} \xi_{4}^{\mathrm{s}} \leq 0 \Rightarrow \xi_{2}^{\mathrm{b}}=1 \mathrm{e} \xi_{1}^{\mathrm{b}}=\xi_{3}^{\mathrm{b}}=\xi_{4}^{\mathrm{b}}=0 \\
& \text { Para } \xi_{1}^{\mathrm{s}}, \xi_{2}^{\mathrm{s}} \mathrm{e} \xi_{4}^{\mathrm{s}} \leq 0 \Rightarrow \xi_{3}^{\mathrm{b}}=1 \mathrm{e} \xi_{1}^{\mathrm{b}}=\xi_{2}^{\mathrm{b}}=\xi_{4}^{\mathrm{b}}=0 \\
& \text { Para } \xi_{1}^{\mathrm{s}}, \xi_{2}^{\mathrm{s}} \mathrm{e} \xi_{3}^{\mathrm{s}} \leq 0 \Rightarrow \xi_{4}^{\mathrm{b}}=1 \mathrm{e} \xi_{1}^{\mathrm{b}}=\xi_{2}^{\mathrm{b}}=\xi_{3}^{\mathrm{b}}=0
\end{aligned}
$$

Observa-se que esse é o caso em que o ponto base é um dos vértices da célula.

Se apenas $\xi_{i}^{s}$ e $\xi_{j}^{s} \leq 0 \Rightarrow a=\xi_{\ell}^{s} / \xi_{k}^{s} ; \xi_{k}^{b}=1 /(a+1) ; \xi_{\ell}^{b}=a \cdot \xi_{k}^{b} ; \xi_{i}^{b}=0 ; \xi_{j}^{b}=0$ 
onde $\ell$ e k são determinados da seguinte forma:

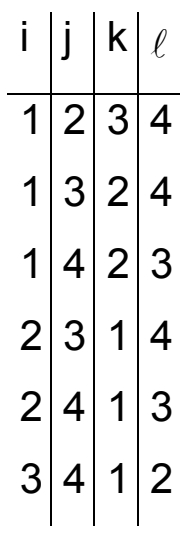

A tabela abaixo contempla todas as possibilidades deste caso:

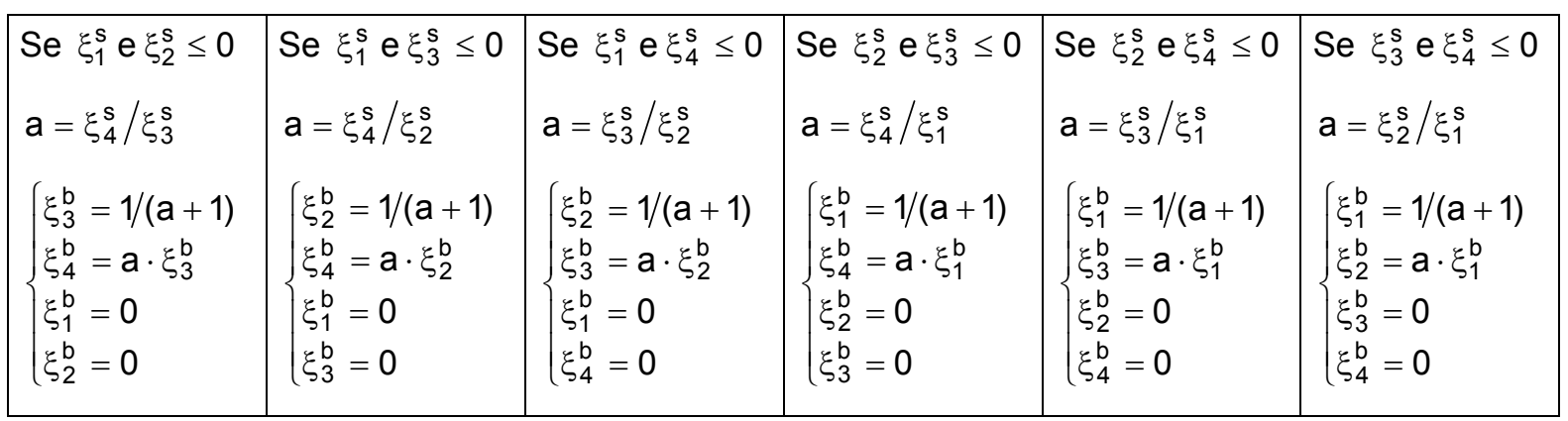

Observa-se que esse é o caso em que o ponto base está localizado em uma das arestas da célula.

Se apenas

$\xi_{i}^{s} \leq 0 \Rightarrow a=\xi_{k}^{s} / \xi_{j}^{s} ; b=\xi_{\ell}^{s} / \xi_{j}^{s} ; \xi_{j}^{b}=1 /(a+b+1) ; \xi_{k}^{b}=a \cdot \xi_{j}^{b} ; \xi_{\ell}^{b}=b \cdot \xi_{j}^{b} ; \xi_{i}^{b}=0$

Com j, $\mathrm{k}$ e $\ell$ determinados pelo esquema cíclico de índices para células:

$\left\{\begin{array}{l}i=1,2,3,4 \\ j=2,3,4,1 \\ k=3,4,1,2 \\ \ell=4,1,2,3\end{array}\right.$ 


\begin{tabular}{|c|c|c|c|}
\hline Se $\xi_{1}^{\mathrm{s}} \leq 0$ & Se $\xi_{2}^{\mathrm{s}} \leq 0$ & Se $\xi_{3}^{\mathrm{s}} \leq 0$ & Se $\xi_{4}^{\mathrm{s}} \leq 0$ \\
$\mathrm{a}=\xi_{3}^{\mathrm{s}} / \xi_{2}^{\mathrm{s}}$ & $\mathrm{a}=\xi_{4}^{\mathrm{s}} / \xi_{3}^{\mathrm{s}}$ & $\mathrm{a}=\xi_{1}^{\mathrm{s}} / \xi_{4}^{\mathrm{s}}$ & $\mathrm{a}=\xi_{2}^{\mathrm{s}} / \xi_{1}^{\mathrm{s}}$ \\
$\mathrm{b}=\xi_{4}^{\mathrm{s}} / \xi_{2}^{\mathrm{s}}$ & $\mathrm{b}=\xi_{1}^{\mathrm{s}} / \xi_{3}^{\mathrm{s}}$ & $\mathrm{b}=\xi_{2}^{\mathrm{s}} / \xi_{4}^{\mathrm{s}}$ & $\mathrm{b}=\xi_{3}^{\mathrm{s}} / \xi_{1}^{\mathrm{s}}$ \\
$\left\{\begin{array}{l}\xi_{2}^{\mathrm{b}}=1 /(\mathrm{a}+\mathrm{b}+1) \\
\xi_{3}^{\mathrm{b}}=\mathrm{a} \cdot \xi_{2}^{\mathrm{b}} \\
\xi_{4}^{\mathrm{b}}=\mathrm{b} \cdot \xi_{2}^{\mathrm{b}} \\
\xi_{1}^{\mathrm{b}}=0\end{array}\right.$ & $\left\{\begin{array}{l}\xi_{3}^{\mathrm{b}}=1 /(\mathrm{a}+\mathrm{b}+1) \\
\xi_{4}^{\mathrm{b}}=\mathrm{a} \cdot \xi_{3}^{\mathrm{b}} \\
\xi_{1}^{\mathrm{b}}=\mathrm{b} \cdot \xi_{3}^{\mathrm{b}} \\
\xi_{2}^{\mathrm{b}}=0\end{array}\right.$ & $\left\{\begin{array}{l}\xi_{4}^{\mathrm{b}}=1 /(\mathrm{a}+\mathrm{b}+1) \\
\xi_{1}^{\mathrm{b}}=\mathrm{a} \cdot \xi_{4}^{\mathrm{b}} \\
\xi_{2}^{\mathrm{b}}=\mathrm{b} \cdot \xi_{4}^{\mathrm{b}} \\
\xi_{3}^{\mathrm{b}}=0\end{array}\right.$ & $\left\{\begin{array}{l}\xi_{1}^{\mathrm{b}}=1 /(\mathrm{a}+\mathrm{b}+1) \\
\xi_{2}^{\mathrm{b}}=\mathrm{a} \cdot \xi_{1}^{\mathrm{b}} \\
\xi_{3}^{\mathrm{b}}=\mathrm{b} \cdot \xi_{1}^{\mathrm{b}} \\
\xi_{4}^{\mathrm{b}}=0\end{array}\right.$ \\
\hline
\end{tabular}

Este é o caso em que o ponto fonte está localizado em uma das faces da célula.

$\operatorname{Se} \xi_{i}^{s}, \xi_{j}^{s}, \xi_{k}^{s}$ e $\xi_{\ell}^{s}>0 \Rightarrow \xi_{i}^{b}=\xi_{i}^{s} ; \xi_{j}^{b}=\xi_{j}^{s} ; \xi_{k}^{b}=\xi_{k}^{s} ; \xi_{\ell}^{b}=\xi_{\ell}^{s}$

Este último é o caso em que o ponto fonte (s) está localizado no domínio da célula, assim, o ponto base (b) coincide com o ponto fonte.

De posse das coordenadas homogêneas do ponto base, pode-se finalmente determinar as coordenadas globais desse ponto utilizando-se as equações abaixo:

$$
\begin{aligned}
& X_{1}^{\mathrm{b}}=\xi_{1}^{\mathrm{b}} \cdot \mathrm{X}_{1}^{1}+\xi_{2}^{\mathrm{b}} \cdot \mathrm{X}_{1}^{2}+\xi_{3}^{\mathrm{b}} \cdot \mathrm{X}_{1}^{3}+\xi_{4}^{\mathrm{b}} \cdot \mathrm{X}_{1}^{4} \\
& \mathrm{X}_{2}^{\mathrm{b}}=\xi_{1}^{\mathrm{b}} \cdot \mathrm{X}_{2}^{1}+\xi_{2}^{\mathrm{b}} \cdot \mathrm{X}_{2}^{2}+\xi_{3}^{\mathrm{b}} \cdot \mathrm{X}_{2}^{3}+\xi_{4}^{\mathrm{b}} \cdot \mathrm{X}_{2}^{4} \\
& \mathrm{X}_{3}^{\mathrm{b}}=\xi_{1}^{\mathrm{b}} \cdot \mathrm{X}_{3}^{1}+\xi_{2}^{\mathrm{b}} \cdot \mathrm{X}_{3}^{2}+\xi_{3}^{\mathrm{b}} \cdot \mathrm{X}_{3}^{3}+\xi_{4}^{\mathrm{b}} \cdot \mathrm{X}_{3}^{4}
\end{aligned}
$$

ou simplesmente:

$$
X_{i}^{b}=\xi_{j}^{b} \cdot X_{i}^{j} \quad i=1,2,3 \quad j=1,2,3,4
$$

onde as coordenadas globais $X_{i}^{j}$ são referentes à célula a ser integrada.

Semelhantemente ao que se fez para o elemento de contorno na seção anterior, executa-se uma transformação de coordenadas, do sistema 
cartesiano global para o sistema adimensional homogêneo e uma divisão genérica do domínio da célula em 4 subdomínios. Figuras 5.14 e 5.15.

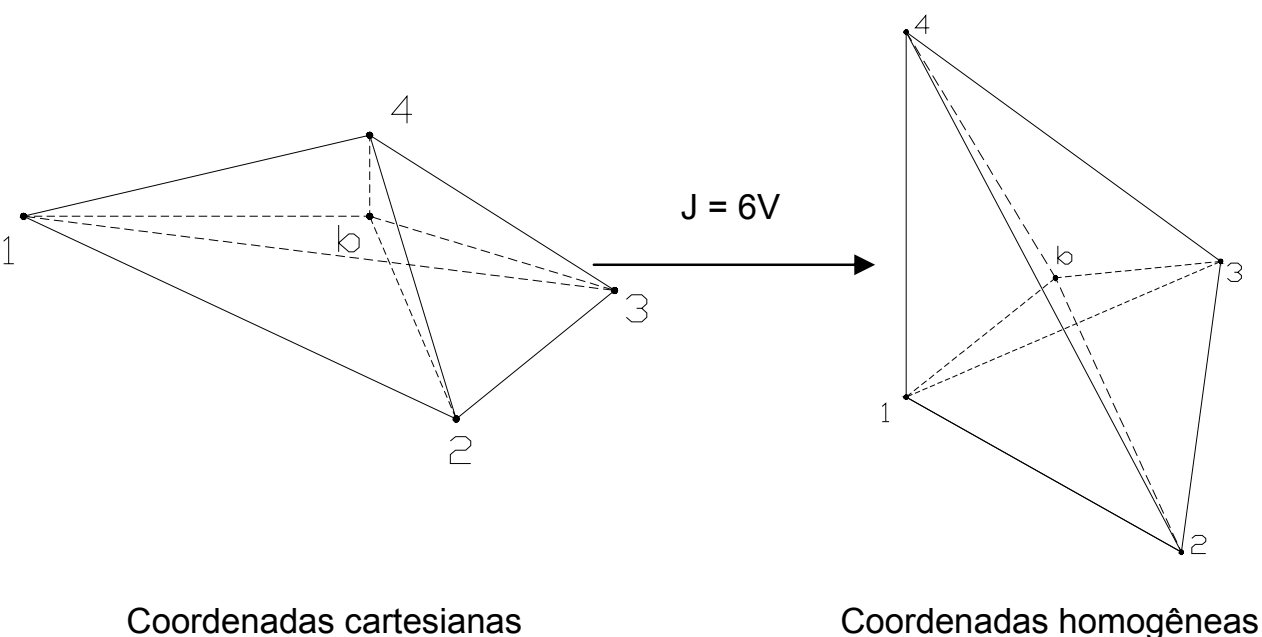

Figura 5.14 - Transformação de coordenadas para células
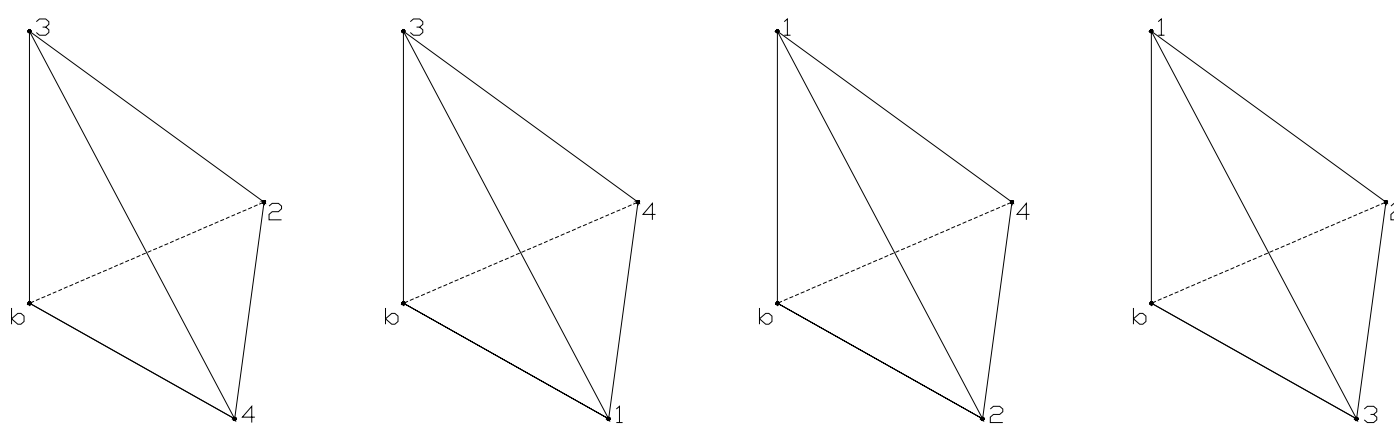

Figura 5.15 - Subdivisão de célula

Deve-se observar que, caso o ponto base esteja em uma face, a transformação de domínio anula automaticamente um subdomínio. Caso esteja em uma aresta, 2 subdomínios são eliminados. Caso esteja em um vértice, sobra apenas um domínio.

Assim:

$I=\int_{V} f d V=6 V \int_{\xi_{3}} \int_{\xi_{2}} \int_{\xi_{1}} f d \xi_{1} d \xi_{2} d \xi_{3}$ 
onde, neste caso, a função f pode ser a função $u_{i j}^{*} \phi_{\ell}$, na montagem da matriz $B$, a função $D_{\mathrm{ijk}} \phi_{\ell}$, na montagem da matriz B' ou a função $\varepsilon_{\mathrm{ijk}}^{*} \phi_{\ell}$, para a matriz $\mathrm{Q}$.

Levando-se em consideração a subdivisão de célula, tem-se:

$I=6 V \sum_{i=1}^{4} \xi_{i}^{b} \int_{0}^{1} \int_{0}^{1-\gamma_{j}} \int_{0}^{1-\gamma_{k}-\gamma_{\ell}} \mathrm{fd} \gamma_{j} \mathrm{~d} \gamma_{k} \mathrm{~d} \gamma_{\ell}$

sendo:

i - o subelemento;

$\xi_{i}^{b} \quad-o$ Jacobiano da transformação;

j, k e $\ell$ - a numeração local dos nós da face oposta ao ponto base (b);

$\gamma \quad$ - a coordenada homogênea no sistema de coordenadas do subelemento.

As coordenadas homogêneas $\xi_{\ell}$ de um ponto qualquer ' $q$ ' pertencente ao subelemento 'i', podem ser calculadas utilizando-se as equações abaixo:

$$
\begin{aligned}
& \text { Para } \mathrm{i}=1 \Rightarrow \xi_{\ell}^{\mathrm{q}}=\xi_{\ell}^{\mathrm{b}} \gamma_{1}+\xi_{\ell}^{2} \gamma_{2}+\xi_{\ell}^{3} \gamma_{3}+\xi_{\ell}^{4} \gamma_{4} \\
& \text { Para } \mathrm{i}=2 \Rightarrow \xi_{\ell}^{\mathrm{q}}=\xi_{\ell}^{1} \gamma_{1}+\xi_{\ell}^{\mathrm{b}} \gamma_{2}+\xi_{\ell}^{3} \gamma_{3}+\xi_{\ell}^{4} \gamma_{4} \\
& \text { Para } \mathrm{i}=3 \Rightarrow \xi_{\ell}^{\mathrm{q}}=\xi_{\ell}^{1} \gamma_{1}+\xi_{\ell}^{2} \gamma_{2}+\xi_{\ell}^{\mathrm{b}} \gamma_{3}+\xi_{\ell}^{4} \gamma_{4} \\
& \text { Para } \mathrm{i}=4 \Rightarrow \xi_{\ell}^{\mathrm{q}}=\xi_{\ell}^{1} \gamma_{1}+\xi_{\ell}^{2} \gamma_{2}+\xi_{\ell}^{3} \gamma_{3}+\xi_{\ell}^{\mathrm{b}} \gamma_{4}
\end{aligned}
$$

onde $\ell$ varia de 1 a 4

A eq.(5.108) pode ser escrita segundo o sistema de coordenadas esféricas homogêneas $(\rho, \alpha, \beta)$ da figura 5.16. 


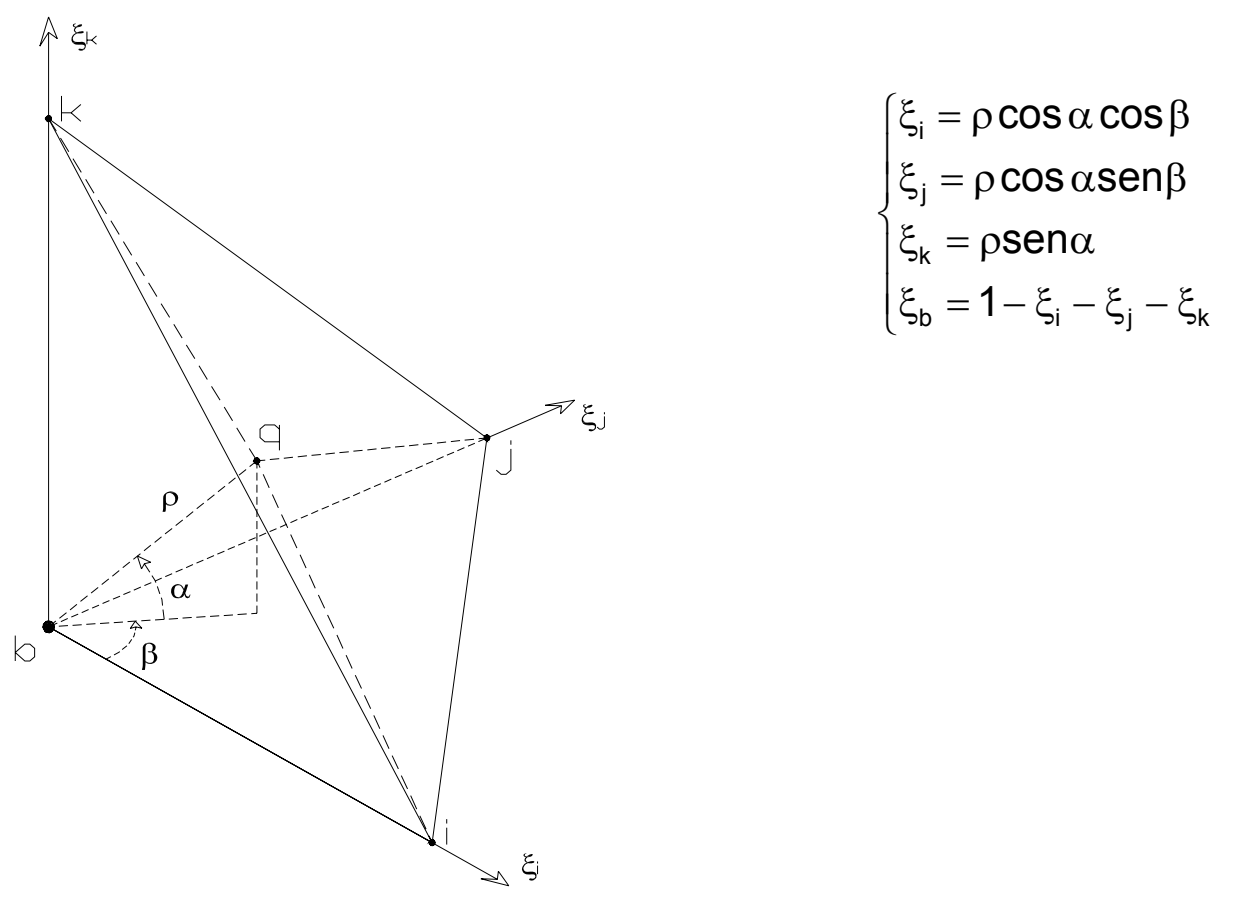

Figura 5.16 - Coordenadas homogêneas para célula tetraédrica

$\mathrm{I}=6 \mathrm{~V} \sum_{\mathrm{i}=1}^{4} \xi_{\mathrm{i}}^{\mathrm{s}} \int_{0}^{\pi / 2} \int_{0}^{\pi / 2} \int_{0}^{\bar{\rho}} \mathrm{f} \cdot \rho^{2} \cos \alpha \mathrm{d} \rho \mathrm{d} \alpha \mathrm{d} \beta$

onde $\rho^{2} \cos \alpha$ é o valor do Jacobiano da transformação, e $\bar{\rho}$ é o valor final de $\rho$ e pode ser calculado considerando-se $\xi_{b}=0$ e escrevendo-se os demais valores de $\xi$ em função de $\rho, \alpha$ e $\beta$ :

$\bar{\rho}(\alpha, \beta)=\frac{1}{\operatorname{sen} \alpha+\cos \alpha(\operatorname{sen} \beta+\cos \beta)}$

Os ângulos $\alpha$ e $\beta$ da eq.(5.113) podem ser transformados para outro sistema de coordenadas adimensional $\left(\zeta_{\alpha}, \zeta_{\beta}\right)$, mais adequado para a quadratura de Gauss:

$I=\frac{3 \pi^{2} \bigvee}{8} \sum_{i=1}^{4} \xi_{i}^{s} \int_{-1}^{1} \int_{-1}^{1} \int_{0}^{\bar{\rho}} f \cdot \rho^{2} \cos \alpha d \rho d \zeta_{\alpha} d \zeta_{\beta}$ 
onde $\frac{\pi^{2}}{16}$ é o Jacobiano da transformação de coordenadas e, de uma forma generalizada:

$$
\begin{aligned}
& \alpha\left(\zeta_{\alpha}\right)=\left(\frac{\alpha_{f}-\alpha_{i}}{2}\right) \zeta_{\alpha}+\frac{\alpha_{f}+\alpha_{i}}{2} \\
& \beta\left(\zeta_{\beta}\right)=\left(\frac{\beta_{f}-\beta_{i}}{2}\right) \zeta_{\beta}+\frac{\beta_{f}+\beta_{i}}{2}
\end{aligned}
$$

Subdividindo-se progressivamente o raio adimensional $\rho$ segundo esquema da figura 5.12, tem-se:

$$
I=\frac{3 \pi^{2} V}{8} \sum_{i=1}^{4} \xi_{i}^{s} \int_{-1}^{1} \int_{-1}^{1} \sum_{z=1}^{n} \frac{\left(\rho_{z}-\rho_{z-1}\right)}{2} \int_{-1}^{1} f \cdot \rho^{2} \cos \alpha d \zeta_{\rho} d \zeta_{\alpha} d \zeta_{\beta}
$$

Resolvendo-se numericamente a eq.(5.118), pode-se escrever:

$$
I=\frac{3 \pi^{2} \bigvee}{16} \sum_{i=1}^{4} \xi_{i}^{b} \sum_{i g \beta=1 \text { ig } \alpha=1 z=1}^{n g \beta} \sum_{z}^{n g \alpha} \sum^{n}\left(\rho_{z-1}\right) \sum_{i g \rho=1}^{n g \rho} f \cdot \rho^{2} \cos \alpha \omega_{i g \rho} \omega_{\text {ig } \alpha} \omega_{i g \beta}
$$

Foram adotados valores de $\mathrm{ng} \rho=3$ e $\mathrm{ng} \alpha=\mathrm{ng} \beta=6$ no programa desenvolvido.

Observa-se que para a dedução da matriz B', a singularidade do núcleo $D_{\text {kij }}\left(1 / r^{2}\right)$ desaparece com a transformação de coordenadas do sistema cartesiano para o sistema de coordenadas esféricas; sendo assim, trata-se de uma integração regular e o processo de integração ora apresentado pode ser utilizado sem problemas.

Para o caso da montagem da matriz $Q$, observa-se que, segundo um sistema de coordenadas esféricas, a integral no elemento a ser resolvida (eq.(5.120)) é não-singular, pois a singularidade do núcleo $\varepsilon_{\mathrm{ijk}}^{*}\left(1 / \mathrm{r}^{2}\right)$ (eq.(3.55) rescrita abaixo) desaparece com o Jacobiano da transformação de coordenadas $\left(r^{2} \cdot \operatorname{sen} \theta\right)$. Ver figura 5.17. Assim, utiliza-se o procedimento de integração não-singular para células da presente seção. 


$$
\begin{aligned}
& I=\int_{\Omega_{\mathrm{e}}} \varepsilon_{\mathrm{ijk}}^{*}(\mathrm{~s}, \mathrm{q}) \phi_{\ell}(\mathrm{q}) \mathrm{d} \Omega_{\mathrm{e}} \sigma_{\mathrm{jk}}^{\mathrm{n} \ell} \\
& \varepsilon_{\mathrm{ijk}}^{*}(\mathrm{~s}, \mathrm{q})=\frac{-1}{16 \pi(1-v) G r^{2}}\left\{(1-2 v)\left(r_{, j} \delta_{i \mathrm{k}}+r_{, \mathrm{k}} \delta_{\mathrm{ij}}\right)-r_{{ }_{\mathrm{i}}} \delta_{\mathrm{jk}}+3 r_{, j} r_{{ }_{\mathrm{k}}} r_{{ }_{\mathrm{i}}}\right\}
\end{aligned}
$$

$$
\begin{aligned}
& \left\{\begin{array}{l}
x=r \cos \theta \cos \phi \\
y=r \cos \theta \operatorname{sen} \phi \\
z=r \operatorname{sen} \theta
\end{array}\right. \\
& d \Omega=r^{2} \operatorname{sen} \theta d r d \theta d \phi
\end{aligned}
$$$$
\theta
$$$$
\phi
$$

Figura 5.17 - Coordenadas esféricas

\subsection{INTEGRAÇÃO SINGULAR PARA CÉLULAS}

Neste trabalho, a única integral de domínio que realmente apresenta singularidade é aquela da eq.(5.121) abaixo, presente na equação integral de contorno para tensões em pontos internos (eq.(3.66)). Deseja-se resolver numericamente aquela integral, referente às tensões não-elásticas.

$\mathrm{I}=\int_{\Omega} \overline{\mathrm{E}}_{\mathrm{i} \ell \mathrm{k}} \sigma_{\ell \mathrm{k}}^{\tilde{\mathrm{n}}}(\mathrm{q}) \mathrm{d} \Omega$

Porém, nesta seção, antes de se resolver a integral singular acima, o autor optou por apresentar a formulação envolvida na consideração da atuação de tensões não-elásticas no cálculo das tensões internas. Para tanto, parte-se 
da eq.(3.65) rescrita abaixo, onde se deseja resolver a última integral da mesma.

$$
\begin{aligned}
& \sigma_{\mathrm{mn}}=\int_{\Gamma}\left\{\frac{2 \mathrm{G} v}{1-2 v} \delta_{\mathrm{mn}} \frac{\partial \mathrm{u}_{\ell \mathrm{k}}^{*}}{\partial \mathrm{x}_{\ell}}+\mathrm{G}\left(\frac{\partial \mathrm{u}_{\mathrm{mk}}^{*}}{\partial \mathrm{x}_{\mathrm{n}}}+\frac{\partial \mathrm{u}_{\mathrm{nk}}^{*}}{\partial \mathrm{x}_{\mathrm{m}}}\right)\right\} \mathrm{p}_{\mathrm{k}} \mathrm{d} \Gamma-\int_{\Gamma}\left\{\frac{2 \mathrm{Gv}}{1-2 v} \delta_{\mathrm{mn}} \frac{\partial \mathrm{p}_{\ell \mathrm{k}}^{*}}{\partial \mathrm{x}_{\ell}}+\mathrm{G}\left(\frac{\partial \mathrm{p}_{\mathrm{mk}}^{*}}{\partial \mathrm{x}_{\mathrm{n}}}+\frac{\partial \mathrm{p}_{\mathrm{nk}}^{*}}{\partial \mathrm{x}_{\mathrm{m}}}\right)\right\} \mathrm{u}_{\mathrm{k}} \mathrm{d} \Gamma+ \\
& +\int_{\Omega}\left\{\frac{2 \mathrm{G} v}{1-2 v} \delta_{\mathrm{mn}} \frac{\partial \mathrm{u}_{\ell \mathrm{k}}^{*}}{\partial \mathrm{x}_{\ell}}+\mathrm{G}\left(\frac{\partial \mathrm{u}_{\mathrm{mk}}^{*}}{\partial \mathrm{x}_{\mathrm{n}}}+\frac{\partial \mathrm{u}_{\mathrm{nk}}^{*}}{\partial \mathrm{x}_{\mathrm{m}}}\right)\right\} \mathrm{b}_{\mathrm{k}} \mathrm{d} \Omega-\mathrm{c} \cdot \int_{\Omega}\left\{\frac{2 \mathrm{Gv}}{1-2 v} \delta_{\mathrm{mn}} \frac{\partial \mathrm{u}_{\ell \mathrm{k}}^{*}}{\partial \mathrm{x}_{\ell}}+\mathrm{G}\left(\frac{\partial \mathrm{u}_{\mathrm{mk}}^{*}}{\partial \mathrm{x}_{\mathrm{n}}}+\frac{\partial \mathrm{u}_{\mathrm{nk}}^{*}}{\partial \mathrm{x}_{\mathrm{m}}}\right)\right\} \dot{\mathrm{u}}_{\mathrm{k}} \mathrm{d} \Omega+ \\
& -\rho \cdot \int_{\Omega}\left\{\frac{2 \mathrm{G} v}{1-2 v} \delta_{\mathrm{mn}} \frac{\partial \mathrm{u}_{\ell \mathrm{k}}^{*}}{\partial \mathrm{x}_{\ell}}+\mathrm{G}\left(\frac{\partial \mathrm{u}_{\mathrm{mk}}^{*}}{\partial \mathrm{x}_{\mathrm{n}}}+\frac{\partial \mathrm{u}_{\mathrm{nk}}^{*}}{\partial \mathrm{x}_{\mathrm{m}}}\right) \ddot{u}_{\mathrm{k}} \mathrm{d} \Omega+\int_{\Omega}\left\{\frac{2 \mathrm{Gv}}{1-2 v} \delta_{\mathrm{mn}} \frac{\partial \varepsilon_{\ell \mathrm{k} \mathrm{k}}^{*}}{\partial \mathrm{x}_{\ell}}+\mathrm{G}\left(\frac{\partial \varepsilon_{\mathrm{mjk}}^{*}}{\partial \mathrm{x}_{\mathrm{n}}}+\frac{\partial \varepsilon_{\mathrm{njk}}^{*}}{\partial \mathrm{x}_{\mathrm{m}}}\right)\right\} \sigma_{\mathrm{jk}}^{\tilde{n}} \mathrm{~d} \Omega+\right. \\
& +\overline{\mathrm{g}}_{\mathrm{mnjk}} \sigma_{\mathrm{jk}}^{\tilde{n}}(\mathrm{~s})-\sigma_{\mathrm{mn}}^{\tilde{n}}(\mathrm{~s})
\end{aligned}
$$

Como se trata de uma integração singular, é necessário que se faça um tratamento especial desta. Seguindo esse pensamento, inicialmente, resolverse-á a seguinte integral, derivada diretamente da equação de deslocamentos para pontos internos:

$\mathrm{I}_{\varepsilon}=\lim _{\varepsilon \rightarrow 0} \frac{\partial}{\partial \mathbf{x}_{\ell}(\mathbf{s})} \int_{\Omega-\Omega_{\varepsilon}} \varepsilon_{\mathrm{ijk}}^{*}(\mathrm{~s}, \mathrm{q}) \sigma_{\mathrm{jk}}^{\tilde{n}}(\mathrm{q}) \mathrm{d} \Omega$

onde:

$\varepsilon \quad-$ é o raio infinitesimal;

$\Omega_{\varepsilon} \quad-$ é o domínio infinitesimal.

A eq.(5.122) pode ser escrita em coordenadas esféricas, segundo figura 5.17:

$$
\mathrm{I}_{\varepsilon}=\int_{0}^{2 \pi} \int_{-\pi / 2}^{\pi / 2}\left[\lim _{\varepsilon \rightarrow 0} \frac{\partial}{\partial \mathrm{x}_{\ell}(\mathrm{s})} \int_{\varepsilon}^{\mathrm{R}} \varepsilon_{\mathrm{ijk}}^{*}(\theta, \phi, r(\mathrm{~s}, \mathrm{q})) \sigma_{\mathrm{jk}}^{\tilde{n}}(\mathrm{q}) \mathrm{r}^{2}(\mathrm{~s}, \mathrm{q}) \mathrm{dr}\right] \cos \theta \mathrm{d} \theta \mathrm{d} \phi
$$

Uma vez que o limite inferior da integração em 'r' e a diferenciação na eq.(5.123) dependem da posição do ponto fonte ' $s$ ', é necessário que se considere a variação do limite de integração com a diferenciação. Esse 
processo foi muito bem apresentado por Coda (2000), em sua seção 1.8.2. Aqui, será apresentada apenas a eq.(5.123) já diferenciada.

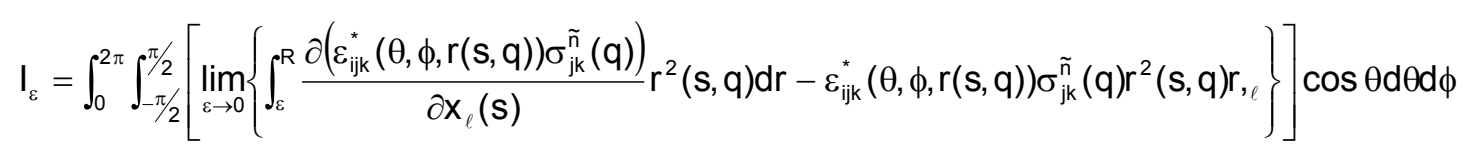

onde:

$r_{\ell}=-\frac{\partial r(s, q)}{\partial x_{\ell}(s)}=\frac{\partial r(s, q)}{\partial x_{\ell}(q)}$

ou:

$r_{1}=\cos \theta \cos \phi$

$r_{, 2}=\cos \theta \operatorname{sen} \phi$

$r_{3}=\operatorname{sen} \theta$

Fazendo-se:

$\mathrm{h}_{\varepsilon}=\varepsilon_{\mathrm{ijk}}^{*}(\theta, \phi, r(s, q)) \sigma_{\mathrm{jk}}^{\tilde{n}}(\mathrm{q})$

e utilizando-se a eq.(3.55), tem-se:

$h_{\varepsilon}=\frac{-1}{16 \pi(1-v) G r^{2}}\left\{(1-2 v)\left(r_{, j} \delta_{i k}+r_{, k} \delta_{i j}\right)-r_{, j} \delta_{j k}+3 r_{, j} r_{k} r_{, j}\right\} \sigma_{j k}^{\tilde{n}}(q)$

Resolvendo-se o limite da eq.(5.124), apenas para a segunda parcela da função, e substituindo-se a eq.(5.130), tem-se:

$\mathrm{I}_{\varepsilon}=\int_{0}^{2 \pi} \int_{-\pi / 2}^{\pi / 2}\left[\lim _{\varepsilon \rightarrow 0}\left\{\int_{\varepsilon}^{R} \frac{\partial \mathrm{h}_{\varepsilon}(\theta, \phi, r(\mathrm{~s}, \mathrm{q}))}{\partial \mathbf{x}_{\ell}(\mathbf{s})} \mathrm{r}^{2}(\mathrm{~s}, \mathrm{q}) \mathrm{dr}\right\}-\mathrm{z}_{\mathrm{ijk}}(\theta, \phi) \sigma_{\mathrm{jk}}^{\tilde{n}}(\mathrm{~s}) \mathrm{r}_{\ell}\right] \cos \theta \mathrm{d} \theta \mathrm{d} \phi$ 
onde:

$$
z_{i j k}(\theta, \phi)=\frac{-1}{16 \pi(1-v) G}\left\{(1-2 v)\left(r_{, j} \delta_{i k}+r_{{ }_{k}} \delta_{i j}\right)-r_{, i} \delta_{j k}+3 r_{, j} r_{{ }_{k}} r_{{ }_{i}}\right\}
$$

Observa-se que:

$$
\frac{\partial \mathrm{h}_{\varepsilon}(\theta, \phi, r(\mathrm{~s}, \mathrm{q}))}{\partial \mathbf{x}_{\ell}(\mathrm{s})}=\frac{\partial \varepsilon_{\mathrm{ijk}}^{*}(\theta, \phi, r(\mathrm{~s}, \mathrm{q}))}{\partial \mathbf{x}_{\ell}(\mathrm{s})} \sigma_{\mathrm{jk}}^{\tilde{n}}(\mathrm{q})
$$

Deseja-se calcular a diferencial de $\varepsilon_{\mathrm{ijk}}^{*}$ com relação a $\mathrm{x}_{\ell}(\mathrm{s})$ da eq.(5.133); assim:

$$
\begin{aligned}
& \frac{\partial \varepsilon_{\mathrm{ijk}}^{*}(\theta, \phi, r(\mathrm{~s}, \mathrm{q}))}{\partial \mathrm{x}_{\ell}(\mathrm{s})}=\frac{-1}{16 \pi(1-v) \mathrm{G}}\left\{3(1-2 v)\left(\mathrm{r}_{{ }_{\mathrm{k}}} \mathrm{r}_{\ell} \delta_{\mathrm{ji}}+\mathrm{r}_{, \mathrm{j}} \mathrm{r}_{\ell} \delta_{\mathrm{ik}}\right)-(1-2 v)\left(\delta_{\ell \mathrm{k}} \delta_{\mathrm{ji}}+\delta_{\mathrm{j} \ell} \delta_{\mathrm{ik}}\right)+\right. \\
& \left.-3 r_{, i} r_{,} \delta_{j k}+\delta_{\ell i} \delta_{j k}-3\left(\delta_{j \ell} r_{, k} r_{, i}+\delta_{k \ell} r_{, j} r_{, i}+\delta_{i \ell} r_{, j} r_{{ }_{k}}\right)+15 r_{, j} r_{, k} r_{, j} r_{, \ell}\right\} \frac{1}{r^{3}}
\end{aligned}
$$

onde, para se chegar à eq.(5.134) acima, utilizou-se a seguinte igualdade:

$$
\frac{\partial \mathrm{r}_{,_{\mathrm{k}}}}{\partial \mathrm{x}_{\ell}(\mathrm{s})}=\frac{1}{\mathrm{r}}\left(-\delta_{\mathrm{k} \ell}+\mathrm{r}_{{ }_{\mathrm{k}}} \mathrm{r}_{\ell}\right)
$$

\section{Fazendo-se:}

$$
\begin{aligned}
& \psi_{\mathrm{ijk} \ell}=\frac{-1}{16 \pi(1-v) G}\left\{3(1-2 v)\left(\mathrm{r}_{{ }_{\mathrm{k}}} \mathrm{r}_{,}, \delta_{\mathrm{ji}}+\mathrm{r}_{\mathrm{j}, \mathrm{r}} \mathrm{r}_{\ell} \delta_{\mathrm{ik}}\right)-(1-2 v)\left(\delta_{\ell \mathrm{k}} \delta_{\mathrm{ji}}+\delta_{\mathrm{j} \ell} \delta_{\mathrm{ik}}\right)+\right. \\
& \left.-3 r_{, j} r_{, \ell} \delta_{j k}+\delta_{\ell i} \delta_{j k}-3\left(\delta_{j \ell} r_{, k} r_{, j}+\delta_{k \ell} r_{, j} r_{, j}+\delta_{i \ell} r_{, j} r_{, k}\right)+15 r,{ }_{, j} r_{, k} r_{, j} r_{, \ell}\right\}
\end{aligned}
$$

a eq.(5.131) pode ser escrita como:

$$
\mathrm{I}_{\varepsilon}=\int_{0}^{2 \pi} \int_{-\pi / 2}^{\pi / 2}\left[\lim _{\varepsilon \rightarrow 0}\left\{\int_{\varepsilon}^{\mathrm{R}} \frac{1}{\mathrm{r}} \psi_{\mathrm{ijk} \ell}(\theta, \phi) \sigma_{\mathrm{jk}}^{\tilde{n}}(\mathrm{q}) \mathrm{dr}\right\}-\mathrm{z}_{\mathrm{ijk}}(\theta, \phi) \mathrm{r}, \ell \sigma_{\mathrm{jk}}^{\tilde{n}}(\mathrm{~s})\right] \cos \theta \mathrm{d} \theta \mathrm{d} \phi
$$


ou:

$\mathrm{I}_{\varepsilon}=\int_{0}^{2 \pi} \int_{-\pi / 2}^{\pi / 2} \lim _{\varepsilon \rightarrow 0}\left\{\int_{\varepsilon}^{R} \frac{1}{\mathrm{r}} \psi_{\mathrm{ijk} \ell}(\theta, \phi) \sigma_{\mathrm{jk}}^{\tilde{n}}(\mathrm{q}) \mathrm{dr}\right\} \cos \theta \mathrm{d} \theta \mathrm{d} \phi+\int_{0}^{2 \pi} \int_{-\pi / 2}^{\pi / 2}-\mathbf{z}_{\mathrm{ijk}}(\theta, \phi) \mathrm{r}, \cos \theta \mathrm{d} \theta \mathrm{d} \phi \sigma_{\mathrm{jk}}^{\tilde{n}}(\mathrm{~s})$

Resolvendo-se a segunda integral da eq.(5.138), tem-se:

$g_{\mathrm{ijk} \ell}(\theta, \phi)=-\int_{0}^{2 \pi} \int_{-\pi / 2}^{\pi / 2} z_{\mathrm{ijk}}(\theta, \phi) \mathrm{r}_{\ell} \cos \theta \mathrm{d} \theta \mathrm{d} \phi$

Substituindo-se a eq.(5.132) na eq.(5.139) acima, tem-se:

$g_{\mathrm{ik} \ell}(\theta, \phi)=-\int_{0}^{2 \pi} \int_{-\pi / 2}^{\pi / 2} \frac{-1}{16 \pi(1-v) \mathrm{G}}\left\{(1-2 v)\left(r_{, j} \delta_{i \mathrm{k}}+r_{, k} \delta_{i j}\right)-r_{, j} \delta_{j k}+3 r_{, j} r_{, k} r_{, j}\right\} r_{, \ell} \cos \theta d \theta d \phi$

ou:

$g_{\mathrm{ik} \ell}(\theta, \phi)=\frac{1}{16 \pi(1-v) \mathrm{G}}\left[\int_{0}^{2 \pi} \int_{-\pi / 2}^{\pi / 2}(1-2 v) \mathrm{r}_{\mathrm{j}}, \mathrm{r}_{\ell} \delta_{\mathrm{ik}} \cos \theta \mathrm{d} \theta \mathrm{d} \phi+\int_{0}^{2 \pi} \int_{-\pi / 2}^{\pi / 2}(1-2 v) \mathrm{r}_{,}, \mathrm{r}_{,}, \delta_{\mathrm{ij}} \cos \theta \mathrm{d} \theta \mathrm{d} \phi+\right.$

$\left.-\int_{0}^{2 \pi} \int_{-\pi / 2}^{\pi / 2} r_{, j} r_{, \ell} \delta_{j k} \cos \theta d \theta d \phi+3 \int_{0}^{2 \pi} \int_{-\pi / 2}^{\pi / 2} r_{, j} r_{, k} r_{, j} r_{, \ell} \cos \theta d \theta d \phi\right]$

Atribuindo-se valores (de 1 a 3 ) a i, j, k e $\ell$, e utilizando-se as eq.(5.126), (5.127) e (5.128), consegue-se chegar a:

$\int_{0}^{2 \pi} \int_{-\pi / 2}^{\pi / 2} r_{j} r_{\ell} \delta_{i k} \cos \theta d \theta d \phi=\frac{4 \pi}{3} \delta_{j \ell} \delta_{i k}$

e

$\int_{0}^{2 \pi} \int_{-\pi / 2}^{\pi / 2} r_{, j} r_{{ }_{k}} r_{, j} r_{, \ell} \cos \theta d \theta d \phi=\frac{4 \pi}{15}\left(\delta_{j k} \delta_{i \ell}+\delta_{j i} \delta_{k \ell}+\delta_{i k} \delta_{j \ell}\right)$ 
Substituindo-se as eq.(5.142) e (5.143) na eq.(5.141), pode-se chegar a:

$g_{\mathrm{ijk} \ell}=\frac{1}{30(1-v) \mathrm{G}}\left[(4-5 v)\left(\delta_{\mathrm{ji}} \delta_{\mathrm{k} \ell}+\delta_{\mathrm{ik}} \delta_{\mathrm{j} \ell}\right)-\delta_{\mathrm{jk}} \delta_{\mathrm{i} \ell}\right]$

A integral referente às tensões não-elásticas da eq.(3.65) pode ser escrita como:

$$
\begin{aligned}
& \sigma_{m n}^{\sigma}(s)=\frac{2 G v}{1-2 v} \delta_{m n}\left[\int_{\Omega} \frac{1}{r^{3}}\left(\psi_{1 j \mathrm{k} 1}+\psi_{2 \mathrm{jk} 2}+\psi_{3 \mathrm{jk} 3}\right) \sigma_{\mathrm{jk}}^{\tilde{n}}(\mathrm{q}) \mathrm{d} \Omega+\left(g_{1 \mathrm{jk} 1}+g_{2 \mathrm{jk} 2}+g_{3 \mathrm{jk} 3}\right) \sigma_{\mathrm{jk}}^{\tilde{n}}(\mathrm{~s})\right]+ \\
& +\mathrm{G}\left[\int_{\Omega} \frac{1}{r^{3}}\left(\psi_{m \mathrm{jkn}}+\psi_{\mathrm{njkm}}\right) \sigma_{\mathrm{jk}}^{\tilde{n}}(\mathrm{q}) \mathrm{d} \Omega+\left(g_{m \mathrm{jkn}}+g_{\mathrm{njkm}}\right) \sigma_{\mathrm{jk}}^{\tilde{n}}(\mathrm{~s})\right]
\end{aligned}
$$

Utilizando-se a eq.(5.136), pode-se chegar a:

$\psi_{1 \mathrm{jk} 1}+\psi_{2 \mathrm{jk} 2}+\psi_{3 \mathrm{jk} 3}=\frac{(1-2 v)}{8 \pi(1-v) \mathrm{G}}\left\{\delta_{\mathrm{jk}}-3 \mathrm{r}_{, \mathrm{j}} \mathrm{r}_{\mathrm{k}}\right\}$

e:

$$
\begin{aligned}
& \psi_{\mathrm{mjkn}}+\psi_{\mathrm{njkm}}=\frac{1}{8 \pi(1-v) G}\left\{(1-2 v)\left(\delta_{\mathrm{mj}} \delta_{\mathrm{kn}}+\delta_{\mathrm{nj}} \delta_{\mathrm{km}}\right)+3 v\left(\mathrm{r}_{\mathrm{m}_{\mathrm{m}}} \mathrm{r}_{\mathrm{j} j} \delta_{\mathrm{kn}}+\mathrm{r}_{{ }_{\mathrm{n}}} \mathrm{r}_{\mathrm{j}_{\mathrm{j}}} \delta_{\mathrm{km}}+\mathrm{r}_{\mathrm{m}} \mathrm{r}_{{ }_{\mathrm{k}}} \delta_{\mathrm{jn}}+\right.\right. \\
& \left.\left.+r_{, n} r_{, k} \delta_{j m}\right)+3\left(r_{m} r_{, n} \delta_{j k}+r_{, j} r_{{ }_{k}} \delta_{m n}\right)-\delta_{m n} \delta_{j k}-15 r_{, j} r_{, k} r_{r_{m}} r_{n}\right\}
\end{aligned}
$$

Utilizando-se a eq.(5.144), tem-se:

$g_{1 \mathrm{jk} 1}+g_{2 \mathrm{jk} 2}+g_{3 \mathrm{jk} 3}=\frac{(1-2 v)}{6(1-v) G} \delta_{\mathrm{jk}}$

e:

$$
g_{m j k n}+g_{n j k m}=\frac{1}{15(1-v) G}\left[(4-5 v)\left(\delta_{m j} \delta_{k n}+\delta_{n j} \delta_{k m}\right)-\delta_{m n} \delta_{j k}\right]
$$


Substituindo-se as eq.(5.146), (5.147), (5.148) e (5.149) na eq.(5.145), pode-se escrever que:

$\sigma_{m n}^{\sigma}(s)=\int_{\Omega} \bar{E}_{m n j k} \sigma_{j k}^{\tilde{n}}(q) d \Omega+\bar{g}_{m n j k} \sigma_{j k}^{\tilde{n}}(s)$

onde:

$$
\begin{aligned}
& \bar{E}_{m n j k}=\frac{1}{8 \pi(1-v) r^{3}}\left\{(1-2 v)\left(\delta_{m j} \delta_{k n}+\delta_{n j} \delta_{k m}-\delta_{m n} \delta_{j k}+3 r_{, j} r_{{ }_{k}} \delta_{m n}\right)+3 v\left(r_{m} r_{, j} \delta_{k n}+r_{, n} r_{, j} \delta_{k m}+\right.\right.
\end{aligned}
$$

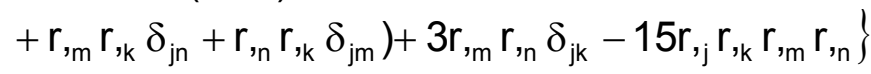

e

$$
\bar{g}_{\mathrm{mnjk}}=\frac{1}{15(1-v)}\left[(4-5 v)\left(\delta_{\mathrm{mj}} \delta_{\mathrm{kn}}+\delta_{\mathrm{nj}} \delta_{\mathrm{km}}\right)-(1-5 v) \delta_{\mathrm{mn}} \delta_{\mathrm{jk}}\right]
$$

Aplicando-se as expressões das soluções fundamentais (eq.(3.43) e (3.44)) e substituindo-se o resultado da integral referente às tensões nãoelásticas, eq.(5.150), na eq.(3.65), pode-se escrever:

$$
\begin{aligned}
& \sigma_{m n}=\int_{\Gamma} D_{k m n} p_{k} d \Gamma-\int_{\Gamma} S_{k m n} u_{k} d \Gamma+\int_{\Omega} D_{k m n} b_{k} d \Omega-c \cdot \int_{\Omega} D_{k m n} \dot{u}_{k} d \Omega-\rho \cdot \int_{\Omega} D_{k m n} \ddot{u}_{k} d \Omega+ \\
& +\int_{\Omega} \bar{E}_{m n j k} \sigma_{j k}^{\tilde{n}}(q) d \Omega+\bar{g}_{m n j k} \sigma_{j k}^{\tilde{n}}(s)-\sigma_{m n}^{\tilde{n}}(s)
\end{aligned}
$$

Deseja-se escrever as tensões não-elásticas $\sigma_{m n}^{\tilde{n}}(s)$ em função de $\sigma_{\mathrm{jk}}^{\tilde{n}}(s)$. Assim:

$$
\begin{aligned}
& \sigma_{m n}^{\tilde{n}}=\frac{1}{2}\left(\sigma_{m n}^{\tilde{n}}+\sigma_{n m}^{\tilde{n}}\right) \\
& \sigma_{m n}^{\tilde{n}}=\frac{1}{2}\left(\sigma_{j k}^{\tilde{n}} \delta_{j m} \delta_{k n}+\sigma_{j k}^{\tilde{n}} \delta_{j n} \delta_{k m}\right) \\
& \sigma_{m n}^{\tilde{n}}=\frac{1}{2}\left(\delta_{j m} \delta_{k n}+\delta_{j n} \delta_{k m}\right) \sigma_{j k}^{\tilde{n}}
\end{aligned}
$$


Substituindo-se a eq.(5.156) na eq.(5.153), pode-se chegar à eq.(3.66):

$$
\begin{aligned}
& \sigma_{\mathrm{ij}}=\int_{\Gamma} D_{\mathrm{kij}} p_{\mathrm{k}} \mathrm{d} \Gamma-\int_{\Gamma} \mathrm{S}_{\mathrm{kij}} \mathrm{u}_{\mathrm{k}} \mathrm{d} \Gamma+\int_{\Omega} D_{\mathrm{kij}} \mathrm{b}_{\mathrm{k}} \mathrm{d} \Omega-\mathrm{c} \cdot \int_{\Omega} D_{\mathrm{kij}} \dot{\mathrm{u}}_{\mathrm{k}} \mathrm{d} \Omega-\rho \cdot \int_{\Omega} D_{\mathrm{kij}} \ddot{\mathrm{u}}_{\mathrm{k}} \mathrm{d} \Omega+ \\
& +\int_{\Omega} \overline{\mathrm{E}}_{\mathrm{ij} \ell \mathrm{k}} \sigma_{\ell \mathrm{k}}^{\tilde{n}}(\mathrm{q}) \mathrm{d} \Omega+\overline{\bar{g}}_{\mathrm{ij} \ell \mathrm{k}} \sigma_{\ell \mathrm{k}}^{\tilde{n}}(\mathrm{~s})
\end{aligned}
$$

onde:

$$
\overline{\bar{g}}_{\mathrm{ij} \ell \mathrm{k}}=\frac{1}{30(1-v)}\left[(-7+5 v)\left(\delta_{\mathrm{i} \ell} \delta_{\mathrm{jk}}+\delta_{\mathrm{ik}} \delta_{\mathrm{j} \ell}\right)-(2-10 v) \delta_{\mathrm{ij}} \delta_{\ell \mathrm{k}}\right]
$$

A notação indicial das eq.(3.66) e (3.70) foram modificadas, apenas com o intuito de se continuar seguindo a notação apresentada na seção 3.6.

Após a dedução da formulação envolvida na consideração de atuação de tensões não-elásticas no cálculo de tensões internas, pode-se voltar à tarefa de resolução numérica da integral referente a tensões não-elásticas.

$$
\mathrm{I}=\int_{\Omega} \overline{\mathrm{E}}_{\mathrm{ij} / \mathrm{k}} \sigma_{\ell \mathrm{k}}^{\tilde{\mathrm{n}}}(\mathrm{q}) \mathrm{d} \Omega
$$

Fazendo-se:

$$
\begin{aligned}
& E_{m n j k}=\bar{E}_{m n j k} \cdot r^{3}=\frac{1}{8 \pi(1-v)}\left\{(1-2 v)\left(\delta_{m j} \delta_{k n}+\delta_{n j} \delta_{k m}-\delta_{m n} \delta_{j k}+3 r,{ }_{, j} r_{, k} \delta_{m n}\right)+3 v\left(r, r_{m} r_{, j} \delta_{k n}+\right.\right.
\end{aligned}
$$

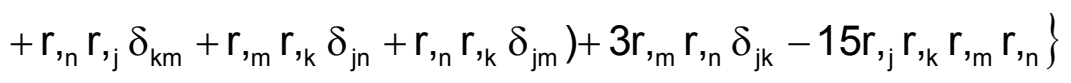

e observando-se a mudança de índices, tem-se

$$
I=\int_{\Omega} E_{i j \ell k} \frac{1}{r^{3}} \sigma_{\ell k}^{\tilde{n}}(q) d \Omega
$$

A eq.(5.158) acima, pode ser transformada para o sistema de coordenadas esféricas; figura 5.17. 
$I=\iint_{\theta} \int_{\phi} \int_{r} E_{i j \ell k} \frac{1}{r} \sigma_{\ell k}^{\tilde{n}}(q) \cos \theta d r d \phi d \theta$

Observa-se na eq.(5.159) uma singularidade de 1/r; é necessário um tratamento especial para tal integral.

As tensões não-elásticas em um ponto qualquer $(q)$ do sólido podem ser escritas em função dos valores das tensões não-elásticas nos nós da célula que contém o ponto q:

$\sigma_{\ell \mathrm{k}}^{\tilde{\mathrm{n}}}(\mathrm{q})=\phi_{\mathrm{t}}(\mathrm{q}) \cdot \sigma_{\ell \mathrm{k}}^{\tilde{\mathrm{nt}}} \quad \mathrm{t}=1,2,3,4$

Substituindo-se a aproximação para célula, eq.(5.160), na eq.(5.159) tem-se:

$$
\mathrm{I}=\int_{\theta} \int_{\phi_{\phi}} \int_{r} E_{i j \ell k} \frac{1}{r} \phi_{t}(q) \cos \theta d r d \phi d \theta \cdot \sigma_{\ell k}^{\tilde{n} t} \quad t=1,2,3,4
$$

Substituindo-se as relações da figura 5.17 na eq.(5.98), tem-se:

$\phi_{t}=\xi_{t}=\frac{1}{6 V}\left[\alpha_{t}+\beta_{t} r \cos \theta \cos \phi+\gamma_{t} r \cos \theta \operatorname{sen} \phi+\delta_{t} r \operatorname{sen} \theta\right]$

ou

$\xi_{t}=\frac{\alpha_{t}}{6 V}+\frac{r}{6 V}\left[\beta_{t} \cos \theta \cos \phi+\gamma_{t} \cos \theta \operatorname{sen} \phi+\delta_{t} \operatorname{sen} \theta\right]$

Como uma simplificação, para o cálculo de tensões internas levando-se em consideração a existência de tensões não-elásticas, não serão permitidos pontos no interior das células de discretização da atuação das tensões nãoelásticas, ou seja, só existem duas possibilidades para a posição do ponto fonte com relação à célula a ser integrada: fora dela, com uma integração regular, segundo procedimento apresentado na seção anterior; ou nos vértices da mesma, com uma integração singular apresentada a seguir. Lembrando-se 
que, para as células de discretização do domínio para a montagem da matriz B (forças volumétricas), nada muda, ou seja, podem existir pontos no interior destas células.

De acordo com a simplificação citada no parágrafo acima, para uma integração singular, a eq.(5.163) pode ser escrita como:

$\xi_{\mathrm{t}}=\delta_{\mathrm{st}}+\frac{\mathrm{r}}{6 \mathrm{~V}}\left[\beta_{\mathrm{t}} \cos \theta \cos \phi+\gamma_{\mathrm{t}} \cos \theta \operatorname{sen} \phi+\delta_{\mathrm{t}} \operatorname{sen} \theta\right]$

onde 's' é a numeração local do ponto fonte.

Substituindo-se a eq.(5.164) na eq.(5.161), tem-se:

$I=\iint_{\theta} \int_{\phi} \int_{\mathrm{r}} E_{\mathrm{ij} \ell \mathrm{k}} \frac{1}{\mathrm{r}} \delta_{\mathrm{st}} \cos \theta \mathrm{drd} \phi \mathrm{d} \theta \cdot \sigma_{\ell \mathrm{k}}^{\tilde{\mathrm{n}} \mathrm{t}}+$

$+\iint_{\theta} \int_{\phi} \int_{\mathrm{r}} E_{\mathrm{ij} \ell \mathrm{k}} \frac{1}{6 \mathrm{~V}}\left[\beta_{\mathrm{t}} \cos \theta \cos \phi+\gamma_{\mathrm{t}} \cos \theta \operatorname{sen} \phi+\delta_{\mathrm{t}} \operatorname{sen} \theta\right] \cos \theta \mathrm{drd} \phi \mathrm{d} \theta \cdot \sigma_{\ell \mathrm{k}}^{\tilde{\mathrm{nt}}}$

ou

$\mathrm{I}=\left(\mathrm{I}_{1}+\mathrm{I}_{2}\right) \cdot \sigma_{\ell \mathrm{k}}^{\tilde{\mathrm{nt}}}$

onde:

$\mathrm{I}_{1}=\int_{\theta} \int_{\phi} \int_{\mathrm{r}} \mathrm{E}_{\mathrm{ij} \ell \mathrm{k}} \frac{1}{\mathrm{r}} \delta_{\mathrm{st}} \cos \theta \mathrm{drd} \phi \mathrm{d} \theta$

e

$\mathrm{I}_{2}=\int_{0} \int_{\phi} \int_{\mathrm{r}} \mathrm{E}_{\mathrm{i} \ell \ell k} \frac{1}{6 \mathrm{~V}}\left[\beta_{\mathrm{t}} \cos \theta \cos \phi+\gamma_{\mathrm{t}} \cos \theta \operatorname{sen} \phi+\delta_{\mathrm{t}} \operatorname{sen} \theta\right] \cos \theta \mathrm{drd} \phi \mathrm{d} \theta$

Apenas a integral $\mathrm{I}_{1}$ é singular, podendo a integral $\mathrm{I}_{2}$ ser resolvida pelo procedimento apresentado na seção anterior. Vale observar que o grau de 
singularidade da função $\bar{E}_{i j \ell k}$ é 3. Esse valor será utilizado na eq.(5.90) para o cálculo do valor do raio adimensional $\rho_{1}$.

Para a resolução da integral de $I_{1}$, primeiramente integra-se em $r$, assim a eq.(5.167) pode ser escrita como:

$\mathrm{I}_{1}=\int_{\theta} \int_{\phi} \mathrm{E}_{\mathrm{ij} \ell \mathrm{k}} \ln \overline{\mathrm{R}}(\theta, \phi) \cdot \delta_{\mathrm{st}} \cos \theta \mathrm{drd} \phi \mathrm{d} \theta$

onde $\bar{R}(\theta, \phi)$ é o raio máximo, do ponto fonte 's' até a face oposta ao mesmo, dado pela eq.(5.170), calculado por um processo semelhante ao que se fez na determinação da eq.(5.114).

$\overline{\mathrm{R}}(\theta, \phi)=\frac{-6 \mathrm{~V}}{\beta_{\mathrm{s}} \cos \theta \cos \phi+\gamma_{\mathrm{s}} \cos \theta \operatorname{sen} \phi+\delta_{\mathrm{s}} \operatorname{sen} \theta}$

No limite inferior $(r \rightarrow 0)$ a integral se anula, pois é feita em uma esfera fechada.

Por fim, optou-se por se realizar a integral da eq.(5.169) nas faces da célula, utilizando-se o processo de integração quase-singular da seção 5.3.

\subsection{EXEMPLOS}

Nesta seção, o autor apresenta dois exemplos numéricos que complementam a validação dos processos de integração desenvolvidos no trabalho.

\subsubsection{Exemplo 5.1}

O exemplo 5.1 trata da análise de um sólido submetido à ação de uma força volumétrica distribuída em todo o seu domínio atuando da direção longitudinal. Figura 5.18. 


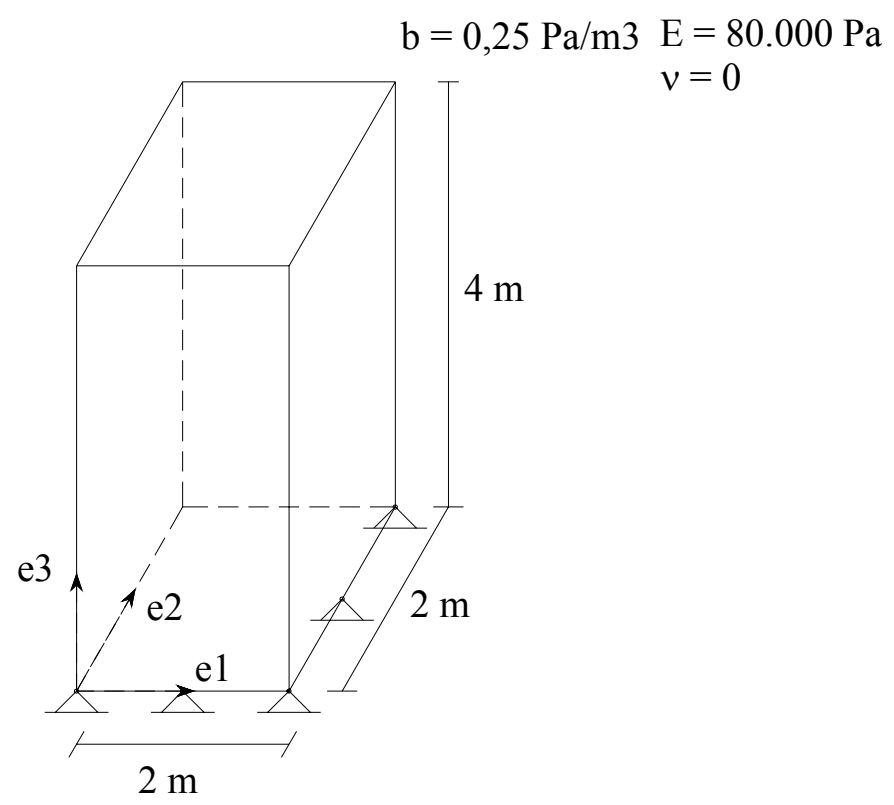

Figura 5.18 - Exemplo 5.1

A solução analítica, para deslocamentos, no caso de uma barra sujeita a uma força uniformemente distribuída ao longo do seu comprimento (figura 5.19), pode ser calculada utilizando-se a integral abaixo:
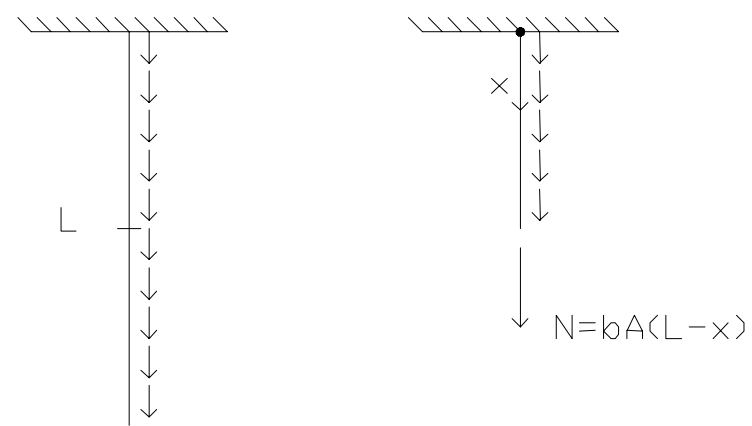

Figura 5.19 - Barra sob força uniformemente distribuída ao longo do comprimento

$$
\begin{aligned}
& u(x)=\int_{0}^{x N} \frac{\bar{N}}{E A} d x \\
& u(x)=\int_{0}^{x} \frac{b A(L-x) \cdot 1}{E A} d x \\
& u(x)=\frac{b}{E} \int_{0}^{x}(L-x) d x \\
& u(x)=\left.\frac{b}{E}\left[L x-\frac{x^{2}}{2}\right]\right|_{0} ^{x}
\end{aligned}
$$


$u(x)=\frac{b}{E}\left(L x-\frac{1}{2} x^{2}\right)$

Para a extremidade da barra $(x=L)$, tem-se:

$u(L)=\frac{b L^{2}}{2 E}$

Substituindo-se os valores envolvidos no problema, tem-se:

$\mathrm{u}=2,5 \times 10^{-5} \mathrm{~m}$

O sólido foi analisado utilizando-se duas discretizações diferentes. Uma delas seria a discretização da figura 5.20, com mesma densidade para a discretização do domínio $(\mathrm{nd} \ell \mathrm{c}=\mathrm{ndpc}=$ ndcc $=2$ ). Ou seja, são 36 nós, 48 elementos de contorno e 48 células. E a outra, uma discretização tipo 2210 , com 108 nós, 176 elementos de contorno e 240 células.
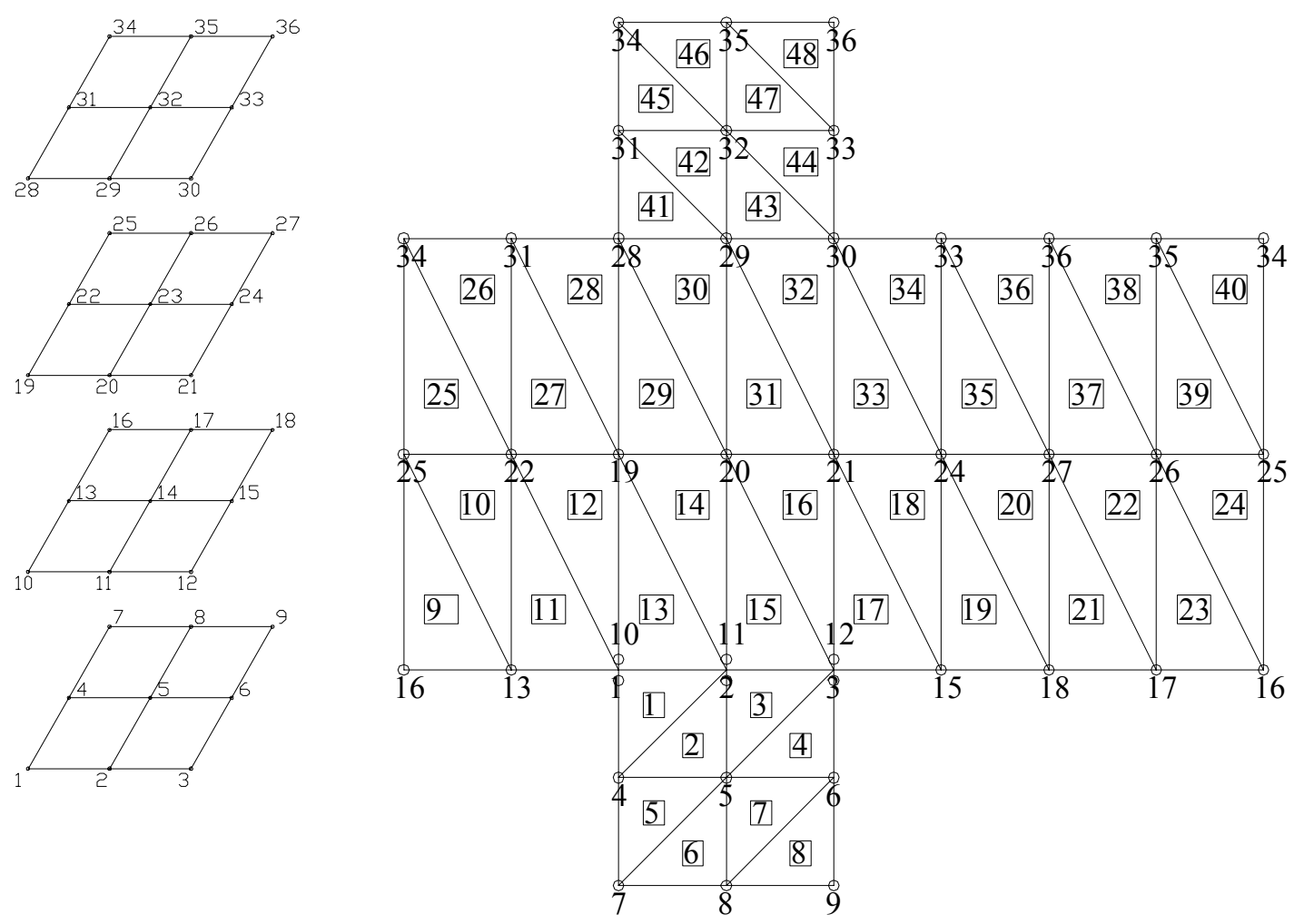

Figura 5.20 - Nós e elementos de contorno 
Os resultados obtidos para os deslocamentos na extremidade livre da barra são apresentados na tabela 5.1 abaixo:

Tabela 5.1 - Deslocamentos na direção longitudinal - exemplo 5.1

\begin{tabular}{||c|c|c|c|c||}
\hline Nó & $\mathbf{2 2 2}$ & erro \% & $\mathbf{2 2 1 0}$ & erro \% \\
\hline$(0,0,4)$ & $2,41200 \mathrm{E}-05$ & $-3,520$ & $2,49330 \mathrm{E}-05$ & $-0,268$ \\
\hline$(1,0,4)$ & $2,36380 \mathrm{E}-05$ & $-5,448$ & $2,49320 \mathrm{E}-05$ & $-0,272$ \\
\hline$(2,0,4)$ & $2,41840 \mathrm{E}-05$ & $-3,264$ & $2,49360 \mathrm{E}-05$ & $-0,256$ \\
\hline$(0,1,4)$ & $2,36330 \mathrm{E}-05$ & $-5,468$ & $2,49310 \mathrm{E}-05$ & $-0,276$ \\
\hline$(1,1,4)$ & $2,34290 \mathrm{E}-05$ & $-6,284$ & $2,49510 \mathrm{E}-05$ & $-0,196$ \\
\hline$(2,1,4)$ & $2,36600 \mathrm{E}-05$ & $-5,360$ & $2,49320 \mathrm{E}-05$ & $-0,272$ \\
\hline$(0,2,4)$ & $2,41710 \mathrm{E}-05$ & $-3,316$ & $2,49340 \mathrm{E}-05$ & $-0,264$ \\
\hline$(1,2,4)$ & $2,36520 \mathrm{E}-05$ & $-5,392$ & $2,49310 \mathrm{E}-05$ & $-0,276$ \\
\hline$(2,2,4)$ & $2,41650 \mathrm{E}-05$ & $-3,340$ & $2,49340 \mathrm{E}-05$ & $-0,264$ \\
\hline $\begin{array}{c}\text { Valor } \\
\text { analítico }\end{array}$ & $\mathbf{2 , 5 0 0 0 0 \mathrm { E } - 0 5}$ & $\mathbf{0}$ & $\mathbf{2 , 5 0 0 0 0 \mathrm { E } - 0 5}$ & $\mathbf{0}$ \\
\hline \hline
\end{tabular}

Da mesma forma, apresentam-se os valores obtidos para as reações de apoio: 
Tabela 5.2 - Reações de apoio - exemplo 5.1

\begin{tabular}{|c|c|c|c|c||}
\hline \hline Nó & $\mathbf{2 2 2}$ & erro \% & $\mathbf{2 2 1 0}$ & erro \% \\
\hline$(0,0,0)$ & $-1,04380 \mathrm{E}+00$ & 4,380 & $-9,97310 \mathrm{E}-01$ & $-0,269$ \\
\hline$(1,0,0)$ & $-9,35830 \mathrm{E}-01$ & $-6,417$ & $-9,96880 \mathrm{E}-01$ & $-0,312$ \\
\hline$(2,0,0)$ & $-1,02380 \mathrm{E}+00$ & 2,380 & $-9,96390 \mathrm{E}-01$ & $-0,361$ \\
\hline$(0,1,0)$ & $-9,31310 \mathrm{E}-01$ & $-6,869$ & $-9,96280 \mathrm{E}-01$ & $-0,372$ \\
\hline$(1,1,0)$ & $-8,96660 \mathrm{E}-01$ & $-10,334$ & $-1,00010 \mathrm{E}+00$ & 0,010 \\
\hline$(2,1,0)$ & $-9,36890 \mathrm{E}-01$ & $-6,311$ & $-9,96570 \mathrm{E}-01$ & $-0,343$ \\
\hline$(0,2,0)$ & $-1,03370 \mathrm{E}+00$ & 3,370 & $-9,97380 \mathrm{E}-01$ & $-0,262$ \\
\hline$(1,2,0)$ & $-9,31100 \mathrm{E}-01$ & $-6,890$ & $-9,96090 \mathrm{E}-01$ & $-0,391$ \\
\hline$(2,2,0)$ & $-1,04290 \mathrm{E}+00$ & 4,290 & $-9,98140 \mathrm{E}-01$ & $-0,186$ \\
\hline $\begin{array}{c}\text { Valor } \\
\text { analítico }\end{array}$ & $-\mathbf{- 1 , 0 0 0 0 0 \mathrm { E } + 0 0}$ & $\mathbf{0}$ & $-\mathbf{1 , 0 0 0 0 0 E}+\mathbf{0 0}$ & $\mathbf{0}$ \\
\hline
\end{tabular}

As tensões na direção longitudinal da barra variam linearmente com a coordenada X3 do problema analisado, assim, na tabela 5.3 são apresentados os valores obtidos para os nós localizados na seção média do sólido $(X 3=2$ $\mathrm{m})$.

Observa-se que os resultados obtidos são bastante satisfatórios, validando, assim, os desenvolvimentos envolvidos. 
Tabela 5.3 - Tensões na direção longitudinal - exemplo 5.1

\begin{tabular}{|c|c|c|c|c||}
\hline Nó & $\mathbf{2 2 2}$ & erro \% & $\mathbf{2 2 1 0}$ & erro \% \\
\hline$(0,0,2)$ & $4,79230 \mathrm{E}-01$ & $-4,154$ & $4,98730 \mathrm{E}-01$ & $-0,254$ \\
\hline$(1,0,2)$ & $4,76090 \mathrm{E}-01$ & $-4,782$ & $4,98750 \mathrm{E}-01$ & $-0,250$ \\
\hline$(2,0,2)$ & $4,80160 \mathrm{E}-01$ & $-3,968$ & $4,98780 \mathrm{E}-01$ & $-0,244$ \\
\hline$(0,1,2)$ & $4,76110 \mathrm{E}-01$ & $-4,778$ & $4,98760 \mathrm{E}-01$ & $-0,248$ \\
\hline$(1,1,2)$ & $4,73290 \mathrm{E}-01$ & $-5,342$ & $4,98790 \mathrm{E}-01$ & $-0,242$ \\
\hline$(2,1,2)$ & $4,76870 \mathrm{E}-01$ & $-4,626$ & $4,98750 \mathrm{E}-01$ & $-0,250$ \\
\hline$(0,2,2)$ & $4,79810 \mathrm{E}-01$ & $-4,038$ & $4,98770 \mathrm{E}-01$ & $-0,246$ \\
\hline$(1,2,2)$ & $4,76290 \mathrm{E}-01$ & $-4,742$ & $4,98750 \mathrm{E}-01$ & $-0,250$ \\
\hline$(2,2,2)$ & $4,79890 \mathrm{E}-01$ & $-4,022$ & $4,98730 \mathrm{E}-01$ & $-0,254$ \\
\hline $\begin{array}{c}\text { Valor } \\
\text { analítico }\end{array}$ & $\mathbf{5 , 0 0 0 0 0 \mathrm { E } - 0 1}$ & $\mathbf{0}$ & $\mathbf{5 , 0 0 0 0 0 \mathrm { E } - 0 1}$ & $\mathbf{0}$ \\
\hline
\end{tabular}

\subsubsection{Exemplo 5.2}

O exemplo 5.2 analisa o mesmo sólido do exemplo anterior, sendo que agora se optou por utilizar discretizações independentes para o contorno e para o domínio. Objetiva-se com este exemplo aferir tal independência de discretizações.

Sendo assim, para o contorno, utilizou-se a discretização 2210 para todas as análises; e para o domínio, utilizaram-se as discretizações do tipo $111,112,113$ e 2210.

As tabelas $5.4,5.5$ e 5.6 abaixo, semelhantemente ao que foi mostrado nas tabelas 5.1, 5.2 e 5.3 do exemplo anterior, apresenta, respectivamente, os deslocamentos na extremidade livre da barra, as reações de apoio e as tensões na direção longitudinal da barra para os nós localizados na seção média do sólido $(X 3=2 \mathrm{~m})$. 
Tabela 5.4 - Deslocamentos na direção longitudinal - exemplo 5.2

\begin{tabular}{||c|c|c|c|c|c|c|c|c||}
\hline \hline Nó & 2210_111 & erro \% & 2210_112 & erro \% & 2210_113 & erro \% & 2210 & erro \% \\
\hline$(0,0,4)$ & $2,49480 \mathrm{E}-05$ & $-0,208$ & $2,49330 \mathrm{E}-05$ & $-0,268$ & $2,49310 \mathrm{E}-05$ & $-0,276$ & $2,49330 \mathrm{E}-05$ & $-0,268$ \\
\hline$(1,0,4)$ & $2,49370 \mathrm{E}-05$ & $-0,252$ & $2,49320 \mathrm{E}-05$ & $-0,272$ & $2,49320 \mathrm{E}-05$ & $-0,272$ & $2,49320 \mathrm{E}-05$ & $-0,272$ \\
\hline$(2,0,4)$ & $2,49420 \mathrm{E}-05$ & $-0,232$ & $2,49390 \mathrm{E}-05$ & $-0,244$ & $2,49390 \mathrm{E}-05$ & $-0,244$ & $2,49360 \mathrm{E}-05$ & $-0,256$ \\
\hline$(0,1,4)$ & $2,49360 \mathrm{E}-05$ & $-0,256$ & $2,49300 \mathrm{E}-05$ & $-0,280$ & $2,49280 \mathrm{E}-05$ & $-0,288$ & $2,49310 \mathrm{E}-05$ & $-0,276$ \\
\hline$(1,1,4)$ & $2,49520 \mathrm{E}-05$ & $-0,192$ & $2,49510 \mathrm{E}-05$ & $-0,196$ & $2,49510 \mathrm{E}-05$ & $-0,196$ & $2,49510 \mathrm{E}-05$ & $-0,196$ \\
\hline$(2,1,4)$ & $2,49310 \mathrm{E}-05$ & $-0,276$ & $2,49330 \mathrm{E}-05$ & $-0,268$ & $2,49340 \mathrm{E}-05$ & $-0,264$ & $2,49320 \mathrm{E}-05$ & $-0,272$ \\
\hline$(0,2,4)$ & $2,49310 \mathrm{E}-05$ & $-0,276$ & $2,49340 \mathrm{E}-05$ & $-0,264$ & $2,49320 \mathrm{E}-05$ & $-0,272$ & $2,49340 \mathrm{E}-05$ & $-0,264$ \\
\hline$(1,2,4)$ & $2,49290 \mathrm{E}-05$ & $-0,284$ & $2,49300 \mathrm{E}-05$ & $-0,280$ & $2,49310 \mathrm{E}-05$ & $-0,276$ & $2,49310 \mathrm{E}-05$ & $-0,276$ \\
\hline$(2,2,4)$ & $2,49290 \mathrm{E}-05$ & $-0,284$ & $2,49350 \mathrm{E}-05$ & $-0,260$ & $2,49370 \mathrm{E}-05$ & $-0,252$ & $2,49340 \mathrm{E}-05$ & $-0,264$ \\
\hline $\begin{array}{c}\text { Valor } \\
\text { analítico }\end{array}$ & $2,50000 \mathrm{E}-05$ & 0 & $2,50000 \mathrm{E}-05$ & 0 & $2,50000 \mathrm{E}-05$ & 0 & $2,50000 \mathrm{E}-05$ & 0 \\
\hline
\end{tabular}

Tabela 5.5 - Reações de apoio - exemplo 5.2

\begin{tabular}{||c|c|c|c|c|c|c|c|c||}
\hline Nó & 2210_111 & erro \% & 2210_112 & erro \% & 2210_113 & erro \% & 2210 & erro \% \\
\hline$(0,0,0)$ & $-9,97550 \mathrm{E}-01$ & $-0,245$ & $-9,97290 \mathrm{E}-01$ & $-0,271$ & $-9,97460 \mathrm{E}-01$ & $-0,254$ & $-9,97310 \mathrm{E}-01$ & $-0,269$ \\
\hline$(1,0,0)$ & $-9,96990 \mathrm{E}-01$ & $-0,301$ & $-9,96830 \mathrm{E}-01$ & $-0,317$ & $-9,96820 \mathrm{E}-01$ & $-0,318$ & $-9,96880 \mathrm{E}-01$ & $-0,312$ \\
\hline$(2,0,0)$ & $-9,96210 \mathrm{E}-01$ & $-0,379$ & $-9,96370 \mathrm{E}-01$ & $-0,363$ & $-9,96420 \mathrm{E}-01$ & $-0,358$ & $-9,96390 \mathrm{E}-01$ & $-0,361$ \\
\hline$(0,1,0)$ & $-9,96420 \mathrm{E}-01$ & $-0,358$ & $-9,96150 \mathrm{E}-01$ & $-0,385$ & $-9,96200 \mathrm{E}-01$ & $-0,380$ & $-9,96280 \mathrm{E}-01$ & $-0,372$ \\
\hline$(1,1,0)$ & $-1,00010 \mathrm{E}+00$ & 0,010 & $-9,99990 \mathrm{E}-01$ & $-0,001$ & $-1,00010 \mathrm{E}+00$ & 0,010 & $-1,00010 \mathrm{E}+00$ & 0,010 \\
\hline$(2,1,0)$ & $-9,96600 \mathrm{E}-01$ & $-0,340$ & $-9,96670 \mathrm{E}-01$ & $-0,333$ & $-9,96540 \mathrm{E}-01$ & $-0,346$ & $-9,96570 \mathrm{E}-01$ & $-0,343$ \\
\hline$(0,2,0)$ & $-9,97460 \mathrm{E}-01$ & $-0,254$ & $-9,97500 \mathrm{E}-01$ & $-0,250$ & $-9,97460 \mathrm{E}-01$ & $-0,254$ & $-9,97380 \mathrm{E}-01$ & $-0,262$ \\
\hline$(1,2,0)$ & $-9,95830 \mathrm{E}-01$ & $-0,417$ & $-9,96120 \mathrm{E}-01$ & $-0,388$ & $-9,96050 \mathrm{E}-01$ & $-0,395$ & $-9,96090 \mathrm{E}-01$ & $-0,391$ \\
\hline$(2,2,0)$ & $-9,99180 \mathrm{E}-01$ & $-0,082$ & $-9,98290 \mathrm{E}-01$ & $-0,171$ & $-9,98200 \mathrm{E}-01$ & $-0,180$ & $-9,98140 \mathrm{E}-01$ & $-0,186$ \\
\hline $\begin{array}{c}\text { Valor } \\
\text { analítico }\end{array}$ & $-1,00000 \mathrm{E}+00$ & 0 & $-1,00000 \mathrm{E}+00$ & 0 & $-1,00000 \mathrm{E}+00$ & 0 & $-1,00000 \mathrm{E}+00$ & 0 \\
\hline \hline
\end{tabular}

Tabela 5.6 - Tensões na direção longitudinal - exemplo 5.2

\begin{tabular}{|c|c|c|c|c|c|c|c|c||}
\hline Nó & 2210_111 & erro \% & 2210_112 & erro \% & 2210_113 & erro \% & 2210 & erro \% \\
\hline$(0,0,2)$ & $4,98990 \mathrm{E}-01$ & $-0,202$ & $4,98650 \mathrm{E}-01$ & $-0,270$ & $4,99110 \mathrm{E}-01$ & $-0,178$ & $4,98730 \mathrm{E}-01$ & $-0,254$ \\
\hline$(1,0,2)$ & $4,98750 \mathrm{E}-01$ & $-0,250$ & $4,98640 \mathrm{E}-01$ & $-0,272$ & $4,98870 \mathrm{E}-01$ & $-0,226$ & $4,98750 \mathrm{E}-01$ & $-0,250$ \\
\hline$(2,0,2)$ & $4,99110 \mathrm{E}-01$ & $-0,178$ & $4,98490 \mathrm{E}-01$ & $-0,302$ & $4,99000 \mathrm{E}-01$ & $-0,200$ & $4,98780 \mathrm{E}-01$ & $-0,244$ \\
\hline$(0,1,2)$ & $4,98450 \mathrm{E}-01$ & $-0,310$ & $4,98890 \mathrm{E}-01$ & $-0,222$ & $4,98580 \mathrm{E}-01$ & $-0,284$ & $4,98760 \mathrm{E}-01$ & $-0,248$ \\
\hline$(1,1,2)$ & $4,98890 \mathrm{E}-01$ & $-0,222$ & $4,98760 \mathrm{E}-01$ & $-0,248$ & $4,98760 \mathrm{E}-01$ & $-0,248$ & $4,98790 \mathrm{E}-01$ & $-0,242$ \\
\hline$(2,1,2)$ & $4,99380 \mathrm{E}-01$ & $-0,124$ & $4,98860 \mathrm{E}-01$ & $-0,228$ & $4,98760 \mathrm{E}-01$ & $-0,248$ & $4,98750 \mathrm{E}-01$ & $-0,250$ \\
\hline$(0,2,2)$ & $4,98450 \mathrm{E}-01$ & $-0,310$ & $4,98970 \mathrm{E}-01$ & $-0,206$ & $4,98540 \mathrm{E}-01$ & $-0,292$ & $4,98770 \mathrm{E}-01$ & $-0,246$ \\
\hline$(1,2,2)$ & $4,98640 \mathrm{E}-01$ & $-0,272$ & $4,98580 \mathrm{E}-01$ & $-0,284$ & $4,98880 \mathrm{E}-01$ & $-0,224$ & $4,98750 \mathrm{E}-01$ & $-0,250$ \\
\hline$(2,2,2)$ & $4,98660 \mathrm{E}-01$ & $-0,268$ & $4,98840 \mathrm{E}-01$ & $-0,232$ & $4,98480 \mathrm{E}-01$ & $-0,304$ & $4,98730 \mathrm{E}-01$ & $-0,254$ \\
\hline $\begin{array}{c}\text { Valor } \\
\text { analítico }\end{array}$ & $5,00000 \mathrm{E}-01$ & 0 & $5,00000 \mathrm{E}-01$ & 0 & $5,00000 \mathrm{E}-01$ & 0 & $5,00000 \mathrm{E}-01$ & 0 \\
\hline \hline
\end{tabular}

Analisando-se as 3 tabelas acima, percebe-se que a discretização do domínio não influencia muito no problema analisado. 


\section{O MÉTODO DOS ELEMENTOS DE CONTORNO NO DOMÍNIO DO TEMPO}

No presente capítulo será apresentada a formulação utilizada para o Método dos Elementos de Contorno no Domínio do Tempo (TDBEM), bem como um exemplo numérico sobre esta técnica. Vale dizer que o conteúdo apresentado tem como base o texto de Livre-docência do orientador, Coda (2000).

O Time Domain Boundary Element Method (TDBEM) ou Método dos Elementos de Contorno no Domínio do Tempo surge como uma alternativa ao chamado Mass Matrix Boundary Element Method (MMBEM), uma vez que, utilizando soluções fundamentais dependentes do tempo, elimina a necessidade da discretização do domínio para a geração de matriz de massa. Sendo assim, em análises elásticas, nenhuma discretização do domínio é necessária.

Vale dizer que o código computacional básico para esta técnica foi desenvolvido pelo orientador do trabalho; porém, a implementação de pontos fonte singulares para os elementos de contorno triangulares planos foi desenvolvida integralmente pelo autor, fazendo-se uso das técnicas comentadas no capítulo anterior. 


\subsection{EQUAÇÃO INTEGRAL DE DESLOCAMENTOS}

Para a dedução da equação integral de deslocamentos originária do TDBEM, optou-se por se partir da equação de equilíbrio dinâmico expressa em termos de tensões, eq. (6.1), escrita abaixo, onde, inicialmente, não se levou em consideração a simetria do tensor de tensões.

$$
\sigma_{i j, i}+b_{j}=\rho \ddot{u}_{j}+c \dot{u}_{j}
$$

Uma vez que o amortecimento viscoso não será considerado no problema, a eq.(6.1) passa a ser escrita como:

$$
\sigma_{\mathrm{ij}, \mathrm{i}}+\mathrm{b}_{\mathrm{j}}=\rho \ddot{u}_{\mathrm{j}}
$$

A eq.(6.2) escrita em termos de deslocamentos torna-se a equação de movimento para corpos elásticos, ou equação de Navier-Cauchy:

$$
G u_{j, m m}+(\lambda+G) u_{m, m j}+b_{j}=\rho \ddot{u}_{j}
$$

Impondo-se a condição de irrotacionalidade, expressa na equação (6.4) abaixo, à eq.(6.3), obtém-se a velocidade de propagação da onda longitudinal (dilatational wave) $\mathrm{C}_{1}$.

$$
\begin{aligned}
& w_{i j}=\frac{1}{2}\left(u_{i, j}-u_{j, i}\right)=0 \\
& C_{1}=\sqrt{(\lambda+2 G) / \rho}
\end{aligned}
$$

Da mesma forma, aplicando-se a condição da eq.(6.6) abaixo à eq.(6.3), chega-se à expressão da velocidade de propagação da onda equivolumial ou distorcional (shear wave) $\mathrm{C}_{2}$, eq.(6.7).

$\varepsilon_{\mathrm{ii}}=\mathrm{u}_{\mathrm{i}, \mathrm{i}}=0$ 


$$
C_{2}=\sqrt{G / \rho}
$$

Observa-se que $\mathrm{C}_{1}>\mathrm{C}_{2}$.

Substituindo-se as expressões de $C_{1}$ e $C_{2}$ na eq.(6.3), tem-se:

$$
\rho\left(C_{1}^{2}-C_{2}^{2}\right) u_{m, m j}+\rho C_{2}^{2} u_{j, m m}+b_{j}=\rho \ddot{u}_{j}
$$

As condições iniciais podem ser escritas como:

$$
\begin{aligned}
& u_{i}\left(x, t_{0}\right)=u_{0 i}(x) \\
& \dot{u}_{i}\left(x, t_{0}\right)=v_{0 i}(x)
\end{aligned}
$$

As condições de contorno ao longo do tempo podem ser escritas como:

$$
\begin{array}{ll}
u_{i}(x, t)=\bar{u}_{i}(x, t) & \text { em } \Gamma_{1} \\
p_{i}(x, t)=\bar{p}_{i}(x, t) & \text { em } \Gamma_{2}
\end{array}
$$

O método dos resíduos ponderados foi utilizado para a dedução da equação integral de deslocamentos; sendo assim, considera-se dois estados dinâmicos, onde o primeiro respeita a eq.(6.2) e o segundo, além da equação anterior, respeita a eq.(6.3).

$1^{\stackrel{\circ}{)})} u_{i}(\tau), \sigma_{i j}(\tau)$

$\left.2^{\underline{o}}\right) u_{i}^{0}(t-\tau), \sigma_{i j}^{0}(t-\tau)$

Multiplicando-se a eq.(6.2), escrita para o primeiro estado, pelo campo de deslocamentos do segundo estado, tem-se:

$$
\left(\sigma_{i j, i}(\tau)+b_{j}(\tau)-\rho \ddot{u}_{j}(\tau)\right) \cdot u_{j}^{0}(t-\tau)=0
$$

Integrando-se em um domínio $\Omega$ do corpo e em um intervalo de tempo $\left[\mathrm{t}_{0}, \mathrm{t}\right]$ : 


$$
\begin{aligned}
& \int_{t_{0}}^{t} \int_{\Omega}\left(\sigma_{i j, i}(\tau)+b_{j}(\tau)-\rho \ddot{u}_{j}(\tau)\right) \cdot u_{j}^{0}(t-\tau) d \Omega d \tau=0 \\
& \int_{t_{0}}^{t} \int_{\Omega} \sigma_{i j, i}(\tau) u_{j}^{0}(t-\tau) d \Omega d \tau+\int_{t_{0}}^{t} \int_{\Omega} b_{j}(\tau) u_{j}^{0}(t-\tau) d \Omega d \tau=\int_{\Omega} \int_{t_{0}}^{t} \rho \ddot{u}_{j}(\tau) u_{j}^{0}(t-\tau) d \tau d \Omega
\end{aligned}
$$

Integrando-se por partes em relação ao tempo o termo do lado direito da eq.(6.15), pode-se escrever:

$\int_{\Omega} \int_{t_{0}}^{t} \rho \ddot{u}_{j}(\tau) u_{j}^{0}(t-\tau) d \tau d \Omega=\int_{\Omega} \rho\left\{\left.\dot{u}_{j}(\tau) u_{j}^{0}(t-\tau)\right|_{t_{0}} ^{t}-\int_{t_{0}}^{t} \dot{u}_{j}(\tau) \dot{u}_{j}^{0}(t-\tau) d \tau\right\} d \Omega$

Integrando-se por partes, também em relação ao tempo, o segundo termo do lado direito da eq.(6.16) acima, tem-se:

$\int_{t_{0}}^{t} \dot{u}_{j}(\tau) \dot{u}_{j}^{0}(t-\tau) d \tau d \Omega=\left.u_{j}(\tau) \dot{u}_{j}^{0}(t-\tau)\right|_{t_{0}} ^{t}-\int_{t_{0}}^{t} u_{j}(\tau) \ddot{u}_{j}^{0}(t-\tau) d \tau d \Omega$

Substituindo-se a eq.(6.17) na eq.(6.16):

$$
\begin{aligned}
& \int_{t_{0}}^{t} \int_{\Omega} \rho \ddot{u}_{j}(\tau) u_{j}^{0}(t-\tau) d \Omega d \tau=\int_{\Omega} \rho\left\{\dot{u}_{j}(\tau) u_{j}^{0}(t-\tau)-u_{j}(\tau) \dot{u}_{j}^{0}(t-\tau)\right\}_{t 0}^{t} d \Omega+ \\
& +\int_{t_{0}}^{t} \int_{\Omega} \rho u_{j}(\tau) \ddot{u}_{j}^{0}(t-\tau) d \Omega d \tau
\end{aligned}
$$

Integrando-se por partes no espaço o primeiro termo da esquerda da eq.(6.15), tem-se:

$$
\int_{\mathrm{t}_{0}}^{\mathrm{t}} \int_{\Omega} \sigma_{\mathrm{ij}, \mathrm{i}}(\tau) \mathrm{u}_{\mathrm{j}}^{0}(\mathrm{t}-\tau) \mathrm{d} \Omega \mathrm{d} \tau=\int_{\mathrm{t}_{0}}^{\mathrm{t}}\left\{\int_{\Gamma} \sigma_{\mathrm{ij}}(\tau) \mathrm{n}_{\mathrm{i}} \mathrm{u}_{\mathrm{j}}^{0}(\mathrm{t}-\tau) \mathrm{d} \Gamma-\int_{\Omega} \sigma_{\mathrm{ij}}(\tau) \mathrm{u}_{\mathrm{j}, \mathrm{i}}^{0}(\mathrm{t}-\tau) \mathrm{d} \Omega\right\} \mathrm{d} \tau
$$

Semelhantemente ao que foi feito na dedução da formulação do MMBEM (capítulo 3), pode-se escrever:

$p_{j}(\tau)=\sigma_{i j}(\tau) n_{i}$ 


$$
\begin{aligned}
& \sigma_{i j}(\tau) u_{i, j}^{0}(t-\tau)=\sigma_{i j}(\tau) \varepsilon_{i j}^{0}(t-\tau) \\
& \sigma_{i j}(\tau)=\sigma_{i j}^{e}(\tau)-\sigma_{i j}^{n}(\tau)
\end{aligned}
$$

Observando-se a eq.(6.22), percebe-se que a utilização da técnica dos resíduos ponderados na construção da formulação transiente do Método dos Elementos de Contorno facilita a introdução de tensões plásticas no equacionamento integral final, tornando possível o tratamento de problemas não-lineares físicos via TDBEM. Porém, como este tema não é tratado na pesquisa, optou-se por, desde já, considerar apenas tensões elásticas no equacionamento em desenvolvimento. Sendo assim, a eq.(6.22) pode ser reescrita como:

$$
\sigma_{\mathrm{ij}}(\tau)=\sigma_{\mathrm{ij}}^{\mathrm{e}}(\tau)
$$

Substituindo-se as eq.(6.20), (6.21) e (6.23) na eq.(6.19), tem-se:

$$
\int_{t_{0}}^{t} \int_{\Omega} \sigma_{i j, i}(\tau) u_{j}^{0}(t-\tau) d \Omega d \tau=\int_{t_{0}}^{t} \int_{\Gamma} p_{j}(\tau) u_{j}^{0}(t-\tau) d \Gamma d \tau-\int_{t_{0}}^{t} \int_{\Omega} \sigma_{i j}(\tau) \varepsilon_{i j}^{0}(t-\tau) d \Omega d \tau
$$

Substituindo-se a eq.(6.24) e a eq.(6.18) na eq.(6.15), tem-se:

$$
\begin{aligned}
& \int_{t_{0}}^{t} \int_{\Gamma} p_{j}(\tau) u_{j}^{0}(t-\tau) d \Gamma d \tau=\int_{\Omega} \rho\left\{\dot{u}_{j}(\tau) u_{j}^{0}(t-\tau)-u_{j}(\tau) \dot{u}_{j}^{0}(t-\tau)\right\}_{t_{0}}^{t} d \Omega+ \\
& -\int_{t_{0}}^{t} \int_{\Omega} b_{j}(\tau) u_{j}^{0}(t-\tau) d \Omega d \tau+\int_{t_{0}}^{t} \int_{\Omega} \sigma_{i j}(\tau) \varepsilon_{i j}^{0}(t-\tau) d \Omega d \tau+\int_{t_{0}}^{t} \int_{\Omega} \rho u_{j}(\tau) \ddot{u}_{j}^{0}(t-\tau) d \Omega d \tau
\end{aligned}
$$

Aplicando-se a propriedade da eq.(6.26) abaixo ao terceiro termo do lado direito da eq.(6.25) e integrando-se por partes em $\Omega$, tem-se:

$$
\begin{aligned}
& \sigma_{\mathrm{ij}}^{\mathrm{e}} \varepsilon_{\mathrm{ij}}^{0}=\varepsilon_{\mathrm{k} \ell} C_{\mathrm{ij}}^{\mathrm{k} \ell} \varepsilon_{\mathrm{ij}}^{0}=\varepsilon_{\mathrm{k} \ell} \sigma_{\mathrm{k} \ell}^{0}=\mathrm{u}_{\ell, \mathrm{k}} \sigma_{\mathrm{k} \ell}^{0}=\mathrm{u}_{\mathrm{j}, \mathrm{i}} \sigma_{\mathrm{ij}}^{0} \\
& \int_{\mathrm{t}_{0}}^{\mathrm{t}} \int_{\Omega} \sigma_{\mathrm{ij}}(\tau) \varepsilon_{\mathrm{ij}}^{0}(\mathrm{t}-\tau) \mathrm{d} \Omega \mathrm{d} \tau=\int_{\mathrm{t}_{0}}^{\mathrm{t}} \int_{\Omega} \mathrm{u}_{\mathrm{j}, \mathrm{i}}(\tau) \sigma_{\mathrm{ij}}^{0}(\mathrm{t}-\tau) \mathrm{d} \Omega \mathrm{d} \tau
\end{aligned}
$$


$\int_{t_{0}}^{t} \int_{\Omega} u_{j, i}(\tau) \sigma_{i j}^{0}(t-\tau) d \Omega d \tau=\int_{t_{0}}^{t} \int_{\Gamma} u_{j}(\tau) n_{i} \sigma_{i j}^{0}(t-\tau) d \Gamma d \tau-\int_{t_{0}}^{t} \int_{\Omega} u_{j}(\tau) \sigma_{i j, i}^{0}(t-\tau) d \Omega d \tau$

Substituindo-se a eq.(6.20) na eq.(6.28) e substituindo-se o resultado na eq.(6.27), pode-se escrever:

$\int_{t_{0}}^{t} \int_{\Omega} \sigma_{i j}(\tau) \varepsilon_{i j}^{0}(t-\tau) d \Omega d \tau=\int_{t_{0}}^{t} \int_{\Gamma} u_{j}(\tau) p_{j}^{0}(t-\tau) d \Gamma d \tau-\int_{t_{0}}^{t} \int_{\Omega} u_{j}(\tau) \sigma_{i j, i}^{0}(t-\tau) d \Omega d \tau$

Substituindo-se a eq.(6.29) na eq.(6.25), tem-se:

$\int_{t_{0}}^{t} \int_{\Gamma} p_{j}(\tau) u_{j}^{0}(t-\tau) d \Gamma d \tau=\int_{\Omega} \rho\left\{\dot{u}_{j}(\tau) u_{j}^{0}(t-\tau)-u_{j}(\tau) \dot{u}_{j}^{0}(t-\tau)\right\}_{t_{0}}^{t} d \Omega+$

$-\int_{t_{0}}^{t} \int_{\Omega} b_{j}(\tau) u_{j}^{0}(t-\tau) d \Omega d \tau-\int_{t_{0}}^{t} \int_{\Omega} u_{j}(\tau)\left\{\sigma_{i j, i}^{0}(t-\tau)-\rho \ddot{u}_{j}^{0}(t-\tau)\right\} d \Omega d \tau+$

$+\int_{t_{0}}^{t} \int_{\Gamma} u_{j}(\tau) p_{j}^{0}(t-\tau) d \Gamma d \tau$

Pela eq.(6.2), observa-se que o penúltimo termo da eq.(6.30) é conhecido.

$$
-\int_{\mathrm{t}_{0}}^{\mathrm{t}} \int_{\Omega} \mathrm{u}_{\mathrm{j}}(\tau)\left\{\sigma_{\mathrm{ij}, \mathrm{i}}^{0}(\mathrm{t}-\tau)-\rho \ddot{u}_{\mathrm{j}}^{0}(\mathrm{t}-\tau)\right\} \mathrm{d} \Omega \mathrm{d} \tau=\int_{\mathrm{t}_{0}}^{\mathrm{t}} \int_{\Omega} \mathrm{u}_{\mathrm{j}}(\tau) \mathrm{b}_{\mathrm{j}}^{0}(\mathrm{t}-\tau) \mathrm{d} \Omega \mathrm{d} \tau
$$

Assim, substituindo-se a eq.(6.31) na eq.(6.30), chega-se à eq.(6.32) abaixo, que é conhecida como Teorema de Graffi:

$$
\begin{aligned}
& \int_{t_{0}}^{t} \int_{\Gamma} p_{j}(\tau) u_{j}^{0}(t-\tau) d \Gamma d \tau+\int_{t_{0}}^{t} \int_{\Omega} b_{j}(\tau) u_{j}^{0}(t-\tau) d \Omega d \tau+\left[\int_{\Omega} \rho u_{j}(\tau) \dot{u}_{j}^{0}(t-\tau) d \Omega\right]_{t_{0}}^{t} \\
& =\int_{t_{0}}^{t} \int_{\Gamma} u_{j}(\tau) p_{j}^{0}(t-\tau) d \Gamma d \tau+\int_{t_{0}}^{t} \int_{\Omega} u_{j}(\tau) b_{j}^{0}(t-\tau) d \Omega d \tau+\left[\int_{\Omega} \rho \dot{u}_{j}(\tau) u_{j}^{0}(t-\tau) d \Omega\right]_{t_{0}}^{t}
\end{aligned}
$$

Após a dedução do Teorema de Graffi, substitui-se o estado elastodinâmico auxiliar $(\cdot)^{0}$ pela solução do problema fundamental da elastodinâmica para corpos infinitos ou semi-infinitos isótropos. Por sua vez, o 
problema fundamental é definido substituindo-se a força volumétrica ' $b_{j}$ ' por um carregamento variável no tempo, aplicado em um ponto fonte ' $s$ ':

$b_{k j}^{*}=\delta_{k j} \delta(s, q) f(\tau)$

onde:

$\mathrm{b}_{\mathrm{kj}}^{*} \quad$ - é a carga fundamental aplicada na direção $\mathrm{k}$;

$\delta_{\mathrm{kj}} \quad-$ é o delta de Kroneker (0 se $\mathrm{k} \neq \mathrm{j}$ e 1 se $\mathrm{k}=\mathrm{j}$ );

$\delta(s, q) \quad-$ é o delta de Dirac (0 se $s \neq q$ e $+\infty$ se $s=q)$;

q - é um ponto de campo;

$f(\tau)$ - é uma função que representa a variação temporal do carregamento.

Assim, semelhantemente ao que se fez para o MMBEM, pode-se escrever:

$$
\sigma_{\mathrm{kij}, \mathrm{i}}^{*}+\mathrm{b}_{\mathrm{kj}}^{*}=\rho \ddot{u}_{\mathrm{kj}}^{*}
$$

Vale dizer que as soluções das equações diferenciais (6.34) são as chamadas funções de Green; para o caso de domínio infinito, estas são chamadas de Solução Fundamental de Stokes, $\left\{\mathrm{u}_{\mathrm{kj}}^{*}, \sigma_{\mathrm{kj}}^{*}\right\}$.

As condições iniciais para se resolver este problema fundamental são de repouso:

$$
\left\{\begin{array}{l}
u_{\mathrm{kj}}^{*}\left(q, t_{0}\right)=0 \\
\dot{u}_{\mathrm{kj}}^{*}\left(q, t_{0}\right)=0
\end{array}\right.
$$

Substituindo-se na eq.(6.32) o estado elastodinâmico auxiliar pelo estado fundamental e adotando-se $t_{0}=0$ : 


$$
\begin{aligned}
& \int_{0}^{t} \int_{\Omega} u_{j}(q, \tau) \delta_{k j} \delta(s, q) f(t-\tau) d \Omega d \tau+\int_{0}^{t} \int_{\Gamma} p_{k j}^{*}(Q, t-\tau ; s / f) u_{j}(Q, \tau) d \Gamma d \tau+ \\
& -\int_{\Omega} \rho \dot{u}_{j}(q, 0) u_{j}^{*}(q, t, s / f) d \Omega=\int_{0}^{t} \int_{\Gamma} u_{k j}^{*}(Q, t-\tau ; s / f) p_{j}(Q, \tau) d \Gamma d \tau+ \\
& +\int_{0}^{t} \int_{\Omega} b_{j}(q, \tau) u_{k j}^{*}(q, t-\tau ; s / f) d \Omega d \tau-\int_{\Omega} \rho u_{j}(q, 0) \dot{u}_{k j}^{*}(q, t ; s / f) d \Omega
\end{aligned}
$$

onde a notação '/f' é referente à função de carga f e as soluções fundamentais tridimensionais são:

$$
\begin{aligned}
& u_{k j}^{*}(q, t, s / f)=\frac{1}{4 \pi \rho}\left\{\left(\frac{3 r_{k} r_{j}}{r^{3}}-\frac{\delta_{k j}}{r}\right)_{C_{1}^{-1}}^{C_{2}^{-1}} \alpha f(t-\alpha r) d \alpha+\right. \\
& \left.+\frac{r_{k} r_{j}}{r^{3}}\left[\frac{1}{C_{1}^{2}} f\left(t-r / C_{1}\right)-\frac{1}{C_{2}^{2}} f\left(t-r / C_{2}\right)\right]+\frac{\delta_{k j}}{r C_{2}^{2}} f\left(t-r / C_{2}\right)\right\}
\end{aligned}
$$

e

$$
\begin{aligned}
& p_{k j}^{*}(q, t, s / f)=\frac{1}{4 \pi}\left\{6 C_{2}^{2} \frac{\left[\delta_{i k} r_{, j}+\delta_{j k} r_{, i}+\delta_{i j} r_{, k}-5 r_{, i} r_{, j} r_{, k}\right]}{r^{2}} \int_{C_{1}^{-1}}^{C_{2}^{-1}} \alpha f(t-\alpha r) d \alpha+\right. \\
& +2 \frac{\left[\delta_{i k} r_{j,}+\delta_{j k} r_{{ }_{j i}}+\delta_{i j} r_{{ }_{k}}-6 r_{{ }_{i j}} r_{, j} r_{{ }_{k}}\right]}{r^{2}}\left[\frac{C_{2}^{2}}{C_{1}^{2}} f\left(t-r / C_{1}\right)-f\left(t-r / C_{2}\right)\right]+ \\
& +\frac{2 r_{, j} r_{, j} r_{, k}}{r C_{2}}\left[\dot{f}\left(t-r / C_{2}\right)-\frac{C_{2}^{3}}{C_{1}^{3}} \dot{f}\left(t-r / C_{1}\right)\right]+ \\
& -\frac{\delta_{i j} r_{, k}}{r^{2}}\left(1-2 \frac{C_{2}^{2}}{C_{1}^{2}}\right)\left[f\left(t-r / C_{1}\right)+\frac{r}{C_{1}} \dot{f}\left(t-r / C_{1}\right)\right]+ \\
& \left.-\frac{\delta_{k j} r_{, j}+\delta_{i k} r_{j}}{r^{2}}\left[f\left(t-r / C_{2}\right)+\frac{r}{C_{2}} \dot{f}\left(t-r / C_{2}\right)\right]\right\} n_{i}
\end{aligned}
$$

Considerando-se as propriedades da distribuição Delta de Dirac, a eq.(6.35) pode ser escrita como: 


$$
\begin{aligned}
& C_{k j} \int_{0}^{t} u_{j}(s, \tau) f(t-\tau) d \tau=\int_{0}^{t} \int_{\Gamma} u_{k j}^{*}(Q, t-\tau ; s / f) p_{j}(Q, \tau) d \Gamma d \tau+ \\
& -\int_{0}^{t} \int_{\Gamma} p_{k j}^{*}(Q, t-\tau ; s / f) u_{j}(Q, \tau) d \Gamma d \tau+\int_{0}^{t} \int_{\Omega} b_{j}(q, \tau) u_{k j}^{*}(q, t-\tau ; s / f) d \Omega d \tau+ \\
& +\int_{\Omega} \rho u_{k j}^{*}(q, t ; s / f) \dot{u}_{j}(q, 0) d \Omega-\int_{\Omega} \rho \dot{u}_{k j}^{*}(q, t ; s / f) u_{j}(q, 0) d \Omega
\end{aligned}
$$

onde $\mathrm{C}_{\mathrm{kj}}$ assume o valor de $\delta_{\mathrm{kj}}$ para pontos fonte internos ao problema estudado e um valor dependente da superfície do problema para pontos fonte no contorno. É muito importante observar que a eq.(6.38) só é válida para pontos fonte internos e no contorno; para pontos fonte externos, fazem-se necessárias algumas considerações adicionais.

Pode-se começar afirmando que quando da aplicação de um carregamento concentrado no ponto fonte ' $\mathrm{s}$ ', após um período de tempo ' $\mathrm{t}$ ' uma certa região é perturbada por este carregamento, figura 6.1.

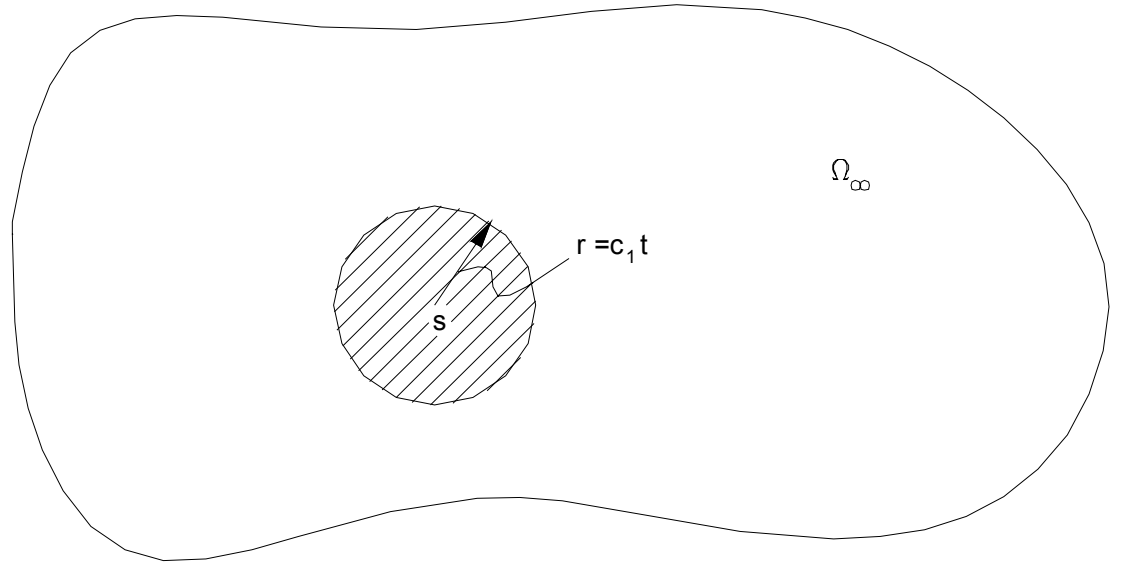

Figura 6.1 - Região perturbada após um período de tempo

Observa-se que na figura 6.1 adotou-se arbitrariamente a velocidade longitudinal $\mathrm{C}_{1}$.

Para pontos fonte localizados no interior ou no contorno do corpo, a convolução representada na eq.(6.38) pode ser esboçada na figura 6.2. 
sentido do movimento

convolutivo da solução

fundamental

Figura 6.2 - Convolução temporal para pontos fonte internos ou no contorno do corpo

Vale observar que no processo convolutivo os valores fundamentais são dependentes de ' $t$ - $\tau$ '; sendo assim, o raio externo das regiões perturbadas são referentes ao instante $\tau=0$ e o ponto fonte ' $s$ ' representa o instante $\tau=\mathrm{t}$. Em outra palavras, 't- $\tau$ ' varia de 't' a '0'.

$$
\left\{\begin{array}{l}
0<\tau<t \\
t<t-\tau<0
\end{array}\right.
$$

A figura 6.3 representa o processo convolutivo para pontos fonte localizados fora do domínio do corpo, onde 'e' é a menor distância entre o ponto fonte 's' e o contorno $\Gamma$ do corpo.

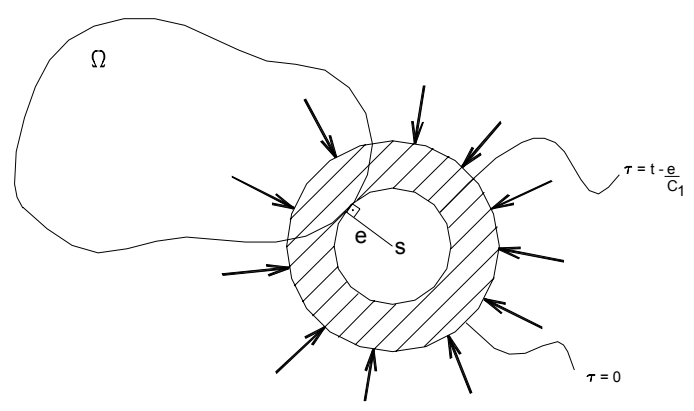

Figura 6.3 - Convolução temporal para pontos fonte externos ao corpo

Neste caso, a eq.(6.38) seria escrita como:

$$
\int_{\Gamma} \int_{0}^{t} p_{k j}^{*}(Q, t-\tau ; s / f) u_{j}(\tau) d \tau d \Gamma=\int_{\Gamma} \int_{0}^{t} u_{k j}^{*}(Q, t-\tau ; s / f) p_{j}(\tau) d \tau d \Gamma
$$

onde, por simplificação, não se escreveu as integrais de volume. 
A eq.(6.39) poderia ser dividida em:

$$
\begin{aligned}
& \int_{\Gamma} \int_{0}^{t-e / c_{1}} p_{k j}^{*}(Q, t-\tau ; s / f) u_{j}(\tau) d \tau d \Gamma+\int_{\Gamma} \int_{t-e / c_{1}}^{t} p_{k j}^{*}(Q, t-\tau ; s / f) u_{j}(\tau) d \tau d \Gamma \\
& =\int_{\Gamma} \int_{0}^{t-e / c_{1}} u_{k j}^{*}(Q, t-\tau ; s / f) p_{j}(\tau) d \tau d \Gamma+\int_{\Gamma} \int_{t-e / c_{1}}^{t} u_{k j}^{*}(Q, t-\tau ; s / f) p_{j}(\tau) d \tau d \Gamma
\end{aligned}
$$

Graficamente:

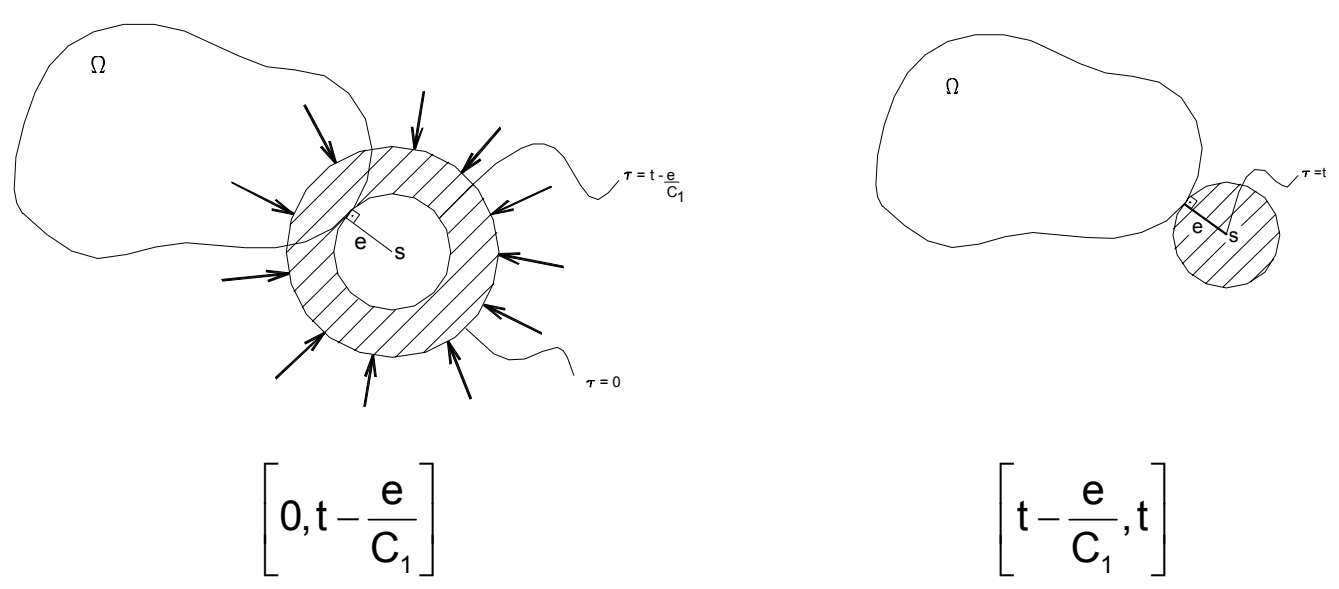

Figura 6.4 - Convolução temporal para pontos fonte externos ao corpo - divisão

O erro encontrado nas referências clássicas do MEC (KARABALIS \& BESKOS, 1984) (ou eq.(6.39)) é a consideração da região do intervalo de tempo $\left[\mathrm{t}-\frac{\mathrm{e}}{\mathrm{C}_{1}}, \mathrm{t}\right]$; anulando-se as integrais neste intervalo, tem-se:

$$
\int_{\Gamma} \int_{0}^{\left(t-e / c_{1}\right)} p_{k j}^{*}(Q, t-\tau ; s / f) u_{j}(\tau) d \tau d \Gamma=\int_{\Gamma} \int_{0}^{\left(t-e / c_{1}\right)} u_{k j}^{*}(Q, t-\tau ; s / f) p_{j}(\tau) d \tau d \Gamma
$$

\section{Fazendo-se:}

$\mathrm{t}-\frac{\mathrm{e}}{\mathrm{C}_{1}}=\mathrm{t}^{\prime}$

e, conseqüentemente: 
$t=t^{\prime}+\frac{e}{C_{1}}$

Pode-se reescrever a eq.(6.42) como:

$$
\int_{\Gamma} \int_{0}^{t^{\prime}} p_{k j}^{*}\left(Q, t^{\prime}+\frac{e}{C_{1}}-\tau ; s / f\right) u_{j}(\tau) d \tau d \Gamma=\int_{\Gamma} \int_{0}^{t^{\prime}} u_{k j}^{*}\left(Q, t^{\prime}+\frac{e}{C_{1}}-\tau ; s / f\right) p_{j}(\tau) d \tau d \Gamma
$$

A eq.(6.43) pode ser entendida como uma translação temporal do instante final da análise.

Finalmente, para pontos fonte exteriores, a eq.(6.38) correspondente é escrita como:

$$
\begin{aligned}
& \int_{0}^{t^{\prime}} \int_{\Gamma} p_{k j}^{*}\left(Q, t^{\prime}+\frac{e}{C_{1}}-\tau ; s / f\right) u_{j}(\tau) d \Gamma d \tau+\int_{\Omega} \rho u_{j}(0) \dot{u}_{k j}^{*}\left(q, t^{\prime}+\frac{e}{C_{1}} ; s / f\right) d \Omega \\
& =\int_{0}^{t} \int_{\Gamma} u_{k j}^{*}\left(Q, t^{\prime}+\frac{e}{C_{1}}-\tau ; s / f\right) p_{j}(\tau) d \Gamma d \tau+\int_{0}^{t} \int_{\Omega} u_{k j}^{*}\left(q, t^{\prime}+\frac{e}{C_{1}}-\tau ; s / f\right) b_{j}(\tau) d \Omega d \tau+ \\
& +\int_{\Omega} \rho \dot{u}_{j}(0) u_{k j}^{*}\left(q, t^{\prime}+\frac{e}{C_{1}} ; s / f\right) d \Omega
\end{aligned}
$$

\subsection{SOLUÇÃO FUNDAMENTAL SUAVE}

A partir deste ponto, faz-se necessário que se assuma um comportamento particular para o carregamento fundamental. Optou-se pela utilização de solução fundamental suave, ou seja, resultante da aplicação do carregamento fundamental distribuído ao longo de um intervalo de tempo. Tal solução fundamental apresenta comportamento numérico bastante satisfatório. Para maiores detalhes, ver Coda (2000).

Antes de se aplicar a solução fundamental suave, é necessário que se apresente uma propriedade que é inerente a todo estado de Stokes ou Lamb. Tal propriedade afirma que um estado de Stokes para uma excitação que se inicia no tempo 0 avaliado no tempo 't- $\tau$ ' é equivalente a outro gerado por uma 
excitação que se inicia no tempo ' $\tau$ ' avaliado no tempo 't'. Sendo assim, podese escrever:

$$
\begin{aligned}
& u_{k j}^{*}(Q, t-\tau ; s, 0)=u_{k j}^{*}(Q, t ; s, \tau) \\
& p_{k j}^{*}(Q, t-\tau ; s, 0)=p_{k j}^{*}(Q, t ; s, \tau)
\end{aligned}
$$

Tomando-se a eq.(6.38) - ou (6.45), quando se tratar de pontos fonte externos ao corpo - modificando o argumento $(\mathrm{q}, \tau ; \mathrm{s} / \mathrm{f})$ para $(\mathrm{q}, \tau ; \mathrm{s}, 0)$, uma vez que a função de carga, eq.(6.33), será considerada a partir do instante inicial $\tau$ $=0 ;$ e utilizando-se a propriedade da solução fundamental de Stokes, representada nas eq.(6.46) e (6.47), a eq.(6.38) pode ser escrita como:

$$
\begin{aligned}
& C_{k j} \int_{0}^{t} u_{j}(s, \tau) f(t-\tau) d \tau=\int_{0}^{t} \int_{\Gamma} u_{k j}^{*}(Q, t ; s, \tau) p_{j}(Q, \tau) d \Gamma d \tau+ \\
& -\int_{0}^{t} \int_{\Gamma} p_{k j}^{*}(Q, t ; s, \tau) u_{j}(Q, \tau) d \Gamma d \tau+\int_{0}^{t} \int_{\Omega} b_{j}(q, \tau) u_{k j}^{*}(q, t ; s, \tau) d \Omega d \tau+ \\
& +\int_{\Omega} \rho u_{k j}^{*}(q, t ; s, 0) \dot{u}_{j}(q, 0) d \Omega-\int_{\Omega} \rho \dot{u}_{k j}^{*}(q, t ; s, 0) u_{j}(q, 0) d \Omega
\end{aligned}
$$

A função mais simples para se satisfazer as condições da solução fundamental em desenvolvimento é:

$$
f(\tau)=\frac{H(\tau)-H\left(\tau-t_{d}\right)}{t_{p}}
$$

onde:

$t_{d} \quad$ é o tempo de duração do impulso resultante;

$H($ ) - é a função Heaveside;

$t_{p} \quad-$ é um valor arbitrário qualquer.

Substituindo-se a eq.(6.49) no primeiro termo da eq.(6.48), tem-se: 
$C_{k j} \int_{0}^{t} u_{j}(s, \tau) \frac{H(t-\tau)-H\left(t-\tau-t_{d}\right)}{t_{p}} d \tau=C_{k j} \int_{t-t_{d}}^{t} \frac{u_{j}(s, \tau) d \tau}{t_{p}}$

Associando-se a duração do carregamento a um número 'd' de intervalos de tempo da análise numérica e fazendo-se ' $t_{p}=\Delta t$ ', tem-se:

$\int_{\mathrm{t}_{\mathrm{nt}}-\mathrm{d} \Delta \mathrm{t}}^{\mathrm{t}_{\mathrm{n}}} \frac{\mathrm{u}_{\mathrm{j}}(\mathrm{s}, \tau)}{\Delta \mathrm{t}} \mathrm{d} \tau=\int_{\mathrm{t}_{\mathrm{nt}}-\mathrm{d} \Delta \mathrm{t}}^{\mathrm{t}_{\mathrm{n}}-\mathrm{d} \Delta \mathrm{t}+\Delta \mathrm{t}} \frac{\mathrm{u}_{\mathrm{j}}(\mathrm{s}, \tau)}{\Delta \mathrm{t}} \mathrm{d} \tau+\ldots+\int_{\mathrm{t}_{\mathrm{nt}}-\Delta \mathrm{t}}^{\mathrm{t}_{\mathrm{nt}}} \frac{\mathrm{u}_{\mathrm{j}}(\mathrm{s}, \tau)}{\Delta \mathrm{t}} \mathrm{d} \tau=\int_{\mathrm{t}_{\theta-1}}^{\mathrm{t}_{\theta}} \frac{\mathrm{u}_{\mathrm{j}}^{\theta}(\mathrm{s}, \tau)}{\Delta \mathrm{t}} \mathrm{d} \tau$

onde:

$\theta \quad$ - é o número do passo de tempo, e varia de 1 a nt;

nt - é o número total de passos de tempo;

$\mathrm{u}_{\mathrm{j}}^{\theta}(\mathrm{s}, \tau)$ - representa o deslocamento para cada ' $\theta$ '.

Assumindo-se que o impulso estará restrito a um único intervalo de tempo, a eq.(6.50) pode ser escrita como:

$\int_{0}^{t} \frac{\mathrm{u}_{\mathrm{j}}(\mathrm{s}, \tau)[\mathrm{H}(\mathrm{t}-\tau)-\mathrm{H}(\mathrm{t}-\tau-\Delta \mathrm{t})]}{\Delta \mathrm{t}} \mathrm{d} \tau=\int_{\mathrm{t}-\Delta \mathrm{t}}^{\mathrm{t}} \frac{\mathrm{u}_{\mathrm{j}}(\mathrm{s}, \tau) \mathrm{d} \tau}{\Delta \mathrm{t}}$

Assim, a variação temporal do carregamento fundamental fica:

$f(\tau)=\frac{H(\tau)-H(\tau-\Delta t)}{\Delta t}$

Substituindo-se a eq.(6.52) na eq.(6.48), tem-se:

$$
\begin{aligned}
& C_{k j} \int_{t_{n t}-\Delta t}^{t_{n t}} \frac{u_{j}^{(n t)}(s, \tau)}{\Delta t} d \tau=\int_{0}^{t} \int_{\Gamma} u_{k j}^{R}(Q, t ; s, \tau) p_{j}(Q, \tau) d \Gamma d \tau+ \\
& -\int_{0}^{t} \int_{\Gamma} p_{k j}^{R}(Q, t ; s, \tau) u_{j}(Q, \tau) d \Gamma d \tau+\int_{0}^{t} \int_{\Omega} b_{j}(q, \tau) u_{k j}^{R}(q, t ; s, \tau) d \Omega d \tau+ \\
& +\int_{\Omega} \rho u_{k j}^{R}(q, t ; s, 0) \dot{u}_{j}(q, 0) d \Omega-\int_{\Omega} \rho \dot{u}_{k j}^{R}(q, t ; s, 0) u_{j}(q, 0) d \Omega
\end{aligned}
$$


onde o índice 'R' é referente a ressalto.

Observa-se que as duas últimas integrais da eq.(6.54) são os termos referentes às condições iniciais do problema, respectivamente, velocidade e deslocamentos iniciais A partir deste ponto, tais condições iniciais serão consideradas nulas. Bem como o termo referente às forças volumétricas (terceiro termo do lado direito da eq.(6.54)). Assim, a eq.(6.54) pode ser reescrita como:

$$
C_{k j} \int_{t_{n t}-\Delta t}^{t_{n t}} \frac{u_{j}^{(n t)}(s, \tau)}{\Delta t} d \tau=\int_{0}^{t} \int_{\Gamma} u_{k j}^{R}(Q, t ; s, \tau) p_{j}(Q, \tau) d \Gamma d \tau-\int_{0}^{t} \int_{\Gamma} p_{k j}^{R}(Q, t ; s, \tau) u_{j}(Q, \tau) d \Gamma d \tau
$$

Para a substituição da função de carga ressalto, eq.(6.53), nas expressões dos valores fundamentais de Stokes, eq.(6.36) e (6.37), optou-se por se decompor a referida função em duas partes:

$$
f(\tau)=\frac{H(\tau)}{\Delta t}-\frac{H(\tau-\Delta t)}{\Delta t}
$$

Desta forma, pode-se escrever:

$$
\begin{aligned}
& u_{k j}^{R}(q, \tau ; s, 0)=u_{k j}^{H}(q, \tau ; s, 0)-u_{k j}^{H}(q, \tau-\Delta t ; s, 0) \\
& p_{k j}^{R}(q, \tau ; s, 0)=p_{k j}^{H}(q, \tau ; s, 0)-p_{k j}^{H}(q, \tau-\Delta t ; s, 0)
\end{aligned}
$$

onde o índice superior 'H' alude a 'Heaveside'.

Sendo assim, os valores fundamentais podem ser escritos como:

$$
\begin{aligned}
& u_{k j}^{H}=\frac{1}{4 \pi \rho \Delta t}\left\{B_{k j}\left[\left(\tau^{2}-\frac{r^{2}}{C_{1}^{2}}\right) H\left(\tau-\frac{r}{C_{1}}\right)-\left(\tau^{2}-\frac{r^{2}}{C_{2}^{2}}\right) H\left(\tau-\frac{r}{C_{2}}\right)\right]+\right. \\
& \left.+\mathbb{C}_{k j}\left[\frac{1}{C_{1}^{2}} H\left(\tau-\frac{r}{C_{1}}\right)-\frac{1}{C_{2}^{2}} H\left(\tau-\frac{r}{C_{2}}\right)\right]+D_{k j} H\left(\tau-\frac{r}{C_{1}}\right)\right\}
\end{aligned}
$$


e

$$
\begin{aligned}
& \mathrm{p}_{\mathrm{kj}}^{\mathrm{H}}(\mathrm{Q}, \tau ; \mathrm{s}, 0)=\frac{\mathrm{n}_{\mathrm{i}}}{4 \pi \Delta t}\left\{\mathrm{E}_{\mathrm{kji}}\left[\left(\tau^{2}-\frac{\mathrm{r}^{2}}{\mathrm{C}_{1}^{2}}\right) \mathrm{H}\left(\tau-\frac{\mathrm{r}}{\mathrm{C}_{1}}\right)-\left(\tau^{2}-\frac{\mathrm{r}^{2}}{\mathrm{C}_{2}^{2}}\right) \mathrm{H}\left(\tau-\frac{\mathrm{r}}{\mathrm{C}_{2}}\right)\right]+\right. \\
& +\mathrm{F}_{\mathrm{kji}}\left[\mathrm{H}\left(\tau-\frac{\mathrm{r}}{\mathrm{C}_{1}}\right)-\frac{\mathrm{C}_{2}^{2}}{\mathrm{C}_{1}^{2}} \mathrm{H}\left(\tau-\frac{\mathrm{r}}{\mathrm{C}_{2}}\right)\right]+\mathrm{G}_{\mathrm{kji}}\left[\delta\left(\tau-\frac{r}{\mathrm{C}_{2}}\right)-\frac{\mathrm{C}_{2}^{3}}{\mathrm{C}_{1}^{3}} \delta\left(\tau-\frac{r}{\mathrm{C}_{1}}\right)\right]+ \\
& \left.+\mathrm{H}_{\mathrm{kji}}\left[H\left(\tau-\frac{r}{\mathrm{C}_{1}}\right)-\frac{r}{\mathrm{C}_{1}} \delta\left(\tau-\frac{r}{\mathrm{C}_{1}}\right)\right]-\mathrm{I}_{\mathrm{kji}}\left[H\left(\tau-\frac{r}{\mathrm{C}_{2}}\right)+\frac{r}{\mathrm{C}_{2}} \delta\left(\tau-\frac{r}{\mathrm{C}_{2}}\right)\right]\right\}
\end{aligned}
$$

onde:

$$
B_{k j}=\frac{3 r{ }_{k} r_{, j}-\delta_{k j}}{2 r^{3}}
$$

$\mathbb{C}_{k j}=\frac{r_{{ }_{k}} r_{{ }_{j}}}{r}$

$D_{k j}=\frac{\delta_{k j}}{C_{2}^{2} r}$

$E_{k j i}=3 C_{2}^{2} \frac{\left(\delta_{i k} r_{, j}+\delta_{i j} r_{{ }_{k}}+\delta_{j k} r_{, j}-5 r_{, j} r_{{ }_{k}} r_{, j}\right)}{r^{4}}$

$F_{k j i}=\frac{2}{r^{2}}\left[6 r_{, i} r_{, j} r_{, k}-\delta_{i k} r_{, j}+\delta_{i j} r_{, k}+\delta_{k j} r_{j i}\right]$

$G_{k j i}=\frac{2 r_{, j} r_{, j} r_{, k}}{r C_{2}}$

$\mathrm{H}_{\mathrm{kji}}=\delta_{\mathrm{ij}} \mathrm{r}_{\mathrm{k}}\left(1-2 \frac{\mathrm{C}_{2}^{2}}{\mathrm{C}_{1}^{2}}\right)$

$I_{k j i}=\frac{\delta_{k j} r_{{ }_{i j}}+\delta_{i k} r_{, j}}{r^{2}}$

De posse da eq.(6.55) e das expressões dos valores fundamentais envolvidos, pode-se aplicar as aproximações temporais para o TDBEM. Sendo assim - com uma certa semelhança com o que se faz nas aproximações espaciais para o MMBEM - substituindo-se as aproximações temporais na 
equação integral eq.(6.55), chega-se à eq.(6.69) abaixo, que representa o processo convolutivo aproximado para o TDBEM:

$\mathrm{C}_{\mathrm{kj}} \int_{\mathrm{t}_{\mathrm{nt}}-\Delta \mathrm{t}}^{\mathrm{t}_{\mathrm{nt}}} \Phi_{(\mathrm{nt})}^{\beta}(\tau, \Delta \mathrm{t}) / \Delta \mathrm{td} \tau \mathrm{U}_{\mathrm{j} \beta \mathrm{nt}}(\mathrm{s})+\int_{\Gamma_{(e)}} \int_{(\theta-1) \Delta \mathrm{t}}^{\theta \Delta \mathrm{t}} \mathrm{p}_{\mathrm{kj}}^{\mathrm{R}}\left(\mathrm{Q}^{\mathrm{e}}, \mathrm{t}_{\mathrm{nt}}, \mathrm{s}, \tau\right) \phi_{\ell}^{\mathrm{e}}(\mathrm{Q}) \Phi_{\theta}^{\beta}(\tau) \mathrm{d} \tau \mathrm{d} \Gamma \mathrm{U}_{\mathrm{j} \beta \theta}^{e \mathrm{e}}$

$=\int_{\Gamma_{(e)}} \int_{(\theta-1) \Delta t}^{\theta \Delta t} u_{k j}^{R}\left(Q^{e}, t_{n t} ; s, \tau\right) \phi_{\ell}^{e}(Q) \Phi_{\theta}^{\beta}(\tau) d \tau d \Gamma P_{j \beta \theta}^{\ell e}$

onde o índices 'e' é referente a elementos de contorno.

\subsection{CONVOLUÇÃO TEMPORAL}

Como a função de carga que se está utilizando é a de ressalto, a colocação temporal é distribuída ao longo do intervalo de tempo atual. Sendo assim, o primeiro termo da equação integral aproximada, eq.(6.69), para aproximação constante - que foi a aproximação temporal utilizada - pode ser escrito como:

$$
\int_{t_{n t}-\Delta t}^{t_{n t}} \phi_{(n t)}^{1}(\tau, \Delta t) / \Delta t d \tau U_{j n t}(s)=U_{j n t}(s)
$$

Desenvolvendo-se de maneira sistemática as convoluções temporais presentes na eq.(6.69), considera-se o instante em análise 't' e o intervalo de tempo $\left[t_{\theta-1}, t_{\theta}\right]$ sobre o qual se pretende realizar as integrais em questão. Ver figura 6.5 . 


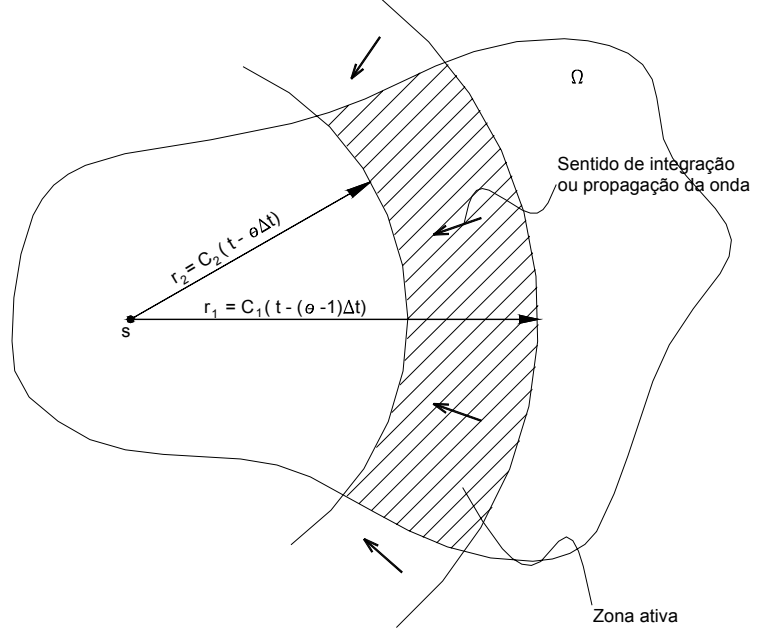

Figura 6.5 - Integração temporal para o intervalo $\left[t_{\theta-1}, t_{\theta}\right]$ para tempo de colocação $t>t_{\theta}$

Observa-se que apenas os elementos de contorno que estão contidos na zona ativa, totalmente ou parcialmente, é que serão integrados espacialmente.

Assumindo-se TA $=\left(\mathrm{t}-\mathrm{r} / \mathrm{C}_{\alpha}\right)-\operatorname{com} \alpha=1,2$, dependendo da velocidade de onda considerada - os valores temporais convoluídos são dados por:

$$
\begin{aligned}
& \mathrm{K} 1_{\alpha}^{\theta}=\int_{\mathrm{t}_{\theta-1}}^{\mathrm{t}_{\theta}}\left[(\mathrm{t}-\tau)^{2}-\frac{\mathrm{r}^{2}}{\mathrm{C}_{\alpha}^{2}}\right] \mathrm{H}\left(\mathrm{t}-\tau-\frac{\mathrm{r}}{\mathrm{C}_{\alpha}}\right) \mathrm{d} \tau \\
& = \begin{cases}\left(\mathrm{t}^{2}-\frac{\mathrm{r}^{2}}{\mathrm{C}_{\alpha}^{2}}\right) \Delta \mathrm{t}-\mathrm{t}\left(\mathrm{t}_{\theta}^{2}-\mathrm{t}_{\theta-1}^{2}\right)+\left(\mathrm{t}_{\theta}^{3}-\mathrm{t}_{\theta-1}^{3}\right) / 3 & \text { para } \mathrm{t}-\mathrm{t}_{\theta}>\frac{\mathrm{r}}{\mathrm{C}_{\alpha}} \\
\left(\mathrm{t}^{2}-\frac{\mathrm{r}^{2}}{\mathrm{C}_{\alpha}^{2}}\right)\left(T A-\mathrm{t}_{\theta-1}\right)-t\left(T A^{2}-\mathrm{t}_{\theta-1}^{2}\right)+\left(T \mathrm{~A}^{3}-\mathrm{t}_{\theta-1}^{3}\right) / 3 & \text { para } \mathrm{t}-\mathrm{t}_{\theta}>\frac{r}{\mathrm{C}_{\alpha}}>\mathrm{t}-\mathrm{t}_{\theta} \\
0 & \text { para } \frac{r}{\mathrm{C}_{\alpha}}>\mathrm{t}-\mathrm{t}_{\theta-1}\end{cases}
\end{aligned}
$$

$$
\mathrm{K} 2_{\alpha}^{\theta}=\int_{t_{\theta-1}}^{t_{\theta}} H\left(t-\tau-\frac{r}{C_{\alpha}}\right) d \tau=\left\{\begin{array}{llc}
\Delta t & \text { para } & t-t_{\theta}>\frac{r}{C_{\alpha}} \\
T A-t_{\theta-1} & \text { para } t-t_{\theta-1}>\frac{r}{C_{\alpha}}>t-t_{\theta} \\
0 & \text { para } & \frac{r}{C_{\alpha}}>t-t_{\theta-1}
\end{array}\right.
$$




$$
\mathrm{K}^{\theta}{ }_{\alpha}^{\theta}=\int_{\mathrm{t}_{\theta-1}}^{\mathrm{t}_{\theta}} \delta\left(\mathrm{t}-\tau-\frac{\mathrm{r}}{\mathrm{C}_{\alpha}}\right) \mathrm{d} \tau= \begin{cases}0 & \text { para } \mathrm{t}-\mathrm{t}_{\theta}>\frac{\mathrm{r}}{\mathrm{C}_{\alpha}} \\ 1 & \text { para } \mathrm{t}-\mathrm{t}_{\theta-1}>\frac{\mathrm{r}}{\mathrm{C}_{\alpha}}>\mathrm{t}-\mathrm{t}_{\theta} \\ 0 & \text { para } \frac{\mathrm{r}}{\mathrm{C}_{\alpha}}>\mathrm{t}-\mathrm{t}_{\theta-1}\end{cases}
$$

A transformação dos coeficientes ' $\mathrm{K} \gamma_{\alpha}^{\theta}$ ' (com $\gamma=1,2,3$ e $\left.\alpha=1,2\right)$ da solução fundamental Heaveside para coeficientes da função ressalto, ' $\overline{\mathrm{K}} \gamma_{\alpha}^{\theta}$, , é feita da seguinte forma:

$$
\overline{\mathrm{K}} \gamma_{\alpha}^{\theta}=\mathrm{K} \gamma_{\alpha}^{\theta}-\mathrm{K} \gamma_{\alpha}^{(\theta-1)}
$$

Substituindo-se os coeficientes transformados da eq.(6.74), na eq.(6.69), tem-se:

$$
\mathrm{C}_{\mathrm{kj}} \mathrm{U}_{\mathrm{jnt}}(\mathrm{s})+\int_{\Gamma_{(\mathrm{e})}} \overline{\mathrm{p}}_{\mathrm{kj}}^{\mathrm{R} \theta}\left(\mathrm{Q}^{(\mathrm{e})}, \mathrm{s}\right) \phi_{\ell}^{\mathrm{e}}(\mathrm{Q}) \mathrm{d} \Gamma \mathrm{U}_{\mathrm{j} \theta}^{\mathrm{ee}}=\int_{\Gamma_{(\mathrm{e})}} \overline{\mathrm{u}}_{\mathrm{kj}}^{\mathrm{R} \theta}\left(\mathrm{Q}^{(\mathrm{e})}, \mathrm{s}\right) \phi_{\ell}^{\mathrm{e}}(\mathrm{Q}) \mathrm{d} \Gamma \mathrm{P}_{\mathrm{j} \theta}^{\ell \mathrm{e}}
$$

onde ' $\theta$ ' varia de 1 a 'nt' e o símbolo $\overline{(\cdot)}$ indica valor da solução fundamental convoluído, explícitos nas eq.(6.76) e (6.77) abaixo.

$$
\overline{\mathrm{u}}_{\mathrm{kj}}^{\mathrm{R} \theta}=\frac{1}{4 \pi \rho \Delta \mathrm{t}}\left\{\mathrm{B}_{\mathrm{kj}}\left[\overline{\mathrm{K}} 1_{1}^{\theta}-\overline{\mathrm{K}} 1_{2}^{\theta}\right]+\mathbb{C}_{\mathrm{kj}}\left[\frac{1}{\mathrm{C}_{1}^{2}} \overline{\mathrm{K}} 2_{1}^{\theta}-\frac{1}{\mathrm{C}_{2}^{2}} \overline{\mathrm{K}} 2_{2}^{\theta}\right]+\mathrm{D}_{\mathrm{kj}} \overline{\mathrm{K}} 2_{1}^{\theta}\right\}
$$

e

$$
\begin{aligned}
& \overline{\mathrm{p}}_{\mathrm{kj}}^{\mathrm{R} \theta}=\frac{\mathrm{n}_{\mathrm{i}}}{4 \pi \Delta \mathrm{t}}\left\{\mathrm{E}_{\mathrm{kji}}\left[\overline{\mathrm{K}} 1_{1}^{\theta}-\overline{\mathrm{K}} 1_{2}^{\theta}\right]+\mathrm{F}_{\mathrm{kji}}\left[\overline{\mathrm{K}} 2_{1}^{\theta}-\frac{\mathrm{C}_{2}^{2}}{\mathrm{C}_{1}^{2}} \overline{\mathrm{K}} 2_{2}^{\theta}\right]+\right. \\
& \left.+\mathrm{G}_{\mathrm{kji}}\left[\overline{\mathrm{K}} 3_{2}^{\theta}-\frac{\mathrm{C}_{2}^{3}}{\mathrm{C}_{1}^{3}} \overline{\mathrm{K}} 3_{1}^{\theta}\right]+\mathrm{H}_{\mathrm{kji}}\left[\overline{\mathrm{K}} 2_{1}^{\theta}-\frac{\mathrm{r}}{\mathrm{C}_{1}} \overline{\mathrm{K}} 3_{1}^{\theta}\right]-\mathrm{I}_{\mathrm{kjl}}\left[\overline{\mathrm{K}} 2_{2}^{\theta}+\frac{\mathrm{r}}{\mathrm{C}_{2}} \overline{\mathrm{K}} 3_{2}^{\theta}\right]\right\}
\end{aligned}
$$




\subsection{INTEGRAÇÃO ESPACIAL}

Pode-se dizer que a eq.(6.75) está pronta para ser integrada espacialmente em seus elementos de contorno, que, bem como suas funções de forma, são os mesmos utilizados para o MMBEM, ou seja: elementos triangulares planos com aproximação linear. Sendo assim, as integrações espaciais presentes na eq.(6.75) são feitas de maneira bastante semelhante ao que foi descrito para o MMBEM (capítulo 5).

Para o caso das integrações singulares, são necessários alguns esclarecimentos. Observando-se as figuras 6.6 e 6.7 abaixo, percebe-se que as integrais singulares só ocorrem no primeiro passo de tempo e podem ser de dois tipos. No primeiro, figura 6.6, o passo de tempo é suficientemente grande para garantir que não ocorra descontinuidade no interior do elemento. Neste caso, o processo de integração singular é suficiente. No segundo tipo, figura 6.7, a descontinuidade no elemento pode ser resolvida de duas formas: subtraindo-se e somando-se a solução fundamental correspondente da estática em todo o elemento singular, obtendo-se uma integral regular (dinâmica menos estática) e outra estática singular e somando-se o resultado; e a adoção de pontos fonte exteriores. Todos esses casos são possíveis no programa desenvolvido.

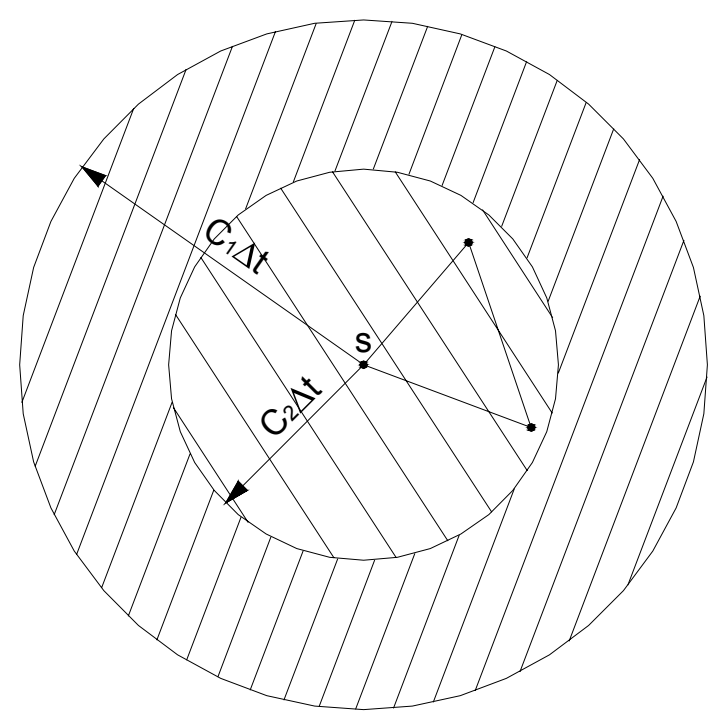

Figura 6.6 - Elemento singular totalmente coberto por zona ativa 


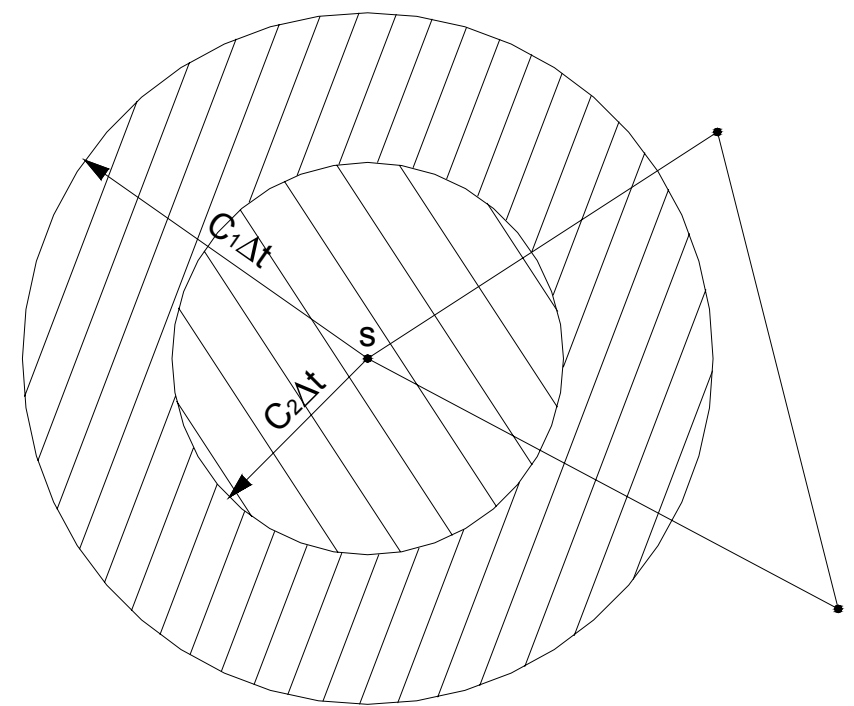

Figura 6.7 - Elemento singular parcialmente coberto pelas zonas ativas

Sendo assim, as integrais presentes na eq.(6.75) podem ser resolvidas:

$$
\mathrm{C}_{\mathrm{ki}} \mathrm{U}_{\mathrm{jn} \mathrm{t}}(\mathrm{s})+\mathrm{h}_{\mathrm{kj}}^{\theta / e} \mathrm{U}_{\mathrm{j} \theta}^{\prime e}=\mathrm{g}_{\mathrm{kj}}^{\theta / e} P_{j \theta}^{\prime e}
$$

onde ' $\theta$ ' varia de 1 até o número de passos de tempo necessários para se atingir o instante de análise; ' $\ell$ ' varia de 1 até o número de nós do elemento ' $e$ '; e 'e' varia de 1 até o número de elementos de contorno.

Adotando-se um número de pontos fonte igual ao número de nós do problema e aplicando-se a eq.(6.78) para cada um deles, para um dado instante da análise, pode-se escrever:

$\mathrm{H}_{\theta}^{\mathrm{n}_{t}} \mathrm{U}_{\theta}=\mathrm{G}_{\theta}^{\mathrm{n}_{\mathrm{t}}} \mathrm{P}_{\theta}$

onde o índice ' $n_{t}$ ' representa o último intervalo de tempo da convolução.

Para um melhor entendimento do processo convolutivo da eq.(6.79), admita-se $n_{t}=1$, onde ' $\theta$ ' só pode ser igual a 1 :

$$
\mathrm{H}_{1}^{1} \mathrm{U}_{1}=\mathrm{G}_{1}^{1} \mathrm{P}_{1}
$$


Após a imposição das condições de contorno do problema, a eq.(6.80) pode ser facilmente resolvida, onde as incógnitas de $U_{1}$ e $P_{1}$ são determinadas e serão utilizadas para o próximo valor de $n_{t}$. Assim, para $n_{t}=2, \theta$ varia de 1 a 2:

$\hat{\mathrm{H}}_{2}^{2} \mathrm{U}_{2}+\mathrm{H}_{1}^{2} \mathrm{U}_{1}=\hat{\mathrm{G}}_{2}^{2} \mathrm{P}_{2}+\mathrm{G}_{1}^{2} \mathrm{P}_{1}$

O índice $(\hat{*})$ que aparece na eq.(6.81) significa que os termos que são escritos com este símbolo são conhecidos de $n_{t}$ anterior; isto, graças à propriedade inerente ao estado de Stokes, expressa nas eq.(6.46) e (6.47), que, para o caso em questão, pode ser escrito como:

$$
\begin{aligned}
& u_{k j}^{*}\left(q, t_{n t} ; s, \tau_{\theta}\right)=u_{k j}^{*}\left(q, t_{n t}+\Delta t ; s, \tau_{\theta+1}\right) \\
& p_{k j}^{*}\left(q, t_{n t} ; s, \tau_{\theta}\right)=p_{k j}^{*}\left(q, t_{n t}+\Delta t ; s, \tau_{\theta+1}\right)
\end{aligned}
$$

Sendo assim, pode-se escrever:

$$
\begin{aligned}
& \hat{\mathrm{H}}_{2}^{2}=\mathrm{H}_{1}^{1} \\
& \hat{\mathrm{G}}_{2}^{2}=\mathrm{G}_{1}^{1}
\end{aligned}
$$

Generalizando:

$$
\begin{aligned}
& \hat{H}_{\theta}^{n_{t}}=H_{\theta-1}^{n_{t}-1} \\
& \hat{G}_{\theta}^{n_{t}}=G_{\theta-1}^{n_{t}-1}
\end{aligned}
$$

Assim, as únicas matrizes que precisam ser calculadas, para cada passo de tempo, são aquelas cujo valor de ' $\theta$ ' é igual à 1. As demais já foram calculadas para o ' $n_{t}$ ' anterior. Então, a eq.(6.81) pode ser escrita como:

$\mathrm{H}_{1}^{1} \mathrm{U}_{2}+\mathrm{H}_{1}^{2} \mathrm{U}_{1}=\mathrm{G}_{1}^{1} \mathrm{P}_{2}+\mathrm{G}_{1}^{2} \mathrm{P}_{1}$ 
ou

$\mathrm{H}_{1}^{1} \mathrm{U}_{2}=\mathrm{G}_{1}^{1} \mathrm{P}_{2}+\mathrm{F}^{1}$

onde

$\mathrm{F}^{1}=\mathrm{G}_{1}^{2} \mathrm{P}_{1}-\mathrm{H}_{1}^{2} \mathrm{U}_{1}$

Genericamente, a eq.(6.89) pode ser escrita como:

$H_{1}^{1} U_{n_{t}}=G_{1}^{1} P_{n_{t}}+F^{n_{t}}$

onde $\mathrm{F}^{\mathrm{n}_{\mathrm{t}}}$ só apresenta valores conhecidos.

Observa-se que, se as condições de contorno não mudarem durante o processo, a inversão da matriz associada à solução do sistema de equações é feita apenas uma vez, reduzindo-se em muito o custo computacional. Observase também, que à medida que $\mathrm{n}_{\mathrm{t}}$ cresce, a área inativa do problema também cresce. Assim, existe um valor de ' $n_{t}$ ' relacionado à maior distância entre dois pontos da discretização, a partir do qual não existirão mais elementos de contorno ativos, conseqüentemente, não haverá mais a necessidade de novas integrações e armazenagem de novas matrizes. O citado valor pode ser calculado por:

$$
\mathrm{n}_{\mathrm{t}}^{0}=\frac{\mathrm{d}_{\max }}{\mathrm{C}_{2} \Delta \mathrm{t}}+1
$$

onde $d_{\max }$ é a maior distância entre dois pontos da discretização. 


\subsection{EXEMPLO}

O único exemplo que o autor gostaria de apresentar a respeito do TDBEM neste capítulo, é aquele da viga submetida à ação de carga súbita de tração - exemplo difícil para essa técnica -, de valor constante com o tempo, exemplo 4.1, onde o MMBEM foi utilizado nas análises realizadas. Outros exemplos sobre o TDBEM, desta vez aplicado à análise de meios infinitos e semi-infinitos, podem ser encontrados no capítulo 10.

A figura 6.8 apresenta os deslocamentos longitudinais do nó localizado no centro da face carregada do sólido, ao longo do tempo de análise. Foi utilizada uma discretização do tipo $2210^{1}$ para todos os casos. Na figura 6.8, além do número de passos de tempo (pt) em que foi dividido o tempo total da análise, variou-se a distância relativa entre o ponto fonte e o ponto geométrico (rd); este parâmetro indica o quanto o ponto fonte foi colocado para fora do elemento, em função do comprimento do maior lado $\left(L_{\text {máx }}\right)$ do elemento a que pertence o nó; assim, a distância absoluta (d) entre o ponto fonte e o ponto geométrico pode ser escrita como:

$\mathrm{d}=\mathrm{rd} \cdot \mathrm{L}_{\text {máx }}$

Observa-se que $r d=0$ significa que o processo de colocação de ponto fonte exterior ao corpo não foi utilizado, e que, por conseguinte, ocorrem integrações singulares no processo de montagem das matrizes $\mathrm{G}$ e $\mathrm{H}$.

${ }^{1}$ Ver capítulo 9. 


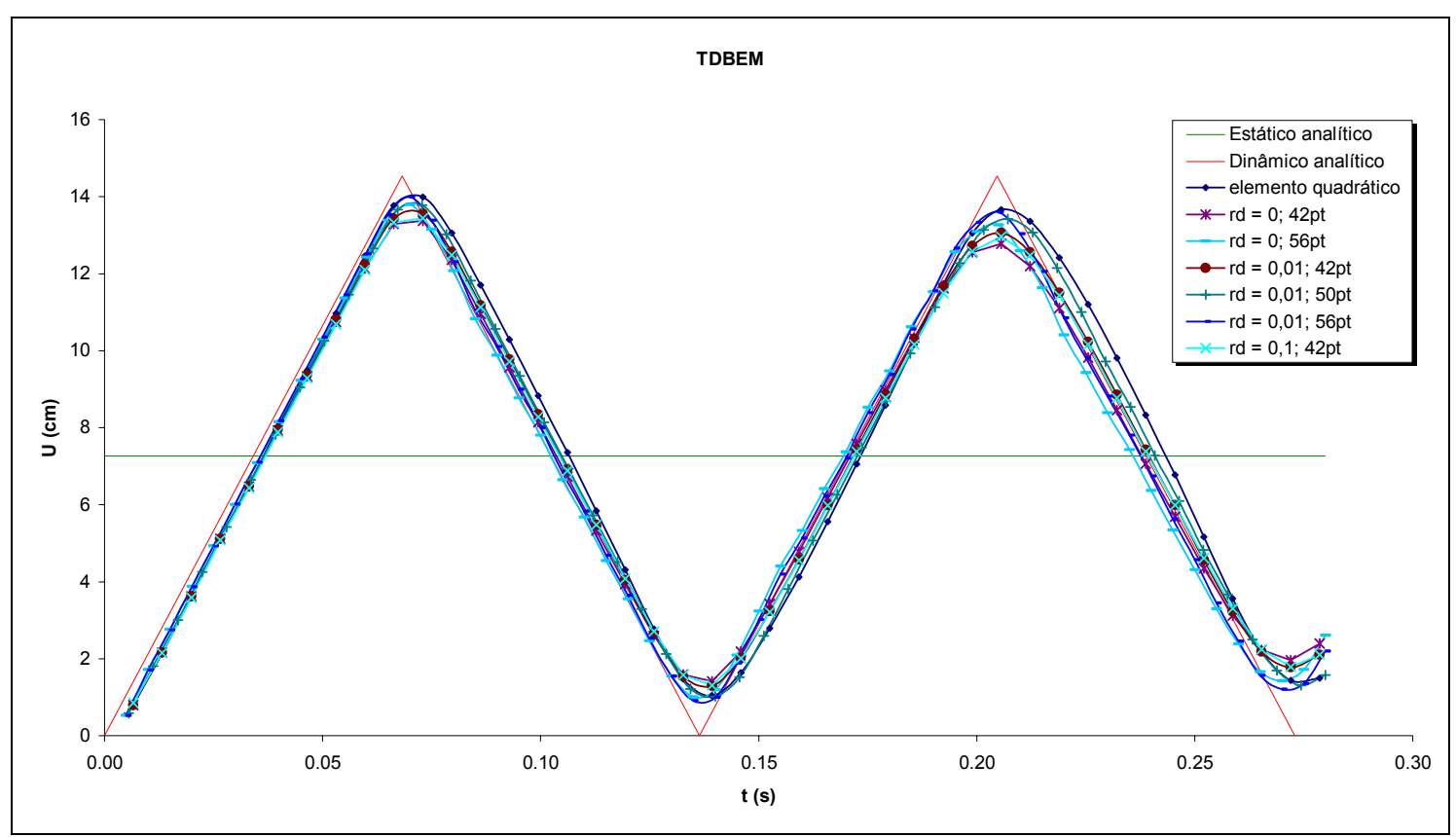

Figura 6.8 - Deslocamentos longitudinais

Analisando-se a figura 6.8 , observa-se primeiramente que todos os resultados foram bastante satisfatórios. Os valores para o elemento quadrático foram fornecidos por programa desenvolvido por Coda (1993). Para rd =0,01, com a diminuição do $\Delta t$ houve uma melhora nos resultados obtidos. Para número de passos de tempo igual a 42 e $r d \neq 0$, observa-se que 0 comportamento para $r d=0,01$ foi melhor que aquele para $r d=0,1$.

A figura 6.9 abaixo apresenta os resultados para as reações de apoio para o nó localizado no centro da face apoiada, para 42 passos de tempo, e elemento quadrático, linear com $\mathrm{rd}=0$ e linear com $\mathrm{rd}=0,1$.

Também se observa uma boa concordância dos resultados obtidos com o comportamento analítico. 


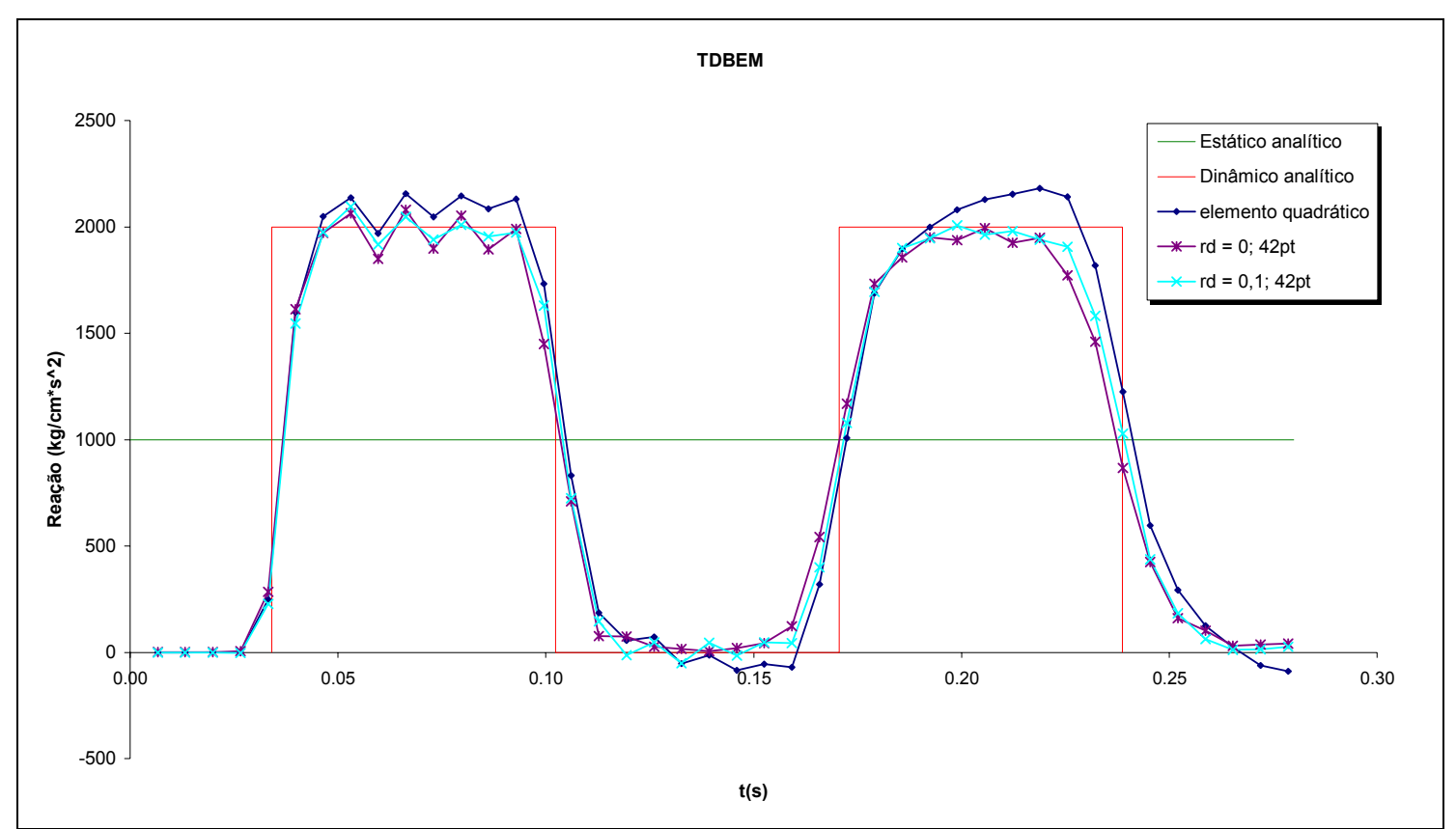

Figura 6.9 - Reações de apoio 


\section{O MÉTODO DOS ELEMENTOS FINITOS}

No presente capítulo, apresenta-se toda a formulação utilizada no trabalho para o Método dos Elementos Finitos (MEF). Esse método tão bem explorado é utilizado aqui para a discretização das superestruturas. Vigas e pilares são modelados por elementos finitos simples de barra, e as cascas, modeladas por elementos finitos simples de casca. Para o tratamento desses elementos estruturais, o Método dos Elementos de Contorno (MEC) não tem apresentado maiores vantagens com relação ao MEF. No final do capítulo, são apresentados 5 exemplos numéricos, onde foram analisados casos estáticos e dinâmicos.

\subsection{EQUACIONAMENTO BÁSICO}

Nesta seção será apresentada uma formulação para o MEF, totalmente voltada ao acoplamento MEC/MEF, feita com base no trabalho de Coda (2000) e Mesquita (2002).

Semelhantemente ao que se fez para o MEC, deseja-se resolver numericamente a equação diferencial abaixo, conhecida como equação de equilíbrio dinâmico do elemento infinitesimal:

$$
\sigma_{\mathrm{kj}, \mathrm{j}}+\mathrm{b}_{\mathrm{k}}=\rho \ddot{u}_{\mathrm{k}}+\mathrm{cu}_{\mathrm{k}} \quad \text { em } \Omega \quad \mathrm{k}, \mathrm{j}=1,2,3
$$

onde a simetria do tensor de tensões já foi aplicada e $\Omega$ representa o domínio do corpo. 
Consideraram-se condições iniciais e de contorno nulas, respectivamente representadas nas eq.(7.2) e (7.3) abaixo:
$\mathrm{u}_{\mathrm{k}}=0$
$\dot{\mathrm{u}}_{\mathrm{k}}=0$
Para $\mathrm{t}=0 \quad \mathrm{em} \Omega$
$\mathrm{u}_{\mathrm{k}}=\overline{\mathrm{u}}_{\mathrm{k}} \quad \forall \mathrm{t}$
em $\Gamma_{1}$
$k=1,2,3$
$\mathrm{p}_{\mathrm{k}}=\overline{\mathrm{p}}_{\mathrm{k}} \quad \forall \mathrm{t}$
em $\Gamma_{2}$
$\mathrm{k}=1,2,3$

onde o símbolo $\bullet$ significa valor prescrito e $\Gamma_{1}+\Gamma_{2}=\Gamma$ representa o contorno do corpo.

Multiplicando-se a eq.(7.1) por uma função ' $u_{k}^{\prime}$ ', representando um campo de deslocamentos qualquer para o corpo, tem-se:

$$
\sigma_{k j, j} u_{k}^{\prime}+b_{k} u_{k}^{\prime}=\rho \ddot{u}_{k} u_{k}^{\prime}+c \dot{u}_{k} u_{k}^{\prime} \quad \text { em } \Omega \quad k, j=1,2,3
$$

Integrando-se a eq.(7.4) no domínio $\Omega$ do corpo, a igualdade da mesma se mantém; assim:

$$
\int_{\Omega} \sigma_{k j, j} u_{k}^{\prime} d \Omega+\int_{\Omega} b_{k} u_{k}^{\prime} d \Omega=\int_{\Omega} \rho \ddot{u}_{k} u_{k}^{\prime} d \Omega+\int_{\Omega} c \dot{u}_{k} u_{k}^{\prime} d \Omega
$$

Integrando-se por partes (ou aplicando-se o teorema da divergência a) o primeiro termo da equação acima, tem-se:

$$
\int_{\Omega} \sigma_{k j, j} u_{k}^{\prime} d \Omega=\int_{\Gamma} \sigma_{k j} n_{j} u_{k}^{\prime} d \Gamma-\int_{\Omega} \sigma_{k j} u_{k, j}^{\prime} d \Omega=\int_{\Gamma} p_{k} u_{k}^{\prime} d \Gamma-\int_{\Omega} \sigma_{k j} \varepsilon_{k j}^{\prime} d \Omega
$$

onde as eq.(7.7) e (7.8) abaixo foram utilizadas no desenvolvimento.

$$
\begin{aligned}
& \sigma_{k j} n_{j}=p_{k} \\
& \sigma_{k j} u_{k, j}^{\prime}=\sigma_{k j} \varepsilon_{k j}^{\prime}
\end{aligned}
$$


Capítulo 7: O método dos elementos finitos

155

Substituindo a eq.(7.6) na eq.(7.5), tem-se:

$$
\int_{\Omega} \sigma_{k j} \varepsilon_{k j}^{\prime} d \Omega+\int_{\Omega} \rho \ddot{u}_{k} u_{k}^{\prime} d \Omega+\int_{\Omega} c \dot{u}_{k} u_{k}^{\prime} d \Omega=\int_{\Omega} b_{k} u_{k}^{\prime} d \Omega+\int_{\Gamma} p_{k} u_{k}^{\prime} d \Gamma
$$

Considerando-se na eq.(7.9) a atuação de forças concentradas (ver figura 7.1), pode-se escrever:

$$
\begin{aligned}
& \int_{\Omega} \sigma_{k j} \varepsilon_{k j}^{\prime} d \Omega+\int_{\Omega} \rho \ddot{u}_{k} u_{k}^{\prime} d \Omega+\int_{\Omega} c \dot{u}_{k} u_{k}^{\prime} d \Omega=\int_{\Omega} b_{k} u_{k}^{\prime} d \Omega+\int_{\Gamma} F_{k}^{d} \delta(s, q) u_{k}^{\prime}(q) d \Gamma+ \\
& +\int_{\Gamma} p_{k} u_{k}^{\prime} d \Gamma+\int_{\Gamma} F_{k}^{c} \delta(S, Q) u_{k}^{\prime}(Q) d \Gamma
\end{aligned}
$$

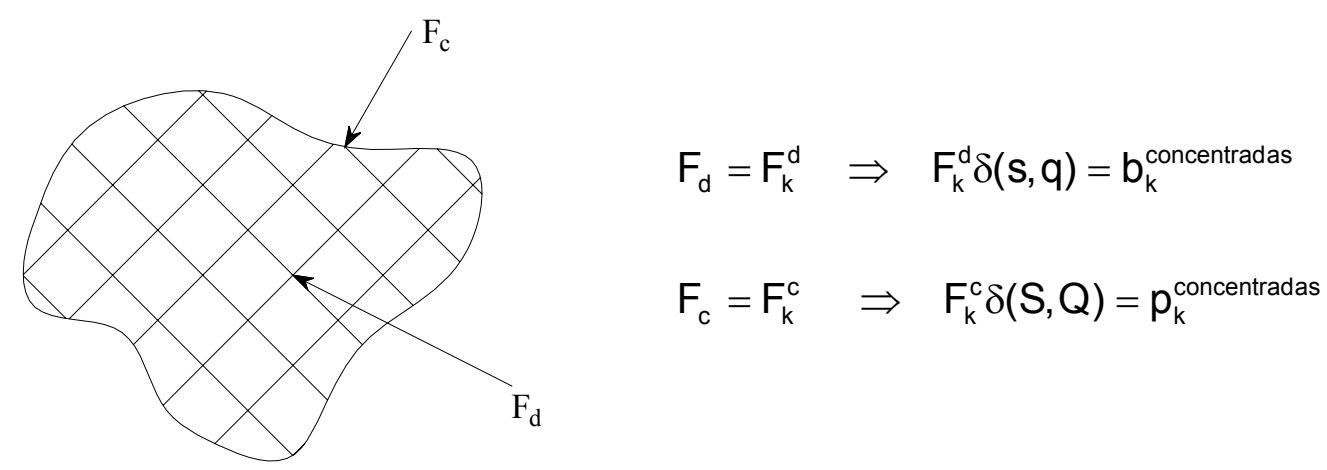

Figura 7.1 - Forças concentradas

Uma das propriedades da função Delta de Dirac é que:

$$
\int_{A} \delta(s, q) f(q) d A=f(s)
$$

onde A pode representar tanto domínio como contorno.

Aplicando a propriedade da função Delta de Dirac na eq.(7.10), pode-se escrever:

$$
\begin{aligned}
& \int_{\Omega} \sigma_{k j} \varepsilon_{k j}^{\prime} d \Omega+\int_{\Omega} \rho \ddot{u}_{k} u_{k}^{\prime} d \Omega+\int_{\Omega} c \dot{u}_{k} u_{k}^{\prime} d \Omega=\int_{\Omega} b_{k} u_{k}^{\prime} d \Omega+\int_{\Gamma} p_{k} u_{k}^{\prime} d \Gamma+ \\
& +F_{k}^{d} u_{k}^{\prime}(s)+F_{k}^{c} u_{k}^{\prime}(S)
\end{aligned}
$$


ou simplesmente:

$$
\int_{\Omega} \sigma_{k j} \varepsilon_{k j}^{\prime} d \Omega+\int_{\Omega} \rho \ddot{u}_{k} u_{k}^{\prime} d \Omega+\int_{\Omega} c \dot{u}_{k} u_{k}^{\prime} d \Omega=\int_{\Omega} b_{k} u_{k}^{\prime} d \Omega+\int_{\Gamma} p_{k} u_{k}^{\prime} d \Gamma+F_{j} u_{j}^{\prime}
$$

A eq.(7.13) acima é conhecida como Princípio dos Trabalhos Virtuais para Problemas Dinâmicos e é a base para a construção do MEF dinâmico. Aplicando-se sobre uma aproximação em elementos finitos tal equação, chegase à seguinte equação matricial:

$\mathrm{KU}+\mathrm{CU}+\mathrm{MÜ}=\mathrm{Bb}+\mathrm{GP}+\mathrm{IF}$

onde I é a matriz identidade, G é a matriz que transforma forças distribuídas em cargas equivalentes, B é a matriz que transforma cargas volumétricas em cargas nodais equivalentes, $M$ é a matriz de massa, $C$ é a matriz de amortecimento e $\mathrm{K}$ é a matriz de rigidez.

\subsection{ELEMENTO FINITO DE BARRA}

Como já foi comentado anteriormente, vigas e pilares serão modelados por elementos finitos de barra geral. A presente seção apresenta o sistema de coordenadas e as matrizes de rigidez $(K)$ e $G$ do elemento finito de barra utilizado na discretização dos citados elementos estruturais. Este elemento segue as hipóteses de Euler/Bernoulli.

O elemento finito de barra utilizado no presente trabalho possui 6 graus de liberdade (gdl) por extremidade, sendo eles: translações em $x_{1}\left(U_{1}\right), x_{2}\left(U_{2}\right)$ e $x_{3}\left(U_{3}\right)$, e rotações em torno de $x_{1}\left(\theta_{1}\right), x_{2}\left(\theta_{2}\right)$ e $x_{3}\left(\theta_{3}\right)$, nesta ordem. Figura 7.2 .

A partir das coordenadas locais dos elementos de barra, pode-se definir seus deslocamentos locais, bem como os esforços internos associados a eles. 


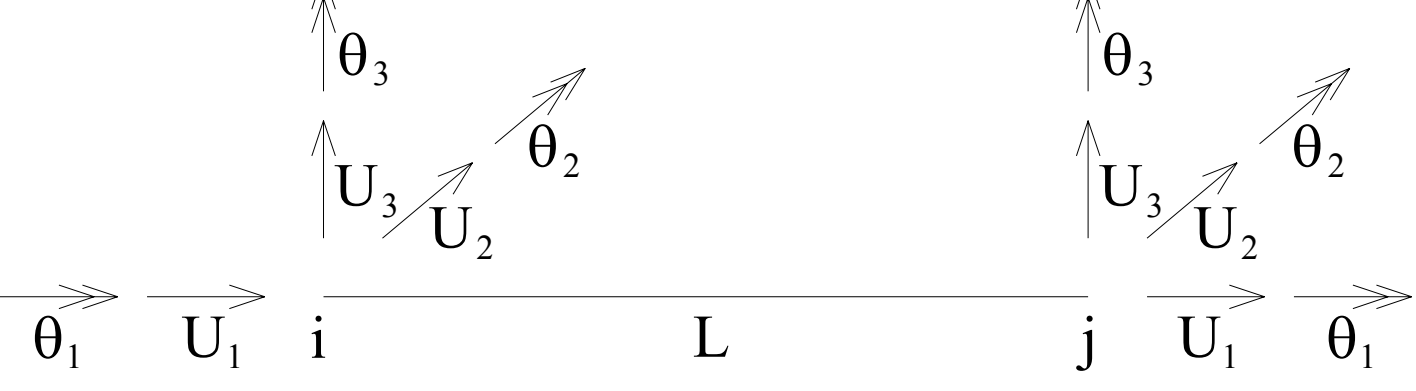

Figura 7.2 - Graus de liberdade do elemento finito de barra

A matriz de rigidez do elemento de barra adotado é bastante conhecida e pode ser escrita da seguinte forma:

$\left[\mathrm{K}_{\mathrm{b} \ell}\right]=\left[\begin{array}{cccccccccccc}\frac{\mathrm{EA}}{\mathrm{L}} & 0 & 0 & 0 & 0 & 0 & \frac{-\mathrm{EA}}{\mathrm{L}} & 0 & 0 & 0 & 0 & 0 \\ 0 & \frac{12 \mathrm{EI}_{3}}{\mathrm{~L}^{3}} & 0 & 0 & 0 & \frac{6 \mathrm{EI}_{3}}{\mathrm{~L}^{2}} & 0 & \frac{-12 \mathrm{EI}_{3}}{\mathrm{~L}^{3}} & 0 & 0 & 0 & \frac{6 \mathrm{EI}_{3}}{\mathrm{~L}^{2}} \\ 0 & 0 & \frac{12 \mathrm{EI}_{2}}{\mathrm{~L}^{3}} & 0 & \frac{-6 \mathrm{EI}_{3}}{\mathrm{~L}^{2}} & 0 & 0 & 0 & \frac{-12 \mathrm{EI}_{2}}{\mathrm{~L}^{3}} & 0 & \frac{-6 \mathrm{EI}_{3}}{\mathrm{~L}^{2}} & 0 \\ 0 & 0 & 0 & \frac{\mathrm{GI}_{1}}{\mathrm{~L}} & 0 & 0 & 0 & 0 & 0 & -\mathrm{GI}_{1} & 0 & 0 \\ 0 & 0 & \frac{-6 \mathrm{EI}_{3}}{\mathrm{~L}^{2}} & 0 & \frac{4 \mathrm{EI}_{2}}{\mathrm{~L}} & 0 & 0 & 0 & \frac{6 \mathrm{EI}_{2}}{\mathrm{~L}^{2}} & 0 & \frac{2 \mathrm{EI}_{2}}{\mathrm{~L}} & 0 \\ 0 & \frac{6 \mathrm{EI}_{3}}{\mathrm{~L}^{2}} & 0 & 0 & 0 & \frac{4 \mathrm{EI}_{3}}{\mathrm{~L}} & 0 & \frac{-6 \mathrm{EI}_{2}}{\mathrm{~L}^{2}} & 0 & 0 & 0 & \frac{2 \mathrm{EI}_{3}}{\mathrm{LA}} \\ \frac{\mathrm{L}}{\mathrm{L}} & 0 & 0 & 0 & 0 & 0 & \frac{\mathrm{EA}}{\mathrm{L}} & 0 & 0 & 0 & 0 & 0 \\ 0 & \frac{-12 \mathrm{EI}_{3}}{\mathrm{~L}^{3}} & 0 & 0 & 0 & \frac{-6 \mathrm{EI}_{2}}{\mathrm{~L}^{2}} & 0 & \frac{12 \mathrm{EI}_{3}}{\mathrm{~L}^{3}} & 0 & 0 & 0 & \frac{6 \mathrm{EI}_{3}}{\mathrm{~L}^{2}} \\ 0 & 0 & \frac{-12 \mathrm{EI}_{2}}{\mathrm{~L}^{3}} & 0 & \frac{6 \mathrm{EI}_{2}}{\mathrm{~L}^{2}} & 0 & 0 & 0 & \frac{12 \mathrm{EI}_{2}}{\mathrm{~L}^{3}} & 0 & \frac{-6 \mathrm{EI}_{3}}{\mathrm{~L}^{2}} & 0 \\ 0 & 0 & 0 & -\mathrm{GI}_{1} & 0 & 0 & 0 & 0 & 0 & \frac{\mathrm{GI}}{\mathrm{L}} & 0 & 0 \\ 0 & 0 & \frac{-6 \mathrm{EI}_{3}}{\mathrm{~L}^{2}} & 0 & \frac{2 \mathrm{EI}_{2}}{\mathrm{~L}} & 0 & 0 & 0 & \frac{-6 \mathrm{EI}_{3}}{\mathrm{~L}^{2}} & 0 & \frac{4 \mathrm{EI}_{2}}{\mathrm{~L}} & 0 \\ 0 & \frac{6 \mathrm{EI}_{3}}{\mathrm{~L}^{2}} & 0 & 0 & 0 & \frac{2 \mathrm{EI}_{3}}{\mathrm{~L}} & 0 & \frac{6 \mathrm{EI}_{3}}{\mathrm{~L}^{2}} & 0 & 0 & 0 & \frac{4 \mathrm{EI}_{3}}{\mathrm{~L}}\end{array}\right]$

onde:
A - área da seção transversal;
E - módulo de elasticidade longitudinal, ou módulo de Young, do material;
G - módulo de elasticidade transversal do material;
$\mathrm{I}_{1} \quad$ - momento de inércia à torção;
$\mathrm{I}_{2} \quad$ - momento de inércia em torno do eixo $\mathrm{x}_{2}$;
$I_{3} \quad-$ momento de inércia em torno do eixo $x_{3}$;
L $\quad$ - comprimento do elemento de barra. 
A matriz de rigidez do elemento da barra apresentada anteriormente foi escrita no sistema de coordenadas locais do elemento; para escrevê-la em termos de coordenadas globais da estrutura é necessário que se determine a sua matriz de incidência cinemática (ou de transformação de coordenadas), o que pode ser feito utilizando-se a eq.(7.15).

$\left\{u_{\mathrm{b} \ell}\right\}=\left[\beta_{\mathrm{b}}\right] \cdot\left\{\mathrm{u}_{\mathrm{bg}}\right\}$

onde:

$\left\{u_{\mathrm{b} \ell}\right\}$ - vetor de deslocamentos do elemento de barra segundo o sistema de coordenadas locais;

$\left[\beta_{\mathrm{b}}\right]$ - matriz de incidência cinemática do elemento de barra;

$\left\{u_{b g}\right\}$ - vetor de deslocamentos do elemento de barra segundo o sistema de coordenadas globais.

Explicitando-se a matriz de incidência cinemática $\left[\beta_{\mathrm{b}}\right]$, tem-se:

$\left[\beta_{\mathrm{b}}\right]=\left[\begin{array}{cccccccccccc}\ell_{1} & \ell_{2} & \ell_{3} & 0 & 0 & 0 & 0 & 0 & 0 & 0 & 0 & 0 \\ \mathrm{~m}_{1} & \mathrm{~m}_{2} & \mathrm{~m}_{3} & 0 & 0 & 0 & 0 & 0 & 0 & 0 & 0 & 0 \\ \mathrm{n}_{1} & \mathrm{n}_{2} & \mathrm{n}_{3} & 0 & 0 & 0 & 0 & 0 & 0 & 0 & 0 & 0 \\ 0 & 0 & 0 & \ell_{1} & \ell_{2} & \ell_{3} & 0 & 0 & 0 & 0 & 0 & 0 \\ 0 & 0 & 0 & \mathrm{~m}_{1} & \mathrm{~m}_{2} & \mathrm{~m}_{3} & 0 & 0 & 0 & 0 & 0 & 0 \\ 0 & 0 & 0 & \mathrm{n}_{1} & \mathrm{n}_{2} & \mathrm{n}_{3} & 0 & 0 & 0 & 0 & 0 & 0 \\ 0 & 0 & 0 & 0 & 0 & 0 & \ell_{1} & \ell_{2} & \ell_{3} & 0 & 0 & 0 \\ 0 & 0 & 0 & 0 & 0 & 0 & \mathrm{~m}_{1} & \mathrm{~m}_{2} & \mathrm{~m}_{3} & 0 & 0 & 0 \\ 0 & 0 & 0 & 0 & 0 & 0 & \mathrm{n}_{1} & \mathrm{n}_{2} & \mathrm{n}_{3} & 0 & 0 & 0 \\ 0 & 0 & 0 & 0 & 0 & 0 & 0 & 0 & 0 & \ell_{1} & \ell_{2} & \ell_{3} \\ 0 & 0 & 0 & 0 & 0 & 0 & 0 & 0 & 0 & \mathrm{~m}_{1} & \mathrm{~m}_{2} & \mathrm{~m}_{3} \\ 0 & 0 & 0 & 0 & 0 & 0 & 0 & 0 & 0 & \mathrm{n}_{1} & \mathrm{n}_{2} & \mathrm{n}_{3}\end{array}\right]$

onde $\ell, \mathrm{m}, \mathrm{n}$ são os versores de direção dos eixos $\mathrm{x}_{1}, \mathrm{x}_{2}$ e $\mathrm{x}_{3}$, respectivamente, com relação aos eixos $X_{1}, X_{2}$ e $X_{3}$ globais. 
Com a matriz de incidência cinemática, pode-se determinar a matriz de rigidez do elemento de barra em coordenadas globais utilizando-se a eq.(7.16).

$\left[\mathrm{K}_{\mathrm{bg}}\right]=\left[\beta_{\mathrm{b}}\right]^{\top}\left[\mathrm{K}_{\mathrm{b} \ell}\right]\left[\beta_{\mathrm{b}}\right]$

A matriz de massa considerada para este elemento é obtida a partir das aproximações em deslocamentos, e é dada em coordenadas locais por:

$\left[\mathrm{M}_{\mathrm{b} \ell}\right]=\frac{\rho \mathrm{AL}}{420}\left[\begin{array}{cccccccccccc}140 & 0 & 0 & 0 & 0 & 0 & 70 & 0 & 0 & 0 & 0 & 0 \\ 0 & 156 & 0 & 0 & 0 & 22 \mathrm{~L} & 0 & 54 & 0 & 0 & 0 & -13 \mathrm{~L} \\ 0 & 0 & 156 & 0 & -22 \mathrm{~L} & 0 & 0 & 0 & 54 & 0 & 13 \mathrm{~L} & 0 \\ 0 & 0 & 0 & \frac{140 \mathrm{I}_{1}}{\mathrm{~A}} & 0 & 0 & 0 & 0 & 0 & \frac{7 \mathrm{I}_{1}}{\mathrm{~A}} & 0 & 0 \\ 0 & 0 & -22 \mathrm{~L} & 0 & 4 \mathrm{~L}^{2} & 0 & 0 & 0 & -13 \mathrm{~L} & 0 & -3 \mathrm{~L}^{2} & 0 \\ 0 & 22 \mathrm{~L} & 0 & 0 & 0 & 4 \mathrm{~L}^{2} & 0 & 13 \mathrm{~L} & 0 & 0 & 0 & -3 \mathrm{~L}^{2} \\ 70 & 0 & 0 & 0 & 0 & 0 & 140 & 0 & 0 & 0 & 0 & 0 \\ 0 & 54 & 0 & 0 & 0 & 13 \mathrm{~L} & 0 & 156 & 0 & 0 & 0 & -22 \mathrm{~L} \\ 0 & 0 & 54 & 0 & -13 \mathrm{~L} & 0 & 0 & 0 & 156 & 0 & 22 \mathrm{~L} & 0 \\ 0 & 0 & 0 & \frac{70 \mathrm{I}_{1}}{4} & 0 & 0 & 0 & 0 & 0 & \frac{140 \mathrm{I}_{1}}{3} & 0 & 0 \\ 0 & 0 & 13 \mathrm{~L} & 0 & -3 \mathrm{~L}^{2} & 0 & 0 & 0 & 22 \mathrm{~L} & 0 & 4 \mathrm{~L}^{2} & 0 \\ 0 & -13 \mathrm{~L} & 0 & 0 & 0 & -3 \mathrm{~L}^{2} & 0 & -22 \mathrm{~L} & 0 & 0 & 0 & 4 \mathrm{~L}^{2}\end{array}\right]$

onde $\rho$ é a densidade do material.

A determinação da matriz de massa em coordenadas globais é obtida seguindo o mesmo procedimento adotado para a matriz de rigidez.

A matriz de amortecimento é assumida de maneira simplificada, tomando-a proporcional às matrizes de massa e rigidez:

$\mathrm{C}=\lambda_{\mathrm{m}} \mathrm{M}+\lambda_{\mathrm{k}} \mathrm{K}$

A matriz G, que, para cada elemento, multiplicando o vetor de cargas distribuídas resulta em um vetor de forças concentradas nas extremidades do elemento, é apresentada a seguir. Independente da presença de cargas distribuídas, a matriz $\mathrm{G}$ é indispensável a analises que utilizam o acoplamento MEC/MEF. 
Para a obtenção da matriz $G$ do elemento de barra, considerou-se aproximação linear para deslocamentos longitudinais e aproximação cúbica para deslocamentos transversais. Sendo assim, pode-se escrever a matriz G em coordenadas locais como:

$\left[\mathrm{G}_{\mathrm{b} \ell}\right]=\left[\begin{array}{cccccccccccc}\frac{\mathrm{L}}{3} & 0 & 0 & 0 & 0 & 0 & \frac{\mathrm{L}}{6} & 0 & 0 & 0 & 0 & 0 \\ 0 & \frac{7 \mathrm{~L}}{20} & 0 & 0 & 0 & -\frac{1}{2} & 0 & \frac{3 \mathrm{~L}}{20} & 0 & 0 & 0 & -\frac{1}{2} \\ 0 & 0 & \frac{7 \mathrm{~L}}{20} & 0 & \frac{1}{2} & 0 & 0 & 0 & \frac{3 \mathrm{~L}}{20} & 0 & \frac{1}{2} & 0 \\ 0 & 0 & 0 & \frac{\mathrm{L}}{3} & 0 & 0 & 0 & 0 & 0 & \frac{\mathrm{L}}{6} & 0 & 0 \\ 0 & 0 & -\frac{\mathrm{L}^{2}}{20} & 0 & \frac{\mathrm{L}}{12} & 0 & 0 & 0 & -\frac{\mathrm{L}^{2}}{30} & 0 & -\frac{\mathrm{L}}{12} & 0 \\ 0 & \frac{\mathrm{L}^{2}}{20} & 0 & 0 & 0 & \frac{\mathrm{L}}{12} & 0 & \frac{\mathrm{L}^{2}}{30} & 0 & 0 & 0 & -\frac{\mathrm{L}}{12} \\ \frac{\mathrm{L}}{6} & 0 & 0 & 0 & 0 & 0 & \frac{\mathrm{L}}{3} & 0 & 0 & 0 & 0 & 0 \\ 0 & \frac{3 \mathrm{~L}}{20} & 0 & 0 & 0 & \frac{1}{2} & 0 & \frac{7 \mathrm{~L}}{20} & 0 & 0 & 0 & \frac{1}{2} \\ 0 & 0 & \frac{3 \mathrm{~L}}{20} & 0 & -\frac{1}{2} & 0 & 0 & 0 & \frac{7 \mathrm{~L}}{20} & 0 & -\frac{1}{2} & 0 \\ 0 & 0 & 0 & \frac{\mathrm{L}}{6} & 0 & 0 & 0 & 0 & 0 & \frac{\mathrm{L}}{3} & 0 & 0 \\ 0 & 0 & \frac{\mathrm{L}^{2}}{30} & 0 & -\frac{\mathrm{L}}{12} & 0 & 0 & 0 & \frac{\mathrm{L}^{2}}{20} & 0 & \frac{\mathrm{L}}{12} & 0 \\ 0 & -\frac{\mathrm{L}^{2}}{30} & 0 & 0 & 0 & -\frac{\mathrm{L}}{12} & 0 & -\frac{\mathrm{L}^{2}}{20} & 0 & 0 & 0 & \frac{\mathrm{L}}{12}\end{array}\right]$

onde L é o comprimento do elemento de barra.

Igualmente ao que foi feito com a matriz de rigidez em coordenadas locais do elemento de barra, deve-se transformar a matriz $G$ da barra em coordenadas locais para coordenadas globais.

\subsection{ELEMENTO FINITO DE PLACA}

É preciso salientar que o elemento finito de casca será considerado como uma composição de um elemento finito de placa e um elemento finito de chapa (ou membrana). Para o elemento finito de placa, decidiu-se utilizar o elemento finito DKT (discrete Kirchhoff triangle) por ter sido um elemento bastante utilizado e que tem apresentado bons resultados; sua matriz de 
rigidez pode ser escrita de forma explícita e trata-se de um elemento triangular com número de graus de liberdade (gdl) mínimo, 9. O desempenho do elemento finito DKT já foi amplamente verificado por diversos autores, seja em casos de placas, cascas, pavimentos e edifícios de múltiplos pavimentos. Além de todos os motivos apresentados anteriormente, deve-se citar também que o autor trabalhou com o elemento finito DKT em sua pesquisa de mestrado para uma análise elastostática de pavimentos de edifícios; sendo assim, a presente seção foi escrita com base em Almeida (1999).

O elemento finito DKT faz parte do grupo dos elementos finitos triangulares de placa com 9 graus de liberdade, sendo 3 por vértice (translação em z $(w)$ e rotações em x $\left(\theta_{x}\right)$ e y $\left.\left(\theta_{y}\right)\right)$. Ver figura 7.3.

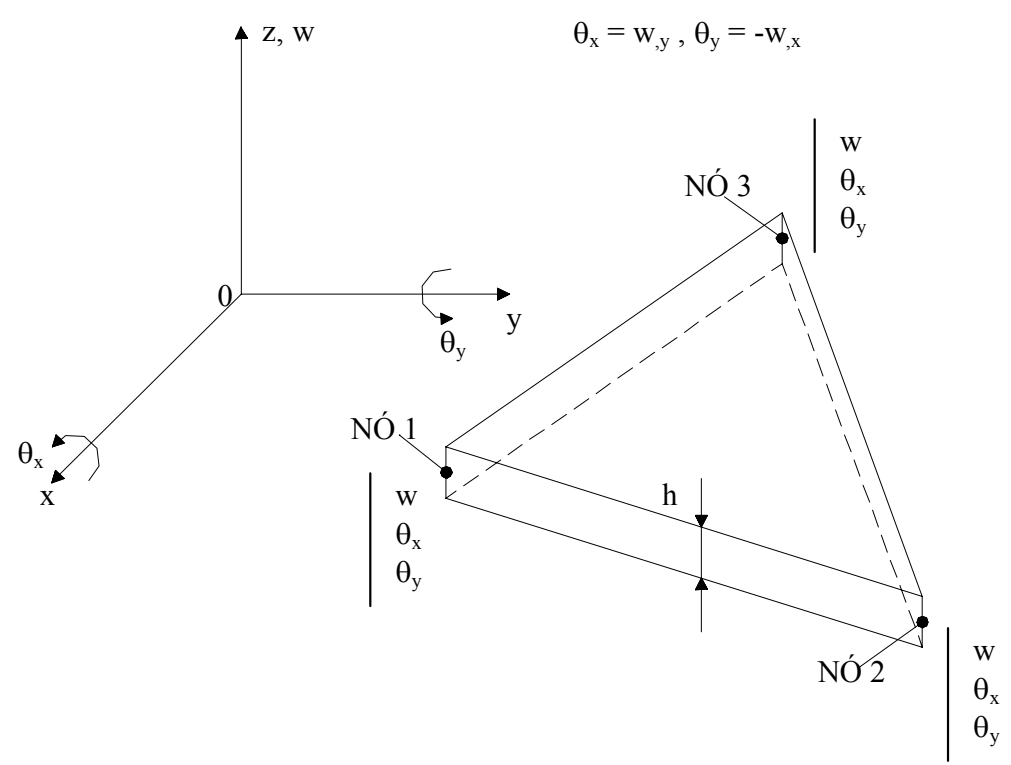

Figura 7.3 - Elemento finito DKT

A teoria de pequenos deslocamentos de placas com deformações por esforço cortante incluídos, também conhecida como teoria das placas de Reissner-Mindlin, é utilizada na formulação do elemento finito DKT. Após as deduções das expressões de energia de deformação e antes de se chegar à matriz de rigidez do elemento DKT, admite-se que o elemento será utilizado na análise de placas delgadas, e assim, as deformações por esforço cortante, e conseqüentemente a energia de deformação causada por esse esforço, são desprezadas quando comparadas com a energia de deformação por flexão. 
A formulação do elemento finito DKT baseia-se nas seguintes hipóteses: as rotações $\beta_{x}$ e $\beta_{y}$ variam quadraticamente no elemento, sendo $\beta_{x}$ e $\beta_{y}$ as rotações da normal ao plano médio indeformado do elemento, segundo os planos $x-z$ e $y-z$, respectivamente; a hipótese de Kirchhoff é imposta discretamente ao longo dos lados do elemento em seus pontos nodais, possibilitando relacionar as rotações com as primeiras derivadas dos deslocamentos transversais; a variação de w é cúbica e definida apenas ao longo dos lados do elemento; impõe-se uma variação linear de $\beta_{\mathrm{n}}$ ao longo dos lados, onde $\beta_{\mathrm{n}}$ é a rotação na direção normal aos lados.

O vetor de cargas nodais equivalentes para um carregamento uniformemente distribuído $(q)$ em um elemento de área $A$ poderia ter sido calculado de forma simplificada, e dado por:

$\{f\}^{\top}=\frac{q A}{3}\left\{\begin{array}{lllllllll}1 & 0 & 0 & 1 & 0 & 0 & 1 & 0 & 0\end{array}\right\}$

Ver figura 7.4

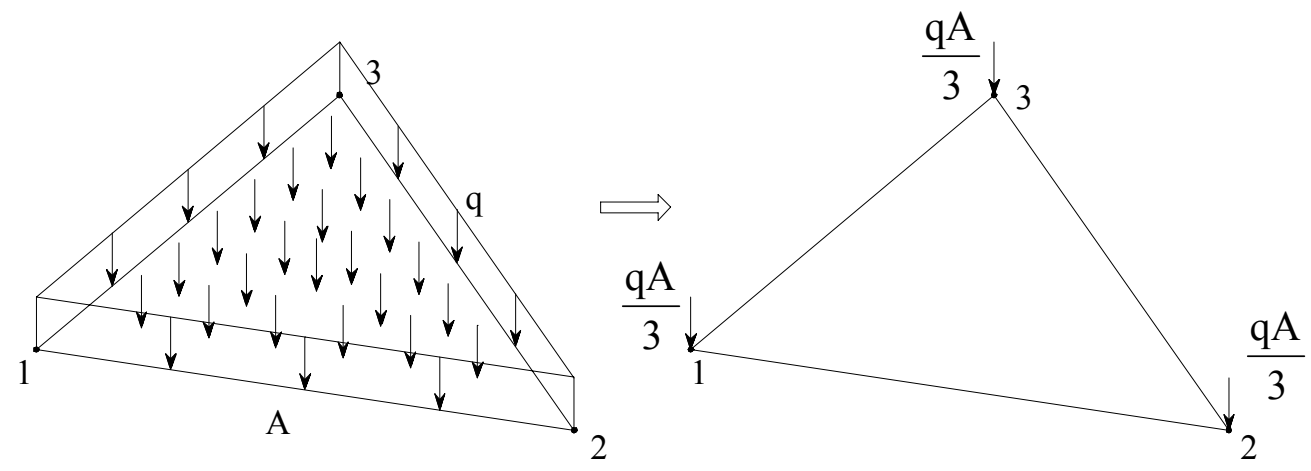

Figura 7.4 - Carregamento uniformemente distribuído no elemento

Todavia, optou-se por uma aproximação linear para tal carregamento, conforme desenvolvimento apresentado posteriormente quando da apresentação da matriz $\mathrm{G}_{\mathrm{p}_{\ell}}$.

Convém citar que a matriz de rigidez do elemento foi calculada utilizando-se o algoritmo apresentado por Jeyachandrabose; Kirkhope; Ramesh Babu (1985).

Utilizou-se um sistema de coordenadas locais segundo a figura 7.5. 
No apêndice C apresenta-se o algoritmo citado nesta seção, tal qual foi utilizado no código computacional implementado.

Após a determinação da matriz de rigidez em coordenadas locais do elemento finito DKT, deve-se transformar essa matriz para o sistema de coordenadas globais da estrutura (ou sub-região) utilizando-se sua matriz de incidência cinemática. É importante salientar que o sistema de coordenadas globais considera os 6 graus de liberdade presentes nos nós de barra, a saber: $\mathrm{U}_{1}, \mathrm{U}_{2}, \mathrm{U}_{3}, \theta_{1}, \theta_{2}, \theta_{3}$, em termos de deslocamentos. Assim, a matriz de rigidez em coordenadas globais possui dimensão 18 x 18, e já pode ser alocada normalmente na matriz de rigidez global da estrutura.

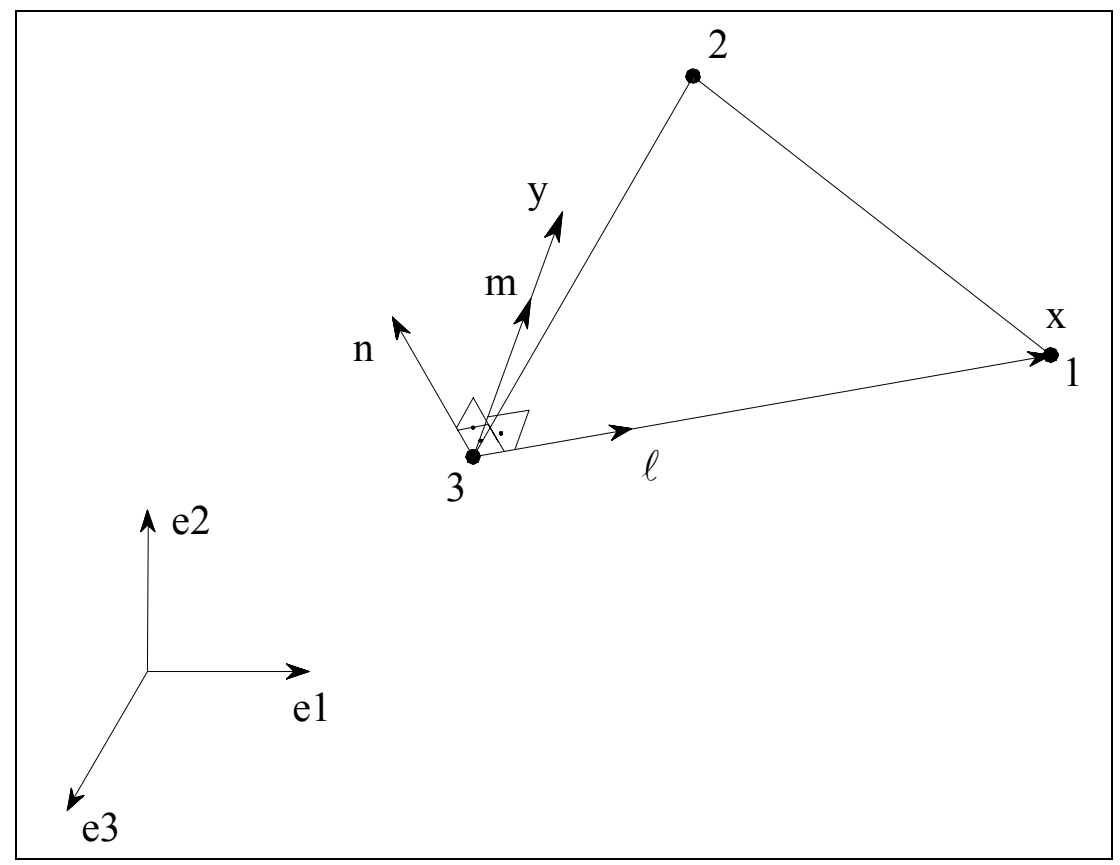

Figura 7.5 - Sistema de coordenadas locais do elemento finito DKT

Considerando-se os co-senos diretores da figura 7.5, a matriz de incidência cinemática para o elemento finito é escrita como: 


$$
\left[\beta_{p}\right]=\left[\begin{array}{cccccccccccccccccc}
\mathrm{n}_{1} & \mathrm{n}_{2} & \mathrm{n}_{3} & 0 & 0 & 0 & 0 & 0 & 0 & 0 & 0 & 0 & 0 & 0 & 0 & 0 & 0 & 0 \\
0 & 0 & 0 & \ell_{1} & \ell_{2} & \ell_{3} & 0 & 0 & 0 & 0 & 0 & 0 & 0 & 0 & 0 & 0 & 0 & 0 \\
0 & 0 & 0 & \mathrm{~m}_{1} & \mathrm{~m}_{2} & \mathrm{~m}_{3} & 0 & 0 & 0 & 0 & 0 & 0 & 0 & 0 & 0 & 0 & 0 & 0 \\
0 & 0 & 0 & 0 & 0 & 0 & \mathrm{n}_{1} & \mathrm{n}_{2} & \mathrm{n}_{3} & 0 & 0 & 0 & 0 & 0 & 0 & 0 & 0 & 0 \\
0 & 0 & 0 & 0 & 0 & 0 & 0 & 0 & 0 & \ell_{1} & \ell_{2} & \ell_{3} & 0 & 0 & 0 & 0 & 0 & 0 \\
0 & 0 & 0 & 0 & 0 & 0 & 0 & 0 & 0 & \mathrm{~m}_{1} & \mathrm{~m}_{2} & \mathrm{~m}_{3} & 0 & 0 & 0 & 0 & 0 & 0 \\
0 & 0 & 0 & 0 & 0 & 0 & 0 & 0 & 0 & 0 & 0 & 0 & \mathrm{n}_{1} & \mathrm{n}_{2} & \mathrm{n}_{3} & 0 & 0 & 0 \\
0 & 0 & 0 & 0 & 0 & 0 & 0 & 0 & 0 & 0 & 0 & 0 & 0 & 0 & 0 & \ell_{1} & \ell_{2} & \ell_{3} \\
0 & 0 & 0 & 0 & 0 & 0 & 0 & 0 & 0 & 0 & 0 & 0 & 0 & 0 & 0 & \mathrm{~m}_{1} & \mathrm{~m}_{2} & \mathrm{~m}_{3}
\end{array}\right]
$$

De posse da matriz de rigidez em coordenadas locais e da matriz de incidência cinemática, pode-se facilmente determinar a matriz de rigidez do elemento DKT em coordenadas globais utilizando-se a seguinte equação matricial:

$\left[\mathrm{K}_{\mathrm{pg}}\right]_{18 \times 18}=\left[\beta_{\mathrm{p}}\right]_{18 \times 9}^{\top}\left[\mathrm{K}_{\mathrm{p} \ell}\right]_{9 \times 9}\left[\beta_{\mathrm{p}}\right]_{9 \times 18}$

Admitindo-se uma aproximação linear para o carregamento distribuído transversal aplicado ao elemento finito de placa, e também se considerando (como aproximação coerente) os deslocamentos transversais médios com comportamento linear, a matriz $\mathrm{G}$ em coordenadas locais para esse elemento pode ser escrita da seguinte forma:

$$
\left[G_{p \ell}\right]=\frac{A}{12}\left[\begin{array}{ccccccccc}
2 & 0 & 0 & 1 & 0 & 0 & 1 & 0 & 0 \\
0 & 2 & 0 & 0 & 1 & 0 & 0 & 1 & 0 \\
0 & 0 & 2 & 0 & 0 & 1 & 0 & 0 & 1 \\
1 & 0 & 0 & 2 & 0 & 0 & 1 & 0 & 0 \\
0 & 1 & 0 & 0 & 2 & 0 & 0 & 1 & 0 \\
0 & 0 & 1 & 0 & 0 & 2 & 0 & 0 & 1 \\
1 & 0 & 0 & 1 & 0 & 0 & 2 & 0 & 0 \\
0 & 1 & 0 & 0 & 1 & 0 & 0 & 2 & 0 \\
0 & 0 & 1 & 0 & 0 & 1 & 0 & 0 & 2
\end{array}\right]
$$

onde A é a área do elemento de placa. 
Como observações adicionais, pode-se dizer que quando a formulação do DKT é aplicada a um elemento de viga, obtém-se a matriz de rigidez exata utilizando um polinômio cúbico de w; a formulação do elemento DKT pode ser estendida a elementos quadrilaterais, sem serem submetidos ao processo de condensação estática, com 12 graus de liberdade (como é o caso do elemento DKQ (discrete Kirchhoff quadrilateral)) e outros elementos poligonais de placa.

A matriz de massa do elemento de placa é obtida a partir da segunda integral da eq.(7.12), considerando-se os campos de aceleração e ponderador lineares (ortogonais no plano do elemento). A densidade será considerada constante ao longo do elemento. Desta forma, numericamente, a matriz resultante é semelhante à obtida para o elemento CST, descrito na próxima seção. As referidas matrizes são transformadas para coordenadas globais segundo a expressão geral (7.16).

\subsection{ELEMENTO FINITO DE CHAPA}

O elemento finito de chapa adotado foi o CST (constant strain triangle), que, como o próprio nome diz, é um elemento triangular que considera aproximação constante para o campo de deformações do elemento, ou, campo de deslocamentos linear, conforme eq.(7.20) e figura 7.6. Esta escolha foi feita com o intuito de facilitar a adequação dos efeitos de membrana ao elemento de placa descrito no item anterior.

$$
\begin{aligned}
& u(x, y)=a_{1}+a_{2} x+a_{3} y \\
& v(x, y)=a_{4}+a_{5} x+a_{6} y
\end{aligned}
$$




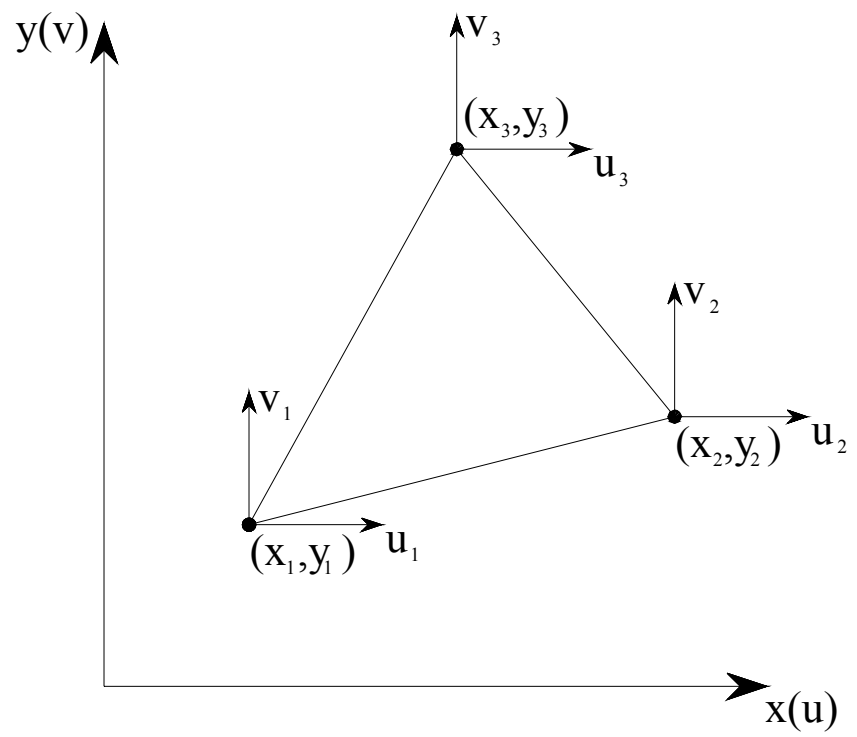

Figura 7.6 - Elemento finito de chapa CST

Observa-se que a eq.(7.20) está escrita em coordenadas cartesianas; em coordenadas adimensionais (ver figura 7.7), a mesma equação poderia ser escrita como:

$\mathrm{u}\left(\xi_{1}, \xi_{2}, \xi_{3}\right)=\xi_{1} u_{1}+\xi_{2} u_{2}+\xi_{3} u_{3}$

$\mathrm{v}\left(\xi_{1}, \xi_{2}, \xi_{3}\right)=\xi_{1} \mathrm{v}_{1}+\xi_{2} \mathrm{v}_{2}+\xi_{3} \mathrm{v}_{3}$

ou:

$\mathrm{u}\left(\xi_{1}, \xi_{2}, \xi_{3}\right)=\sum_{\mathrm{i}=1}^{3} \xi_{\mathrm{i}} \mathrm{u}_{\mathrm{i}}$
$\mathrm{v}\left(\xi_{1}, \xi_{2}, \xi_{3}\right)=\sum_{\mathrm{i}=1}^{3} \xi_{\mathrm{i}} \mathrm{v}_{\mathrm{i}}$

ou ainda, em notação de Einstein (indicial), simplesmente:

$\mathrm{u}=\xi_{\mathrm{i}} \mathrm{u}_{\mathrm{i}}$
$\mathrm{v}=\xi_{\mathrm{i}} \mathrm{v}_{\mathrm{i}}$

$i=1,2,3$ 


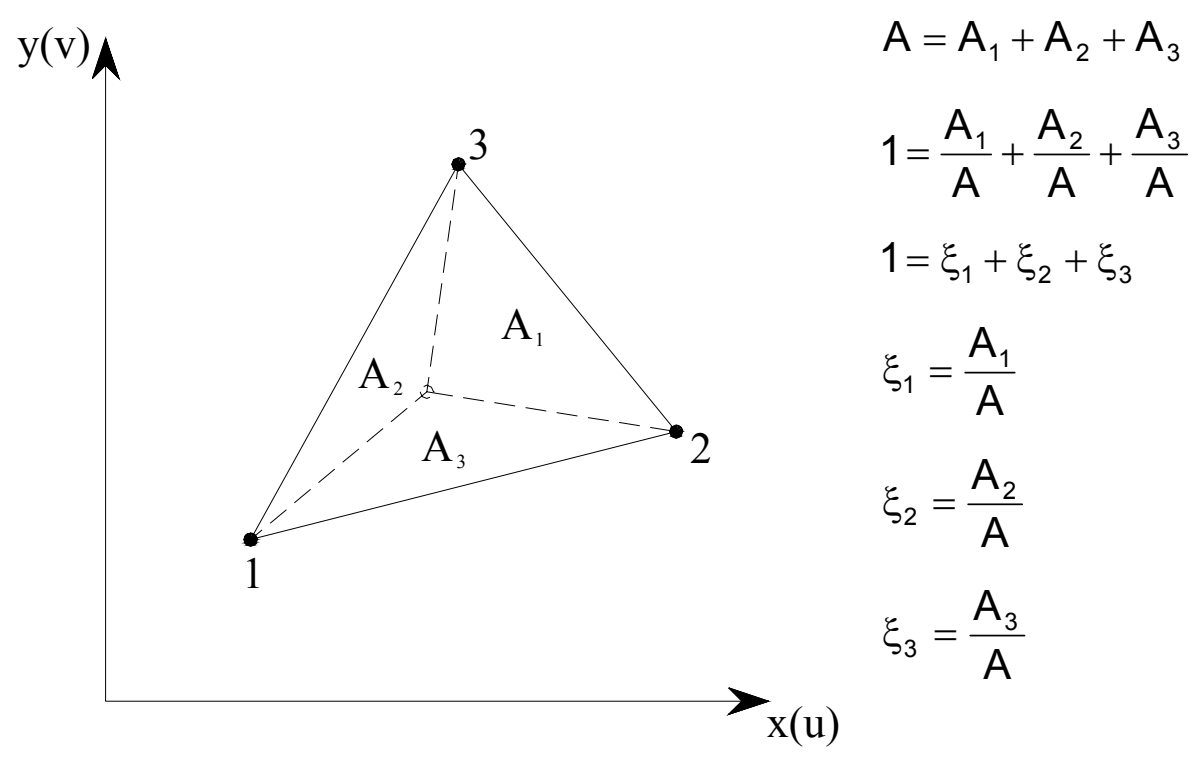

Figura 7.7 - Coordenadas adimensionais

Matricialmente, a eq.(7.21) pode ser escrita como:

$$
\left\{\begin{array}{l}
u \\
v
\end{array}\right\}=\left[\begin{array}{cccccc}
\xi_{1} & \xi_{2} & \xi_{3} & 0 & 0 & 0 \\
0 & 0 & 0 & \xi_{1} & \xi_{2} & \xi_{3}
\end{array}\right]\left\{\begin{array}{l}
u_{1} \\
u_{2} \\
u_{3} \\
v_{1} \\
v_{2} \\
v_{3}
\end{array}\right\}
$$

A matriz de rigidez para este elemento é dada por:

$$
\left[K_{c l}\right]=\frac{E h}{4 A\left(1-v^{2}\right)}\left[\begin{array}{cccccc}
b_{1}^{2}+a_{1}^{2} \beta & a_{1} b_{1}(v+\beta) & b_{1} b_{2}+a_{1} a_{2} \beta & v a_{2} b_{1}+a_{1} b_{2} \beta & b_{1} b_{3}+a_{1} a_{3} \beta & v a_{3} b_{1}+a_{1} b_{3} \beta \\
& a_{1}^{2}+b_{1}^{2} \beta & v a_{1} b_{2}+a_{2} b_{1} \beta & a_{1} a_{2}+b_{1} b_{2} \beta & v a_{1} b_{3}+a_{3} b_{1} \beta & a_{1} a_{3}+b_{1} b_{3} \beta \\
& & b_{2}^{2}+a_{2}^{2} \beta & a_{2} b_{2}(v+\beta) & b_{2} b_{3}+a_{2} a_{3} \beta & v a_{3} b_{2}+a_{2} b_{3} \beta \\
& & & a_{2}^{2}+b_{2}^{2} \beta & v a_{2} b_{3}+a_{3} b_{2} \beta & a_{2} a_{3}+b_{2} b_{3} \beta \\
& \text { simétrica } & & & b_{3}^{2}+a_{3}^{2} \beta & a_{3} b_{3}(v+\beta) \\
& & & & & a_{3}^{2}+b_{3}^{2} \beta
\end{array}\right]
$$

onde $h$ é a espessura do elemento; $a_{i}$ e $b_{i}$ são calculados pelas eq.(7.25) e (7.26), respectivamente; e $\beta$ é dado pela eq.(7.27).

$$
\begin{aligned}
& a_{i}=x_{k}-x_{j} \\
& b_{i}=y_{j}-y_{k}
\end{aligned}
$$


$\beta=\frac{(1-v)}{2}$

Observa-se que i, j, k possui variação cíclica.

A matriz de massa é obtida como descrito para o elemento de placa e é dada por:

$\left[\mathrm{M}_{\mathrm{c} \ell}\right]=\frac{\rho A h}{12}\left[\begin{array}{llllll}2 & 1 & 1 & 0 & 0 & 0 \\ 1 & 2 & 1 & 0 & 0 & 0 \\ 1 & 1 & 2 & 0 & 0 & 0 \\ 0 & 0 & 0 & 2 & 1 & 1 \\ 0 & 0 & 0 & 1 & 2 & 1 \\ 0 & 0 & 0 & 1 & 1 & 2\end{array}\right]$

A matriz de incidência cinemática do elemento finito de chapa utilizado no trabalho pode ser escrita segundo a igualdade abaixo:

$\left[\beta_{\mathrm{c}}\right]=\left[\begin{array}{cccccccccccccccccc}\ell_{1} & \ell_{2} & \ell_{3} & 0 & 0 & 0 & 0 & 0 & 0 & 0 & 0 & 0 & 0 & 0 & 0 & 0 & 0 & 0 \\ \mathrm{~m}_{1} & \mathrm{~m}_{2} & \mathrm{~m}_{3} & 0 & 0 & 0 & 0 & 0 & 0 & 0 & 0 & 0 & 0 & 0 & 0 & 0 & 0 & 0 \\ 0 & 0 & 0 & 0 & 0 & 0 & \ell_{1} & \ell_{2} & \ell_{3} & 0 & 0 & 0 & 0 & 0 & 0 & 0 & 0 & 0 \\ 0 & 0 & 0 & 0 & 0 & 0 & \mathrm{~m}_{1} & \mathrm{~m}_{2} & \mathrm{~m}_{3} & 0 & 0 & 0 & 0 & 0 & 0 & 0 & 0 & 0 \\ 0 & 0 & 0 & 0 & 0 & 0 & 0 & 0 & 0 & 0 & 0 & 0 & \ell_{1} & \ell_{2} & \ell_{3} & 0 & 0 & 0 \\ 0 & 0 & 0 & 0 & 0 & 0 & 0 & 0 & 0 & 0 & 0 & 0 & \mathrm{~m}_{1} & \mathrm{~m}_{2} & \mathrm{~m}_{3} & 0 & 0 & 0\end{array}\right]$

onde os co-senos diretores $\ell_{i}$ e $m_{i}$ são determinados da mesma forma que aquela utilizada para o elemento DKT. Ver Figura 7.5.

Observa-se que a utilização da matriz de incidência cinemática como foi apresentada acima, também implica em uma matriz em coordenadas globais de dimensão 18 x 18, já pronta para ser alocada na matriz global da estrutura, como foi feito com o elemento finito de placa, alocando as contribuições de membrana à casca.

Para o elemento finito de chapa, deduziram-se dois tipos de matrizes G: uma denominada de G1, relativa a carregamento distribuído, com aproximação linear, aplicado longitudinalmente nos lados do elemento finito; e uma matriz 
G2 referente a uma aproximação linear de carregamento distribuído, também longitudinal, sendo que na área do elemento, ou seja, carregamento cisalhante na superfície do elemento. A matriz G1 é comumente utilizada quando da aplicação de carregamento distribuído ao longo dos lados de um elemento, enquanto que a matriz G2 é utilizada na formação da matriz $G$ total dos diversos meios acoplados.

Assim, a matriz G1 pode ser escrita em função do comprimento dos lados em que existe carregamento distribuído aplicado (ver figura 7.8):

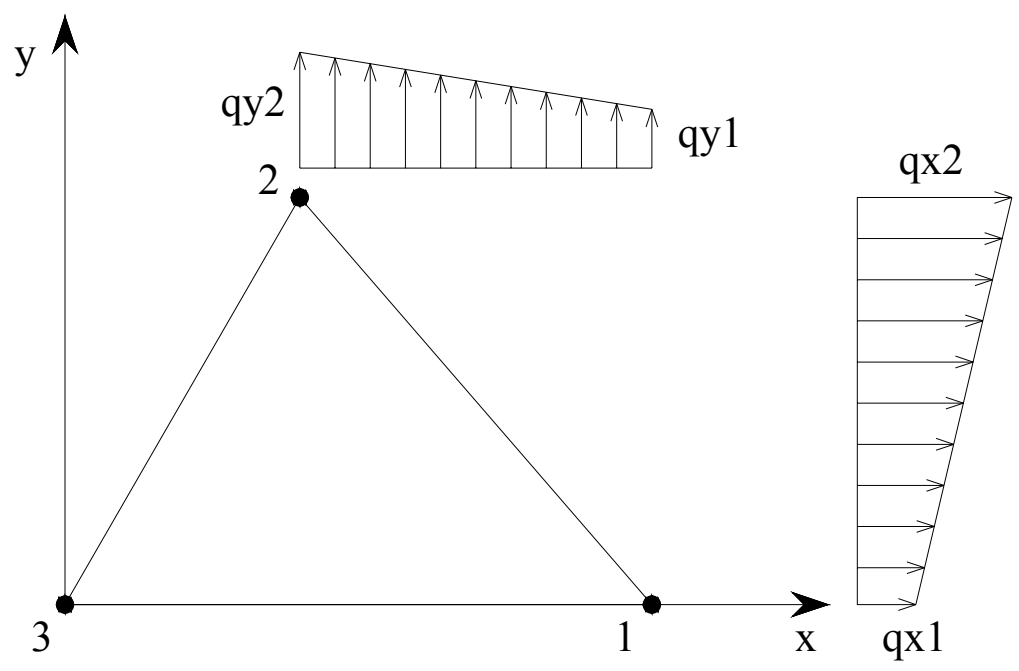

Figura 7.8 - Carregamento distribuído em um lado do elemento finito de chapa CST

$$
\left\{\begin{array}{c}
F_{x}^{1} \\
F_{y}^{1} \\
F_{x}^{2} \\
F_{y}^{2} \\
F_{x}^{3} \\
F_{y}^{3}
\end{array}\right\}=\left[\begin{array}{cccccc}
\frac{\ell_{12}+\ell_{31}}{3} & 0 & \frac{\ell_{12}}{6} & 0 & \frac{\ell_{31}}{6} & 0 \\
0 & \frac{\ell_{12}+\ell_{31}}{3} & 0 & \frac{\ell_{12}}{6} & 0 & \frac{\ell_{31}}{6} \\
\frac{\ell_{12}}{6} & 0 & \frac{\ell_{12}+\ell_{23}}{3} & 0 & \frac{\ell_{23}}{6} & 0 \\
0 & \frac{\ell_{12}}{6} & 0 & \frac{\ell_{12}+\ell_{23}}{3} & 0 & \frac{\ell_{23}}{6} \\
\frac{\ell_{31}}{6} & 0 & \frac{\ell_{23}}{6} & 0 & \frac{\ell_{31}+\ell_{23}}{3} & 0 \\
0 & \frac{\ell_{31}}{6} & 0 & \frac{\ell_{23}}{6} & 0 & \frac{\ell_{31}+\ell_{23}}{3}
\end{array}\right]\left\{\begin{array}{l}
q_{x}^{1} \\
q_{y}^{1} \\
q_{x}^{2} \\
q_{y}^{2} \\
q_{x}^{3} \\
q_{y}^{3}
\end{array}\right\}
$$

ou:

$\left\{\mathrm{F}_{\mathrm{c} \ell}\right\}=\left[\mathrm{G}_{\mathrm{c} \ell}\right]\left\{\mathrm{q}_{\mathrm{c} \ell}\right\}$ 
onde:

$F_{i}^{j} \quad$ - força aplicada ao nó 'j' na direção 'i';

$q_{i}^{j} \quad$ - valor do carregamento distribuído nó 'j' na direção 'i';

$\ell_{\mathrm{ij}} \quad$ - comprimento do lado ij.

Deve-se atentar para o fato de que, em G1, o valor de $\ell_{\mathrm{ij}}$ deve ser considerado nulo quando tal lado não possuir carregamento distribuído.

Já a matriz G2, em coordenadas locais, é deduzida de maneira semelhante ao que se fez para a matriz $G$ do elemento finito DKT. Desta forma, pode-se escrever $\mathrm{G} 2$ como:

$$
\left[G 2_{c \ell}\right]=\frac{A}{12}\left[\begin{array}{cccccc}
2 & 0 & 1 & 0 & 1 & 0 \\
0 & 2 & 0 & 1 & 0 & 1 \\
1 & 0 & 2 & 0 & 1 & 0 \\
0 & 1 & 0 & 2 & 0 & 1 \\
1 & 0 & 1 & 0 & 2 & 0 \\
0 & 1 & 0 & 1 & 0 & 2
\end{array}\right]
$$

O elemento de casca é obtido pela superposição dos DKT e CST; cuidado adicional deve ser tomado para o cálculo de cascas abatidas não acopladas.

Cabe observar que a presente seção foi escrita com base no livro de Assan (1999) e nos trabalhos de Coda $(1993,2000)$.

\subsection{ALGORITMO DE NEWMARK $\beta$ PARA INTEGRAÇÃO TEMPORAL}

A integração temporal do problema dinâmico é feita pelo método de Newmark, considerado um dos melhores para aplicações estruturais via MEF.

$\mathrm{Na}$ presente seção, pretende-se expor de forma resumida e direta o método de Newmark $\beta$ tal qual este foi utilizado no código desenvolvido pelo 
autor. Mais detalhes podem ser encontrados, por exemplo, no trabalho de Coda (2000).

Partindo então da eq.(7.14), aqui escrita como eq.(7.28), não se considerando a presença de forças volumétricas, pode-se escrever:

$\mathrm{KU}+\mathrm{CU}+\mathrm{MU}=\mathrm{GP}+\mathrm{IF}$

O algoritmo de Newmark $\beta$ considera as seguintes aproximações para velocidade e deslocamento no instante de tempo atual, escrito por ' $s+1$ ':

$$
\begin{aligned}
& \dot{U}_{s+1}=\dot{U}_{s}+\frac{1}{2} \Delta t\left[\ddot{U}_{s}+\ddot{U}_{s+1}\right] \\
& U_{s+1}=U_{s}+\Delta t \dot{U}_{s}+\left(\frac{1}{2}-\beta\right) \Delta t^{2} \ddot{U}_{s}+\beta \Delta t^{2} \ddot{U}_{s+1}
\end{aligned}
$$

onde:

$\Delta t \quad$ - intervalo de tempo;
$\beta \quad$ - parâmetro de calibração do método.

Deve-se comentar que o valor de $\beta$ introduz amortecimento numérico no sistema, que em certos casos de acoplamento podem ser benéficos na eliminação de "ruídos" desestabilizadores.

A expressão da aceleração no instante de tempo ' $s+1$ ' pode ser determinada a partir da eq.(7.30):

$$
\ddot{U}_{s+1}=\frac{1}{\beta \Delta t^{2}}\left\{U_{s+1}+a_{s}\right\}
$$

onde:

$$
a_{s}=\left(\beta-\frac{1}{2}\right) \Delta t^{2} \ddot{U}_{s}-\Delta t \dot{U}_{s}-U_{s}
$$


Substituindo-se a expressão para a aceleração da eq.(7.31) na eq.(7.29), tem-se uma nova expressão para a velocidade, dada pela eq.(7.33) abaixo.

$\dot{U}_{s+1}=\frac{1}{2 \beta \Delta t}\left\{U_{s+1}+v_{s}\right\}$

onde:

$\mathrm{v}_{\mathrm{s}}=\left(2 \beta-\frac{1}{2}\right) \Delta \mathrm{t}^{2} \ddot{\mathrm{U}}_{\mathrm{s}}+(2 \beta-1) \Delta \mathrm{t} \dot{\mathrm{U}}_{\mathrm{s}}-\mathrm{U}_{\mathrm{s}}$

Substituindo-se as eq.(7.31) e (7.33) na eq.(7.28), para o instante de tempo atual, pode-se escrever:

$\overline{\mathrm{K}} \mathrm{U}_{\mathrm{s}+1}=\beta \Delta \mathrm{t}^{2} \mathrm{GP} \mathrm{s}+1+\overline{\mathrm{F}}_{\mathrm{s}}$

onde:

$\bar{K}=M+\frac{\Delta t}{2} C+\beta \Delta t^{2} K$

$\overline{\mathrm{F}}_{\mathrm{s}}=\beta \Delta \mathrm{t}^{2} \mathrm{IF} \mathrm{s}_{\mathrm{s}+1}-\frac{\Delta \mathrm{t}}{2} \mathrm{Cv}_{\mathrm{s}}-\mathrm{Ma}_{\mathrm{s}}$

Como foi citado na seção 7.2, a matriz de amortecimento C é proporcional às matrizes de massa e rigidez, segundo a eq.(7.17) reescrita abaixo:

$\mathrm{C}=\lambda_{\mathrm{m}} \mathrm{M}+\lambda_{\mathrm{k}} \mathrm{K}$

Substituindo-se a eq.(7.17) na eq.(7.36), tem-se: 


$$
\overline{\mathrm{K}}=\left(1+\frac{\Delta \mathrm{t}}{2} \lambda_{\mathrm{m}}\right) \mathrm{M}+\left(\beta \Delta \mathrm{t}^{2}+\frac{\Delta \mathrm{t}}{2} \lambda_{\mathrm{k}}\right) \mathrm{K}
$$

Sendo assim, a eq.(7.35) pode ser escrita como:

$$
\overline{\mathrm{K}} \mathrm{U}_{\mathrm{s}+1}=\beta \Delta \mathrm{t}^{2} \mathrm{GP} \mathrm{s}_{\mathrm{s}+1}+\beta \Delta \mathrm{t}^{2} \mathrm{I}_{\mathrm{s+1}}+\overline{\overline{\mathrm{F}}}_{\mathrm{s}}
$$

onde:

$$
\begin{aligned}
& \overline{\bar{F}}_{s}=\left\{\Delta t^{3}(0,25-\beta) \lambda_{k} K+\left(\Delta t^{3}(0,25-\beta) \lambda_{m}+\Delta t^{2}(0,5-\beta)\right) M\right\} \ddot{U}_{s}+ \\
& +\left\{\Delta t^{2}(0,5-\beta) \lambda_{k} K+\left(\Delta t^{2}(0,5-\beta) \lambda_{m}+\Delta t\right) M \dot{U}_{s}+\left\{0,5 \Delta t \lambda_{k} K+\left(0,5 \Delta t \lambda_{m}+1\right) M\right\} U_{s}\right.
\end{aligned}
$$

Deve-se mencionar que foram consideradas condições iniciais nulas para deslocamentos e velocidades. Porém, as acelerações no instante inicial podem não ser nulas e, portanto devem ser calculadas resolvendo-se o sistema da eq.(7.41).

$M \ddot{U}_{0}=\mathrm{GP}_{0}+\mathrm{IF}_{0}$

\subsection{EXEMPLOS}

Nesta seção serão apresentados 5 exemplos utilizando exclusivamente o Método dos Elementos Finitos, para casos estáticos e dinâmicos.

\subsubsection{Exemplo 7.1}

O primeiro exemplo apresenta a análise dinâmica de um pórtico composto por uma viga e dois pilares sujeito a um carregamento senoidal segundo figura 7.9 . 


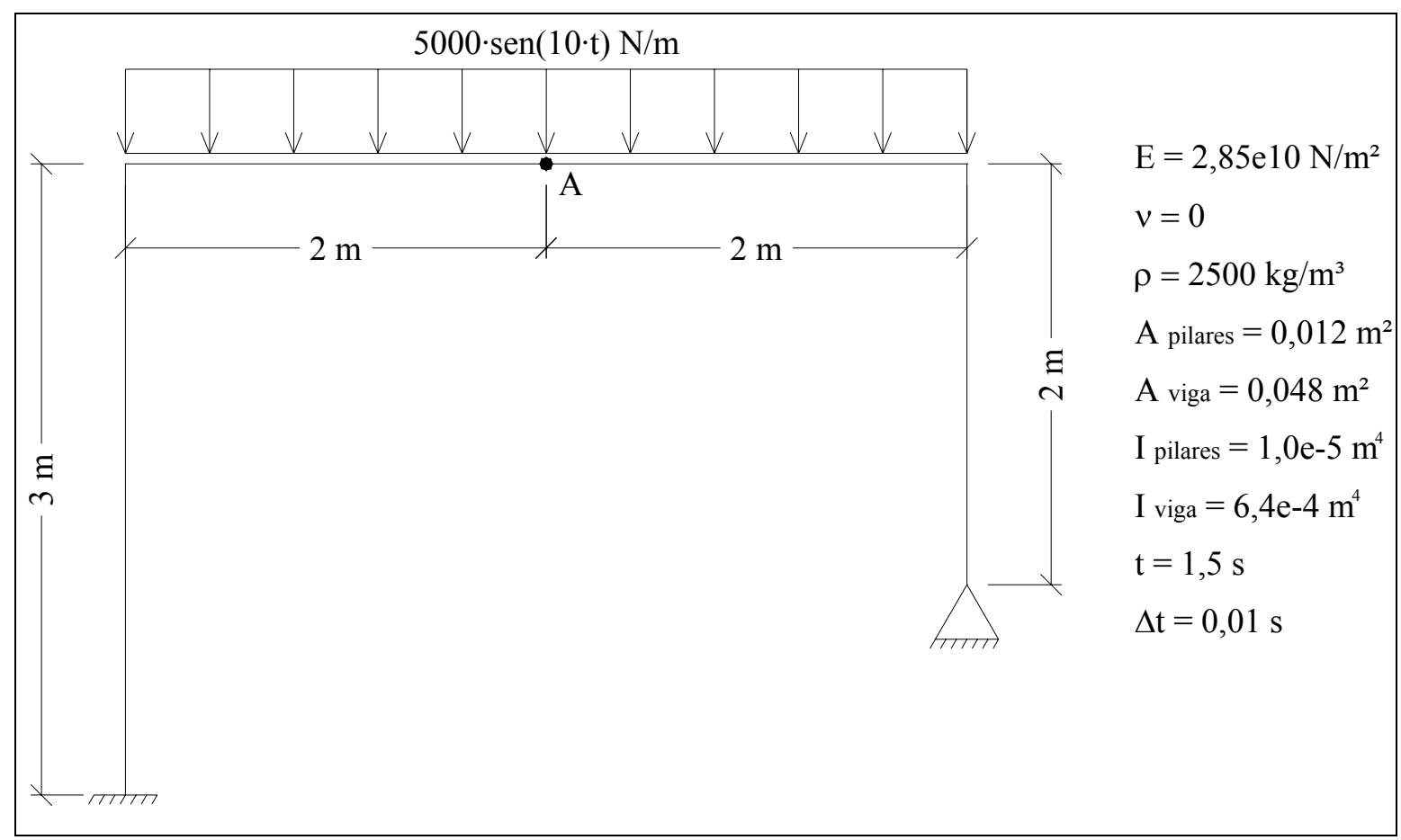

Figura 7.9 - Exemplo 7.1

Para a discretização de cada pilar utilizou-se dois elementos de barra de iguais comprimentos, e para a viga, quatro elementos. Foram feitas duas análises: uma sem amortecimento e outra com amortecimento, onde se utilizaram os seguintes parâmetros de amortecimento:

$$
\left\{\begin{array}{l}
\lambda_{\mathrm{m}}=40 \mathrm{~s}^{-1} \\
\lambda_{\mathrm{k}}=0,07 \mathrm{~s}
\end{array}\right.
$$

A figura 7.10 mostra os deslocamentos verticais em função do tempo para o nó 'A' (ver figura 7.9), considerando-se ou não o amortecimento dinâmico. Para o caso amortecido, observa-se, como o esperado, uma mudança de fase no comportamento da estrutura. 


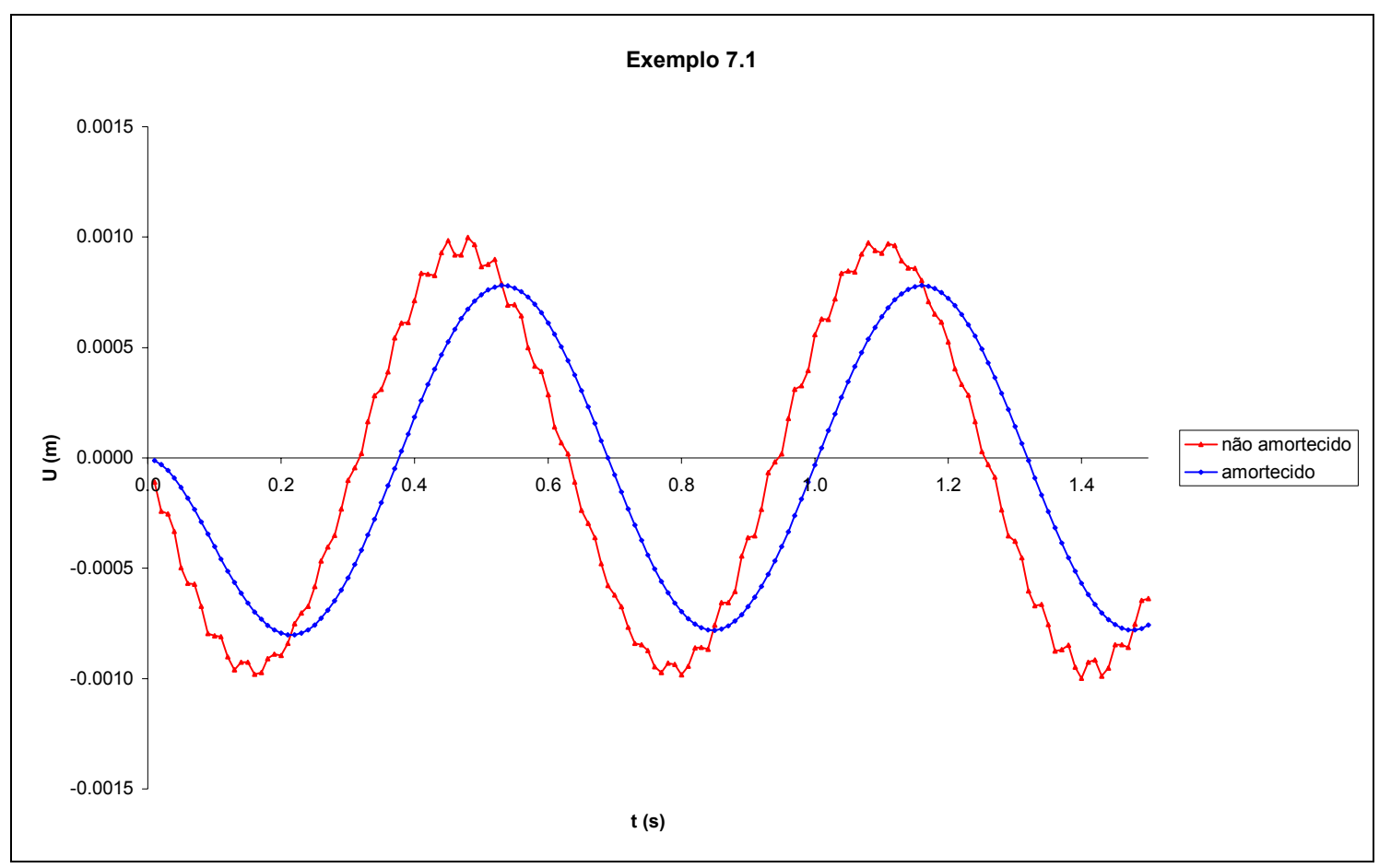

Figura 7.10 - Resultados do exemplo 7.1

\subsubsection{Exemplo 7.2}

O segundo exemplo também é referente à análise dinâmica de um pórtico, agora com apenas um pilar e uma viga, submetido à ação de uma carga de impacto distribuída na viga, conforme figura 7.11.

Utilizou-se uma discretização com um elemento de barra para o pilar e dois elementos de igual comprimento para a viga. 


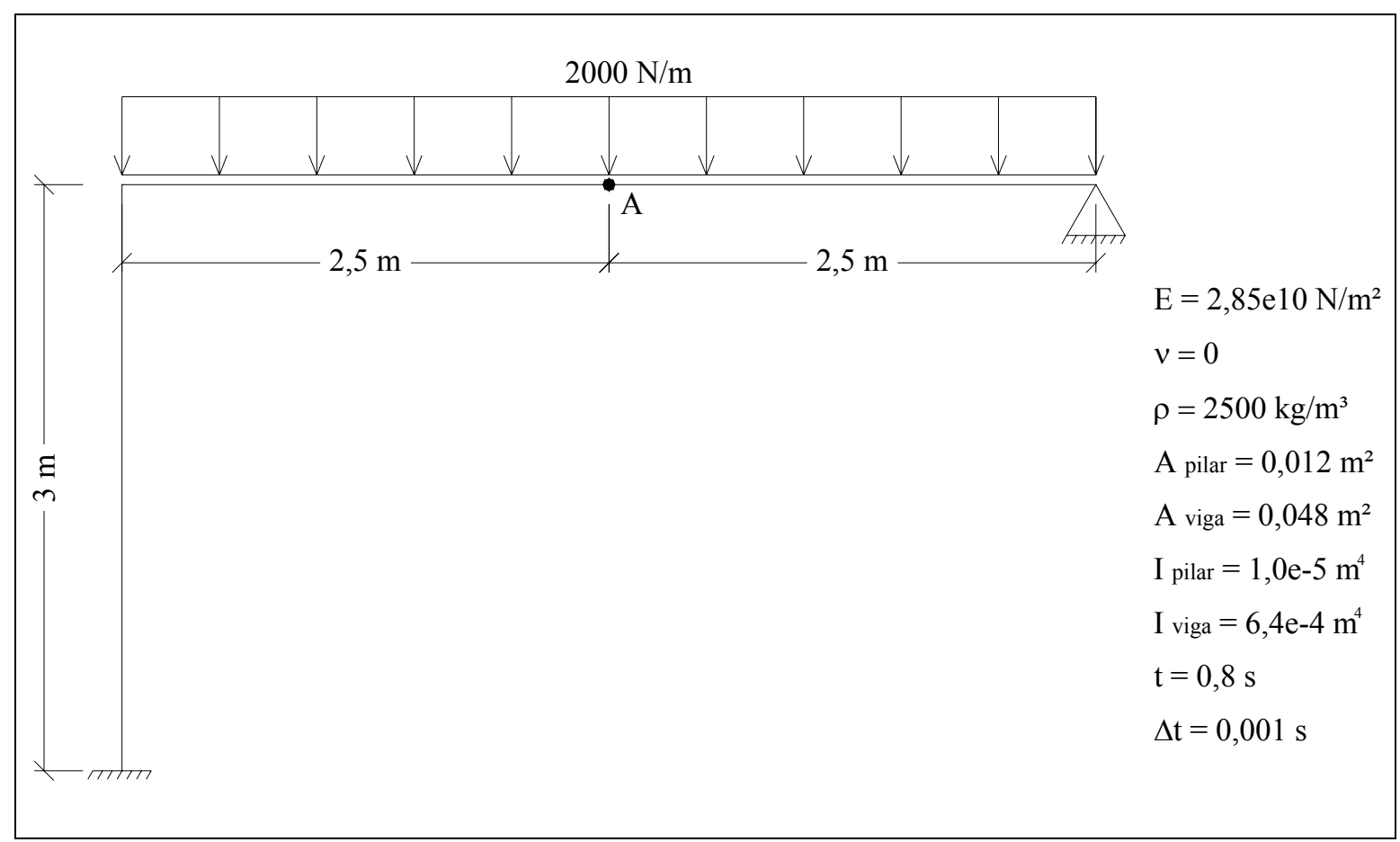

Figura 7.11 - Exemplo 7.2

A figura 7.12 apresenta os deslocamentos verticais para o ponto A em função do tempo para uma análise com amortecimento e outra sem, onde os parâmetros de amortecimento utilizados foram:

$$
\left\{\begin{array}{l}
\lambda_{m}=2 s^{-1} \\
\lambda_{k}=0,001 s
\end{array}\right.
$$

Observando-se a figura 7.12, vê-se que os deslocamentos para o comportamento sem amortecimento oscilam em torno de seu valor estático. $O$ mesmo acontece para o comportamento amortecido, e observa-se ainda que os deslocamentos neste caso convergem perfeitamente para o valor estático com o passar do tempo.

Deve-se comentar que estes resultados coincidem exatamente com os apresentados por programas similares disponíveis no Departamento de Engenharia de Estruturas da EESC/USP para problemas bidimensionais, de onde se conclui que o programa desenvolvido está resolvendo com bastante precisão problemas elastodinâmicos, com ou sem amortecimento, modelados por elementos finitos de barra. 


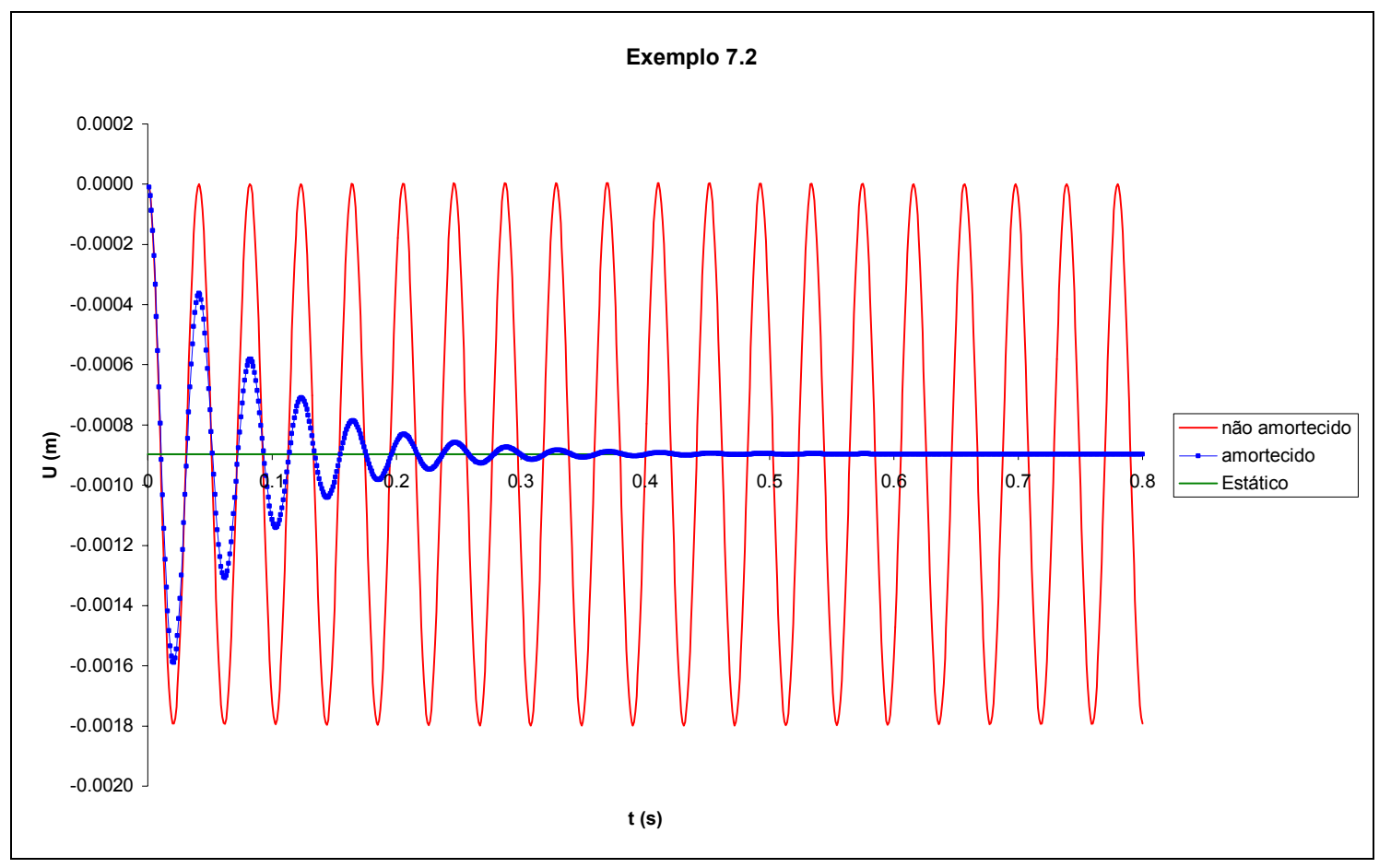

Figura 7.12 - Resultados do exemplo 7.2

\subsubsection{Exemplo 7.3}

O terceiro exemplo foi retirado da dissertação de mestrado do autor (ALMEIDA, 1999) e consiste da análise estática de uma placa quadrada, onde foram considerados os seguintes casos:

- placa simplesmente apoiada nas bordas submetida a carregamento uniformemente distribuído;

- placa engastada nas bordas submetida a carregamento uniformemente distribuído;

- placa simplesmente apoiada nas bordas submetida a carga concentrada no centro;

- placa engastada nas bordas submetida a carga concentrada no centro.

A figura 7.13 apresenta os demais dados envolvidos nas análises da placa, inclusive as discretizações utilizadas. Deve-se observar que, por questão de simetria, apenas um quarto da placa foi discretizado, onde para os lados $A B$ e $A D$ foi imposto rotação tangente nula, e para a carga concentrada, quando considerada, deve ser utilizado apenas um quarto de seu valor. 
Para a montagem dos arquivos de dados utilizou-se o pré-processador intitulado de PEC, de Sousa Junior (1996).

A tabela 7.1 apresenta os valores para o deslocamento transversal no centro da placa (ponto 'A'), bem como os erros associados àqueles, para os diversos casos de carregamento e condição de contorno. Os valores analíticos foram colhidos de Martins e Sabino (1997). Observa-se que os números de graus de liberdade ( $\mathrm{gdl}$ ) referidos na tabela são apenas aqueles dos elementos finitos DKT, sem se considerar os outros graus de liberdade do sistema total, uma vez que este possui 6 graus de liberdade por nó.

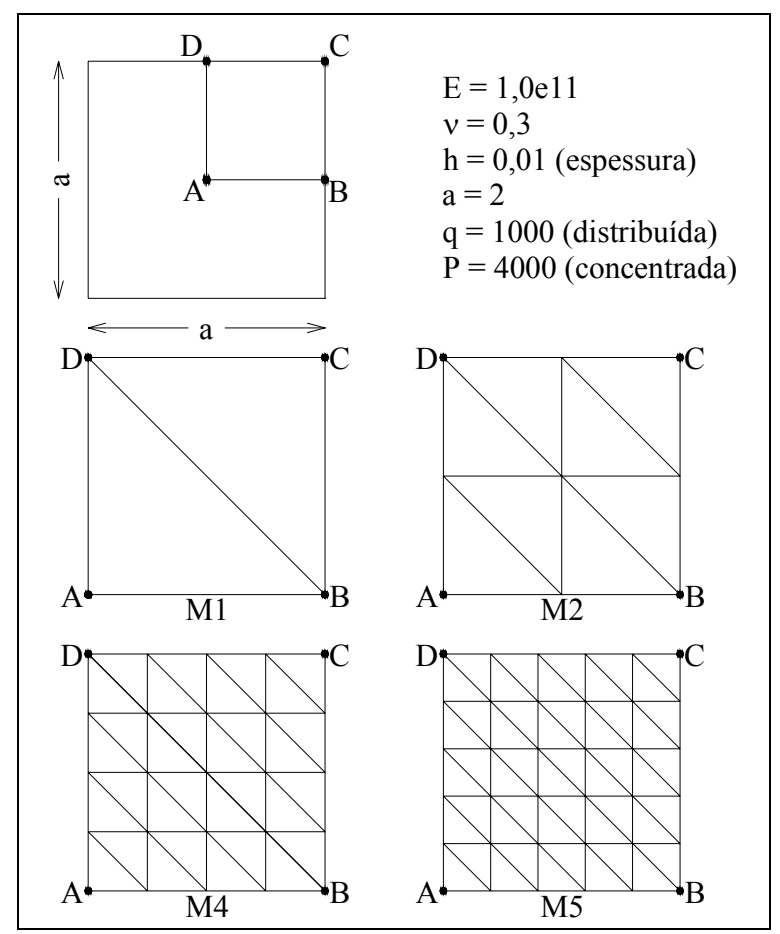

Figura 7.13 - Exemplo 7.3

Tabela 7.1 - Deslocamento transversal (U) para placa quadrada

\begin{tabular}{|c|c|c|c|c|c|c|c|c|c|c|}
\hline \multirow{3}{*}{ malha } & \multirow{3}{*}{ elementos } & \multirow{3}{*}{ gdl } & \multicolumn{4}{|c|}{ carga distribuída } & \multicolumn{4}{|c|}{ carga concentrada } \\
\hline & & & \multicolumn{2}{|c|}{ apoiada } & \multicolumn{2}{|c|}{ engastada } & \multicolumn{2}{|c|}{ apoiada } & \multicolumn{2}{|c|}{ engastada } \\
\hline & & & U.1000 & erro (\%) & $U \cdot 1000$ & erro (\%) & $U \cdot 1000$ & erro (\%) & $U \cdot 1000$ & erro (\%) \\
\hline M1 & 2 & 12 & 4,086 & $-42,436$ & 1,811 & $-18,096$ & 24,516 & 20,953 & 10,866 & 10,821 \\
\hline M2 & 8 & 27 & 6,437 & $-9,320$ & 2,134 & $-3,483$ & 22,464 & 10,829 & 11,146 & 13,677 \\
\hline M4 & 32 & 75 & 6,945 & $-2,158$ & 2,198 & $-0,579$ & 20,972 & 3,468 & 10,329 & 5,344 \\
\hline M5 & 50 & 108 & 7,001 & $-1,368$ & 2,203 & $-0,362$ & 20,748 & 2,363 & 10,172 & 3,743 \\
\hline \multicolumn{3}{|c|}{ valor analítico $\cdot 1000$} & 7,098 & 0 & 2,211 & 0 & 20,269 & 0 & 9,805 & 0 \\
\hline
\end{tabular}


A figura 7.14 foi feita a partir dos resultados apresentados na tabela 7.1, onde se pode visualizar a boa convergência do elemento finito DKT.

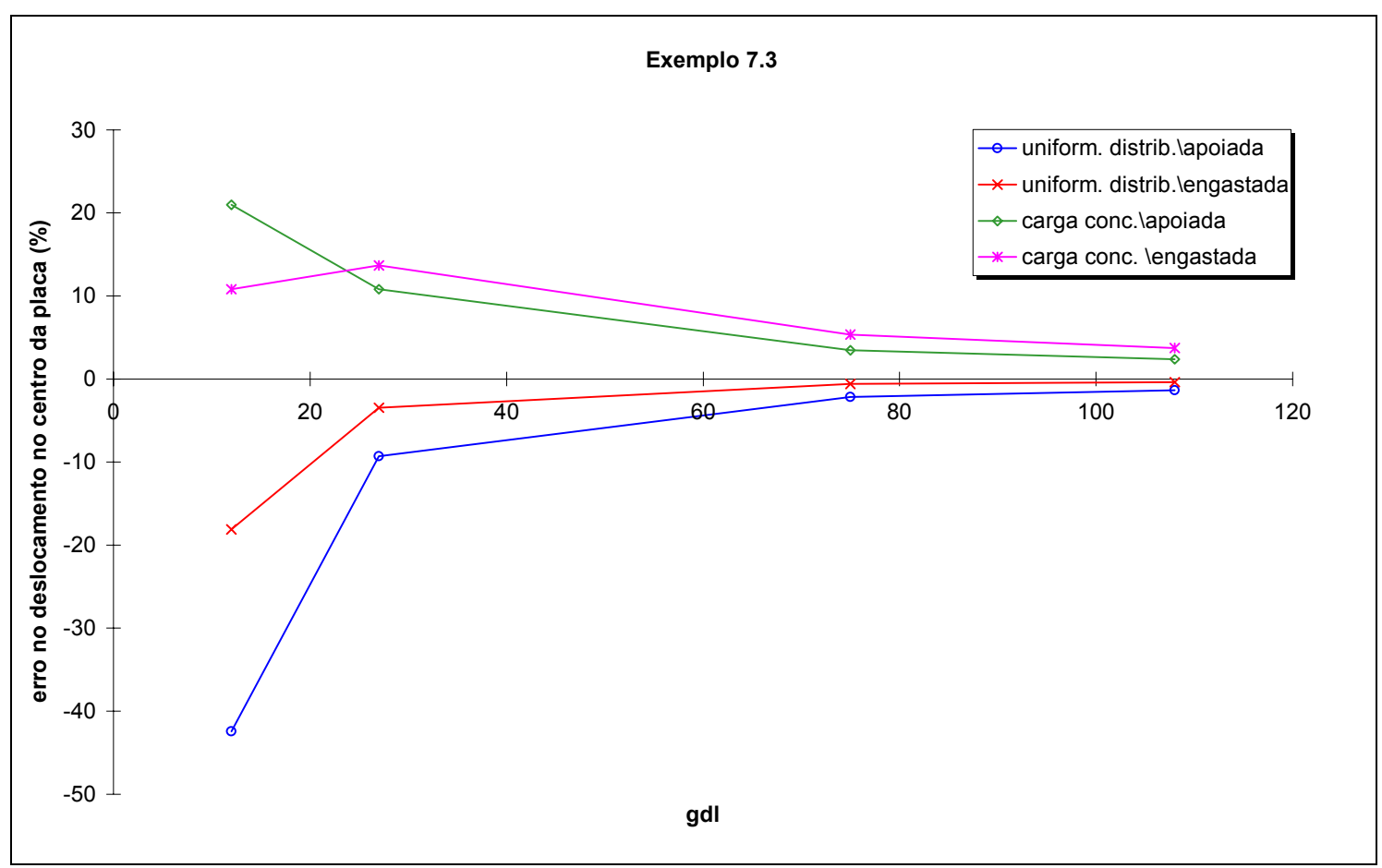

Figura 7.14 - Resultados do exemplo 7.3

\subsubsection{Exemplo 7.4}

O exemplo 7.4 analisa, estática e dinamicamente, uma placa quadrada enrijecida com vigas nas bordas e apoiada em quatro pilares. Ver figura 7.15. A análise estática do exemplo também foi retirada do trabalho de mestrado do autor (ALMEIDA, 1999), onde se pode encontrar mais detalhes a respeito do problema analisado.

Observa-se que o momento de inércia fornecido para as vigas é aquele de flexão; a rigidez à torção das mesmas foi desprezada. Observa-se também que o carregamento foi aplicado apenas na placa, e que os pilares foram considerados apoios rígidos, ou seja, os deslocamentos nas três direções foram considerados nulos naqueles pontos.

A tabela 7.2 apresenta os resultados para o deslocamento transversal calculado no centro do pavimento. As discretizações utilizadas seguem 0 mesmo esquema da figura 7.13 do exemplo 7.3, sendo que neste caso, a discretização foi para toda a placa. O valor analítico foi extraído de Timoshenko 
e Woinowsky-Krieger (1959). A mesma observação que se fez com relação ao número de graus de liberdade da tabela 7.1 do exemplo 7.3 , é válida para este caso.

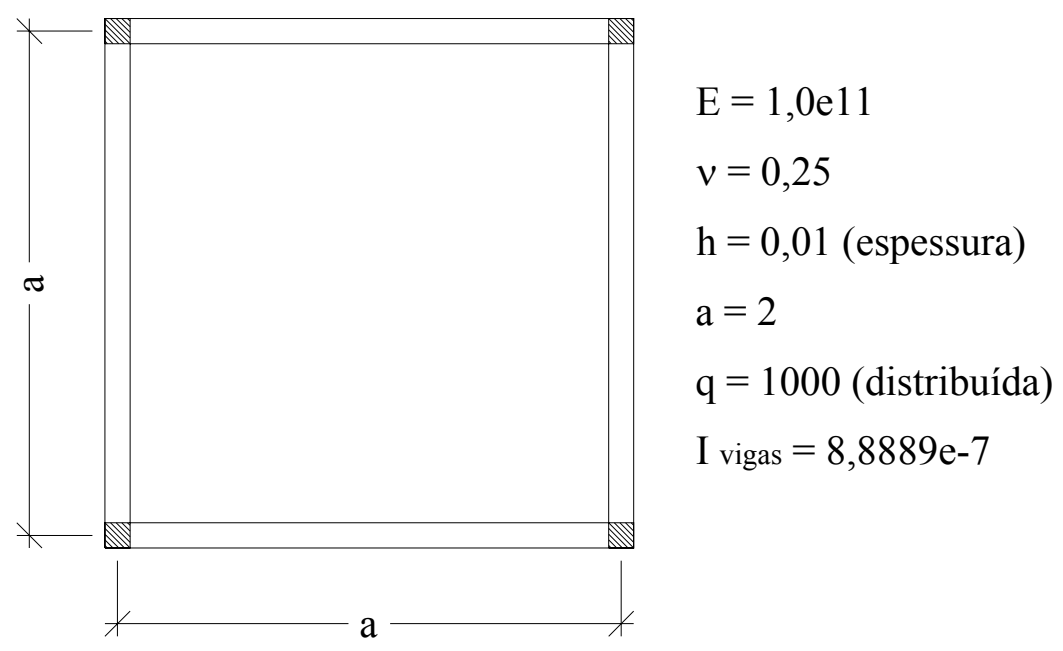

Figura 7.15 - Exemplo 7.4

Tabela 7.2 - Deslocamento transversal $(U)$ para placa apoiada em vigas

\begin{tabular}{||c|c|c|c|c||}
\hline malha & elementos & gdl & U.1000 & erro (\%) \\
\hline M2 & 8 & 27 & 7,663 & $-17,973$ \\
\hline M4 & 32 & 75 & 8,971 & $-3,971$ \\
\hline M10 & 200 & 363 & 9,300 & $-0,450$ \\
\hline \multicolumn{2}{|c|}{ valor analítico·1000 } & 9,342 & 0 \\
\hline
\end{tabular}

A figura 7.16, por sua vez, apresenta os resultados da tabela 7.2, onde se visualiza a convergência monotônica da modelagem adotada para o problema. 


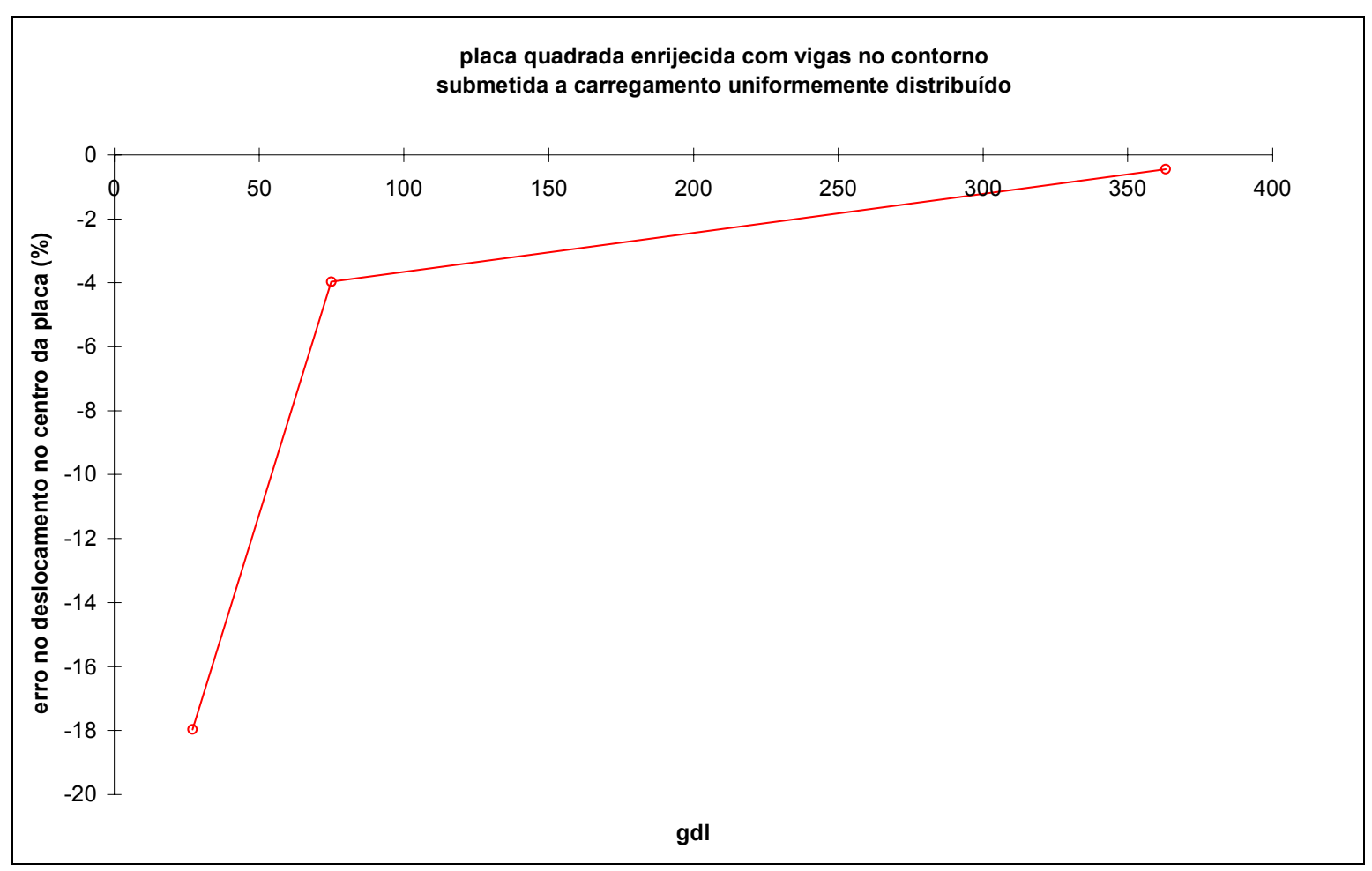

Figura 7.16 - Análise estática do exemplo 7.4

O pavimento deste exemplo também foi analisado dinamicamente, com e sem amortecimento, onde foi submetido a um carregamento de impacto de valor igual ao do problema estático. A discretização utilizada foi a M4, com 32 elementos DKT. Os demais dados envolvidos na análise dinâmica são:

$$
\left\{\begin{array}{l}
\rho=2500 \\
\lambda_{m}=8 \\
\lambda_{k}=0,004 \\
t=0,8 \\
\Delta t=0,001
\end{array}\right.
$$

A figura 7.17 apresenta os deslocamentos obtidos no centro da placa em função do tempo, tanto para o caso amortecido como para o caso sem amortecimento. Observa-se, como era de se esperar, que em ambos os casos o comportamento dinâmico oscila em torno do valor estático. Para o caso amortecido, o comportamento dinâmico converge para o valor estático, e observam-se os modos altos de vibração com bastante precisão. 


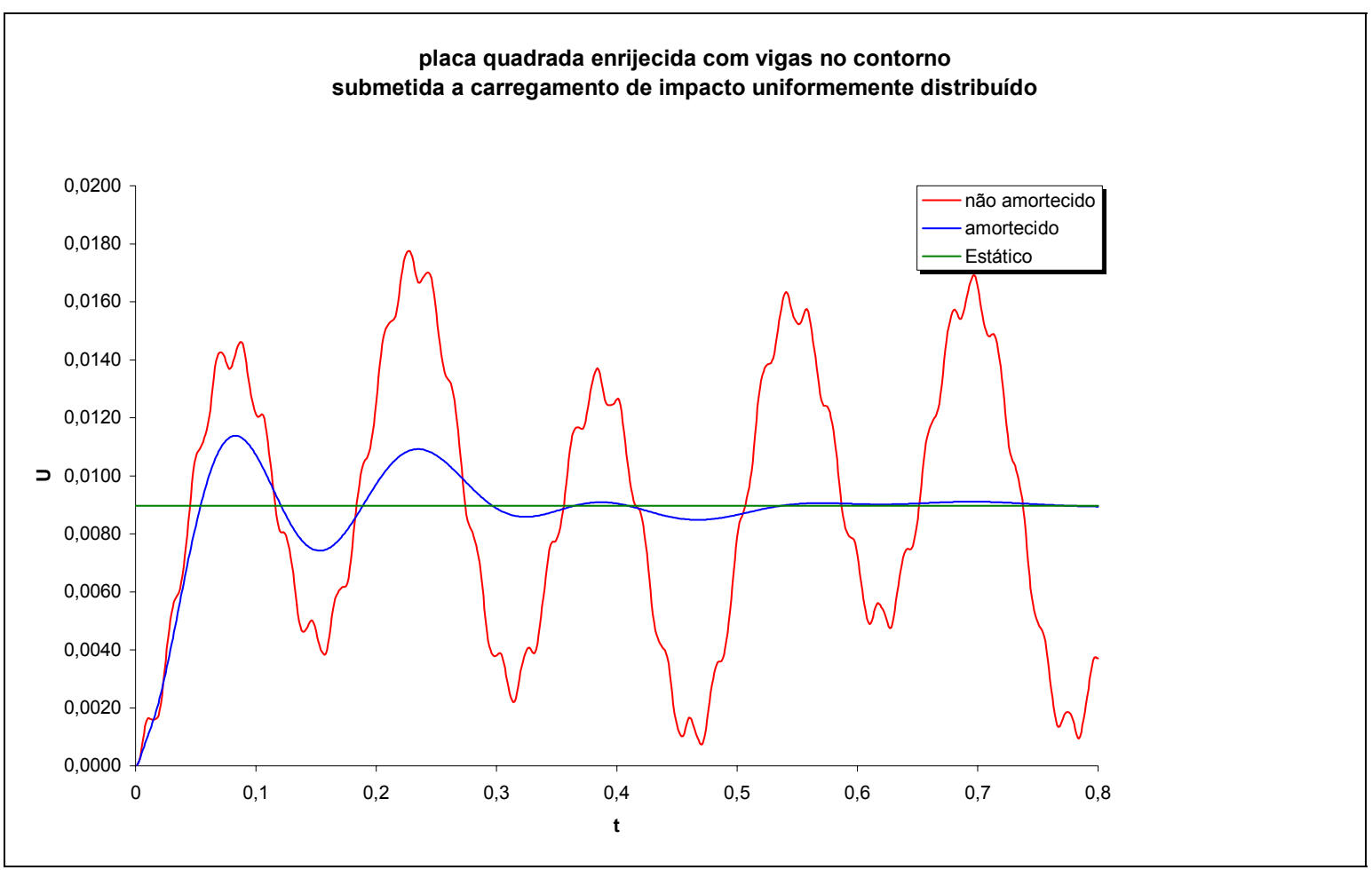

Figura 7.17 - Análise dinâmica do exemplo 7.4

\subsubsection{Exemplo 7.5}

O exemplo de número cinco é de Assan (1999) e trata da análise de uma chapa engastada sujeita a carregamento de flexão aplicado de duas formas distintas: carga concentrada nos nós superior e inferior da extremidade livre e carregamento distribuído no lado superior da chapa. As discretizações utilizadas nas análises realizadas foram as mesmas de Assan (1999). Ver figura 7.18 .

As tabelas 7.3 e 7.4 abaixo apresentam os valores para os deslocamentos transversais para o ponto ' $A$ ' da chapa, respectivamente para carga concentrada e para o carregamento distribuído. O valor analítico foi calculado levando-se em consideração o efeito de força cortante, igualmente ao que foi feito por Assan (1999). Observa-se que para as discretizações M1 e M3 o valor foi calculado como sendo a média entre os valores obtidos no nó inferior e superior da extremidade livre da chapa. Para as demais discretizações, o valor calculado foi aquele no próprio nó. 


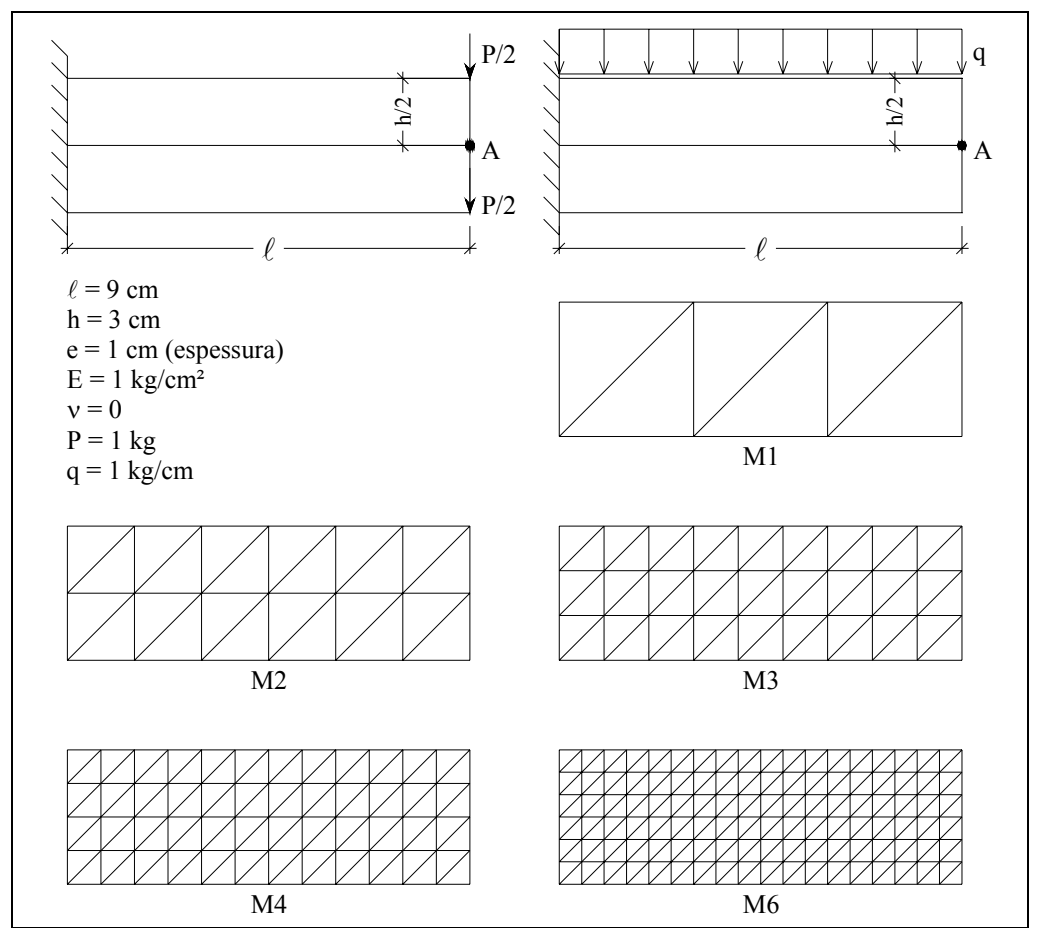

Figura 7.18 - Exemplo 7.5

Tabela 7.3 - Deslocamento transversal (U) para chapa - carga concentrada

\begin{tabular}{||c|c|c|c|c|c|c||}
\hline malha & elementos & gdl & valor & \multicolumn{3}{|c||}{ U } \\
\cline { 5 - 7 } & & & analítico & inferior & superior & valor \\
\hline M1 & 6 & 16 & 115,200 & 29,769 & 29,436 & 29,603 \\
\hline M2 & 24 & 42 & 115,200 & 63,630 & 63,412 & 63,092 \\
\hline M3 & 54 & 80 & 115,200 & 84,349 & 84,190 & 84,270 \\
\hline M4 & 96 & 130 & 115,200 & 95,705 & 95,571 & 94,722 \\
\hline M6 & 216 & 266 & 115,200 & 106,210 & 106,090 & 104,840 \\
\hline \hline
\end{tabular}

Tabela 7.4 - Deslocamento transversal (U) para chapa - carga distribuída

\begin{tabular}{||c|c|c|c|c|c|c||}
\hline malha & elementos & gdl & valor & \multicolumn{3}{|c|}{ U } \\
\cline { 5 - 8 } & & & analítico & inferior & superior & valor \\
\hline M1 & 6 & 16 & 396,900 & 107,360 & 108,280 & 107,820 \\
\hline M2 & 24 & 42 & 396,900 & 220,560 & 221,890 & 220,850 \\
\hline M3 & 54 & 80 & 396,900 & 289,790 & 291,240 & 290,515 \\
\hline M4 & 96 & 130 & 396,900 & 327,580 & 329,060 & 327,840 \\
\hline M6 & 216 & 266 & 396,900 & 362,110 & 363,610 & 362,330 \\
\hline
\end{tabular}


As figuras 7.19 e 7.20 mostram, respectivamente, os resultados apresentados nas tabelas 7.3 e 7.4 .

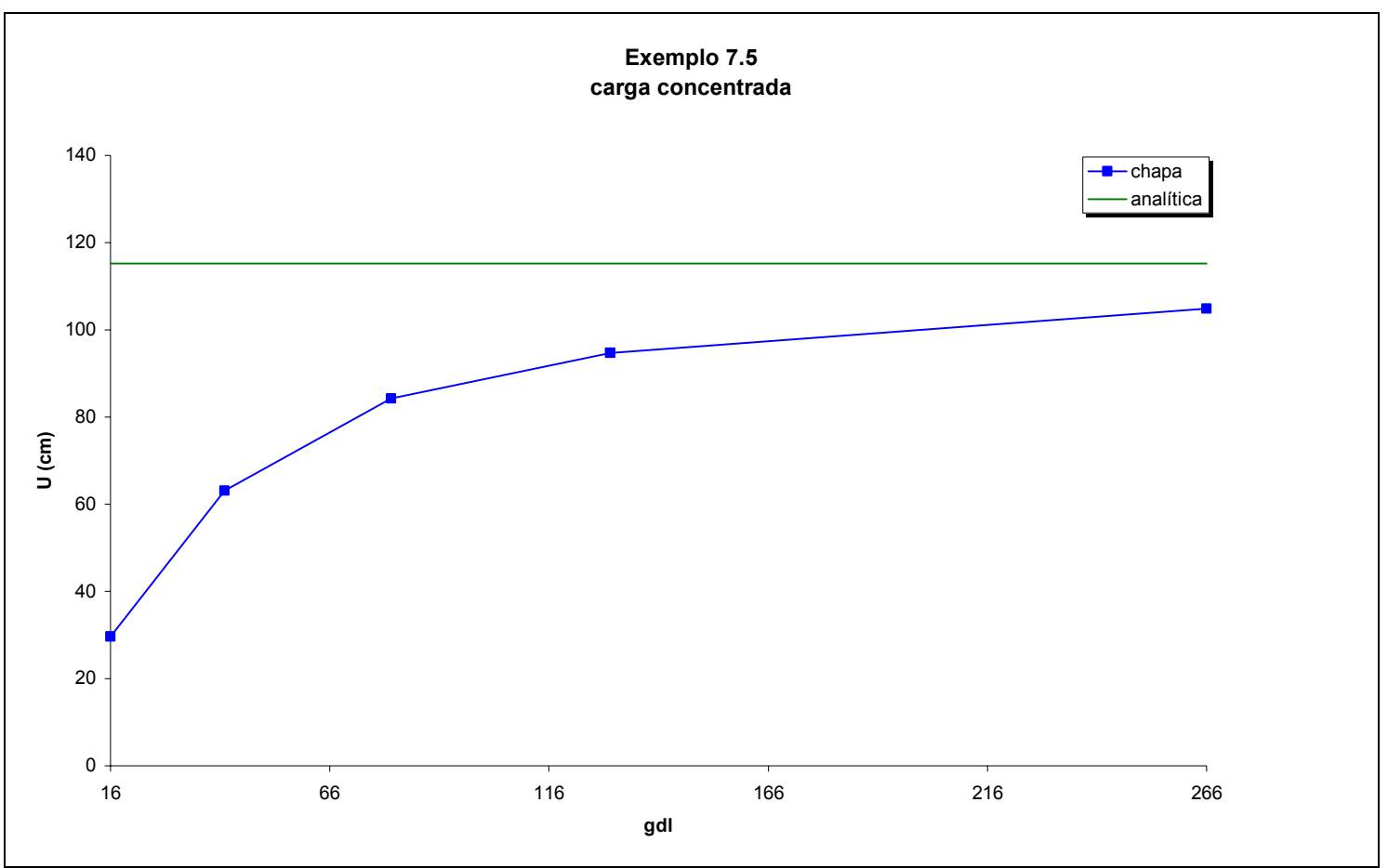

Figura 7.19 - Exemplo 7.5 - carga concentrada

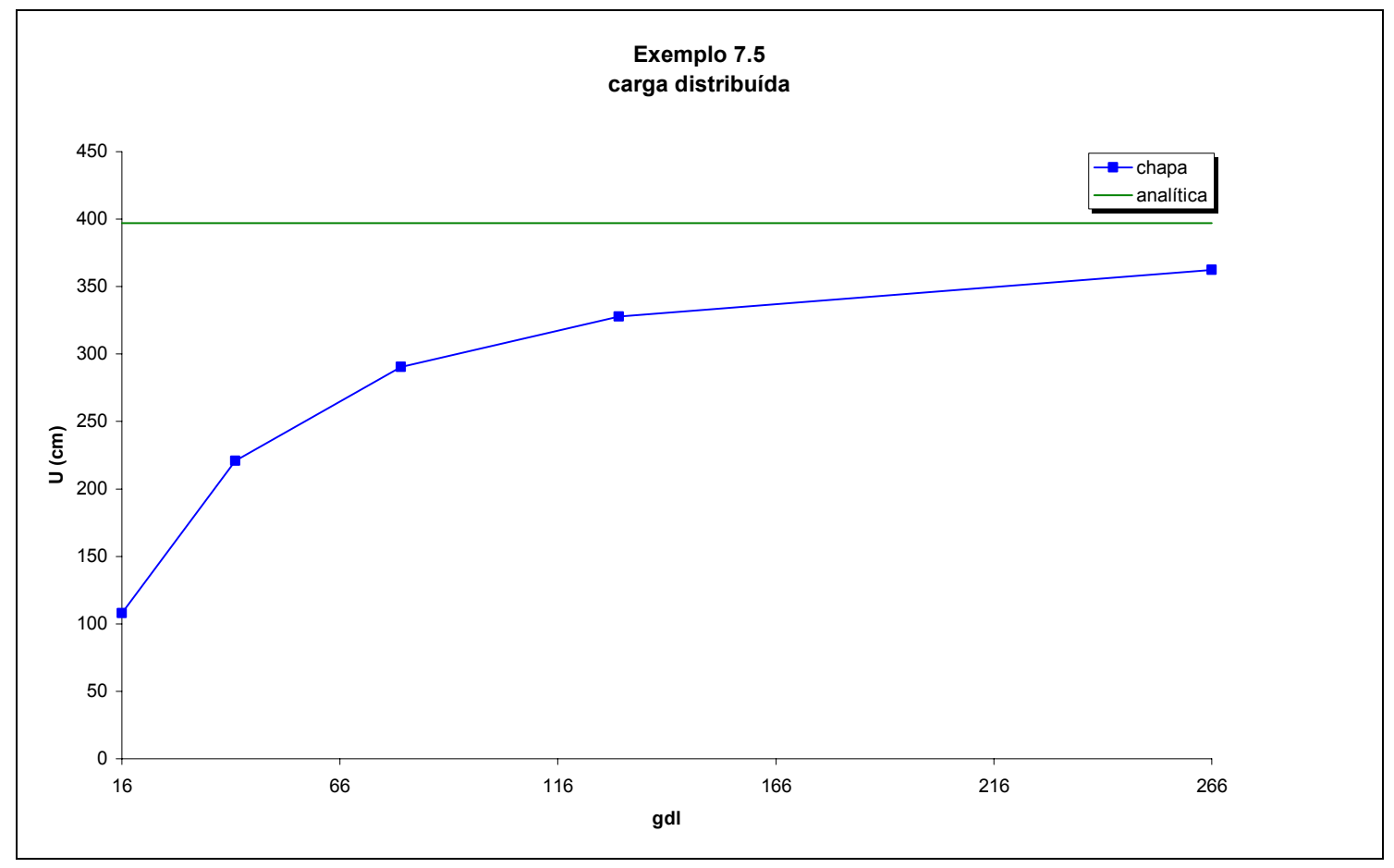

Figura 7.20 - Exemplo 7.5 - carga distribuída 
Analisando-se as figuras 7.19 e 7.20 (ou as tabelas 7.3 e 7.4) observase que o elemento de chapa utilizado (CST) apresenta convergência monotônica para a análise de problemas de flexão, apesar de bastante lenta. $\mathrm{Na}$ verdade, poder-se-ia dizer que tal elemento finito não é indicado para a solução de problema de flexão; melhor seria a utilização de outro elemento finito de chapa (como por exemplo o LST - Linear Strain Triangle), ou de um elemento finito de placa.

Complementando o exemplo de Assan (1999), analisou-se dinamicamente a chapa do presente exemplo sujeita ao carregamento concentrado, sendo que agora de impacto. Foi utilizado densidade $\rho=0,01$ $\mathrm{kg} / \mathrm{cm}^{3}, \Delta \mathrm{t}=1 \mathrm{~s}$, amortecimento nulo e discretização M6.

A figura 7.21 apresenta os deslocamentos transversais no nó ' $A$ ' em função dos passos de tempo da análise. Observa-se que a análise também foi feita com elementos finitos de barra (com uma discretização de 18 elementos finitos). As retas tracejadas representam o dobro do deslocamento estático analítico, calculado com e sem a contribuição das deformações por forças cortantes. Vê-se que os resultados obtidos, tanto com a análise utilizando elementos finitos de chapa como os de barra, foram mais próximas ao valor sem a consideração da força cortante. Esse fato era diretamente esperado da modelagem com o elemento finito de barra, pois este não considera efeitos de esforço cortante. Para o caso da chapa, além da rigidez excessiva do elemento CST, o movimento vibratório de um meio bidimensional apresenta duas ondas de propagação: uma longitudinal e outra transversal. A onda longitudinal, que se propaga mais rapidamente, irá compor efetivamente o movimento de flexão mostrado na figura, sendo que a onda transversal cisalhante é mais dispersiva e sua influência é importante no início do movimento. Assim, os picos menores para o modelo de chapa eram esperados, tal como ocorreu no modelo tridimensional de elementos de contorno. 


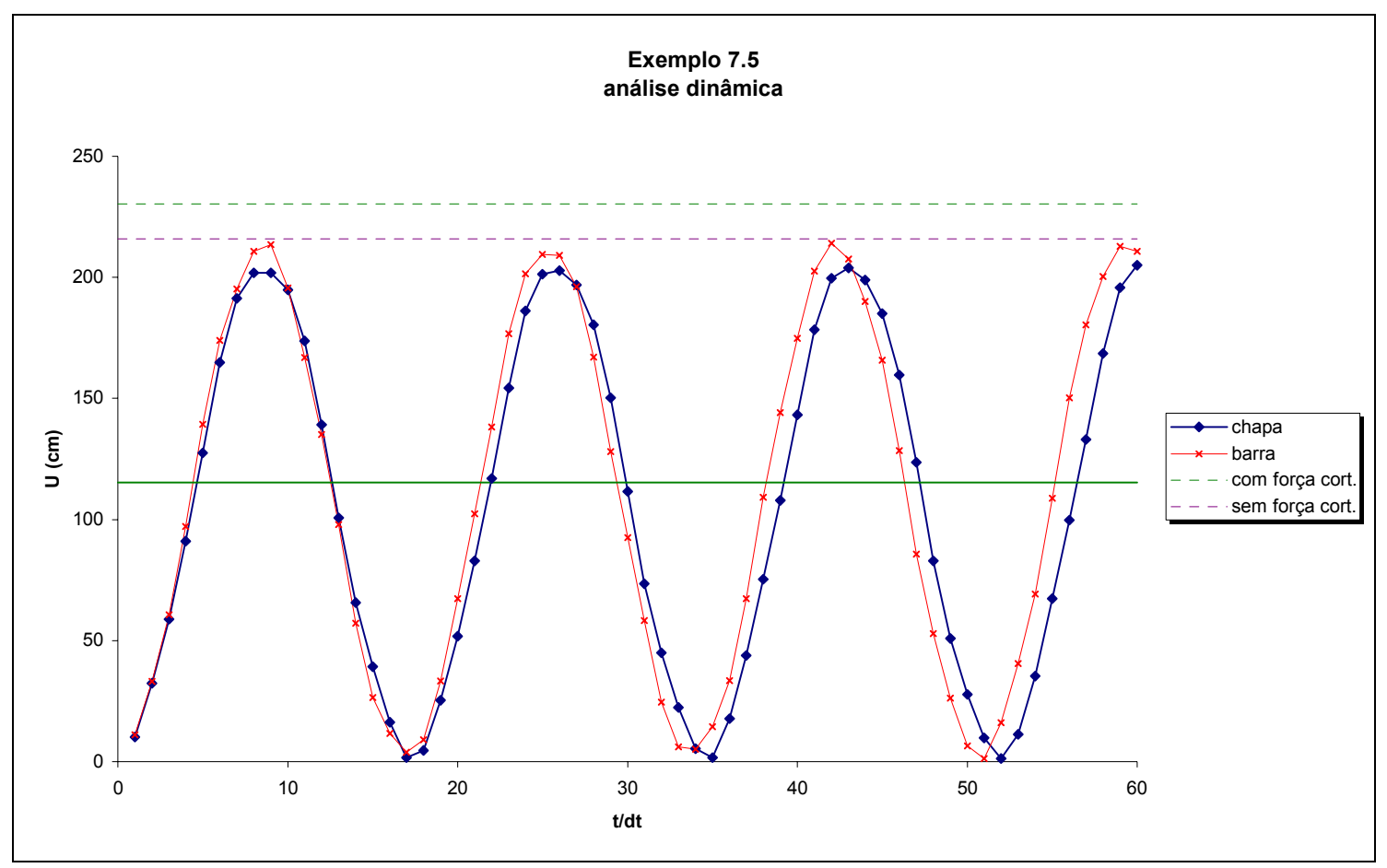

Figura 7.21 - Exemplo 7.5 - análise dinâmica 


\section{O ACOPLAMENTO ENTRE OS MÉTODOS}

Neste capítulo será descrita a forma de acoplamento utilizada no trabalho, e apresentado um exemplo numérico da técnica empregada. A partir desse desenvolvimento, viabiliza-se não apenas o modelo de interação soloestrutura, mas também outros tipos de modelos, como por exemplo: interação estrutura-estrutura, meios contínuos reforçados por fibras, deslizamento de fibras no interior de meios contínuos, entre outros. A inovação em relação aos trabalhos realizados no departamento onde se desenvolveu o presente doutorado, aplicadas a problemas similares - Almeida (2003), Coda (1993, 2000), Ferro (1998), Komatsu (1995), Mendonça (2002), Mesquita (2002), Ramalho (1990) e Tejerina Calderón (1996) -, reside na generalização do processo de acoplamento para multiregiões plastificadas.

Como já foi citado, o acoplamento entre os diferentes meios será feito aplicando-se a técnica de sub-regiões.

\subsection{TÉCNICA DE SUB-REGIÕES}

Para o MEC e MEF dinâmico, pode-se escrever, respectivamente, as seguintes equações matriciais:

$$
\begin{aligned}
& H U+C \dot{U}+M \ddot{U}=G P+B b+Q \sigma^{p} \\
& K U+\bar{C} \dot{U}+M \ddot{U}=G P+B b+Q \sigma^{p}+I F
\end{aligned}
$$


onde I é a matriz identidade.

Na eq.(8.1) pode-se fazer $F:=B b+Q \sigma^{p}$; como da mesma forma pode-se fazer $F:=B b+Q \sigma^{p}+I F$ na eq.(8.2), onde o símbolo := quer dizer "recebe". Assim, pode-se escrever:

$$
\begin{aligned}
& H U+C \dot{U}+M U ̈=G P+F \\
& K U+\bar{C} \dot{U}+M \ddot{U}=G P+F
\end{aligned}
$$

Para um instante atual, simbolizado por ' $t_{s+1}$ ', pode-se escrever:

$$
\begin{aligned}
& \overline{\mathrm{H}} \mathrm{U}_{\mathrm{s}+1}=\Delta \mathrm{t}^{2} \mathrm{GP} \mathrm{s}_{\mathrm{s}+1}+\Delta \mathrm{t}^{2} \mathrm{Q} \sigma_{\mathrm{s}+1}^{\mathrm{p}}+\mathrm{F}_{\mathrm{s}} \\
& \overline{\mathrm{K}} \mathrm{U}_{\mathrm{s}+1}=\beta \Delta \mathrm{t}^{2} \mathrm{GP}_{\mathrm{s}+1}+\beta \Delta \mathrm{t}^{2} \mathrm{Q} \sigma_{\mathrm{s}+1}^{\mathrm{p}}+\mathrm{F}_{\mathrm{s}}
\end{aligned}
$$

onde $\beta$ é parâmetro de Newmark.

Inicialmente, fazendo-se $\Delta t^{2} G=\bar{G}$ e $\Delta t^{2} Q \sigma_{s+1}^{p}+F_{s}=\bar{F}_{s+1}$ na eq.(8.5), tem-se:

$$
\overline{\mathrm{H}} \mathrm{U}_{\mathrm{s}+1}=\overline{\mathrm{G}}_{\mathrm{s}+1}+\overline{\mathrm{F}}_{\mathrm{s}+1}
$$

Da mesma forma, fazendo-se $\beta \Delta t^{2} G=\bar{G}$ e $\beta \Delta t^{2} Q \sigma_{s+1}^{p}+F_{s}=\bar{F}_{s+1}$ na eq.(8.6), pode-se escrever:

$$
\overline{\mathrm{K}} \mathrm{s}_{\mathrm{s}+1}=\overline{\mathrm{GP}}_{\mathrm{s}+1}+\overline{\mathrm{F}}_{\mathrm{s}+1}
$$

Em termos gerais, podem-se escrever as eq.(8.7) e (8.8) como:

$$
H U_{s+1}=G_{s+1}+F_{s+1}
$$


Com o objetivo de exemplificar a técnica de sub-regiões, considere-se o problema de uma viga engastada em uma extremidade e sujeita a um carregamento de tração concentrado na outra extremidade, como mostra a figura 8.1. Considere-se também que a viga foi dividida em duas sub-regiões.

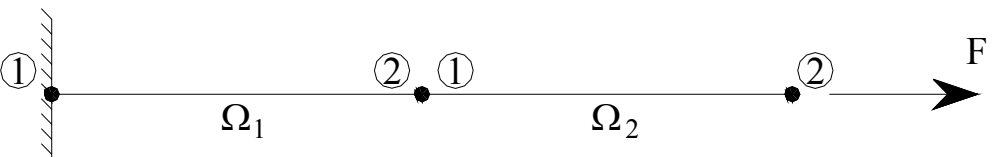

Figura 8.1 - Viga analisada pela técnica de sub-regiões

Para o citado problema, podem-se escrever as seguintes equações matriciais para cada sub-região:

$$
\begin{aligned}
& {\left[\begin{array}{ll}
h_{11}^{1} & h_{12}^{1} \\
h_{21}^{1} & h_{22}^{1}
\end{array}\right]\left\{\begin{array}{l}
U_{1}^{1} \\
U_{2}^{1}
\end{array}\right\}=\left[\begin{array}{ll}
g_{11}^{1} & g_{12}^{1} \\
g_{21}^{1} & g_{22}^{1}
\end{array}\right]\left\{\begin{array}{l}
P_{1}^{1} \\
P_{2}^{1}
\end{array}\right\}+\left\{\begin{array}{l}
F_{1}^{1} \\
F_{2}^{1}
\end{array}\right\}} \\
& {\left[\begin{array}{ll}
h_{11}^{2} & h_{12}^{2} \\
h_{21}^{2} & h_{22}^{2}
\end{array}\right]\left\{\begin{array}{l}
U_{1}^{2} \\
U_{2}^{2}
\end{array}\right\}=\left[\begin{array}{ll}
g_{11}^{2} & g_{12}^{2} \\
g_{21}^{2} & g_{22}^{2}
\end{array}\right]\left\{\begin{array}{l}
P_{1}^{2} \\
P_{2}^{2}
\end{array}\right\}+\left\{\begin{array}{l}
F_{1}^{2} \\
F_{2}^{2}
\end{array}\right\}}
\end{aligned}
$$

Observa-se que o índice superior é referente à sub-região.

Como $U_{1}^{1}$ é conhecido (no caso, nulo), a equação matricial referente ao meio 1 (eq.(8.10)) pode ser escrita da seguinte forma:

$$
\left[\begin{array}{ll}
-g_{11}^{1} & h_{12}^{1} \\
-g_{21}^{1} & h_{22}^{1}
\end{array}\right]\left\{\begin{array}{l}
P_{1}^{1} \\
U_{2}^{1}
\end{array}\right\}=\left[\begin{array}{ll}
-h_{11}^{1} & g_{12}^{1} \\
-h_{21}^{1} & g_{22}^{1}
\end{array}\right]\left\{\begin{array}{l}
U_{1}^{1} \\
P_{2}^{1}
\end{array}\right\}+\left\{\begin{array}{l}
F_{1}^{1} \\
F_{2}^{1}
\end{array}\right\}
$$

Independentemente do tipo de técnica adotada, as seguintes condições precisam ser satisfeitas:

$$
\left\{\begin{array}{l}
\text { compatibilidade geométrica (cinemática) }: U_{2}^{1}=U_{1}^{2} \\
\text { equilíbrio }: P_{2}^{1}=-P_{1}^{2}
\end{array}\right.
$$


Com o objetivo de acoplar, ou seja, combinar numericamente, as eq.(8.12) e (8.11), aplica-se as condições descritas acima ao problema. Assim, podem-se escrever as seguintes equações:

$$
\begin{aligned}
-g_{11}^{1} P_{1}^{1}+h_{12}^{1} U_{2}^{1} & =-h_{11}^{1} U_{1}^{1}+g_{12}^{1} P_{2}^{1}+F_{1}^{1} \\
-g_{21}^{1} P_{1}^{1}+h_{22}^{1} U_{2}^{1} & =-h_{21}^{1} U_{1}^{1}+g_{22}^{1} P_{2}^{1}+F_{2}^{1} \\
h_{11}^{2} U_{2}^{1}+h_{12}^{2} U_{2}^{2} & =-g_{11}^{2} P_{2}^{1}+g_{12}^{2} P_{2}^{2}+F_{1}^{2} \\
h_{21}^{2} U_{2}^{1}+h_{22}^{2} U_{2}^{2} & =-g_{21}^{2} P_{2}^{1}+g_{22}^{2} P_{2}^{2}+F_{2}^{2}
\end{aligned}
$$

Vale observar que a força de contato entre o meio 1 e o meio 2 pode ser acrescida de uma força aplicada ao nó 2 do meio 1 (ou nó 1 do meio 2), sendo assim, pode-se escrever:

$\mathrm{P}_{2}^{1}=\overline{\mathrm{P}}_{2}^{1}+\mathrm{P}_{21}^{1}$

ou

$\mathrm{P}_{1}^{2}=\overline{\mathrm{P}}_{1}^{2}+\mathrm{P}_{12}^{2}$

onde, na eq.(8.14):

$\overline{\mathrm{P}}_{2}^{1}$ - força aplicada no nó 2 do meio 1 (conhecida);

$\mathrm{P}_{21}^{1} \quad$ - força de contato entre o meio 1 e o meio 2 (antigo $P_{2}^{1}$ );

valendo a mesma analogia para a eq.(8.15).

A equação de equilíbrio pode ser escrita agora como:

$P_{21}^{1}=-P_{12}^{2}$

Aplicando-se a eq.(8.14) às eq.(8.13) e colocando-se os valores conhecidos à direita e os incógnitos à esquerda, pode-se escrever: 


$$
\begin{aligned}
-g_{11}^{1} P_{1}^{1}+h_{12}^{1} U_{2}^{1}-g_{12}^{1} P_{21}^{1} & =-h_{11}^{1} U_{1}^{1}+g_{12}^{1} \bar{P}_{2}^{1}+F_{1}^{1} \\
-g_{21}^{1} P_{1}^{1}+h_{22}^{1} U_{2}^{1}-g_{22}^{1} P_{21}^{1} & =-h_{21}^{1} U_{1}^{1}+g_{22}^{1} \bar{P}_{2}^{1}+F_{2}^{1} \\
h_{11}^{2} U_{2}^{1}+h_{12}^{2} U_{2}^{2}+g_{11}^{2} P_{21}^{1} & =g_{11}^{2} \bar{P}_{1}^{2}+g_{12}^{2} P_{2}^{2}+F_{1}^{2} \\
h_{21}^{2} U_{2}^{1}+h_{22}^{2} U_{2}^{2}+g_{21}^{2} P_{21}^{1} & =g_{21}^{2} \bar{P}_{1}^{2}+g_{22}^{2} P_{2}^{2}+F_{2}^{2}
\end{aligned}
$$

Matricialmente:

$$
\begin{aligned}
& \begin{array}{lllll}
\mathrm{H}_{\mathrm{e}}^{1} & \mathrm{H}_{\mathrm{i}}^{1} & -\mathrm{G}_{\mathrm{i}}^{1} & \mathrm{G}_{\mathrm{e}}^{1} & \mathrm{G}_{\mathrm{i}}^{1}
\end{array}
\end{aligned}
$$

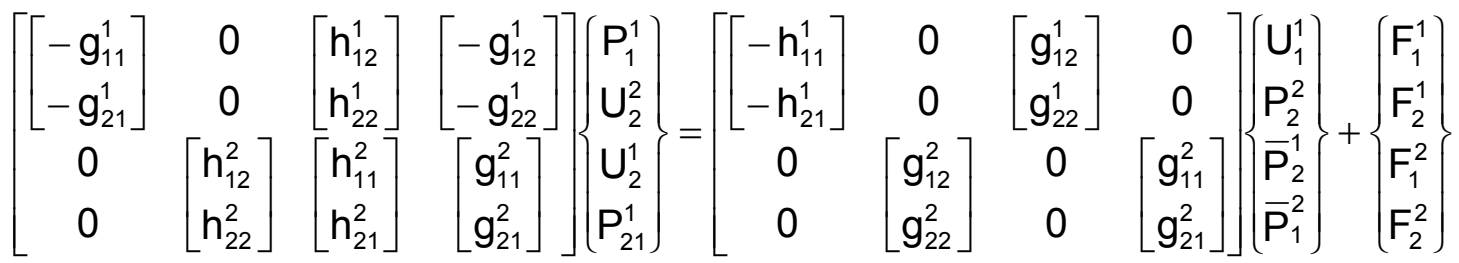

$$
\begin{aligned}
& \begin{array}{lllll}
\mathrm{H}_{\mathrm{e}}^{2} & \mathrm{H}_{\mathrm{i}}^{2} & \mathrm{G}_{\mathrm{i}}^{2} & \mathrm{G}_{\mathrm{e}}^{2} & \mathrm{G}_{\mathrm{i}}^{2}
\end{array}
\end{aligned}
$$

ou simplesmente:

$$
\left[\begin{array}{cccc}
H_{e}^{1} & 0 & H_{i}^{1} & -G_{i}^{1} \\
0 & H_{e}^{2} & H_{i}^{2} & G_{i}^{2}
\end{array}\right]\left\{\begin{array}{c}
P_{e}^{1} \\
U_{e}^{2} \\
U_{i}^{1} \\
P_{i}^{1}
\end{array}\right\}=\left[\begin{array}{cccc}
G_{e}^{1} & 0 & G_{i}^{1} & 0 \\
0 & G_{e}^{2} & 0 & G_{i}^{2}
\end{array}\right]\left\{\begin{array}{c}
U_{e}^{1} \\
P_{e}^{2} \\
P_{i}^{1} \\
P_{i}^{2}
\end{array}\right\}+\left\{\begin{array}{l}
F^{1} \\
F^{2}
\end{array}\right\}
$$

onde os índices 'e' e 'i' são referentes a 'externo' e 'interno', respectivamente, ou a 'não-ligado' e 'ligado'. Deve-se observar que na eq.(8.18) as condições de contorno já foram aplicadas.

Consideram-se as duas sub-regiões apresentadas na figura 8.2.

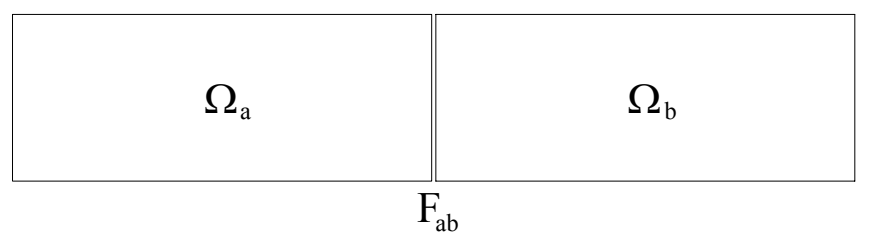

Figura 8.2 - Duas sub-regiões

Para as duas sub-regiões pode-se escrever: 
$\Omega_{\mathrm{a}} \rightarrow \mathrm{H}^{\mathrm{a}} \mathrm{U}^{\mathrm{a}}=\mathrm{G}^{\mathrm{a}} \mathrm{P}^{\mathrm{a}}+\mathrm{F}^{\mathrm{a}}$

$\Omega_{\mathrm{b}} \rightarrow \mathrm{H}^{\mathrm{b}} \mathrm{U}^{\mathrm{b}}=\mathrm{G}^{\mathrm{b}} \mathrm{P}^{\mathrm{b}}+\mathrm{F}^{\mathrm{b}}$

$\left[\begin{array}{ll}H_{e}^{a} & H_{i}^{a}\end{array}\right]\left\{\begin{array}{l}U_{e}^{a} \\ U_{i}^{a}\end{array}\right\}=\left[\begin{array}{ll}G_{e}^{a} & G_{i}^{a}\end{array}\right\}\left\{\begin{array}{c}P_{e}^{a} \\ P_{i}^{a}+\bar{P}_{i}^{a}\end{array}\right\}+\left\{F^{a}\right\}$

$\left[\begin{array}{ll}H_{e}^{b} & H_{i}^{b}\end{array}\right]\left\{\begin{array}{l}U_{e}^{b} \\ U_{i}^{b}\end{array}\right\}=\left[\begin{array}{ll}G_{e}^{b} & G_{i}^{b}\end{array}\right]\left\{\begin{array}{c}P_{e}^{b} \\ P_{i}^{b}+\bar{P}_{i}^{b}\end{array}\right\}+\left\{\begin{array}{l}F^{b} \\ y^{b}\end{array}\right.$

$H_{e}^{a} U_{e}^{a}+H_{i}^{a} U_{i}^{a}=G_{e}^{a} P_{e}^{a}+G_{i}^{a} P_{i}^{a}+G_{i}^{a} \bar{P}_{i}^{a}+F^{a}$

$H_{e}^{b} U_{e}^{b}+H_{i}^{b} U_{i}^{b}=G_{e}^{b} P_{e}^{b}+G_{i}^{b} P_{i}^{b}+G_{i}^{b} \bar{P}_{i}^{b}+F^{b}$

Aplicando-se as condições de contato, tem-se:

$\left\{\begin{array}{c}U_{i}^{a}=U_{i}^{b} \\ P_{i}^{a}=-P_{i}^{b}\end{array} \Rightarrow \begin{array}{l}H_{e}^{a} U_{e}^{a}+H_{i}^{a} U_{i}^{a}=G_{e}^{a} P_{e}^{a}+G_{i}^{a} P_{i}^{a}+G_{i}^{a} \bar{P}_{i}^{a}+F^{a} \\ H_{e}^{b} U_{e}^{b}+H_{i}^{b} U_{i}^{a}=G_{e}^{b} P_{e}^{b}-G_{i}^{b} P_{i}^{a}+G_{i}^{b} \bar{P}_{i}^{b}+F^{b}\end{array}\right.$

Matricialmente:

$\left[\begin{array}{cccc}H_{e}^{a} & 0 & H_{i}^{a} & -G_{i}^{a} \\ 0 & H_{e}^{b} & H_{i}^{b} & G_{i}^{b}\end{array}\right]\left\{\begin{array}{c}U_{e}^{a} \\ U_{e}^{b} \\ U_{i}^{a} \\ P_{i}^{a}\end{array}\right\}=\left[\begin{array}{cccc}G_{e}^{a} & 0 & G_{i}^{a} & 0 \\ 0 & G_{e}^{b} & 0 & G_{i}^{b}\end{array}\right]\left\{\begin{array}{l}P_{e}^{a} \\ P_{e}^{b} \\ \bar{P}_{i}^{a} \\ \bar{P}_{i}^{b}\end{array}\right\}+\left\{\begin{array}{l}F^{a} \\ F^{b}\end{array}\right\}$

Para três ou mais meios (figura 8.3) a técnica de sub-regiões segue o esquema abaixo:

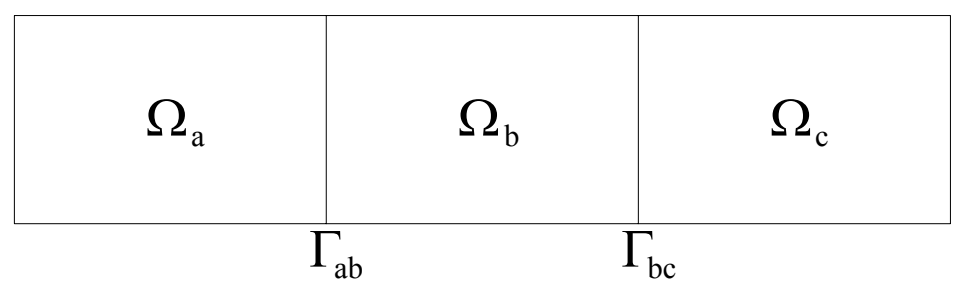

Figura 8.3 - Três sub-regiões 


$$
\begin{aligned}
& H_{e}^{a} U_{e}^{a}+H_{i}^{a} U_{i}^{a b}=G_{e}^{a} P_{e}^{a}+G_{i}^{a} \bar{P}_{i}^{a b}+G_{i}^{a} P_{i}^{a b}+F^{a} \\
& H_{e}^{b} U_{e}^{b}+H_{i}^{b a} U_{i}^{b a}+H_{i}^{b c} U_{i}^{b c}=G_{e}^{b} P_{e}^{b}+G_{i}^{b a} \bar{P}_{i}^{b a}+G_{i}^{b a} P_{i}^{b a}+G_{i}^{b c} \bar{P}_{i}^{b c}+G_{i}^{b c} P_{i}^{b c}+F^{b} \\
& H_{e}^{c} U_{e}^{c}+H_{i}^{c} U_{i}^{c b}=G_{e}^{c} P_{e}^{c}+G_{i}^{c} \bar{P}_{i}^{c b}+G_{i}^{c} P_{i}^{c b}+F^{c}
\end{aligned}
$$$$
\left[\begin{array}{ccccccc}
H_{e}^{a} & 0 & 0 & H_{i}^{a} & -G_{i}^{a} & 0 & 0 \\
0 & H_{e}^{b} & 0 & H_{i}^{b a} & G_{i}^{b a} & H_{i}^{b c} & -G_{i}^{b c} \\
0 & 0 & H_{e}^{c} & 0 & 0 & H_{i}^{c} & G_{i}^{c}
\end{array}\right]\left\{\begin{array}{c}
U_{e}^{a} \\
U_{e}^{b} \\
U_{e}^{c} \\
U_{i}^{a b} \\
P_{i}^{a b} \\
U_{i}^{b c} \\
P_{i}^{b c}
\end{array}\right\}=\left[\begin{array}{ccccccc}
G_{e}^{a} & 0 & 0 & G_{i}^{a} & 0 & 0 & 0 \\
0 & G_{e}^{b} & 0 & 0 & G_{i}^{b a} & G_{i}^{b c} & 0 \\
0 & 0 & G_{e}^{c} & 0 & 0 & 0 & G_{i}^{c}
\end{array}\right]\left\{\begin{array}{l}
P_{e}^{a} \\
P_{e}^{b} \\
P_{e}^{c} \\
P_{i}^{a b} \\
P_{i}^{b a} \\
\bar{P}_{i}^{b c} \\
\bar{P}_{i}^{c b}
\end{array}\right\}+\left\{\begin{array}{l}
F^{a b} \\
F^{b} \\
F^{c}
\end{array}\right\}
$$

e assim sucessivamente.

Deve-se observar que as equações do tipo da eq.(8.25) estão escritas para um determinado instante da análise; quando a plastificação ocorre, a solução da mesma deve se dar de forma iterativa. Os resíduos plásticos são incluídos nos vetores de carga externa como mostrado nas simplificações descritas após a eq.(8.2).

\subsection{EXEMPLO}

O problema estudado neste exemplo já foi apresentado no capítulo 4, onde se utilizou unicamente o MMBEM, e no capítulo 6, onde o TDBEM foi aplicado; analisa-se o caso de uma viga engastada em uma extremidade e sujeita à ação de uma carga constante de tração aplicada subitamente à outra extremidade. Ver figura 8.4 abaixo.

Desta vez, analisou-se o problema descrito acima de diversas formas: via elementos finitos de barra, elementos finitos de chapa, acoplamento MEF/MEF entre sub-regiões de elementos finitos de barra, acoplamento MEC/MEC e acoplamento MEC/MEF entre sub-regiões de elementos de contorno e elementos finitos de barra. Neste exemplo, onde se lê MEC, deve ficar claro que se trata do MMBEM. Para a análise com elementos finitos de 
barra, utilizaram-se 10 elementos de barra para a discretização da viga; para a análise com elementos finitos de chapa, foram utilizados 40 elementos CST; para a análise utilizando o processo de acoplamento MEF/MEF, dividiu-se a viga em duas sub-regiões de elementos de barra (cada uma com 5 elementos) e acoplou-se os 6 graus de liberdade da interface entre elas; para o acoplamento MEC/MEC, a viga foi dividida em duas sub-regiões de elementos de contorno - cada qual com uma discretização do tipo $225^{1}$, ou seja, 2 divisões na largura $(\ell), 2$ divisões na profundidade (prof) e 5 divisões no comprimento $(L)$ - onde todos os graus de liberdade da interface foram acoplados; por fim, para o acoplamento MEC/MEF, dividiu-se a viga em duas sub-regiões: uma discretizada por elementos de contorno e outra discretizada por elementos finitos de barra, e entre elas, uma casca com características especiais, funcionando como "elemento de ligação". Coda (1993) também analisou este problema, utilizando o acoplamento MEC/MEF, em seu exemplo 8.4.2. Maiores detalhes podem ser vistos na figura 8.5.

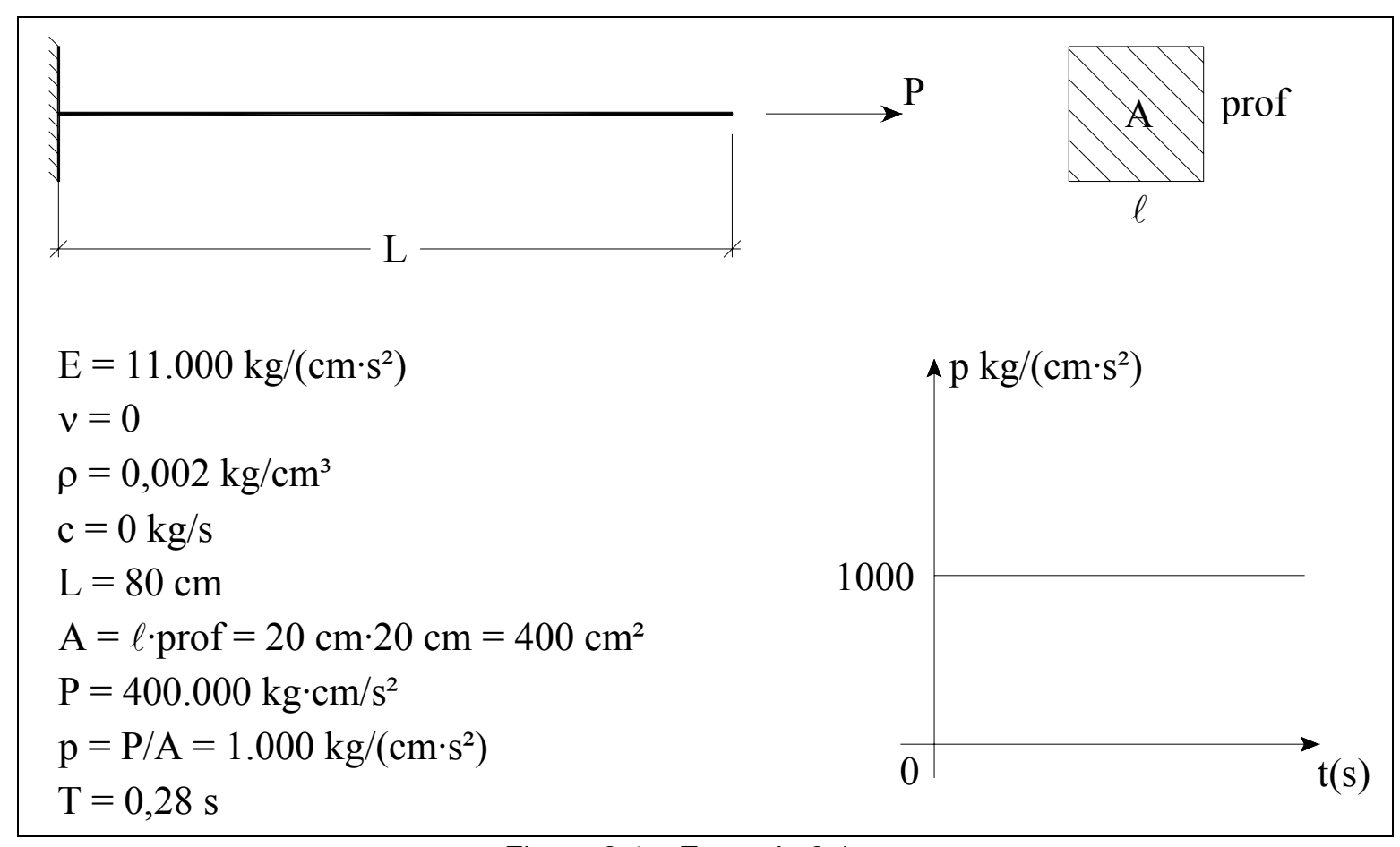

Figura 8.4 - Exemplo 8.1 


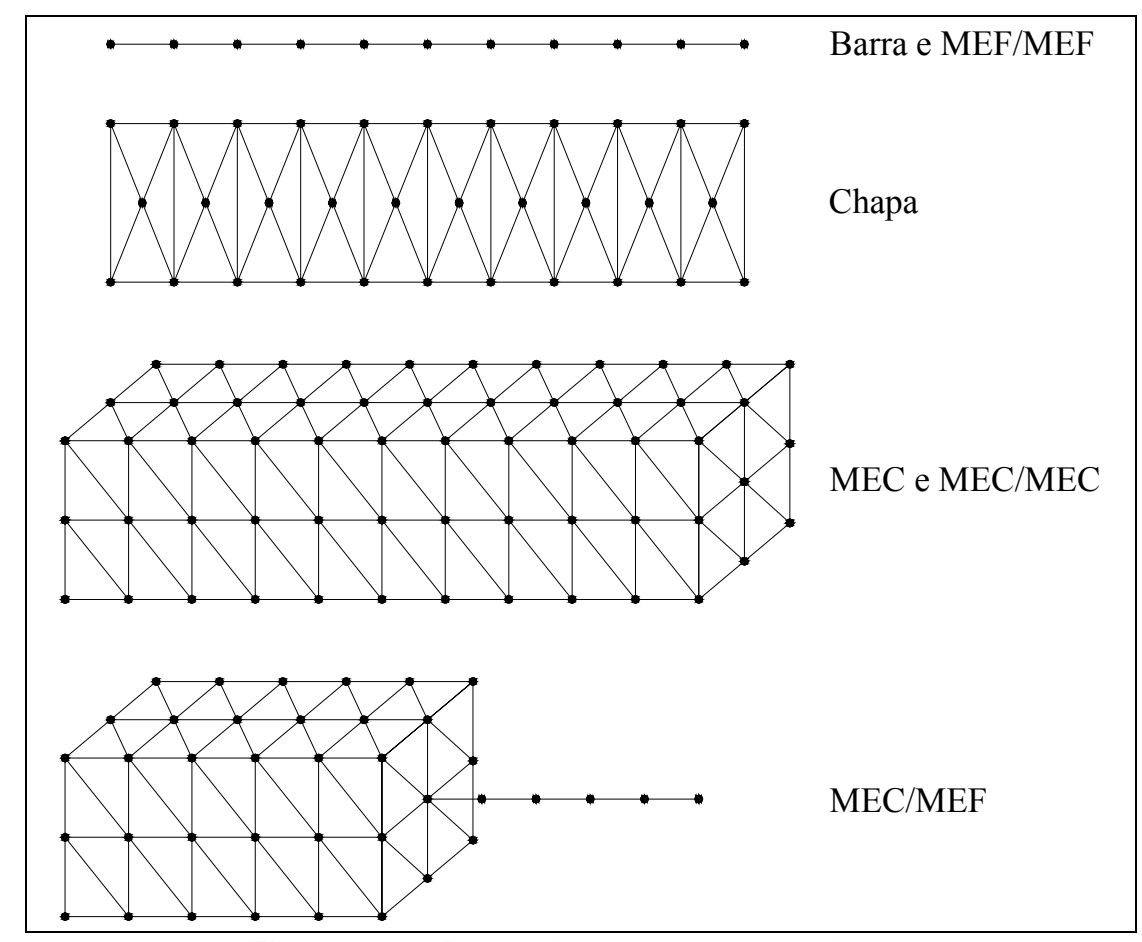

Figura 8.5 - Discretizações do exemplo 8.1

Mais algumas observações são necessárias ao entendimento das análises realizadas. Para o acoplamento MEF/MEF, vale dizer que a matriz da sub-região a ser alocada na matriz total $G$ é a matriz $\beta \Delta t^{2}$ I e não a matriz $\beta \Delta t^{2} G$. Conseqüentemente, o vetor a ser alocado no vetor $P_{s+1}$ total será o vetor $\mathrm{y}_{\mathrm{s}+1}$ (vetor dos valores conhecidos no instante atual), e o vetor a ser alocado no vetor de cargas total $\left(\mathrm{F}_{\mathrm{s}+1}\right)$ será o vetor $\beta \Delta \mathrm{t}^{2} \mathrm{GP}_{\mathrm{s}+1}+\overline{\overline{\mathrm{F}}}_{\mathrm{s}}$. Para maiores detalhes, ver seções 7.5 e 8.1. Para a análise utilizando elementos finitos de chapa, o carregamento foi aplicado uniformemente no lado livre da viga. Para a análise utilizando o acoplamento $M E C / M E F$, a sub-região de elementos finitos é composta, além dos elementos de barra, por uma casca rígida de ligação; para tal, considerou-se módulo de elasticidade $E=1,1 e+10$ $\mathrm{kg} /\left(\mathrm{cm} \cdot \mathrm{s}^{2}\right)$, espessura $\mathrm{h}=1 \mathrm{~cm}$ e densidade nula $(\rho=0)$ para a casca. Além disso, neste tipo de análise, deve-se considerar o primeiro elemento finito de barra, ligado à casca como um "elemento de topo", ou seja, a contribuição da matriz $G$ deste elemento não deve ser computada na montagem da matriz $G$ da subestrutura, evitando a avaliação errônea das forças de contato. 
A figura 8.6 apresenta os deslocamentos longitudinais na extremidade livre da viga em função dos passos de tempo da análise. Os resultados analíticos são de Mansur e Brebbia (1984). Observa-se que os resultados foram todos bastante semelhantes e próximos do comportamento analítico.

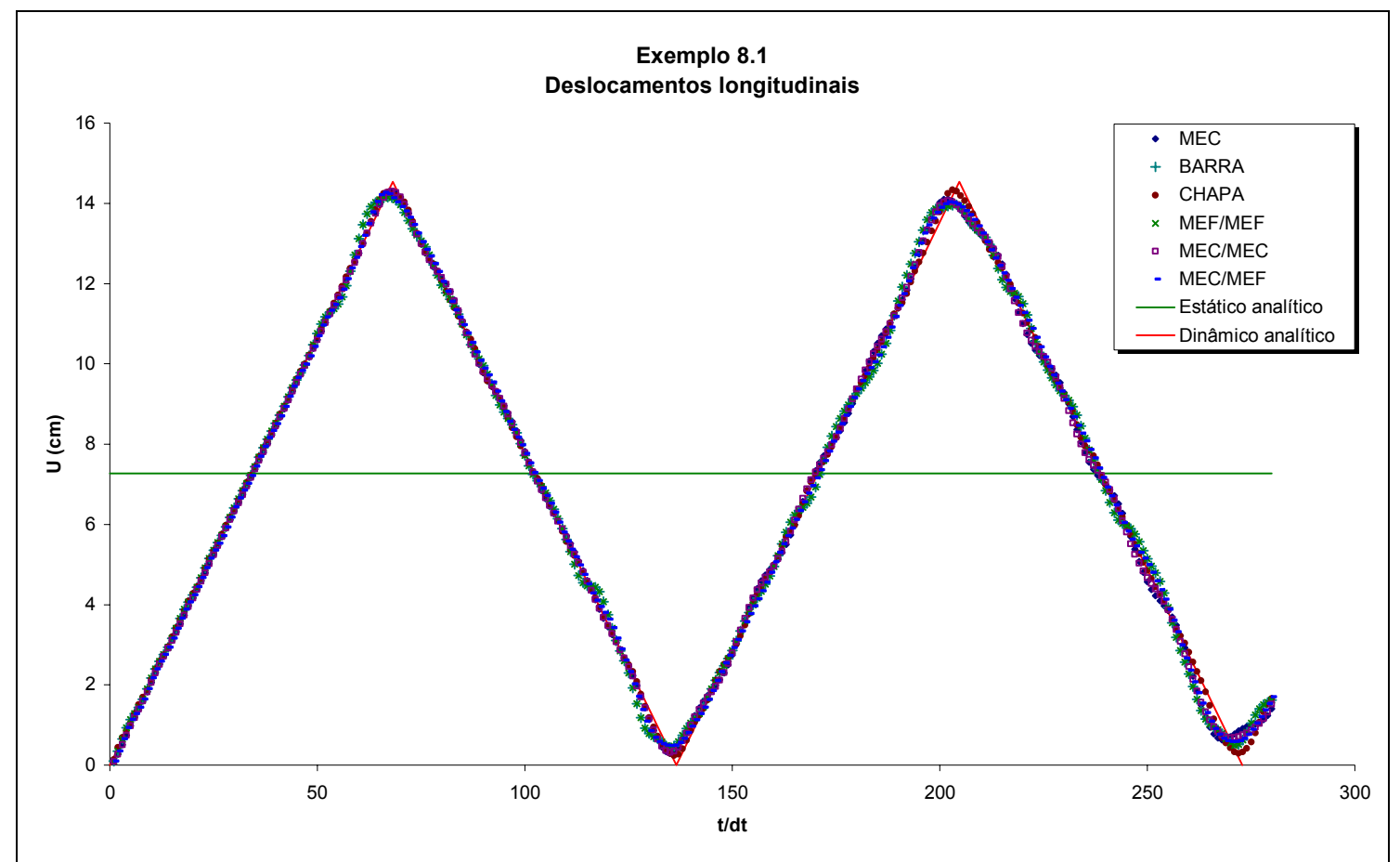

Figura 8.6 - Deslocamentos longitudinais do exemplo 8.1

A figura 8.7 abaixo apresenta os valores das reações de apoio da viga do presente exemplo em função dos passos de tempo da análise. Observa-se que se padronizou o valor das reações de apoio em força de superfície; assim, para as análises por barras e MEF/MEF os valores das reações foram divididos pela área ' $A$ ', e para a análise utilizando-se elementos de chapa, dividiu-se os valores da reação por metade da área 'A', uma vez que a reação fornecida pelo programa no nó de apoio é a metade da reação de apoio propriamente dita, já que se tem dois nós de apoio na discretização utilizada.

Observando-se a figura 8.7, vê-se que os resultados obtidos com as diversas formas de análise foram bastante satisfatórios tendo em vista o comportamento de alta freqüência, as refrações, reflexões e dispersões agregadas a um problema de propagação de uma frente de onda ressalto em meio tridimensional. Deve-se observar que esse seria o exemplo mais difícil de se obter resultados satisfatórios com as formulações aplicadas. 


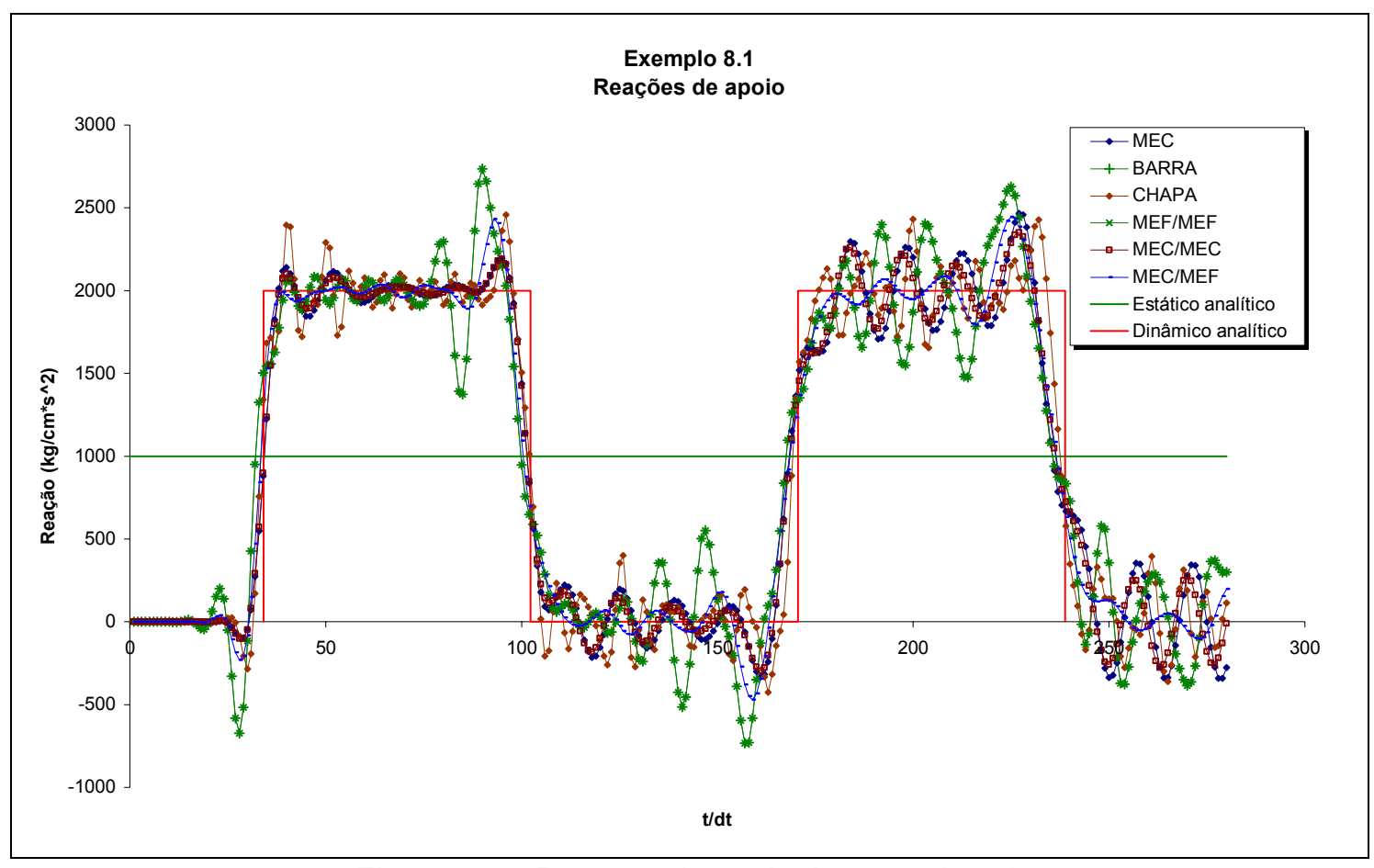

Figura 8.7 - Reações de apoio do exemplo 8.1

Por meio do presente exemplo, observa-se que a técnica de acoplamento empregada está funcionando corretamente e apresenta bons resultados. 


\section{O PROGRAMA COMPUTACIONAL}

Com base nos desenvolvimentos apresentados nos capítulos anteriores, elaborou-se o programa computacional proposto como validação dos desenvolvimentos teóricos da presente tese de doutoramento. Este capítulo nono tem como finalidade descrever as principais características do código computacional desenvolvido, em termos gerais, e suas sub-rotinas mais importantes, bem como comentar a respeito da entrada e saída de dados.

\subsection{GENERALIDADES}

O programa computacional desenvolvido, intitulado como SIMBOLICK, foi implementado em linguagem de programação FORTRAN 77 utilizando-se o software Visual Fortran Professional Edition 6.0.

Quando da implementação do processo de acoplamento, optou-se pela divisão do código completo em diversos programas fonte, cada qual com uma atividade específica, e todos chamados por um programa principal. Sendo assim, os programas fonte são: 


\begin{tabular}{|c|c|}
\hline principal.for & $\begin{array}{l}\text { programa principal; faz a leitura dos dados gerais à análise; } \\
\text { chama os demais programas fonte; gerencia a análise dinâmica; } \\
\text { resolve o sistema total da análise }\end{array}$ \\
\hline mef. for & $\begin{array}{l}\text { lê os dados das sub-regiões do } \mathrm{MEF} ; \text { monta e grava matrizes e } \\
\text { vetores do } \mathrm{MEF} \text {; aloca as matrizes } \overline{\mathrm{K}} \text { e } \beta \Delta \mathrm{t}^{2} \mathrm{G} \text {, respectivamente } \\
\text { nas matrizes } \mathrm{H} \text { e G do sistema total }\end{array}$ \\
\hline mec. for & $\begin{array}{l}\text { lê os dados das sub-regiões do MMBEM; lê ou monta e grava } \\
\text { matrizes do MMBEM; aloca as matrizes } \bar{H} \text { e } \\
\text { respectivamente nas matrizes } \mathrm{H} \text { e G do sistema total }\end{array}$ \\
\hline tabem. for & $\begin{array}{l}\text { lê os dados das sub-regiões do TDBEM; lê ou monta e grava } \\
\text { matrizes de TDBEM; aloca as matrizes h e g, respectivamente } \\
\text { nas matrizes H e G do sistema total }\end{array}$ \\
\hline vff.for & $\begin{array}{l}\text { monta os vetores } \mathrm{F} \text { e } \mathrm{P} \text { no instante de tempo atual para as } \\
\text { sub-regiões do MEF; aloca esses vetores em seus } \\
\text { correspondentes do sistema total }\end{array}$ \\
\hline$v f C$ for & $\begin{array}{l}\text { monta os vetores } \mathrm{F} \text { e } \mathrm{P} \text { no instante de tempo atual para as } \\
\text { sub-regiões do MMBEM; aloca esses vetores em seus } \\
\text { correspondentes do sistema total }\end{array}$ \\
\hline vftabem.for & $\begin{array}{l}\text { monta os vetores } \mathrm{F} \text { e } \mathrm{P} \text { no instante de tempo atual para as } \\
\text { sub-regiões do TDBEM; aloca esses vetores em seus } \\
\text { correspondentes do sistema total }\end{array}$ \\
\hline anlc.for & $\begin{array}{l}\text { calcula } \dot{U} \text { e } \ddot{U} \text { para o instante de tempo atual para as sub- } \\
\text { regiões do MMBEM; calcula } \sigma \text { para estas sub-regiões; realiza a } \\
\text { análise não-linear iterativa }\end{array}$ \\
\hline posf.for & $\begin{array}{l}\text { monta o vetor U e reações das sub-regiões do MEF a partir do } \\
\text { vetor solução total; calcula U̇ e Ü para o instante de tempo } \\
\text { atual; escreve resultados em arquivos de dados }\end{array}$ \\
\hline posc.for & $\begin{array}{l}\text { monta o vetor U e reações das sub-regiões do MMBEM a partir } \\
\text { do vetor solução total; calcula } \dot{U} \text {, } \ddot{U} \text { e } \varepsilon \text { para o instante de } \\
\text { tempo atual; escreve resultados em arquivos de dados }\end{array}$ \\
\hline postdbem. for & $\begin{array}{l}\text { monta o vetor U e reações das sub-regiões do TDBEM a partir } \\
\text { do vetor solução total; escreve resultados em arquivo de } \\
\text { dados }\end{array}$ \\
\hline
\end{tabular}

Além desses 11 subprogramas, aqueles que tratam diretamente com o TDBEM - tdbem.for, vftdbem.for e postdbem.for - ainda fazem chamada a um bloco de declaração de variáveis contidas em blocofixo.for, que funciona como uma dependência do programa como um todo.

\subsection{ENTRADA DE DADOS}

A entrada de dados para cada análise é feita por meio de arquivos, sendo 1 arquivo de entrada para os dados gerais e 1 arquivo para cada subregião.

$\mathrm{Na}$ interface com o usuário é necessário informar o nome do arquivo de entrada de dados gerais (com extensão e tamanho máximo de 100 caracteres) e escolher se as matrizes do MMBEM serão calculadas (c ou C) ou lidas (I ou L). Observa-se que esta opção também existe para o TDBEM, sendo que é feita no próprio arquivo de entrada de dados para este método; para o MEF, a 
citada opção não foi implementada, pois a montagem das matrizes envolvidas possui custo computacional baixo. Observa-se também que, uma vez escolhida a opção para o MMBEM, esta será válida para todas as sub-regiões modeladas por este método.

\subsubsection{Entrada de dados gerais}

$\mathrm{O}$ arquivo de dados gerais deve ser escrito de acordo com o esquema abaixo:

NSR, NL

BT, T, NPT, NMIT

OP, ET, ED

$1 \quad \operatorname{KODESR}(1) \quad \mathrm{VNARQSR}(1) \quad \mathrm{NNSR}(1) \quad$ SYSR(1) NTDBEM

NSR KODESR(NSR) VNARQSR(NSR) NNSR(NSR) SYSR(NSR) NTDBEM

$1 \quad \mathrm{NGLL}(1) \quad \mathrm{SRI}(1) \quad \operatorname{MGLAI}(1) \quad \ldots \quad \operatorname{MGLAI}(\mathrm{NGLL}(1))$

SRJ(1) MGLAJ(1) ‥ MGLAJ(NGLL(1))

NL NGLL(NL) SRI(NL) MGLAI(NL) $\quad \cdots \quad$ MGLAI(NGLL(NL))

$\operatorname{SRJ}(\mathrm{NL}) \quad \mathrm{MGLAJ}(\mathrm{NL}) \quad \cdots \quad \operatorname{MGLAJ}(\mathrm{NGLL}(\mathrm{NL}))$

onde:

NSR - é o número de sub-regiões;

$\mathrm{NL} \quad-$ é o número de ligações de sub-regiões;

BT - é o parâmetro $\beta$ do método de Newmark;

T - é o tempo total da análise (pode ser 1 para análises elastostáticas e igual a NPT para análises estáticas nãolineares);

NPT - é o número de passos de tempo (para análises dinâmicas) ou número de incrementos de carga (para análises estáticas nãolineares); 
NMIT

- é o número máximo de iterações (admitido 100 nas análises realizadas);

OP - caracter que indica se a análise é linear (I ou L) ou não-linear (n ou $\mathrm{N})$;

ET - parâmetro de verificação de convergência por tensão;

ED - parâmetro de verificação de convergência por deslocamento;

$\operatorname{KODESR}(\mathrm{I})$ - código da sub-região I (1- MEF; 2-MMBEM; 3- TDBEM);

VNARQSR(I) - nome do arquivo de entrada de dados da sub-região I;

NNSR(I) - número de nós da sub-região I;

SYSR(I) - tensão de plastificação da sub-região I (basta fornecer qualquer valor diferente de 0 quando houver plastificação na sub-região);

NTDBEM - número de um nó da sub-região de TDBEM que se queira determinar deslocamentos e forças de superfície ao longo do tempo; deve ser diferente de 0 quando houver alguma sub-região de TDBEM; na verdade, só será armazenado o último valor fornecido, para a última sub-região;

NGLL(I) - número de graus de liberdade ligados na ligação I;

SRI(I), SRJ(I) - sub-regiões ligadas na ligação I;

MGLAI(I) - grau de liberdade acoplado da sub-região SRI(I), na ligação I;

$\operatorname{MGLAJ}(\mathrm{I}) \quad$ - grau de liberdade acoplado da sub-região SRJ(I), na ligação I;

\subsubsection{Entrada de dados do MEF}

Para as sub-regiões modeladas pelo Método dos Elementos Finitos, a entrada de dados é feita segundo o esquema a seguir:

NN, NNP, NEB, NEP, NECP, NNDP, NNCC, NEC, NEPC, NECPC

NETB, NETP, NETCH

ROB, ROP, ROCH, \$LM, \$LK, NA

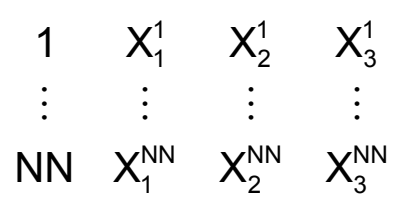


$\begin{array}{cccc}1 & X_{1}^{1} & X_{2}^{1} & X_{3}^{1} \\ \vdots & \vdots & \vdots & \vdots \\ N N P & X_{1}^{N N P} & X_{2}^{N N P} & X_{3}^{N N P}\end{array}$

$\begin{array}{llllllllll}1 & \mathrm{~KB}_{1}^{1} & \mathrm{~KB}_{2}^{1} & \mathrm{~KB}_{3}^{1} & \mathrm{~A}(1) & \mathrm{E}(1) & \mathrm{G}(1) & \mathrm{l}_{1}^{1} & \mathrm{I}_{2}^{1} & \mathrm{I}_{3}^{1}\end{array}$

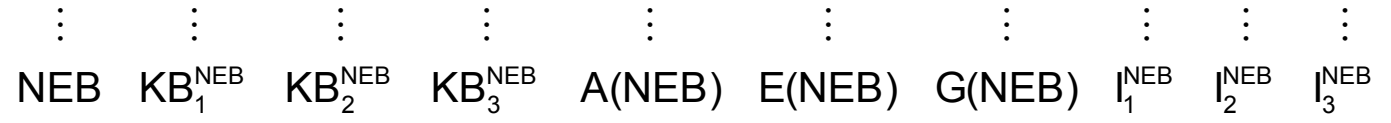

$\begin{array}{lllllll}1 & \mathrm{KP}_{1}^{1} & \mathrm{KP}_{2}^{1} & \mathrm{KP}_{3}^{1} & \mathrm{EP}(1) & \mathrm{H}(1) & \mathrm{PNU}(1)\end{array}$

$\begin{array}{ccccccc}\vdots & \vdots & \vdots & \vdots & \vdots & \vdots & \vdots \\ \text { NEP } & K P_{1}^{N E P} & K P_{2}^{N E P} & K P_{3}^{N E P} & E P(N E P) & H(N E P) & P N U(N E P)\end{array}$

$\begin{array}{lllllll}1 & \mathrm{KCP}_{1}^{1} & \mathrm{KCP}_{2}^{1} & \mathrm{KCP}_{3}^{1} & \mathrm{ECP}(1) & \mathrm{HCP}(1) & \mathrm{PNUCP}(1)\end{array}$

NECP $\mathrm{KCP}_{1}^{\mathrm{NECP}} \mathrm{KCP}_{2}^{\mathrm{NECP}} \mathrm{KCP}_{3}^{\mathrm{NECP}} \mathrm{ECP}(\mathrm{NECP}) \mathrm{HCP}(\mathrm{NECP}) \mathrm{PNUCP}(\mathrm{NECP})$ $\begin{array}{llllllllllllll}\text { NÓ1D } & 0 / 1 & 0 / 1 & 0 / 1 & 0 / 1 & 0 / 1 & 0 / 1 & \text { VDP } & \text { VDP } & \text { VDP } & \text { VDP VDP VDP }\end{array}$

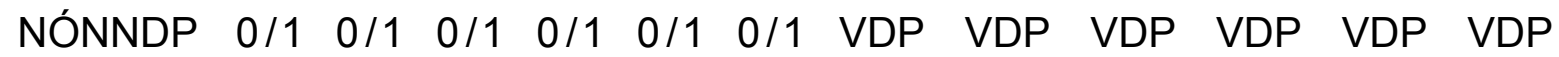
$\begin{array}{llllllllllllll}\text { NÓ1F } & 0 / 1 & 0 / 1 & 0 / 1 & 0 / 1 & 0 / 1 & 0 / 1 & F & F & F & F & F & F\end{array}$

$\begin{array}{lllllllllllll}\text { NÓNNCC } & 0 / 1 & 0 / 1 & 0 / 1 & 0 / 1 & 0 / 1 & 0 / 1 & F & F & F & F & F & F\end{array}$

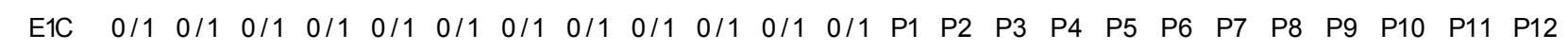

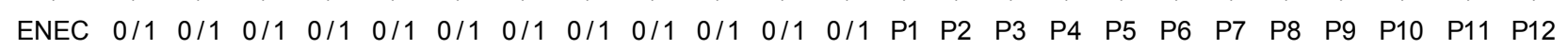

$\{$ se NEC $\neq 0\}$

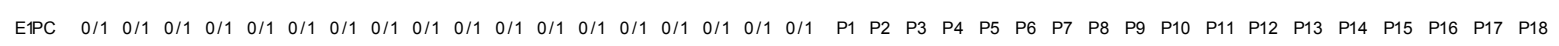

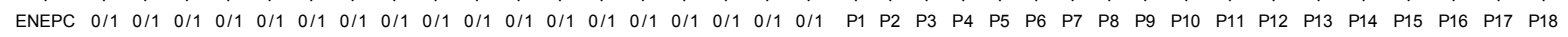
$\{$ se NEPC $\neq 0\}$

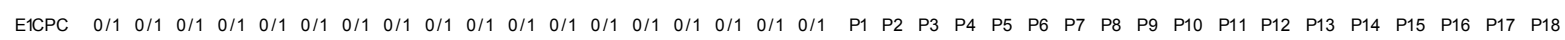

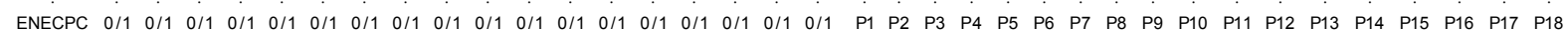
$\{$ se NECPC $\neq 0$ \}

CF1, CF2, CF3, CF4, CF5, CF6, CF7, CF8, CF9, CF10 \{se NNCC $\neq 0$ \}

CP1, CP2, CP3, CP4, CP5, CP6, CP7, CP8, CP9, CP10 \{se NEC, NEPC ou

$\mathrm{NECPC} \neq 0\}$

ETB1

$\vdots$

ETBNETB

ETP1

$\vdots$

ETPNETP 


\section{ETCP1 \\ ETCPNETCP}

onde:

NN - é o número de nós da sub-região;

NNP - é o número de nós auxiliares (usados para definir o plano do elemento de barra);

NEB - é o número de elementos de barra;

NEP - é o número de elementos de placa;

NECP - é o número de elementos de chapa;

NNDP - é o número de nós com deslocamento prescrito;

NNCC - é o número de nós com carga concentrada;

NEC - é o número de elementos de barra carregados;

NEPC - é o número de elementos de placa carregados;

NECPC - é o número de elementos de chapa carregados;

NETB - é o número de elementos de topo de barra (elementos de topo são aqueles que não contribuirão com a montagem da matriz $\mathrm{G}$ da sub-região);

NETP - é o número de elementos de topo de placa;

$\mathrm{NETCH} \quad-$ é o número de elementos de topo de chapa;

ROB - densidade para os elementos de barra (deve ser 0 para análises estáticas);

ROP - densidade para os elementos de placa (deve ser 0 para análises estáticas);

$\mathrm{ROCH} \quad$ - densidade para os elementos de chapa (deve ser 0 para análises estáticas);

\$LM - parâmetro que multiplica a matriz M para montar a matriz C;

\$LK - parâmetro que multiplica a matriz K para montar a matriz C;

NA - número de um nó que se deseje escrever os resultados para deslocamentos e reações ao longo do tempo (pode ser 0);

$X_{i}^{j} \quad-$ coordenada i do nó j; 
$\mathrm{KB}_{1}^{\mathrm{j}}$ e $\mathrm{KB}_{2}^{\mathrm{j}} \quad$ - nó inicial e nó final do elemento de barra j;

$\mathrm{KB}_{3}^{\mathrm{j}} \quad-$ nó auxiliar do elemento de barra j;

$\mathrm{A}(\mathrm{j}) \quad$ - área da seção transversal do elemento de barra j;

$\mathrm{E}(\mathrm{j}) \quad-$ módulo de elasticidade longitudinal do elemento de barra j;

$\mathrm{G}(\mathrm{j}) \quad$ - módulo de elasticidade transversal do elemento de barra j;

$\mathrm{I}_{1}^{\mathrm{j}} \quad-$ momento de inércia à torção do elemento de barra j;

$\mathrm{I}_{2}^{\mathrm{j}}$ e $\mathrm{I}_{3}^{\mathrm{j}} \quad-$ momento de inércia em torno dos eixos $\mathrm{x}_{2}$ e $\mathrm{x}_{3}$ (locais) do elemento de barra j;

$\mathrm{KP}_{\mathrm{i}}^{\mathrm{j}} \quad$ - nó i do elemento de placa $\mathrm{j}$;

EP(j) - módulo de elasticidade do elemento de placa j;

$\mathrm{H}(\mathrm{j}) \quad$ - espessura do elemento de placa $\mathrm{j}$;

PNU(j) - coeficiente de Poisson do elemento de placa j;

$\mathrm{KCP}_{\mathrm{i}}^{\mathrm{j}} \quad-$ nó i do elemento de chapa j;

ECP(j) - módulo de elasticidade do elemento de chapa j;

HCP(j) - espessura do elemento de chapa j;

PNUCP(j) - coeficiente de Poisson do elemento de chapa j;

NÓ1D - primeiro nó com deslocamento prescrito;

0/1 - 0 para deslocamento livre e 1 para prescrito;

VDP - valor do deslocamento para cada grau de liberdade do nó;

NÓNNDP - último nó com deslocamento prescrito;

NÓ1F - primeiro nó carregado;

$\mathrm{F}$

- valor da força concentrada para cada grau de liberdade do nó;

NÓNNCC - último nó carregado;

E1C - primeiro elemento de barra carregado;

$\mathrm{Pi}$

- valor do carregamento distribuído para cada grau de liberdade (para elemento de barra, para $\mathrm{i} \leq 6$, nó inicial da barra, para $\mathrm{i} \geq 7$, nó final da barra; para elementos de placa ou chapa, i de 1 a 6 , nó 1 do elemento, de 7 a 12, nó 2 do elemento, de 13 a 18, nó 3 do elemento);

ENEC - último elemento de barra carregado;

E1PC - primeiro elemento de placa carregado;

ENEPC - último elemento de placa carregado; 


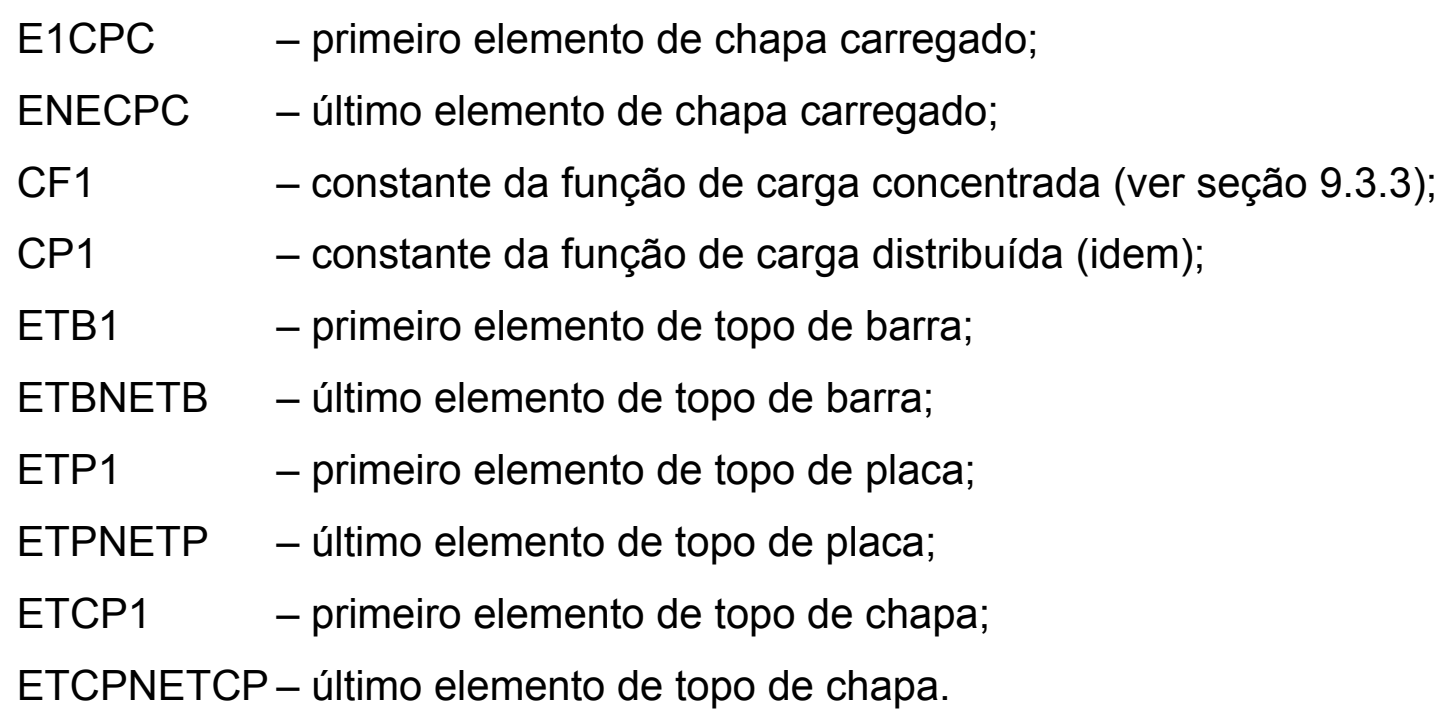

\subsubsection{Entrada de dados do MMBEM}

Para as sub-regiões modeladas pelo MMBEM, a entrada de dados segue o esquema abaixo:

NU, E, ALFA, GRADT, SY, H, PHI

RO, C

NNT, NE, NC, NCN, NA

NNDP, NNFSP

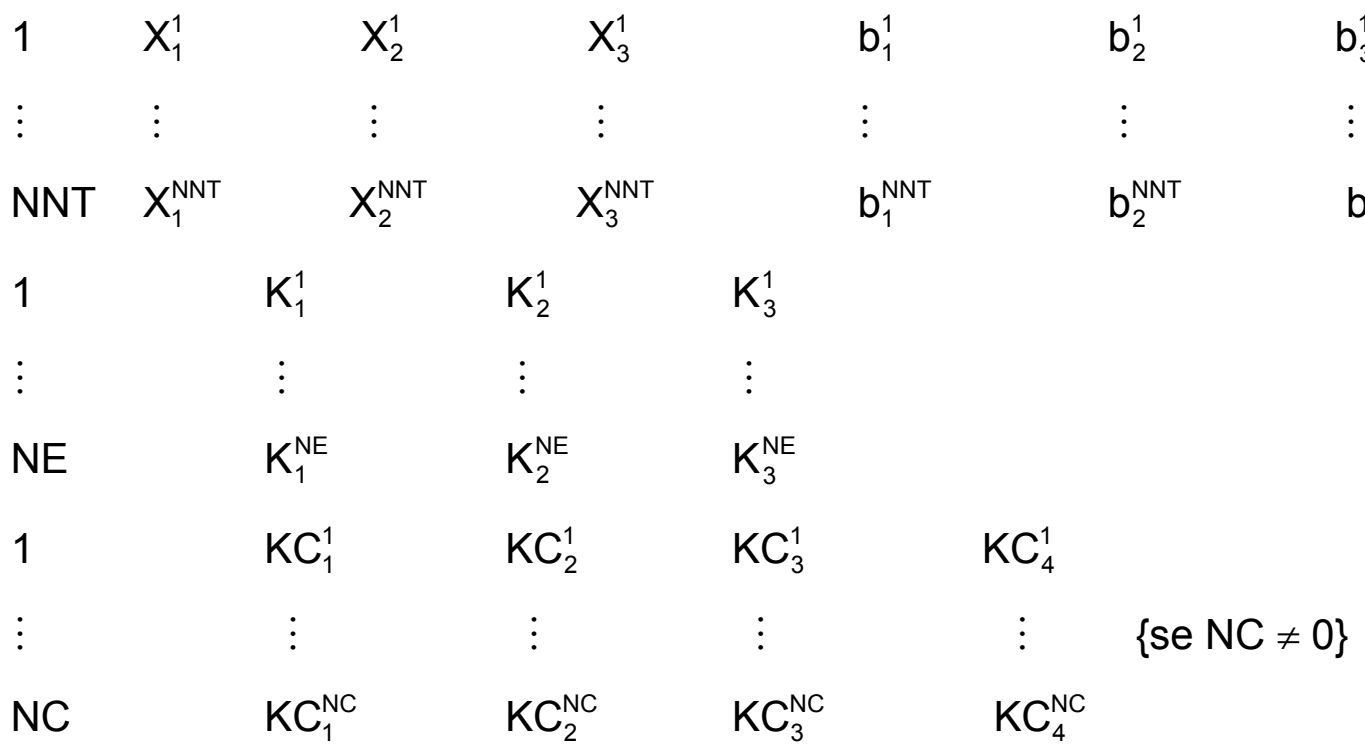




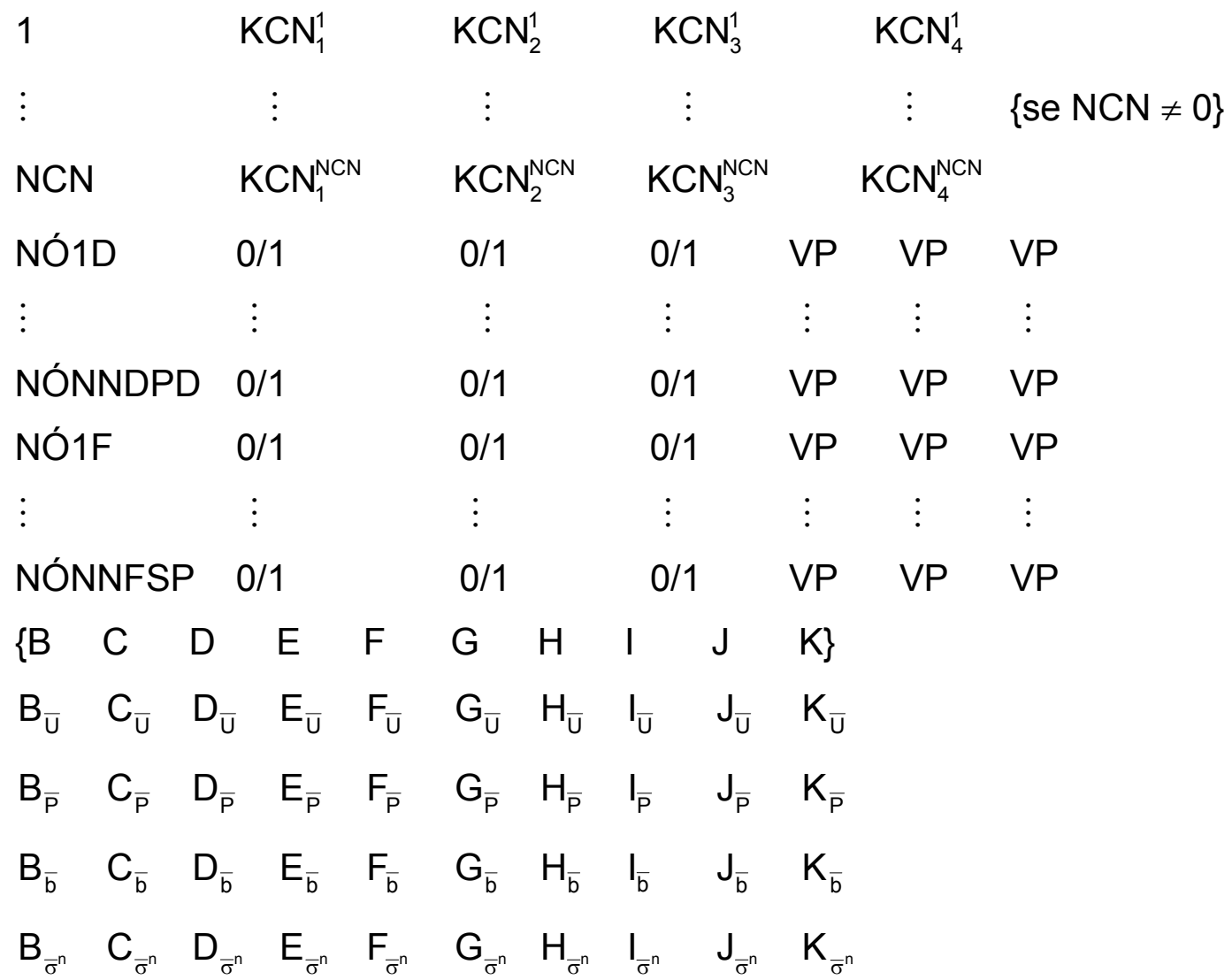

onde:

NU

E

ALFA

GRADT

SY

$\mathrm{H}$

$\mathrm{PHI}$

RO

C

NNT

NE

NC
- coeficiente de Poisson;

- módulo de elasticidade longitudinal ou módulo de Young;

- coeficiente da dilatação térmica na direção longitudinal;

- gradiente de temperatura (para problemas de tensão inicial);

- tensão de plastificação, para o critério de von Mises, e dobro da coesão c, para o critério de Drucker Prager (deve ser atribuído valor 0 para análises elásticas);

- valor do hardening;

- ângulo de atrito interno $\phi$ para o critério de Drucker Prager (deve ser 0 para o critério de von Mises);

- densidade $\rho$;

- amortecimento c;

- número de nós total (no domínio e no contorno);

- número de elementos de contorno;

- número de células para análise dinâmica; 


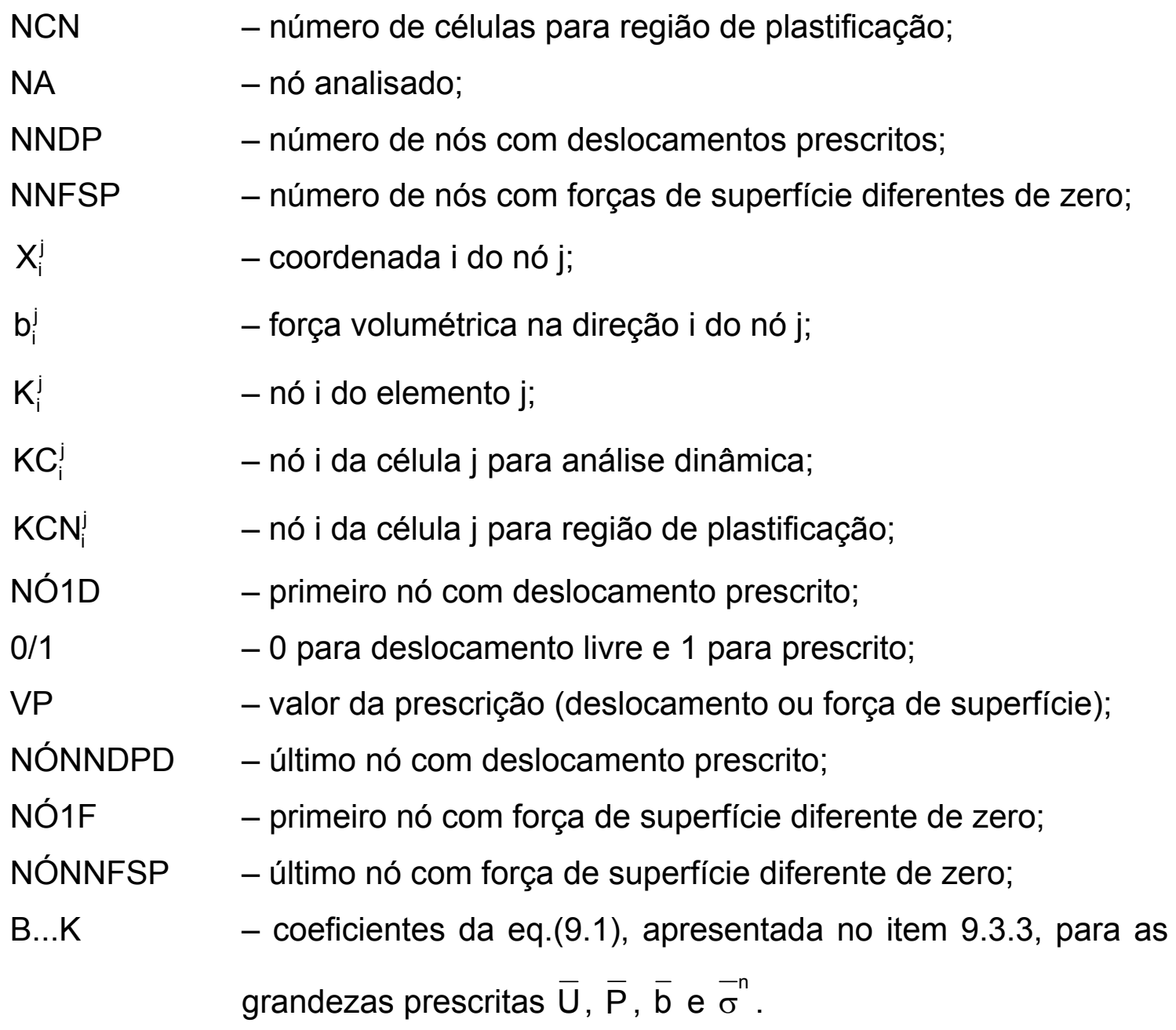

Cabem mais algumas explicações a respeito da entrada de dados apresentada acima. O coeficiente de dilatação térmica ALFA foi utilizado para análise de problemas de dilatação térmica (tensões iniciais). Por uma questão de simplificação, analisaram-se apenas casos de dilatação na direção longitudinal do sólido (direção 3). A discretização de domínio utilizada para a montagem das matrizes da análise dinâmica é independente daquela utilizada para a determinação das matrizes da análise não-linear.

\subsubsection{Entrada de dados do TDBEM}

A entrada de dados para as sub-regiões tratadas pelo TDBEM é semelhante ao que foi feito anteriormente para as outras técnicas. Porém, considera-se a presença de linhas de comentário. Sendo assim, para o TDBEM, apresenta-se um exemplo de arquivo de entrada de dados para uma 
sub-região tratada por esta técnica, ao invés do esquema que se vinha apresentando anteriormente.

Desta forma, considera-se a entrada de dados, para a sub-região modelada pelo TDBEM, de um problema de semi-espaço infinito sujeito a carregamento de impacto constante. Observa-se que os comentários estão entre chaves e que poderiam ser linhas em branco no arquivo.

\begin{tabular}{|c|c|c|c|}
\hline \multicolumn{4}{|c|}{$\begin{array}{l}\quad 49720 \\
\{\text { coordenadas dos nós }\} \\
\{\text { nó, } x 1, x 2, x 3\}\end{array}$} \\
\hline $\begin{array}{l}1 \\
2\end{array}$ & 20.0000000000 & 0.00000000000 & ח \\
\hline 3 & 3.33333333333 & 0.00000000000 & 0.00000000000 \\
\hline 4 & 6.66666666667 & 0.00000000000 & 0.00000000000 \\
\hline 5 & 10.0000000000 & 0.00000000000 & 0.00000000000 \\
\hline 6 & 13.3333333333 & 0.00000000000 & 0.00000000000 \\
\hline 7 & 16.6666666667 & 0.00000000000 & 0.00000000000 \\
\hline 8 & 20.0000000000 & 20.0000000000 & 0.00000000000 \\
\hline 9 & 20.0000000000 & 3.33333333333 & 0.00000000000 \\
\hline 10 & 20.0000000000 & 6.66666666667 & 0.00000000000 \\
\hline 11 & 20.0000000000 & 10.0000000000 & 0.00000000000 \\
\hline 12 & 20.0000000000 & 13.3333333333 & 0.00000000000 \\
\hline 13 & 20.0000000000 & 16.6666666667 & 0.00000000000 \\
\hline 14 & 0.00000000000 & 20.0000000000 & 0.00000000000 \\
\hline 15 & 16.6666666667 & 20.0000000000 & 0.00000000000 \\
\hline 16 & 13.3333333333 & 20.0000000000 & 0.00000000000 \\
\hline 17 & 10.0000000000 & 20.0000000000 & 0.00000000000 \\
\hline 18 & 6.66666666667 & 20.0000000000 & 0.00000000000 \\
\hline 19 & 3.33333333333 & 20.0000000000 & 0.00000000000 \\
\hline 20 & 0.00000000000 & 16.6666666667 & 0.00000000000 \\
\hline 21 & 0.00000000000 & 13.3333333333 & 0.00000000000 \\
\hline 22 & 0.00000000000 & 10.0000000000 & 0.00000000000 \\
\hline 23 & 0.00000000000 & 6.66666666667 & 0.00000000000 \\
\hline 24 & 0.00000000000 & 3.33333333333 & 0.00000000000 \\
\hline 25 & 16.6666666667 & 16.6666666667 & 0.00000000000 \\
\hline 26 & 16.6666666667 & 13.3333333333 & 0.00000000000 \\
\hline 27 & 16.6666666667 & 10.0000000000 & 0.00000000000 \\
\hline 28 & 16.6666666667 & 6.66666666667 & 0.00000000000 \\
\hline 29 & 16.6666666667 & 3.33333333333 & 0.00000000000 \\
\hline 30 & 13.3333333333 & 16.6666666667 & 0.00000000000 \\
\hline 31 & 13.3333333333 & 13.3333333333 & 0.00000000000 \\
\hline 32 & 13.3333333333 & 10.0000000000 & 0.00000000000 \\
\hline 33 & 13.3333333333 & 6.66666666667 & 0.00000000000 \\
\hline 34 & 13.3333333333 & 3.33333333333 & 0.00000000000 \\
\hline 35 & 10.0000000000 & 16.6666666667 & 0.00000000000 \\
\hline 36 & 10.0000000000 & 13.3333333333 & 0.00000000000 \\
\hline 37 & 10.0000000000 & 10.0000000000 & 0.00000000000 \\
\hline 38 & 10.0000000000 & 6.66666666667 & 0.00000000000 \\
\hline 39 & 10.0000000000 & 3.33333333333 & 0.00000000000 \\
\hline 40 & 6.66666666667 & 16.6666666667 & 0.00000000000 \\
\hline 41 & 6.66666666667 & 13.3333333333 & 0.00000000000 \\
\hline 42 & 6.66666666667 & 10.0000000000 & 0.00000000000 \\
\hline 43 & 6.66666666667 & 6.66666666667 & 0.00000000000 \\
\hline 44 & 6.66666666667 & 3.33333333333 & 0.00000000000 \\
\hline 45 & 3.33333333333 & 16.6666666667 & 0.00000000000 \\
\hline 46 & 3.33333333333 & 13.3333333333 & 0.00000000000 \\
\hline 47 & 3.33333333333 & 10.0000000000 & 0.00000000000 \\
\hline 48 & 3.33333333333 & 6.66666666667 & 0.00000000000 \\
\hline 49 & 3.33333333333 & 3.33333333333 & 0.00000000000 \\
\hline
\end{tabular}




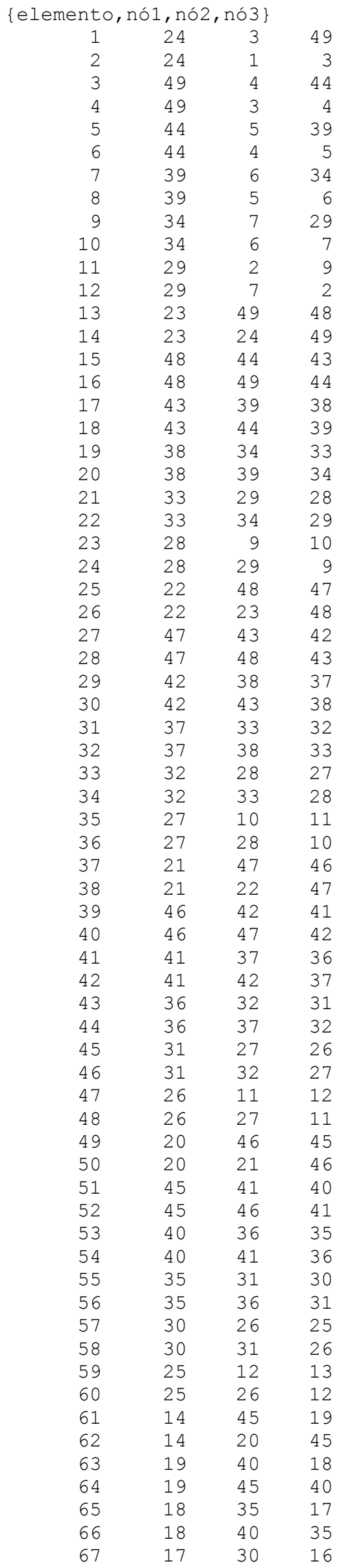




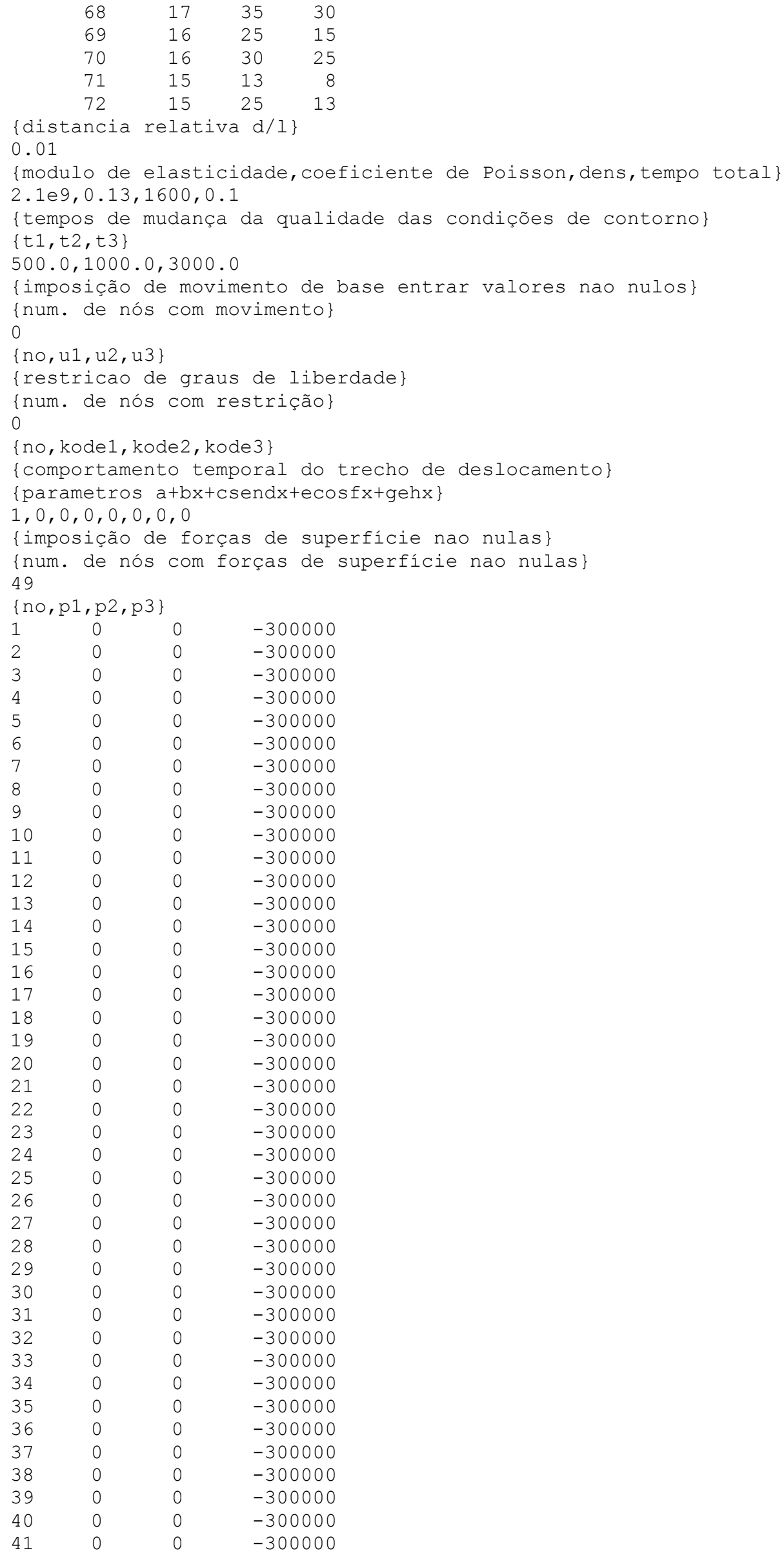




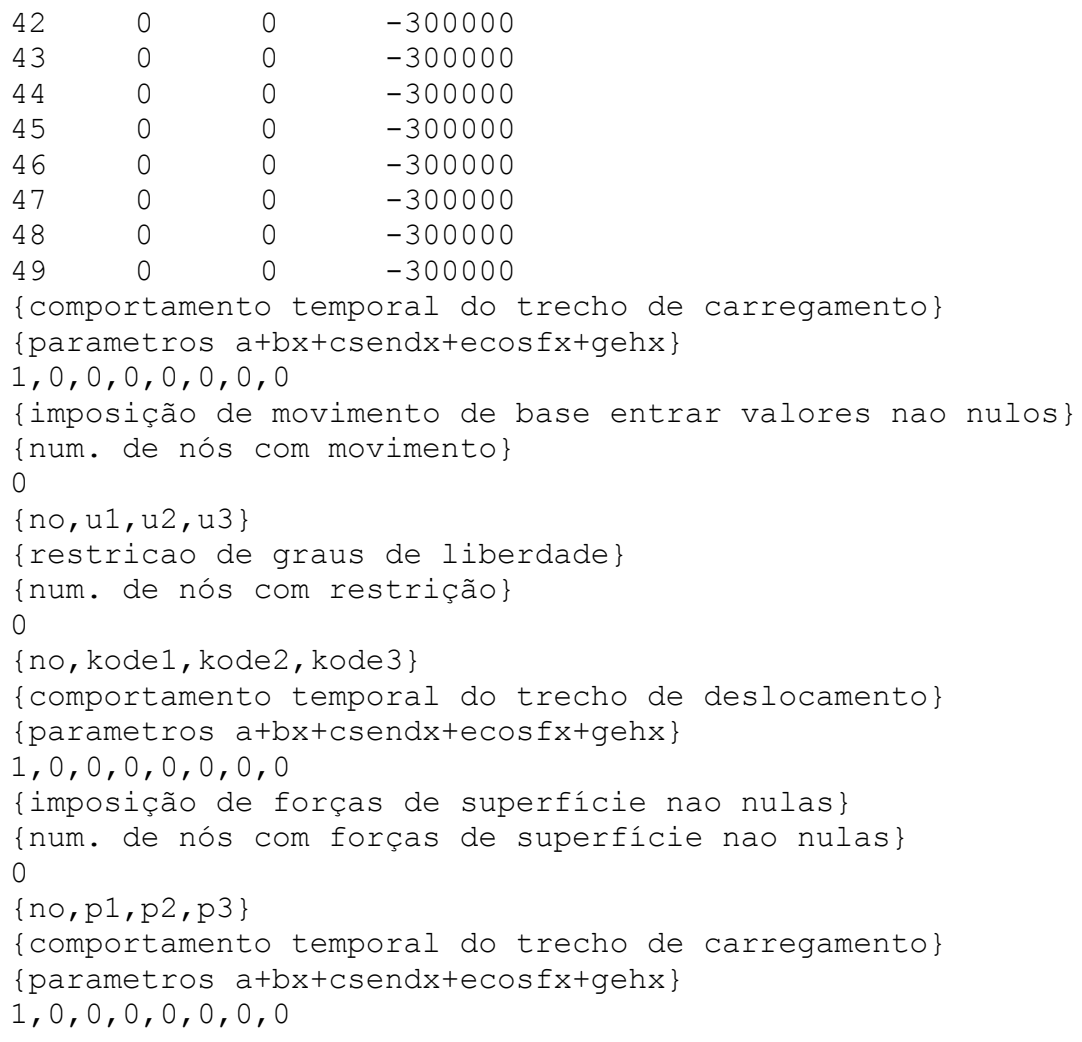

Fazem-se necessárias algumas explicações a respeito do arquivo de dados apresentado acima. A opção 'ler' - primeira linha - é referente às matrizes $\mathrm{h}$ e g; se a opção for ' 0 ', as citadas matrizes são calculadas normalmente, se a opção for '1', estas são lidas. Para um valor de distância relativa nulo, considera-se o ponto fonte no contorno. Se não houver mudanças na qualidade das condições de contorno - observa-se que são possíveis até 3 mudanças -, deve-se fornecer tempos de mudança da qualidade de tais condições maiores que o tempo total da análise.

\subsection{CARACTERÍSTICAS COMPLEMENTARES}

Nesta seção, apresentam-se resumidamente mais algumas características do código computacional desenvolvido. 


\subsubsection{Gravação de matrizes do MMBEM}

Analisando-se os tempos de processamento de cada etapa do MMBEM, observou-se que a montagem das matrizes envolvidas no problema é uma etapa que consome parcela de tempo substancial. Com o objetivo de se agilizar o processamento das análises, resolveu-se gravar as matrizes $G, H, B$, Q, G', H', B' e Q' diretamente em disco rígido. Assim, para cada análise, deve-se fornecer ao programa se essas matrizes serão calculadas ou lidas (em acesso direto em disco). O algoritmo abaixo ilustra o que foi feito no código computacional.

SE (OP = ' $\ell$ ' OU OP = 'L') ENTÃO

LÊ AS MATRIZES G, H, B, Q, G', H', B' e Q' SENÃO

CALCULA AS MATRIZES G, H, B, Q, G', H', B' e Q' GRAVA AS MATRIZES G, H, B, Q, G', H', B' e $\mathrm{Q}^{\prime}$

FIM SE

Com esse procedimento, após se calcular as citadas matrizes uma vez, pode-se realizar várias análises sem que elas sejam calculadas novamente, inclusive com modificações do valor das constantes $\Delta t, \rho$ e c e das condições de contorno, carregamento do problema, tensões limite de plastificação e superfícies de plastificação.

\subsubsection{Solução do sistema de equações}

Inicialmente a resolução do sistema de equações lineares era feita utilizando-se a sub-rotina DLSLRG, que é uma sub-rotina interna do software Visual Fortran Professional Edition 6.0 de resolução de sistema de equações lineares de números reais e geral. Porém, internamente, essa sub-rotina executa duas outras: a sub-rotina DLFCRG, de fatorização LU, e a sub-rotina DLFSRG, de solução do sistema linear. O que se fez posteriormente, também com o objetivo de se obter economia de tempo de processamento, foi substituir 
a sub-rotina DLSLRG, pelas duas outras citadas: a primeira delas, chamada imediatamente antes do início da análise dinâmica não-linear, ou seja, uma única vez; enquanto que a segunda, chamada internamente ao processo de análise dinâmica não-linear. Caso houvesse mudança de qualidade de condições de contorno em deslocamento, realizar-se-ia a primeira etapa novamente.

\subsubsection{Carregamentos e condições de contorno dinâmicos}

Para os MMBEM e MEF, os carregamentos e deslocamentos prescritos são calculados dinamicamente segundo a expressão abaixo:

$$
f(t)=A+B t+C t^{2}+D \cdot \operatorname{sen}(E t)+F \cdot \cos (G t)+H \cdot e^{I t}+J \cdot e^{-K t}
$$

A leitura dos coeficientes de $\mathrm{B}$ a $\mathrm{K}$ da eq.(9.1), para cada um dos 4 vetores do MMBEM $\left(\bar{P}(t), \bar{U}(t), b(t)\right.$ e $\left.\bar{\sigma}^{n}(t)\right)$ e 2 do MEF $(\bar{F}(t), \bar{P}(t))$, é feita junto com a leitura do arquivo de entrada de dados de cada uma dessas técnicas. $O$ valor independente A seria o inicial fornecido, para $\mathrm{t}=0$.

Para o TDBEM, a equação utilizada é a eq.(9.2) abaixo:

$$
f(t)=A+B t+C \cdot \operatorname{sen}(D \cdot t)+E \cdot \cos (F \cdot t)+G \cdot e^{H t}
$$

Para esta técnica, todos os coeficientes são lidos, e o valor de A multiplica o valor inicial da função $f(t)$.

\subsection{SAÍDA DE DADOS}

A saída de dados também é feita por meio de arquivos, para cada subregião. Assim como foi feito para a entrada dos dados, cada método tem seu próprio tipo de arquivo de saída. Dessa forma, apresenta-se a seguir os arquivos de saída de dados fornecidos pelo código computacional desenvolvido, para cada tipo de sub-região. 


\subsubsection{Saída de dados para o MEF}

Para o MEF, existem 3 tipos de arquivos de saída de dados. O próprio sistema cria o nome desses arquivos a partir do nome do arquivo de entrada de dados da sub-região. Sendo assim, para uma sub-região de elementos finitos cujo nome do arquivo de entrada seja "analise1_fem.ent", os nomes dos arquivos de saída criados seriam:

analise1_fem.des deslocamentos para o nó analisado; analise1_fem.rea. reações para o nó analisado; analise1_fem.sai deslocamentos e reações para a sub-região.

Observa-se que os dois primeiros arquivos só são criados se o nó analisado (NA) for diferente de zero; e que o terceiro arquivo só é criado se o número de passos de tempo (NPT) for igual a 1.

\subsubsection{Saída de dados para o MMBEM}

Para as sub-regiões tratadas pelo MMBEM, a saída de dados é realizada por 4 arquivos, também criados a partir do nome do arquivo de entrada. Considerando-se que o nome do arquivo seja "analise1_mmbem.ent", os nomes dos arquivos de saída seriam:

analise1_mmbem.na1...... deslocamentos e forças de superfície para o NA; analise1_mmbem.na2. tensões para o NA; analise1_mmbem.na3. deformações para o NA; analise1_mmbem.sol deslocamentos, forças de superfície, tensões e deformações para a sub-região.

Semelhantemente ao que acontece para o MEF, os arquivos com extensão na1, na2 e na3 só são escritos se o nó analisado (NA) for diferente de zero; e o arquivo com extensão sol, só é criado se o número de passos de tempo for igual a 1 . 


\subsubsection{Saída de dados para o TDBEM}

A saída de dados para o TDBEM é um pouco mais limitada que aquelas apresentadas anteriormente. Apenas um arquivo é criado, incondicionalmente, com extensão sai. Como já foi dito, o valor fornecido para o nó analisado deve ser diferente de zero, quando houver sub-região modelada pelo TDBEM. $O$ arquivo - cujo nome é aquele da entrada de dados da sub-região, com terminação sai - fornece, além de outros dados complementares, os deslocamentos e forças de superfície para o nó analisado.

\subsection{GERADOR DE MALHAS}

Nesta seção será apresentado um gerador de discretizações desenvolvido para a análise de sólidos paralelepipédicos. Esse gerador foi desenvolvido de maneira bastante simplificada e fornece as coordenadas dos nós do problema, as conectividades dos elementos de contorno e as conectividades das células. Leva em consideração a presença de nós duplos na base e no topo do sólido.

Como dados de entrada, deve-se fornecer os comprimentos nas 3 direções (largura, profundidade e comprimento) e o número de divisões para cada uma dessas direções, para os elementos de contorno (nd $\ell$, ndp e ndc) e para as células (nd $\ell$, ndpc e ndcc). Deve haver uma relação inteira entre os números de divisões, nas 3 direções, para a discretização do contorno e das células. Assim, definem-se as 3 relações abaixo:

$$
\begin{aligned}
& \operatorname{ir} \ell=\mathrm{nd} \ell / \mathrm{nd} \ell \mathrm{c} \\
& \operatorname{irp}=\mathrm{ndp} / \mathrm{ndpc} \\
& \operatorname{irc}=\mathrm{ndc} / \mathrm{ndcc}
\end{aligned}
$$

O número de nós total, o número de elementos e o número de células são calculados utilizando-se respectivamente as equações abaixo: 


$$
\begin{aligned}
& \mathrm{nn}=(\mathrm{nd} \ell+1) \cdot(\mathrm{ndp}+1) \cdot(\mathrm{ndc}+3) \\
& \mathrm{ne}=(\mathrm{nd} \ell \cdot \mathrm{ndc}+\mathrm{ndp} \cdot \mathrm{ndc} \cdot \mathrm{nd} \ell \cdot \mathrm{ndp}) \cdot 4 \\
& \mathrm{nc}=\mathrm{nd} \ell \mathrm{c} \cdot \mathrm{ndpc} \cdot \mathrm{ndcc} \cdot 6
\end{aligned}
$$

A geração da disposição dos nós, inclusive nós duplos, e a geração dos elementos de contorno, podem ser ilustradas pela figura 9.1, onde é apresentado um exemplo de discretização $\operatorname{com} \mathrm{nd} \ell=\mathrm{ndp}=\mathrm{ndc}=2$.

Deve-se ter um cuidado especial para com os nós 14 e 32 (duplos dos nós 5 e 41, respectivamente) do exemplo da figura 9.1. Na verdade, esse tipo de nó é completamente dispensável na análise, apenas aparecendo na discretização como uma simplificação do processo de geração de malhas desenvolvido pelo autor. Porém, a presença desses nós não acarretará uma inviabilidade da análise, desde que o programa os entenda como nós do contorno, apesar de não serem nós de elementos de contorno. Isso é facilmente realizado pela formulação desenvolvida no trabalho.

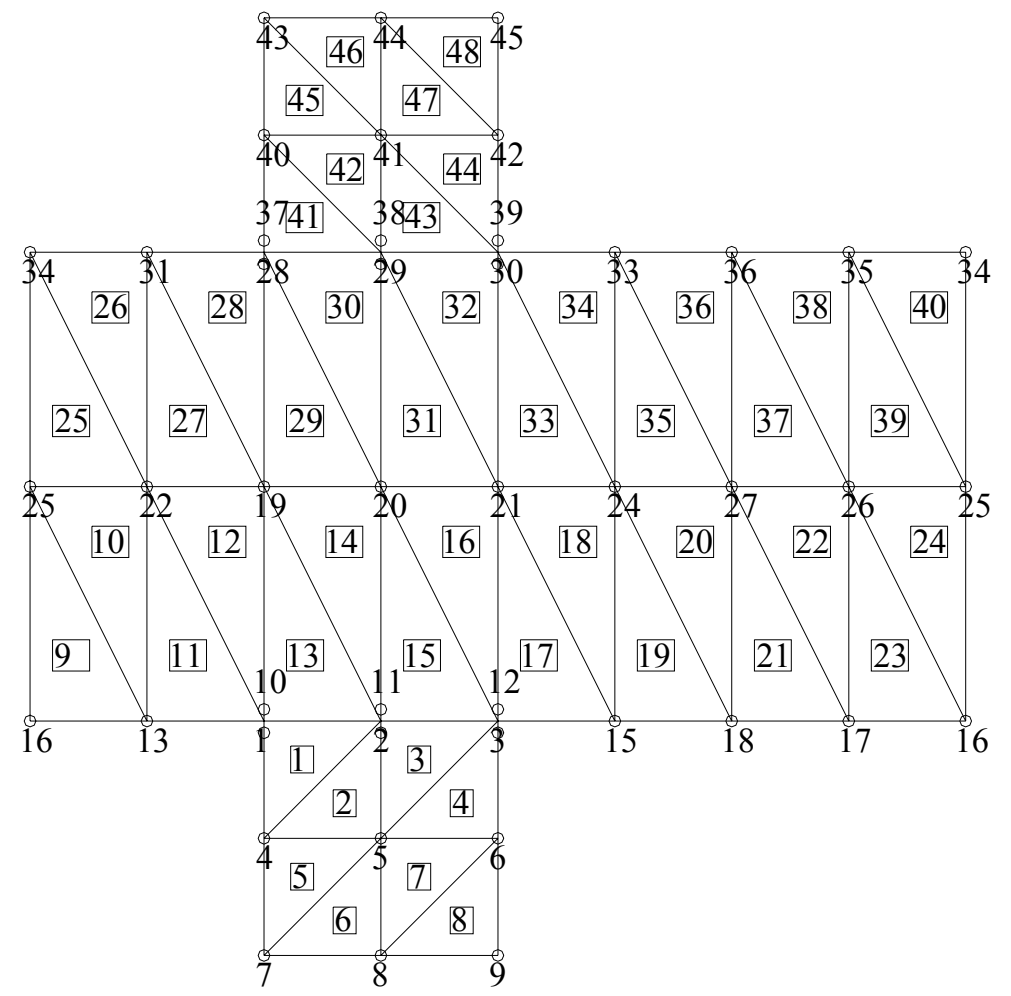

Figura 9.1 - Nós e elementos de contorno 
Com relação às células, estas são geradas de acordo com o esquema da figura 9.2, onde se poderia dizer que se é apresentada uma divisão nd $\ell c=$ $\mathrm{ndpc}=\mathrm{ndcc}=1$.

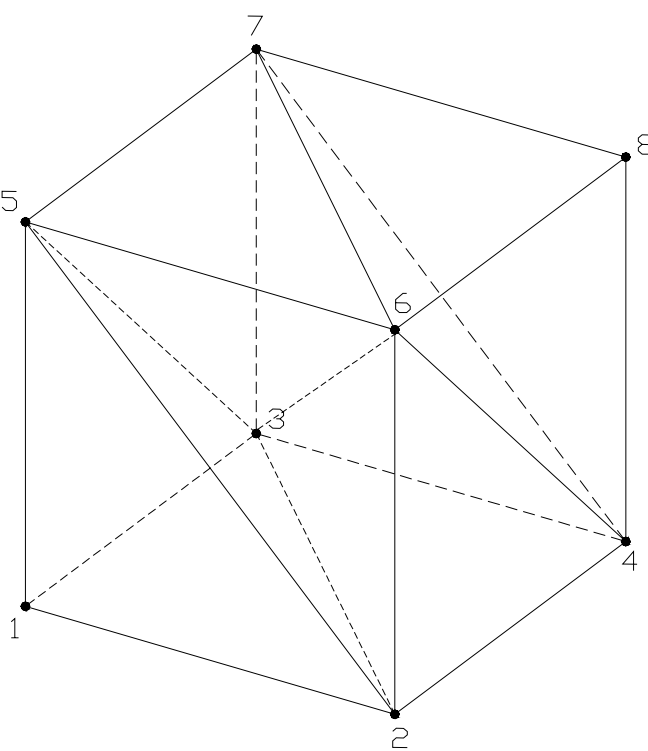

Figura 9.2 - Paralelogramo de células

As conectividades das 6 células da figura 9.2 são:

\begin{tabular}{|c|c|c|c|c|}
\hline Célula & Nó 1 & Nó 2 & Nó 3 & Nó 4 \\
\hline 1 & 1 & 2 & 3 & 5 \\
\hline 2 & 2 & 4 & 3 & 6 \\
\hline 3 & 3 & 7 & 5 & 6 \\
\hline 4 & 3 & 5 & 2 & 6 \\
\hline 5 & 4 & 7 & 6 & 8 \\
\hline 6 & 3 & 7 & 6 & 4 \\
\hline
\end{tabular}

A discretização da figura 9.1 , caso se tivesse $n d \ell c=n d p c=n d c c=2$, teria 8 "paralelogramos de células" como aquele mostrado na figura 9.2.

Deve-se comentar também, que, para problemas mais complexos, a geração da geometria, células internas e elementos de contorno foi realizada utilizando-se o programa comercial ANSYS. Nesse caso, é necessário, após a 
geração automática, interferência manual na criação dos nós duplos - quando houver - pertinentes às análises. 


\section{EXEMPLOS FINAIS}

Neste capítulo de exemplos finais, três problemas são estudados com o intuito principal de mostrar a aplicação dos desenvolvimentos descritos nos capítulos anteriores, bem como a potencialidade do código computacional desenvolvido na pesquisa. Considera-se que a aferição das técnicas apresentadas no trabalho foi realizada por meio dos exemplos simples presentes no final dos capítulos relativos a cada técnica.

Para todas as análises realizadas nestes exemplos, utilizou-se um microcomputador com processador Amd 2200 XP, 2Gb de memória RAM e HD de $120 \mathrm{~Gb}$, adquirido com recurso de reserva técnica da bolsa de doutoramento do autor, financiada pela Fundação de Amparo à Pesquisa do Estado de São Paulo - FAPESP.

\subsection{EXEMPLO 10.1}

No primeiro exemplo, analisa-se um sólido apoiado em uma face, sujeito a um carregamento súbito, uniformemente aplicado na face oposta. O material do sólido apresenta características não-lineares físicas. Ver figura 10.1.

O sólido do presente exemplo foi analisado de duas formas distintas. $\mathrm{Na}$ primeira, utilizou-se o MMBEM para todo o sólido, e na segunda, utilizou-se o acoplamento MMBEM/TDBEM/FEM, onde a sub-região modelada pelo MMBEM correspondeu à metade do sólido e a outra metade foi igualmente dividida entre as sub-regiões do TDBEM e do FEM. Para a modelagem do acoplamento, vale dizer que a região de plastificação do problema ficou naturalmente confinada na região do MMBEM. A sub-região modelada pelo 
MEF (ou FEM) compreende não apenas a barra, mas também uma casca de ligação, como foi feito no exemplo 8.1. A figura 10.2 apresenta as discretizações utilizadas para ambos os casos.

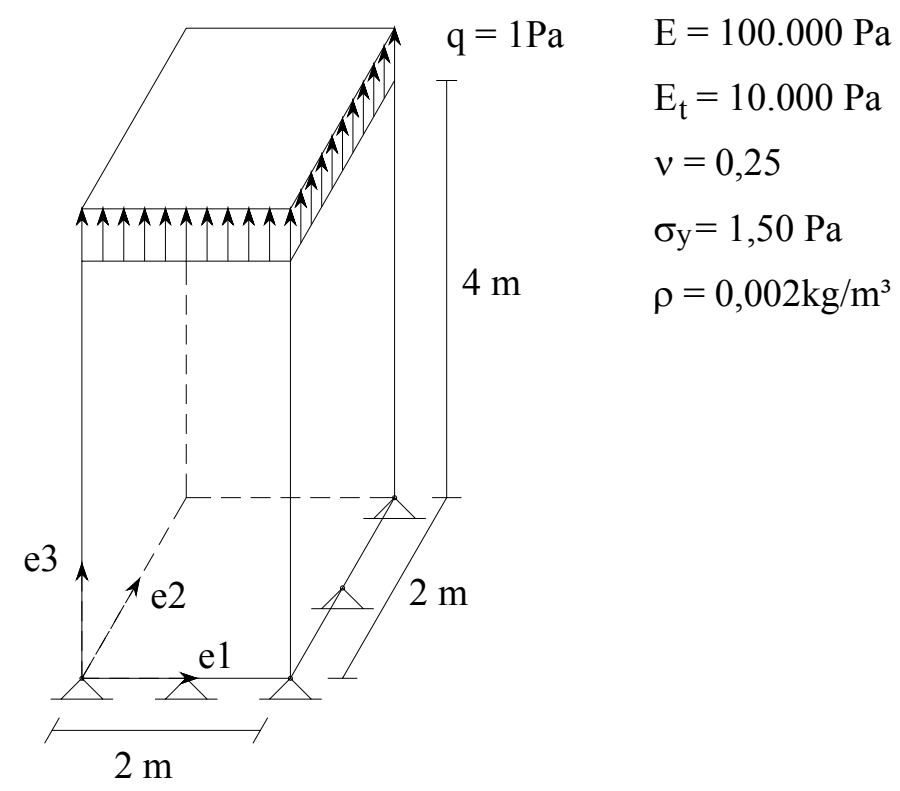

Figura 10.1 - Exemplo 10.1
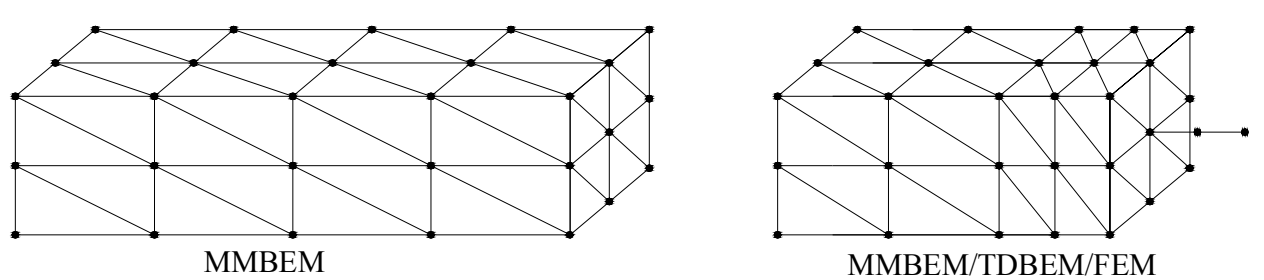

Figura 10.2 - Discretizações do exemplo 10.1

É necessário ainda dizer que o passo de tempo $(\Delta t)$ adotado para a análise com o MMBEM foi de 0,00012s, e para o acoplamento MMBEM/TDBEM/FEM, 0,0002s. Esta diferença é justificada pelas condições de estabilidade para o TDBEM quando tratando de corpos finitos, e por questões de amortecimento numérico do MMBEM, já bem conhecidas.

A figura 10.3 apresenta os resultados obtidos para o deslocamento longitudinal no nó localizado no centro da face carregada. 


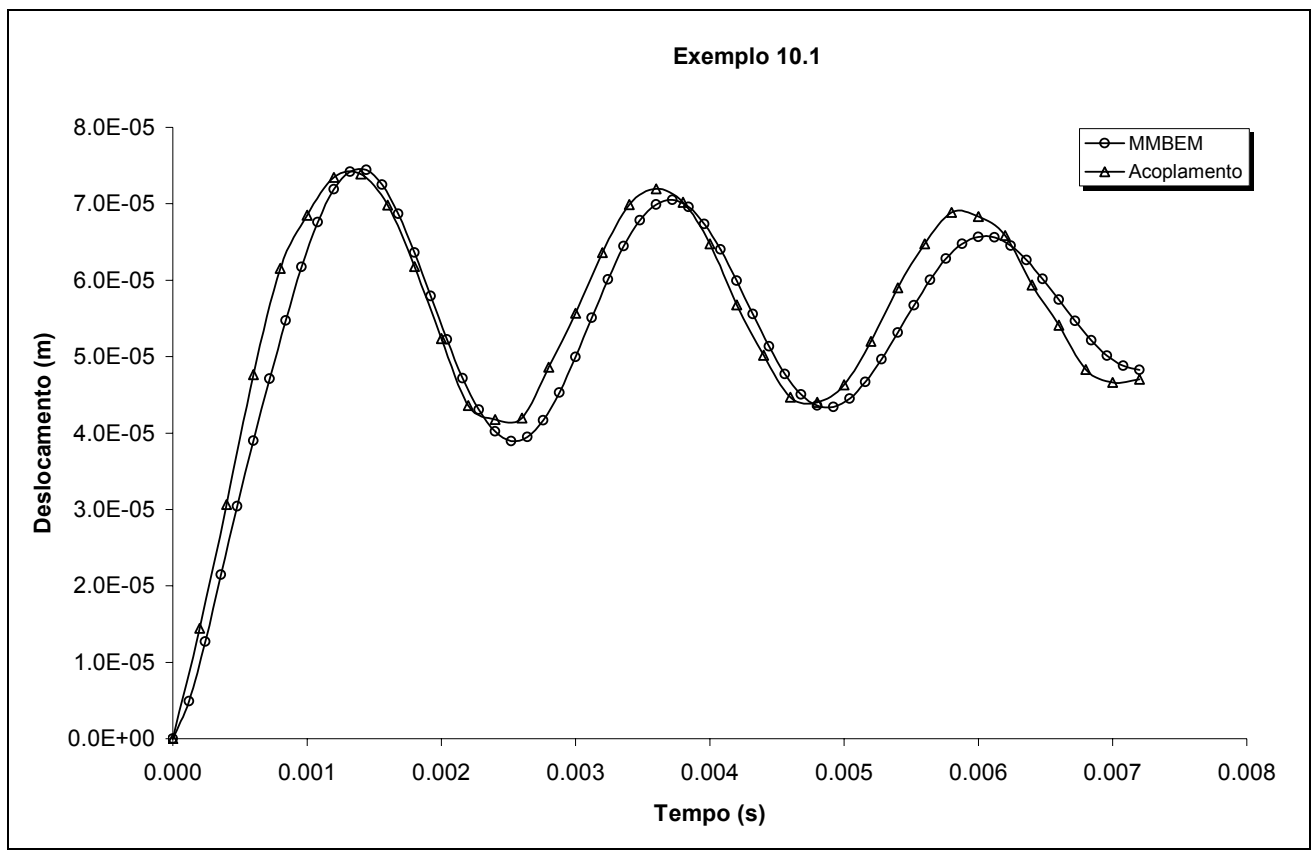

Figura 10.3 - Deslocamento longitudinal do exemplo 10.1

Pode-se dizer que os resultados apresentaram ótima concordância, apesar da diferença das modelagens adotadas. Conclui-se que o acoplamento MMBEM/TDBEM/FEM para análises dinâmicas não-lineares foi implementado com sucesso. Observa-se ainda que este exemplo é particularmente difícil de ser analisado pela utilização do TDBEM - por se tratar de meio finito mostrando que a técnica foi bem implementada e se apresenta precisa e estável. Graças ao grau de dificuldade deste exemplo, a formulação resultante se mostra uma ferramenta poderosa para o estudo de problemas de interação solo-estruturas gerais e outros tipos de problema, como por exemplo, meios reforçados.

\subsection{EXEMPLO 10.2}

O exemplo 10.2 é um pouco mais elaborado que todos aqueles apresentados anteriormente nesta tese. Nele, um solo fictício é analisado sob diversas condições de carregamento, modelagens e comportamento físico. Cada consideração será apresentada ao longo da apresentação do exemplo. Sendo assim, por uma questão organizacional, optou-se por se dividir o mesmo em seções terciárias como segue. 


\subsubsection{Placa quadrada sobre semiplano infinito}

O primeiro caso trata da análise de uma placa quadrada de lado ' $L$ ' sobre um semiplano infinito sujeita a um carregamento transversal uniformemente distribuído em toda a sua área ' $p$ '.

O solo foi modelado inicialmente pelo TDBEM e a placa, pelo MEF, com elementos de casca. A discretização utilizada neste tipo de análise para a placa e para o solo, bem como os demais dados envolvidos no problema podem ser vistos na figura 10.4 abaixo. Vale dizer que os acoplamentos realizados foram feitos nos 3 graus de liberdade possíveis, para cada nó, porém, é possível escolher direções livres, caso se deseje.

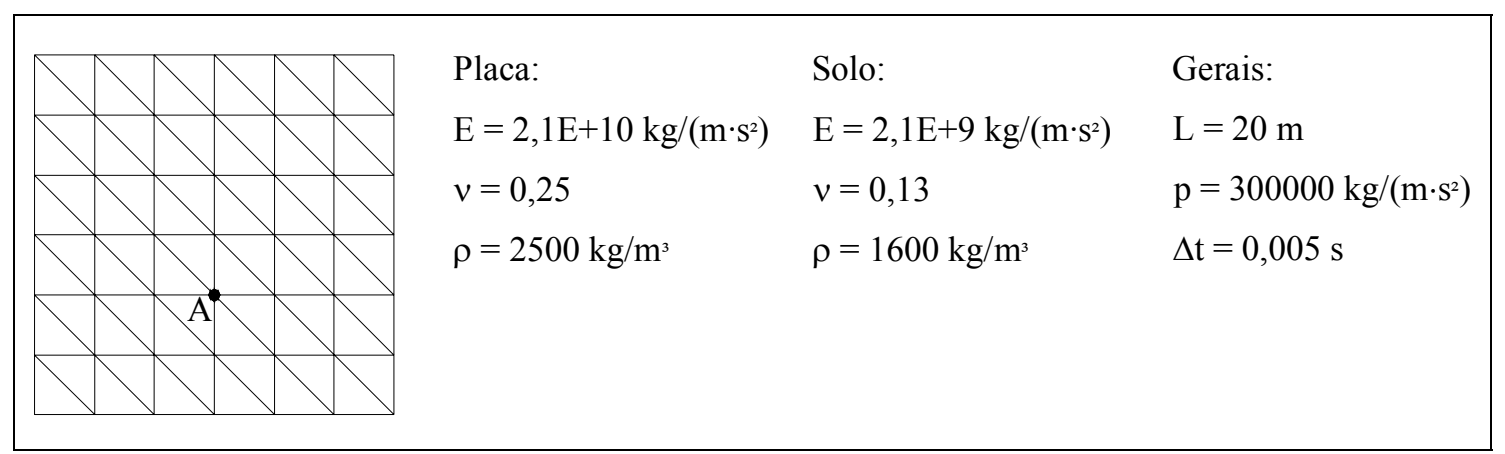

Figura 10.4 - Exemplo 10.2.1

A figura 10.5 abaixo apresenta os resultados para os deslocamentos transversais do ponto $A$, onde a espessura ' $h$ ' da placa variou de 0 a $5 \mathrm{~m}$ na análise. Para ' $h=0$ ', apenas a sub-região do TDBEM foi considerada, e a carga foi aplicada diretamente no solo.

Pela figura 10.5, observa-se que aumentando a espessura da placa, e conseqüentemente a rigidez da mesma, os deslocamentos diminuem, o que era totalmente esperado. Observa-se também que as estabilidades obtidas para as diversas análises foram bastante satisfatórias.

$\mathrm{Na}$ figura 10.6 são apresentadas diversas análises para o problema exposto acima, considerando-se $\mathrm{h}=0$, ou seja, a inexistência da placa. Algumas explicações são necessárias com relação às análises realizadas. Em primeiro lugar, a análise estática denominada "BEM estendido" foi feita unicamente com elementos de contorno, onde se discretizou uma região quadrada de lado igual a $200 \mathrm{~m}$, e a região carregada foi localizada no meio da região discretizada. 
Figura 10.7. A análise "TDBEM 12x12" e "TDBEM 24x24" foi feita apenas com elementos do TDBEM na região carregada com 12 e 24 divisões em cada lado, respectivamente; sendo assim, a análise "TDBEM" poderia ser escrita também como "TDBEM 6×6". A análise "TDBEM estendido" seria igual àquela apresentada na figura 10.7, sendo que dinâmica, modelada pelo TDBEM. A análise dinâmica intitulada "MMBEM estendido" pode ser visualizada na figura 10.8, onde se utilizou, além das células em todo o domínio discretizado, elementos de contorno apenas na superfície do semiplano. Por último, a discretização "MMBEM+TDBEM" foi feita da seguinte forma: a sub-região modelada pelo MMBEM compreende um cubo (de arestas iguais a $20 \mathrm{~m}$ ) de elementos de contorno (em todas as faces) e células, e é acoplada a uma subregião tratada pelo TDBEM, conforme figura 10.9, onde se observa tal discretização parcialmente por baixo da mesma. O autor gostaria de citar que os elementos da matriz dos termos livres da equação de deslocamentos para elementos de contorno foram determinados considerando a sub-região do TDBEM como um corpo fechado (com a criação de alguns nós e elementos de contorno fictícios e temporários, conhecidos como enclosing elements) e realizando-se então movimento de corpo rígido para o citado sólido virtual (AHMAD e BANERJEE, 1988; ARAÚJO, NISHIKAVA e MANSUR, 1997; CARRION, 2002; CARRION, MESQUITA NETO e ROMANINI, 2001).

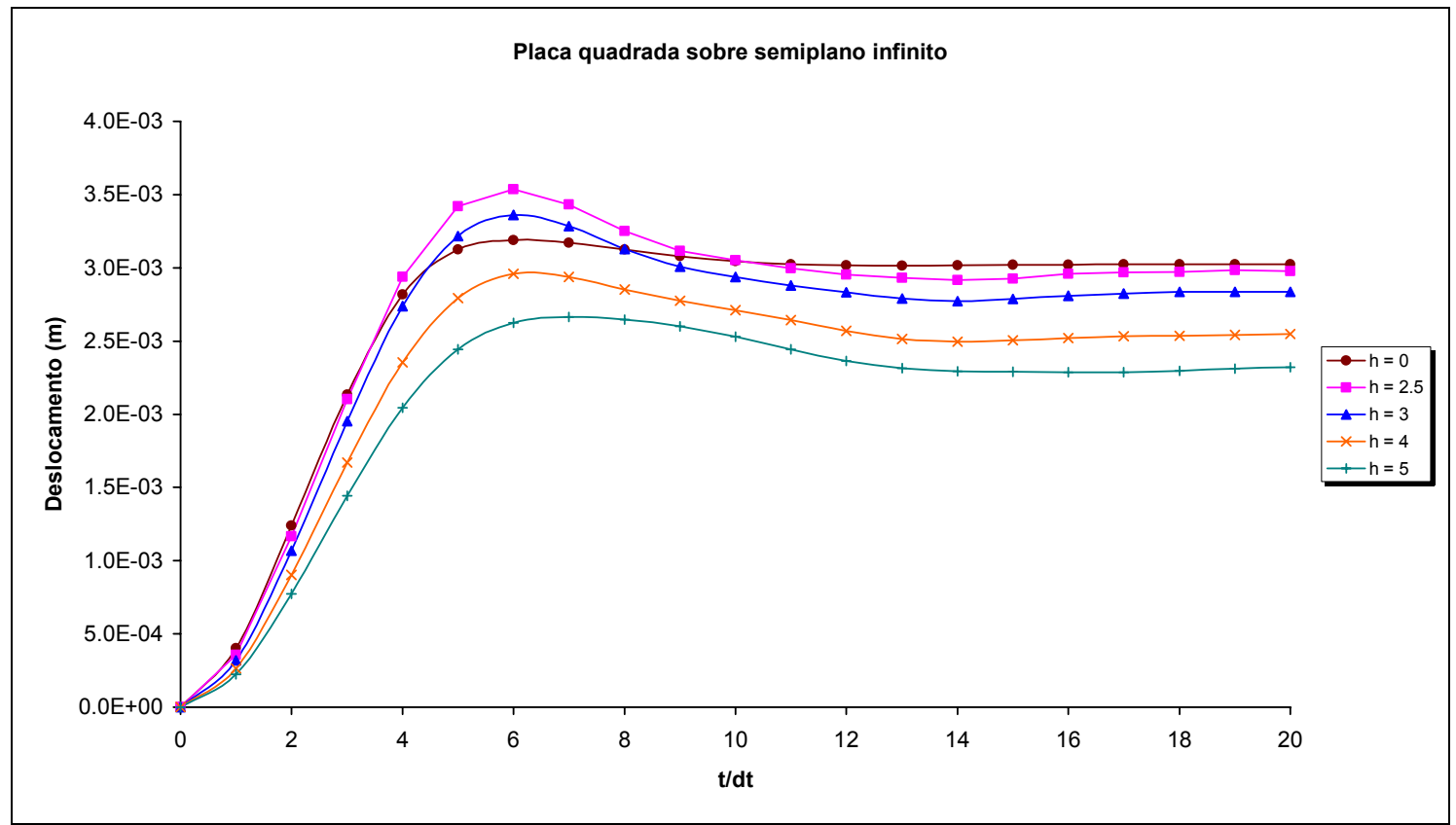

Figura 10.5 - Deslocamentos para o exemplo 10.2.1 
Na figura 10.6, observa-se a boa concordância entre os valores obtidos com as diversas análises utilizadas, onde as dinâmicas convergem para o valor estático, obtido com a utilização do MEC, após certo tempo. Comparando as análises realizadas unicamente pelo TDBEM, observa-se que a extensão da malha não fornece diferença significativa no comportamento do problema de superfície livre.

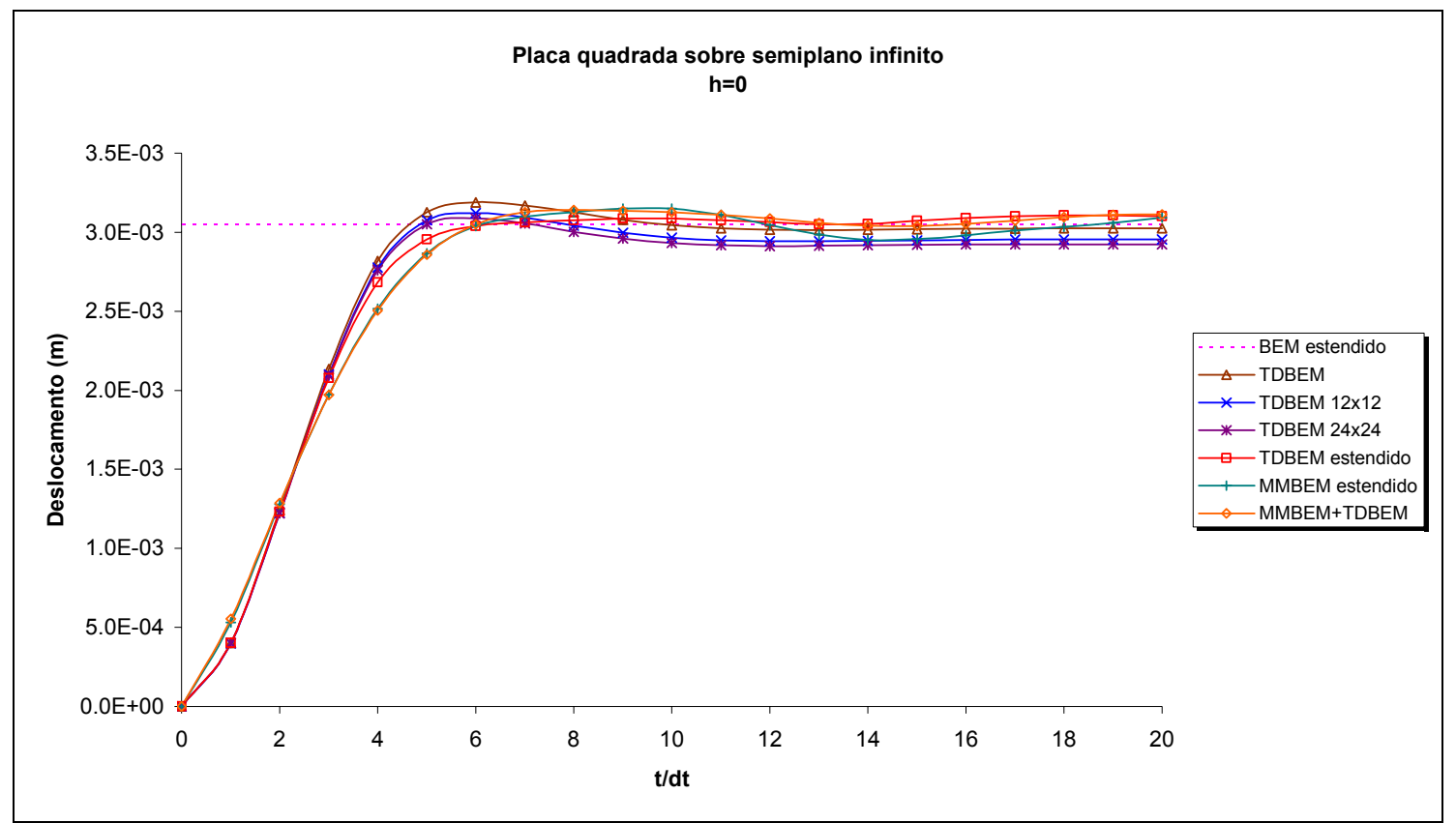

Figura 10.6 - Exemplo 10.2.1 - análise elastodinâmica para h=0

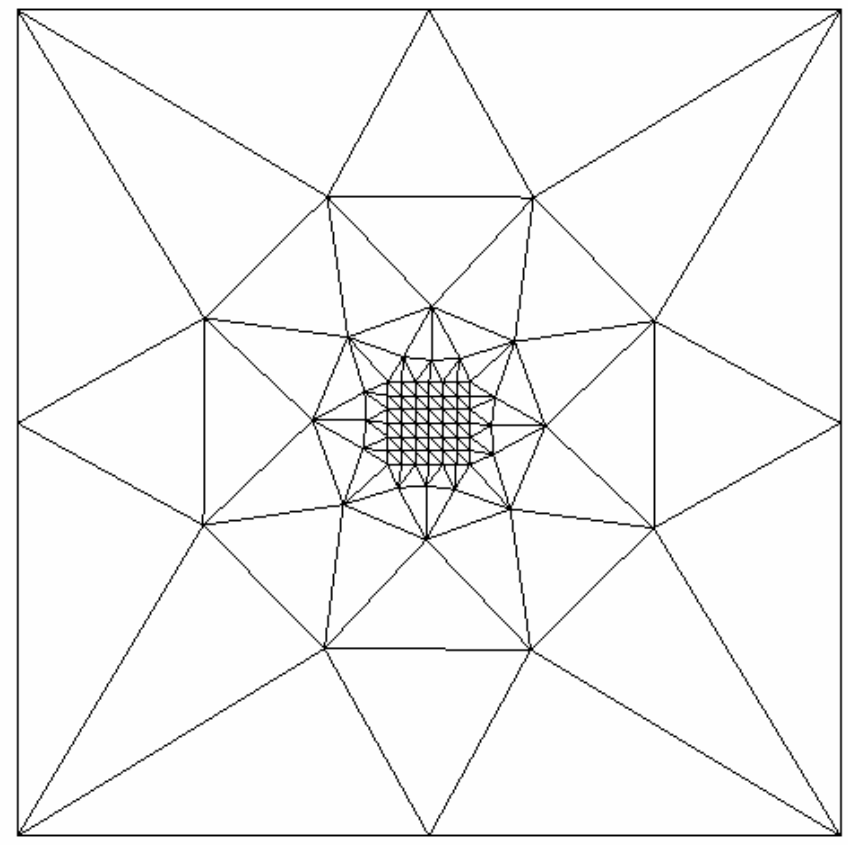

Figura 10.7 - BEM estendido 


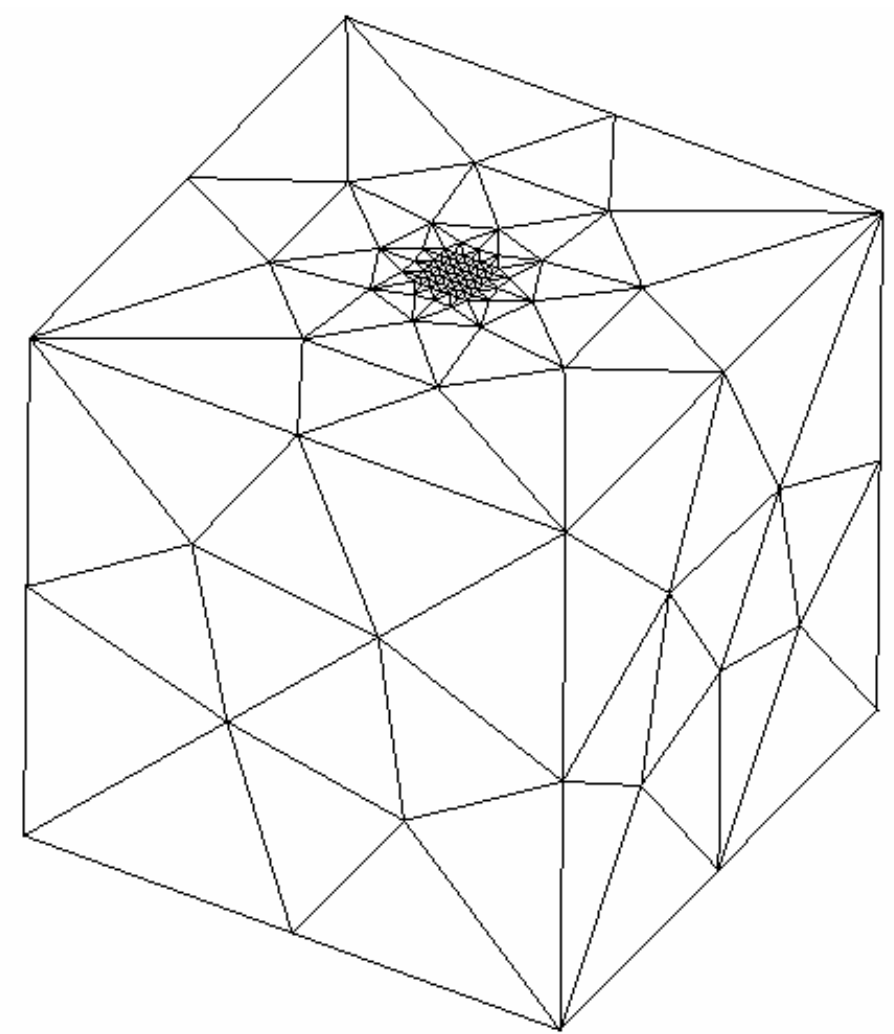

Figura 10.8 - MMBEM estendido

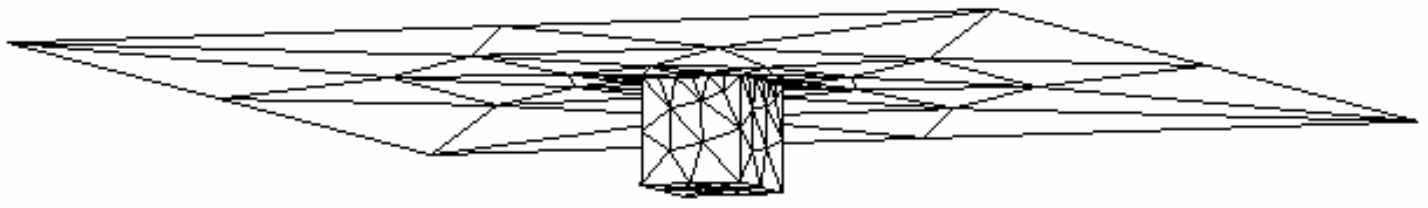

Figura 10.9 - MMBEM+TDBEM

As figuras 10.10 a 10.13 apresentam os mesmos resultados que a figura 10.6, sendo que agora se considerando a presença da placa, onde a espessura $\mathrm{h}$ da mesma variou de 0,2 a $5 \mathrm{~m}$.

$\mathrm{Na}$ figura 10.10 , considerou-se $\mathrm{h}=20 \mathrm{~cm}$. Comparando-se os resultados obtidos com aqueles apresentados na figura 10.6, observa-se que o ganho de rigidez do conjunto placa-solo é pequeno, uma vez que a espessura da placa é pequena em comparação com o comprimento do seu lado, aparecendo, entretanto, movimento ondulatório inerente à vibração da placa.

$\mathrm{Na}$ figura 10.11, apresenta-se os resultados obtidos com a placa de espessura $\mathrm{h}=1,5 \mathrm{~m}$ para as mesmas modelagens consideradas na figura 10.10. Também se observa uma boa concordância entre os resultados, principalmente aqueles obtidos com o TDBEM $6 \times 6$ e 12x12. As observações feitas para a figura 10.11 valem para a seguinte, onde se adotou $h=2,5 \mathrm{~m}$. 


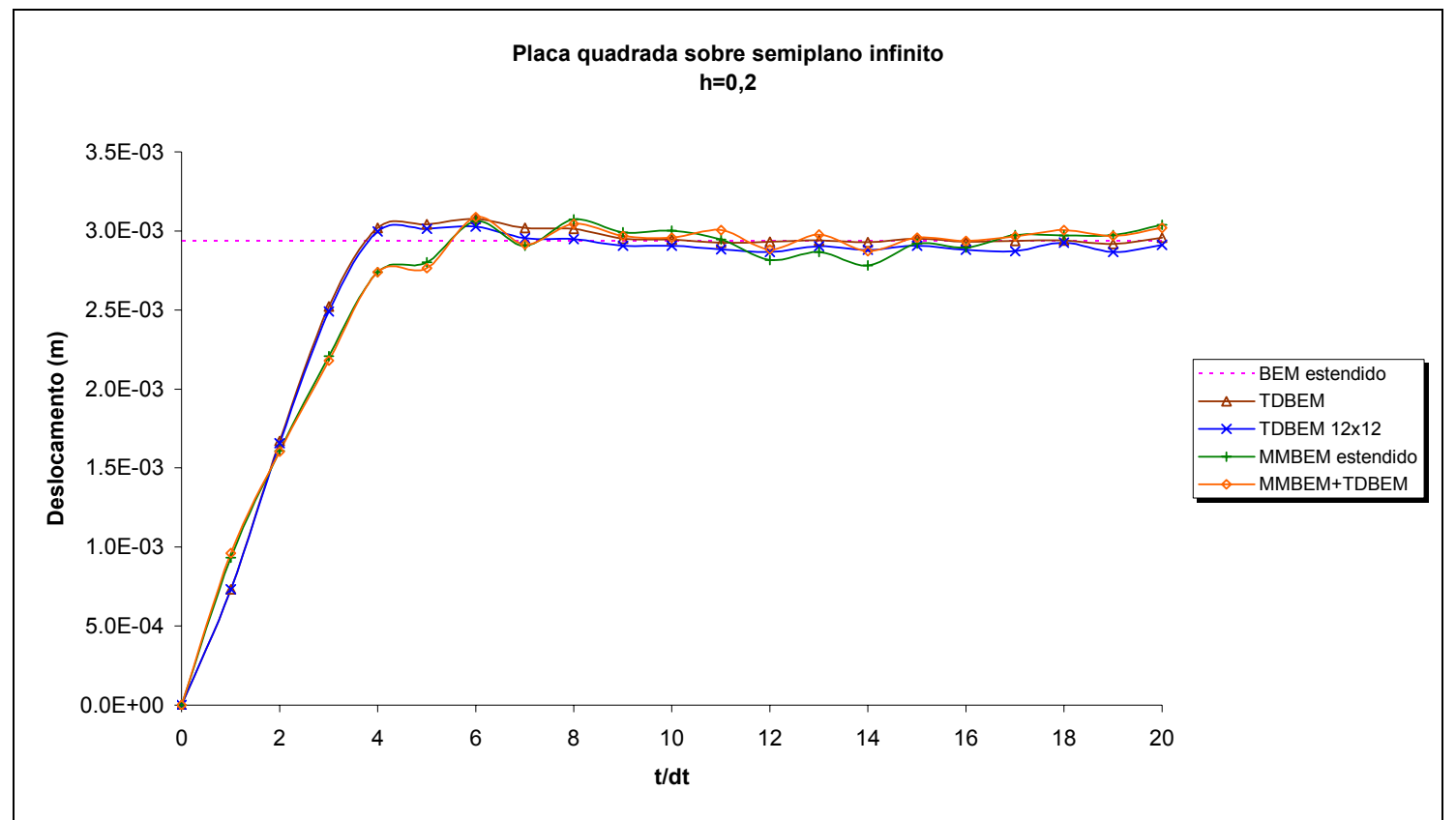

Figura 10.10 - Exemplo 10.2.1 - análise elastodinâmica para $\mathrm{h}=0,2$

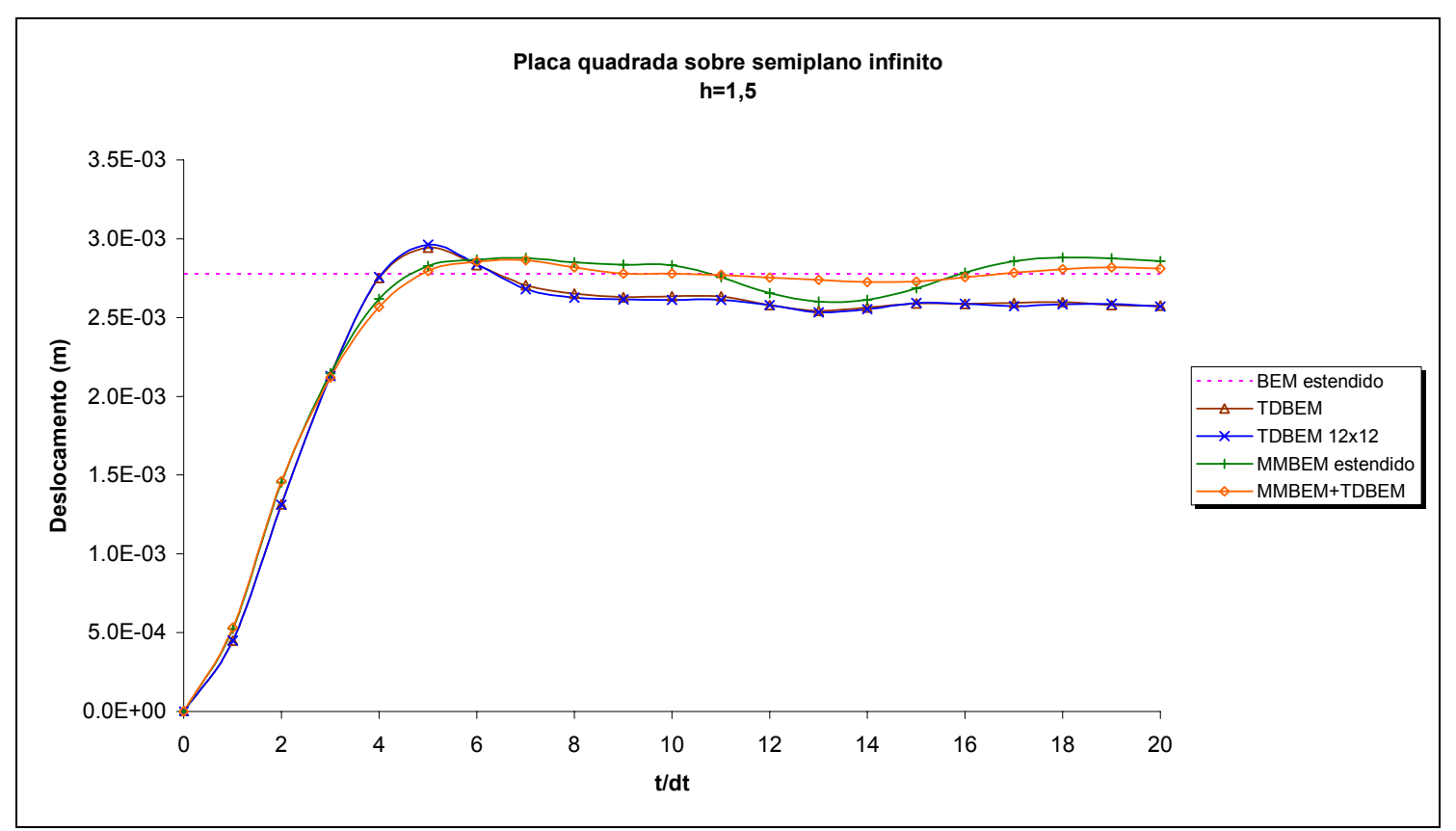

Figura 10.11 - Exemplo 10.2.1 - análise elastodinâmica para $\mathrm{h}=1,5$ 


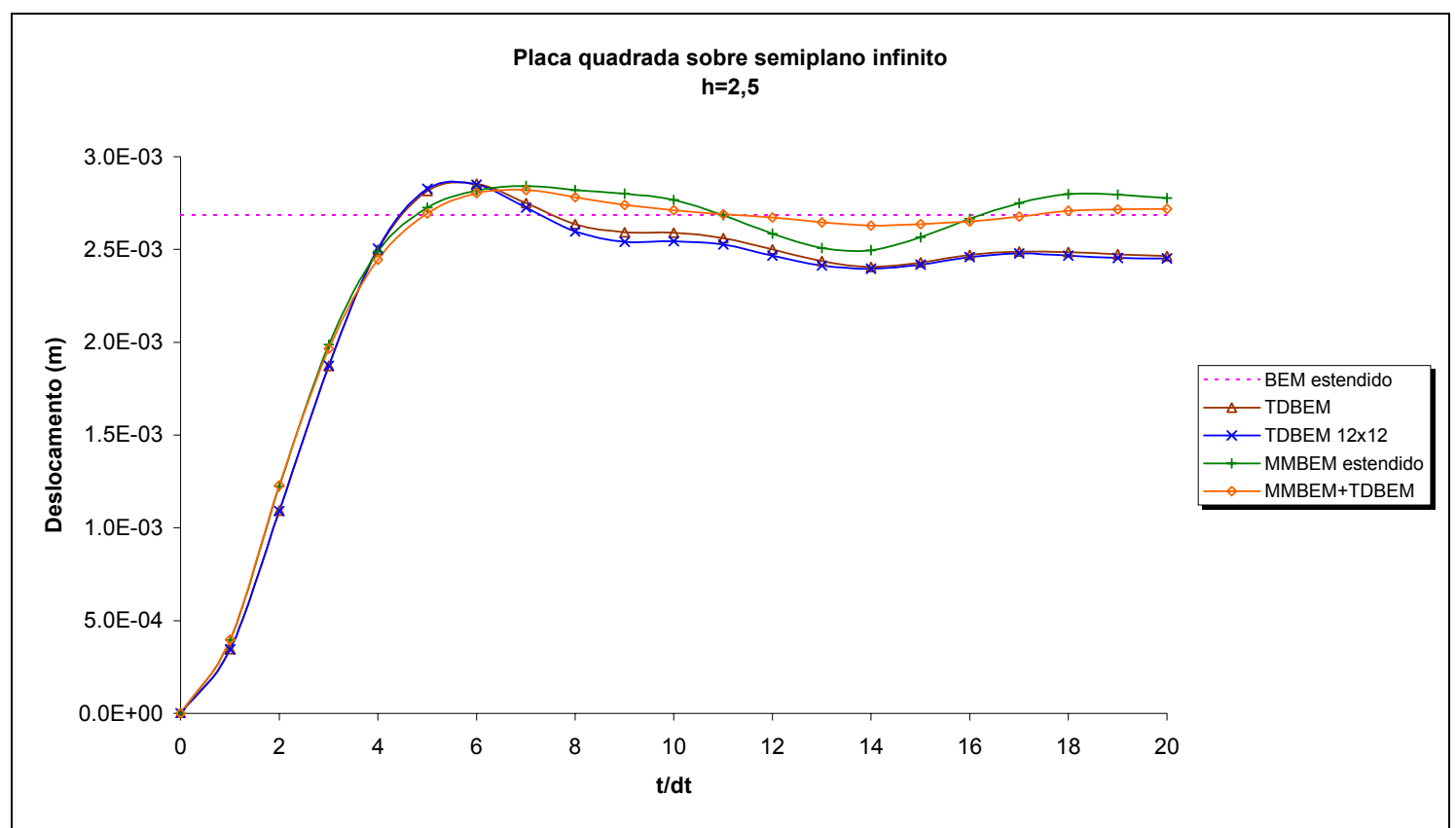

Figura 10.12 - Exemplo 10.2.1 - análise elastodinâmica para h=2,5

Para $h=5 m$, figura 10.13 , observa-se um pico inicial mais elevado em relação ao valor final, além de uma maior suavidade nas curvas apresentadas. Tal fato pode ser explicado pela maior força de inércia (massa) conferida pela maior espessura da casca. Observa-se também que, para este caso, considerou-se ainda a modelagem do TDBEM 24×24.

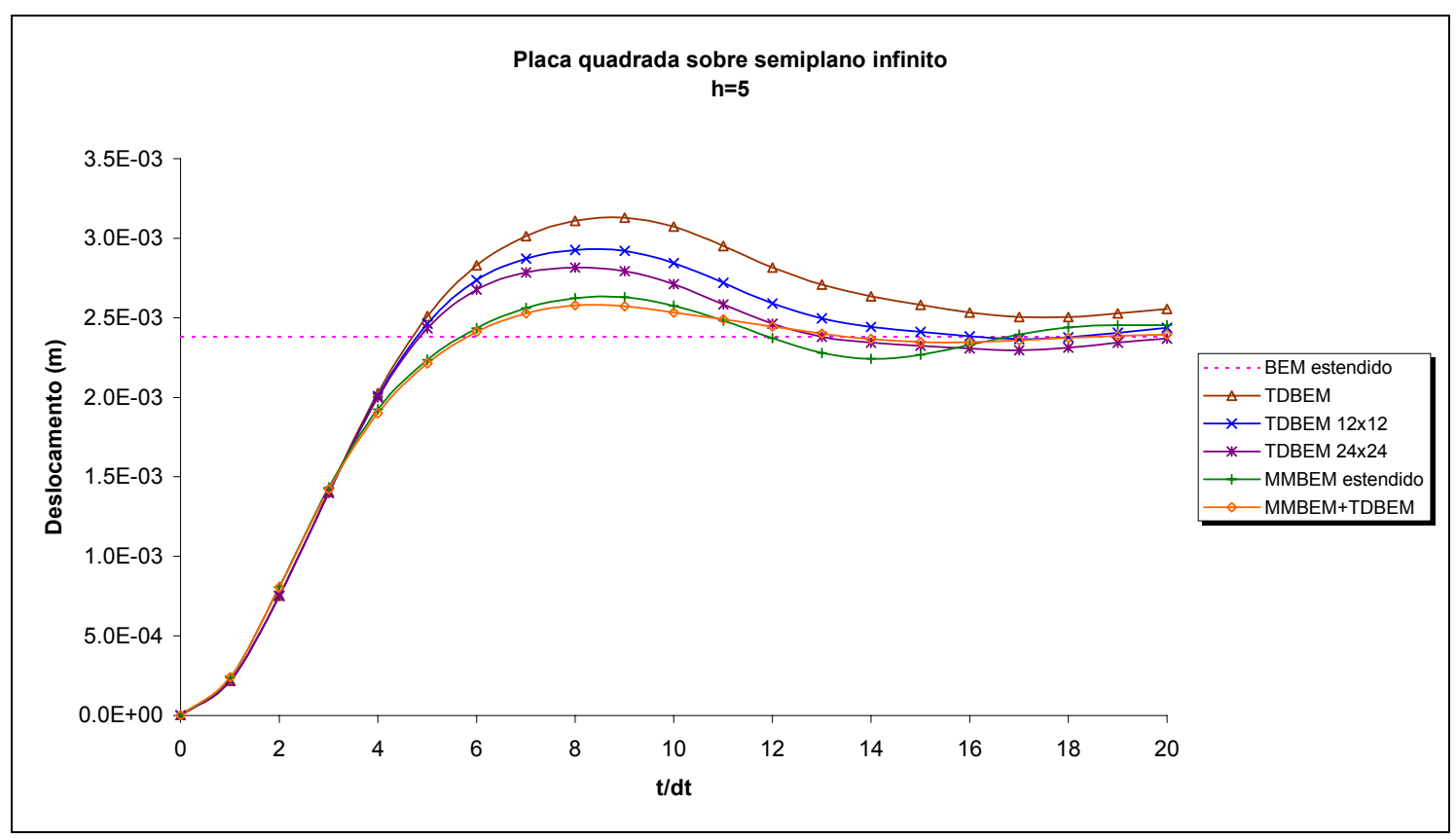

Figura 10.13 - Exemplo 10.2.1 - análise elastodinâmica para h=5 
Comparando-se as 4 figuras anteriores com a figura 10.6, nota-se que as análises para a modelagem do TDBEM estendido foram suprimidas. Assim foi feito porque tais análises sofreram perda de estabilidade em determinados momentos. Procurou-se resolver tal problema com um aumento no valor de $\beta$ do algoritmo de integração temporal do MEF (Newmark) e, por conseguinte, gerando um adiantamento da contribuição das forças inerciais das sub-regiões modeladas por esta técnica; esta alternativa se baseia no trabalho de $\mathrm{Hu}$ (1997), relacionado com algoritmos de integração especiais para problemas de impacto. Os resultados foram bastante interessantes. Para a placa muito delgada da figura $10.10(\mathrm{~h}=0,2 \mathrm{~m})$, para $\beta=0,5$, também ocorreu perda de estabilidade, porém a parte estável da análise foi maior que aquela para o valor usual de $\beta$, ou seja, 0,25 . Ver figura 10.14. Para os demais valores de $h$, considerando-se $\beta=0,5$, não se pode falar em perda de estabilidade numérica para o tempo de análise em questão. Figuras 10.15 a 10.17. Donde se conclui que o problema de estabilidade no acoplamento TDBEM/FEM pode ser contornado pela utilização de valores mais apropriados para o parâmetro $\beta$ do método de Newmark.

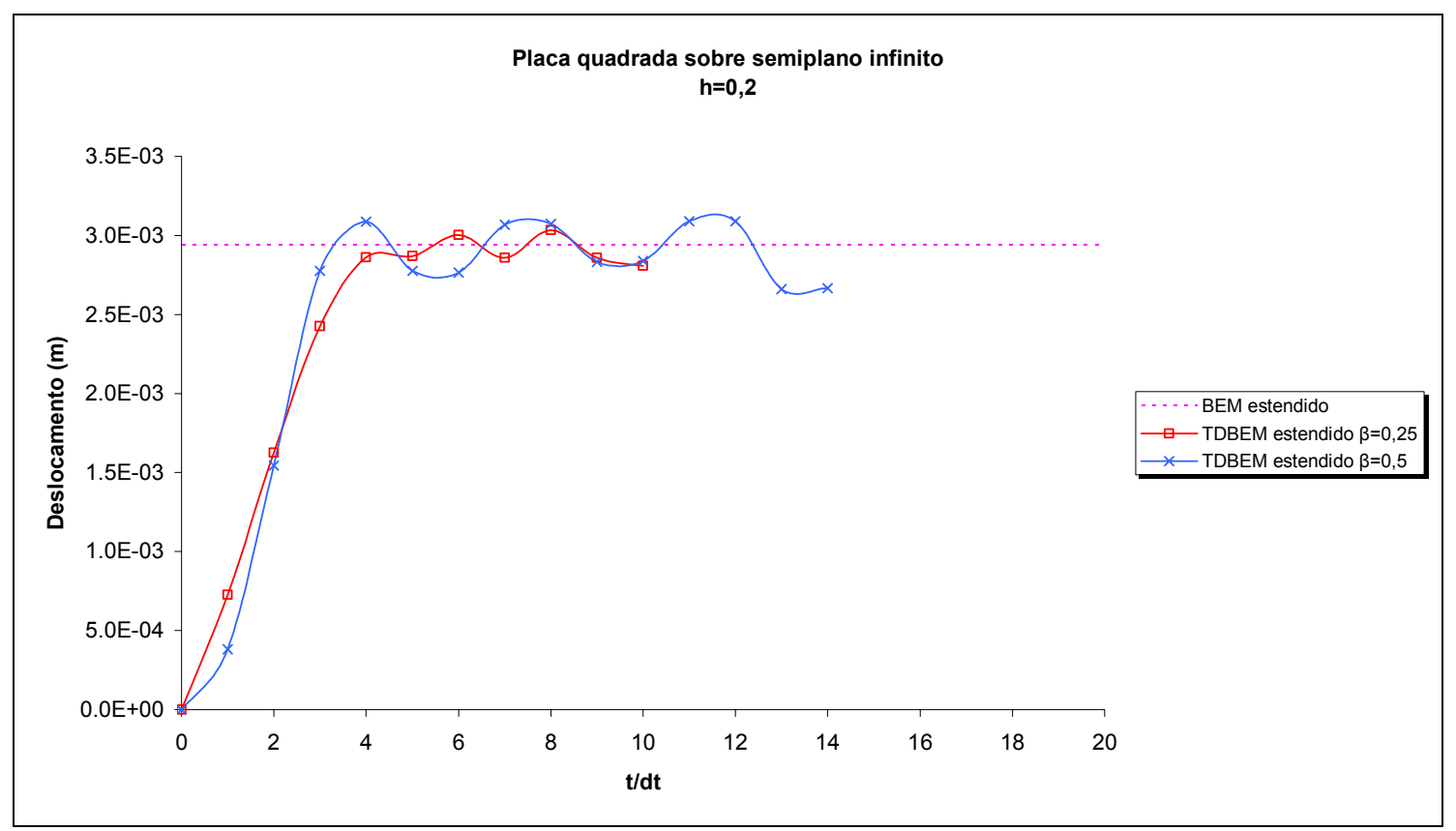

Figura 10.14 - Exemplo 10.2.1 - TDBEM estendido para $\mathrm{h}=0,2$ 


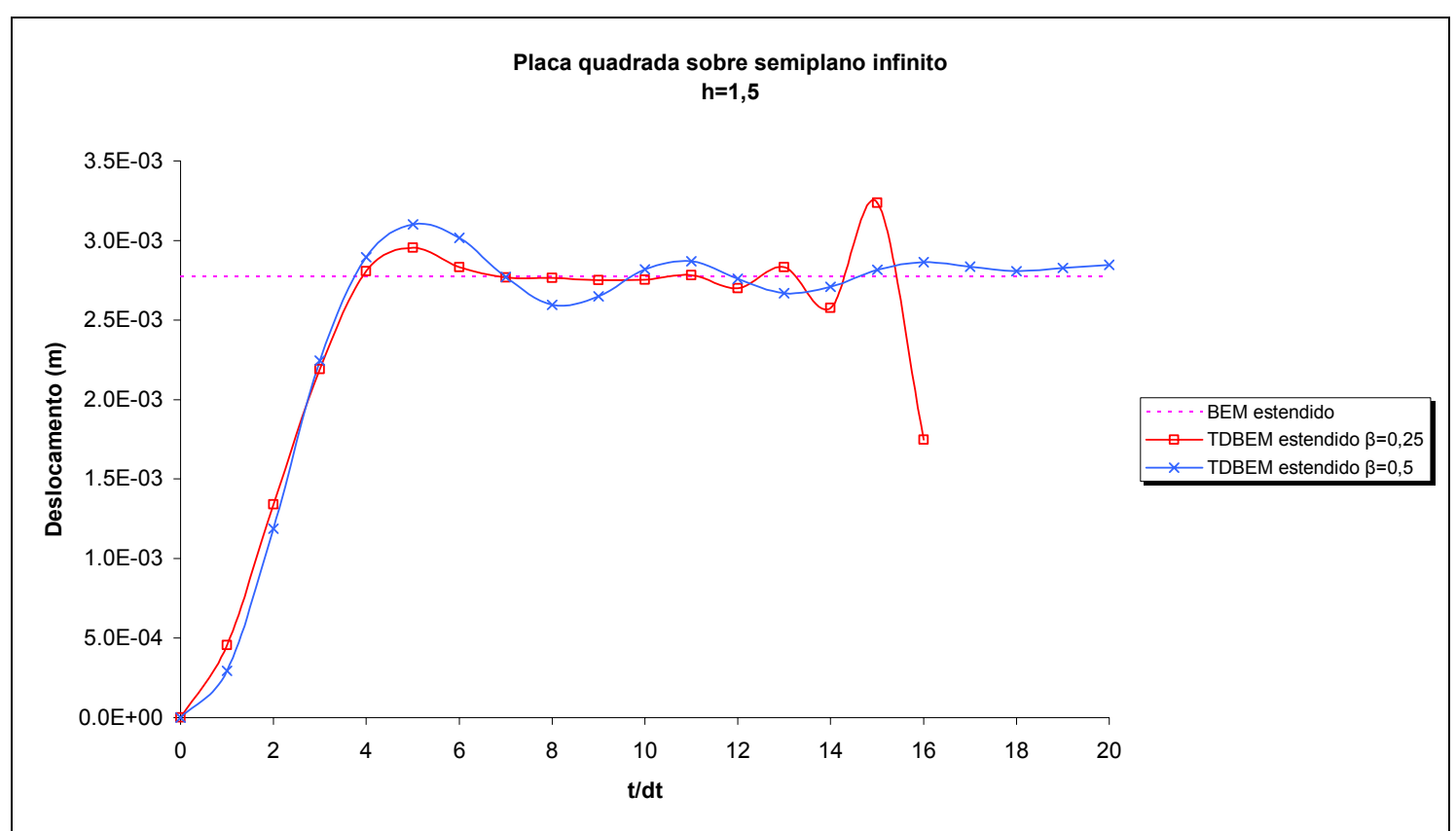

Figura 10.15 - Exemplo 10.2.1 - TDBEM estendido para $\mathrm{h}=1,5$

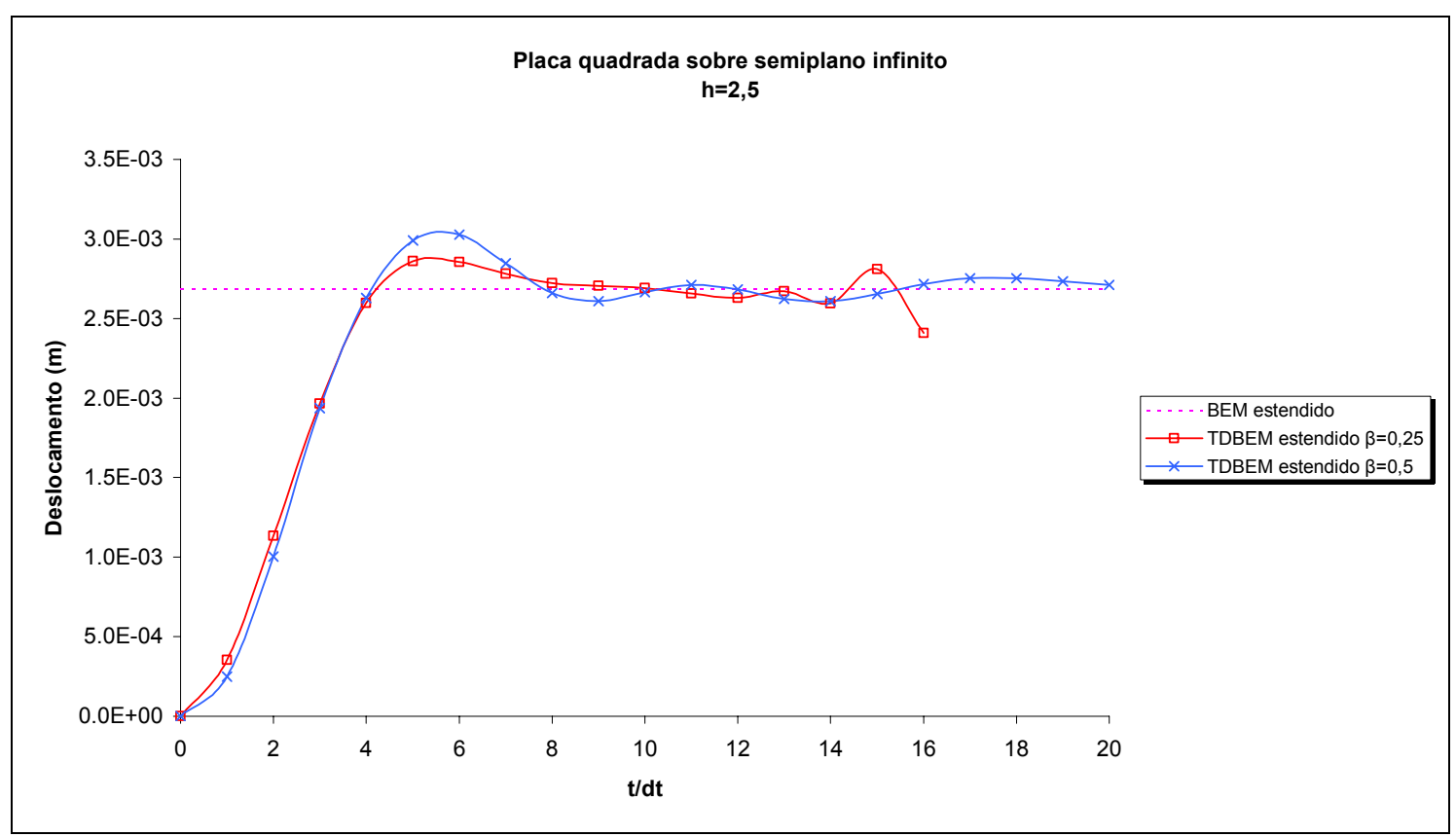

Figura 10.16 - Exemplo 10.2.1 - TDBEM estendido para $\mathrm{h}=2,5$

Deve-se observar que, para valores relativos maiores entre o módulo de elasticidade do radier e do solo, a calibração do parâmetro $\beta$ pode ser necessária para a estabilização do processo, mesmo com discretizações finitas, no TDBEM, ver exemplo 10.3. 


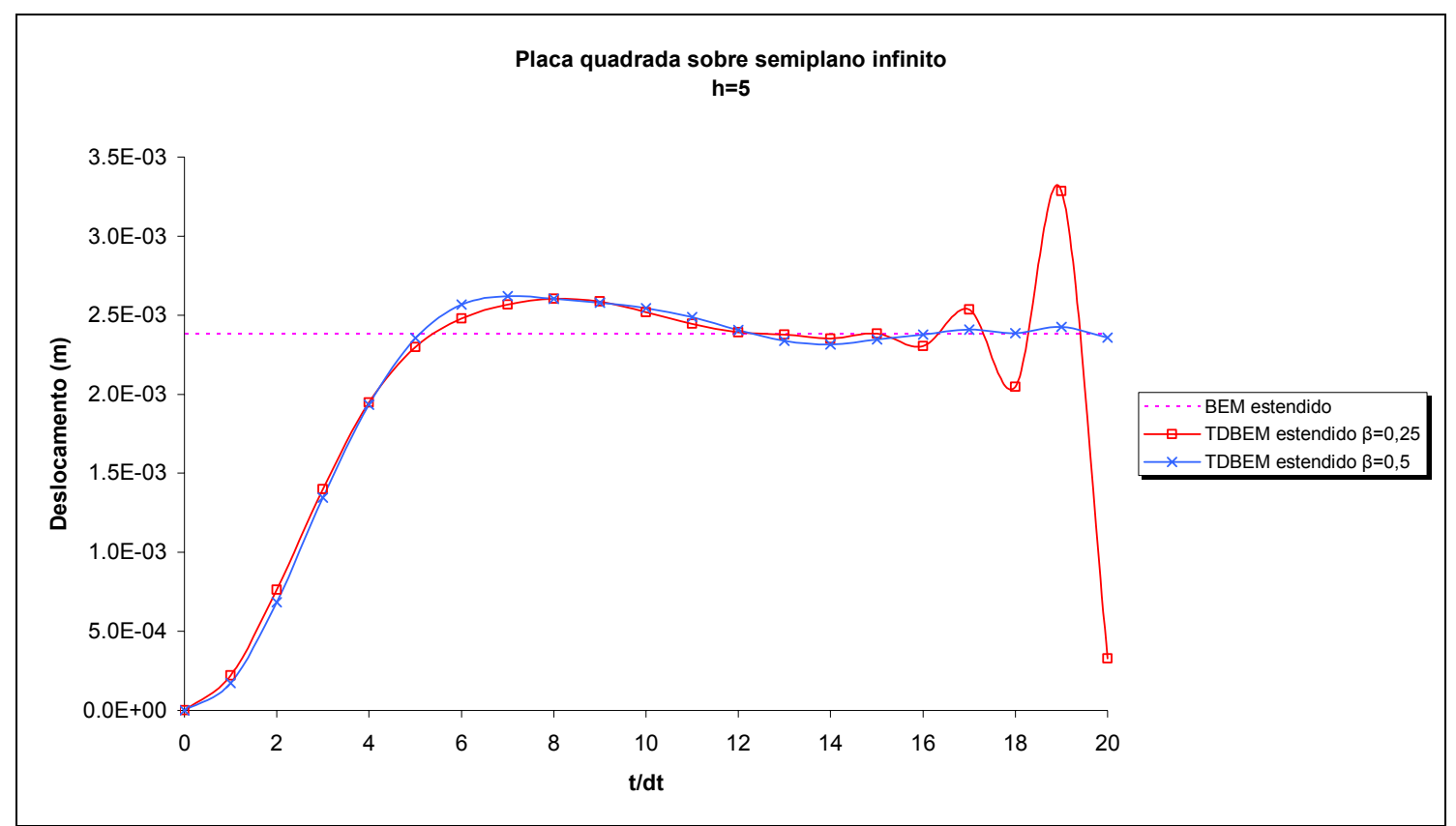

Figura 10.17 - Exemplo 10.2.1 - TDBEM estendido para h=5

Após as análises elásticas apresentadas anteriormente, partiu-se para análises elastoplásticas do caso de interação solo-estrutura em questão, inicialmente estáticas e posteriormente dinâmicas. Considerou-se uma nova dimensão de placa desta vez $(13,33 \times 13,33 \mathrm{~m})$ localizada no meio da face livre do cubo sólido, com uma discretização 4x4, apenas com o intuito de manter as discretizações do MMBEM e se deixar a distância de 1 lado de elemento de contorno para que a plastificação fosse melhor representada pelo cubo das modelagens utilizadas. Tais modelagens foram: uma denominada MMBEM+BEM, que seria semelhante à MMBEM+TDBEM, sendo que com células de plastificação ao invés das células da análise dinâmica na sub-região do MMBEM e elementos de contorno do BEM no lugar de elementos do TDBEM; e outra denominada MMBEM+TDBEM EPD (elastoplástico dinâmico), onde, para o cubo modelado pelo MMBEM, além das células da matriz de massa, existem, coincidentemente, as células de plastificação. As modelagens MMBEM+BEM e MMBEM+TDBEM EPD foram utilizadas respectivamente para os casos elastoplástico estático e elástoplástico dinâmico.

Para as análises elastoplásticas estáticas, considerou-se o critério de plastificação de von Mises com tensão de plastificação de $150000 \mathrm{~kg} /\left(\mathrm{m} \cdot \mathrm{s}^{2}\right)$, coeficiente de endurecimento (hardening) de $2,1 \cdot 10^{8} \mathrm{~kg} /\left(\mathrm{m} \cdot \mathrm{s}^{2}\right)$ e 30 passos de carga. 
A figura 10.18 apresenta as curvas de deslocamento no ponto central ao invés do ponto $A$, como foi feito até então - pelos passos de carga, para valores de espessura de placa de 1,5; 2,5 e 5m. O carregamento foi aplicado de duas formas distintas: distribuído na placa (d), com valor igual àquele utilizado anteriormente, e uma carga concentrada no centro da placa (c), com valor equivalente à resultante do carregamento distribuído na nova placa.

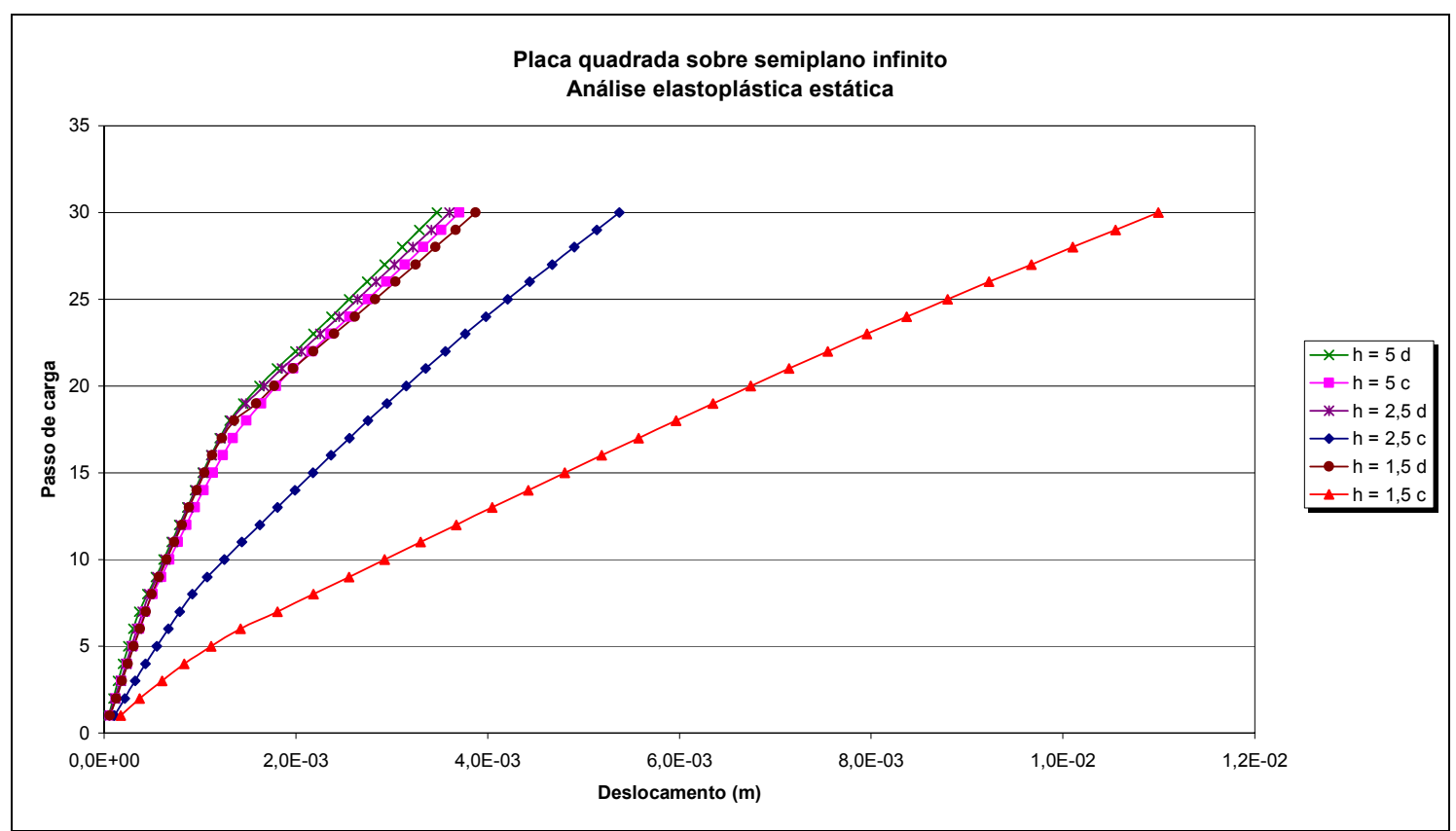

Figura 10.18 - Exemplo 10.2.1 - análise elastoplástica estática

Analisando-se a figura 10.18, observa-se à primeira vista que, quanto menor a espessura da placa, maior a diferença entre os valores obtidos com as cargas distribuída e concentrada. Isto ocorre porque com o aumento da espessura da placa as forças de contato entre estrutura e solo ficam semelhantes para ambos os casos. A figura 10.19 apresenta as forças de contato entre a placa e o solo do último passo de carga, para a espessura $1,5 \mathrm{~m}$, onde são comparados os resultados para carregamento concentrado e distribuído. Observa-se que os valores negativos, presentes para o carregamento concentrado, são de tração. A partir da figura 10.19 pode-se perceber que, para a carga concentrada, as tensões de contato são de compressão no centro e de tração nos vértices. Nota-se também os elevados valores, principalmente na região comprimida. Já para a carga distribuída, só existem tensões de compressão. Agora, analisando-se a figura 10.20, vê-se 
perfeitamente que, para $\mathrm{h}=5 \mathrm{~m}$, em primeiro lugar, só existem forças de contato de compressão, e os valores de tais forças são bastante semelhantes.

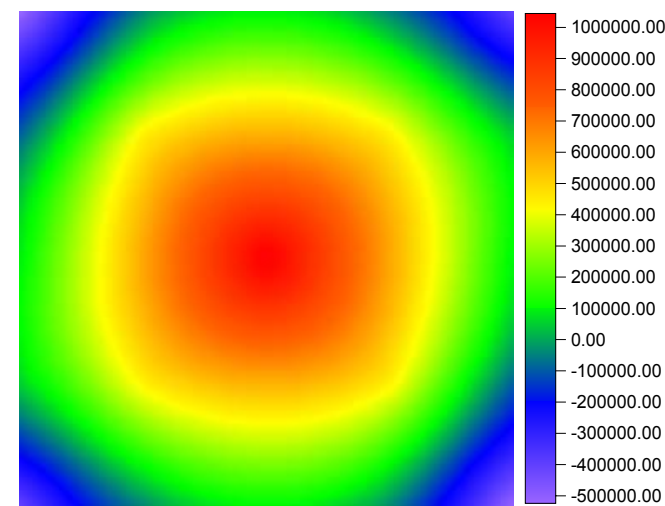

Carga concentrada

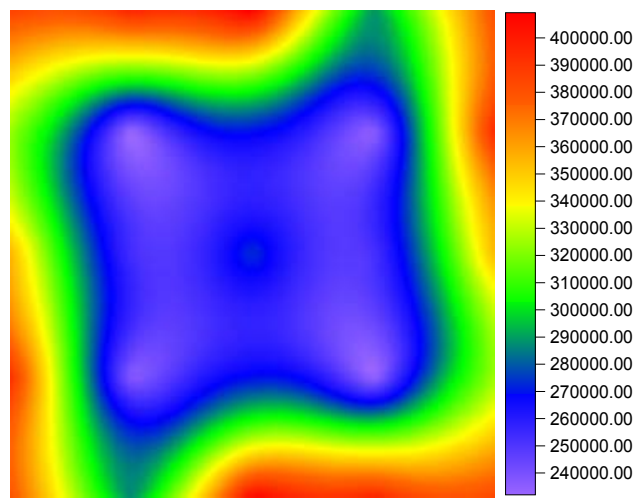

Carga distribuída

Figura 10.19 - Exemplo 10.2.1 - força de contato para $\mathrm{h}=1,5$

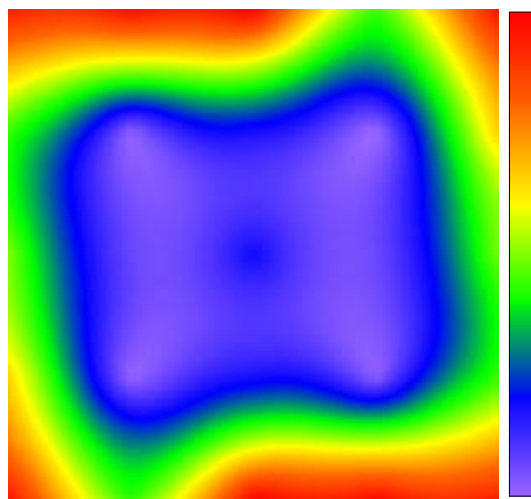

Carga concentrada

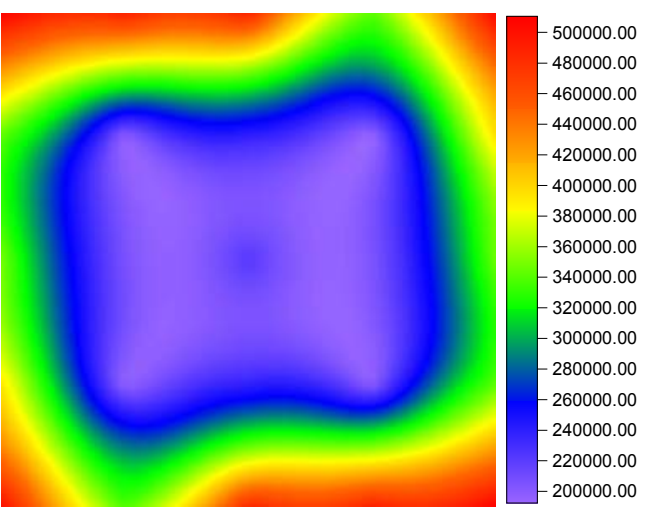

Carga distribuída

Figura 10.20- Exemplo 10.2.1 - força de contato para $\mathrm{h}=5$

Para a análise elastoplástica dinâmica, as figuras 10.21 a 10.23 apresentam a comparação entre os deslocamentos transversais do nó localizado no centro da placa ao longo dos passos de tempo, com e sem a plastificação, para as espessuras $h=5 m, h=2,5 m$ e $h=1,5 m$, respectivamente. Na legenda das figuras, "ED” quer dizer elastodinâmico e "EPD”, elastoplástico dinâmico. Observa-se que a consideração de plastificação no solo, de acordo com os parâmetros fornecidos anteriormente, aumenta para 3 a 4 vezes os valores dos deslocamentos sem a consideração da mesma. A figura 10.24 mostra apenas as curvas para as análises elastoplásticas para os 3 valores de h. 


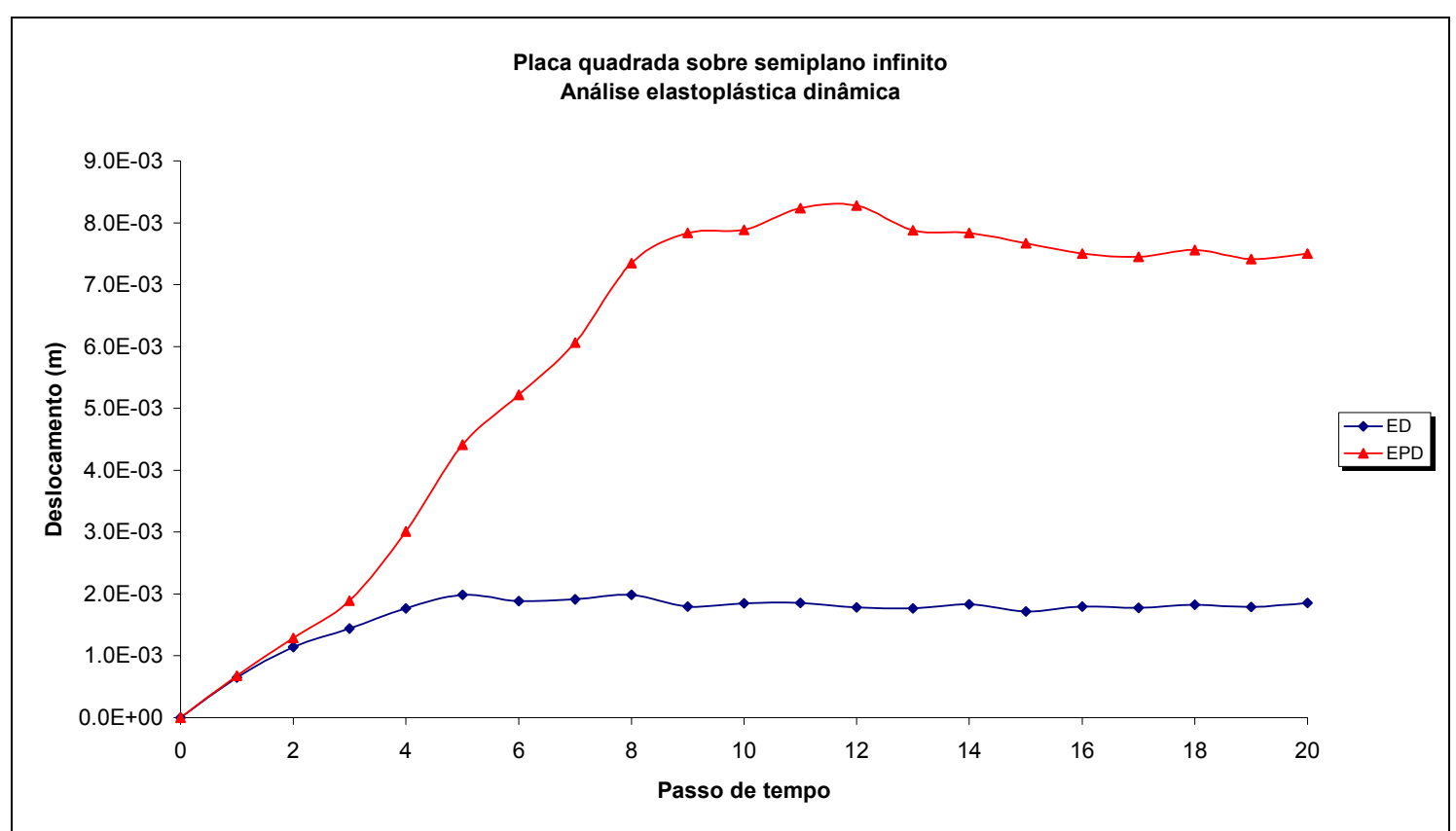

Figura 10.21 - Exemplo 10.2.1 - análise elastoplástica dinâmica para h=5

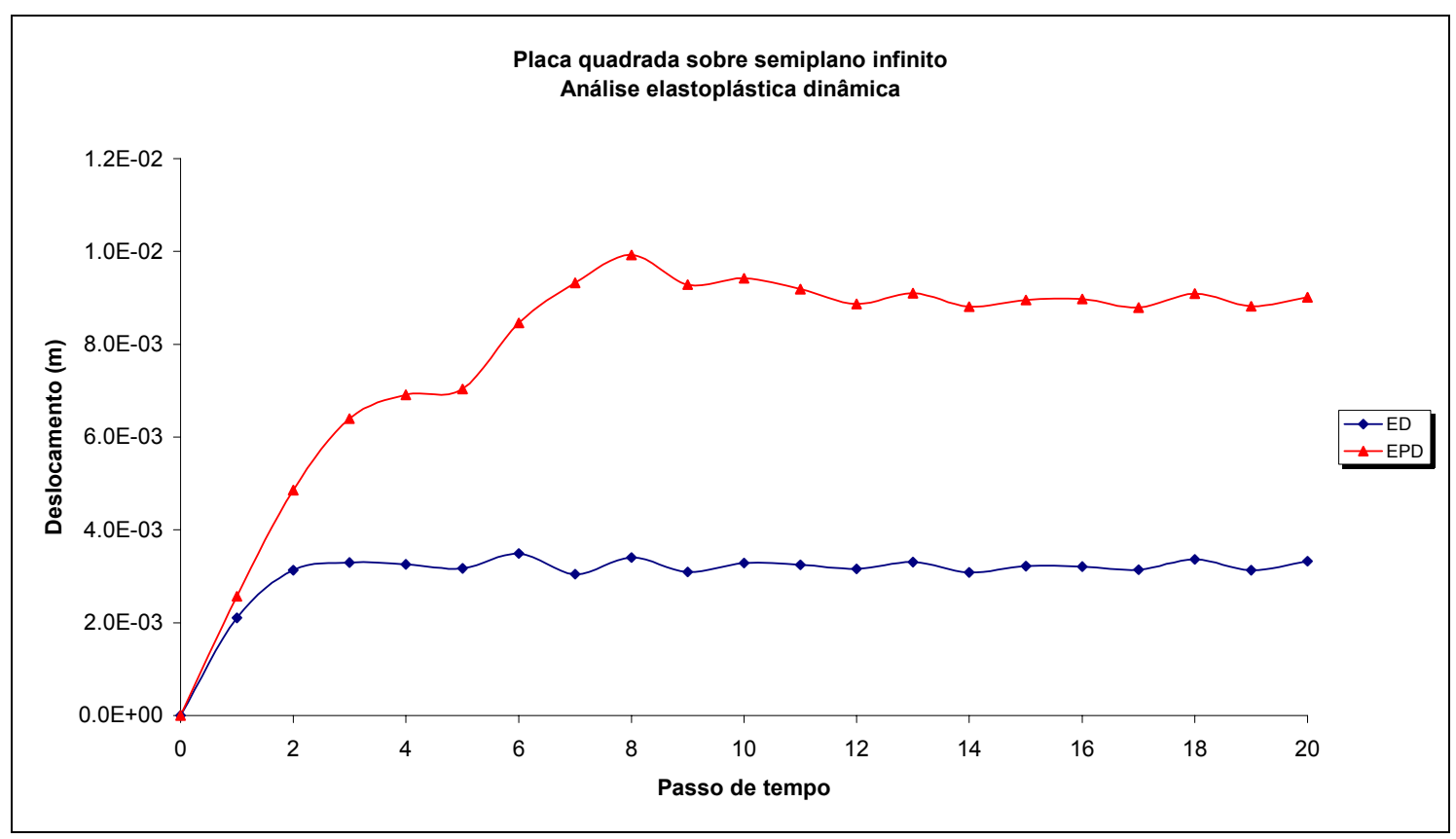

Figura 10.22 - Exemplo 10.2.1 - análise elastoplástica dinâmica para h=2,5 


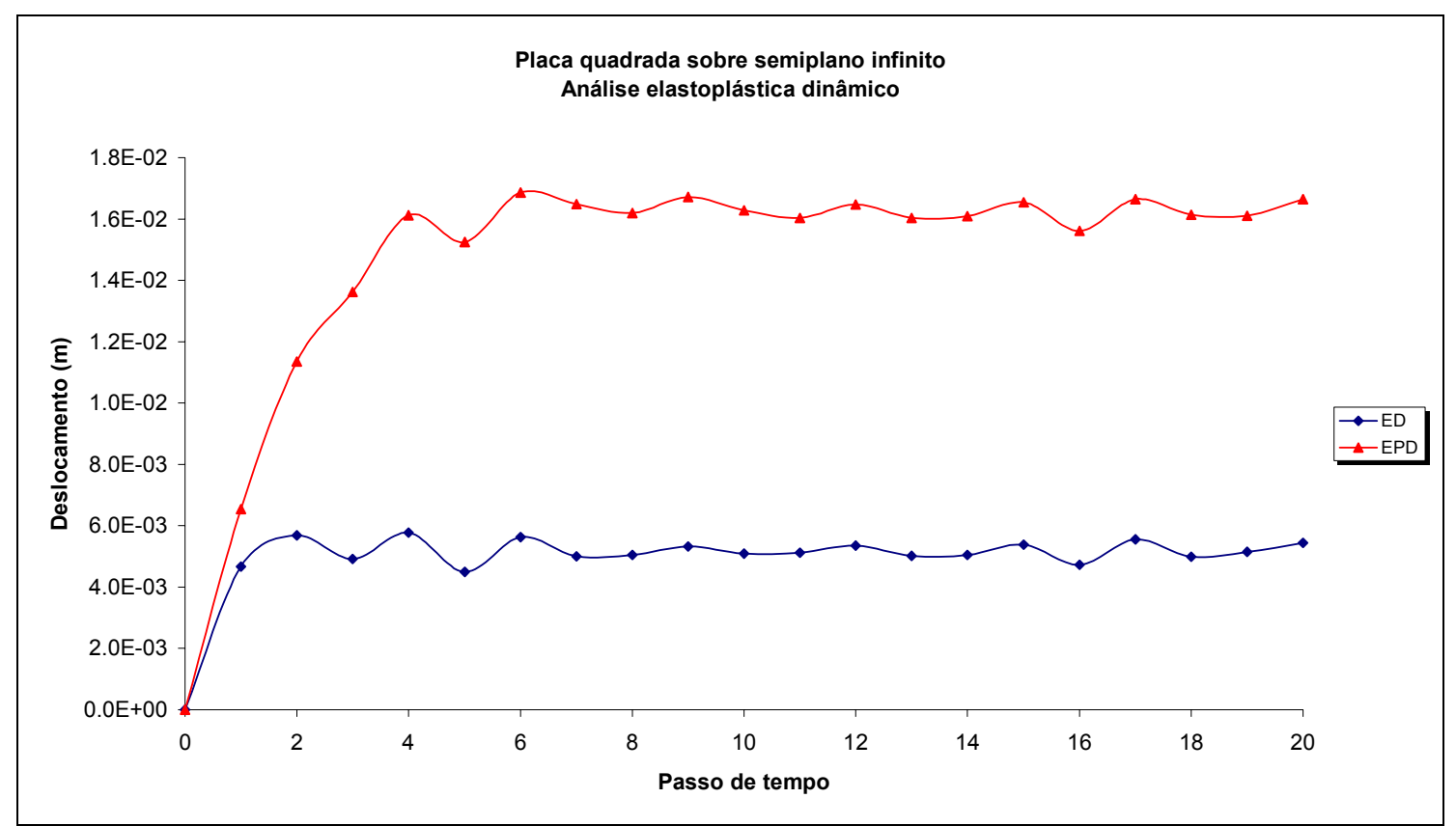

Figura 10.23 - Exemplo 10.2.1 - análise elastoplástica dinâmica para h=1,5

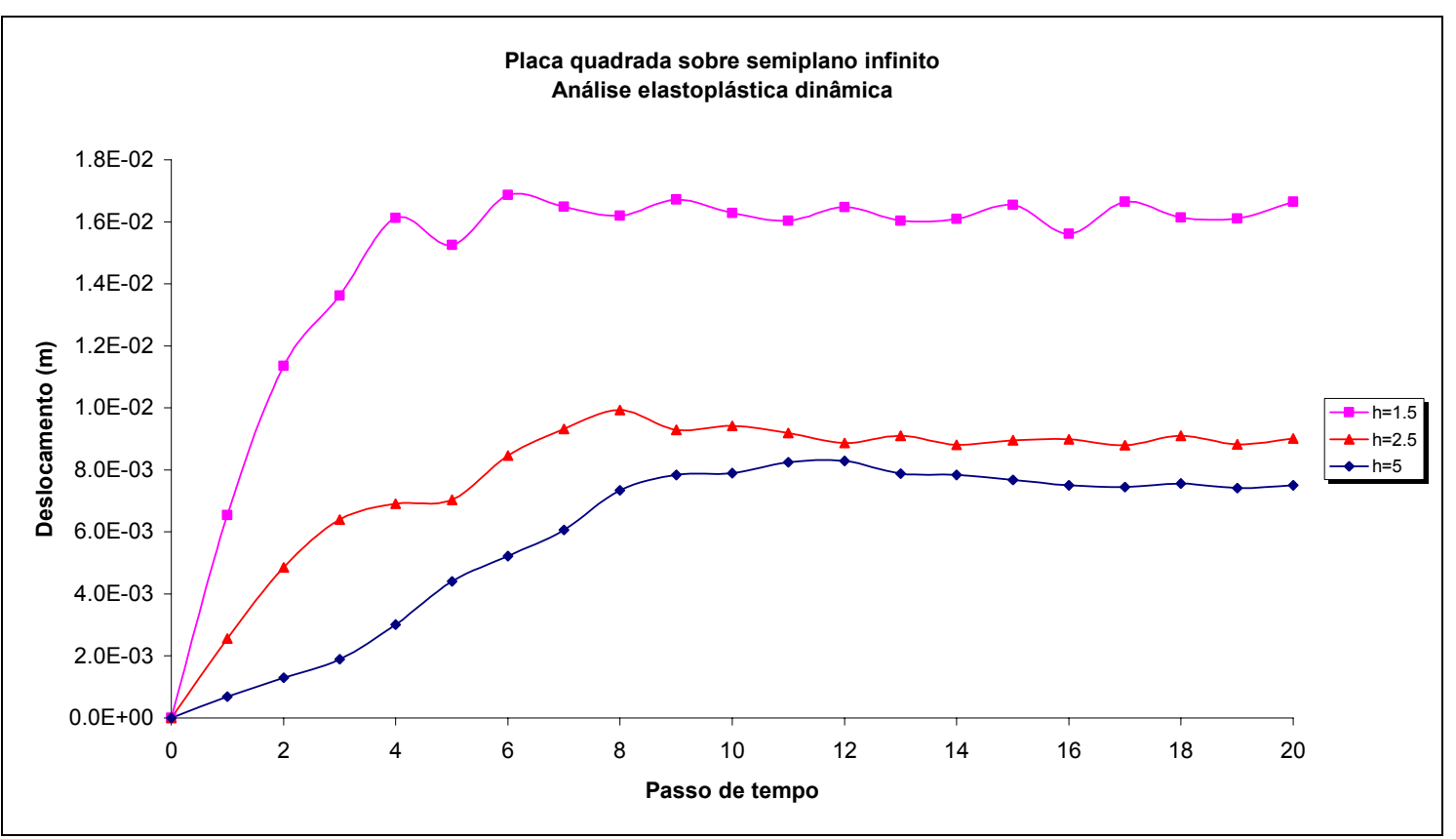

Figura 10.24 - Exemplo 10.2.1 - análise elastoplástica dinâmica

\subsubsection{Placa quadrada com barra sobre semiplano infinito}

Neste segundo estágio do exemplo 2, considera-se a sub-região de elementos finitos composta não apenas pela placa, mas também por uma barra localizada no centro da mesma. Como, para este estágio, foram realizadas análises elastoplásticas, utilizou-se a placa quadrada com dimensão 
$13,33 \times 13,33 \mathrm{~m}$ e $1,5 \mathrm{~m}$ de espessura. Os demais dados para a barra envolvidos no problema são apresentados no quadro abaixo.

$$
\begin{aligned}
& \rho=2500 \mathrm{~kg} / \mathrm{m}^{3} \\
& L=40 \mathrm{~m} \\
& A=2 \mathrm{~m} \times 2 \mathrm{~m}=4 \mathrm{~m}^{2} \\
& E=2,1 E+10 \mathrm{~kg} /\left(\mathrm{m} \cdot \mathrm{s}^{2}\right) \\
& v=0,25
\end{aligned}
$$

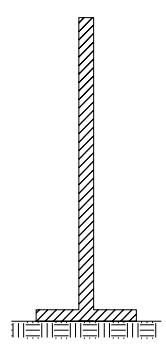

Em todas as análises mostradas a seguir, considerou-se a atuação do peso próprio da placa.

A figura 10.25 apresenta os deslocamentos estáticos horizontais na extremidade livre da barra pela força horizontal no mesmo ponto, onde o solo foi modelado pelo acoplamento MMBEM+BEM estendido. Observa-se que, para as análises com hardening $\left(\mathrm{H}=2,1 \mathrm{E}+8 \mathrm{~kg} /\left(\mathrm{m} \cdot \mathrm{s}^{2}\right)\right)$, aplicou-se, além do peso próprio da placa, uma carga concentrada horizontal na extremidade livre da barra, de valor $1250000 \mathrm{Kg} \cdot \mathrm{m} / \mathrm{s}^{2}$. Para as demais análises, considerou-se controle de deslocamento horizontal no topo da barra, onde se calculou a força necessária para tal deslocamento. Observando-se a legenda da figura 10.25, as análises "perf." foram feitas com coeficiente de hardening nulo $(\mathrm{H}=0)$, ou seja, plasticidade perfeita; as análises "soft.", foram realizadas com $\mathrm{H}=-2,1 \mathrm{E}+8$ $\mathrm{kg} /\left(\mathrm{m} \cdot \mathrm{s}^{2}\right)$, ou seja, com softening neste valor; para os critérios de Drucker Prager, o valor de porcentagem que segue indica quantos por cento o valor da tensão de plastificação por tração é da tensão de plastificação por compressão, sendo esta última $150000 \mathrm{~kg} /\left(\mathrm{m} \cdot \mathrm{s}^{2}\right)$. Pode-se observar que as análises com hardening e plasticidade perfeita foram semelhantes, porém com a consideração de softening, houve colapso frágil da estrutura. Até mesmo para a plasticidade perfeita com o critério de Drucker Prager $10 \%$, as forças necessárias para a obtenção dos deslocamentos prescritos são menores, indicando já um início de colapso da estrutura.

Na figura 10.26, vê-se os resultados para o deslocamento vertical no meio da placa para as mesmas análises realizadas na figura anterior, acrescidas da resposta elástica. Os valores positivos indicam deslocamento 
para baixo. Igualmente ao que foi observado na figura 10.25 , as 3 modelagens que levaram em consideração softening nas análises entraram em colapso rapidamente e em ordem proporcional ao valor da tensão de plastificação por tração, do menor ao maior. Observa-se também que, para a análise com endurecimento, critério de plastificação de Drucker Prager e tensão de plastificação à tração de $10 \%$, o ponto estudado se movimentou em sentido contrário ao obtido utilizando-se o critério de Von Mises. Também se observa que, para os 3 valores de coeficiente de hardening, quanto maior a tensão de plastificação à tração, menores são os deslocamentos.

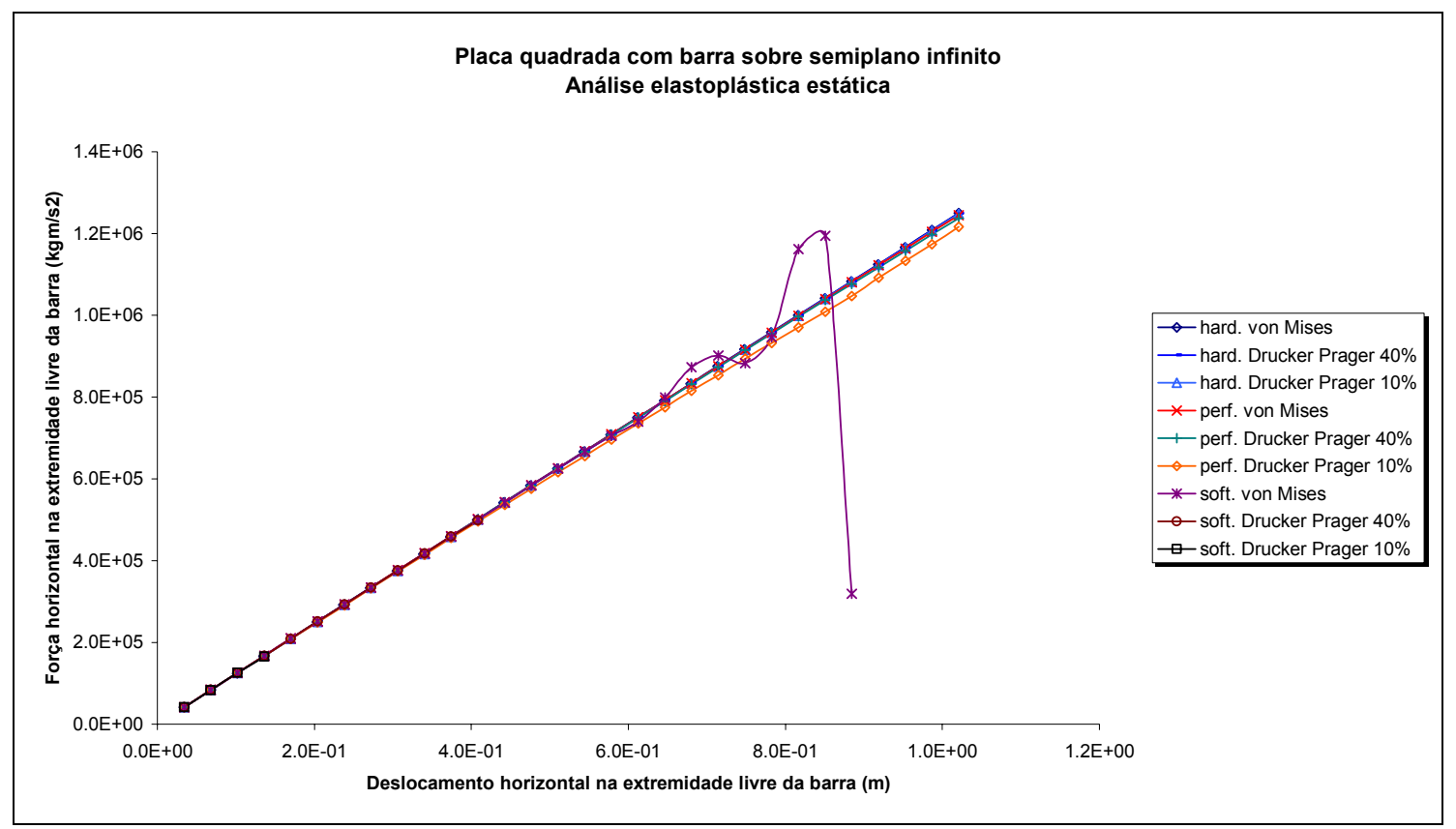

Figura 10.25 - Exemplo 10.2.2 - análise elastoplástica estática - deslocamento horizontal

O caso em questão também foi analisado dinamicamente, com aplicação súbita/constante das cargas atuantes, onde se utilizou a modelagem MMBEM+TDBEM EPD para o tratamento do solo. Apenas o critério de plastificação de Von Mises foi empregado, onde se variou o coeficiente de endurecimento $\mathrm{H}$.

Observando-se a figura 10.27, vê-se que, para valores de $\mathrm{H}$ positivos (hardening), as análises em questão são viáveis; para valores de $\mathrm{H} \leq 0$ (plasticidade perfeita ou softening), os modelos apresentam colapso logo no início das análises, o que era de se esperar, uma vez que, para um 
carregamento de impacto, o pico de tensão ocorre no início da análise. $\mathrm{Na}$ legenda da figura citada, "EE" representa o comportamento elastostático.

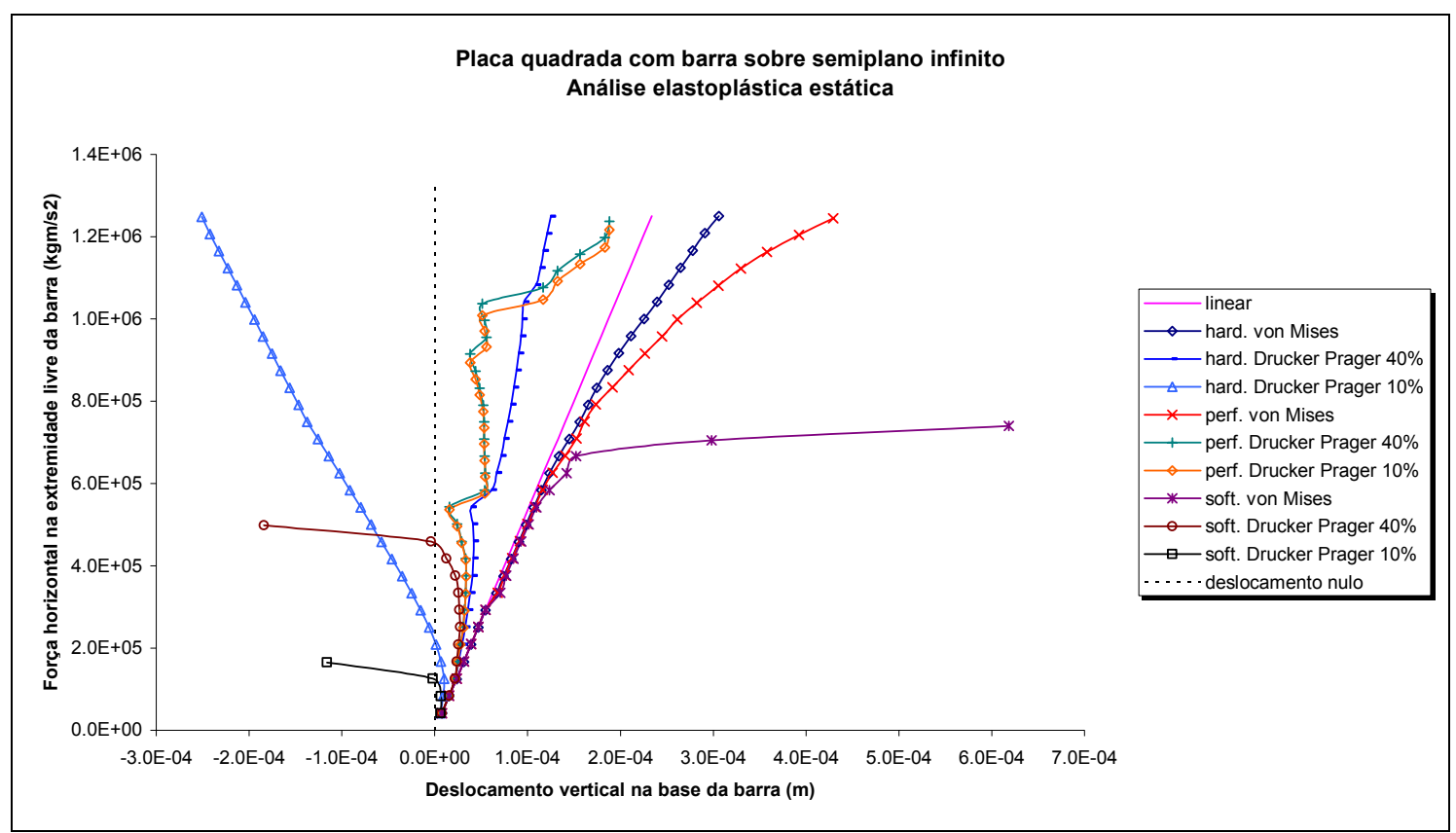

Figura 10.26 - Exemplo 10.2.2 - análise elastoplástica estática - deslocamento vertical

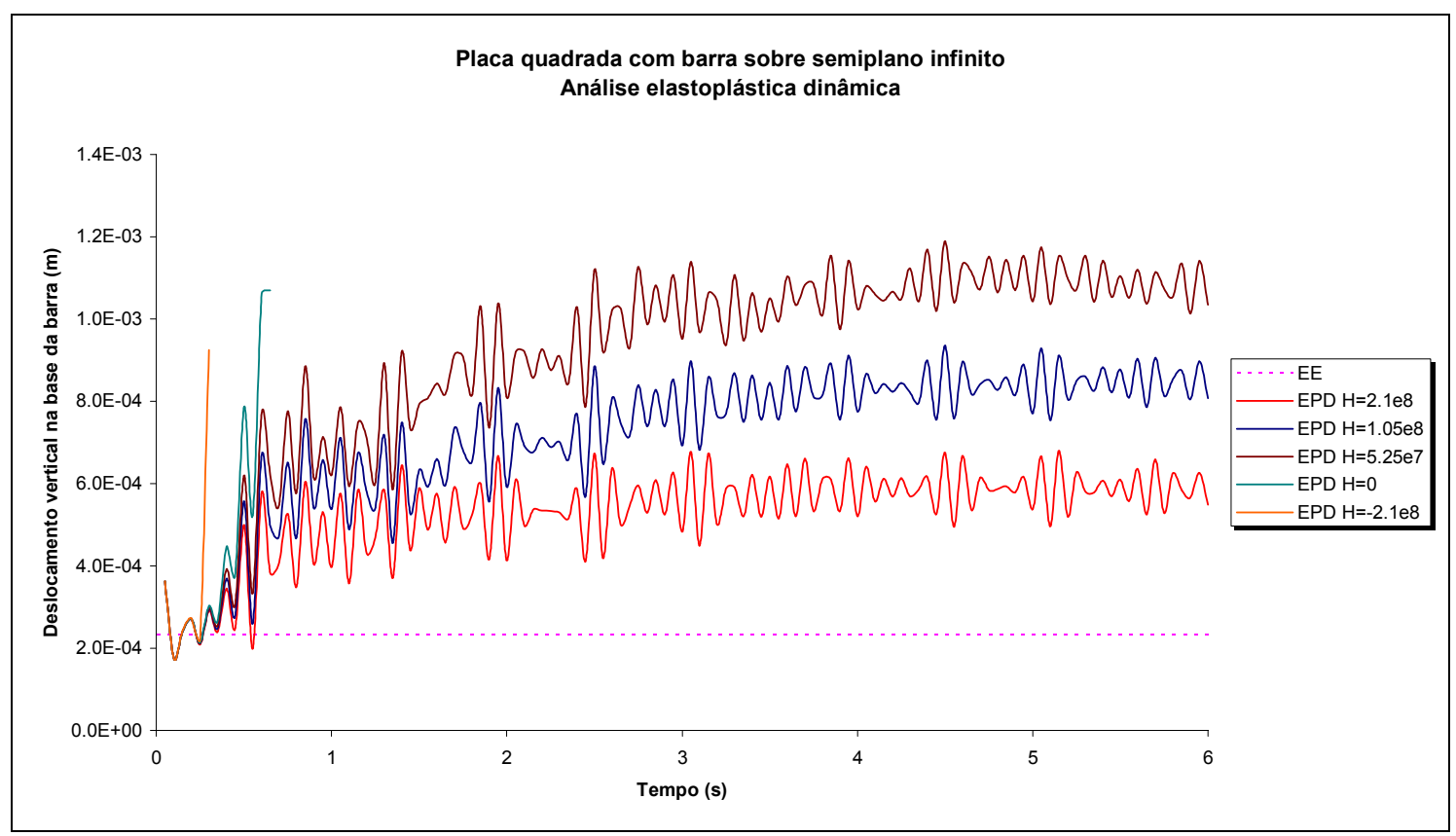

Figura 10.27 - Exemplo 10.2.2 - análise elastoplástica dinâmica - deslocamento vertical 
Já a figura 10.28, apresenta os deslocamentos horizontais no topo da barra ao longo do tempo. O comportamento "ED" representa as respostas elastodinâmicas, e a curva "MEF" foi construída a partir de uma análise apenas com elementos finitos, onde a barra foi considerada engastada ao solo. Observa-se as mesmas perdas de estabilidade presentes na figura anterior, e os menores deslocamentos obtidos para a modelagem puramente com elementos finitos. Este último fato, juntamente com o menor período deste movimento, é justificado pela não consideração da deformação do solo.

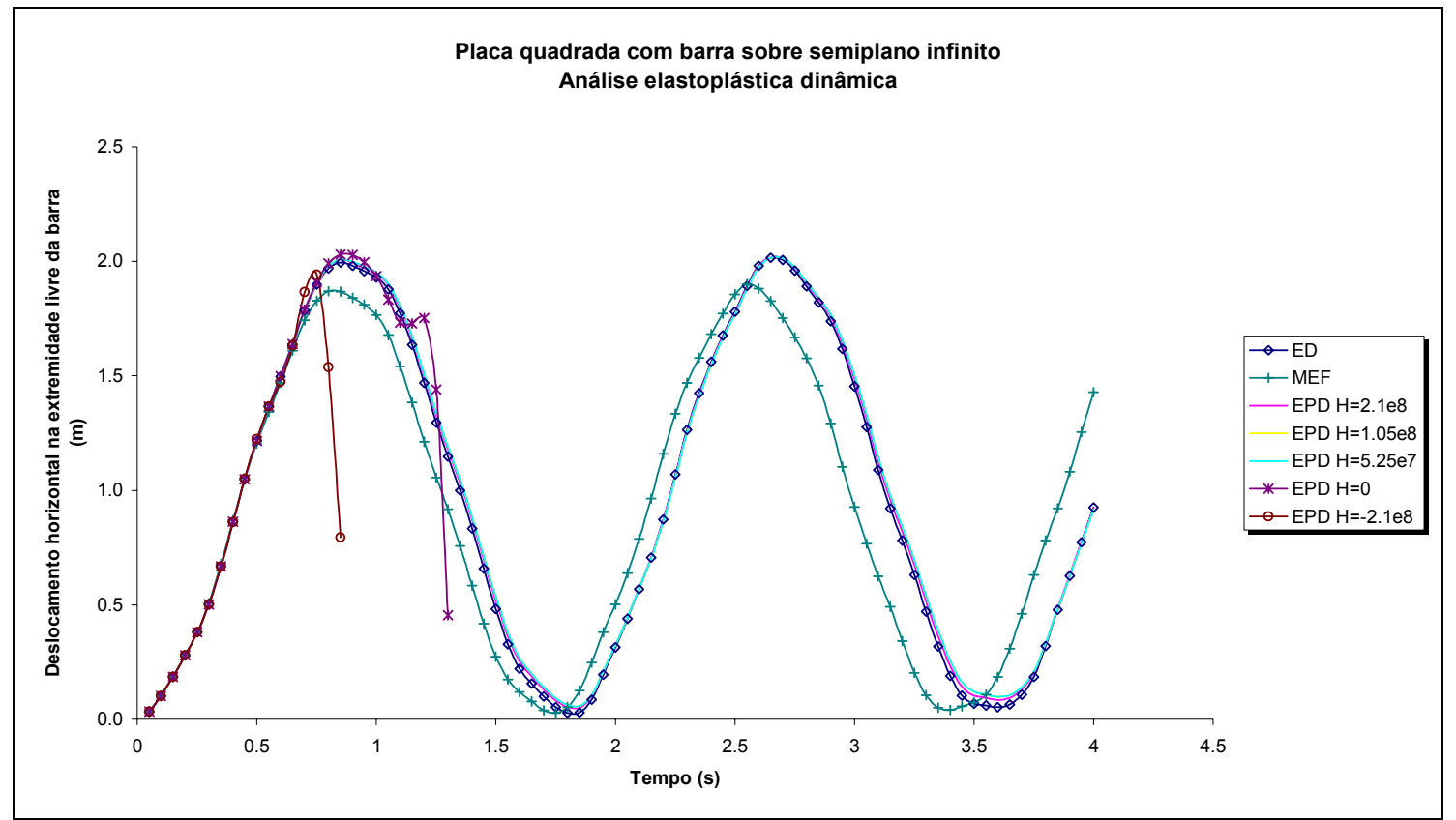

Figura 10.28 - Exemplo 10.2.2 - análise elastoplástica dinâmica - deslocamento horizontal

Preocupado em se verificar o que acontece em um tempo maior para os casos com plasticidade perfeita e amolecimento, optou-se por se considerar a aplicação das cargas apenas para o primeiro passo de tempo, onde para os demais passos, estas são consideradas nulas.

A figura 10.29 apresenta os deslocamentos verticais para diversos valores de $\mathrm{H}$, considerando-se a aplicação das cargas conforme o parágrafo anterior. Observa-se que, para a consideração de plasticidade perfeita, a análise se tornou possível; para os casos com softening, apesar das análises se estenderem por um tempo maior, ainda ocorre colapso frágil.

A figura 10.30 mostra o deslocamento horizontal para as análises da figura anterior, onde se chega às mesmas conclusões. 


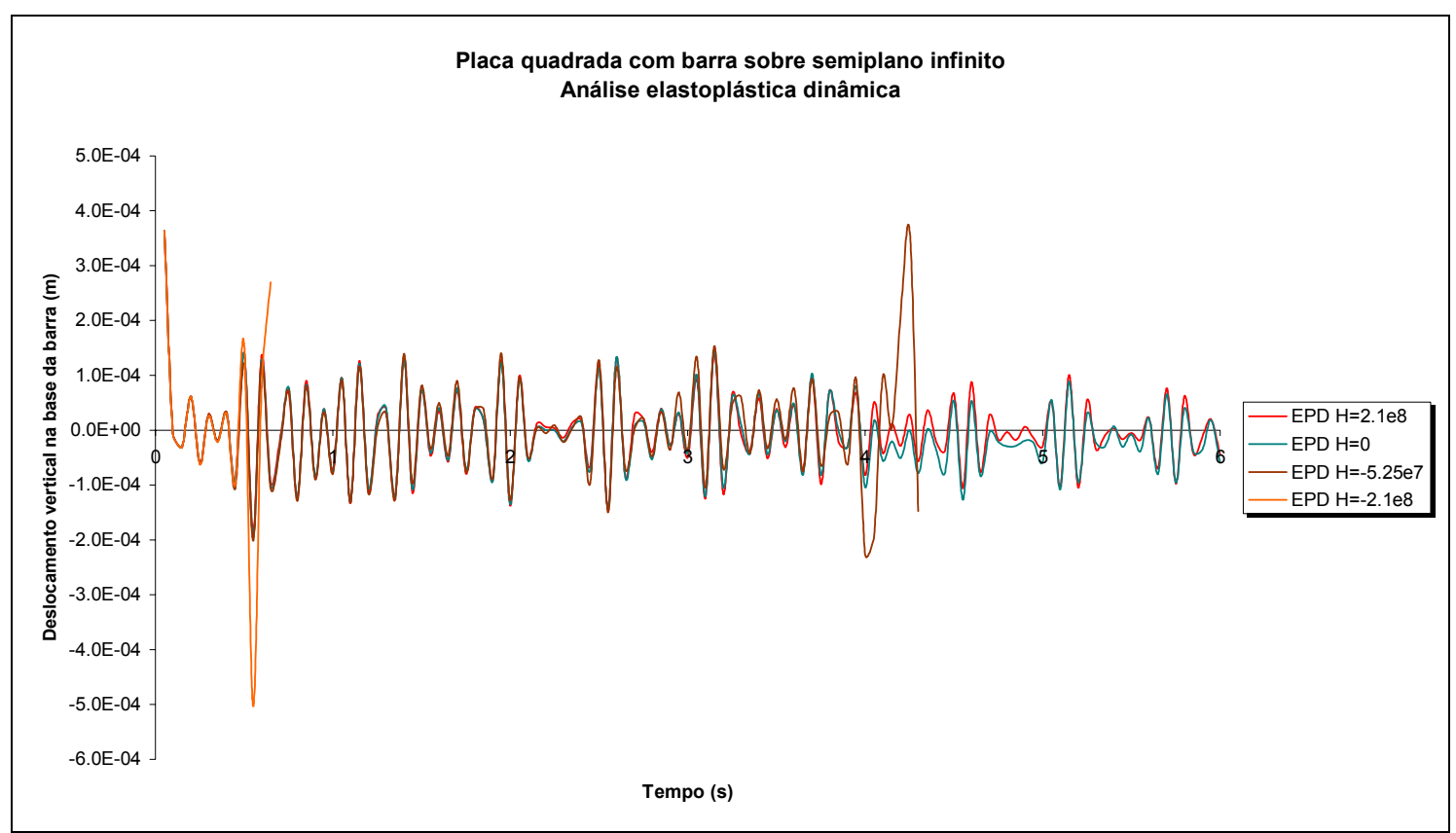

Figura 10.29 - Exemplo 10.2.2 - análise elastoplástica dinâmica - deslocamento vertical corte

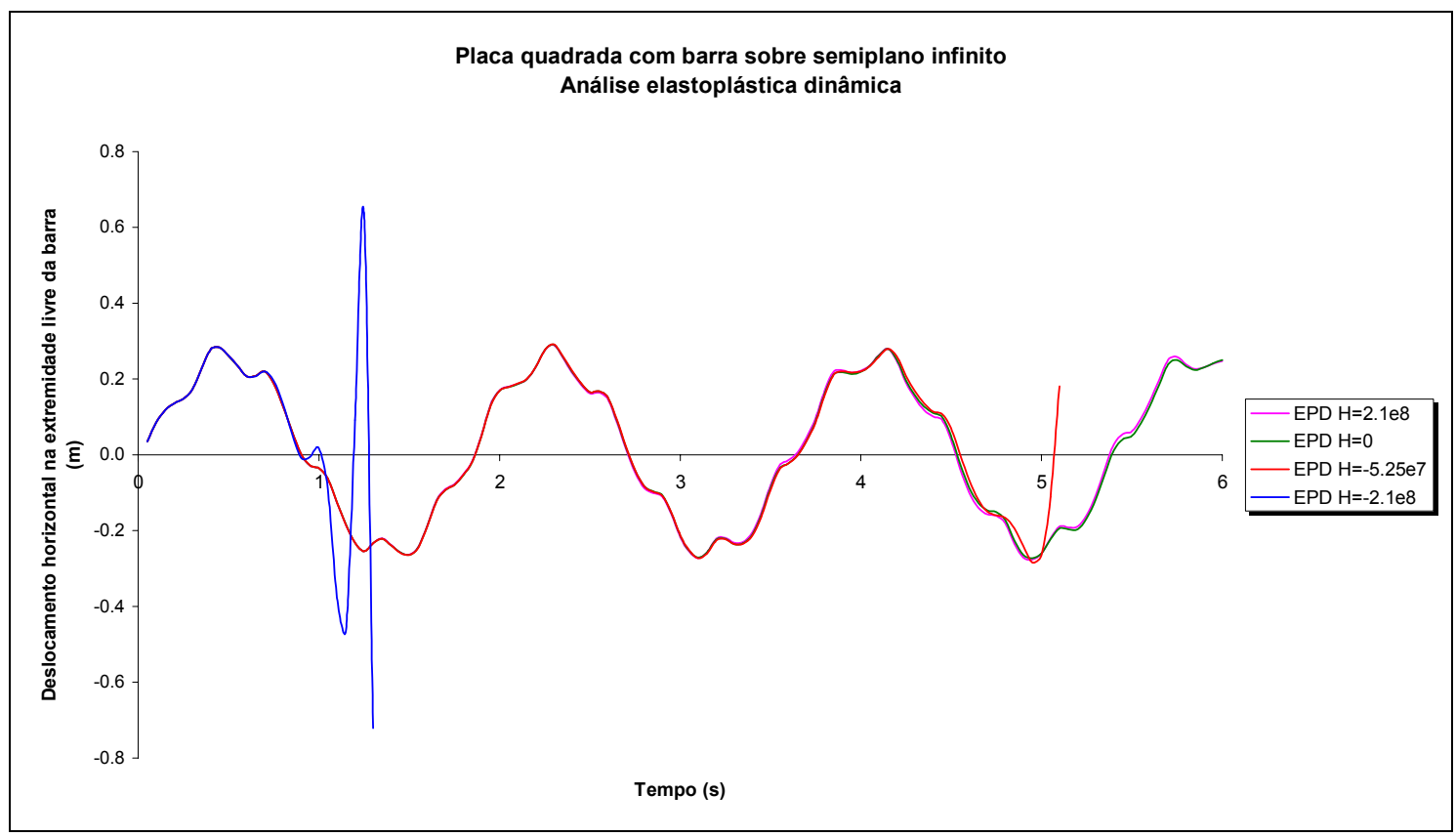

Figura 10.30 - Exemplo 10.2.2 - análise elastoplástica dinâmica - deslocamento horizontal corte

Desta forma, no dimensionamento de estruturas sobre radiers, recomenda-se a limitação das tensões dinâmicas a valores inferiores às tensões de colapso, tal como para análises estáticas, pois o sistema de fundação é incapaz de recuperar o equilíbrio, mesmo para ações de curta duração. 


\subsubsection{Reservatório sobre semiplano infinito}

O terceiro e último caso do exemplo 2 é o estudo de um reservatório sobre o semiplano em questão. A superestrutura, modelada por elementos finitos, foi ligada a uma placa com as mesmas dimensões do caso anterior $(13,33 \times 13,33 \times 1,5 m)$ em uma única sub-região do MEF. Ver figura 10.31. Os dados relativos à superestrutura são dados pela figura 10.32, onde se observa que os pilares vão da placa até o fundo do reservatório.

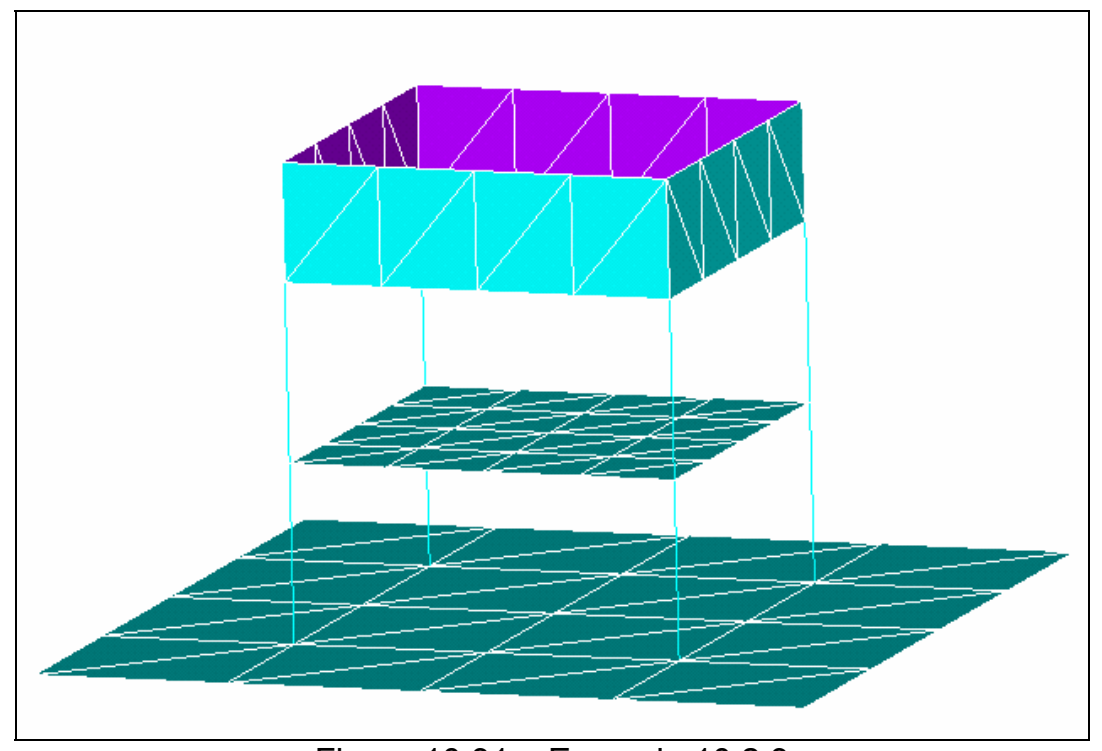

Figura 10.31 - Exemplo 10.2.3

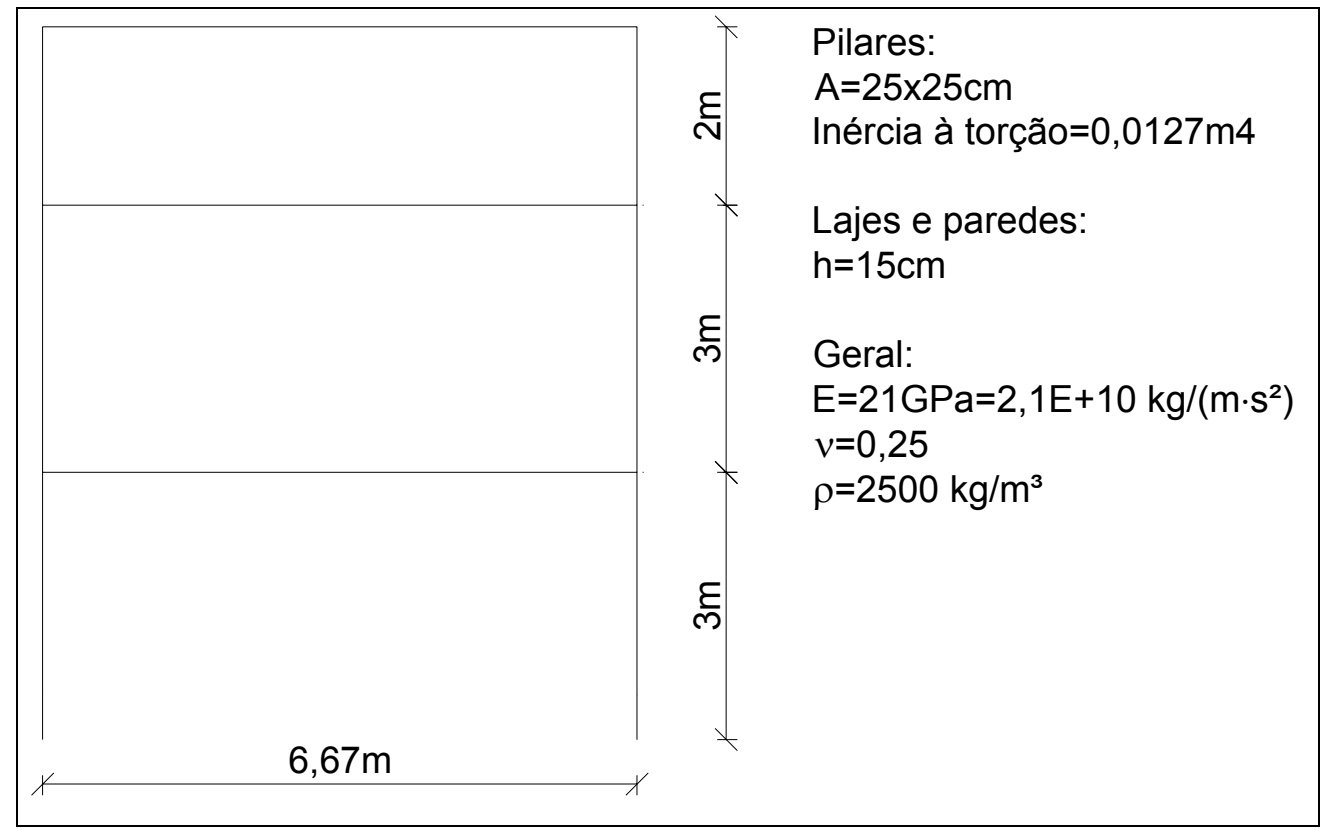

Figura 10.32 - Dados do exemplo 10.2.3 
Considerou-se como carregamento aplicado à estrutura a ação de uma carga concentrada (F) no valor de $20 \mathrm{kN}$ (ou $2 \mathrm{E}+4 \mathrm{~kg} \cdot \mathrm{m} / \mathrm{s}^{2}$ ) aplicada em uma das quinas superiores do reservatório, perpendicular à parede em que a carga é aplicada; e a ação vertical do carregamento da água, considerando o reservatório cheio (q).

Uma análise preliminar estática foi realizada apenas para a superestrutura, considerada engastada no solo, onde, aplicando-se apenas a carga da água, o deslocamento máximo foi de $1,62 \mathrm{~cm}$ no meio da laje do reservatório. Considerando-se a carga concentrada sozinha, o deslocamento máximo foi de $1,13 \mathrm{~cm}$, horizontal, ou seja, $0,14 \%$ da altura do reservatório.

Diversas análises comparativas foram realizadas, onde, mais uma vez, para aquelas elastostáticas, o MMBEM+BEM foi utilizado para a consideração do solo, e para as análises elastoplásticas dinâmicas empregou-se o MMBEM+TDBEM EPD.

As 4 próximas figuras apresentam resultados de análises elastodinâmicas para deslocamento horizontal no ponto de aplicação da carga, em sua direção, ao longo do tempo.

Nas figuras 10.33 e 10.34, observa-se a influência do solo no deslocamento em questão, para a consideração da força concentrada separadamente (F) e do conjunto força concentrada+água $(F+q)$, respectivamente. Primeiramente, percebe-se que os deslocamentos para 0 acoplamento solo-estrutura são maiores que aqueles onde se considerou engaste nos pilares (MEF). A freqüência dos movimentos, porém, não apresentaram grandes diferenças. É importante dizer neste momento que a aplicação da massa da água foi considerada confinada nas paredes do reservatório. Isto foi facilmente realizado considerando-se uma densidade fictícia para os elementos da parede do reservatório com valor calculado como sendo a massa total do conjunto parede+água divida pelo volume das paredes. 


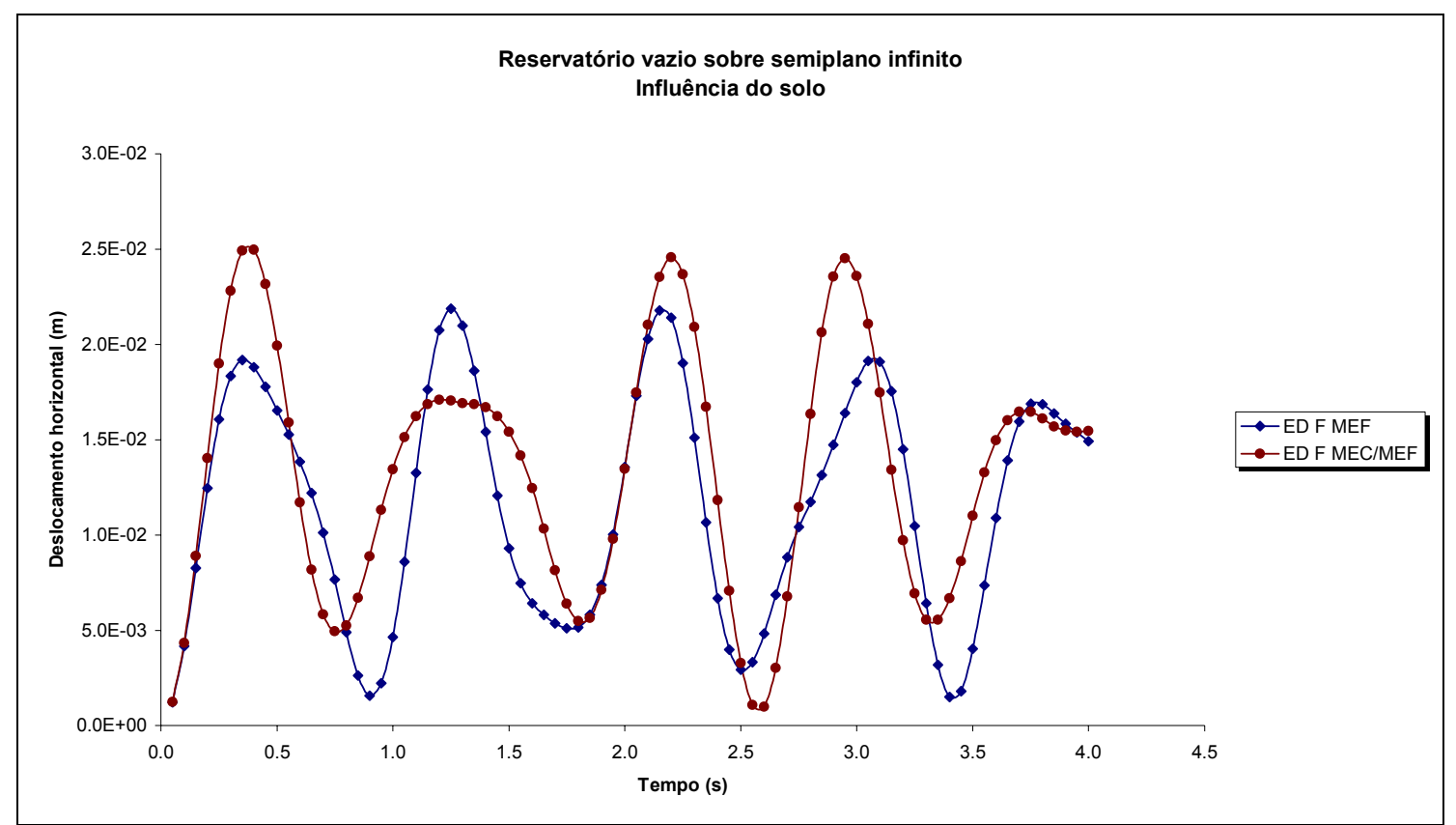

Figura 10.33 - Exemplo 10.2.3 - influência do solo para carga concentrada

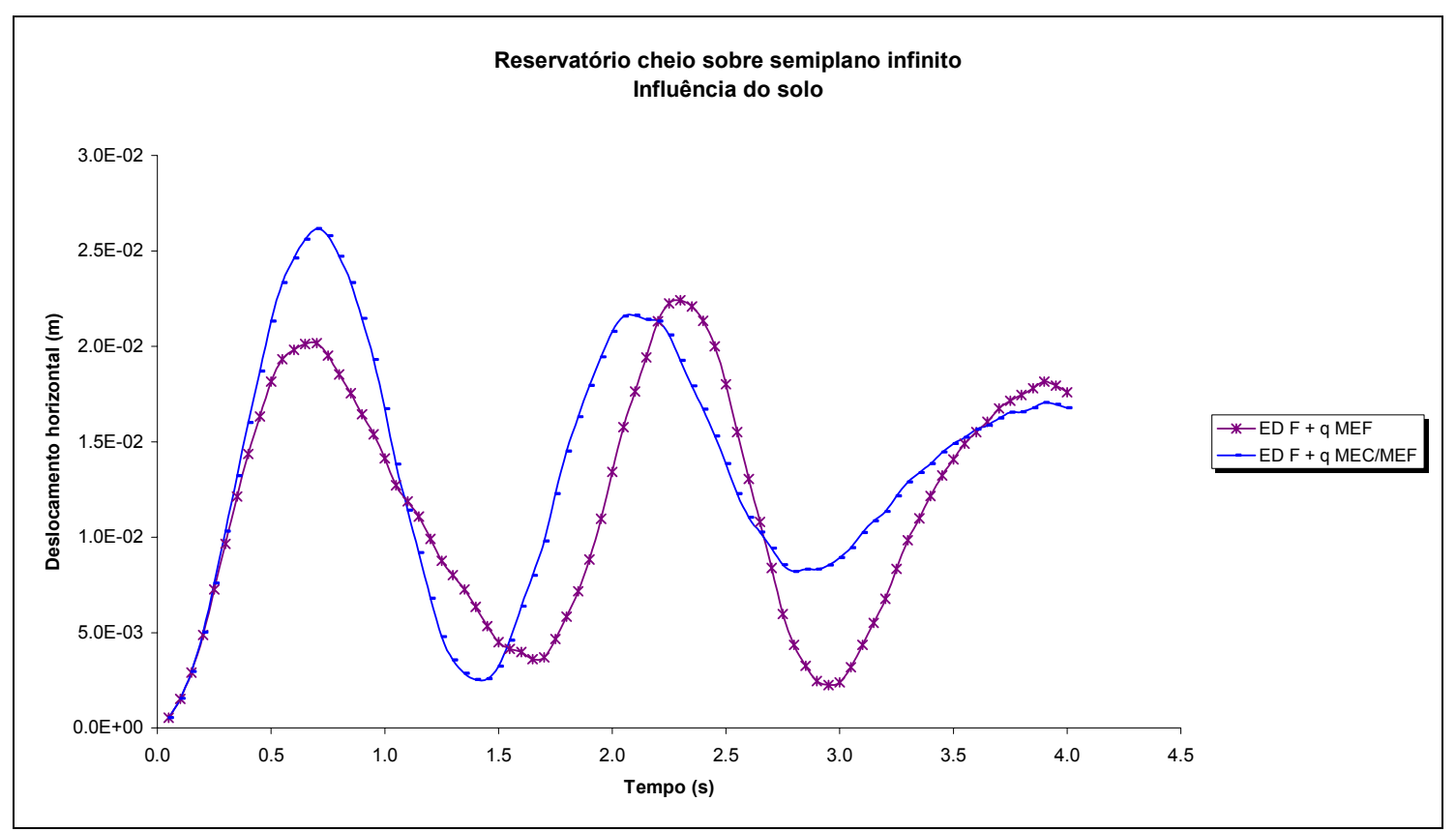

Figura 10.34 - Exemplo 10.2.3 - influência do solo para carga concentrada+água

As figuras 10.35 e 10.36 mostram a influência da água para a análise via MEF e via acoplamento MEC/MEF, respectivamente. O fato que mais se destaca nas duas figuras é o aumento no período do movimento com a consideração da inércia da água. Secundariamente, observa-se que, para a amplitude inicial, os valores obtidos com a consideração do reservatório cheio, são maiores; donde se conclui que, para este último caso, a estrutura leva um 
tempo maior para atingir os deslocamentos máximos, porém, estes são mais elevados que aqueles onde não se considerou a ação da água.

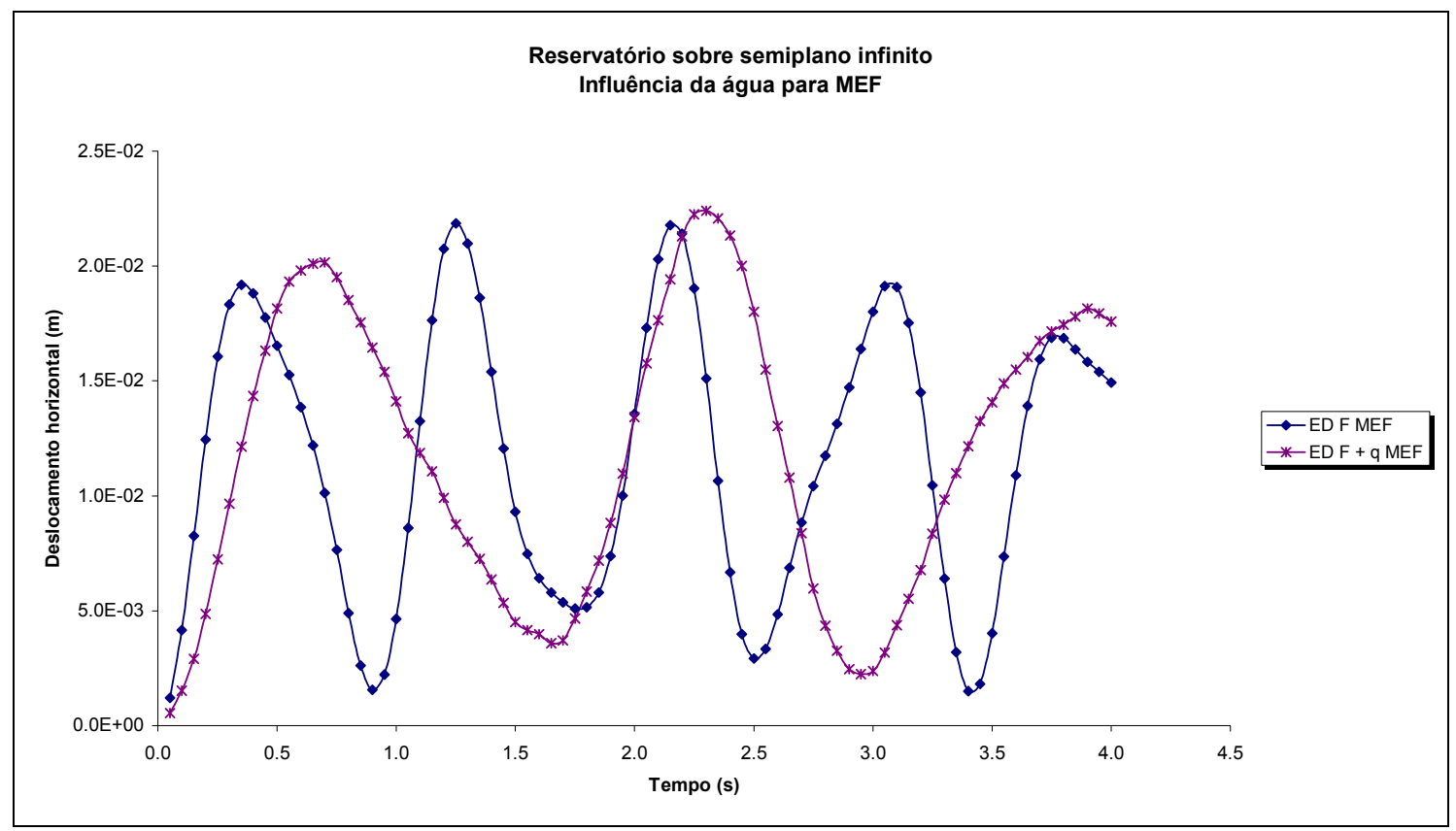

Figura 10.35 - Exemplo 10.2.3 - influência da água para o MEF

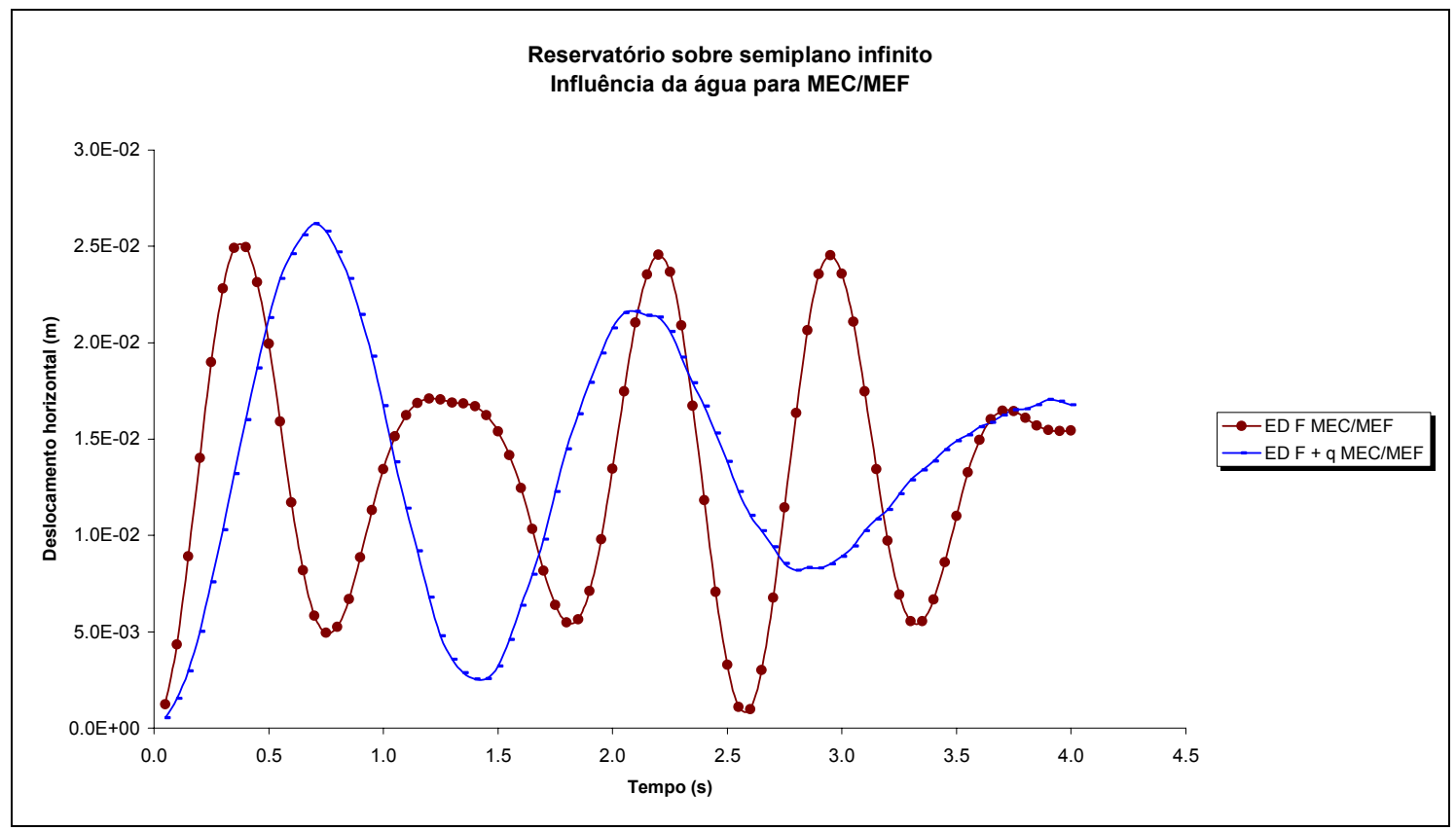

Figura 10.36 - Exemplo 10.2.3 - influência da água para o acoplamento MEC/MEF 
A figura 10.37 contempla análises dinâmicas elastoplásticas do problema em estudo. Inicialmente, considerou-se a análise elastodinâmica da situação mais desfavorável, ou seja, F+q com acoplamento, e se determinou as máximas tensões de compressão e tração no contato solo-estrutura, sendo elas:

$\left\{\begin{array}{l}\sigma_{\text {máx.compr. }}=34485 \mathrm{~kg} / \mathrm{m} \cdot \mathrm{s}^{2} \\ \sigma_{\text {máx. tração }}=16197 \mathrm{~kg} / \mathrm{m} \cdot \mathrm{s}^{2}\end{array}\right.$

De posse destes valores, considerou-se a tensão de plastificação à compressão como sendo $70 \%$ da tensão máxima de compressão, e a tensão de plastificação à tração como sendo $30 \%$ da máxima tensão de tração, no critério de Drucker Prager. Considerou-se ainda, hardening de 10\% do valor do módulo de elasticidade longitudinal do solo, plasticidade perfeita e softening como valor negativo do hardening. A figura 10.37 apresenta os valores para os deslocamentos verticais para o meio do radier em função do tempo para as considerações feitas anteriormente. Diferentemente do que foi considerado até então, os valores negativos indicam deslocamentos para baixo. Desta vez, apenas a análise com softening apresentou colapso.

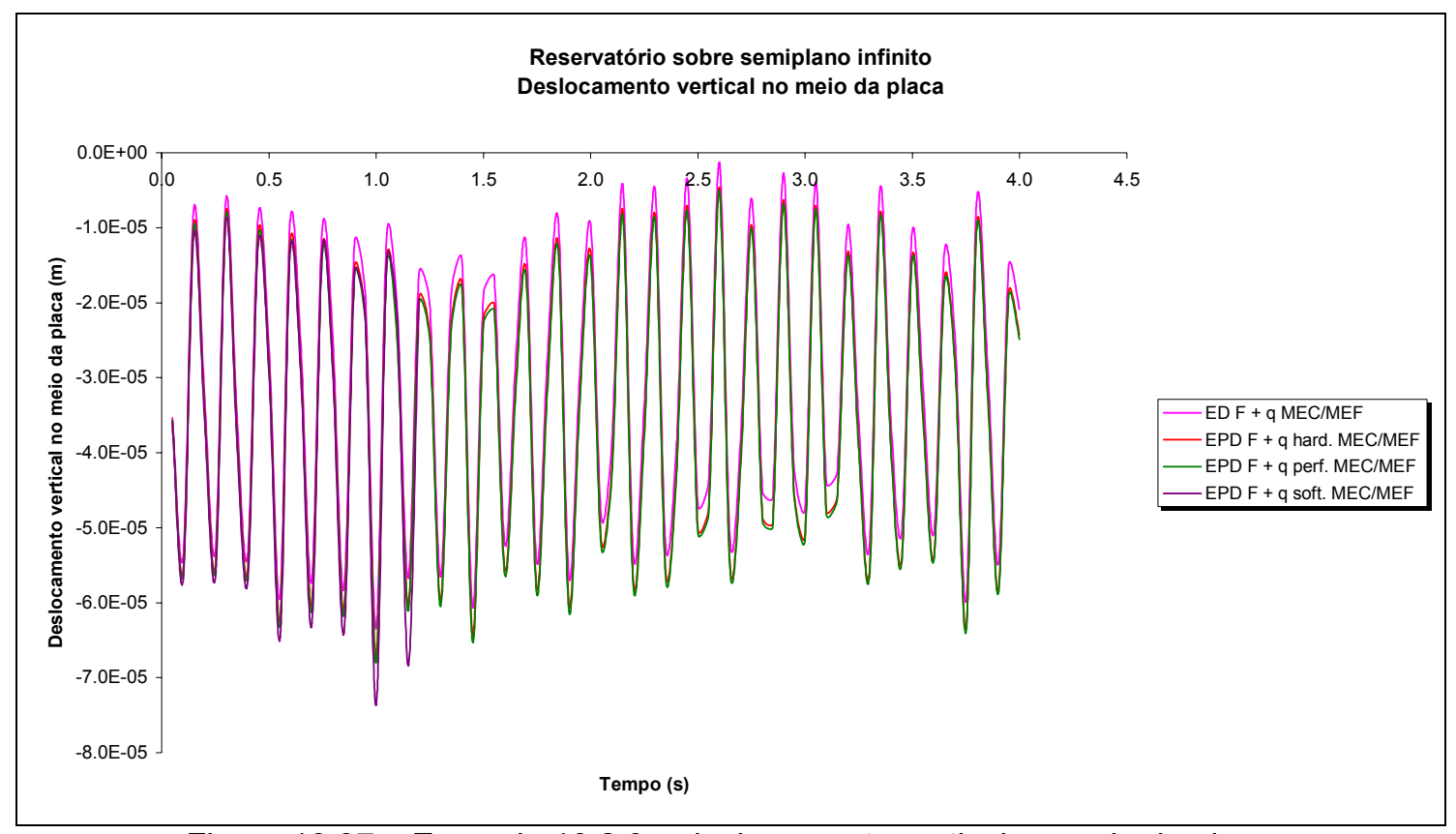

Figura 10.37 - Exemplo 10.2.3 - deslocamento vertical no meio da placa 


\subsection{EXEMPLO 10.3}

O último exemplo geral estuda o problema de uma cavidade esférica em um meio infinito sujeita a uma pressão interna conforme figura 10.38 abaixo, onde não se considerou unidade nos valores apresentados.

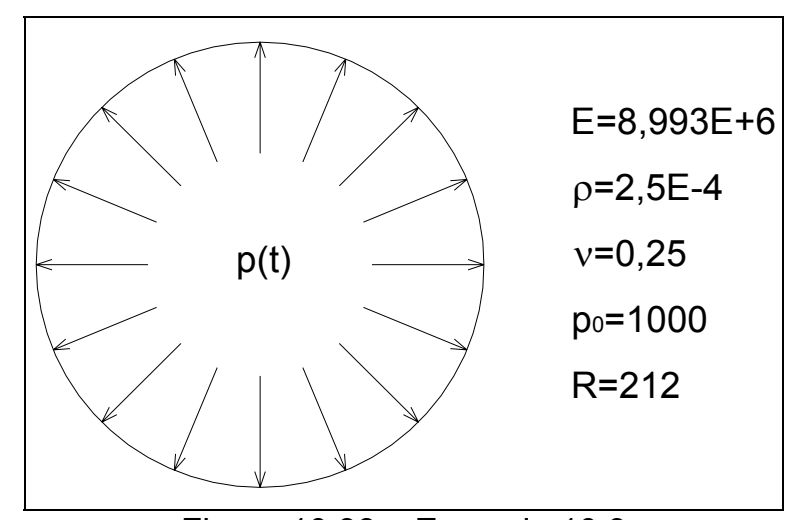

Figura 10.38 - Exemplo 10.3

Foram realizadas diversas análises do problema proposto, onde se considerou: carga $p(t)$ de impacto, aplicação de reforço à parede da cavidade e plastificação da região próxima à cavidade. Para o reforço da parede, considerou-se uma casca de espessura $t=5$ e densidade $\rho$ e coeficiente de Poisson $v$ iguais aos do meio infinito, e variou-se o valor do módulo de elasticidade da casca. As várias análises podem ser agrupadas em 10 tipos, por modelagem utilizada, descritos a seguir.

1ㅇ) TDBEM elastodinâmico

EC TDBEM

Foram utilizados 480 elementos de contorno para a discretização da cavidade; dois valores para $\Delta t$ foram testados: 0,0003 e 0,0005. 
2º) FEM+TDBEM elastodinâmico

Trata-se da mesma modelagem apresenta anteriormente, sendo que com a aplicação do reforço, com o mesmo número de elementos. Consideraram-se dois valores para o módulo de elasticidade do material da casca:

$E c=\left\{\begin{array}{l}10 \cdot E s=8,993 \cdot 10^{7} \\ 100 \cdot E s=8,993 \cdot 10^{8}\end{array}\right.$

sendo Ec o módulo de elasticidade da casca, e Es o módulo de elasticidade do meio infinito. A partir deste segundo tipo de análise, para as análises dinâmicas, utilizou-se apenas $\Delta \mathrm{t}=0,0005$.

$3^{\text {}) ~ B E M ~ e l a s t o s t a ́ t i c o ~}$

EC BEM

Neste tipo de análise, utilizou-se 480 elementos de contorno do BEM para a discretização da cavidade.

4ํㅜ) FEM+BEM elastostático

Trata-se da análise anterior mais a casca de reforço. 
5ํ) MMBEM+TDBEM elastodinâmico

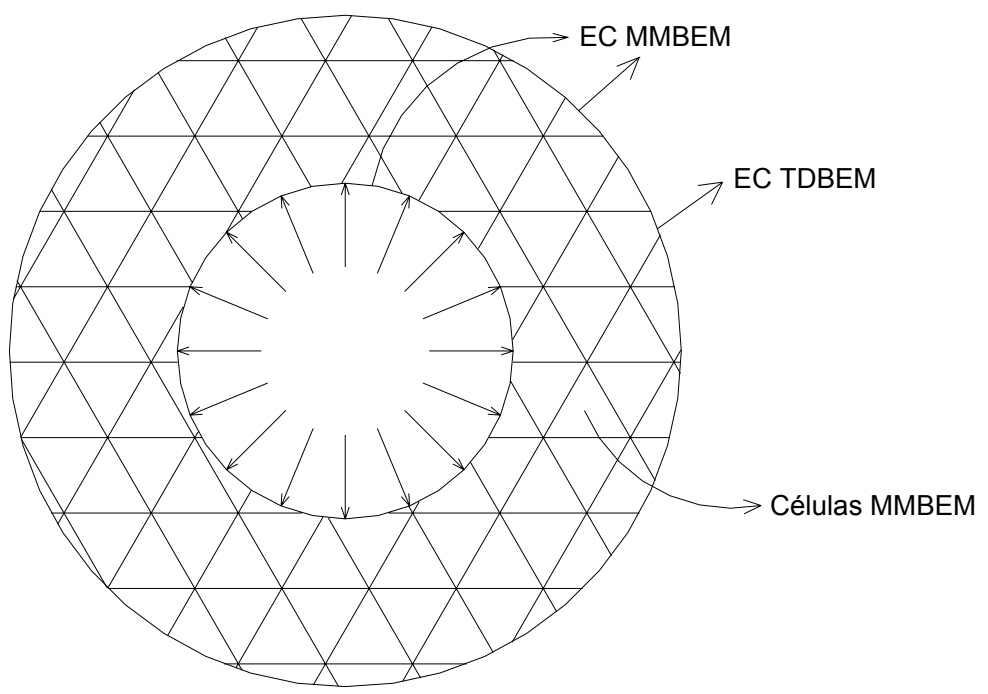

Nesta análise, utilizou-se 922 células do MMBEM, e 74 elementos de contorno para a superfície de acoplamento MMBEM/TDBEM, onde seu raio foi de $2 R$.

6ํ) FEM+MMBEM+TDBEM elastodinâmico

Trata-se da mesma modelagem anterior, sendo que com o reforço.

$7^{0}$ ) MMBEM elastoplástico estático

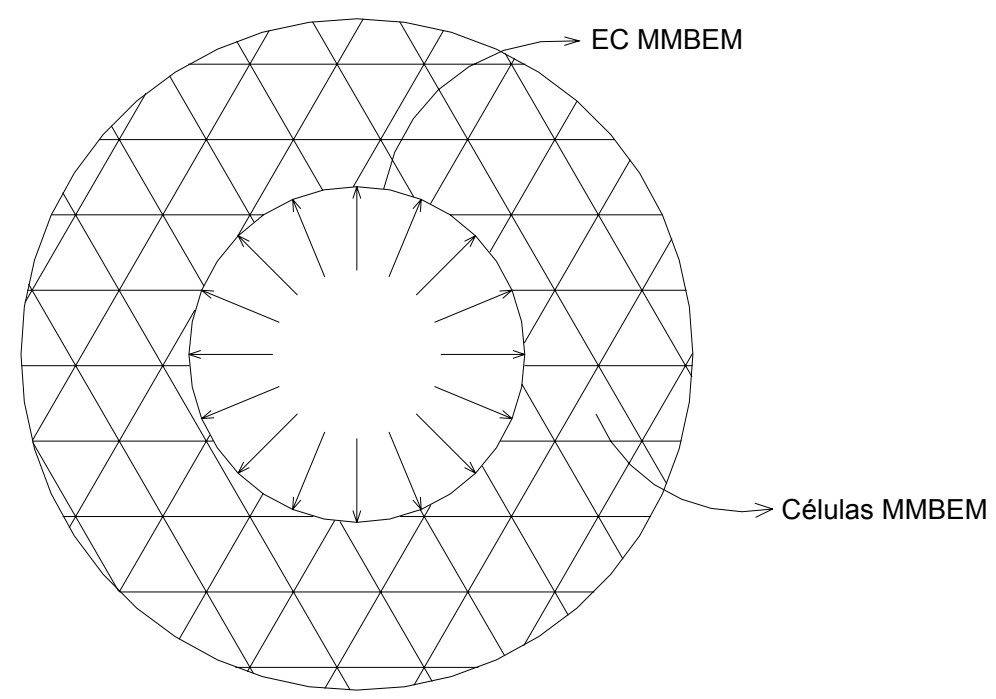


No lugar das células da análise dinâmica do tipo anterior, são consideradas células de plastificação para o MMBEM. Utilizou-se o critério de plastificação de Von Mises, onde se adotou tensão de plastificação de valor 700 e módulo de elasticidade tangente $\left(E_{t}\right)$ de $10 \%$ do valor de $E$ do meio infinito.

$8^{\circ}$ ) FEM+MMBEM elastoplástico estático

Consideração do reforço na modelagem anterior.

9ํ) MMBEM+TDBEM elastoplástico dinâmico

Trata-se da modelagem 5 considerando-se a plastificação da sub-região do MMBEM, para tanto, são consideradas as células de plastificação, coincidentes com as da montagem da matriz de massa.

10으) FEM+MMBEM+TDBEM elastoplástico dinâmico

Modelagem anterior reforçada.

A figura 10.39 abaixo apresenta os resultados de deslocamento radial ao longo do tempo para as análises elásticas. A resposta analítica, sem a consideração de reforço, foi colhida de Timoshenko e Goodier (1980). Observase inicialmente que todos os valores dinâmicos convergiram para os seus correspondentes estáticos. Para as análises com o TDBEM, não houve grandes diferenças para os dois valores de $\Delta$ t utilizados. Nas análises com o FEM, o valor de $\beta=0,25$ não forneceu resultados estáveis; os valores que são apresentados na figura 10.39 , foram obtidos com $\beta=0,32$. O comportamento obtido para a modelagem FEM+TDBEM se mostrou mais suave que aquele apresentado para a modelagem FEM+MMBEM+TDBEM. Deve-se comentar que o estudo relativo à estabilidade do acoplamento TDBEM/FEM é uma contribuição original deste trabalho. Também se pode comentar, como era 
esperado, que o reforço da casca forneceu maior rigidez ao sistema, gerando assim, menores deslocamentos radiais.

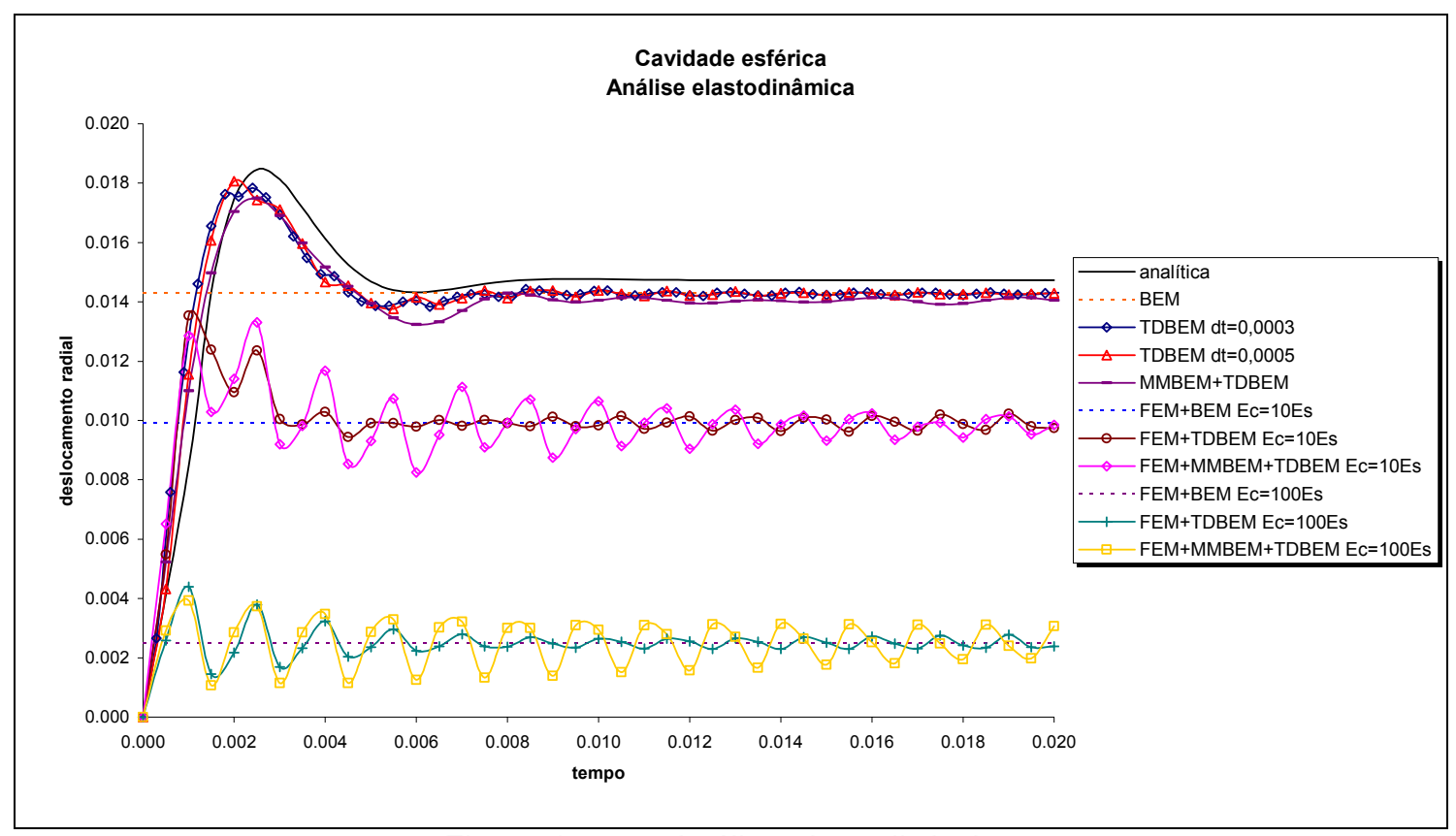

Figura 10.39 - Análise elastodinâmica

A figura 10.40 apresenta os resultados de tensão $\mathrm{x}$ deformação radiais para as análises elastoplásticas estáticas, com e sem reforço. É interessante observar o quanto a ação do reforço diminui as deformações plásticas, chegando até à supressão da plastificação para a casca mais rígida. O autor gostaria de informar que, para a análise sem reforço, apenas 3 nós - dos 39 da superfície mais externa (nós de célula, uma vez que nesta região não existem elementos de contorno) chegaram a plastificar. Pelos resultados, observa-se que o efeito de explosão submersa (túneis) em edificações distantes será fortemente influenciado pela presença ou não de reforços.

A figura 10.41 apresenta os deslocamentos radiais com a aplicação da pressão interna. 


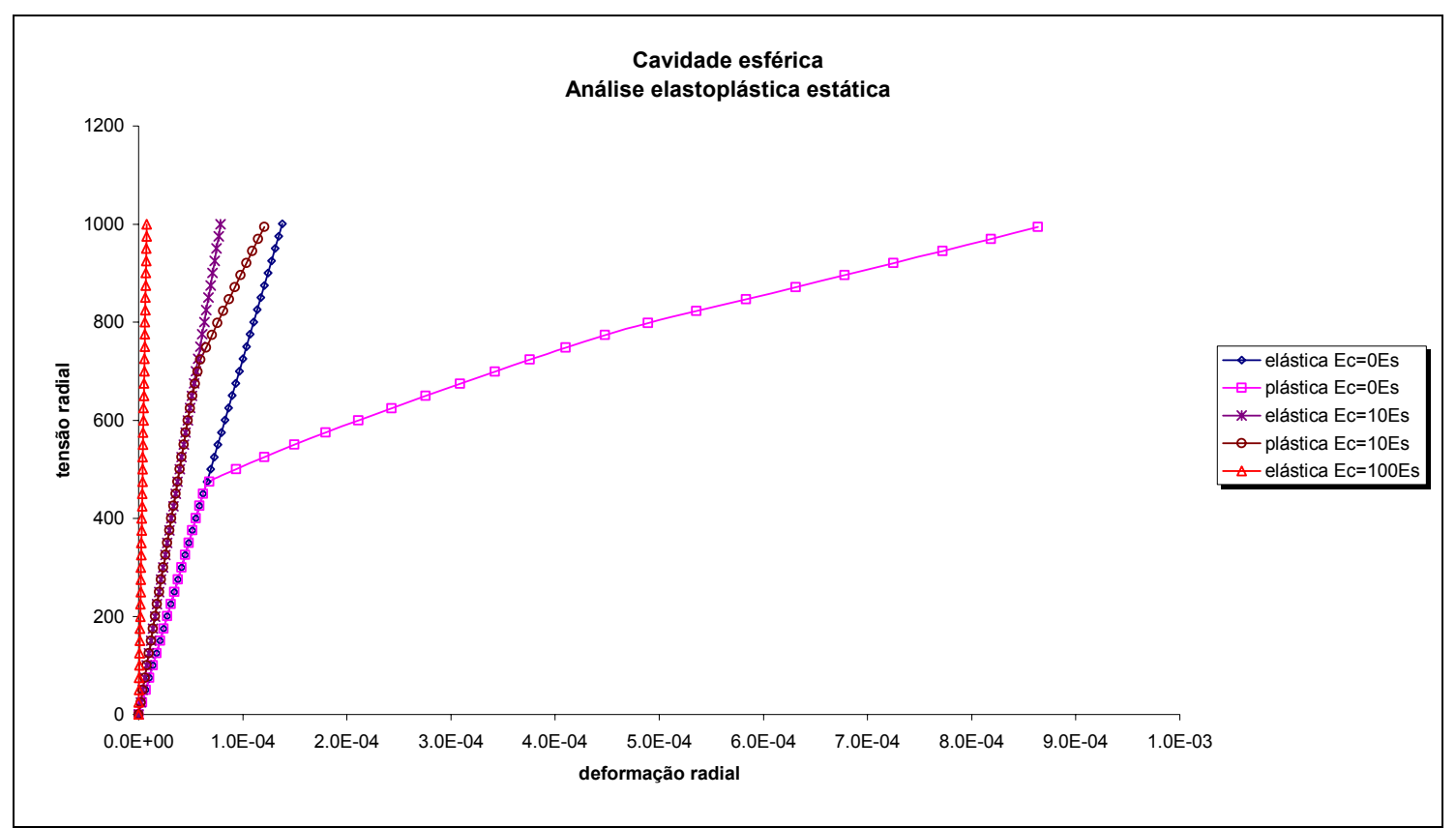

Figura 10.40 - Análise elastoplástica estática - tensão x deformação

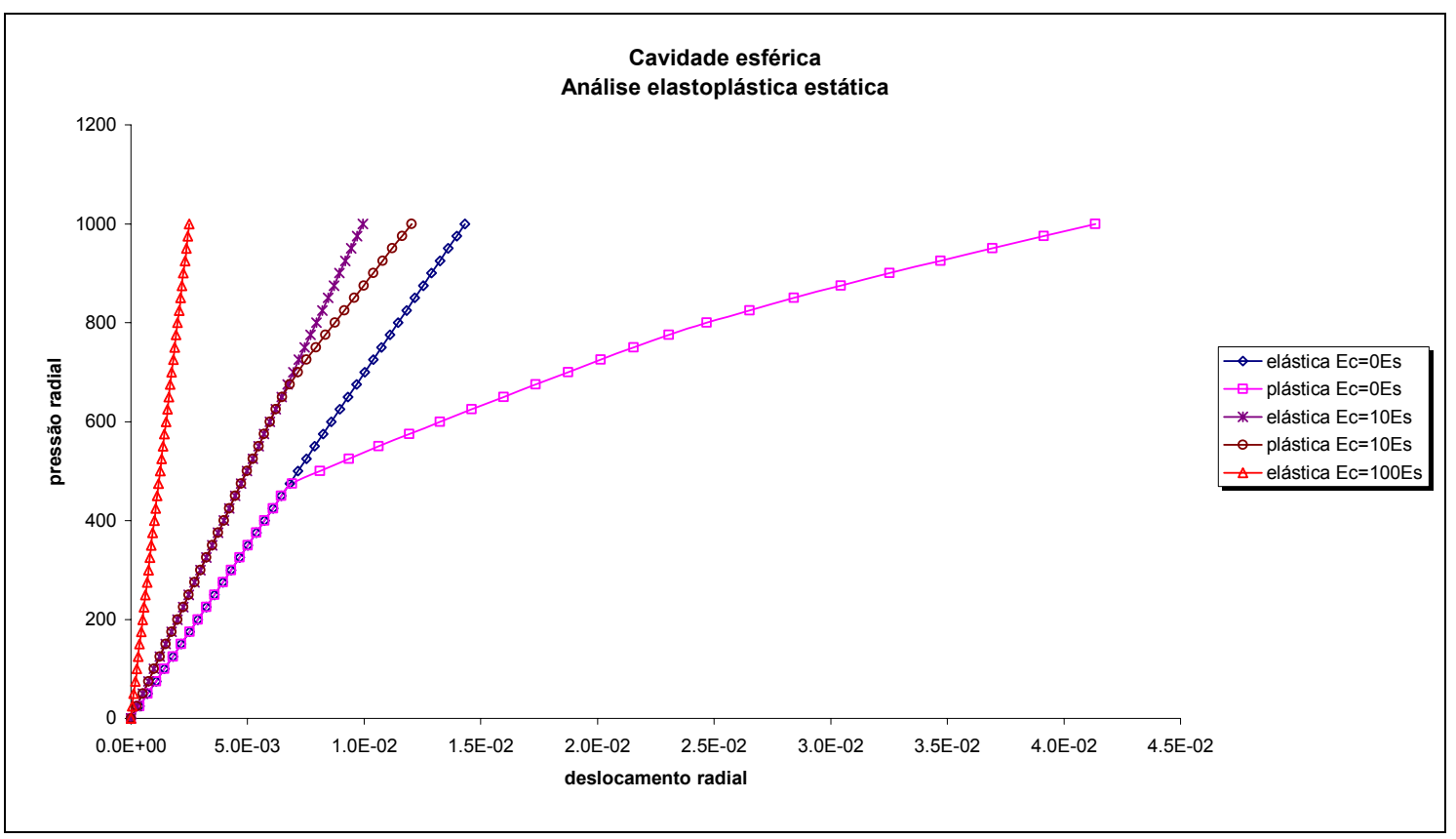

Figura 10.41 - Análise elastoplástica estática - pressão $\mathrm{x}$ deslocamento 
Por fim, a figura 10.42 apresenta os deslocamentos radiais em função do tempo para uma aplicação súbita do carregamento. Observa-se que a não consideração de reforço, para análise elastoplástica, apresenta valores elevados de deslocamento, em comparação com aqueles obtidos para a análise elástica. Com a consideração do reforço para Ec=10Es esta diferença diminui, e, para a casca mais rígida, a diferença nem é perceptível, embora aconteça a plastificação. Como uma informação adicional, pode-se dizer que, para a casca menos rígida, a partir do sexto passo de tempo a análise foi elástica; e, para a casca mais rígida, a partir do décimo primeiro passo.

Pode-se afirmar ainda, a respeito do parágrafo anterior, que, se naquelas análises fosse considerada a não-linearidade física do reforço (MEF), os resultados poderiam ser bem diferentes.

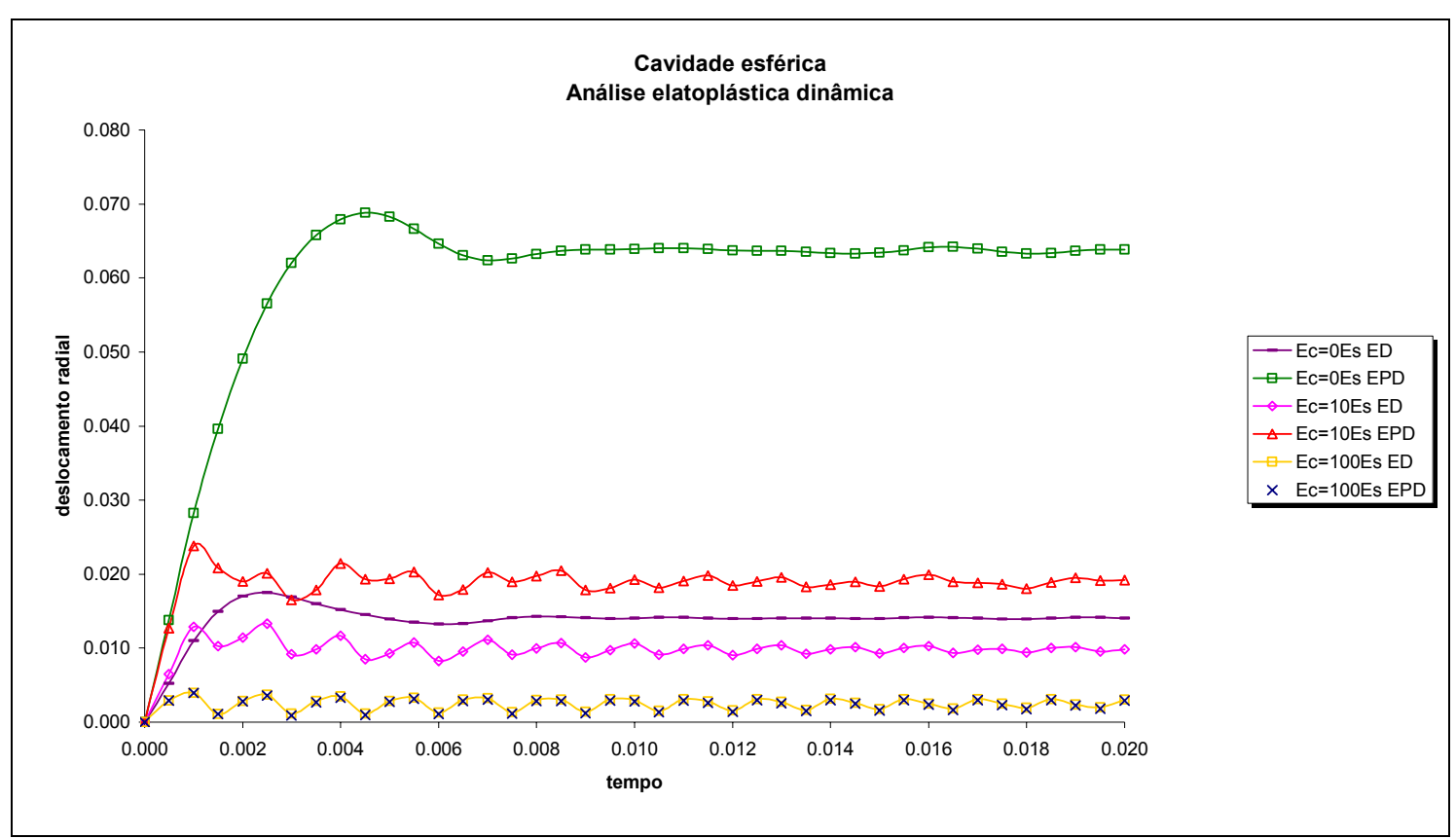

Figura 10.42 - Análise elastoplástica dinâmica 


\section{CONCLUSÃO}

Neste último capítulo, são apresentadas as conclusões da presente tese de doutoramento e sugestões para a continuação da pesquisa.

Optou-se por se dividir o capítulo em subseções com a intenção de proporcionar uma melhor apresentação dos tópicos abordados no mesmo.

\subsection{GENERALIDADES}

O principal objetivo do trabalho foi o desenvolvimento de um código computacional que possibilitasse a análise de estruturas tridimensionais em regime elástico-linear acopladas a meios infinitos ou semi-infinitos (como é o caso do solo), que foram tratados como físico não-lineares. Objetivou-se também a implementação de todo o procedimento estático equivalente.

Analisando-se todas as aplicações apresentadas na tese, principalmente os exemplos simples de final de capítulos de descrição das diversas técnicas numéricas empregadas, funcionando como benchmarks, pode-se chegar à principal conclusão do trabalho, que é a de que os desenvolvimentos metodológicos propostos e utilizados conseguem descrever com boa aproximação os fenômenos estudados na pesquisa - sejam eles, a mecânica e dinâmica de estruturas elásticas acopladas a meios elastoplásticos infinitos ou semi-infinitos, como comentado acima - e que o código computacional elaborado está funcionando perfeitamente e apresentando bons resultados.

Deve-se comentar que, para se atingir de forma completa o objetivo material mencionado anteriormente, foram necessárias implementações numéricas e análises muitas vezes inéditas, bem como a utilização de outras 
desenvolvidas por diferentes autores. Na seção seguinte, estas contribuições são esmiuçadas e permeadas com considerações conclusivas, comentando os comportamentos das respostas numéricas obtidas.

\subsection{CONCLUSÕES E DISCUSSÕES}

Como conclusões complementares do trabalho, pode-se começar comentando a respeito da eficiência dos dois algoritmos empregados para as integrações temporais: Newmark $\beta$, para o Método dos Elementos Finitos (MEF), e Houlbolt, para o Método dos Elementos de Contorno aplicando matriz de massa, ou Mass Matrix Boundary Element Method (MMBEM). O emprego com sucesso deste último tinha sido verificado por vários outros pesquisadores, conforme capítulo 4, sendo que para análises bidimensionais. A presente pesquisa vem trazer contribuições ao estudo do citado método aplicado a problemas 3D. Porém, é importante mencionar que, a depender da discretização utilizada e do intervalo de tempo $(\Delta t)$ fornecido na análise, o algoritmo de Houbolt pode apresentar perda de estabilidade numérica. Isto ocorre porque $\Delta \mathrm{t}^{2}$ pode assumir valores muito pequenos e, quando multiplicado pela matriz $\mathrm{G}$, cujos elementos também podem ser muito pequenos, levam à perda de precisão no processo numérico que relaciona esta matriz com a matriz $\overline{\mathrm{H}}$; para maiores detalhes, ver exemplo 4.1. Outro problema observado nas análises elastodinâmicas pelo MMBEM, é o considerável amortecimento numérico do método, em comparação com o MEF unidimensional. Ver exemplo 4.2. Porém, sabe-se que, apesar de não citado explicitamente na literatura, o algoritmo de Newmark $\beta$ é instável para aplicações com o MEC. Além disso, também se deve comentar o aparecimento de movimento em outras direções que não a principal, devido à análise ser tridimensional. Estes movimentos causam uma redistribuição da energia envolvida no movimento principal do sólido, ajudando na redução do pico inicial da formulação.

Ainda sobre a análise elastodinâmica de sólidos pelo MMBEM, pôde-se concluir que, de maneira geral, o comportamento para um problema de tensão normal longitudinal súbita é fortemente influenciado pela densidade de células na direção predominante do movimento (longitudinal). Pôde-se afirmar também 
que a discretização do contorno do sólido influencia significativamente neste tipo de análise. Porém, para problemas de baixa freqüência (flexão), a dependência em relação à densidade de células é menor que a influência da discretização do contorno.

Outra conclusão importante do trabalho é com relação à utilização do Método dos Elementos de Contorno (MEC) à modelagem de problemas nãolineares físicos. Já se chegou a afirmar que o MEC não se apresentava como uma técnica apropriada ao estudo de tais problemas. A partir da observação dos desenvolvimentos apresentados para o tratamento de plasticidade, bem como dos resultados das implementações realizadas (inclusive com diferentes tipos de encruamento), pode-se concluir que o MEC também é uma ferramenta muito precisa para o tratamento de problemas inelásticos. A contribuição da presente pesquisa é ainda mais valorizada quando se considera que o estudo do tema em discussão foi feito tridimensionalmente, inclusive com a apresentação de diversos exemplos. Com relação à utilização de células tetraédricas, observa-se a possibilidade de variação linear de tensões (4 nós), o mesmo só seria possível no MEF para elementos com 8 nós. Além disso, para problemas onde uma pequena porção do corpo plastifica, tal como para o solo que circunda uma estrutura de fundação, apenas naquela região é necessária a utilização de células de plastificação. Ainda se pode afirmar que a escolha da direção da previsão elástica para o retorno à superfície de plastificação, bem como a obtenção de forma fechada (explícita) do multiplicador plástico se mostraram bastante eficientes. Ainda com relação aos problemas inelásticos, a formulação numérica encontrada na literatura bidimensional foi corrigida em Coda e Venturini (2000), e no presente trabalho foi implementada de forma original para problemas tridimensionais.

Outra grande contribuição do trabalho foi 0 desenvolvimento, implementação e descrição detalhada dos diversos processos de integração utilizados na pesquisa: singular, não-singular e quase-singular para elementos de contorno triangulares planos com aproximação linear, e singular e nãosingular para as células tetraédricas, também com aproximação linear. Ver capítulo 5 .

Com relação ao Método dos Elementos de Contorno no Domínio do Tempo ou Time Domain Boundary Element Method (TDBEM), o autor gostaria 
de dizer que esta foi a única técnica não implementada exclusivamente por ele, tendo recebido código computacional básico desenvolvido pelo orientador; porém, a implementação de pontos fonte singulares para os elementos de contorno triangulares planos foi desenvolvida integralmente nesse trabalho, fazendo-se uso das técnicas comentadas anteriormente. Observou-se no trabalho que a aplicação do TDBEM a problemas de corpos fechados é bastante sensível ao valor do intervalo de tempo $\Delta t$ adotado. Para problemas infinitos ou semi-infinitos, esta dependência já se mostrou menor. Considerando o caso da placa sobre base elástica, observou-se que, para colocação de ponto fonte exterior, quanto maior a distância entre o ponto fonte e o ponto geométrico, mais flexível se apresenta o modelo, confirmando a importância das implementações de pontos fonte singulares realizadas aqui. Comparando-se os resultados obtidos com e sem o esquema de ponto fonte exterior, não foram percebidas grandes diferenças. Para o mesmo caso, também foi observado que com o aumento do refinamento da malha empregada o modelo ganha rigidez, fornecendo assim, resultados menores.

A respeito do acoplamento entre o TDBEM e o MEF, observou-se em algumas análises perda de estabilidade. Tal problema foi resolvido com um aumento no valor de $\beta$ do algoritmo de integração temporal do MEF (Newmark). Esta alteração gera um adiantamento da contribuição das forças inerciais das sub-regiões modeladas por esta técnica. O autor considera tal descoberta simples e ao mesmo tempo muito importante, uma vez que tal problema já chegou a ser encarado como um entrave ao acoplamento entre as duas técnicas.

Uma conclusão a respeito do MEF, principalmente observando-se os exemplos do capítulo 7 , onde o método foi utilizado exclusivamente, é a de que os elementos finitos escolhidos se mostraram bastante eficientes para a análise dos elementos estruturais correspondentes. $O$ algoritmo de Newmark $\beta$ também se mostrou uma excelente opção para as análises dinâmicas onde o MEF foi empregado. Para a análise dinâmica de uma chapa sujeita a carregamento de flexão, observou-se um certo amortecimento nos deslocamentos obtidos. Tal fenômeno pode ser justificado pelo fato do movimento vibratório de um meio bidimensional apresentar duas ondas de 
propagação: uma longitudinal e outra transversal. A onda longitudinal, que se propaga mais rapidamente, irá compor efetivamente o movimento de flexão, sendo que a onda transversal cisalhante é mais dispersiva e sua influência é importante no início do movimento. Assim, os picos menores eram esperados, tal como ocorreu no modelo tridimensional de elementos de contorno.

Com relação ao código computacional desenvolvido, algumas observações podem ser feitas. A primeira delas seria com relação à entrada de dados, onde a mesma é feita através de arquivos de entrada independentes, ou seja, um arquivo geral, onde são fornecidos os dados comuns à análise, e um arquivo para cada sub-região. Esta divisão apresenta-se como uma alternativa bastante interessante, uma vez que permite que um mesmo arquivo de uma determinada sub-região seja utilizado em diferentes análises. Esta característica é ainda mais vantajosa se se levar em consideração que as matrizes envolvidas no MEC (MMBEM e TDBEM), uma vez calculadas, podem ser lidas em outras análises, economizando um considerável tempo de processamento. A leitura de matrizes para o MEF não foi implementada, porém o tempo gasto para a determinação de tais matrizes é bastante inferior àqueles envolvidos no MEC. A divisão do esquema de solução do sistema de equações gerais em duas etapas - fatorização LU e retrosubstituição - também trouxe ganhos na redução do tempo de processamento, já que a etapa de triangularização é realizada uma única vez.

Por fim, discutem-se as conclusões específicas a respeito dos exemplos numéricos finais analisados. Para o caso da análise elastoplástica estática de placa sobre semiplano infinito sujeita a carregamento concentrado no centro do radier e distribuído, separadamente, observou-se que, quanto menor a espessura do radier, maior a diferença entre os valores obtidos com as cargas distribuída e concentrada. Isto ocorre porque com o aumento da espessura da placa as forças de contato entre estrutura e solo são mais uniformemente distribuídas. Para um radier delgado, pôde-se perceber que, para a carga concentrada, as tensões de contato são de compressão no centro e podem chegar a valores de tração nas bordas. Notaram-se também, valores elevados, principalmente na região comprimida. Já para a carga distribuída, só existem tensões de compressão. Para um radier espesso só existem forças de contato de compressão e os valores de tais forças são bastante semelhantes. Também 
se observou que, em estruturas acopladas ao solo com consideração de amolecimento (softening) no solo, pode-se chegar a um rápido colapso do sistema, mesmo para cargas rápidas (dinâmica). Tal observação alerta para o cuidado que se deve ter em construções em solos com tais características (colapsíveis), que é o caso do solo da cidade de São Carlos e região, que apresenta inclusive grande permeabilidade.

Para o exemplo onde se estudou dinamicamente um reservatório sobre semiplano, observou-se que os deslocamentos para o acoplamento soloestrutura são maiores que aqueles onde se considerou engaste nos pilares, sendo a estrutura discretizada exclusivamente por elementos finitos. A freqüência dos movimentos, porém, não apresentou grandes diferenças. Também se comparou a influência da água no reservatório. Observou-se o aumento no período do movimento com a consideração da ação da água. Este fenômeno ocorre devido às forças inerciais envolvidas no grande volume de água considerado. Também se observou que, para a amplitude inicial, os valores obtidos com a consideração do reservatório cheio, são maiores; donde se conclui que, para este último caso, a estrutura leva um tempo maior para atingir os deslocamentos máximos, porém, estes são mais elevados que aqueles onde não se considerou a ação da água, sendo esta a situação mais desfavorável.

Finalmente, para a análise elastoplástica estática da cavidade esférica em meio infinito sujeita a carga de pressão interna, concluiu-se que a ação de reforço na parede da cavidade pode diminuir bastante as deformações plásticas no solo, podendo chegar até à supressão da plastificação. Para a análise elastoplástica dinâmica, concluiu-se que a diferença entre os deslocamentos radiais obtidos, com e sem a consideração de plastificação do meio infinito, é inversamente proporcional à rigidez do reforço, lembrando-se que, se nestas análises fosse considerada a não-linearidade física do reforço (MEF), os resultados poderiam ser bem diferentes. 


\subsection{SUGESTÕES PARA A CONTINUAÇÃO DA PESQUISA}

Como foi dito no capítulo introdutório do trabalho, acredita-se que o desenvolvimento do código computacional para a simulação numérica tridimensional dinâmica de estruturas conectadas ao solo suposto elastoplástico foi de grande importância para a engenharia, pois não se dispunha até então de ferramenta tão geral para se fazer tal tipo de análise. Os programas com os quais se poderiam tentar tais simulações estão elaborados em elementos finitos, o que limita muito o seu emprego devido ao volume de informações que se precisa gerar e aos problemas relacionados à simulação de meios infinitos ou semi-infinitos quando de análises dinâmicas envolvendo propagação de ondas. Mesmo assim, diversas sugestões podem ser dadas para a continuação da pesquisa. A seguir, apresentam-se as mais relevantes.

> Implementação de comportamento viscoelástico, plástico e viscoplástico para a superestrutura (MEF). Estendendo-se, assim, as aplicações, para casos ainda mais realistas, onde as não-linearidades físicas possam ocorrer tanto no meio contínuo (solo) quanto na superestrutura.

Implementação de função de Green transiente (solução fundamental de Lamb) que contempla condições de contorno conhecidas como "superfície livre". Este procedimento reduziria em muito o consumo computacional da implementação da versão do MEC no domínio do tempo (TDBEM), com estruturas enterradas, além de possibilitar um avanço significativo das formulações existentes para a análise de meios infinitos no domínio do tempo.

Inclusão da viscoelasticidade no comportamento da região não-plastificada, também modelada pela versão do MEC no domínio do tempo (TDBEM).

Estudo da utilização de algoritmos alternativos para a integração temporal do MMBEM. 
Estudo e implementação do acoplamento fluido-estrutura para a modelagem dinâmica de reservatórios e barragens.

Aprimoramento ou mesmo otimização do sofisticado programa desenvolvido para realização da análise dinâmica não-linear da interação de estruturas com o solo, onde se daria especial ênfase à forma computacional de processamento, utilizando-se o conceito de paralelismo (uso de clusters).

O autor acredita concretizar o estudo e implementação de tais sugestões em projetos de pesquisa futuros. 


\section{REFERÊNCIAS}

ADAM, M.; PFLANZ, G.; SCHMID, G. (2000). Two- and three-dimensional modelling of half-space and train-track embankment under dynamic loading. Soil Dynamics and Earthquake Engineering, v.19, n.8, p.559-73.

ADOLPH, M. (2002). Soluções transientes de problemas viscoelastodinâmicos em meios ilimitados através da transformada rápida de Fourier-FFT. Dissertação (Mestrado) - Faculdade de Engenharia Mecânica, Universidade Estadual de Campinas, Campinas. 2002.

AHMAD, S.; BANERJEE, P.K. (1988a). Multi-domain BEM for two-dimensional problems of elastodynamic. International Journal for Numerical Methods in Engineering, v.26, p.891-911.

AHMAD, S.; BANERJEE, P.K. (1988b). Time-domain transient elastodynamic analysis of 3-D solids by BEM. International Journal for Numerical Methods in Engineering, v.26, p.1709-28.

ALMEIDA, F.P.A. (1999). Análise comparativa de resultados de diferentes discretizações para as lajes de pavimentos utilizando os elementos finitos DKT e P15N. 126p. Dissertação (Mestrado) - Escola de Engenharia de São Carlos, Universidade de São Paulo, São Carlos. 1999.

ALMEIDA, F.P.A.; ANTUNES, H.M.C.C. (2000). Aplicação do elemento finito $\mathrm{P} 15 \mathrm{~N}$ na análise de pavimentos de edifícios. In: JORNADAS SUDAMERICANAS DE INGENIERÍA ESTRUCTURAL, 29., 2000, Punta Del Este, UR. Memória. (CD ROM). Montevideo, Instituto de Estructuras y Transporte/Faculdad de Ingeniería, Universidad de la República/ASAIE. 12p.

ALMEIDA, V.S. (2003). Análise da interação solo não-homogêneo/estrutura via acoplamento MEC/MEF. Tese (Doutorado) - Escola de Engenharia de São Carlos, Universidade de São Paulo, São Carlos. 2003.

ANTUNES, H.M.C.C.; ALMEIDA, F.P.A. (1999). Análise comparativa de resultados de diferentes discretizações para as lajes de pavimentos de edifícios. In: IBERIAN LATIN-AMERICAN CONGRESS ON COMPUTATIONAL METHODS IN ENGINEERING, 20., 1999, São Paulo. Anais. (CD ROM). 20p.

ARAÚJO, F.C.; NISHIKAVA, L.K.; MANSUR, W.J. (1997). On the consideration of enclosing elements in 3D elastodynamic analysis with the BEM and BE/FE coupled process. In: IBERIAN LATIN-AMERICAN CONGRESS ON COMPUTATIONAL METHODS IN ENGINEERING, 18., 1997, Brasília. Anais. v.1, p.423-31.

ARGYRIS, J.; MLEJNEK, H.P. (1991). Dynamics of structures. Texts on Computational Mechanics, v.5, p.484-90. 
ASSAN, A.E. (1999). Método dos elementos finitos: primeiros passos. Campinas, Editora da UNICAMP. (Coleção Livro-Texto)

BANERJEE, P.K.; CATHIE, D.N. (1980). A direct formulation and numerical implementation of the boundary element method for two-dimensional problems of elasto-plasticity. International Journal of Mechanical Sciences, v.22, n.4, p.233-45.

BARBIRATO, J.C.C. (1999). Método dos elementos de contorno com a reciprocidade dual para a análise transiente tridimensional da mecânica do fraturamento. 246p. Tese (Doutorado) - Escola de Engenharia de São Carlos, Universidade de São Paulo, São Carlos. 1999.

BARROS, P.L.A.; MESQUITA, E. (1999). Elastodynamic Green's functions for orthotropic plane strain continua with inclined axis of symmetry. International Journal of Solids and Structures, v.36, n.31, p.4767-88.

BARROS, R.M. (2001). Funções de Green e de influência para meios viscoelásticos transversalmente isotrópicos no domínio da freqüência. Tese (Doutorado) - Faculdade de Engenharia Mecânica, Universidade Estadual de Campinas, Campinas. 2001.

BATHE, K.J. (1975). ADINA - a finite element program for automatic dynamic incremental nonlinear analysis. Acoustics and Vibration Lab., Rep. 82448-1; Dept. of Mech. Eng., MIT.

BATOZ, J.L.; BATHE, K.J.; HO, L.W. (1980). A study of three-node triangular plate bending elements. International Journal for Numerical Methods in Engineering, v.15, n.12, p.1771-812, Dec..

BELYTSCHKO, T.; CHANG, H.S.; LU, Y.Y. (1989). A variationally coupled finite element-boundary element method. Computers \& Structures, v.33, n.1, p.17-20.

BESKOS, D.E. (1987). Boundary element methods in dynamic analysis. Applied Mechanics Reviews, v.40, n.1, p.1-23.

BESKOS, D.E. (1997). Boundary element methods in dynamic analysis: part II (1986-1996). Applied Mechanics Reviews, v.50, n.3, p.149-97, Mar..

BREBBIA, C.A.; DOMINGUEZ, J. (1992). Boundary elements: an introductory course. 2.ed. Southampton, McGraw-Hill. (Computational mechanics publications).

CARRER, J.A.M.; MANSUR, W.J. (1999). Stress and velocity in 2D transient elastodynamic analysis by the boundary element method. Engineering Analysis with Boundary Elements, v.23, n.3, p.233-45.

CARRER, J.A.M.; TELLES, J.C.F. (1992). A boundary element formulation to solve transient dynamic elastoplastic problems. Computers \& Structures, v.45, n.4, p.707-13. 
CARRION, R. (2002). Uma implementação do Método dos Elementos de Contorno para problemas viscoelastodinâmicos estacionários tridimensionais em domínios abertos e fechados. Tese (Doutorado) - Faculdade de Engenharia Mecânica, Universidade Estadual de Campinas, Campinas. 2002.

CARRION, R.; MESQUITA NETO, E.; ROMANINI, E. (2001). On the modeling of dynamic stationary behavior of 3D elastic half-spaces by the direct Boundary Element Method using enclosing elements. In: IBERIAN LATIN-AMERICAN CONGRESS ON COMPUTATIONAL METHODS IN ENGINEERING, 22., Campinas, 2001. Anais. (CD ROM). 16p.

CHUEIRI, L.H.M. (1994). Formulação do método dos elementos de contorno para análise elastoplástica de placas. Tese (Doutorado) - Escola de Engenharia de São Carlos, Universidade de São Paulo, São Carlos. 1994.

CISILINO, P.A. (1997). Boundary element formulation for 3d analysis of fatigue crack growth in linear and non-linear fracture mechanics. Ph.D. Thesis Wessex Institute of Technology, UK. 1997.

CISILINO, P.A.; ALIABADI, M.H. (1999). Three-dimensional boundary element analysis of fatigue crack growth in linear and non-linear fracture. Engineering Fracture Mechanics, v.63, n.6, p.713-33.

CODA, H.B. (1990). Análise da vibração livre de meios elásticos bidimensionais pelo método dos elementos de contorno. 130p. Dissertação (Mestrado) Escola de Engenharia de São Carlos, Universidade de São Paulo, São Carlos. 1990.

CODA, H.B. (1993). Análise tridimensional de estruturas pela combinação entre o método dos elementos de contorno e o método dos elementos finitos. 202p. Tese (Doutorado) - Escola de Engenharia de São Carlos, Universidade de São Paulo, São Carlos. 1993.

CODA, H.B. (2000). Contribuição à análise dinâmica transiente de meios contínuos pelo Método dos Elementos de Contorno. 381p. Tese (Livredocência) - Escola de Engenharia de São Carlos, Universidade de São Paulo, São Carlos. 2000.

CODA, H.B. (2001). Dynamic and static non-linear analysis of reinforced media: a BEM/FEM coupling approach. Computers \& Structures, v.79, n.31, p.2751-65.

CODA, H.B.; VENTURINI, W.S. (1995a). Non-singular time-stepping BEM for transient elastodynamic analysis. Engineering Analysis with Boundary Elements, v.15, n.1, p.11-8.

CODA, H.B.; VENTURINI, W.S. (1995b). Three-dimensional transient BEM analysis. Computers \& Structures, v.56, n.5, p.751-68.

CODA, H.B.; VENTURINI, W.S. (1996a). Further improvements on three dimensional transient BEM elastodynamic analysis. Engineering Analysis with Boundary Elements, v.17, p.231-43. 
CODA, H.B.; VENTURINI, W.S. (1996b). A simple comparison between two 3D time domain elastodynamic boundary element formulations. Engineering Analysis with Boundary Elements, v.17, n.1, p.33-44.

CODA, H.B.; VENTURINI, W.S. (1999a). On the coupling of 3D BEM and FEM frame model applied to elastodynamic analysis. International Journal of Solids and Structures, v.36, p.4789-804.

CODA, H.B.; VENTURINI, W.S. (1999b). BEM/FEM non-linear model applied to transient analysis with viscous damping. J. of the Braz. Soc. Mechanical Sciences, v.21, n.3, p.519-36.

CODA, H.B.; VENTURINI, W.S. (2000). Dynamic non-linear stress analysis by the mass matrix BEM. Engineering Analysis with Boundary Elements, v.24, p.623-32.

CODA, H.B.; VENTURINI, W.S.; ALIABADI, M.H. (1999). A general 3D BEM/FEM coupling applied to elastodinamic continua/frame structures interaction analysis. International Journal for Numerical Methods in Engineering, v.46, n.5, p.695-712.

FERRO, N.C.P. (1998). Uma combinação MEC/MEF para análise de interação solo-estrutura. Tese (Doutorado) - Escola de Engenharia de São Carlos, Universidade de São Paulo, São Carlos. 1998.

FRANGI, A.; MAIER, G. (1999). Dynamic elastic-plastic analysis by a symmetric Galerkin boundary element method with time-independent kernels. Computer Methods in Applied Mechanics and Engineering, v.171, p.281-308.

FUDOLI, C.A. (1999). Formulação do método dos elementos de contorno e plasticidade com gradiente. Tese (Doutorado) - Escola de Engenharia de São Carlos, Universidade de São Paulo, São Carlos. 1999.

GUAN, F.; NOVAK, M. (1994). Transient response of an elastic homogeneous half-space to suddenly applied rectangular loading. Journal of Applied Mechanics, v.61, p.256-63, June.

HU, N. (1997). A solution method for dynamic contact problems. Computers \& Structures, v.63, p.1053-63.

ISRAIL, A.S.M.; BANERJEE, P.K. (1992). Advanced development of boundary element method for two-dimensional dynamic elasto-plasticity. International Journal of Solids and Structures, v.29, n.11, p.1433-51.

JEYACHANDRABOSE, C.; KIRKHOPE, J.; RAMESH BABU, C. (1985). An alternative explicit formulation for the DKT plate-bending element. International Journal for Numerical Methods in Engineering, v.21, p.1289-93.

KARABALIS, D.L.; BESKOS, D.E. (1984). Dynamic response of 3-D rigid surface foundations by time domain element method. Earthquake Engineering and Structural Dynamics, v.12, n.1, p.73-93. 
KARABALIS, D.L.; BESKOS, D.E. (1986). Dynamic response of 3-D foundations by the boundary element method. Computer Methods in Applied Mechanics and Engineering, v.56, n.1, p.91-119.

KOMATSU, J.S. (1995). Estudo de problemas de escavação através de combinação de elementos de contorno e elementos finitos. Tese (Doutorado) Escola de Engenharia de São Carlos, Universidade de São Paulo, São Carlos. 1995.

KONTONI, D.P.N.; BESKOS, D.E. (1988). Boundary element formulation for dynamic analysis of nonlinear systems. Engineering Analysis, v.5, n.3, p.11425.

KONTONI, D.P.N.; BESKOS, D.E. (1993). Transient dynamic elastoplastic analysis by the dual reciprocity BEM. Engineering Analysis with Boundary Elements, v.12, p.1-16.

LAETHEM, M.V.; BACKX, E.; WYNENDAELE, H.; CAESTECKER, F.; DHONDT, G. (1984). The use of boundary elements to represent the far field in soil-structure interaction. Nuclear Engineering and Design, v.78, p.313-27.

LAIER, J.E.; BARREIRO, J.C. (1983). Complementos de resistência dos materiais. São Carlos, EESC.

MANSUR, W.J.; BREBBIA, C.A. (1984). Further developments on the solution of the transient scalar wave equation, In: Topics in Boundary Element Research: Time-dependent and Vibration Problems, Spriger-Verlag, Berlin.

MARTÍN, D.; ALIABADI, M.H. (1998). Boundary element analysis of twodimensional elastoplastic contact problems. Engineering Analysis with Boundary Elements, v.21, n.4, p.349-60.

MARTINS, R.A.F.; SABINO, J. (1997). A simple and efficient triangular finite element for plate bending. Engineering Computations, v.14, n.8, p.883-900.

MATOS FILHO, R.F. (1999). Análise da interação estaca-solo via uma combinação do MEF com o MEC. Dissertação (Mestrado) - Escola de Engenharia de São Carlos, Universidade de São Paulo, São Carlos. 1999.

MENDONÇA, A.V. (1997). Análise da interação placa-estaca-solo via combinação do método dos elementos finitos com o método dos elementos de contorno. Dissertação (Mestrado) - Escola de Engenharia de São Carlos, Universidade de São Paulo, São Carlos. 1997.

MENDONÇA, A.V. (2002). Estudo de estruturas compostas por lâminas planas de espessuras constantes: uma abordagem pelo método dos elementos de contorno. 291p. Tese (Doutorado) - Escola de Engenharia de São Carlos, Universidade de São Paulo, São Carlos. 2002.

MENDONÇA, A.V.; PAIVA, J.B. (2000). A boundary element method for the static analysis of raft foundations on piles. Engineering Analysis with Boundary Elements, v.24, p.237-47. 
MESQUITA, A.D. (2002). Novas metodologias e formulações para o tratamento de problemas inelásticos com acoplamento progressivo MEC/MEF. Tese (Doutorado) - Escola de Engenharia de São Carlos, Universidade de São Paulo, São Carlos. 2002.

MESQUITA, E. (1989). Zur dynamischen wechse/wirkung von fundamenten auf dem viskoelastischen halbraum (Sobre a interação dinâmica de fundações sobre um semi-espaço viscoelástico). VDI Fortschrittberichte, Reihe 11: Schwingungstechnik, NR 120, VDI-Verlag Duesseldorf, 1989 ISBN 3-18142011-5.

MESQUITA, E.; ADOLPH, M.; ROMANINI, E. (2002). Transient response of three dimensional rigid structures interacting with visco-elastic soil profiles. In: ENGINEERING MECHANICS DIVISION CONFERENCE OF THE ASCE, 15., New York, 2002. Proceedings. Columbia University, NY. p.1-8, June.

MESQUITA, E.; ROMANINI, E. (1992). Green's function approach versus direct boundary element scheme to model the dynamic interaction of foundations resting on a viscoelastic layer over a bedrock. In: INTERNATIONAL CONFERENCE ON BOUNDARY ELEMENT METHODS, 14., Seville, 1992. Proceedings. Seville, Spain. v.2, p.107-21, Nov..

MON-MA, M.L.; VENTURINI, W.S.; CODA, H.B. (1996). A simple technique to evaluate quasi-singular integrals. In: BRAZILIAN SEMINAR ON THE BOUNDARY ELEMENT METHOD IN ENGINEERING, 1., Rio de Janeiro, 1996. Anais. Rio de Janeiro, p.45-8.

NOVAK, M.; EL HIFNAWY, L. (1983). Effect of soil-structure interaction on damping of structures. Earthquake Engineering and Structural Dynamics, v.11, p.595-621.

PAN, G.; OKADA, H.; ATLURI, S.N. (1994). Nonlinear transient dynamic analysis of soil-pavement interaction under moving load: a coupled BEM-FEM approach. Engineering Analysis with Boundary Elements, v.14, p.99-112.

PANZECA, T.; POLIZZOTTO, C.; ZITO, M. (1994). Dynamic plasticity analysis by boundary/interior elements. Engineering Analysis with Boundary Elements, v.14, p.113-20.

PAVLATOS, G.D.; BESKOS, D.E. (1994). Dynamic elastoplastic analysis by BEM/FEM. Engineering Analysis with Boundary Elements, v.14, n.1, p.51-63.

PROVIDAKIS, C.P.; BESKOS, D.E. (1986). Dynamic analysis of beams by the boundary element method. Computers \& Structures, v.22, n.6, p.957-64.

RAMALHO, M.A. (1990). Sistema para análise de estruturas considerando interação com meio elástico. Tese (Doutorado) - Escola de Engenharia de São Carlos, Universidade de São Paulo, São Carlos. 1990.

SIMO, J.C.; HUGHES, T.J.R. (1986). Elastoplasticity and viscoplasticity: computational aspects. 
SOUSA JUNIOR, E. (1996). Um aplicativo para o ambiente windows para aquisição de dados para análise de pavimentos de edifícios via método dos elementos finitos. 99p. Dissertação (Mestrado) - Escola de Engenharia de São Carlos, Universidade de São Paulo, São Carlos. 1996.

SOUZA, V.J.B. (2001). Algoritmos de integração eficientes para o método dos elementos de contorno tridimensional. 135p. Dissertação (Mestrado) - Escola de Engenharia de São Carlos, Universidade de São Paulo, São Carlos. 2001.

SPIEGEL, M.R. (1973). Manual de fórmulas e tabelas matemáticas. Tradução de Roberto Chioccarello. São Paulo, Mc-Graw-Hill do Brasil.

SPYRAKOS, C.C.; BESKOS, D.E. (1986). Dynamic response of rigid stripfoundations by a time-domain boundary element method. International Journal for Numerical Methods in Engineering, v.23, n.8, p.1547-65.

STAMOS, A.A.; BESKOS, D.E. (1995). Dynamic analysis of large 3-D underground structures by BEM. Earthquake Engineering and Structural Dynamics, v.24, n.6, p.917-34.

TANRIKULU, A.H.; YERLI, H.R.; TANRIKULU, A.K. (2001). Application of the multi-region boundary element method to dynamic soil-structure interaction analysis. Computers and Geotechnics, v.28, n.4, p.289-307.

TEJERINA CALDERÓN, E. (1996). Sobre o uso do método dos elementos de contorno - MEC para o estudo de interação de placas com o meio contínuo. 247p. Tese (Doutorado) - Escola de Engenharia de São Carlos, Universidade de São Paulo, São Carlos. 1996.

TELLES, J.C.F.; BREBBIA, C.A. (1981a). The boundary element method in plasticity. Applied Mathematical Modelling, v.5, n.4, p.275-81.

TELLES, J.C.F.; BREBBIA, C.A. (1981b). Boundary elements: new developments in elastoplastic analysis. Applied Mathematical Modelling, v.5, n.5, p.376-82.

TELLES, J.C.F.; CARRER, J.A.M. (1994). Static and transient dynamic nonlinear stress analysis by the boundary element method with implicit techniques. Engineering Analysis with Boundary Elements, v.14, n.1, p.65-74.

TELLES, J.C.F.; CARRER, J.A.M.; MANSUR, W.J. (1999). Transient dynamic elastoplastic analysis by the time-domain BEM formulation. Engineering Analysis with Boundary Elements, v.23, p.479-86.

TIMOSHENKO, S.P.; GOODIER, J.N. (1980). Teoria da elasticidade. 3.ed. Rio de Janeiro: Guanabara Dois.

TIMOSHENKO, S.P.; WOINOWSKY-KRIEGER, S. (1959). Theory of plates and shells. 2.ed. New York, McGraw-Hill International Editions. (Engineering mechanics series). 
TRIANTAFYLLIDIS, T. (1991). 3-D time domain BEM using half-space Green's functions. Engineering Analysis with Boundary Elements, v.8, n.3, p.115-24.

VENTURINI, W.S. (1982). Application of the boundary element method to solve geomechanical problems. Ph.D. Thesis - Wessex Institute of Technology, UK. 1982.

VENTURINI, W.S. (1988). Um estudo sobre o método dos elementos de contorno e suas aplicações em problemas de engenharia. Tese (Livredocência) - Escola de Engenharia de São Carlos, Universidade de São Paulo, São Carlos. 1988.

VILLAÇA, S.F.; GARCIA, L.F.T. (1996). Introdução à teoria da elasticidade. Rio de Janeiro, COPPE/UFRJ.

VON ESTORFF, O. (1991). Dynamic response of elastic blocks by time domain BEM and FEM. Computers \& Structures, v.38, n.3, p.289-300.

VON ESTORFF, O.; KAUSEL, E. (1989). Coupling of boundary and finite elements for soil-structure interaction problems. Earthquake Engineering and Structural Dynamics, v.18, p.1065-75.

WEARING, J.L.; BURSTOW, M.C. (1994). Elasto-plastic analysis using a coupled boundary element finite element technique. Engineering Analysis with Boundary Elements, v.14, n.1, p.39-49.

YU, G.; MANSUR, W.J.; CARRER, J.A.M.; LIE, S.T. (2001). A more stable scheme for BEM/FEM coupling applied to two-dimensional elastodynamics. Computers \& Structures, v.79, n.8, p.811-23.

ZIENKIEWICZ, O.C.; KELLY, D.W.; BETTESS, P. (1977). The coupling of the finite element method and boundary solution procedures. International Journal for Numerical Methods in Engineering, v.11, p.355-75. 


\section{BIBLIOGRAFIA COMPLEMENTAR}

ALIABADI, M.H.; HALL, W.S.; PHEMISTER, T.G. (1985). Taylor expansions for singular kernels in the boundary element method. International Journal for Numerical Methods in Engineering, v.21, p.2221-36.

BARBIRATO, J.C.C. (1991). Formulação do método dos elementos de contorno para sólidos elásticos tridimensionais; baseada na solução fundamental de Mindlin. Dissertação (Mestrado) - Escola de Engenharia de São Carlos, Universidade de São Paulo, São Carlos. 1991.

CHAVES, A.P.; RIBEIRO, G.O. (2002). An assessment of the numerical algorithms for weakly singular integral evaluation in the BEM applied to 3-D elastoplastic problems. In: IBERIAN LATIN-AMERICAN CONGRESS ON COMPUTATIONAL METHODS IN ENGINEERING, 23., 2002, Giulianova, IT. Anais. (CD ROM).

CLOUGH, R.W.; PENKIEN, J. (1993). Dynamics of structures. 2.ed. Singapore, McGraw-Hill. (Civil engineering series).

FINLAYSON, B.A. (1972). The method of weighted residuals and variational principles: with application in fluid mechanics, heat and mass transfer. Cap. 1-2, p.3-39. (Mathematics in science and engineering, 87).

GANGMING, L. (1989). A new boundary element method coupled with FEM packages. Communications in Applied Numerical Methods, v.5, p.365-71.

GAO, X.W.; DAVIES, T.G. (2000). An effective boundary element algorithm for 2D and 3D elastoplastic problems. International Journal of Solids and Structures, v.37, n.36, p.4987-5008.

JOHNSON, L.R. (1974). Green's function for Lamb's problem. Geophys. J.R. astr. Soc., v.37, p.99-131.

KARABALIS, D.L.; BESKOS, D.E. (1990). Letter to the editor: time-domain transient elastodynamic analysis of 3-D solids by BEM. International Journal for Numerical Methods in Engineering, v.29, n.1, p.211-5.

LU, S.; YE, T.Q. (1991). Direct evaluation of singular integrals in elastoplastic analysis by the boundary element method. International Journal for Numerical Methods in Engineering, v.32, n.2, p.295-311.

PALERMO JUNIOR, L.; FOLTRAN, C.E.; MESQUITA NETO, E. (2002). On the analysis of the thin walled structures using the boundary element method. In: IBERO LATIN AMERICAN CONGRESS ON COMPUTATIONAL METHODS FOR ENGINEERING, 23., 2002, Giulianova, IT. Anais. (CD ROM). 
TELLES, J.C.F. (1987). A self-adaptive co-ordinate transformation for efficient numerical evaluation of general boundary element integrals. International Journal for Numerical Methods in Engineering, v.24, p.959-73.

TELLES, J.C.F.; BREBBIA, C.A. (1981a). Boundary element solution for halfplane problems. International Journal of Solids and Structures, v.17, n.12, p.1149-58.

UNIVERSIDADE DE SÃO PAULO. Escola de Engenharia de São Carlos. Serviço de Biblioteca (2002). Diretrizes para elaboração de dissertações e teses na EESC-USP. 3.ed. São Carlos.

YU, G.; MANSUR, W.J.; CARRER, J.A.M.; GONG, L. (1998). Time weighting in time domain BEM. Engineering Analysis with Boundary Elements, v.22, n.3, p.175-81. 


\section{APÊNDICE A - Tensões e direções principais}

Neste primeiro apêndice, apresenta-se de forma objetiva o cálculo das tensões e direções principais a partir de um estado de tensões cartesianas, bem como a transformação inversa, ou seja, a partir de um estado de tensões principais a determinação das tensões em coordenadas cartesianas. Para a implementação da determinação das direções principais, o autor, juntamente com seu orientador, desenvolveu um procedimento próprio para tal fim; enquanto que para o cálculo das tensões principais, tomou-se como base os trabalhos de Laier e Barreiro (1983), Spiegel (1973) e Villaça e Garcia (1996). Vale ressaltar que vários exemplos numéricos foram testados para aferir o código computacional elaborado, principalmente ao que se refere à determinação das direções principais, e pode-se dizer que os resultados encontrados foram excelentes.

\section{A.1 DETERMINAÇÃO DAS TENSÕES PRINCIPAIS}

Nesta seção será, como já foi dito, apresentado de maneira bastante prática o procedimento para o cálculo das tensões principais a partir de um estado de tensões em coordenadas cartesianas. Sendo assim, considera-se o seguinte tensor de tensões, já levando em consideração sua simetria:

$[\sigma]=\left[\begin{array}{lll}\sigma_{11} & \sigma_{12} & \sigma_{13} \\ \sigma_{12} & \sigma_{22} & \sigma_{23} \\ \sigma_{13} & \sigma_{23} & \sigma_{33}\end{array}\right]$

Pode-se dizer que as tensões principais são as raízes da seguinte equação: 
$\sigma_{0}^{3}-I_{1} \cdot \sigma_{0}^{2}+I_{2} \cdot \sigma_{0}-I_{3}=0$

onde os $\mathrm{l}_{\mathrm{j}}$ são chamados de invariantes do estado de tensão, e são dados por

$$
\begin{aligned}
& \mathrm{I}_{1}=\sigma_{11}+\sigma_{22}+\sigma_{33} \\
& \mathrm{I}_{2}=\sigma_{11} \cdot \sigma_{22}+\sigma_{11} \cdot \sigma_{33}+\sigma_{22} \cdot \sigma_{33}-\sigma_{12}^{2}-\sigma_{13}^{2}-\sigma_{23}^{2} \\
& \mathrm{I}_{3}=\sigma_{11} \cdot \sigma_{22} \cdot \sigma_{33}-\sigma_{11} \cdot \sigma_{23}^{2}-\sigma_{22} \cdot \sigma_{13}^{2}-\sigma_{33} \cdot \sigma_{12}^{2}+2 \cdot \sigma_{12} \cdot \sigma_{13} \cdot \sigma_{23}
\end{aligned}
$$

Resolvendo-se a eq.(A.2) pode-se chegar a:

$$
\begin{aligned}
& Q_{0}=\frac{3 I_{2}-I_{1}^{2}}{9} \\
& R=\frac{-9 I_{1} I_{2}+27 I_{3}+2 I_{1}^{3}}{54} \\
& D=Q_{0}^{3}+R^{2} \\
& Q=\sqrt{-D}
\end{aligned}
$$$$
\theta=\operatorname{atan}\left(\frac{\mathrm{Q}}{\mathrm{R}}\right) \quad\left\{\begin{array}{l}
\text { Para } \mathrm{R}>0 \Rightarrow 0<\theta<\frac{\pi}{2} \\
\text { Para } \mathrm{R}<0 \Rightarrow \frac{\pi}{2}<\theta<\pi
\end{array}\right.
$$$$
\sigma_{1}=2 \sqrt{-Q_{0}} \cdot \cos \left(\frac{\theta}{3}\right)+\frac{l_{1}}{3}
$$$$
\sigma_{2}=2 \sqrt{-Q_{0}} \cdot \cos \left(\frac{2 \pi-\theta}{3}\right)+\frac{I_{1}}{3}
$$$$
\sigma_{3}=2 \sqrt{-Q_{0}} \cdot \cos \left(\frac{2 \pi+\theta}{3}\right)+\frac{I_{1}}{3}
$$

onde $\sigma_{1}, \sigma_{2}$ e $\sigma_{3}$ são as 3 tensões principais. 


\section{A.2 DETERMINAÇÃO DAS DIREÇÕES PRINCIPAIS}

A determinação das direções principais parte da solução do seguinte sistema de equações:

$$
\left[\begin{array}{ccc}
\left(\sigma_{11}-\sigma_{p}\right) & \sigma_{12} & \sigma_{13} \\
\sigma_{12} & \left(\sigma_{22}-\sigma_{p}\right) & \sigma_{23} \\
\sigma_{13} & \sigma_{23} & \left(\sigma_{33}-\sigma_{p}\right)
\end{array}\right] \cdot\left\{\begin{array}{l}
\ell_{p} \\
m_{p} \\
n_{p}
\end{array}\right\}=\left\{\begin{array}{l}
0 \\
0 \\
0
\end{array}\right\}
$$

onde p varia de 1 a 3 e $\ell$, m e n são as componentes do versor de cada direção principal.

Pelo desenvolvimento proposto no trabalho, supõem-se $\ell_{p}=0$, assim:

$$
\left[\begin{array}{cc}
\sigma_{12} & \sigma_{13} \\
\left(\sigma_{22}-\sigma_{p}\right) & \sigma_{23} \\
\sigma_{23} & \left(\sigma_{33}-\sigma_{p}\right)
\end{array}\right] \cdot\left\{\begin{array}{l}
m_{p} \\
n_{p}
\end{array}\right\}=\left\{\begin{array}{l}
0 \\
0 \\
0
\end{array}\right\}
$$

A propriedade da eq.(A.16) abaixo mostra que se $\ell_{p}=0$, a solução $m_{p}=$ 0 e $n_{p}=0$ é impossível.

$$
\ell_{\mathrm{p}}^{2}+\mathrm{m}_{\mathrm{p}}^{2}+\mathrm{n}_{\mathrm{p}}^{2}=1
$$

Tomando-se duas equações quaisquer da eq.(A.15), pode-se escrever que:

$$
A_{2 \times 2} \cdot x_{2 \times 1}=0_{2 \times 1}
$$

onde:

$$
x_{2 \times 1}=\left\{\begin{array}{c}
m_{p} \\
n_{p}
\end{array}\right\}
$$


Como $x_{2 \times 1} \neq 0_{2 \times 1} \Rightarrow \operatorname{det}\left(A_{2 \times 2}\right)=0$.

Se o determinante $\operatorname{de} A\left(\operatorname{det}\left(A_{2 \times 2}\right)\right)$ for igual a 0 , então $\ell_{p}$ realmente é igual 0 ; senão $\ell_{\mathrm{p}}$ é diferente de 0 .

Admitindo-se que $\ell_{p}$ é realmente igual a 0 , apenas um dos 3 casos pode acontecer:

i) $\ell_{p}=0\left(\operatorname{det}\left(A_{2 \times 2}\right)=0\right)$ e $m_{p}=0 \Rightarrow n_{p}=1$

$$
\left\{\begin{array}{c}
\sigma_{13} \\
\sigma_{23} \\
\sigma_{33}-\sigma_{p}
\end{array}\right\}=\left\{\begin{array}{l}
0 \\
0 \\
0
\end{array}\right\} \quad \Rightarrow \quad \begin{aligned}
& \sigma_{13}=\sigma_{23}=0 \mathrm{e} \\
& \sigma_{33}=\sigma_{\mathrm{p}}
\end{aligned}
$$

Ou seja, pode-se dizer simplesmente que, se $\ell_{p}=0$ e $\sigma_{p}=\sigma_{33} \Rightarrow m_{p}=0$ e $n_{p}=1$.

ii) $\ell_{p}=0\left(\operatorname{det}\left(A_{2 \times 2}\right)=0\right)$ e $n_{p}=0 \Rightarrow m_{p}=1$

$$
\left\{\begin{array}{c}
\sigma_{12} \\
\sigma_{22}-\sigma_{\mathrm{p}} \\
\sigma_{23}
\end{array}\right\}=\left\{\begin{array}{l}
0 \\
0 \\
0
\end{array}\right\} \quad \Rightarrow \quad \begin{aligned}
& \sigma_{12}=\sigma_{23}=0 \mathrm{e} \\
& \sigma_{22}=\sigma_{\mathrm{p}}
\end{aligned}
$$

Da mesma forma, se $\ell_{p}=0$ e $\sigma_{p}=\sigma_{22} \Rightarrow n_{p}=0$ e $m_{p}=1$.

iii) $m_{p} \neq 0$ e $n_{p} \neq 0$

Se ambos $m_{p}$ e $n_{p}$ são diferentes de 0 , ou seja, $\sigma_{p} \neq \sigma_{22}$ e $\sigma_{p} \neq \sigma_{33}$, então se pode supor $m_{p}=1$. Substituindo-se essa igualdade em qualquer uma das equações do sistema da eq.(A.15), pode-se obter um valor para $n_{p}$. Para que o vetor $(\ell, m, n)$ seja o versor que se estava procurando determinar, basta que esse seja normalizado: 
$\mathrm{N}_{\mathrm{p}}=\sqrt{\ell_{\mathrm{p}}+\mathrm{m}_{\mathrm{p}}+\mathrm{n}_{\mathrm{p}}}$

$\left\{\begin{array}{l}\ell_{\mathrm{p}}:=\frac{\ell_{\mathrm{p}}}{\mathrm{N}_{\mathrm{p}}} \\ \mathrm{m}_{\mathrm{p}}:=\frac{\mathrm{m}_{\mathrm{p}}}{\mathrm{N}_{\mathrm{p}}} \\ \mathrm{n}_{\mathrm{p}}:=\frac{\mathrm{n}_{\mathrm{p}}}{\mathrm{N}_{\mathrm{p}}}\end{array}\right.$

O desenvolvimento apresentado da eq.(A.15) à eq.(A.20) foi considerando que $\ell_{p}=0\left(\operatorname{det}\left(A_{2 \times 2}\right)=0\right)$, mas se esta igualdade não for satisfeita, então se pode supor $\ell_{p}=1$; assim, a eq.(A.14) pode ser escrita como:

$\left[\begin{array}{ccc}\left(\sigma_{11}-\sigma_{p}\right) & \sigma_{12} & \sigma_{13} \\ \sigma_{12} & \left(\sigma_{22}-\sigma_{p}\right) & \sigma_{23} \\ \sigma_{13} & \sigma_{23} & \left(\sigma_{33}-\sigma_{p}\right)\end{array}\right] \cdot\left\{\begin{array}{l}1 \\ m_{p} \\ n_{p}\end{array}\right\}=\left\{\begin{array}{l}0 \\ 0 \\ 0\end{array}\right\}$

ou

$$
\left[\begin{array}{cc}
\sigma_{12} & \sigma_{13} \\
\left(\sigma_{22}-\sigma_{p}\right) & \sigma_{23} \\
\sigma_{23} & \left(\sigma_{33}-\sigma_{p}\right)
\end{array}\right] \cdot\left\{\begin{array}{l}
m_{p} \\
n_{p}
\end{array}\right\}=\left\{\begin{array}{c}
\sigma_{p}-\sigma_{11} \\
-\sigma_{12} \\
-\sigma_{13}
\end{array}\right\}
$$

Tomando-se duas equações quaisquer da eq.(A.22), pode-se escrever:

$$
A_{2 \times 2} \cdot x_{2 \times 1}=y_{2 \times 1}
$$

Para encontrar o versor $(\ell, m, n)$ basta resolver o sistema da eq.(A.23) (apenas um é necessário) e normalizar o vetor encontrado utilizando as eq.(A.19) e (A.20).

Com o objetivo de facilitar o entendimento do desenvolvimento apresentado anteriormente, apresenta-se o algoritmo utilizado para a determinação das direções principais. 


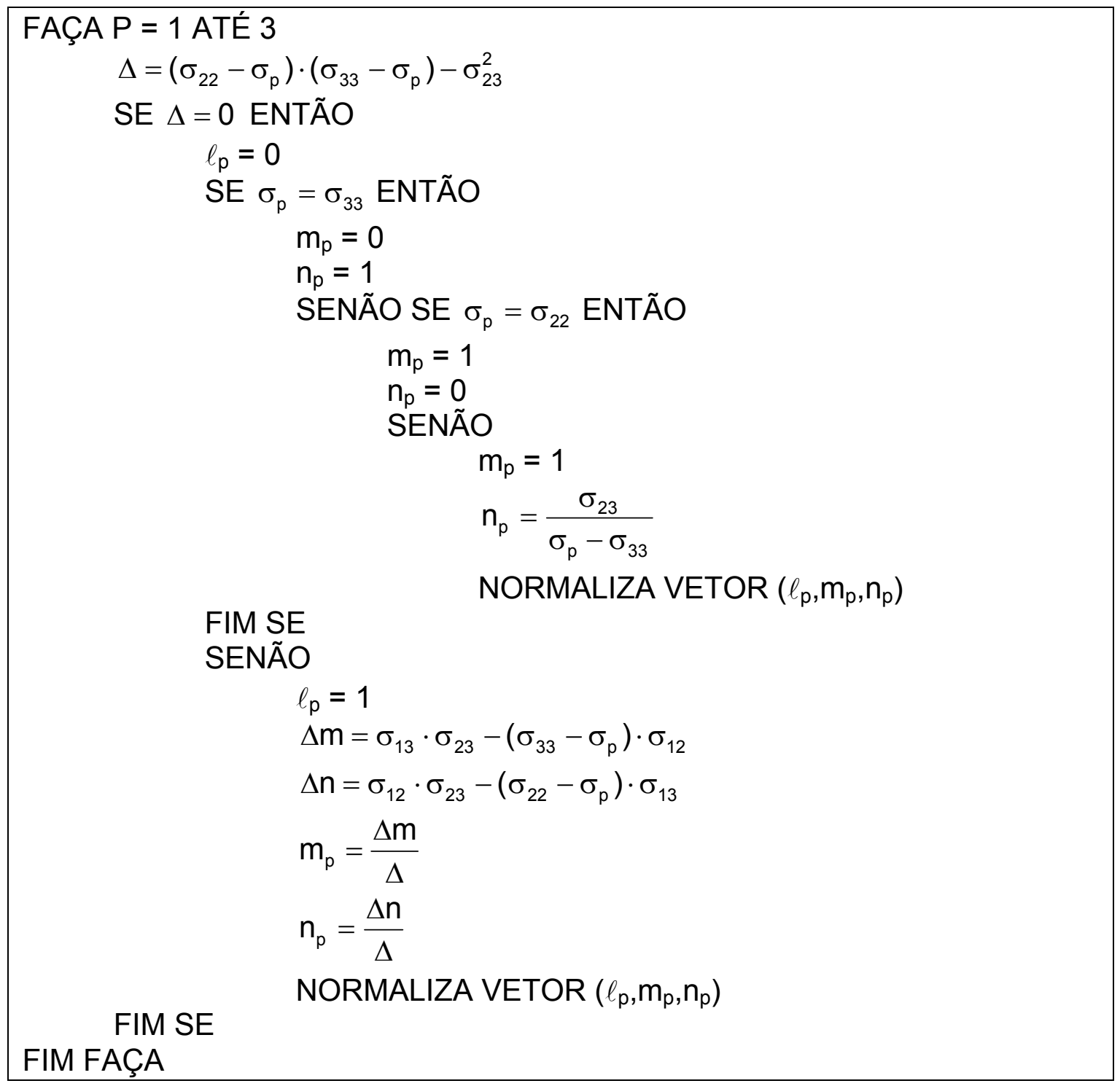

Com relação ao algoritmo apresentado acima, faz-se 2 observações: a primeira é que no cálculo de $\Delta$, escolheu-se as duas últimas equações; a segunda observação é que se utilizou o método de Krammer para a solução do sistema de equações (se $\ell_{p} \neq 0$ ).

\section{A.3 TRANSFORMAÇÃO DE COORDENADAS}

De posse das direções principais, pode-se transformar um vetor do espaço das tensões principais de volta para o espaço das tensões cartesianas utilizando-se a eq.(A.24) abaixo. 
$\left[\begin{array}{lll}z_{11} & z_{12} & z_{13} \\ z_{21} & z_{22} & z_{23} \\ z_{31} & z_{32} & z_{33}\end{array}\right]=\left[\begin{array}{ccc}\ell_{1} & \ell_{2} & \ell_{3} \\ m_{1} & m_{2} & m_{3} \\ n_{1} & n_{2} & n_{3}\end{array}\right] \cdot\left[\begin{array}{ccc}z_{1} & 0 & 0 \\ 0 & z_{2} & 0 \\ 0 & 0 & z_{3}\end{array}\right] \cdot\left[\begin{array}{ccc}\ell_{1} & m_{1} & n_{1} \\ \ell_{2} & m_{2} & n_{2} \\ \ell_{3} & m_{3} & n_{3}\end{array}\right]$

Tomando-se partido da simetria do tensor escrito em coordenadas cartesianas, pode-se simplesmente escrever:

$$
\begin{aligned}
& z_{11}=\ell_{1}^{2} \cdot z_{1}+\ell_{2}^{2} \cdot z_{2}+\ell_{3}^{2} \cdot z_{3} \\
& z_{12}=\ell_{1} \cdot m_{1} \cdot z_{1}+\ell_{2} \cdot m_{2} \cdot z_{2}+\ell_{3} \cdot m_{3} \cdot z_{3} \\
& z_{13}=\ell_{1} \cdot n_{1} \cdot z_{1}+\ell_{2} \cdot n_{2} \cdot z_{2}+\ell_{3} \cdot n_{3} \cdot z_{3} \\
& z_{22}=m_{1}^{2} \cdot z_{1}+m_{2}^{2} \cdot z_{2}+m_{3}^{2} \cdot z_{3} \\
& z_{23}=m_{1} \cdot n_{1} \cdot z_{1}+m_{2} \cdot n_{2} \cdot z_{2}+m_{3} \cdot n_{3} \cdot z_{3} \\
& z_{33}=n_{1}^{2} \cdot z_{1}+n_{2}^{2} \cdot z_{2}+n_{3}^{2} \cdot z_{3}
\end{aligned}
$$




\section{APÊNDICE B - Dedução da constante $k$}

Considerando-se um corpo tridimensional infinitesimal solicitado apenas por uma tensão normal em uma das 3 direções cartesianas (ex.: direção 1) (ver figura B.1), pode-se escrever a seguinte relação para as deformações elásticas que aparecem, normais às 3 direções:

$$
\left\{\begin{array}{l}
\varepsilon_{1} \\
\varepsilon_{2} \\
\varepsilon_{3}
\end{array}\right\}=\frac{1}{\mathrm{E}} \cdot\left[\begin{array}{ccc}
1 & -v & -v \\
-v & 1 & -v \\
-v & -v & 1
\end{array}\right] \cdot\left\{\begin{array}{c}
\sigma_{1} \\
0 \\
0
\end{array}\right\}
$$

ou simplesmente:

$$
\begin{aligned}
& \varepsilon_{1}=\left(\frac{1}{E}\right) \sigma_{1} \\
& \varepsilon_{2}=\left(\frac{-v}{E}\right) \sigma_{1} \\
& \varepsilon_{3}=\left(\frac{-v}{E}\right) \sigma_{1}
\end{aligned}
$$

A norma Euclidiana de $\varepsilon$ pode ser calculada pela eq.(B.5) abaixo:

$$
|\varepsilon|=\sqrt{\varepsilon_{1}^{2}+\varepsilon_{2}^{2}+\varepsilon_{3}^{2}}
$$




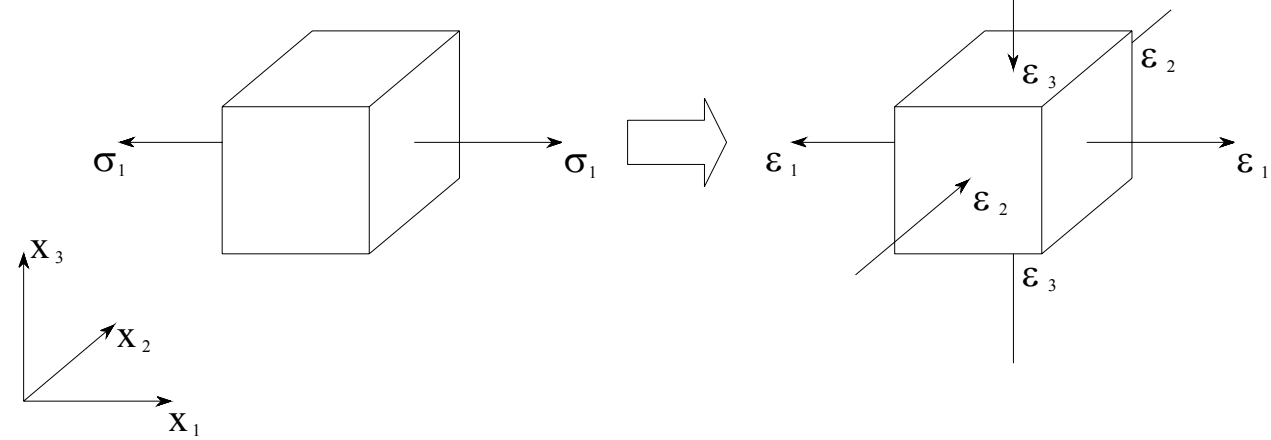

Figura B.1 - Carga normal aplicada a um corpo tridimensional infinitesimal

Substituindo-se as eq.(B.2), (B.3) e (B.4) na eq.(B.5), tem-se:

$|\varepsilon|=\sqrt{\sigma_{1}^{2} \cdot\left(\frac{1}{E^{2}}+\frac{v^{2}}{E^{2}}+\frac{v^{2}}{E^{2}}\right)}$

$|\varepsilon|=\sqrt{\frac{\sigma_{1}^{2}}{E^{2}} \cdot\left(1+2 v^{2}\right)}$

$|\varepsilon|=\frac{\sqrt{1+2 v^{2}}}{E} \cdot \sqrt{\sigma_{1}^{2}}$

$|\varepsilon|=\frac{\sqrt{1+2 v^{2}}}{E} \cdot|\sigma|$

$|\sigma|=\frac{E}{\sqrt{1+2 v^{2}}} \cdot|\varepsilon|$

Sabe-se que, para o caso uniaxial,

$\left|\sigma_{1}\right|=\mathrm{E} \cdot\left|\varepsilon_{1}\right|$

Assim, para que a norma referente ao estado tridimensional $|\varepsilon|$ seja igual à norma referente ao caso uniaxial $\left|\varepsilon_{1}\right|$, tem-se: 
$\left|\varepsilon_{1}\right|=\frac{1}{\sqrt{1+2 v^{2}}} \cdot|\varepsilon|=\mathrm{k}(v) \cdot|\varepsilon|$

onde:

$k(v)=\frac{1}{\sqrt{1+2 v^{2}}}$

Observa-se que, levando-se em consideração que o material seja incompressível $(v=0,5)$ o valor de $\mathrm{k}$ é de $\sqrt{\frac{2}{3}}$, tão conhecido em análises nãolineares físicas.

$$
k(0,5)=\frac{1}{\sqrt{1+2(0,5)^{2}}}=\frac{1}{\sqrt{1+2 \cdot 0,25}}=\frac{1}{\sqrt{1+0,5}}=\frac{1}{\sqrt{1,5}}=\frac{1}{\sqrt{3 / 2}}=\sqrt{\frac{2}{3}}
$$




\section{APÊNDICE C - Algoritmo para o cálculo da matriz de rigidez em coordenadas locais do elemento finito de placa DKT}




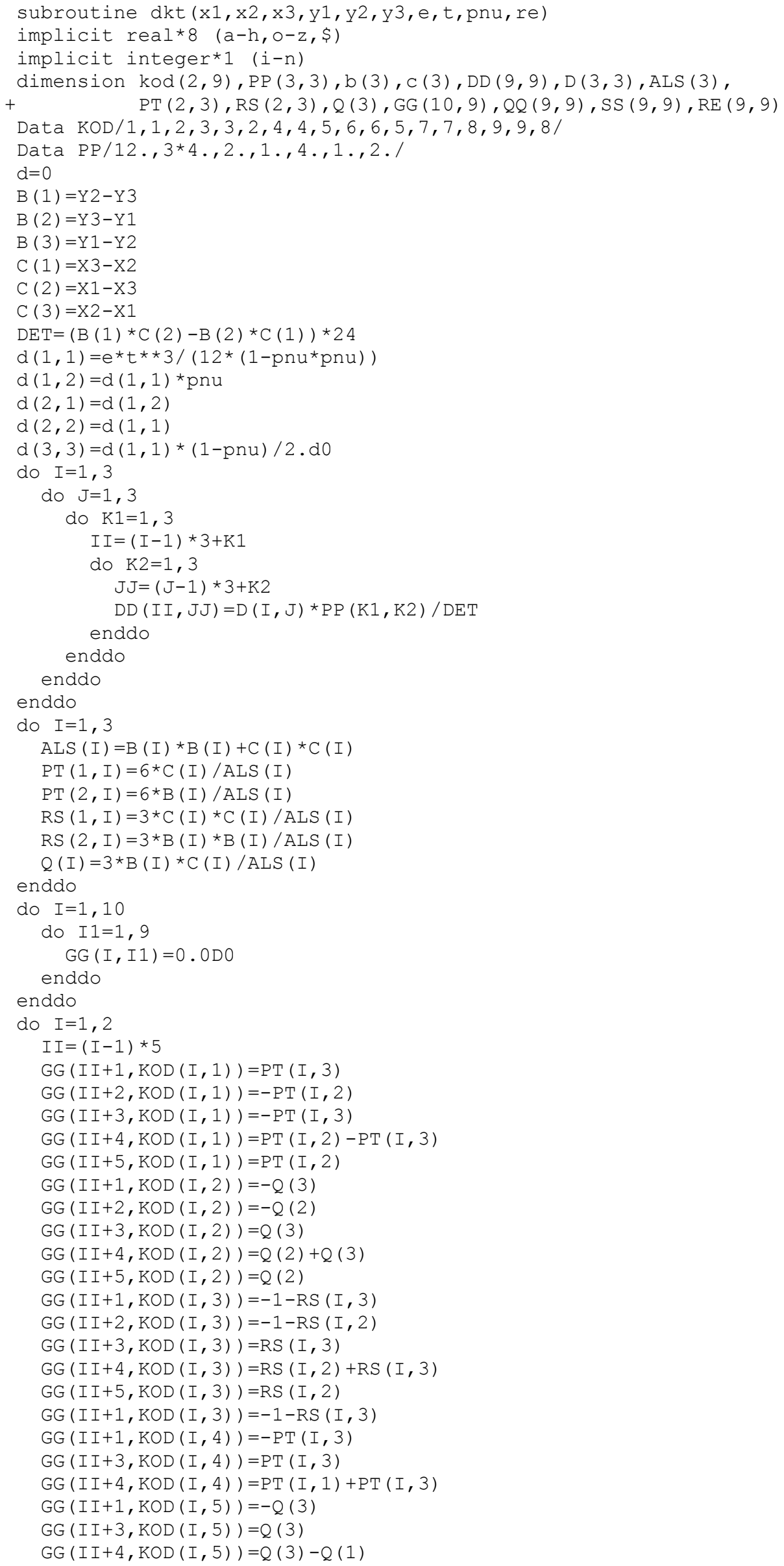




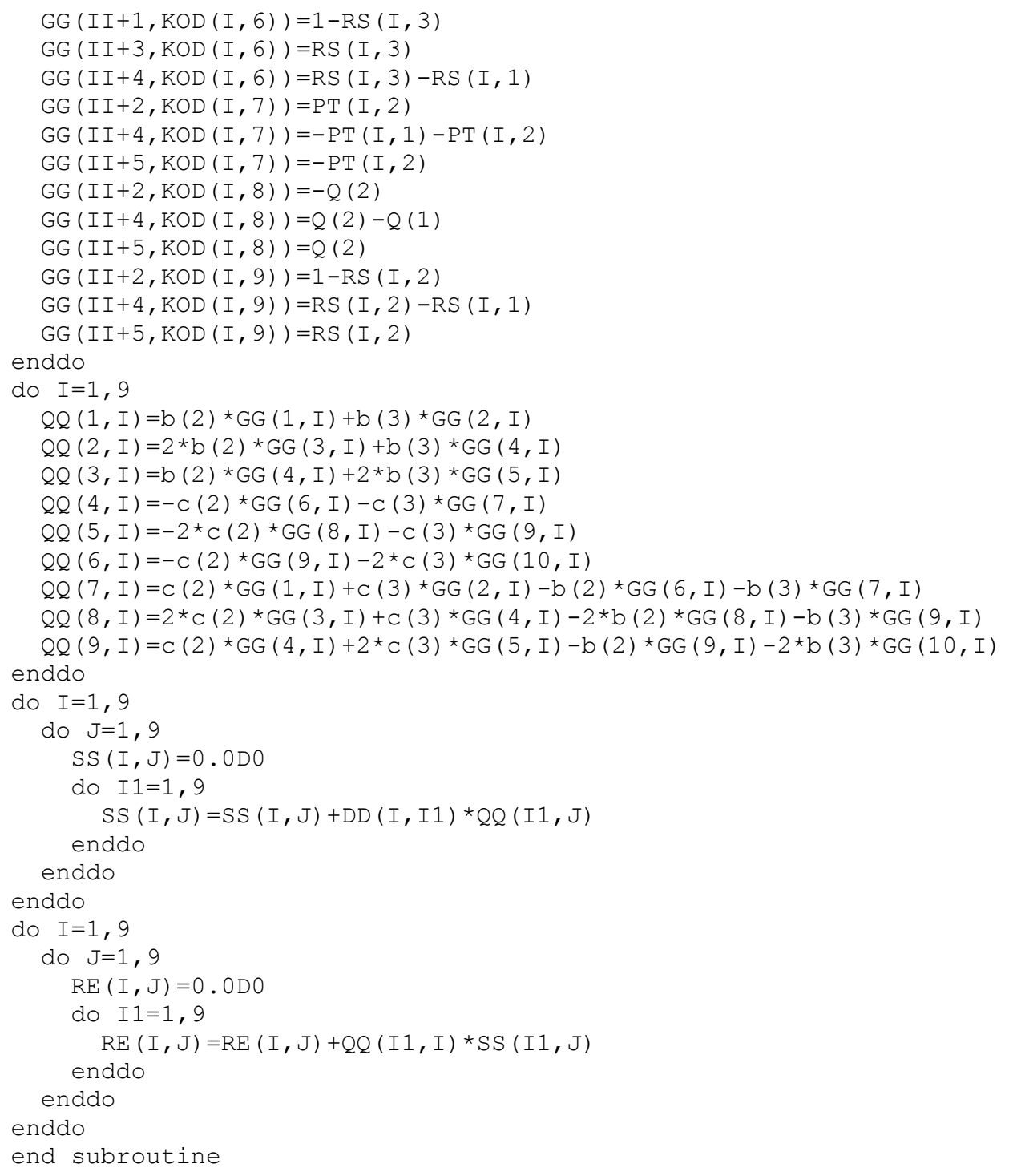

onde:

e $\quad$ - módulo de elasticidade;

t - espessura;

pnu - coeficiente de Poisson;

re $\quad$ - matriz de rigidez do elemento em coordenadas locais. 


\section{APÊNDICE D - Equacionamento para critério de plastificação de Drucker Prager}

No capítulo 4, para fins de equacionamento, considerou-se a superfície de plastificação como sendo a de von Mises. No presente apêndice, apresentam-se, de forma bastante direta, as equações pertinentes ao critério de plastificação de Drucker Prager, também implementado pelo autor no presente trabalho. Como referência para este apêndice, cita-se o trabalho de Mesquita (2002).

Sendo assim, parte-se da equação da superfície de plastificação do critério abordado neste apêndice.

$$
f(\sigma, \kappa)=\bar{f}(\sigma)-\bar{\sigma}(\kappa)=0
$$

onde:

$$
\begin{array}{ll}
\sigma & - \text { representa o estado de tensão; } \\
\kappa & - \text { o parâmetro de encruamento; } \\
\bar{f} & - \text { uma função do estado de tensão } \sigma_{\mathrm{ij}} ; \\
\bar{\sigma} & - \text { uma tensão equivalente. }
\end{array}
$$

Para o critério de plastificação de Druker Prager:

$$
f=\bar{f}(\sigma)-\sigma_{0}-\frac{H}{\sqrt{3}} \kappa
$$

onde $\mathrm{H}$ é o módulo plástico e: 
$\sigma_{0}=\frac{6 c \cos \phi}{\sqrt{3}(3+\sin a l \cdot \operatorname{sen} \phi)}$

sendo 'c' a coesão do material, ' $\phi$ ' o ângulo de atrito interno e 'sinal' dado por:

$\sin a l=\left\{\begin{array}{l}1 \text { para superfície interna a Mohr Coulomb } \\ -1 \text { para superfície interna a Mohr Coulomb }\end{array}\right.$

e

$\bar{f}(\sigma)=\|\sigma\|+\alpha \mu^{\top} \sigma$

com:

$\|\sigma\|=\frac{1}{\sqrt{3}} \sqrt{\sigma_{1}^{2}+\sigma_{2}^{2}+\sigma_{3}^{2}-\sigma_{1} \sigma_{2}-\sigma_{1} \sigma_{3}-\sigma_{2} \sigma_{3}}$

$\alpha=\frac{2 \operatorname{sen} \phi}{\sqrt{3}(3+\sin a l \cdot \operatorname{sen} \phi)}$

$\mu^{\top} \sigma=\sigma_{1}+\sigma_{2}+\sigma_{3}$

Substituindo a eq.(D.4) na eq.(D.2), pode-se escrever:

$f=\|\sigma\|+\alpha \mu^{\top} \sigma-\sigma_{0}-\frac{H}{\sqrt{3}} \kappa$

Por fim, apresenta-se a expressão para $\Delta \lambda$ :

$$
\Delta \lambda=\frac{f^{\text {ten }}}{\left(\frac{\left\|\sigma^{\text {ten }}\right\|+\alpha \mu^{\top} \sigma^{\text {ten }}}{\left|\left(C^{e}\right)^{-1} \cdot \sigma^{\text {ten }}\right|}+\frac{H}{\sqrt{3+6 v^{2}}}\right)}
$$


Uma última observação precisa ser feita: as constantes ' $c$ ' e ' $\phi$ ' também podem ser escritas em função das tensões limites de compressão $\left(\sigma_{c}\right)$ e tração $\left(\sigma_{t}\right)$ do material.

Para superfície interna a Mohr Coulomb:

$$
\begin{aligned}
& \text { sinal }=+1 \\
& \operatorname{sen} \phi=\frac{3\left(\sigma_{c}-\sigma_{t}\right)}{\sigma_{c}+3 \sigma_{t}} \\
& \text { C }=\frac{2 \sigma_{c} \cdot \sigma_{t}}{\cos \phi\left(\sigma_{c}+3 \sigma_{t}\right)}
\end{aligned}
$$

Para superfície externa a Mohr Coulomb:

$$
\begin{aligned}
& \text { sinal }=-1 \\
& \operatorname{sen} \phi=\frac{3\left(\sigma_{c}-\sigma_{t}\right)}{3 \sigma_{c}+\sigma_{t}} \\
& \text { c }=\frac{2 \sigma_{c} \cdot \sigma_{t}}{\cos \phi\left(3 \sigma_{c}+\sigma_{t}\right)}
\end{aligned}
$$

